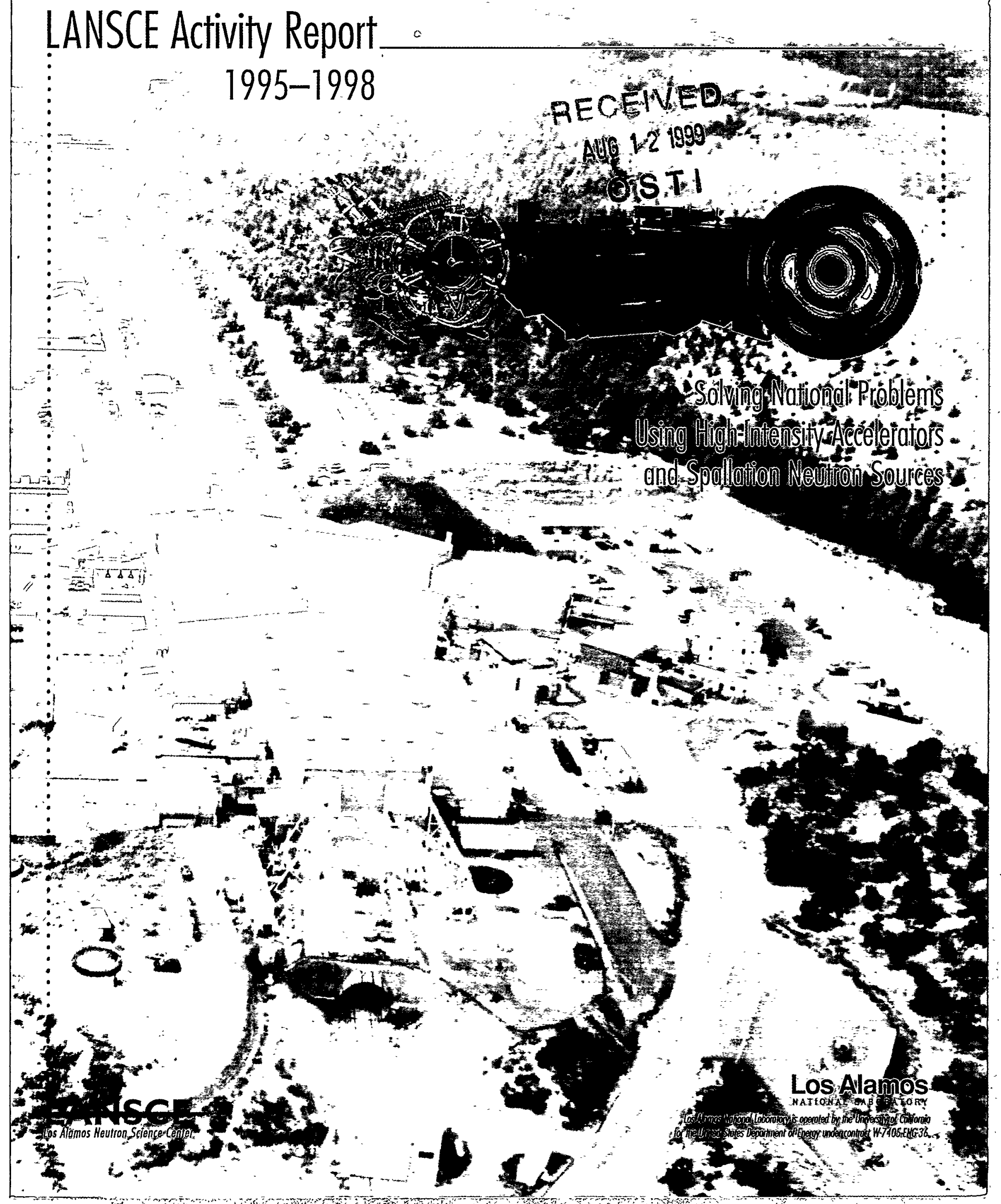


Managing Editor: Amy Robinson

Art Director and Design: Garth Tietjen

Composition: Barbara Maes

Illustration: Barbara Maes and Garth Tietjen

Assistant Managing Editor: Sue Harper

Editing: Mable Amador, Lynne Atencio, Sue Harper,

Ann Mauzy, Sheila Molony, Cynthia Phillips,

Amy Robinson, Jan Torline, and Kyle Wheeler.

The editors acknowledge the valuable contributions of

Laboratory subject matter experts who provided technical

information and guidance.

Technical Review Board: Audrey Archuleta, Gene Farnum,

Geoff Greene, Earl Hoffman, Ken Johnson, Paul Lewis,

Roger Pynn, Joyce Roberts, Stan Schriber, Steve Sterbenz,

Dan Strottman, and Steve Wender.

CD Integrated Design and Production: Jim Cruz,

Rick de Saussure, and Christoper J. Vigil.

Printing Coordination: CIC-9 Imaging Services

This is the first report in this series.

An Affirmative Action/Equal Opportunity Employer

This report was prepared as an account of work sponsored by an agency of the United States Government. Neither the Regents of the University of Califomia, the United States Government nor any agency thereof, nor any of their employees make any warranty, express or implied, or assume any legal liability or responsibility for the accuracy, completeness, or usefulness of any information, apparatus, product, or process disclosed, or represent that its use would not infringe privately owned rights. Reference herein to any specific commercial product, process, or service by trade name, trademark, manufacturer, or otherwise does not necessarily constitute or imply its endorsement, recommendation, or favoring by the Regents of the University of Califomia, the United States Government, or any agency thereof. Los Alamos National Laboratory strongly supports academic freedom and a researcher's right to publish; as an institution, however, the Laboratory does not endorse the viewpoint of a publication or guarantee its technical correctness.

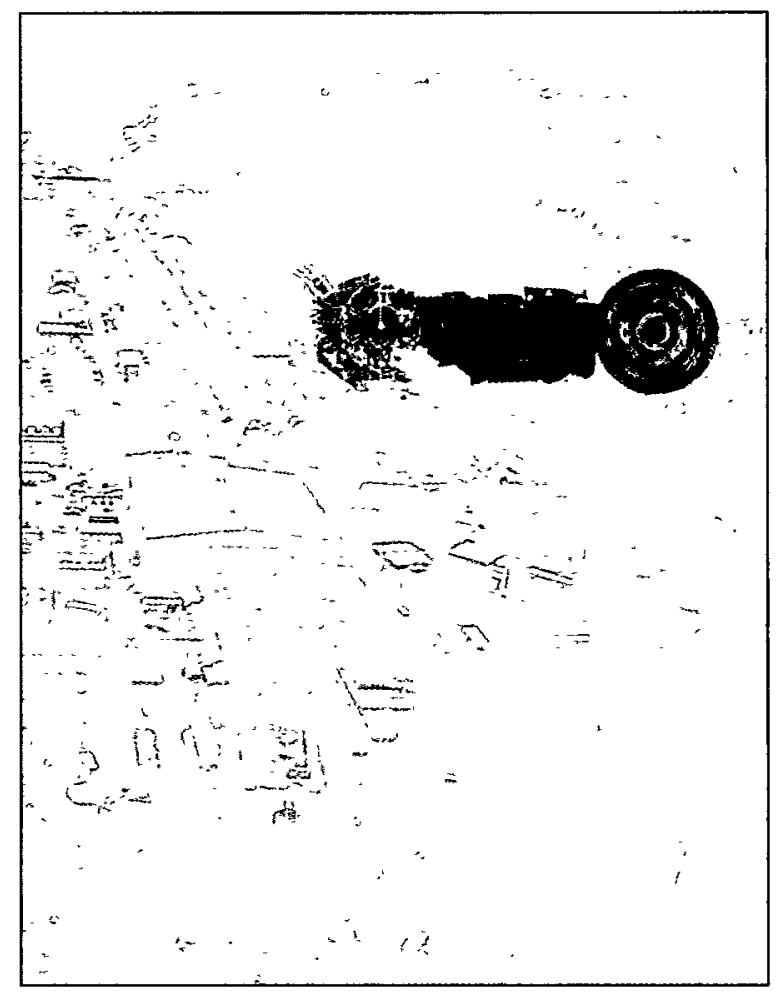

About the cover. The bockground is o photogroph of the Los Alamos Neution Science Center looking from the east to the west. The colloge image (from left to right) is a section of an accelerotor, the wide-angle voccum vessel on PHAROS, and a pole figure from texture dato token on HIPD. 


\section{DISCLAIMER}

This report was prepared as an account of work sponsored by an agency of the United States Government. Neither the United States Government nor any agency thereof, nor any of their employees, make any warranty, express or implied, or assumes any legal liability or responsibility for the accuracy, completeness, or usefulness of any information, apparatus, product, or process disclosed, or represents that its use would not infringe privately owned rights. Reference herein to any specific commercial product, process, or service by trade name, trademark, manufacturer, or otherwise does not necessarily constitute or imply its endorsement, recommendation, or favoring by the United States Government or any agency thereof. The views and opinions of authors expressed herein do not necessarily state or reflect those of the United States Government or any agency thereof. 


\section{DISCLAIMER}

Portions of this document may be illegible in electronic image products. Images are produced from the best available original document. 
LA-13547-SR

Status Report

Issued: February 1999

LANSCE Activity Report 1995-1998

Solving National Problems

Using High-Intensity Accelerators

and Spallation Neutron Sources

Compiled by

Audrey Archuleta

Gene Farnum

Geoff Greene

Sue Harper

Earl Hoffman

Ken Johnson

Paul Lewis

Barbara Maes

Roger Pynn

Joyce Roberts

Amy Robinson

Stan Schriber

Steve Sterbenz

Dan Strottman

Garth Tietjen

Steve Wender

\section{Los Alamos}

NATIONAL LABORATORY

Los Alamos, New Mexico 87545 


\title{
LANSCE Activity Report 1995-1998
}

Solving National Problems

Using High-Intensity Accelerators

and Spallation Neutron Sources

\begin{abstract}
The Los Alamos Neutron Science Center Activity Report describes scientific and technological progress and achievements in LANSCE Division during the period of 1995 to 1998 . This report includes a message from the Division Director, an overview of LANSCE, sponsor overviews, research highlights, advanced projects and facility upgrades achievements, experimental and user program accomplishments, news and events, and a list of publications. The research highlights cover the areas of condensed-matter science and engineering, accelerator science, nuclear science, and radiography. This report also contains a compact disk that includes an overview, the Activity Report itself, LANSCE operations progress reports for 1996 and 1997, experiment reports from LANSCE users, as well as a search capability.
\end{abstract}


Division Director's Introduction

Overview

Research Highhlights w

Condensed-Motter Science ond Engineering -

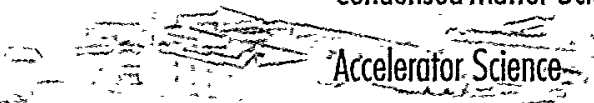

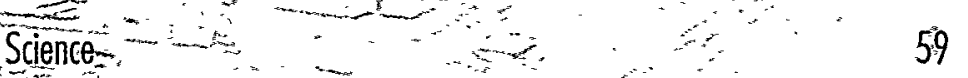

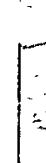

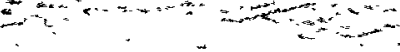

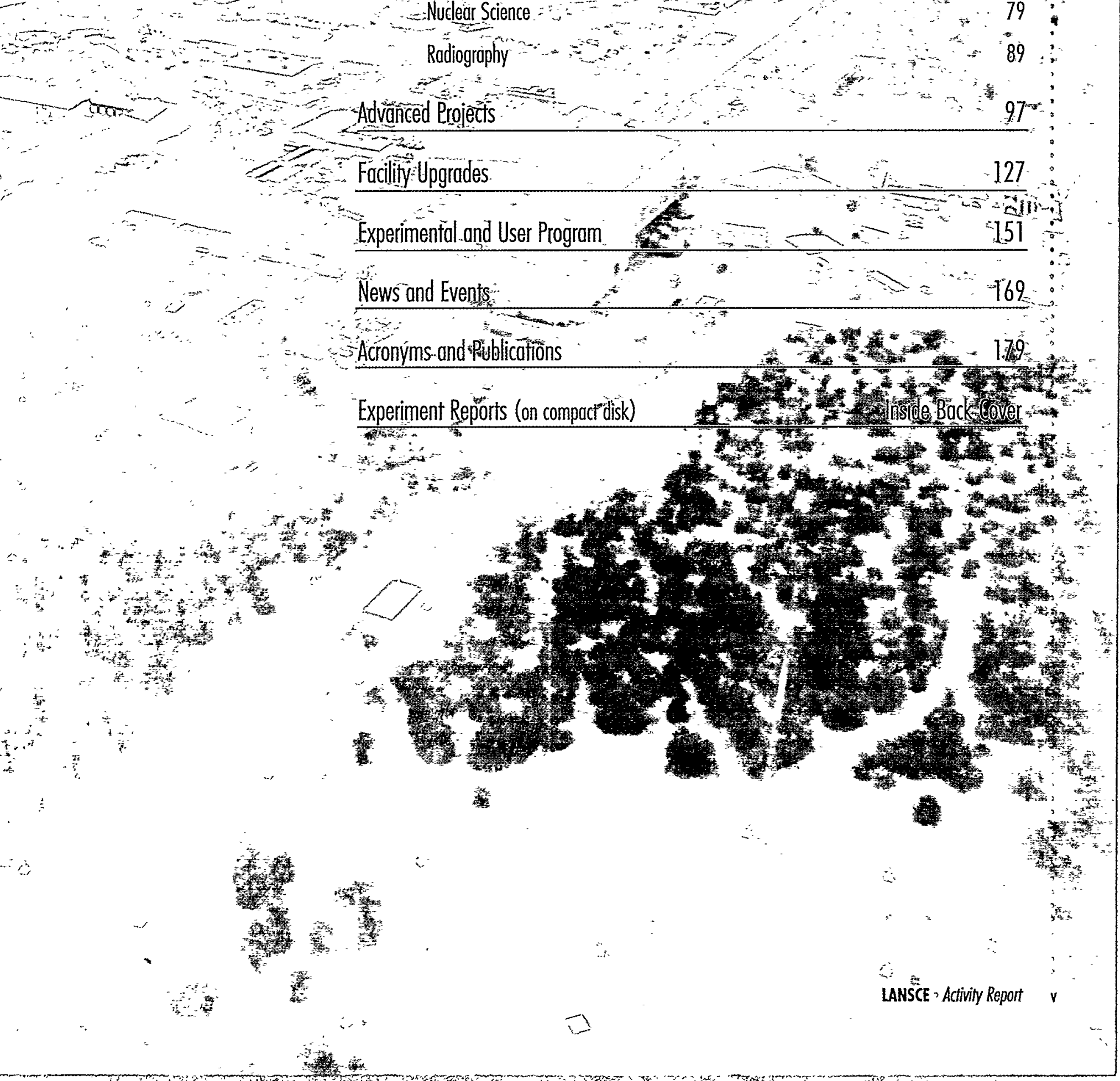





\section{A Division with a Mission}

Nearly 5 years ago, when John Browne became the Laboratory's Program Director for LANSCE and Energy Research (LER) programs, the task of redirecting the activity of the Technical Area 53 mesa from meson physics to neutron science seemed a daunting one. In those early days, John and I wrote a program plan that articulated the case for neutron science playing a central role in the U.S. Stockpile Stewardship program, while Stan Schriber-then Division Director of the Accelerator Operations and Technology (AOT) Division - worked hard to reduce the cost of running our accelerator.

The last 5 years have brought striking progress. The Accelerator Production of Tritium (APT) project has been funded and its low-energy demonstration accelerator (LEDA), constructed with strong support from LANSCE staff, will soon be operational at a beam current never before seen. In 1995 (actually on my 50th birthday!) a conference was held in Los Alamos to affirm the importance of neutrons to stockpile stewardship. Among the plenary speakers were leaders from each of the weapons laboratories as well as Vic Reis, Assistant Secretary of Energy for Defense Programs, and "Marcy" Greenwood, Associate Director of the White House Office of Science and Technology Policy. In the past few years, Chris Morris from the Physics Division and his collaborators have developed proton radiography to a point where it is seen as a viable technology for a future hydrotest facility; in our 1994 program plan it was barely mentioned.

The Department of Defense funded the LANSCE Reliability Improvement Project, which began in 1994 and finished in 1998 and has already resulted in greatly increased facility reliability - $84 \%$ in 1997 compared with $70 \%$ (and sometimes less!) in prior years. The Department of Energy (DOE) Offices of Defense Programs and Basic Energy Sciences have recognized their synergistic, longterm needs for neutrons and are collaborating on a project-the Short-Pulse Spallation Source Enhancement Project-that will increase beam current to the Lujan Center to $200 \mu \mathrm{A}$ and provide several new spectrometers for the U.S. scientific community. These spectrometers are being built by teams from universities, industries, and federal laboratories, following a new model that will likely form the basis for spectrometer construction at the Oak Ridge Spallation Neutron Source (SNS). As a full partner in the SNS project, LANSCE will be responsible for the construction of its linac and for implementing its control systems. Congress approved construction funding for the SNS project recently. In separate actions, Congress has appropriated funds to build a "spur" to the LANSCE linac that will enable production of radioisotopes vital to medical research and diagnostics, and also seems set to support new nuclear energy projects, including our Accelerator-Driven Transmutation of Waste (ATW) activity.

Based on these successes, the Laboratory decided last year to reorganize the LER Program Office and AOT Division into one organization-LANSCE Division-whose mission is to solve national problems using high-power accelerators and spallation neutron sources. Our principal sponsors are the DOE's Offices of Defense Programs and Basic Energy Sciences, which have synergistic, long-term needs for the neutron and accelerator science that is at the heart of LANSCE. Our customers are scientists from the broad U.S. community, many of whom access our facilities through a peer-review process to carry out experiments in defense and civilian science. As a National User Facility, LANSCE is part of a network of such facilities that are built and operated by the DOE for the nation's scientists. We play a role for Los Alamos that includes attracting talented scientists to the Laboratory and providing a tool for scientists in many fields with diverse scientific and technical interests. We are, as Vic Reis has said on several occasions, the "anchor store in the mall" that can bring new people to work on the challenging problems of maintaining a safe, secure, and reliable nuclear stockpile.

This activity report includes much more detail on the advances I have described, as well as descriptions of many other, but equally significant, contributions that comprise a fabric of scientific and technical contributions made over the past 5 years. I would like to take this opportunity to thank all of those who participated in these scientific and technical activities. You have much to be proud of.

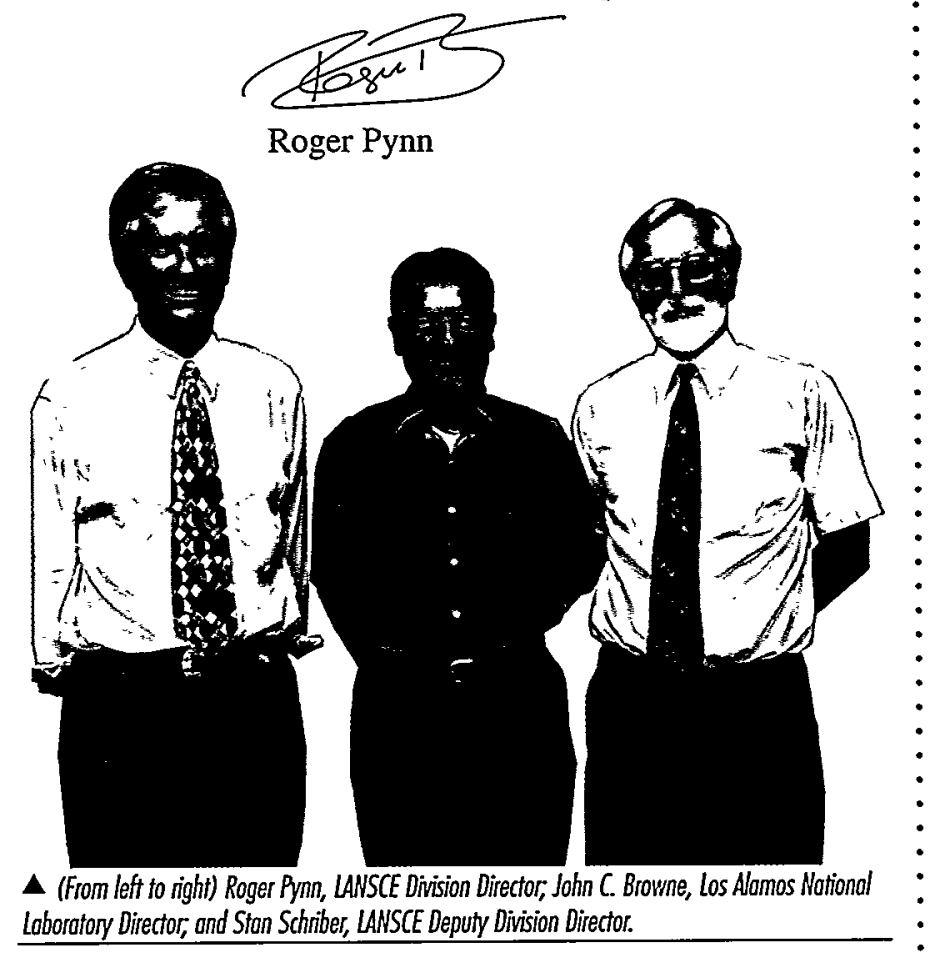

LANSCE - Activity Report 
- - 


\section{LANSCE at a Glânce}

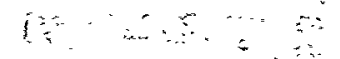

A National User Focility

Particle Beam Productioñ

Experimental Facilities

Programs and Projects

\section{ts}

$\because$

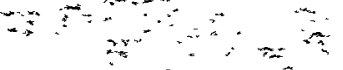

$\div=$
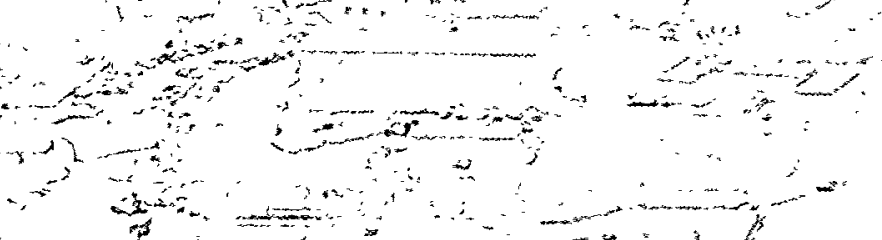

$$
\frac{-\infty=1}{-B}
$$

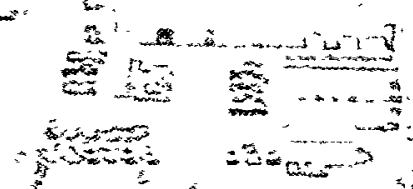

$+3 .=0$
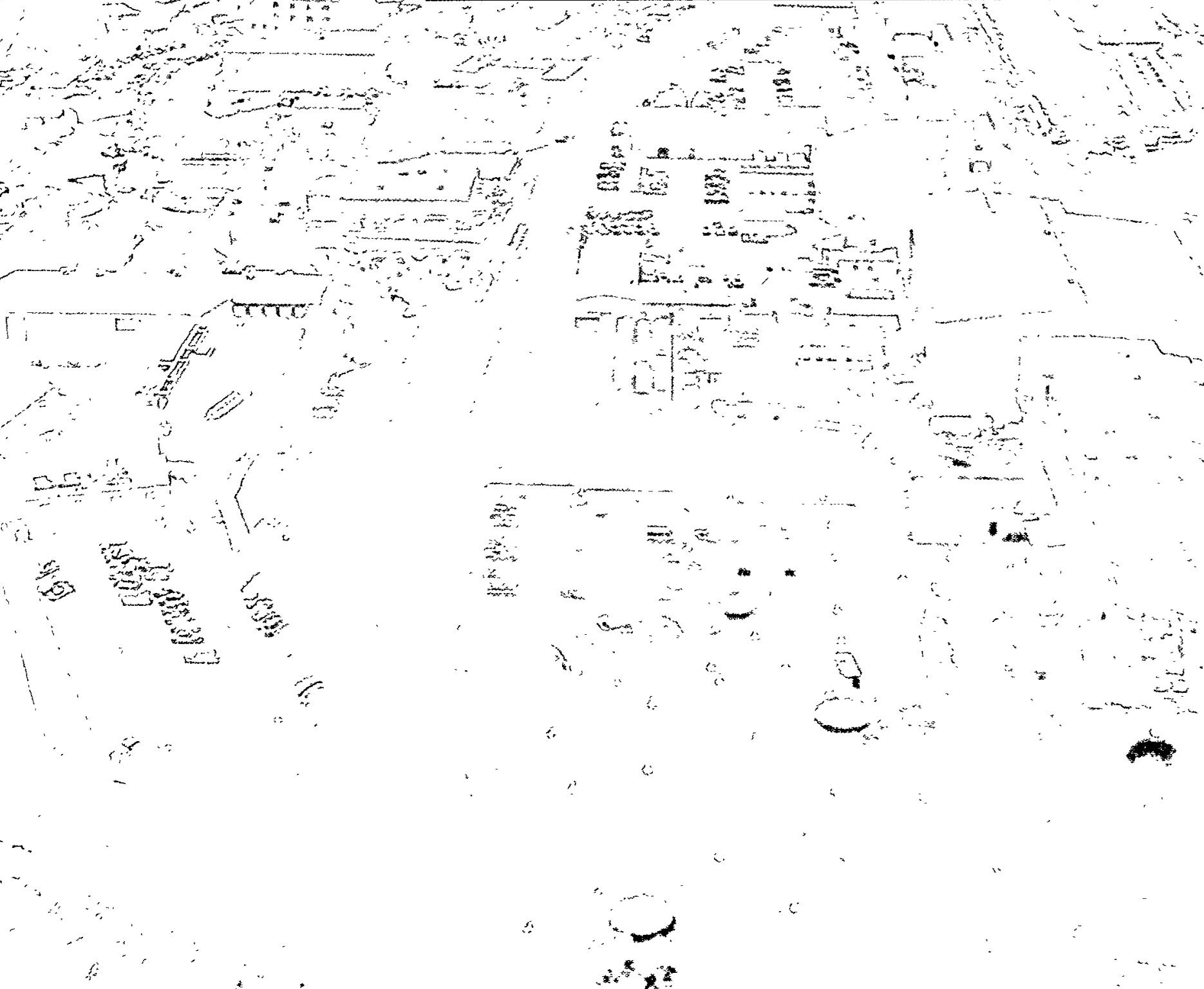


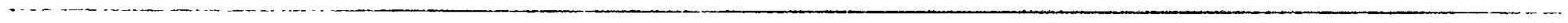




\section{LANSCE at a Glance}

In October 1995, with the closeout of the nuclear physics program and the increased national need for neutron research, Los Alamos National Laboratory refocused the mission of its linear accelerator complex. The new mission calls for the development and use of spallation neutron sources for research and applications. As a result, the accelerator complex formerly known as the Los Alamos Meson Physics Facility (LAMPF) is now called the Los Alamos Neutron Science Center (LANSCE). LANSCE, an acronym that formerly referred to the Manuel Lujan Jr. Neutron Scattering Center (now the Lujan Center), comprises a high-power, $800-\mathrm{MeV}$ proton linear accelerator (linac); a Proton Storage Ring (PSR); neutron production targets at the Lujan Center and the Weapons Neutron Research (WNR) facility; a proton radiography facility; a high-power materials irradiation area called the Los Alamos Spallation Radiation Effects Facility (LASREF); an isotope production facility (IPF); and a variety of spectrometers.

\section{LANSCE is ideal for research in radiography, condensed-matter science and engineering, accelerator science, and nuclear science.}

With the ability to produce protons and neutrons using the world's most powerful proton linac, LANSCE is ideal for research in radiography, condensed-matter science and engineering, accelerator science, and nuclear science. LANSCE uses these research capabilities to contribute to the Department of Energy (DOE) Stockpile Stewardship Program and to support a National User Program open to scientists from universities, industry, and federal laboratories.
Neutron or proton beams ot LANSCE can be used to...

- measure structures of materials, with particular advantage in mognetic materials and hydrogenous materials such as polymers, organic materials, and biological molecules

- measure density fluctuations of materials; for example, phonons, magnetic spin waves, and crystal field excitations

- charecterize otomic and magnetic densities close to interfaces and surfaces

- study cosmic radiation effects on integrated circuits

- meosure stroin and texture

- image hydrogenous materiol such as water or oil in parts or components

- image defects in light materials that lie inaccessibly beneath heavy moterials, well beyond the penetration range of xrays

- determine the atomic structure of materials under extreme conditions of pressure

- provide accurate, noninevasive dynamic measurements of velocity and temperature of materials in a shocked state

- produce neutron-poor radioisotopes

- radiograph systems that evolve rapidly in time

- measure nudear cross sections over a large ronge of energies

- perform fundamental physics experiments using ultracold neutrons

\section{A National User Facility}

As a national facility for defense and civilian research in radiography, nuclear science, and condensed-matter science, LANSCE hosts scientists from universities, industry, the Laboratory, and other research facilities from around the world. Scientists may apply for beam time by completing a proposal, which is subjected to appropriate peer review before beam time is granted. Once beam time is granted,

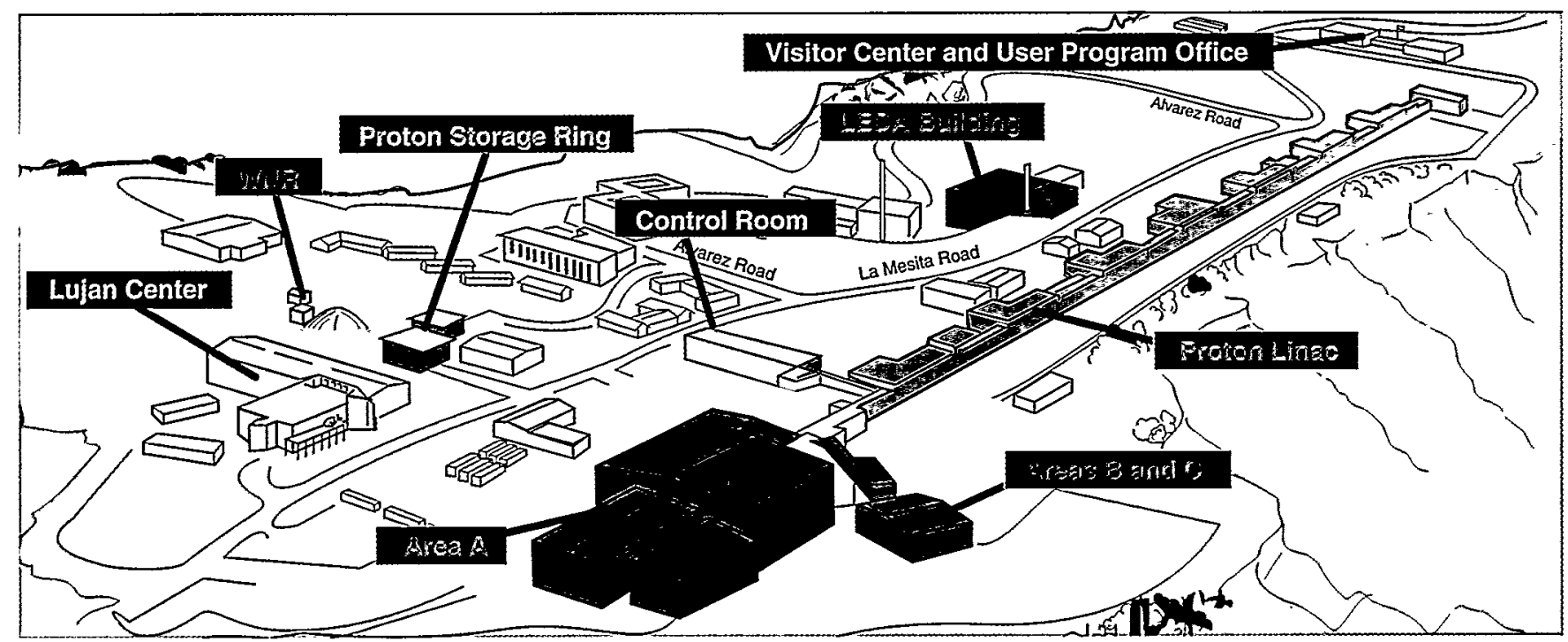

$\Delta$ The Los Alomos Neutron Science Center. 
the experiment is reviewed for technical and safety issues. Information about LANSCE and a blank proposal form are available on the World Wide Web at lansce.lanl.gov.

Scientists who use the facility are also encouraged to join the LANSCE User Group, which enables users to influence operating policies, future instrumentation, and other factors that impact users. Additional information about the LANSCE User Group is available in the "Experimental and User Program" section of this report.

\section{LANSCE hosts scientists from universities, industry, the Laboratory, and other research facilities from around the world.}

Financial support for the operation of LANSCE is provided by the DOE Office of Defense Programs (DP) and by the Office of Basic Energy Sciences (OBES). These two offices have long-term, synergistic needs for the neutrons that LANSCE provides.

\section{Particle Beam Production}

\section{High-Intensity Proton Linear Accelerator}

LANSCE is the world's most versatile spallation neutron source. Its high-intensity, 1-megawatt (MW) proton linear accelerator is the heart of many LANSCE activities.

To understand how the LANSCE linac works, remember that a hydrogen atom contains only one electron orbiting a nucleus with a single proton. When that electron is stripped off, a positive hydrogen ion $\left(\mathrm{H}^{+}\right)$, or proton, remains. Adding a second electron to a hydrogen atom produces a negative ion $\left(\mathrm{H}^{-}\right)$. The LANSCE high-intensity proton linac can simultaneously accelerate $\mathrm{H}^{+}$and $\mathrm{H}^{-}$ions to energies of 800-million-electron volts (MeV). The three-stage, half-milelong linac provides $\mathrm{H}^{+}$beam with an average current of up to
1 milliampere $(\mathrm{mA})$ at a repetition rate of 100 hertz $(\mathrm{Hz})$ and a peak of $17 \mathrm{~mA}$. The $\mathrm{H}^{-}$beam produced by the linac has an average current of up to 100 microamperes $(\mu \mathrm{A})$ at a repetition rate of $20 \mathrm{~Hz}$ and a peak of $20 \mathrm{~mA}$.

The first stage of the accelerator contains injector systems for each kind of particle $\left(\mathrm{H}^{+}\right.$and $\left.\mathrm{H}^{-}\right)$. Each injector system has a 750-kilo-electron volt (keV) Cockroft-Walton generator and an ion source. The two ion sources produce $\mathrm{H}^{+}$and $\mathrm{H}^{-}$particles inside high-voltage domes. When the particles leave the injectors, the achieved velocity is $4 \%$ of the speed of light. The two ion beams are merged, bunched, and matched into a $201.25-\mathrm{MHz}$ drift-tube linac for further acceleration to $100 \mathrm{MeV}$ (43\% of the speed of light). The third and longest stage of the accelerator ( 800 meters) is the side-coupled-cavity linac, where particles are accelerated to their final energy of $800 \mathrm{MeV}$ ( $84 \%$ of the speed of light).

The particle beams from the linac are separated and directed down three main beam lines leading to several experimental areas including Area A, Areas B and C, the Lujan Center, and the WNR facility. Operators can control the $\mathrm{H}^{+}$ and $\mathrm{H}^{-}$beams separately, allowing most experiments to run simultaneously.

\section{Proton Storage Ring}

The PSR converts $\mathrm{H}^{-}$linac macropulses that are approximately 750 milliseconds (ms) long into short $(0.27 \mu \mathrm{s})$, intense proton $\left(\mathrm{H}^{+}\right)$bursts that provide the capability for precise neutron time-of-flight measurements for a variety of experimental programs. $\mathrm{H}^{-}$beam is converted to $\mathrm{H}^{+}$by removing its two electrons using a stripper foil in the injector section of the PSR. The injected proton beam has a substructure of several thousand micropulses that arises from the acceleration process. The PSR collects these micropulses into one high-intensity pulse and ejects pulses toward the Lujan Center neutron target 20 times a second.

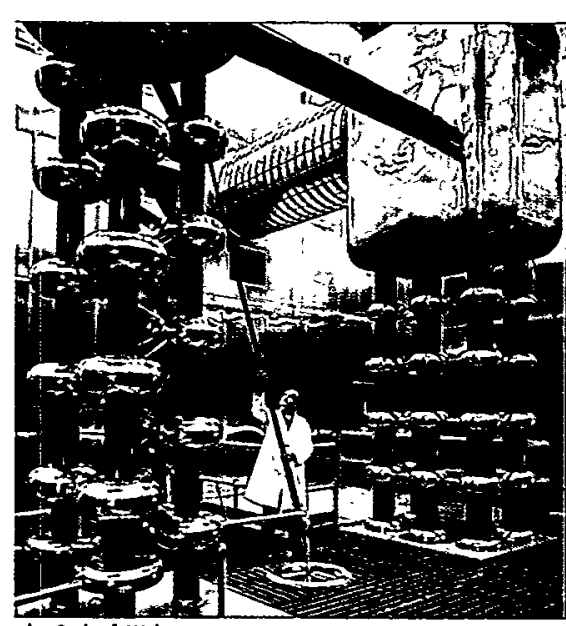

$\Delta$ Cockroft-Walton injector.

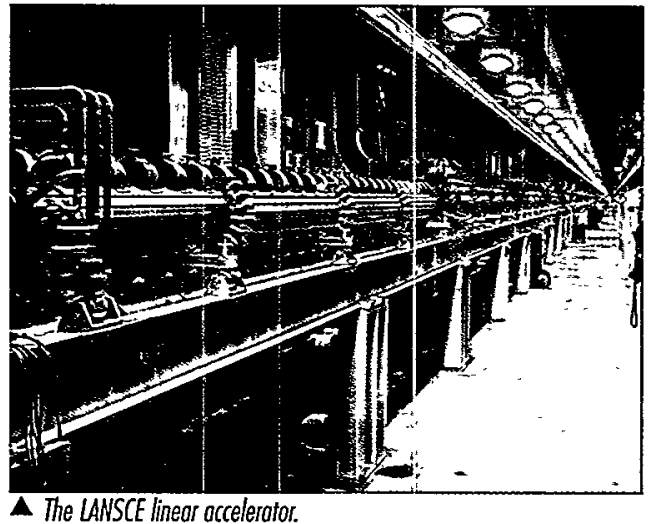

A The LANSCE lineor occelerator.

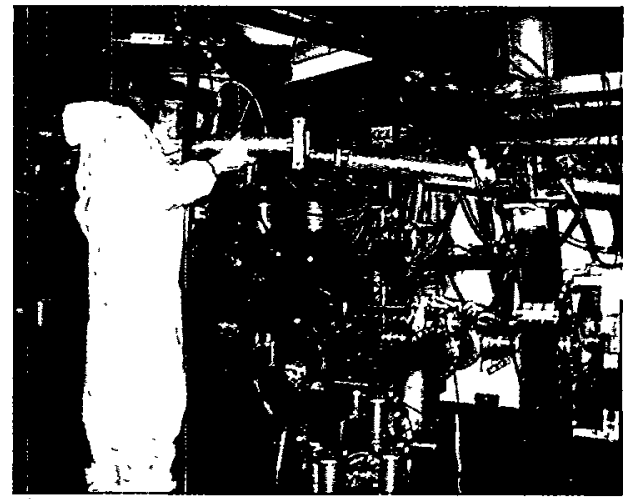

$\boldsymbol{A}$ Proton Storage Ring upgrade work.

8 LANSCE • Activity Report 


\section{Neutron Production}

The spallation process occurs when protons strike heavymetal targets such as tungsten and drive neutrons from the nuclei of the target atoms. For the $800-\mathrm{MeV}$ proton beams used at LANSCE, about 20 neutrons per proton are ejected. The short, highly intense bursts of spallation neutrons are used in neutron scattering and nuclear science experiments. The neutrons produced have energies up to hundreds of million electron volts, which are the right energies for nuclear physics experiments at WNR and some experiments at the Lujan Center.

\section{Moderators}

For the majority of the work in neutron scattering at the Lujan Center, the initial spallation neutron energies are too high, and correspondingly their wavelengths are too short for investigating condensed matter. For this reason the neutrons must be "cooled down" before being used for scattering experiments. This process is accomplished by allowing the neutrons to interact with a moderator-a material with a large scattering cross section, such as water or liquid hydrogen. Neutrons enter the moderator, which is placed close to the neutron source, and in a series of collisions, lose energy to moderator atoms. After a few tens of collisions, the neutron energies are similar to the thermal energy of the moderator. Thus a beam of thermal neutrons is emitted from the moderator with an average energy determined by the moderator temperature. The average energy of the neutrons from a water moderator is approximately 25 milli-electron volts ( $\mathrm{meV}$ ), while the average energy from the liquid hydrogen moderator at $20 \mathrm{~K}$ is approximately $5 \mathrm{meV}$. The energies and wavelengths (around 1.8 angstroms for $25 \mathrm{meV}$ ) of these neutrons match the excitation energies and the interatomic spacings of condensed matter and are thus very useful for neutron scattering experiments.

\section{Experimental Facilities}

\section{Manual Lujan Jr. Neutron Scattering Center}

At the Lujan Center, moderated spallation neutrons are used for condensed-matter science and engineering and nuclear science research. Because of the unique design of the split target and its flux-trap moderators, the Lujan Center yields a higher peak neutron flux than any other spallation neutron source used for condensed-matter science and engineering.

\section{Instruments and Flight Paths}

Most of the flight paths at the Lujan Center are equipped with spectrometers for determining the atomic, molecular, and magnetic structures as well as the vibrational and magnetic excitations of materials. Of the sixteen flight paths, which currently provide seventeen independent neutron beams, seven have instruments for condensed-matter science and engineering, three are used for nuclear science research, and the remainder are being instrumented.

\section{Weapons Neutron Research Facility}

At the WNR facility, high-energy, unmoderated neutrons and protons are used for basic and applied research in nuclear science and weapons-related measurements. The WNR facility consists of two target areas: Target 2 and Target 4, and their associated flight paths. The neutron beams produced at WNR complement those produced at the Lujan Center because they are of much higher energy and have shorter pulse duration. With both capabilities, LANSCE is able to deliver neutrons with energies ranging from small fractions of an electron volt to $800 \mathrm{MeV}$.

\section{Instruments and Flight Paths}

At Target 2, also known as the Blue Room, proton-induced reactions can be studied using the linac or the PSR proton beam. In addition, the Blue Room is used for a variety of proton irradiation experiments. This target consists of a low-background room with seven flight paths (one is unused). Experiments in the Blue Room can exploit the variable-energy feature of the linac using proton beams from 250 to $800 \mathrm{MeV}$.
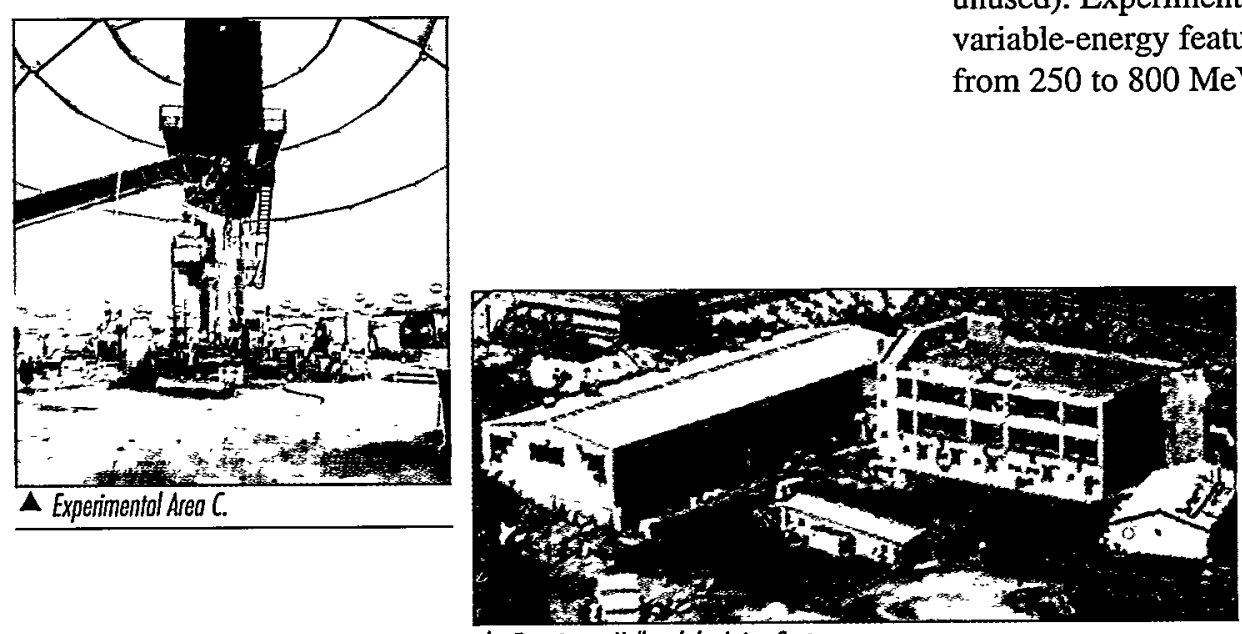

A Experiment Holl and the Lujon Center.

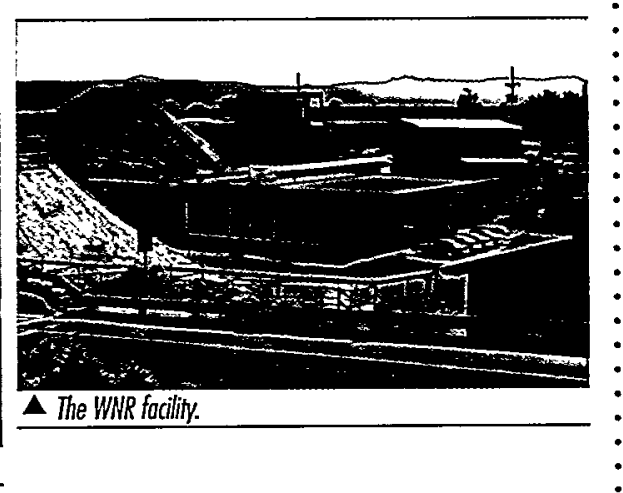

LANSCE - Activity Report 


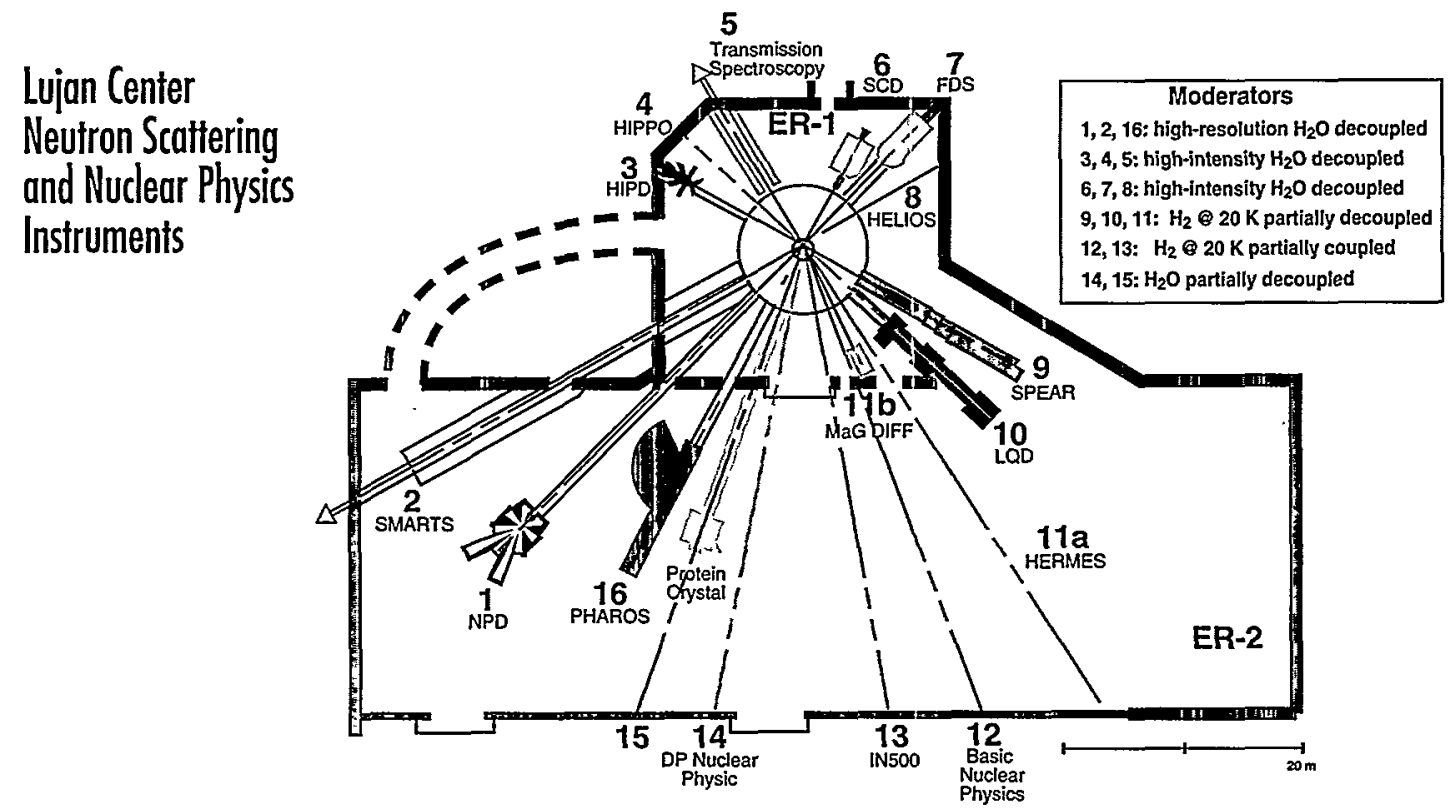

Neutron Powder Diffractometer (MPD) used for powder diffraction studies of strain distributions and refinement of structures of polycystalline materials. Mark Bourke $¥ 505-665-1386 ¥$ bourke@lanl.gov

2 Flight path used for nucleor physics experiments. During October 1998 through March 1999 , this flight path will be used to study neutron capture on small quantities of radioactive torgets. John Ullmann $¥ 505-667-2517$ \# vllmann@lanl.gov

IIEN, to be commissioned in 1999: Spectrometer for Moterials Research at Temperature and Siress (SMARTS) is a new neutron powder diffractometer instrument for strain measurements. Compared with NIPD (which will still be in operation), SMARTS will have a 30 times increase in count rate, an extensive array of in-silu copabilities for sample environments, the ability to make measurements on both smoll and very lorge samples, and easy access to and control of the sample position. Mark Bourke ¥505-665-1386 ¥ bourke@lanl.gov

High Intensity Powder Diffractometer (HIPD) used for pouder diffraction studies of texture, small somples, or polycrystolline materials subiected to pressures up to $30 \mathrm{GPa}$. Robert Von Dreele $¥ 505-667-3630 ¥$ vondreele@lanl.gov

NHEW, to be commissioned in 1999: High-Pressure and Preferred Orientation (HIPPO) diffractometer is a new high-intensity powder diffractometer for texture measurements. Compared with HIPD (which will still be in operation), HIPPO offers an increased number of detectors ( 10 to 20 times over current capability), induding backscaftering detectors, a better sample environment, and an order of magnilude increase in sample throughput. Kristin Bennett ¥ 505-665-4047 ¥ bennetf@lanl.gov

5 : $A$ generalpurpose flight path used to study the Doppler broodening of lowenergy neutron resonances resulting from environmental changes (i.e., temperature, pressure). Vincent Yuan $¥ 505$-667-3939 ¥vyuan@lanl.gov

6 Single-[rystal Diffractometer (SCD) used for measuring the atomic structure of single cystals and texture. Yusheng Zhoo ¥ 505-667-3886 ¥ yzhro@lanl.gov

7 Filter Difference Spectrometer (FDS) used for vibrational spectroscopy. Juergen Eckert $¥ 505$-665-2374 \# juergen@lanl.gov

Flight path will be the site of a new high-intensity chopper spectrometer, HELlOS. The delails of this project are currently in the planning stage.

Thom Hason, Oak Ridge National Laboratory ¥ 423-241-1499 ¥ moson!@ornl.gov Collin Broholm, Johns Hopkins University ¥410-516-7890¥ broholm@ihu.edu

Brent Fultz, California Institufe of Technology ¥ 626-395-2170 ¥btf@hyperfine.callech.edu

Surface Profile Anolysis Reflectometer (SPEAR) used for determining nuclear and mognetic density profiles near solid or liquid interfoces. Unpolarized neutron beam: Gregory Smith $¥ 505$-665-2842 $¥$ gsmith@lanl.gov Polarized neutron beam: Michael Fitzsimmons $¥ 505-665-4045 ¥$ fizz@lanl.gov
Low-Q Difffoctometer (LOD) used for smallongle scottering studies of biological entities, polymers, colloids, gels, porous materiols, oggregates, and metallic alloys. Rex Hjelm $¥ 505$-665-2372 ¥ hjelm@lanl.gov Jaroslow Majewski 505-667-8840 ¥ jorek@lonl.gov

DTFFlight path used for cold neutron radiography experiments, $n+p \rightarrow \delta+\gamma$, and experiments to measure the neutron electric dipole moment. Thomas MeDonald $¥$ 505-665-7294 ¥medonald@lanl.gov

Flight path Ilo will be the site of a new high-resolution backscattering spectrometer, HERMES. The details of this project are currently in the planning stage. John Larese, Brookhoven National Loboratory $¥ 516-344-4349$ ¥ jzl@bnl.gov

[1ib] Brogg Rotor ultracold neutron source used to study the fundamental properties of the neutron. Experiments include neutron beto decay osymmetry and ultrocold neutron depolarization studies.

Thomas Bowles $¥ 505$-667-3937 $¥$ jib@lanl.gov Sue Seestrom $¥$ 505-667-0156 $¥$ seestrom@lanl.gov

Flight path $11 \mathrm{~b}$ will be the site of a new magnetism diffractometer for use with the new 30-tesla pulsed mognet and for a polarized reflectometer. Michael Fitzsimmons $¥$ 505-665-4045 $¥$ fitz@lanl.gov

Flight poth used for a fundomental nucleor physics effort to precisely measure the asymmetry of the emission of gammo roys from the copture of polorized neutrons or by protons. David Bovman $¥$ 505-667-4363 ¥ bowman@lanl.gov

113 Flight path will be used for the development of new neutron scattering techniques for inelastic neutron scattering. Ferenc Mezei ¥ 505-667-7633 ¥mezei@lanl.gov

11 New in 1999: Flight path will be used for the study of neutron copture on radioactive nuclei, as well as providing useful information for nudear astrophysics. John Ullmann $¥$ 505-667-2517 ¥ ullmann@lanl.gov

15 New, to be commissioned in 2000: The profein crystallography station will be a new diffraction instrument for protein crysiallography ond fibre and membrane diffraction. Benno Schoenborn $¥ 505$-665-2033 ¥ schoenborn@lonl.gov Paul Langon $¥$ 505-665-4105 ¥ langan_paul@lanl.gov

III PHAROS is used for inelastic scottering experiments, including phonon densities of states, magnetic excitations, momentum distribution, crystal field levels, and chemical spectroscopy. Robert Robinson $¥ 505-667-3626$ \# rrobinson@lanl.gov Robert McQueeney ¥ 505-665.0841 ¥ mcqueeney@lanl.gov 
Target 4 is the most intense high-energy neutron source in the world. At this target, the proton beam from the linac is used to produce neutrons for the study of neutron-induced reactions. Target 4 consists of a "bare" unmoderated neutron production target and six flight paths that have flight path distances ranging from 10 to 90 meters at angles of $15^{\circ}$ to $90^{\circ}$ with respect to the proton beam. The shape of the neutron spectrum ranges from a hard (high-energy) spectrum at $15^{\circ}$ to a softer (low-energy) spectrum at $90^{\circ}$. The time structure of the proton beam can be modified for particular experiments.

Each flight path has a name that tells the source of the flight path and its direction with respect to the proton beam. For example, 4FP15R is a flight path (FP) that starts at Target 4 (thus "4FP") and is 15 degrees to the right $(15 \mathrm{R})$ of the incoming proton beam.

\section{Los Alamos Spallation Radiation Effects Facility}

In response to the need for a complete understanding of radiation damage and corrosion phenomena at operating and proposed spallation sources, the LASREF offers a prototypical environment for testing materials for spallation environments at the 1-MW beam stop of the LANSCE linac. This capability is currently being used for materials certification experiments in support of the Accelerator Production of Tritium (APT) project. The huge database that will result from this activity will also be important for

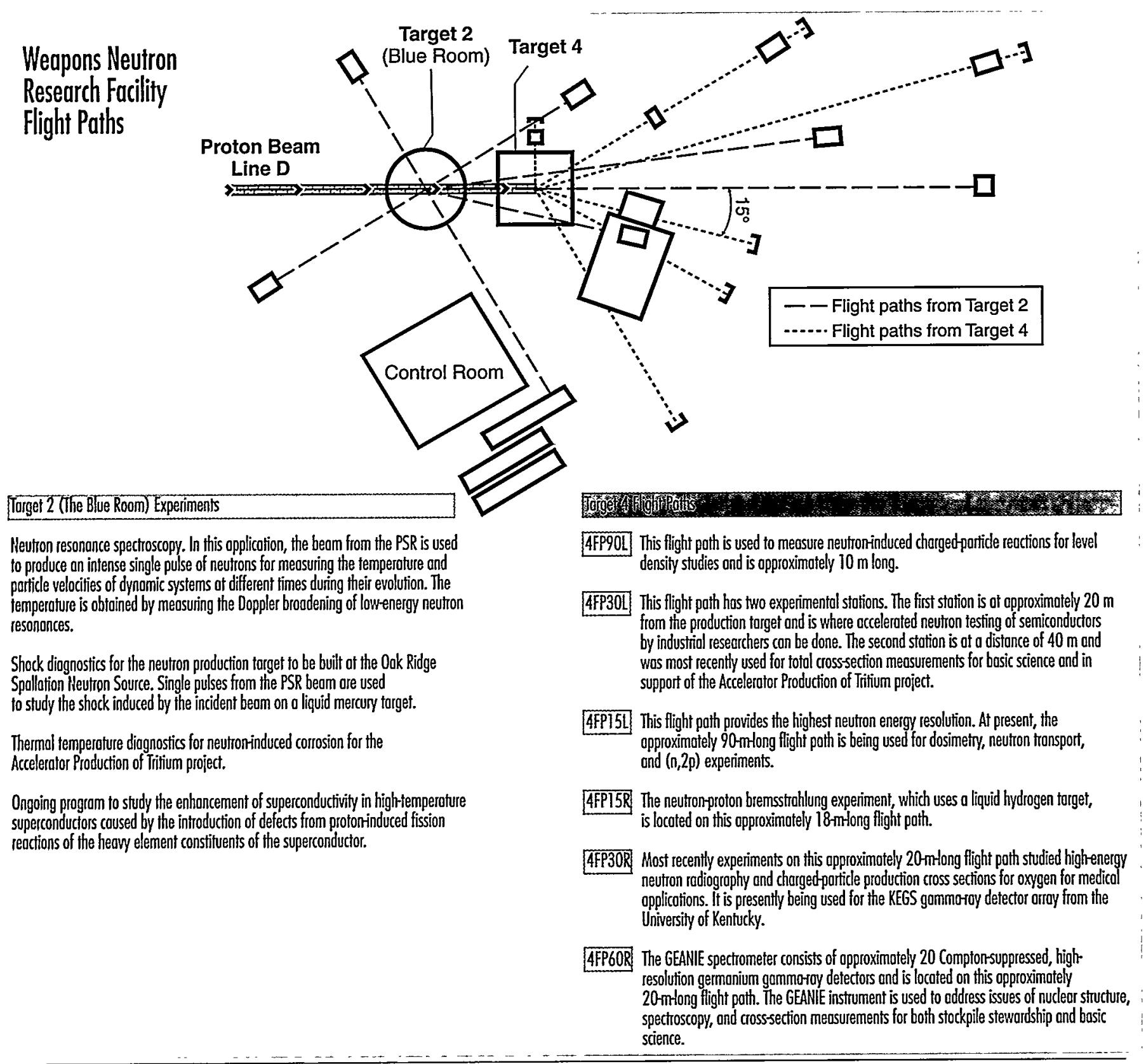




\section{Examples of Commercial Radioisotopes}

${ }^{68} \mathrm{Ge}$ This isotope is the parent of $68 \mathrm{Ga}$, which is a positron emilter. The major use of this isotope is for calibration and absorption correction sources for clinical positron-emitter-tomography (PET) scanners.

82St This isotope is the parent of 82Rb, which is a positron emitter. The major use of this isotope is for clinical cardiac imaging by PET.

${ }^{109} \mathrm{Cd}$ This isotope is used in portable $\mathrm{x}$-ray fluorescence instruments used on the floors of metallurgical shops for the analysis of steels.

${ }^{67 \mathrm{Cu}}$ Research activities using this isotope include studies of copper metabolism, investigations of autoimmune diseases such as myosthenio gravis, and early detection and treament of lung cancer, as well as diagnosis and treatment of lymphoma and colon cancer.

72Se This isotope is the parent of 72As, which is a positron emitter. This isotope holds promise for a wide variety of clinical diagnostic PET procedures without the necessity of an on-site cyclotron.

88Y: This isotope is generated from its parent $88 Z$ Zr. $88 \%$ is used for chemical development of monoclonol antibody lobeling with ${ }^{90 Y}$.

26Al: This rodioisotope is used for understanding the role of aluminum in Alzheimer's Disease.

$325 \mathrm{i}$ This long-lived radioisotope of silicon is used for environmental research.

148Gd This is a lowenergy alpha source used for physics experiments.

Other isotopes, either ovoilable or under development, include: $73 \mathrm{Ar}$, 74Ar, $7 \mathrm{Be},{ }^{2078 \mathrm{Bi}}, 67 \mathrm{Ga}, 172 \mathrm{HF} / 172 \mathrm{Lu}, 194 \mathrm{Hg} / 194 \mathrm{Au}, 145 \mathrm{Pr}, 146 \mathrm{Pm}$, 101Rh, $102 \mathrm{Rh}, 83 \mathrm{Rb}, 75 \mathrm{Se}, 108 \mathrm{mAg}, 22 \mathrm{Na},{ }^{85 \mathrm{ST},} 179 \mathrm{Ta}, 95 \mathrm{mTC}, 44 \mathrm{Ti} / 44 \mathrm{Sc}$, $48 \mathrm{~V}, 49 \mathrm{~V}, 65 \mathrm{Zn}, 88 \mathrm{Zr}$. the Spallation Neutron Source (SNS) that will be built at Oak Ridge National Laboratory.

\section{Isotope Production Facility}

The medical radioisotopes research and production programs make use of $800-\mathrm{MeV}$ protons from the LANSCE linac to produce neutron-poor radioisotopes of demonstrated or potential value in nuclear medicine and/or biomedical research. With the intense beam available at LANSCE, radioisotopes can be produced in quantities not available anywhere else in the world. The automated insertion and retrieval system at the LANSCE beam stop allows the insertion of target materials, including metals, alloys, and salts,

\section{With the intense beam available at LANSCE, radioisotopes can be produced in quantities not available anywhere else in the world.}

into the 1-MW proton beam. The target and irradiation schedules are determined by the requirements of commercial customers and the nuclear-medicine research community. After irradiation, the targets are transported to a hot-cell facility and processed for the radioisotope products.

LANSCE is one of several facilities worldwide that contribute to an uninterrupted supply of neutron-poor isotopes.

\section{Proton Radiography}

In Area $\mathrm{C}$, scientists use $\mathrm{H}^{-}$beam from the linac as a viable, new radiographic probe for creating multiple highresolution images of imploding or exploding objects on a submicrosecond time scale. Protons interact through strong electromagnetic forces allowing the simultaneous measurement of different material properties, such as material density and composition distributions.
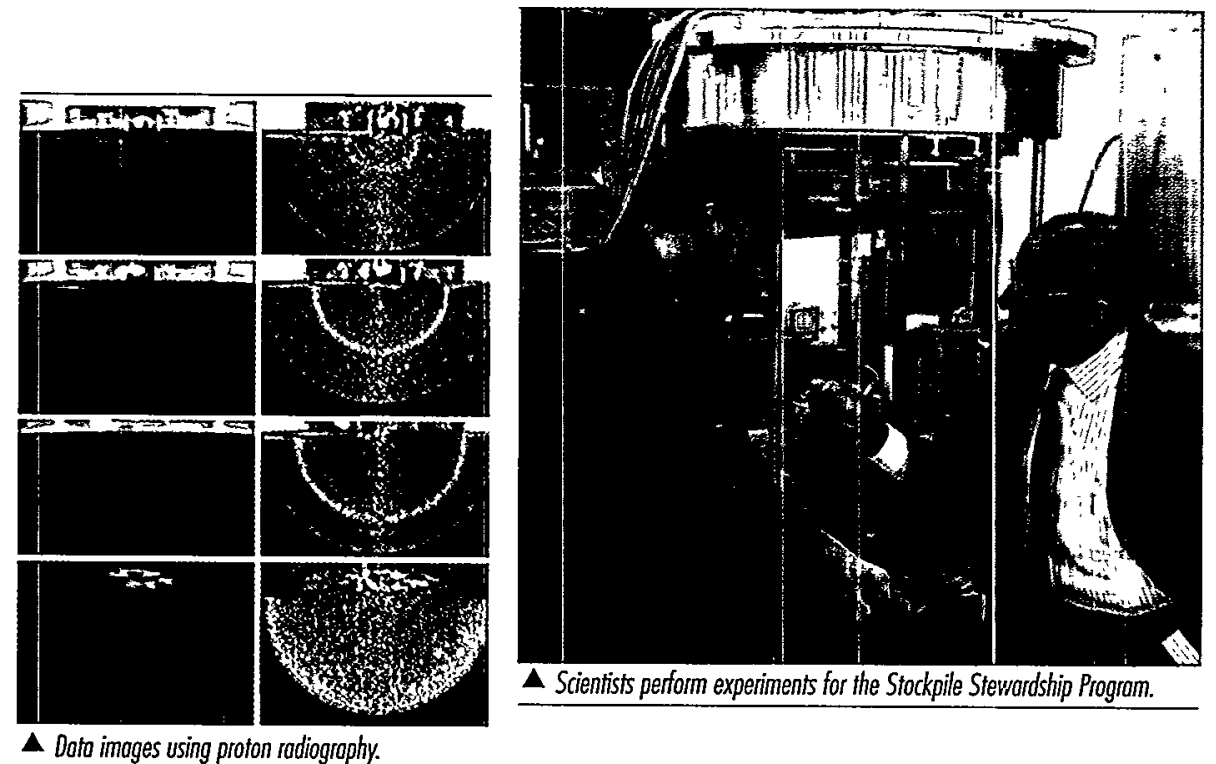

A Scientists perform experiments for the Stockpile Stewardship Program.

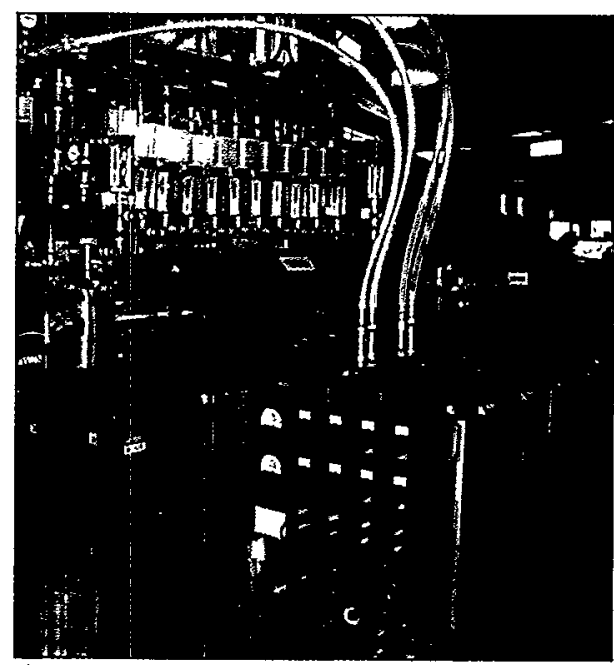

A An SPSS enhancement-the refubished if buncher. 
Protons have several properties that are advantageous for these types of experiments:

- high penetrating power

- high detection efficiency

- very little scattered background

- inherent multipulse capability

- permit large distances from the test object and the containment vessel for the incoming and transmitted beams

Los Alamos scientists are developing proton radiography as a tool for studying stockpile stewardship problems. They have developed magnetic optics; fast, integrating, largearea detectors; and containment vessels to lay the groundwork for advanced radiographic capability.

\section{Programs and Projects}

\section{Stockpile Stewardship}

The DOE/DP Stockpile Stewardship Program contributes to the need for the United States to maintain a safe and reliable nuclear weapons stockpile with the cessation of full-scale nuclear testing. Providing dependable stewardship of the enduring stockpile without testing will require a thorough understanding of the physical processes involved in a nuclear explosion and of the effects of aging on safety and reliability. LANSCE's capabilities in radiography and neutron science can play a very important role in that mission.

\section{LANSCE Reliability Improvement Project}

The goal of the LANSCE Reliability Improvement Project (LRIP), funded by the Department of Defense, is to provide a reliable, $20-\mathrm{Hz}, 100-\mu \mathrm{A}$ proton beam from the PSR to the Lujan Center target-moderator-reflector system (TMRS). The reliability goal is $85 \%$, operating at 8 months per year, measured against scheduled time for experiments.

\section{A Multi-Use Facility}

With multiple research and development activities related to neutron ond accelerator science carried out simulfoneously, LANSCE allows synergism between different disciplines and encourages the flow of ideas between the ocademic, industrial, and defense communities.

The two primary sponsors of LANSCE-the U.S. Department of Energy (DOE) Offices of Defense Programs and Basic Energy Sciences-redlized how LANSCE can meet their synergistic, long-term requirements. The DOE Office of Defense Programs operates LANSCE as port of its research and development activities that support stockpile stewordship. Through the Stockpile Stewardship Program, scientists from Sandia National Laboratories, Lawrence Livermore Notional Laboratory, and Los Alamos use LANSCE facilities to help ensure that the U.S. nudlear weapons stockpile remains sofe, secure, and reliable without nuclear testing. LANSCE also operates a national neutron scattering user program supported by the DOE Office of Basic Energy Sciences.

The pie chart shows that funding for research and project development at LANSCE is olso provided by the U.S. Department of Defense, Loborotory institutional support, and other federal agencies.

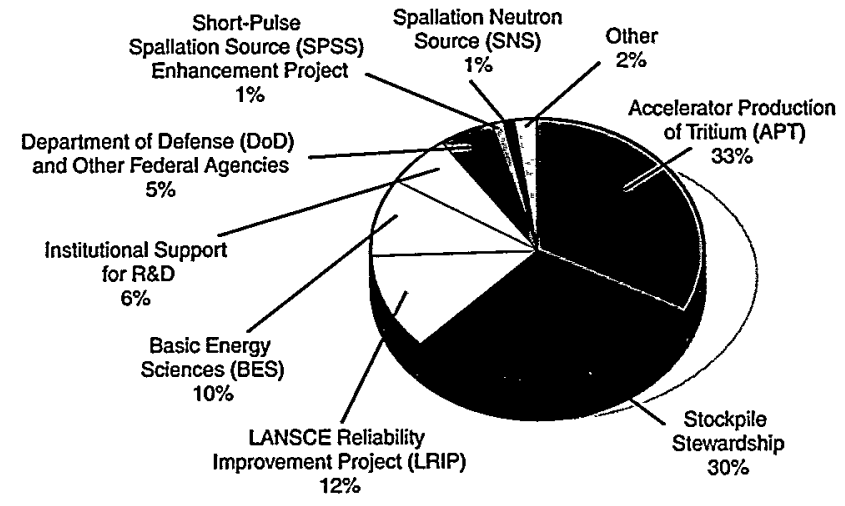

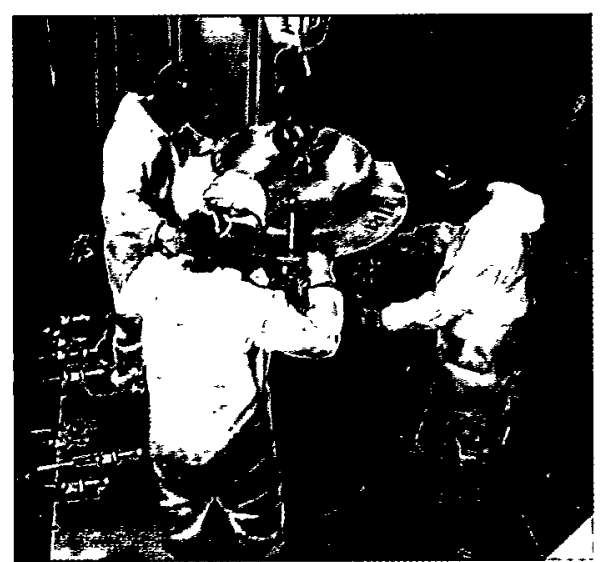

$\triangle$ LRIP enhancements-installation of the TMRS.

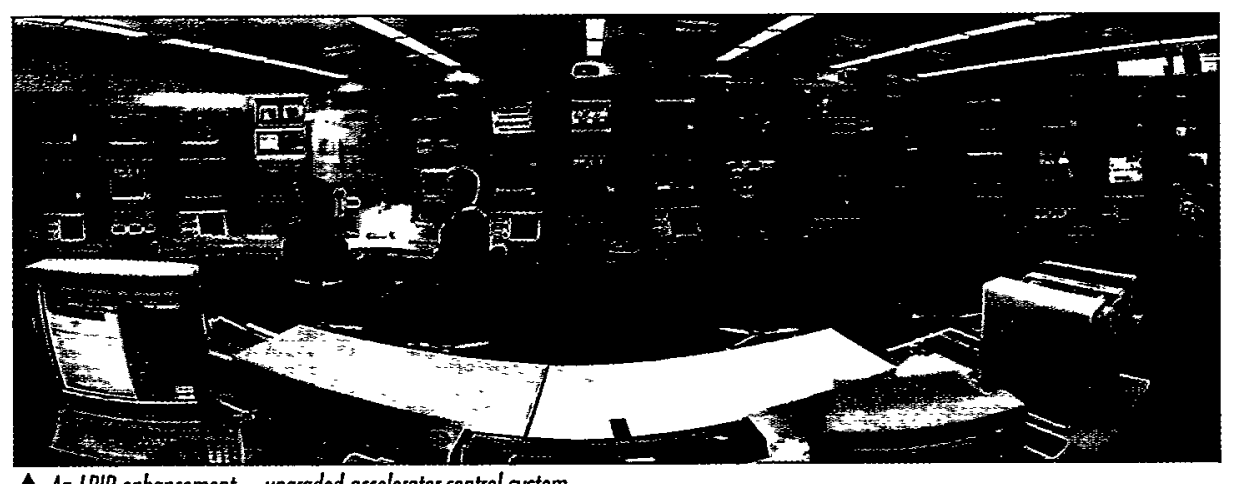

$\Delta$ An LRIP enhoncement-upgraded occeleratorcontrol system. 
The new TMRS installed during the summer of 1998 is designed to handle $200 \mu \mathrm{A}$ or $160 \mathrm{~kW}$ of beam power. To allow new spectrometers to be built on four beam holes added in a 1998 upgrade of the Lujan Center, two new moderators are part of the new system, one water and one liquid hydrogen. The new $\mathrm{H}^{-}$injection scheme into the PSR will contribute to the reduction in beam losses in the ring that limited operation in the past. In the summer and fall of 1998, LANSCE staff commissioned the modified PSR, delivered beam to the new TMRS, and increased the beam current to the $100-\mu \mathrm{A}$ level.

\section{Short-Pulse Spallation Source Enhancement Project}

The goal of this collaborative project involving DOE/DP and DOE/OBES is to significantly upgrade LANSCE capabilities by increasing the neutron source intensity and by constructing additional neutron scattering spectrometers. DOE/DP will fund the accelerator upgrade to provide $200 \mu \mathrm{A}$ of $\mathrm{H}^{-}$ beam current to the Lujan Center target. The major components of the accelerator upgrades are a new $\mathrm{H}^{-}$ion source and injector system and an upgraded radio-frequency (rf) bunching system in the PSR. These improvements will result in greater neutron flux for experiments at the Lujan Center. DOE/OBES will fund several advanced neutron scattering instruments at the Lujan Center.

\section{The new instruments at the Lujan Center will be built by teams of scientists from academia, industry, and federal laboratories.}

The new instruments at the Lujan Center will be built by teams of scientists from academia, industry, and federal laboratories and will be used for research by both the teams' members and general users. Two instruments, SMARTS and HIPPO, are underway; and another two, HELIOS and HERMES, have been recommended for construction.

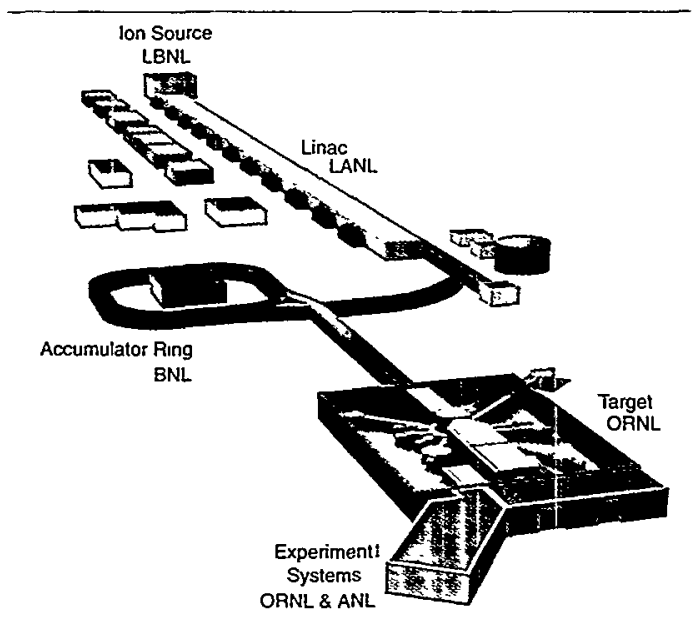

$\triangle$ Antist's conception of the SNS facility.
The DOE Office of Biological and Environmental Research is funding a fifth instrument for protein crystallography. The SPSS Enhancement Project is scheduled to be completed in 2002. Two more spectrometers are being built with internal Laboratory funds.

\section{Accelerator Production of Tritium}

DOE is now investigating the use of a high-power accelerator to produce tritium for the nuclear weapons stockpile. Use of an accelerator offers advantages in safety, waste production, efficiency, and cost over current reactor production techniques. The Accelerator Production of Tritium (APT) project will use a high-power (100-MW) continuous proton accelerator to produce a high flux of neutrons by the spallation reaction on a tungsten target. These neutrons are multiplied and moderated in a surrounding lead blanket containing tubes of helium-3 gas. Tritium is produced by neutron capture on helium-3. APT preliminary design and an aggressive engineering development and demonstration (ED\&D) program is underway at Los Alamos. Final design and construction will be carried out by Burns and Roe, Inc., and General Atomics industrial partners. If approved, the plant will be built at the DOE Savannah River site in South Carolina for operation as early as 2007 . LANSCE provides much of the technical staff, expertise, and ED\&D facilities to the APT project.

To prepare for the construction, scientists are completing several important ED\&D and prototyping activities at LANSCE. They are building and will soon operate a fullpower, low-energy demonstration accelerator (LEDA) to provide design confirmation and operational experience with the front end of the APT accelerator. LEDA will prototype all stages through design, construction, and commissioning and will demonstrate the integrated continuous-wave

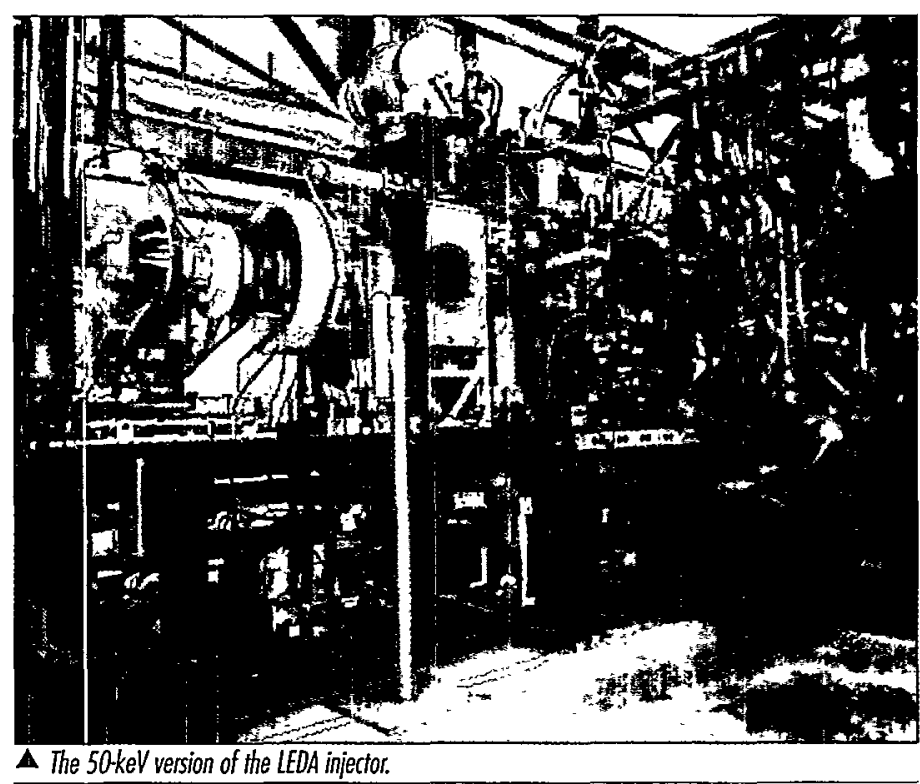


operation of the injector (ion source), radio-frequency quadrupole, and coupled-cavity drift-tube linac up to an energy of approximately $20 \mathrm{MeV}$. Since part of the APT accelerator will involve superconducting technology, ED\&D activities are also underway to design and test superconducting rf cavities at LANSCE. An aggressive program of materials and other studies at LASREF will characterize the performance of the target/blanket assembly.

\section{Spallation Neutron Source}

The SNS project is a major DOE initiative designed to provide a $1-\mathrm{MW}, 60-\mathrm{Hz}$, state-of-the-art, intense pulsed neutron source for neutron scattering experiments and material science studies. The SNS, which is to be constructed at Oak Ridge National Laboratory, will consist of a high-intensity, negative-hydrogen ion source, a 1-giga-electron volt ( $\mathrm{GeV}$ ) linear accelerator, a pulsestacking storage ring, a neutron production target, a set of neutron scattering instruments, and the conventional facilities required to house the systems. The project is a collaboration of Argonne National Laboratory, Brookhaven National Laboratory, Lawrence Berkeley National Laboratory, Los Alamos National Laboratory, and Oak Ridge National Laboratory. Los Alamos and LANSCE are responsible for the linac, which accelerates the $\mathrm{H}^{-}$beam to $1 \mathrm{GeV}$ and for the overall facility control system.

\section{Long-Pulse Spallation Source}

LANSCE is examining the possible installation of a 1-MW spallation neutron production target in the existing building at the end of the $800-\mathrm{MeV}$ linac. Because the linac provides proton pulses of $1 \mathrm{msec}$ in duration, the new source would be a long-pulse spallation source (LPSS).

The main emphasis of this new facility would be in the area of neutron scattering with cold neutrons; i.e., it would use liquid hydrogen or other low-temperature moderators.

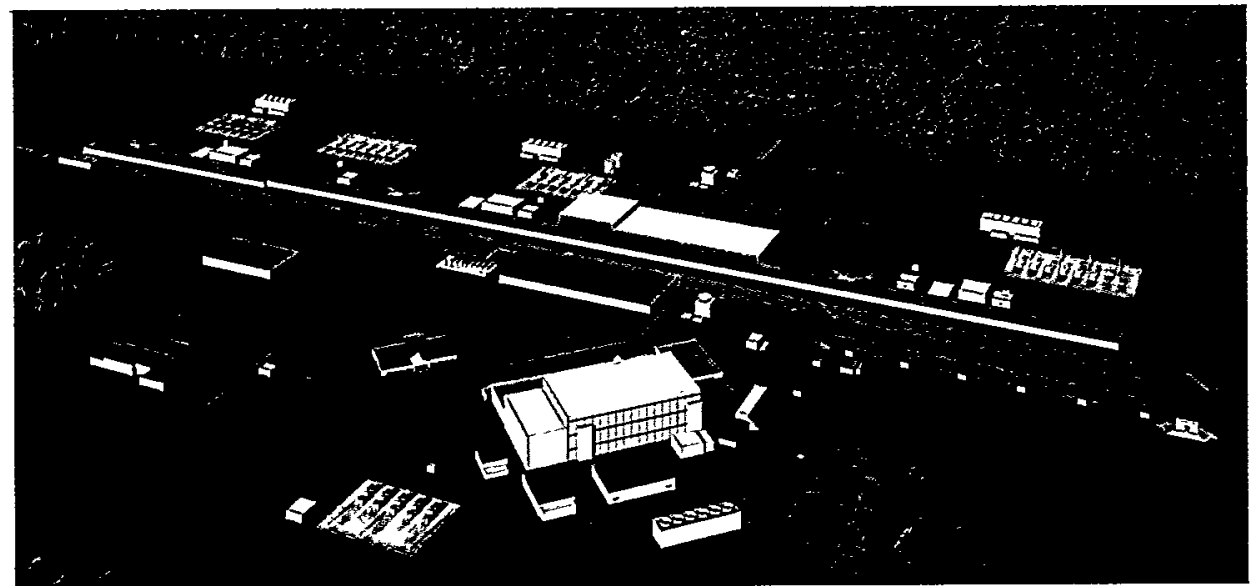

$\Delta$ Artist's rendering of the proposed APT focility.

\section{Current APT Activities}

High-Power Materials Studies

Mechanical test samples of materials used in high-power spallation targets are undergoing long-erm irradiation of LASREF. Over 5000 samples have been tested to date, providing first-of-r-kind fundamental mechanical data for the accelerator and the fusion industries. Somple andysis is conducted by a widespread collaboration of laboratories in the United States and Europe. Additional samples of prototypic elements for specific spallation targets are also being irrodiated and tested.

\section{Corrosion Studies}

Corrosion of materials in irradiated cooling water is being investigated by electrochemical impedance spectroscopy and related techniques. Mitigation of corrosion effects is being tested by chonging water pH and hydrogen content.

\section{Spallation Product Charactenization}

A key design problem in accelerator applications is the production and control of radionuclides in cooling woter and gas streams. Isotopes such os ${ }^{7} \mathrm{Be}$ and ${ }^{22} \mathrm{Na}$ are of speciol concern and are not adequately addressed by currently availoble reactor data. Experiments are underway to measure radionuclide content, sufface plate out, and cleanup techniques in gas and water streams for various experiments mounted in LASREF.

\section{Decay Heat Characterization}

The design of cooling systems in high-power spallation torgets is predicated on exact knowledge of energy deposited in the apparatus, as well as heat generoted from decoying isotopes after the beom is turned off. Unique on-line measurements of decay heat are underway in LASREF by detecting temperature changes in inlet and outlet cooling water.

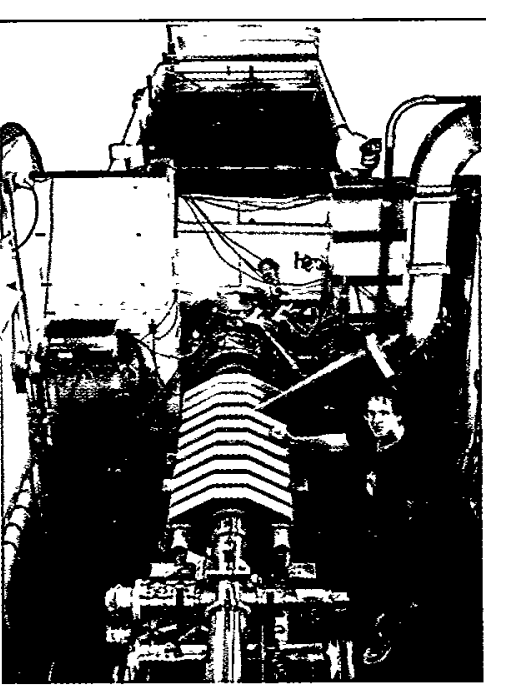

$\Delta$ LANSCE has highrpower microwave and freeelection loser copobilities. 


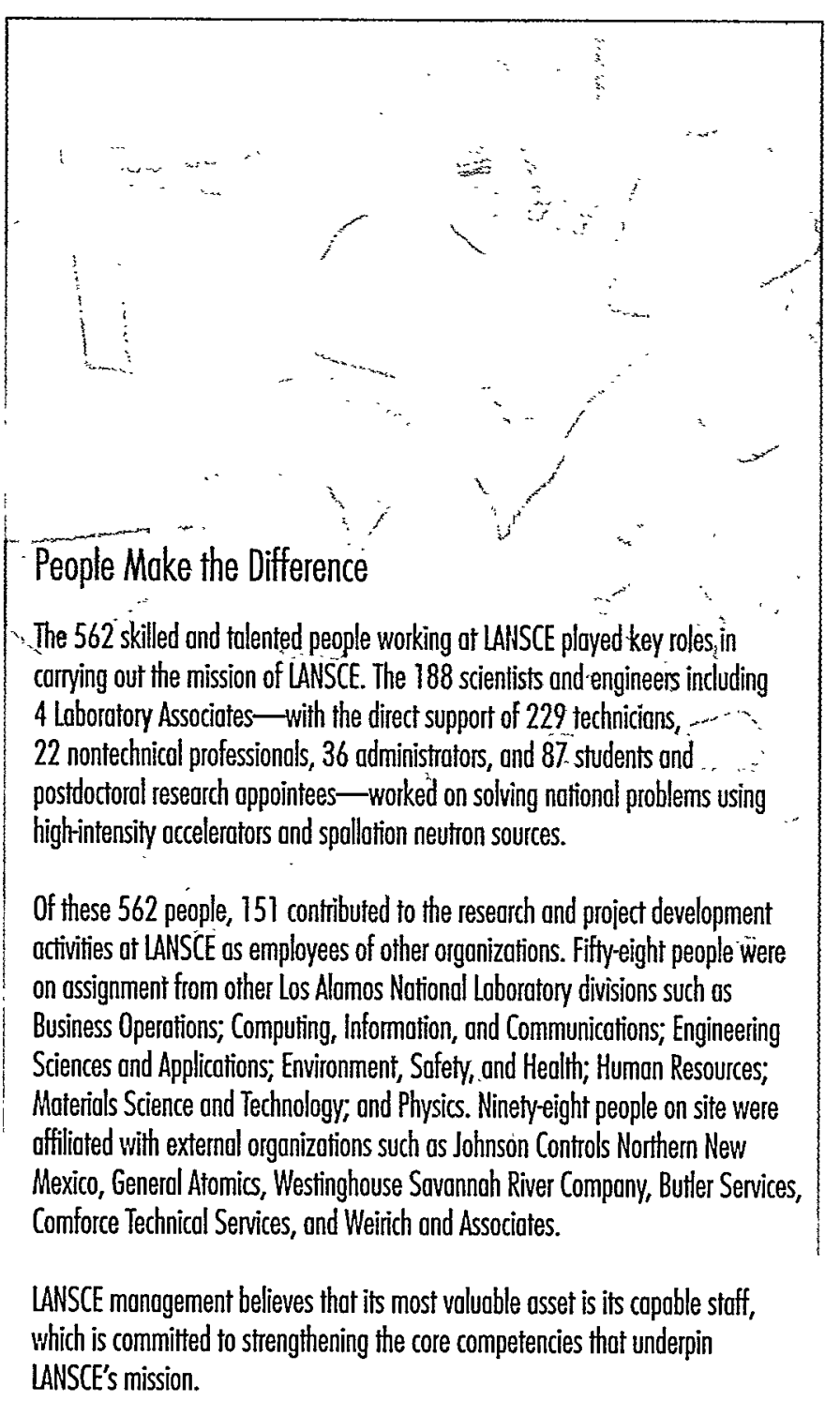

This proposed LPSS would provide complementary capabilities to the current short-pulse Lujan Center. The combined facilities would offer the possibilities for a variety of applications, including materials science research, structural biology, and fundamental physics.

\section{Accelerator-Driven Transmutation of Waste}

The Accelerator-Driven Transmutation of Waste (ATW) project at LANSCE is studying concepts for plutonium disposition, nuclear waste transmutation, and subcritical nuclear energy production. The Laboratory plans to conduct demonstration experiments for these concepts with the high-power proton beam provided by the $800-\mathrm{MeV}$ linear accelerator at LANSCE. Such experiments cannot be mounted elsewhere in the world.

\section{Neutrino Oscillations}

A collaboration of the University of California at Riverside, University of California at San Diego, University of California at Santa Barbara, Embry-Riddle Aeronautical University, Los Alamos National Laboratory, Louisiana State University, Louisiana Tech University, Southern University, and Temple University is probing the small neutrino mass by searching for neutrino oscillations. If neutrinos have nonzero mass, a neutrino of one type may spontaneously transform into a neutrino of another type. The liquid scintillator neutrino detector (LSND) experiment at Los Alamos was designed to search with high sensitivity for neutrino oscillations. The LANSCE accelerator is an intense source of lowenergy neutrinos because of its 1-mA proton intensity and $800-\mathrm{MeV}$ energy.

The LSND detector consists of an approximately cylindrical tank $8.3 \mathrm{~m}$ long by $5.7 \mathrm{~m}$ in diameter. The center of the detector is $30 \mathrm{~m}$ from the neutrino source, the LANSCE linac beam stop. On the inside surface of the tank, 1220 Hamamatsu phototubes provide $25 \%$ photocathode coverage. The tank is filled with 167 metric tons of liquid consisting of mineral oil with a low concentration of scintillator. The LSND experiment observes excesses of events for neutrino oscillations. This implies that at least one neutrino has a mass greater than $0.4 \mathrm{eV}$.

\section{Advanced Concepts and Applications}

LANSCE addresses important national problems by performing research and development on high-power microwave sources and advanced pulsed-power systems for both defense and advanced accelerator applications. Other research areas include the interaction of microwave radiation and matter, microwave-driven chemistry, and development of advanced electro-dynamic systems, including the advanced free-electron laser and the high-brightness, subpicosecond bunch-electron accelerator. Theoretical research

\section{LANSCE performs research and development on high-power microwave sources and advanced pulsed-power systems for both defense and advanced accelerator applications.}

is conducted on intense electron beam physics issues including coherent synchrotron radiation and emittance growth mechanisms in high-current linear induction accelerators. By tackling many difficult problems for the Departments of Energy and Defense, other federal agencies, and industry, our research stays at the scientific forefront that in turn advances the state of the art of the accelerator technology needed for next-generation accelerators. 


\section{LDRD-Funded Projects}

The Laboratory Directed Research and Development (LDRD) Program supports internally-proposed, innovative research and development that extends the Laboratory's science and technology capabilities. The selection process is highly competitive, with a comprehensive review by peers or Laboratory managers and selection based on innovation and scientific merit in a mission context. The LDRD program has two major components: Competency Development (CD) and Individual Projects (IP).

The CD component funds research related to the technical competencies that underpin the Laboratory's ability to execute its missions and respond to new DOE and national initiatives. Therefore, the CD component invests in large multidisciplinary projects that tackle significant missionrelated problems from several viewpoints, often combining theory and experiment and drawing on scientists from several technical divisions.

The IP component invests in the most far-reaching and basic research proposed by the technical staff. This component has a primary goal of funding research to extend the Laboratory's science and technology knowledge base. Therefore, IP projects tend to be fundamental and exploratory in nature, often mapping out uncharted territory. They involve both high risk and high potential returns.

LANSCE has many projects that owe much of their success to LDRD "seed money" support. One example of a current LANSCE project is the Regenerative Amplifier Free-Electron Laser (RAFEL), which is now in the program development phase with several major aerospace companies for application as a high-power, tunable light source.

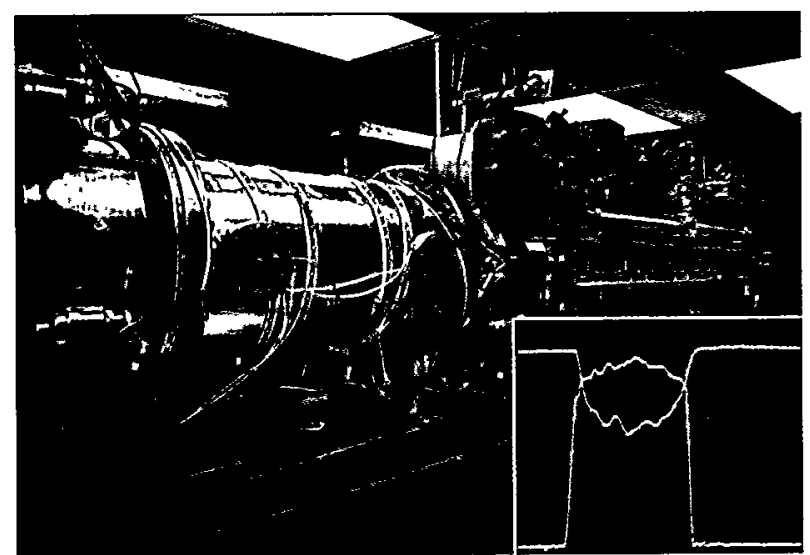

A Highrgain, selfomplified spontaneous emission and regenerative amplifier freetectron laser show promise for short wovelength ond high-power generotion of light.
In FYs 1997 and 1998, LANSCE Division had the following LDRD Projects...

Competency Development (CD)

- Fundamental Studies of Radiation Damage in Two-Phose Oxide Composites

- Structure, Dynamics, and Function of Biomolecules

- Advanced Biomolecular Moterials Based on MembraneProtein/Polymer Complexation

- Neutron Scattering from Correlated Electron Systems (see page 40)

- Microstructures of Transition Metol Oxides Characterized with Neutron and X-Ray Scattering

- A Neutron Diffraction and Computational Micromechanics Study of Deformation in Advanced Materials

- Neutron Scattering Studies on Shear-Induced Structure in Polymers

- Dynamics of Polymers at Interfaces

- Liquid-Lead and Lead-Bismuth Technology for Use in Subcritical Systems Applied to Nuclear Waste Destruction (see page 123)

- Molten Salt and Seporations Technologies Evaluation

- Energy Transfer In Molecular Solids (see page 56)

- Manipulation of Residual Stresses to Improve Material Properties (see page 28)

- Veside and Lomellar Phase Stability: An Experimental Approach to a Problem Centrol to the Theory of Complex Fluids

- Actinide Crystol Structures with an Emphasis on Plutonium Alloys (see page 38)

- Advonced Modeling of High Intensity Accelerators

- Advanced Research Copabilities for Neutron Science and TechnologyNew Polarizers

- Advanced Research Capabilities for Neutron Scattering

- Comporative Investigation of Spin, Charge, and Lattice Degrees of Freedom in Three Classes of Colossal Magnetoresistive Materials

- Proliferation-Resistant, Low-Environmental Impoct Treatment Processes for Nuclear Woste Destruction (see page 123)

Individual Projects (IP)

- High-Power, High-Frequency, Annular-Beam FreeElectron Loser

- Sub-picosecond Electron Bunch Diognostic (see page 70)

- Plasma-Wakefield Accelerator

- A Compact Compton Backscatter X-Ray Source for Mammography and Coronary Angiography

- Production and Trapping of Ultrar-Cold Neutons in Superfluid Helium

- High-Pressure Crystal Chemistry of Hydrous Minerals

- Pulse Shaping in Explosive Pulsed Power Systems (see page 72)

- Self-Amplified Spontoneous-Emission Reloted Science Studies (see pages 68 and 76) 


\section{LANSCE Addresses Important Issues for Stockpile Stewardship}

The Stockpile Stewardship Program at LANSCE supports the operation of the $800-\mathrm{MeV}$ proton accelerator and its neutron-generating targets as a National User Facility for users from the defense, basic, and applied research communities. Defense research takes advantage of a wide range of LANSCE's capabilities to provide the DOE Defense Programs (DP) community with information critical to the national stockpile stewardship mission: maintaining nuclear deterrence without nuclear testing. Among the capabilities being exploited at LANSCE are neutron scattering, proton and neutron radiography, neutron resonance spectroscopy, and neutron-induced nuclear reaction measurements.

A stockpile stewardship research program at LANSCE has been designed to bring the traditional weapons design and engineering community into closer contact with LANSCE capabilities. LANSCE researchers provide fundamental data that will inform and guide the development of full-fidelity physics modeling of nuclear explosions. This modeling is being implemented on a new generation of supercomputers-the fastest in the world-at the DP laboratories as part of the Accelerated Strategic Computing Initiative (ASCI). In addition, LANSCE research is contributing to the development of advanced manufacturing processes that will be important for stockpile refurbishment activities in the future. The current stockpile stewardship research projects, which have enhanced the visibility of LANSCE in the nuclear weapons community, are as follows:

- Proton radiography has demonstrated the ability to make multiple-exposure radiographs of dynamic systems driven by high explosives on time scales of tens of nanoseconds. Image resolution and contrast are sufficient, for example, to observe shock fronts in a detonating explosive. At LANSCE, $800-\mathrm{MeV}$ protons have been used to image these shock fronts as a function of initial temperature. This work has shed important light on the mechanisms involved in highexplosives burn. At Brookhaven National Laboratory, a LANSCE and Lawrence Livermore National Laboratory team is demonstrating the potential for using much higher energy protons $(25 \mathrm{GeV})$ to generate radiographs of relatively large objects. This research is important in the development of proton radiography, which provides multiple-time, high-resolution tomography of implosion systems used in stockpile stewardship hydrodynamics testing. (See page 94.)

-Weapons materials science investigations of microstructural changes in aged materials and in materials manufactured by new processes are leading to a better understanding of weapons performance as weapons age beyond their design lifetimes. Basic studies of the atomic vibrations in plutonium are leading to new equations-of-state models for weapons design codes. Residual stress and texture measurements are showing how manufacturing changes affect subsequent corrosion and cracking resistance as well as anisotropic response to deformation and vibration. Aging in high explosives is being investigated with small-angle neutron scattering together with $x$-ray and light scattering to elucidate binder segregation and explosive/binder interface changes. The ability to tailor contrast of various hydrogen bonds in the explosive by selective deuteration is a powerful capability of neutron scattering. Neutron reflectometry is helping Sandia National Laboratories develop better coatings and adhesives for weapons components. Neutron scattering is being used in materials subjected to accelerated aging techniques to determine microscopic changes that presage changes in mechanical properties. Finally, thermal and cold neutrons are being used to detect defects in weapons parts and to construct three-dimensional tomographs of the defect structure. (See pages 24, 30, 38, 46, 56,90 , and 92 .)

- Weapons nuclear science is a project that focuses on providing high-precision nuclear data needed for performance assessment of the aging stockpile. Weapons nuclear data measurements are providing information that will be used to interpret the radiochemical data that has been archived from the weapons testing program using modern ASCI tools. Measurements are being carried out at the Lujan Center's moderated neutron source and at WNR's unmoderated, high-energy neutron source. The recently installed GEANIE (Germanium Array for Neutron-Induced Excitations) instrument at WNR will provide more accurate cross sections for high-energy neutron interactions with actinide elements. At the Lujan Center, first-ever measurements of the neutron-capture cross sections for very shortlived isotopes that were used or produced in weapons tests will provide information that could only be estimated previously with theory and calculations. (See pages 80,82 , and 86.)

- Neutron Resonance Spectroscopy (NRS) is taking advantage of the brightness of LANSCE's Proton Storage Ring and using the very narrow absorption resonances known in specific isotopes (such as ${ }^{171} \mathrm{~W}$ ) to determine the temperature and particle velocity in explosives and in materials accelerated or shocked by explosives. Changes in the width and energy of absorption peaks reflect changes in the temperature and velocity of the absorbing material. Samples of interest are doped or "tagged" with an isotope with appropriate resonance characteristics. Great strides have been made in reducing the noise levels and developing the theoretical analysis tools needed to make this technique more precise. NRS measurements on dynamic systems are yielding information on shockwave properties of weapons materials and will soon be used to study the equation-of-state of high explosives after detonation. (See page 84.) 


\section{LANSCE Is a National User Facility for Neutron Scattering}

The Department of Energy Office of Basic Energy Sciences/ Energy Research supports an in-house program of basic research and the operation and development of the Lujan Center as a National User Facility for neutron scattering. The research programs encompass materials science, condensedmatter physics, chemical physics, and crystallography.

Current research topics in materials science (see pages 26, $30,32,44$, and 48) include

- study of internal strain in engineering materials using neutron diffraction

- intermolecular forces in complex fluids

- shear-induced changes in polymers

- study of conducting polymer films

- reinforcement of rubber by carbon black

- self-assembly of colloids

- structure of macromolecular systems

- participation in a collaborative access team at the Advanced Photon Source for the study of complex fluids

- an in-house rotating-anode $x$-ray scattering capability to support thin film and strain measurements

In condensed-matter physics (see pages 40 and 42 ), the emphasis is on f-electron and magnetic systems and Brillouin scattering including

- interfacial magnetism in spatially limited materials

- magnetism in uranium intermetallics

- heavy-fermion compounds

- colossal magnetoresistive materials

- crystal-field levels in actinide oxides

- excitations in amorphous materials

Chemical physics studies (see page 50) center around

- molecular vibrations in novel organo-metallic compounds and macromolecular systems

- dihydrogen-metal bonds

- long-range, non-dissipative energy transports in biological molecules

- adsorption of halocarbons in zeolites

Crystallography activities (see pages 34 and 38) include

- the development of the Generalized Structure Analysis System (GSAS)

- interpretation of hkl-dependent strains

- analysis of texture and high-pressure studies
As a National User Facility, funds are included for the operation and development of the Proton Storage Ring; operation and development of the Lujan Center neutronproduction target; operation and development spectrometers and data acquisition systems; and interactions with users.

Projects (see pages $64,66,100,119,133,138,141$, and 157 ) in this part of the program include

- designing and constructing new spectrometers including HIPPO, SMARTS, HELIOS, and HERMES

- modeling and improving the radiation shielding at the Lujan Center

- modeling the target/moderator performance and comparison with measured time and energy distributions

- addressing the radionuclide inventory in targets, in air emissions, and induced radioactivity in neutron scattering samples

- upgrading neutron scattering spectrometers, such as the wide-angle bank of PHAROS, increasing detector coverage on several spectrometers, developing YBCO cryogenic neutron flippers, and developing new data acquisition and chopper control systems

- increasing the variety of sample environments by purchasing a 12-tesla superconducting magnet, developing a 30-tesla pulsed magnet, and building a high-pressure/temperature apparatus

- developing a Monte Carlo tool for instrument simulation

- implementing radial collimators for improved measurement of spatially resolved strain

- improving reliability through upgrades of Personnel Access Control Systems, aging power supplies, and timing systems

- studying parameters for the proposed Long-Pulse Spallation Source operations

- studying the PSR instability threshold and developing improved stripping foils

- administering a full LANSCE User Program 

Condensed-Matter Science and Engineering

Nuclear Science: 



\section{Condensed-Matter Science and Engineering}

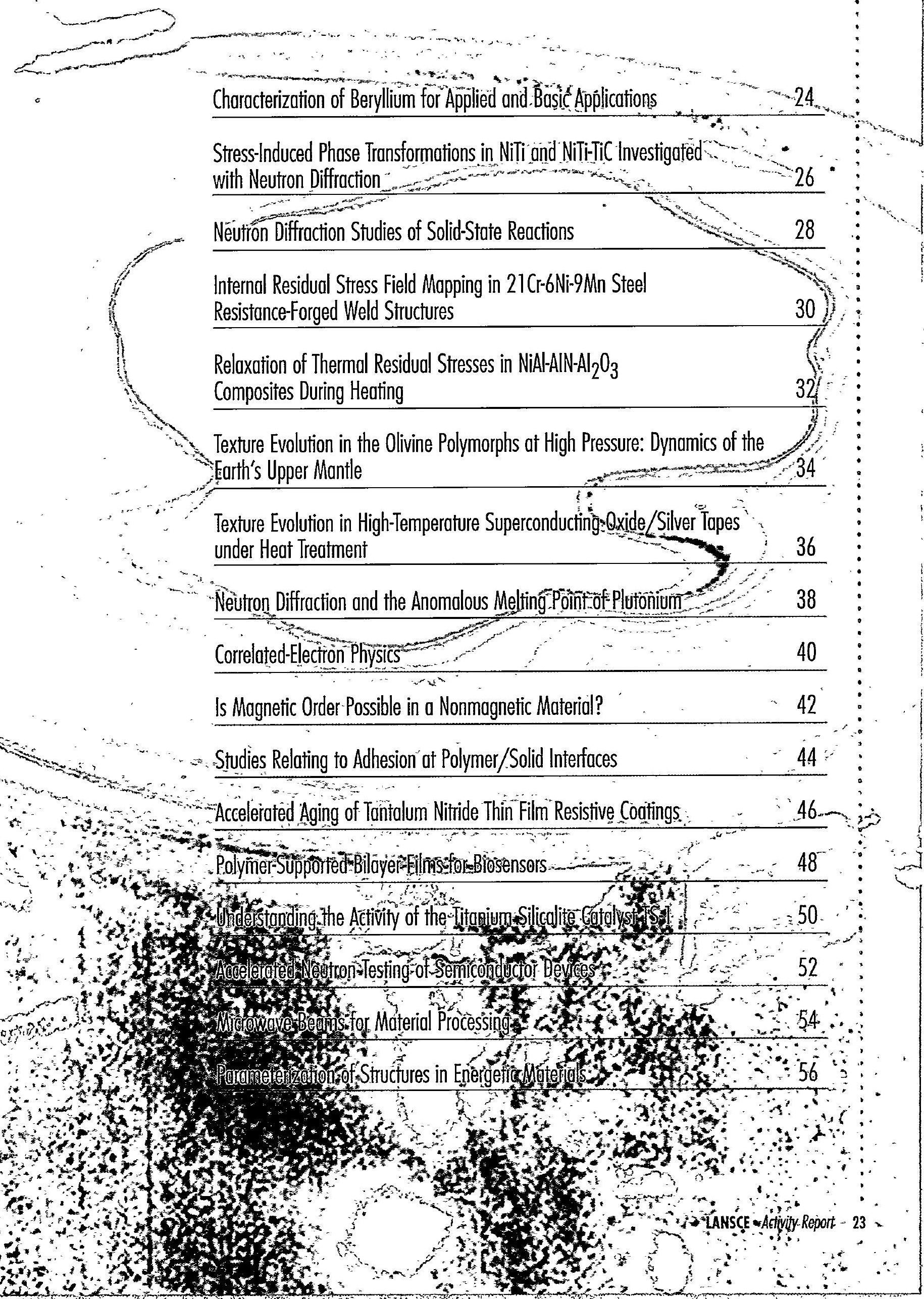




\title{
Characterization of Beryllium for Applied and Basic Applications
}

\author{
M. A. M. Bourke (Materials Science and Technology Division and LANSCE Division), R. Varma \\ (Materials Science and Technology Division), M. R. Daymond (LANSCE Division), D. H. Carter, C. Tome \\ (Materials Science and Technology Division)
}

\begin{abstract}
Catastrophic failure, stress corrosion, and fatigue are all issues that impact the use of beryllium components. In turn, these physical processes are impacted by the presence of residual strain and texture in polycrystalline beryllium. Neutron diffraction is a powerful tool for characterizing beryllium because it can produce both spatially resolved strain and texture maps. The results can validate advanced finite element continuum mechanics models or improve our understanding of polycrystalline deformation processes.
\end{abstract}

Part of the Enhanced Surveillance/Dismantlement program at Los Alamos involves the characterization of beryllium, which by virtue of its large scattering cross section for thermal neutrons, is an important element both in the nuclear power industry and for the weapons program. In either application, it is often welded to provide particular shapes. However, welds generally exhibit residual stresses that impact service lives because of their influence (beneficial or detrimental) on fatigue performance. One subsidiary issue is that beryllium in its solid form is relatively innocuous but as a powder it is toxic. This makes conventional hole-drilling methods for measuring residual stress difficult and the use of a nondestructive technique like neutron diffraction attractive.

At the Lujan Center, we used the spatially resolved strainmeasurement capability on NPD to define a $(3-\mathrm{mm})^{3}$ sampling volume and to profile residual strain distributions in a welded beryllium ring. The ring is shown mounted between two radial collimators in Figure 1. Several strain profiles suggested modest circumferential strain variations.

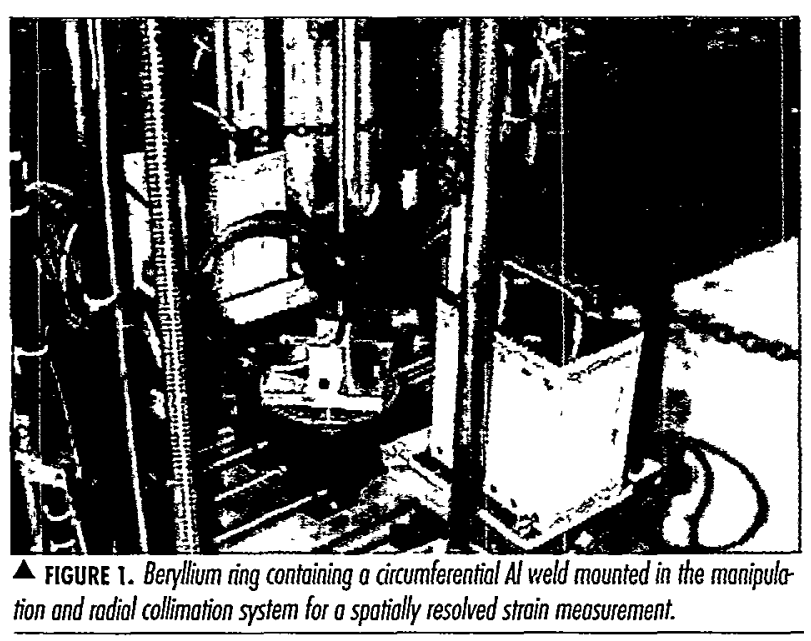

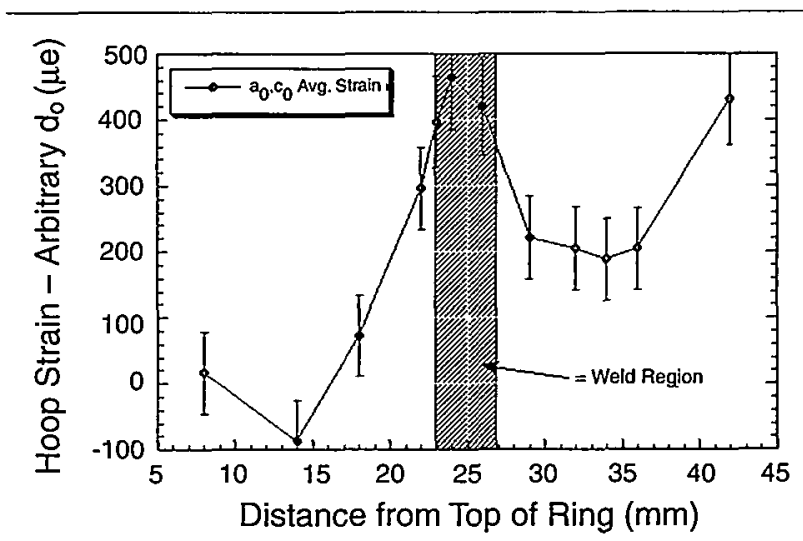

A FIGURE 2. Neutron diffraction meosurements of the residud hoop stroin as the sumpling vot ume is scanned ocross on AlSi waist weld.

Moreover, as seen in Figure 2, there is evidence that the stress distribution was not symmetric about the weld plane, contrary to the natural symmetric assumption.

In Figure 2, the hoop strain is the average of the $\mathrm{a}_{0}$ and $\mathrm{c}_{0}$ lattice parameters. This was chosen as an empirical measure of the bulk response for comparison with continuum mechanics (finite element programs), which take no account of possible polycrystalline anisotropies. As a first approximation, this is defensible, but reality at the level of the microstructure is more complicated, with strongly heterogeneous strain fields varying across adjacent grains.

To explore the intricacies of the "real" strain fields in the welded sample, we examined a more controlled situation, namely, a loaded compact tension (CT) specimen. By applying a known load, what we hoped would be a wellcharacterized strain field was introduced from which the lattice plane and spatial dependence of the strains could be examined. From these results we expected to more accurately interpret the measurements in the waist weld. To apply the load, a fixture was fabricated to allow clevis pins in the specimen to be forced apart by a bolt. The force was monitored using a load cell.

A specimen was fatigued to induce a stable and symmetric crack. Unfortunately, beryllium is sometimes vulnerable to unstable fracture during fatigue in which case the crack propagates at $45^{\circ}$ to the expected direction. This occurred without our knowledge, so the strain field shown in Figure 3, which we had expected to be symmetric about $(0,0)$, initially was inexplicable. However, when we subsequently loaded the specimen to failure, the reason for the nonsymmetric strain field became apparent (Figure 4). The 


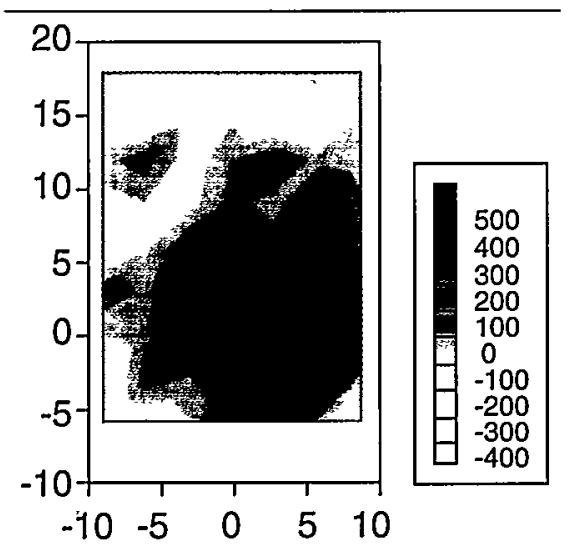

$\triangle$ FIGURE 3. Spatially resolved "residual" stroin map in a looded, crocked but unbroken $C T$ specimen (units are microstrain, positive is tensile).

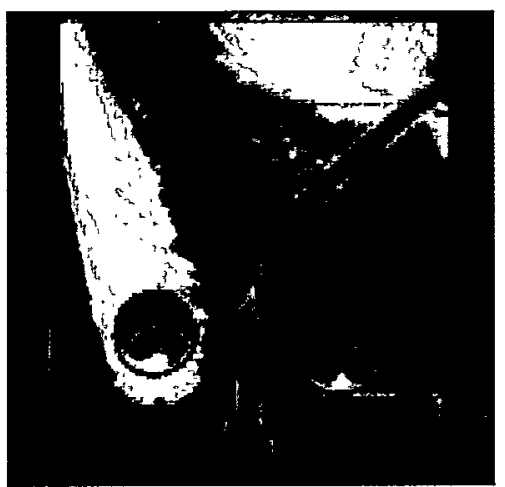

A FIGURE 4. Beryllium $C T$ specimen ofter loading to failure on completion of the neutron profile shown in Figure 4.

explanation for the proclivity of $\mathrm{CT}$ specimens to sometimes fracture at $45^{\circ}$ appears to be related to the texture of the starting beryllium - this was also measured at LANSCE using HIPD.

Close examination of the lattice strain-specific strains in both the CT and the weld specimens showed large anisotropy and fluctuations from tension to compression in the same regions. This led us to perform a uniaxial test on an HIP'd (hot isostatically pressed) sample. The specific lattice reflection strains recorded during this load test are shown in Figure 5. Note the wide spread and in particular the atypical behavior of the 0002 (basal planes).

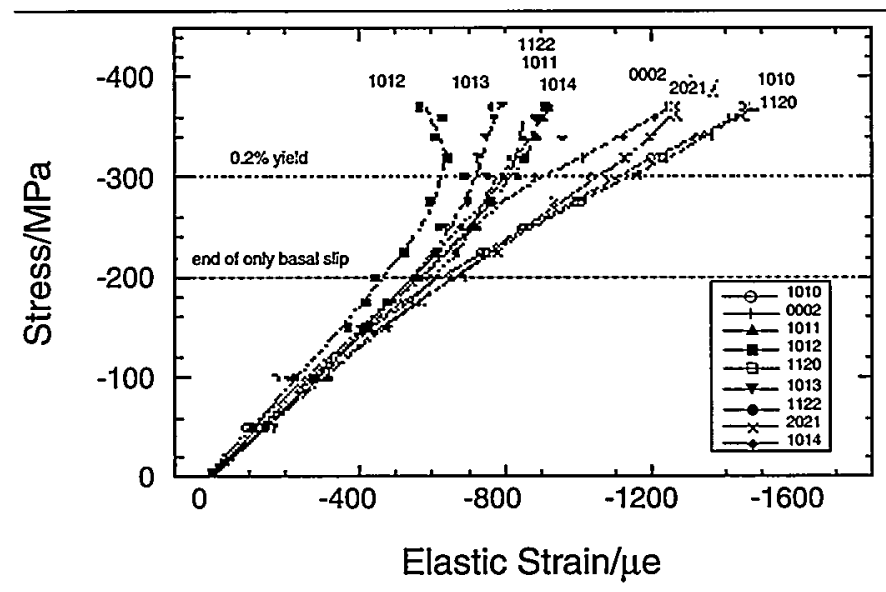

$\Delta$ FIGURE 5. Elastic strain parallel to the looding direction for different lattice reflections from a beryllium cylinder (24 mm long, $10 \mathrm{~mm}$ in diameter) during a compression test.

Our next step is to use elastic plastic models of a polycrystalline aggregate that employ the Hill ${ }^{1,2}$ self-consistent approach (first implemented by Hutchinson ${ }^{3}$ ) to elucidate the dependence of different lattice plane orientations on different inelastic deformation processes. In the model, a population of grains is chosen with orientations appropriate for the measured texture. Each single grain, assumed ellipsoidal, is modeled with anisotropic elastic properties and single crystal slip mechanisms. Interaction between the individual grain and the surrounding medium, which has properties of the average of all the grains, is carried out using an elastoplastic, Eshelby-type, self-consistent approach. Because the properties of the medium are given by the average of all the grains, it is initially undetermined and must therefore be solved by iteration. Small total deformations are assumed (typically less than $4 \%$ ), and no lattice rotation or texture development is assumed. Using these models, we are striving towards a more comprehensive interpretation of the diffraction data obtained in both the $\mathrm{CT}$ and the weld specimens.

\section{References}

1. R. Hill, J. Mech. Phys. Solids, 13, 89-101 (1965).

2. R. Hill, J. Mech. Phys. Solids, 13, 213-222 (1965).

3. J. W. Hutchinson, Proc. Roy. Soc. Lond. A 319, 247-272 (1970). 


\title{
Stress-Induced Phase Transformations in NiTi and NiTi-TiC Investigated with Neutron Diffraction
}

\author{
R. Vaidyanathan (Massachusetts Institute of Technology), M. A. M. Bourke (Materials Science and \\ Technology Division and LANSCE Division), D. C. Dunand (Northwestern University)
}

Superelastic NiTi alloys in the austenitic phase can undergo a phase transformation to the martensitic phase if subjected to stresses. We used neutron diffraction to study this reversible stress-induced phase transformation in NiTi and NiTi reinforced with TiC particles. We obtained neutron spectra of austenitic NiTi at room temperature to investigate the discrete phase strains, martensite phase fractions, and texture evolution at various stress levels during mechanical loading and unloading. To model the data, we used Rietveld refinements that incorporated a spherical harmonic texture formulation. The series of experiments helped to understand (a) the stressinduced transformation in NiTi, (b) composites where the matrix deforms by a stress-induced transformation, and (c) changes in the transformation due to stress cycling.

The shape-memory and superelastic effect exhibited by near-stoichiometric NiTi-based alloys originates from the thermoelastic phase transformation between an ordered, high-temperature cubic phase B2 (austenite) and an ordered, low-temperature monoclinic phase B19' (martensite). Neutron diffraction measurements can be successfully used to monitor discrete phase strains, bulk texture, and phase fractions during such transformations. ${ }^{1,2}$ At room temperature, nickel-rich austenitic NiTi alloys show the superelastic (or pseudoelastic) behavior; i.e., during mechanical loading, tensile strains as high as $8 \%$ result from the formation of stress-induced martensite. During subsequent unloading, the martensite reverts to austenite, with recovery of all the accumulated macroscopic strain. ${ }^{3}$ Since the NiTi phase transformation is thermoelastic, it is expected to be dependent on internal and external stresses. Stresses produced by an elastic reinforcing second phase in NiTi-based metal matrix composites can thus affect the matrix's transformation and deformation behavior.

We applied uniaxial compressive stresses to an unreinforced superelastic sample (NiTi) and a composite superelastic sample containing 10 vol.\% TiC particles (NiTi$10 \mathrm{TiC}$ ) and collected neutron diffraction spectra during the deformation. Figure 1 shows the stress-strain response of superelastic NiTi and NiTi-10TiC. The marked points indicate the stress levels at which the mechanical cycle was halted and neutron diffraction data obtained for two such experiments. The load axis was placed in a horizontal plane at $45^{\circ}$ to the incident neutron beam, and three detectors recorded diffraction patterns at $\pm 90^{\circ}$ and $+32^{\circ}$ from the incident neutron beam.

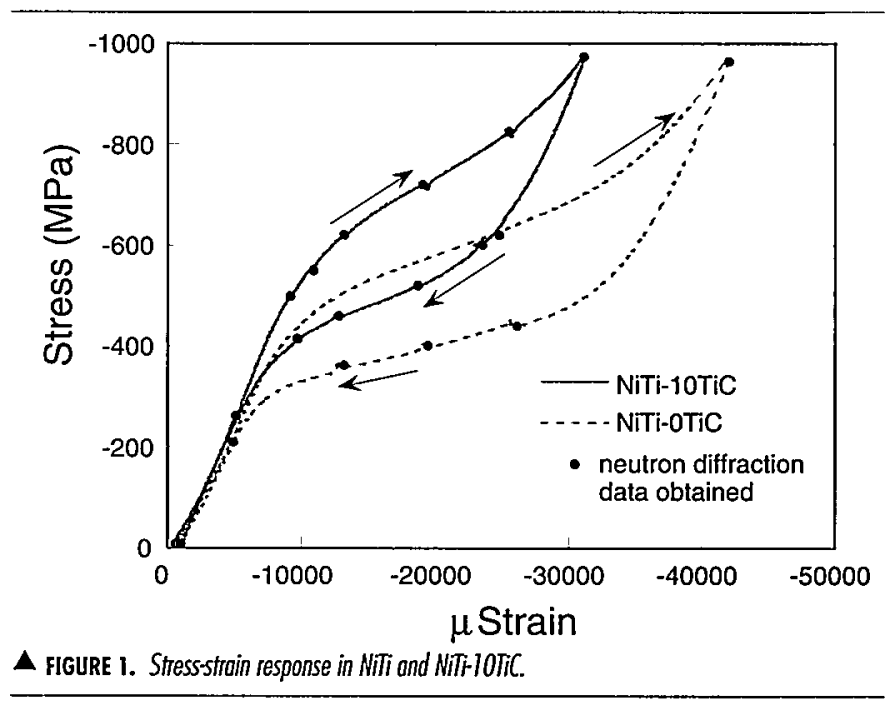

The experiments yielded in situ measurements of the thermoelastic stress-induced transformation. Figure 2 shows that, as stress increases, the austenite (100) peak disappears while the martensite (100) peak appears. When stress is removed, the martensite reverts back to austenite, and the austenite (100) peak reappears as the martensite (100) peak disappears. Figure 3 shows that the superelastic strain due to the transformation (elastic strain subtracted from macroscopic strain in Figure 1) is linearly related to the volume of austenite transformed to martensite, which is obtained from a Rietveld refinement using the Generalized Structure Analysis System (GSAS) code. ${ }^{4,5}$ In both NiTi and NiTi10TiC, this linear relationship is maintained for loading and unloading, indicating that the hysteresis in Figure 1 is due to stress, not strain. However, NiTi-TiC exhibits more

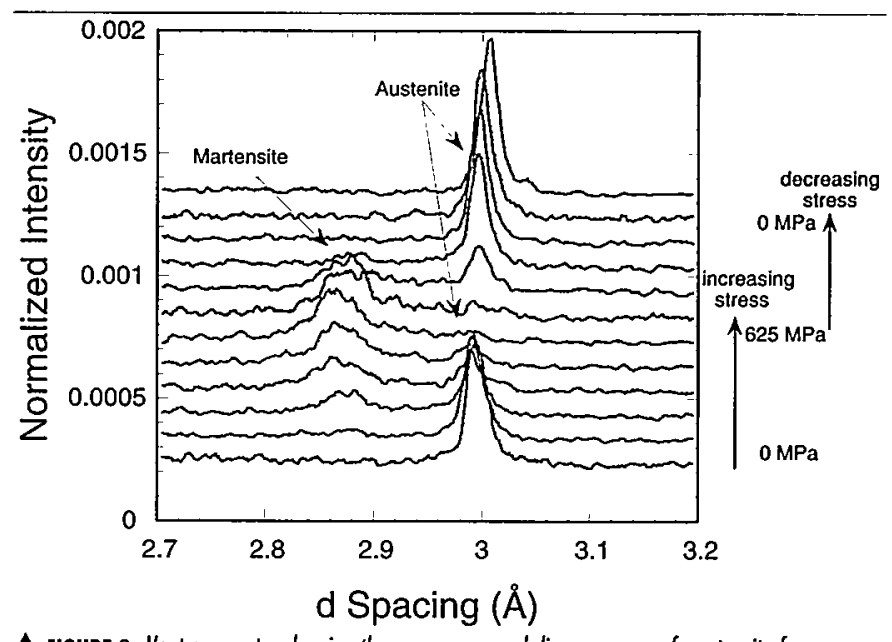

A FIGURE 2. Neutron spectro showing the appearonce and disappearance of mostensite from oustenite as a function of opplied stress. 


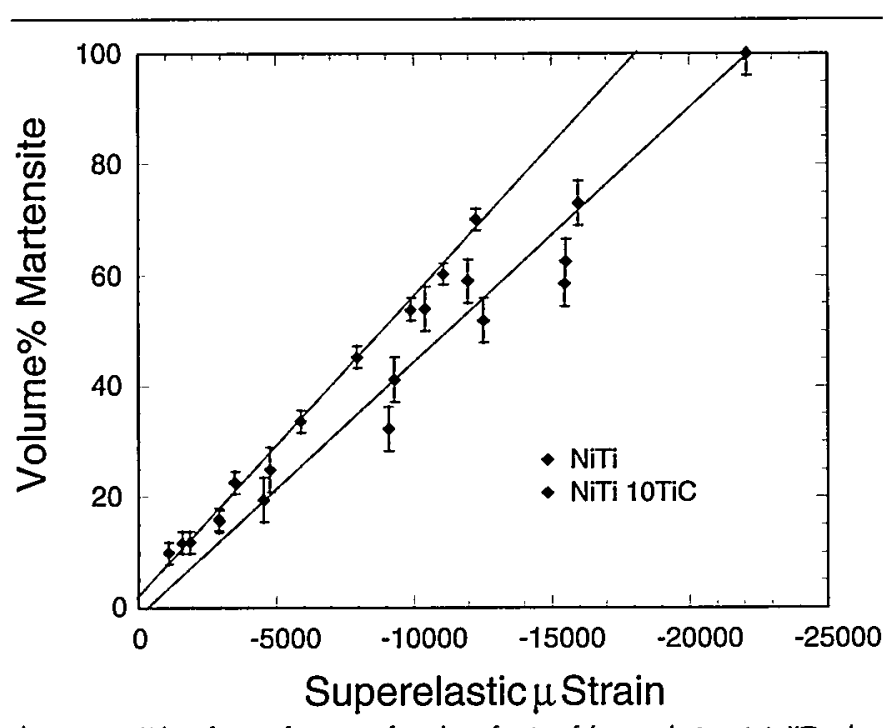

A FIGURE 3. Volume froction of mortensite formed as a function of the superelastic strain in NiTi and NiTHOTIC.

martensite for a given macroscopic superelastic strain than $\mathrm{NiTi}$, probably because of lower texture and the need to accommodate the mismatching $\mathrm{TiC}$ particulates.

We determined by GSAS the elastic strains in the austenite, the martensite, and the reinforcing $\mathrm{TiC}$ particles at various stresses. These strains are used to investigate load transfer in composites where the matrix undergoes a stress-induced phase transformation, ${ }^{6}$ and to indicate that elastic load transfer is observed analogous to composites where the matrix deforms by slip.

A quantitative description of the texture is obtained from GSAS by using a spherical harmonic texture description, which gives significant qualitative differences compared to the less-precise March-Dollase texture formulation. The texture of the originally isotropic austenitic phase increases as it transforms into martensite: the increase can be explained by the crystallographic relationship between the parent austenite phase and the stress-induced martensite, which is highly textured as it forms to produce the maximal strain in the direction of the applied load. Figure 4

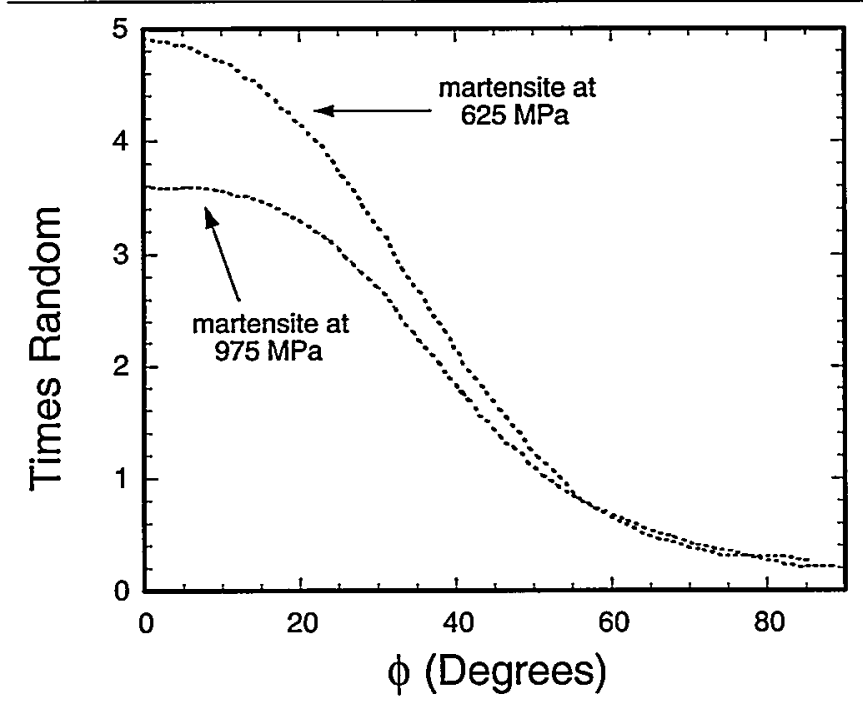

$\Delta$ FIGURE 4. Texture evolution in martensite.

shows martensite texture for applied stresses of $625 \mathrm{MPa}$ and $975 \mathrm{MPa}$, where the $y$-axis is a measure of the number of crystals that are oriented at an angle $\phi$ between the normal to the (100) planes and the loading axis, compared to a randomly oriented polycrystal. Thus, Figure 4 indicates that the martensite has most of its (100) planes aligned perpendicular to the loading axis and that martensite texture is more pronounced at $625 \mathrm{MPa}$ (the beginning of the transformation), than at $975 \mathrm{MPa}$, when most of the martensite has formed. This finding can be explained by the accommodation needed between martensite variants.

Finally, we investigated the effect of stress cycling on $\mathrm{NiTi}$ by performing multiple times the mechanical cycle shown in Figure 1 and collecting data during the 1st, 2nd, and 100th cycles: The critical stress for martensite formation drops and the stress-strain gradient during the transformation increases; this macroscopic evolution of the stressstrain curve is reflected in texture changes as measured by neutron diffraction. ${ }^{7}$

\section{References}

1. D. C. Dunand, D. Mari, M. A. M. Bourke, and J. A. Roberts, "NiTi and NiTi-TiC Composites .4. Neutron-Diffraction Study of Twinning and Shape-Memory Recovery," Metall. Mater. Trans. 27A, 2820 (1996).

2. M. A. M. Bourke, R. Vaidyanathan, and D. C. Dunand, "Neutron-Diffraction Measurement of Stress-Induced Transformation in Superelastic NiTi," Appl. Phys. Lett. 69, 2477 (1996).

3. Engineering Aspects of Shape Memory Alloys, T. W. Duerig, K. N. Melton, D. Stoeckel, and C. M. Wayman, Eds. (Butterworth-Heinemann, London, 1990).

4. A. C. Larson and R. B. Von Dreele, "General Structure Analysis System (GSAS)," Los Alamos National Laboratory report LA-UR-86-748 (1986).

5. R. Vaidyanathan, M. A. M. Bourke, and D. C. Dunand, "Analysis of Neutron Diffraction Spectra Obtained During Stress-Induced Transformations in Superelastic NiTi," to be submitted to J. Appl. Phys.

6. R. Vaidyanathan, M. A. M. Bourke, and D. C. Dunand, paper to be submitted to Acta Mater.

7. R. Vaidyanathan, M. A. M. Bourke, and D. C. Dunand, paper to be submitted to Metall. Mater. Trans. 


\section{Neutron Diffraction Studies of Solid-State Reactions}

\section{E. Üstündag (California Institute of Technology), B. Clausen (LANSCE Division), M. A. M. Bourke (Materials Science and Technology Division and LANSCE Division)}

We study solid-state reactions and phase transitions in ceramics that involve a volume change and generate residual stresses. Our aim is not only to prevent the deleterious effects of these stresses but also to use them to enhance properties. To achieve this, we need to understand the mechanisms that control stress generation. We have thus far investigated the reduction of $\mathrm{NiAl}_{2} \mathrm{O}_{4}$ (which undergoes a volume shrinkage) as a model system. We built a unique controlled-atmosphere furnace to follow, for the first time, the evolution of phases and stresses in situ with neutron diffraction. We found that residual compressive stresses can be generated by this reaction that can place ceramics under compression leading to improved fracture resistance (tempering). We also obtained additional data about the reaction that allowed us to better understand its evolution.

Ceramic materials possess attractive properties such as high strength, stiffness, and hardness; excellent abrasion, creep, corrosion, and oxidation resistance; low density; and unique electrical properties. However, they are brittle and not structurally reliable. To overcome this disadvantage, one method employed is to place the surfaces of ceramics, where most cracks nucleate, under compression so that crack propagation is inhibited. This is called tempering and is used extensively for glass. One objective of this study is to examine the feasibility of tempering using solid-state reactions that can generate residual stresses. The understanding of how these stresses evolve will help avoid their sometimes deleterious effects. This is also a fundamental study of residual stress generation during reactions and phase transformations in ceramics.

The first reaction we investigated was the partial reduction of $\mathrm{NiAl}_{2} \mathrm{O}_{4}$ spinel to a metal ceramic composite of nickel (Ni) and aluminum oxide $\left(\mathrm{Al}_{2} \mathrm{O}_{3}\right){ }^{1-5}$ The volume change that accompanies the reduction is predicted to be about $-18 \%$, theoretically. ${ }^{3}$ The residual stresses are generated here from the constraint imposed by the unreduced part of spinel. If not relaxed or controlled, these stresses can crack specimens. 1,2 The cracking does prove, however, that strain energy is generated from the volume change during reduction.

Our aim in this study was to follow the reduction in situ using neutron diffraction to see how phases and strains evolve. This would help us to understand the operative mechanisms so that we could better manipulate the residual stresses. This is the first study of its kind and proves the extreme advantage of neutron diffraction in obtaining fundamental information about solid-state reactions.

We used the NPD at the Lujan Center for this study. We built a special furnace for this project (Figure 1) with a retort tube made of amorphous silica. The experiment started by heating a spinel sample (Figure 2) in nitrogen up to the reduction temperature.

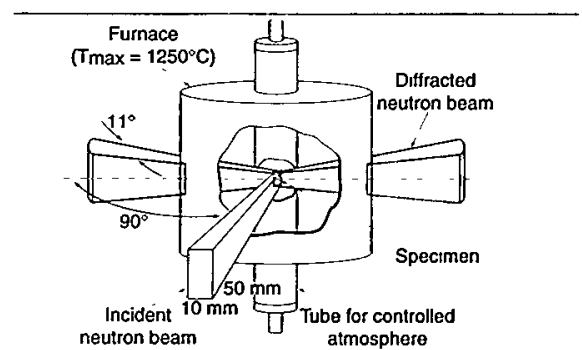

$\Delta$ FIGURE 1. The special funnace provides a controlled atmosphere and allows in situ reaction studies at high temperalures.

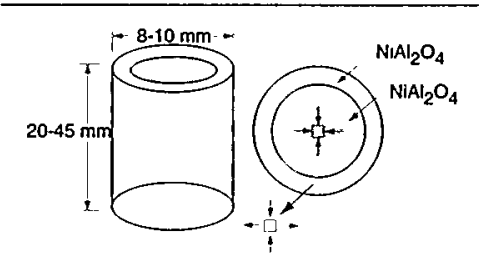

A FIGURE 2. The specimen geometry fogether with the expected stress stote during reduction.

We performed data acquisition during heating and at the reduction temperature. This way, we determined the "strain-free" lattice constant of spinel before the reaction started. We then initiated the reduction reaction by introducing a $\mathrm{CO} / \mathrm{CO}_{2}$ mixture into the furnace. This mixture established a reducing atmosphere with oxygen partial pressures on the order of $10^{-12}$ to $10^{-14}$ (Ref. 3). We acquired data at 0.5 to $1 \mathrm{hr}$ intervals to monitor phase and strain evolution.

The Rietveld refinement technique ${ }^{6}$ we used in the data analysis provides a wealth of information about the reaction. For instance, we can determine the volume fractions of phases as they evolve with a precision of 2 to 3 vol\%. From this, we can calculate the thickness of the reduced layer. Because of the frequent data collection intervals (approximately every $15 \mathrm{~min}$ utes), such data are very valuable in determining the rate controlling step of the reaction. As seen in Figure 3, the NPD results yield many more data points along kinetics curves than the more traditional methods (cf. the three upper curves with the lowest one). A diffusion-controlled reaction will show a parabolic variation of reaction layer thickness versus time,

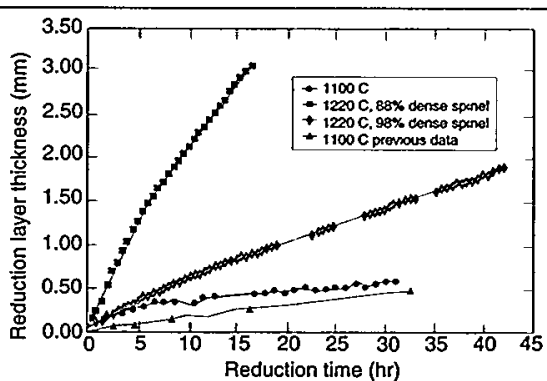

$\Delta$ FIGURE 3. The three upper curves have mony more dato points, which were token on NPD, compared to the lowest curve obtained by internpted tests and microstructurol examination. Such frequent data are voluable in determining the rote confrolling step of the reaction. 
whereas interface-reaction-controlled kinetics is expected to be a linear function of time. Our preliminary results suggest that both types of kinetics are operative, although the diffusion mechanism appears to be more effective. Figure 3 also shows that the reduction temperature and the initial density of spinel are very potent parameters that influence the kinetics.

Figures 4 and 5 illustrate the evolution of strain in unreduced spinel and the reduced composite for two different samples. Using Figure 2, one can visualize a pseudo-hydrostatic compressive strain in spinel. This strain will increase as the reaction proceeds (assuming no relaxation). The strain (and stress) state in the reduced composite, however, is not so straightforward to interpret. Looking down the axis of the sample, radial compression and hoop tension is predicted in this region (Figure 2). However, since the whole specimen is sampled by the neutron beam, an appropriate averaging scheme is needed to see which one of these is more dominant. For this reason, we developed finite element models of the reaction. Figure 6 shows one result where we studied the effect of porosity in the initial spinel. Since the Young's modulus of spinel is a strong function of porosity, a porous spinel will have a low modulus.

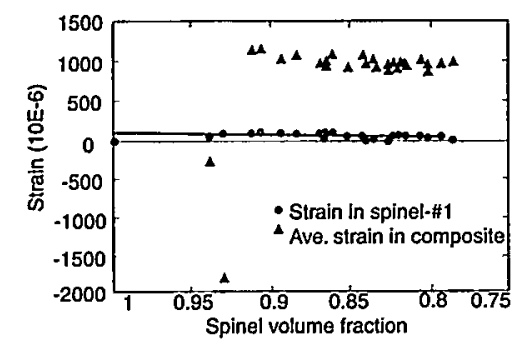

$\Delta$ FIGURE 4. The evolution of stroin in unreduced spinel and the reduced composite. No oppreciable stroin wos buit into sample.

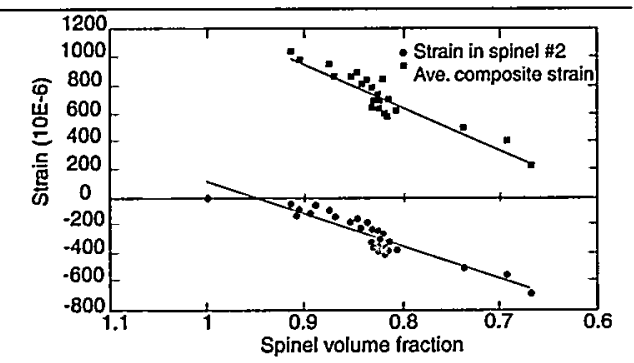

$\Delta$ FIGURE 5. The evolution of stroin in unreduced spinel and the reduced composite. Sample shows compressive stroin buildup in spinel.

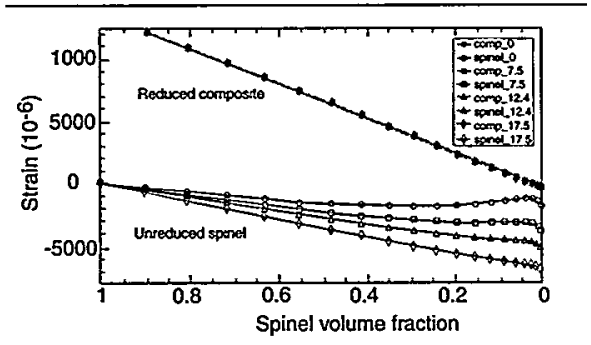

A FIGURE 6. Finite element colculation of stroin generated during reduction as a function of porosity in spinel.

This, in turn, will reduce the constraint exerted by unreduced spinel on the reduction layer and affect strain evolution. The model, overall, predicts a high average tensile strain in the composite at the beginning of the reaction. The unreduced spinel, on the other hand, starts with zero strain and experiences increasing compression with time.

When we compare these predictions with the NPD results (Figures 4 and 5), we realize that no appreciable strain was built in the first sample. We must point to the fact that we did not have a true stress-free reference for the reduced composite, and we used literature data instead. Therefore, the absolute values of strains measured in it are unknown. The trend in the composite, though, supports the observation in spinel. After retrieving the sample, we realized that it had cracked during the reduction, instantaneously relieving all the residual stresses. The NPD results were, therefore, fully supported. The second sample (Figure 5) did show compressive strain buildup in spinel and the composite behavior was collaborative. The maximum stress we calculated from the measured strain values was about $-300 \mathrm{MPa}$.

Based on these preliminary results, we conclude that significant compressive stresses can be generated during the reduction of spinel. Therefore, using this reaction, it may be possible to place ceramic surfaces under compression.

Our current work is aimed at completing the data analyses of several additional reductions performed in NPD. We are also modifying our finite element models to include high-temperature inelastic deformation mechanisms such as creep to obtain a more realistic simulation of the reaction.

This study was supported by an LDRD project at Los Alamos and the Powell Foundation at Caltech.

\section{References}

I. E. Üstündag, R. Subramanian, R. Dieckmann, and S. L. Sass, "In Situ Formation of Metal-Ceramic Microstructures in the Ni-Al-O System by Partial Reduction Reactions," Acta Metall. 43, 383 (1995).

2. E. Üstïndag, P. Ret, R. Subramanian, R. Dieckmann, and S. L. Sass, "In Situ Metal-Ceramic Microstructures by Partial Reduction Reactions in the Ni-Al-O System and the Role of $\mathrm{ZrO}_{2}, "$ Mat. Sci. Eng. A195, 39 (1995).

3. E. Üstündag, "Processing, Structure and Characterization of In-Situ-Formed Metal-Ceramic Composites Obtained by Partial Reduction Reactions," Ph.D. thesis, Cornell University (1995).

4. Z. Zhang, E. Üstündag, and S. L. Sass, "Partial Reduction of $\mathrm{NiAl}_{2} \mathrm{O}_{4}-$ Mechanism and the Influence of Doping" in: Thermodynamics and Kinetics of Phase Transformations, MRS Proceedings, J.S. Im et al., Eds., (MRS, Pittsburgh, Pennsylvania, 1996) Vol. 398 , p. 489.

5. E. Üstündag et al., "Influence of Residual Stresses on the Evolution of Microstructure during the Partial Reduction of $\mathrm{NiAl}_{2} \mathrm{O}_{4}$," Mat. Sci. Eng. A238, 50 (1997).

6. A.C. Lawson and R.B. Von Dreele, "GSAS-General Structure Analysis System," Los Alamos National Laboratory report LA-UR 86-748 (1986). 


\title{
Internal Residual Stress Field Mapping in 21Cr-6Ni-9Mn Steel Resistance-Forged Weld Structures
}

\author{
R. Varma (Materials Science and Technology Division), M. Bourke (Materials Science and Technology \\ Division and LANSCE Division), S. Ellis, J. Newmeyer (Engineering Sciences and Applications Division), \\ P. Brand (National Institute of Standards and Technology)
}

The welds and the weld braze zones in components in the enduring stockpile may have high levels of microstrains and may be prone to microcracks in the presence of hydrogen and corrosive agents. We conducted neutron diffraction strain experiments on welded steel specimens at the National Institute of Standards and Technology (NIST) and also at LANSCE and compared them with finite element calculations of residual strains. There appears to be quantitative similarity between the experimental results and the results of our finite element (FE) calculations, but further refinement of the cooling models in the FE codes are needed before quantitative predictions can be realized.

Residual stress in welded structures may result from volume changes during solidification of the melt zone and shrinkage during cooling in the melt region and in surrounding heat-affected zones. This effect is particularly

important in resistance-forged welded steel components. The resistance-forged welding process uses a temperature of $900^{\circ} \mathrm{C}$ at $6,000 \mathrm{psi}$ of pressure and causes large thermal gradients with shallow thermal penetration into the neighboring regions because of the low thermal conductivity of steel. High residual strains are possible in and around the welds. In some cases, these regions may be exposed to hydrogen with subsequent hydrogen embrittlement. The welds and the weld braze zones in components in the enduring stockpile may have high levels of microstrains and may be prone to microcracks in the presence of hydrogen and corrosive agents. Under such situations, the residual stress in combination with the design stress for the component may lead to fracture at lower stress thresholds at certain locations in and around the welds and may lead to catastrophic failure in the components.

We conducted neutron diffraction strain experiments on welded steel specimens at NIST [Figures 1(a), 1(b), and 1(c)] and also at LANSCE. In the work that was completed

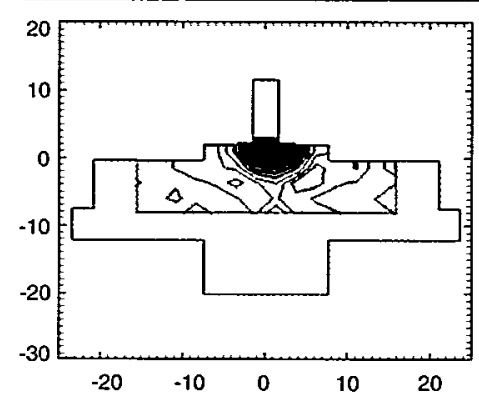

(a)
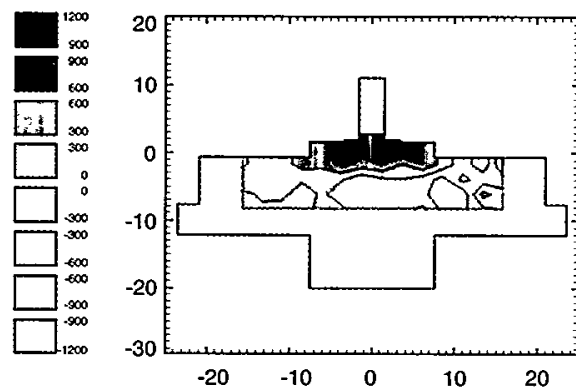

(D)

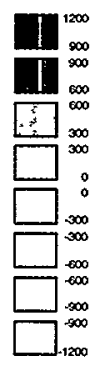

FIGURE 1. Neutron diffraction meosurements of the (a) hoop, (b) rodial, and (c) axiol stroins in a resistance-forged velded 27-6-9- steel port. Measurements were mode at NIST.

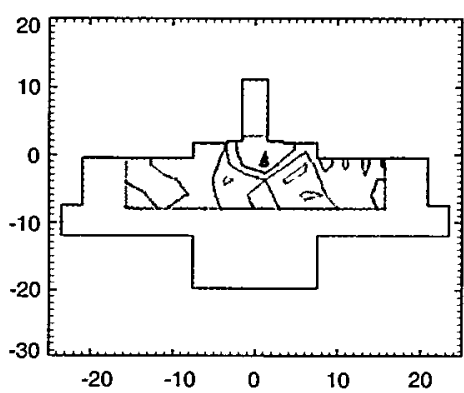

(c)

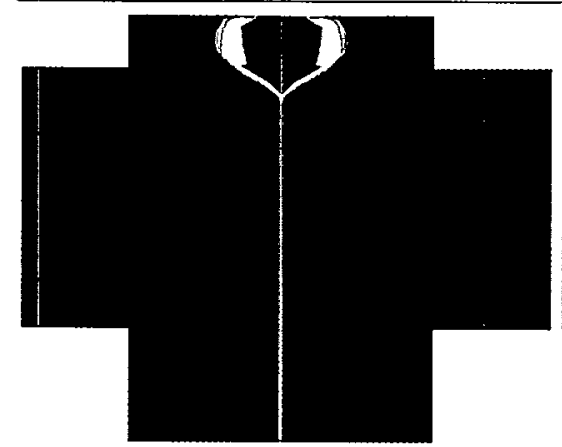

(a)

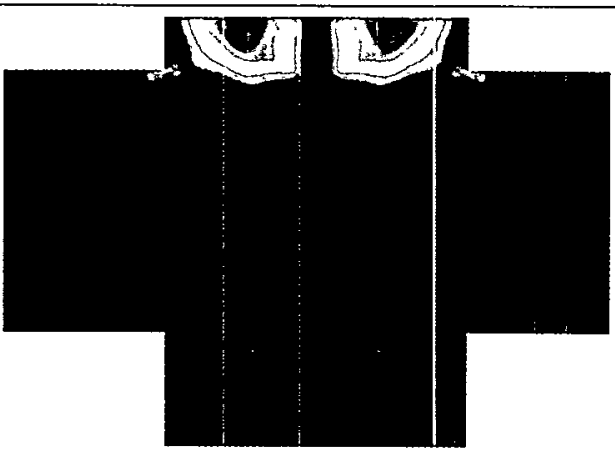

(b)

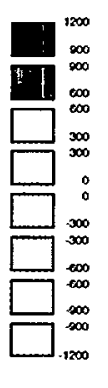
.

西

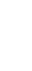


at NIST, using a monochromatic neutron beam from the reactor, we used a gauge volume of $\sim 8 \mathrm{~mm}^{3}$ (2 $\mathrm{mm}$ in each direction) to measure the strain maps for the hoop, radial, and axial strains in the side-welded structure, shown in Figure 1, as well as in a bottom-welded version not shown. Conversion of atomic d-spacing to elastic strains in a neutron diffraction measurement requires the determination of the unstrained lattice plane spacing. Strain fields fall off rapidly away from the welds, hence the d-spacing measured in regions remote from the weld can be considered to be unstrained values $\left(d_{0}\right)$ for our calculations. Conversion of measured lattice plane spacing to stress values requires two further measured values, i.e., the modulus (218.8 GPa) and the Poisson's ratio (0.2845). These values were taken from prior published measurements of 21-6-9 steel.

Finite element modeling of the elastic strain for the experimental grids $(-2 \mathrm{~mm} \times 2 \mathrm{~mm} \times 2 \mathrm{~mm})$ used in the neutron diffraction measurements were made for the hoop, radial, and azimuthal directions. The finite element analyses shown in Figures 2(a), 2(b), and 2(c) may be compared with the experimental measurements shown in Figures 1(a), I(b), and $1(\mathrm{c})$, respectively. There appears to be qualitative similarity between the experimental results and the results of our FE calculations, but further refinement of the cooling models in the $\mathrm{FE}$ codes are needed before quantitative predictions can be realized. 


\title{
Relaxation of Thermal Residual Stresses in NiAl-AIN-Al $\mathrm{O}_{3}$ Composites During Heating
}

\author{
H. Choo, P. Nash (Illinois Institute of Technology), M. Daymond (ISIS, Rutherford Appleton Laboratory), \\ M. A. M. Bourke (Materials Science and Technology Division and LANSCE Division)
}

The nickel-aluminide-based composite containing both AlN dispersion particles and $\mathrm{Al}_{2} \mathrm{O}_{3}$ fibers shows good high-temperature strength and is a potential replacement for current nickel-base superalloys. To better understand the strengthening mechanisms of this composite, we characterized the thermal residual stresses as a function of temperature using the neutron powder diffractometer (NPD). Results show that the residual stresses developed during cooling from the processing temperature relax at around $1200 \mathrm{~K}$ upon reheating. Hence, we conclude that the mechanical properties of this composite are not directly affected by the thermal residual stress above this temperature.

The ordered intermetallic alloy nickel aluminide (NiAl) has potential advantages over current superalloys for use in high-temperature structural applications. These advantages include a high melting temperature $(1922 \mathrm{~K}$-about $300 \mathrm{~K}$ higher than the nickel-base superalloys); low density $\left(5.9 \mathrm{~g} / \mathrm{cm}^{3}\right.$ - approximately $2 / 3$ that of the superalloys); high thermal conductivity; and excellent oxidation resistance. ${ }^{1}$ However, cast polycrystalline NiAl suffers from poor room temperature fracture toughness and poor creep resistance at intended service temperatures (1300-1400 K). Such inadequate mechanical properties pose a major challenge for the alloy designer who wishes to take advantage of those properties that are superior to current superalloys. To address these problems, we developed a hybrid NiAl composite containing both AIN dispersion particles and $\mathrm{Al}_{2} \mathrm{O}_{3}$ fibers. ${ }^{2}$ We intended to achieve a synergistic improvement in mechanical properties, especially creep resistance, by combining the two different strengthening mechanisms: (1) fine AlN dispersion particles (typically less than $1 \mu \mathrm{m}$ ), distributed homogeneously both along grain boundaries and within grains, will inhibit dislocation movement, and (2) long fibers improve strength by load transfer and by constraining macroscopic flow over a wide strain rate range. Mechanical testing at $1300 \mathrm{~K}$ revealed that this composite is one of the strongest NiAl-based alloys developed to date, approaching the level of NASAIR 100 , a first-generation Ni-base single-crystal superalloy. ${ }^{2}$

Most metal-matrix composites reinforced by a ceramic phase have a considerable difference in the coefficient of thermal expansion between the matrix $(\mathrm{NiAl})$ and the reinforcements (both AlN and $\mathrm{Al}_{2} \mathrm{O}_{3}$ ). Thus, on cooling from the fabrication temperature $(1473 \mathrm{~K})$, thermal residual stresses develop as a result of differential thermal contrac- tions of the three phases. These stresses are known to influence mechanical properties such as yield strength ${ }^{3}$ and fatigue resistance. ${ }^{4}$ Furthermore, since these composites are designed for high-temperature application (e.g., turbine blades), understanding the characteristics of thermal residual stresses at elevated temperatures and the effect on creep behavior is critical.

We characterized the thermal residual stresses in the NiAl-AIN-5\% $\mathrm{Al}_{2} \mathrm{O}_{3}$ composite using NPD as a function of temperature. Figure 1 shows the diffraction pattern comprising raw data and Rietveld refinement. In the diffraction pattern, the crosses represent the data and the solid line overlapping the data points is the fit from the refinement. Tick marks identify the refined peak positions of the $\mathrm{Al}_{2} \mathrm{O}_{3}$ (rhombohedral), AlN (hexagonal), and $\mathrm{NiAl}$ (cubic) phases at the bottom of each graph, and the difference curve between measured data and refinement is shown below these. We measured each phase's lattice parameters as well as standards from room temperature to $1500 \mathrm{~K}$ and calculated the lattice strain and corresponding stress from the lattice parameter. We also estimated the residual stresses using a finite element model (FEM). Figure 2 shows the NiAl-AIN$5 \% \mathrm{Al}_{2} \mathrm{O}_{3}$ composite mesh. We calculated the residual stress using $\mathrm{ABAQUS},{ }^{5}$ assuming two-dimensional axisymmetry and a perfectly bonded matrix/reinforcement interface. We included plasticity and creep for the matrix but treated the AlN particles and $\mathrm{Al}_{2} \mathrm{O}_{3}$ fibers as purely elastic.

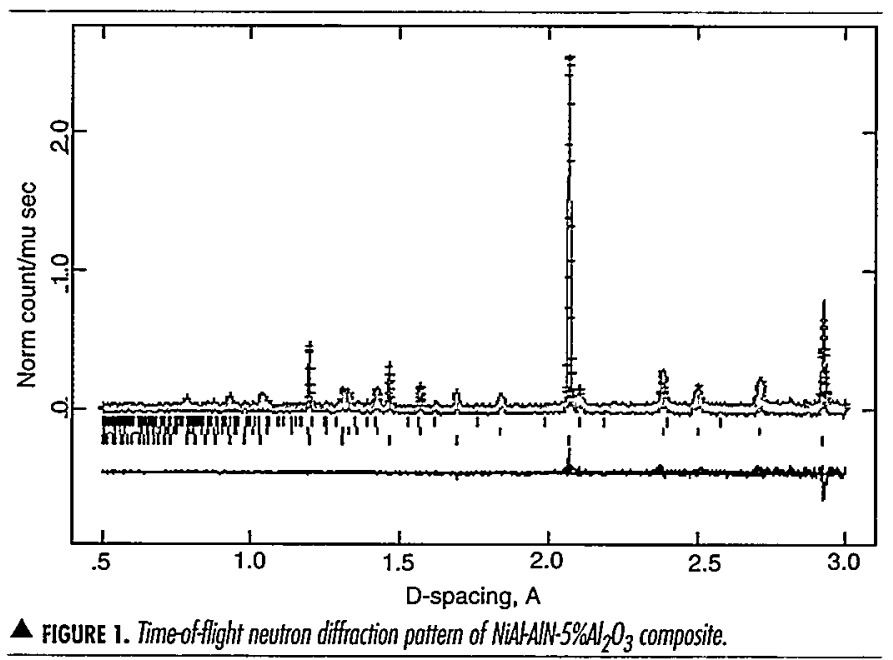

In Figure 3, the data points represent the thermal residual stresses in each phase in NiAl-AIN-5\% $\mathrm{Al}_{2} \mathrm{O}_{3}$ measured with NPD as a function of temperature. The lines in Figure 3 represent the FEM results and are in good agreement with the experimental data. The tensile residual stress 


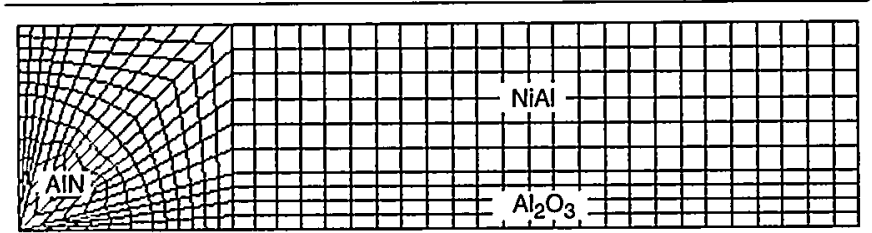

A FIGURE 2. Schematic of the finite element model showing the NiALAIN-5\% $\mathrm{Al}_{2} \mathrm{O}_{3}$ composite mesh.

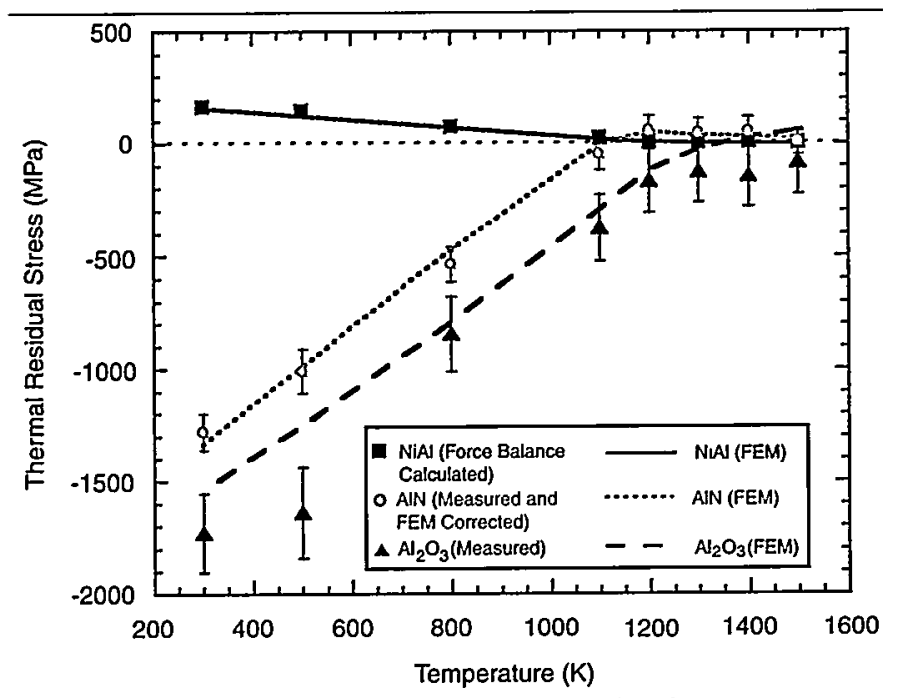

$\triangle$ FIGURE 3. Hydrostatic thermal residuol stresses in NiAl, $\mathrm{ANN}$, and $\mathrm{Al}_{2} \mathrm{O}_{3}$ phases in NiALAN-5\% $\% \mathrm{Al}_{2} \mathrm{O}_{3}$ composite.

developed in the NiAl phase during cooling from the processing temperature decreased as the temperature increased and completely relaxed at around $1200 \mathrm{~K}$, which is significantly lower than the processing temperature $(1473 \mathrm{~K})$. The magnitude of the compressive residual stress in the AIN phase decreased as the temperature increased and developed a small tensile stress above $1100 \mathrm{~K}$. The relaxation temperature of $\mathrm{Al}_{2} \mathrm{O}_{3}$ phase is not quite clear due to uncertainty in the experimental data. However, the FEM result indicates that the residual stress relaxes at around $1300 \mathrm{~K}$. Relaxation of the residual stress at temperatures below the processing temperature and the change of the sign in stress in AlN and $\mathrm{Al}_{2} \mathrm{O}_{3}$ are primarily due to the creep (either dislocation glide or diffusion) of the matrix during the heating cycle. At high temperatures where thermally activated process is important, the thermal residual stresses are known to relax by time-dependent deformation such as creep, which results in a dynamic evolution of the residual stress at high temperatures.

Considering the relaxation of thermal residual stress in the matrix above $1200 \mathrm{~K}$, we conclude that the residual stress above this temperature will not directly affect this composite's creep properties. However, the thermal residual stresses will have some influence on the low and intermediate temperature mechanical properties such as strength and fracture toughness.

\section{References}

I. R. Darolia, "NiAl Alloys for High Temperature Structural Applications," J. Metal 43, 44-49 (1991).

2. H. Choo, P. Nash, and M. Dollar, "Mechanical Properties of NiAl-AlN- $\mathrm{Al}_{2} \mathrm{O}_{3}$ Composites," Materials Science and Engineering $A$ 239-240, 464-471 (1997).

3. R. J. Arsenault and M. Taya, "Thermal Residual Stress in Metal Matrix Composite," Acta Metall. 35, 651-659 (1987).

4. P. Rangaswamy, M. A. M. Bourke, P. K. Wright, N. Jayaraman, E. Kartzmark, and J. A. Roberts, "The Influence of Thermal-Mechanical Processing on Residual Stresses in Titanium Matrix Composites," Materials Science and Engineering A 224, $200-209$ (1997).

5. ABAQUS User's Manual (Hibbitt, Karlsson \& Sorensen, Inc., Pawtucket, RI, 1996). 


\title{
Texture Evolution in the Olivine Polymorphs at High Pressure: Dynamics of the Earth's Upper Mantle
}

\author{
K. A. Bennett, R. B. Von Dreele (LANSCE Division), H. W. Green II, L. Dobrynyetsinaya, E. Riggs \\ (University of California at Riverside), P. C. Burnley (Georgia State University)
}

It is well established that the seismic anisotropy of the shallow upper mantle beneath the oceans is a result of preferred orientations (texture) of plastically deformed $\alpha$-olivine crystals produced from convection. With progressive increases in pressure, such as during subduction, $\alpha$-olivine undergoes a polymorphic phase transformation to wadsleyite ( $\beta$-phase). To understand the seismic structure and dynamics of the deeper upper mantle, we report the first quantitative texture data measured in bulk polycrystalline specimens of experimentally deformed $\alpha$ - and $\beta$-phases that were measured by neutron diffraction. Samples were $\mathrm{Mn}_{2} \mathrm{GeO}_{4}$ that crystallizes in both the olivine and wadsleyite structures and thus serves as a useful analogue of upper mantle material on either side of the 410-km seismic discontinuity. By neutron diffraction on $H I P D$, we find $(100)_{\alpha} / /(001)_{\beta} / /$ max. compression; $(010)_{\alpha} / /(100)_{\beta}$ and $(001)_{\alpha} / /(010)_{\beta}$ perpendicular to compression.

Texture (preferred crystallographic orientation) in olivine has long been known to be responsible for the observed seismic anisotropy in the shallow part of the Earth's upper mantle. ${ }^{1}$ Large-scale shear processes caused by large-scale convection cells deform olivine crystals plastically, which causes them to develop strong preferred orientations. These natural textures can be seen on the surface of the Earth when samples are thrust up to the surface at continental/oceanic convergent zones or, for example, blasted out in volcanic pipes.

With increases in pressure, orthorhombic olivine undergoes a polymorphic phase transformation to wadsleyite ( $\beta$-phase) with a modified spinel structure and then to its true cubic spinel form, ringwoodite $\left(\gamma\right.$-phase). ${ }^{2}$ The $\alpha \rightarrow \beta$ transition is widely considered to be responsible for the prominent seismic discontinuity occurring at the top of the mantle transition zone at $\sim 410 \mathrm{~km}$ depth, and the $\beta-\gamma$ transition may be responsible for the discontinuity sometimes seen at $\sim 520 \mathrm{~km}$ depth. ${ }^{3}$ Little is known about the texture that might be produced by phase transformation or plastic flow of the high pressure polymorphs of olivine in these transition zones mostly because these natural samples are never transported untransformed to the surface. One study of preferred orientation of wadsleyite has been published, ${ }^{4}$ but the experiment involved transformation of a single crystal of olivine into a polycrystal of wadsleyite, hence it is not clear whether the texture that developed was controlled by deformation or crystallography.
In this work we present the first measured textures of $\beta$-phase coexisting in experimentally deformed bulk $\alpha$-olivine samples. ${ }^{5}$ In all experiments we used a germanium analogue to silicate olivine that can be produced at lower pressures in the laboratory ( $5 \mathrm{GPa}$ instead of $13 \mathrm{GPa}$ ) to undergo phase change and simulate mantle conditions.

We investigated quantitatively the texture in both undeformed and deformed $\mathrm{Mn}_{2} \mathrm{GeO}_{4} \alpha$-olivine and two specimens partially transformed to the $\beta$-phase. Using neutron diffraction on HIPD at the Lujan Center, we measured the textures resulting from phase change by time-of-flight neutron diffraction using a generalized spherical harmonic method combined with Rietveld analysis to calculate complete pole figures. ${ }^{6}$ This method of neutron diffraction combined with Rietveld analysis was especially important in this texture study because the coarse-grained multiphase samples contained low symmetry phases and multiple peak overlaps (Figure 1) so that the traditional method of $\mathrm{x}$-ray diffraction would be impossible. With the Rietveld method we were able to extract orientation distribution coefficients and complete pole figures from any number of reflections from all phases in the bulk (3-mm diameter $\times 3-\mathrm{mm}$ long, $1 / 2$ cylinder) sample.

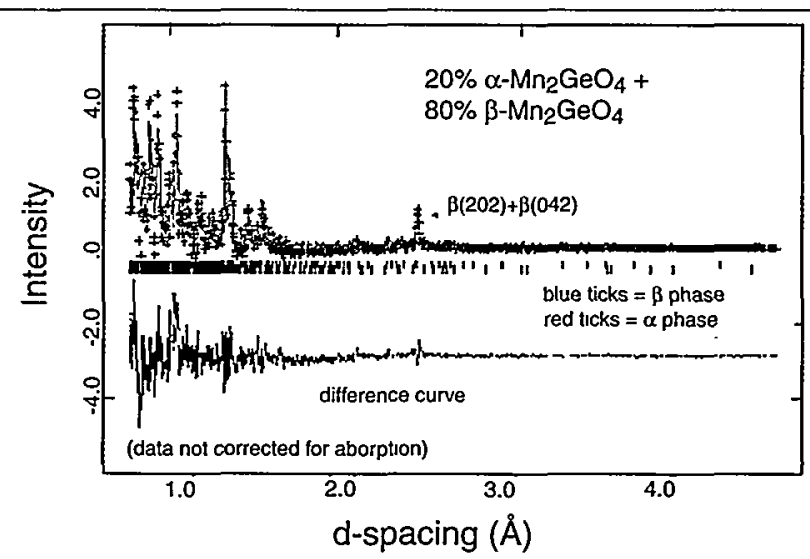

$\Delta$ FIGURE 1. Neutron diffraction spectrum of $\alpha-\beta / \mathrm{An}_{2} \mathrm{GeO}_{4}$ demonstrating multiple peak overlops. Dota meosured on HIPD on a 3-mm-diameter $1 / 2$ cylinder for approximately 1 hour.

Note that the wt\% present and the pole figures for both phases were successfully extracted from the data even when one phase constituted only $20 \%$ of the total weight of these small specimens. As expected, undeformed samples were nearly random (without preferred orientation). Pole figures from the transformed and deformed samples (Figure 2) showed strong preferred orientation with $(100)_{\alpha} / /(001)_{\beta}$ parallel to compression; $(010)_{\alpha} / /(100)_{\beta}$ and $(001)_{\alpha} / /(010)_{\beta}$ perpendicular to compression. 


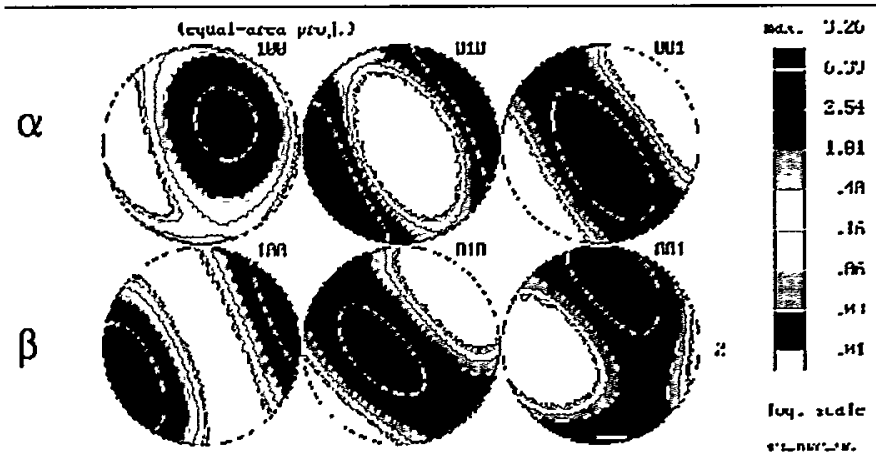

$\triangle$ FIGURE 2. Texture results of $\alpha-\beta \mathrm{Mn}_{2} 6 \mathrm{eO} \mathrm{O}_{4}(20$ wt. $\% \beta$ ). $\alpha$ and $\beta$ pole figures coexisting in a polycrystolline specimen $20 \%$ hansformed from $\alpha$ to $\beta$. The direction of compression is in the center of the pole figure.

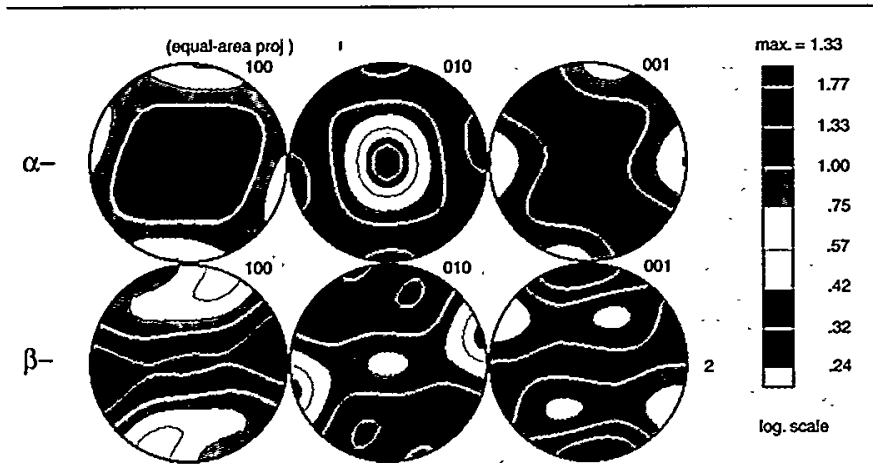

$\Delta$ FIGURE 3. Texture resuls of $\alpha-\beta \mathrm{Mn}_{2} \mathrm{GeO}_{4}(70$ w. $\% \beta$ ). $\alpha$ and $\beta$ pole figures coexisting in a polycrystolline specimen $70 \%$ tronsformed from $\alpha$ to $\beta$. The direction of compression is in the cen ter of the pole figure. The $\beta$ phose (100) pole forms o girdle about (010).

Figure 3 shows moderate preferred orientation with $(100)_{\alpha}$ parallel to compression. $(100)_{\beta}$ forms a double belt perpendicular to the compressor axis. The texture we produced in $\alpha-\mathrm{Mn}_{2} \mathrm{GeO}_{4}$ is the same as that developed by deformation in silicate olivine, both in the laboratory and in nature (note that because of a curiosity of crystallographic nomenclature, $\mathrm{Mn}_{2} \mathrm{GeO}_{4}$ is not indexed the same as silicate olivine; e.g., the $\alpha$-axis in $\mathrm{Mn}_{2} \mathrm{GeO}_{4}$ is the largest d-spacing, making it analogous to the $\beta$-axis of silicate olivine). These preliminary results demonstrate the power of time-of-flight neutron diffraction and Rietveld analysis to obtain complete texture determinations from very small amounts of material.
However, because of the complicated histories of the transformation experiments, it was not possible to determine exact mechanisms of texture development in the $\beta$-phase. Texture could have been the result of deformation, or it could have arisen from either selective growth based upon previously induced texture in the $\alpha$-phase or selective nucleation based upon preferential deformation of certain orientations during the early deformation of the $\alpha$-phase. We are investigating these questions in more detailed deformation and transformation experiments.

\section{References}

1. A. Nicolas and N. I. Christensen, "Formation of Anisotropy in Upper Mantle Peridotites," reviewed in Composition, Structure and Dynamics of the Lithosphere-Asthenosphere System, K. Fuchs and C. Froidevaux, Eds., Geodynamics 16, 111-123 (1987).

2. H. W. Green II, T. E. Young, D. Walker, and C. H. Scholz, "The Effect of Nonhydrostatic Stress on the $\alpha \rightarrow \beta$ and $\alpha \rightarrow \gamma$ Olivine Phase Transformations," in High-Pressure Research: Application to Earth and Planetary Science, Y. Syono and M. H. Manghnani, Eds., Geophys. Monog. Series 67, 229-235 (1992).

3. M. P. Flanagan and P. M. Shearer, "Global Mapping of Topography on Transition Zone Velocity Discontinuities by Stacking SS Precursers," J. Geophys. Res. 103, 2673-2692 (1998).

4. G. Y. Bussod, T. Katsura, and T. G. Sharp, "A View into the Earth's Transition Zone: Experimental Deformation of $\beta-\mathrm{Mg}_{1.8} \mathrm{Fe}_{0.2} \mathrm{SiO}_{4}$ at $\mathrm{High}$ Pressures and High Temperatures," TERRA Nova 6: Abs. Suppl. 1, 9 (1994).

5. K. Bennett, H. W. Green II, Q. Bai, and L. Dobrzhinetskaya, "Preferred Orientation Development in $\alpha$ - and $\beta$ - $\mathrm{Mn}_{2} \mathrm{GeO}_{4}$ : $\mathrm{Implications} \mathrm{for}$ Flow in the Mantle Transition Zone," in preparation.

6. R. B. Von Dreele, "Quantatative Texture Analysis by Reitveld Refinement," J. Appl. Crystallogr. 30, 517-525 (1997). 


\title{
Texture Evolution in High-Temperature Superconducting Oxide/Silver Tapes under Heat Treatment
}

\author{
R. B. Von Dreele (LANSCE Division), J. F. Bingert (Materials Science and Technology Division), \\ H.-R. Wenk (University of California at Berkeley), G. N. Riley (American Superconductor Corp.)
}

Certain mixed metal oxides become superconductors when their temperatures reach the boiling point of liquid nitrogen. With the possibility of commercial use, a superconducting wire or tape is being developed that encases the rather brittle oxide in a silver metal sheath. To carry large electrical currents the superconductor must be highly oriented within the tape. This orientation is affected by a combination of mechanical and heat treatments. We have developed a means of determining this orientation in situ by use of neutron scattering techniques that give a means of following the development of the orientation during processing.

Since the discovery ${ }^{1}$ that certain mixed metal oxides exhibit superconducting behavior at temperatures close to the boiling point of liquid nitrogen, there has been considerable effort to develop commercially useful components using these materials. The development of useful superconducting wire or tape is perhaps the most important of these. One particularly fruitful approach to this problem has been to encase the oxide in a silver metal sheath to provide a means of protecting the rather brittle oxide. The silver sheath also gives the resulting tape sufficient flexibility so that it can be wound in a solenoid for use, for example, in a superconducting magnet. However, the superconducting property of the oxide material is strongly dependent on the direction that the electrical current flows through the crystal structure. For the $\mathrm{Bi}_{2} \mathrm{Sr}_{2} \mathrm{Ca}_{2} \mathrm{Cu}_{3} \mathrm{O}_{\mathrm{x}}$ " $\mathrm{Bi}-2223$ " superconductor (Figure 1), the best conduction is understood to occur in the square $\mathrm{CuO}$ planes at the ends and in the center of the unit cell and in a direction that is perpendicular to the long axis of the unit cell. Thus, to achieve high currentcarrying capacity in these wires, the Bi-2223 oxide crystals must have a strongly developed preferred orientation or texture so that the long unit cell axis is normal to the tape axis. ${ }^{2}$

Over the past few years, manufacturing techniques involving rolling and subsequent heat treatments ${ }^{3}$ have been developed that enhance the supeconducting current-carrying capacity of these wires. However, direct measurement of either the texture or composition of the oxide has been difficult to achieve because of the complexity of the materials and interference from the silver sheath. Consequently, the connection between composition and texture with superconductivity properties could not be established as a function of processing conditions.
Recently, we have developed a technique 4 to determine the texture of polycrystalline components that employs the neutron time-of-flight (TOF) diffraction data obtained on HIPD at the Lujan Center. In this approach, a polycrystalline sample is positioned at several different angular orientations with the TOF diffraction patterns being recorded at each one. The texture causes the peak intensities in these diffraction patterns to be different from what would be expected if the crystallites that make up the sample were oriented in a random way. The texture information is extracted by a modeling and refinement computer program ${ }^{5}$ by processing the entire suite of several tens of these diffraction patterns. The texture effect is modeled by making use of spherical harmonics whose coefficients form part of the set of refined parameters. Other aspects of the sample (crystal structure, phase composition, etc.) are also modeled and refined as part of this procedure. This method is particularly useful for the superconducting tape problem because of the relatively high penetration of neutrons (compared with $\mathrm{x}$-rays) through the silver sheath and superconducting oxide. Moreover, the method can deal successfully with the complexity of these diffraction patterns that result from the several crystalline phases that exist in these materials.

In a preliminary study we successfully examined three silver-sheathed Bi-2223 superconducting tapes using neutron diffraction on HIPD. One was in the "as-rolled" condition, but the other two had been subjected to part of the heat treatment needed to produce highly oriented superconductors. One of the diffraction 
patterns is shown in Figure 2. The analysis showed the development of very strong texture in the desired orientation (Figure 3) after the second step in the heat treatment along with essentially complete conversion of a precursor oxide $\mathrm{Bi}_{2} \mathrm{Sr}_{2} \mathrm{CaCu}_{2} \mathrm{O}_{\mathrm{x}}$ " $\mathrm{Bi}-2212$ " to the desired superconducting $\mathrm{Bi}-2223$ oxide. The as-rolled material showed a nearly random orientation for the $\mathrm{Bi}-2223$ superconductor but a relatively strong texture for the precursor $\mathrm{Bi}-2212$ material. The silver sheath showed a very typical texture obtained from the rolling of a metal sheet. Because the $\mathrm{Bi}$ 2223 texture only develops under heat treatment, it may be that the already existing texture in the precursor $\mathrm{Bi}-2212$ formed in the rolling process acts as a template for the formation of an oriented superconductor. We expect to further clarify this mechanism for the production of highly textured, silver-sheathed Bi-2223 superconductor tape as well as characterize the role of temperature treatments.

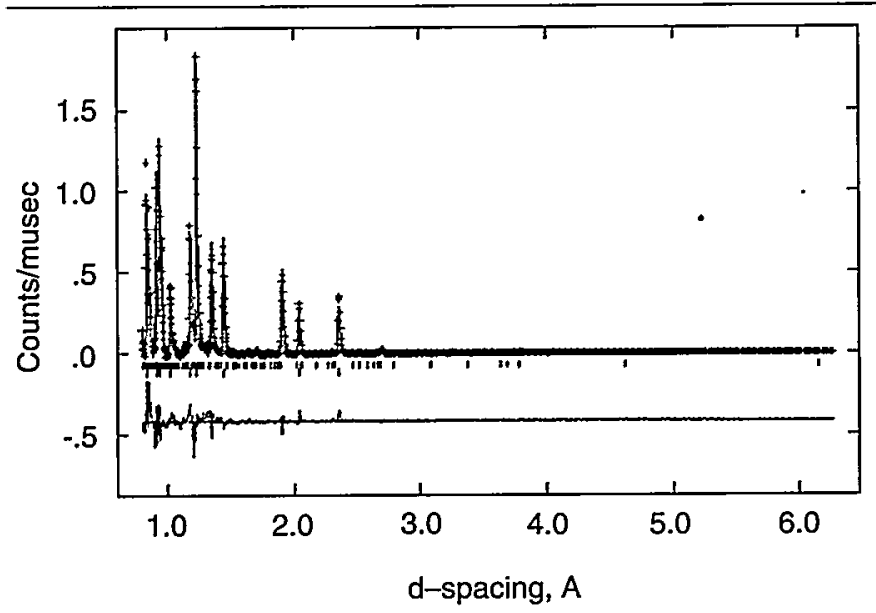

A FIGURE 2. Example neutron diffraction poltem from silversheathed $B i-2223$ tope. The symbol (t) represents the observed intensity; the line is calculated from a model refinement, the fic makks show reflection positions for Bi-2223 ond silver. The bockground contribution has been subtracted for darity.

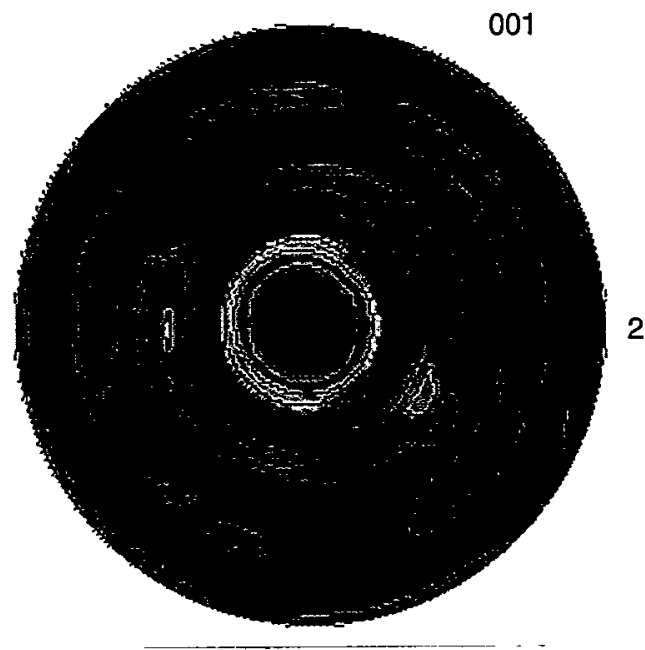

58.27
16.89
4.89
1.42
.41
.12
.03
.01
Log. scale
$29-J u l-97$

$\triangle$ FIGURE 3. Pole figure showing angular distribution of bosal plane normals (001) about a normol to the silversheathed Bi2223 superconductor tope. Rings are residual artifacts from the analysis.

\section{References}

1. J. G. Bednorz and K. A. Müller, "Possible High Tc Superconductivity in the Ba-La-Cu-O System," $Z$. Phys. B 64, 198 (1986).

2. D. Dimos, P. Chaudhari, J. Mannhart, and F. K. Legoues, "Orientation Dependence of Grain-Boundary Critical Currents in $\mathrm{YBa}_{2} \mathrm{Cu}_{3} \mathrm{O}_{7}-\delta$

Bicrystals," Phys. Rev. Lett. 61, 219 (1988).

3. K. H. Sandage, Jr., G. N. Riley, Jr., and W. L. Carter, "Critical Issues in the OPIT Processing of High-Jc BSCCO Superconductors," J. Met. 43, 31 (1991).

4. R. B. Von Dreele, "Quantitative Texture Analysis by Rietveld Refinement," J. App. Crystallogr. 30, 517 (1997).

5. A. C. Larson and R. B. Von Dreele, "General Structure Analysis System - GSAS," Los Alamos National Laboratory report LA-UR 86-748 (1994). 


\section{Neutron Diffraction and the Anomalous Melting Point of Plutonium}
A. C. Lawson (Materials Science and Technology Division), B. Cort (Nuclear Materials Technology Division), J. A. Roberts (LANSCE Division), B. I. Bennett (Applied Theoretical and Computational Physics)

\section{A rule for the melting point of materials, devised by F. A. Lindemann in 1910, was based on Einstein's theory on the heat capacity of materials. Using Einstein's theory, Lindemann was able to devise a simple formula to estimate the temperature at which atoms begin to collide with their neighbors, thus relating the characteristic Einstein temperature to the melting temperature of materials. In this report, we explain how Lindemann's rule can be used with neutron diffraction data on the light actinides to understand the melting points of these materials.}

No comprehensive theory for the melting points of materials has ever been proposed. The best thing we have is a rule that was devised by F. A. Lindemann in 1910.1 Lindemann was inspired by the recent publication of Einstein's theory on the heat capacity of materials. $\mathrm{He}$ wanted to devise a way of estimating the characteristic Einstein temperature, the materials property on which the predictions of Einstein's theory is based. Lindemann realized that the amplitudes of the thermal atomic vibrations, which also depend on the Einstein temperature, could not become too large before the material would shake itself apart. The mean-square amplitude of thermal vibration is directly proportional to the absolute temperature and inversely proportional to the square of the Einstein temperature, so Lindemann could use Einstein's theory to estimate the temperature at which the atoms would begin to experience violent collisions with their neighbors. Thus he was able to devise a simple formula that related the Einstein temperature to the melting temperature. His paper was published just before the invention of the Debye theory in 1914 , which is still the basis of the modern theory of heat capacities and atomic vibrations.

It is easiest to understand Lindemann's rule in terms of $\kappa_{\mathrm{atom}}$, the atomic spring constant of the material. $\kappa_{\mathrm{atom}} \delta \mathrm{x}$ is the force required to increase the separation of two atoms in the crystal by a distance $\delta x$. At high enough temperatures, the mean-square amplitude of atomic vibration, $\left\langle u^{2}\right\rangle$, is proportional to the temperature according to

$$
<u^{2}>=\frac{k_{B} T}{\kappa_{\text {atom }}}
$$

(In this formula, $\mathrm{k}_{\mathrm{B}}$ is the Boltzmann constant.) The Lindemann rule, as modified by Gilvarry, ${ }^{2}$ reads

$$
T_{\text {melt }}=(0.083)^{2} \kappa_{\text {atom }} \Omega^{3}{ }_{k_{B}}^{2},
$$

where $\Omega$ is the atomic volume. The significance of the factor 0.083 is that the material is suppose to melt when the vibrational amplitude reaches $8.3 \%$ of the interatomic spacing. The atomic spring constants can be obtained from measurements of the Debye temperature, the modern successor to the Einstein temperature, with the formula

$$
\kappa_{\text {atom }}=\frac{m}{3}\left(\frac{k_{B} \Theta_{\text {Debye }}}{h}\right)^{2} .
$$

Debye temperatures, $\Theta_{\text {Debye }}$, are usually determined from low-temperature heat-capacity measurements. Numerical values can be obtained in any textbook on solid-state physics.

At Los Alamos, the Science-Based Stockpile Stewardship effort has focused our attention on the light actinides, plutonium in particular. For $\mathrm{Pu}, \Theta_{\text {Debye }}=132 \mathrm{~K}$, so $\mathrm{K}=40.3$ $\mathrm{N} / \mathrm{m}$. The atomic springs are comparable in strength to those on a garage door mechanism. We can also calculate the melting point using the Lindemann rule: for plutonium, $\Omega=24.9 \AA$, so $T_{\text {melt }}=1716 \mathrm{~K}$. This is too high by a factor of two! The actual melting point is $914 \mathrm{~K}$, and this has long been considered anomalously low. ${ }^{3}$ The discrepancies for the other light actinides (except thorium) are even larger.

Does this discrepancy mean that something is wrong with the Lindemann criterion? No, the problem comes from the temperature dependence of $\kappa_{\text {atom }}$, which is strong enough that a large correction must be made to get the correct melting point. $\Theta_{\mathrm{DW}}$ decreases quite strongly with temperature-so strongly that its value is only $89 \mathrm{~K}$ at the melting point. The corresponding $\kappa_{\text {atom }}$ is only $18.3 \mathrm{~N} / \mathrm{m}$. When this value is used, a value of $779 \mathrm{~K}$ is obtained for the

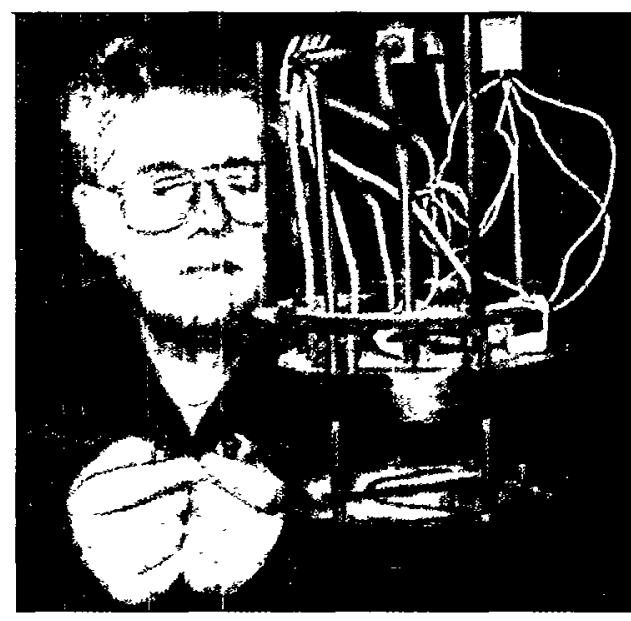

$\Delta$ FIGURE 1. A. Lawson demonstrofes the special furnace used of LANSCE for meosurements of $\Theta_{\text {Debye }}$ of plutonium. 
melting point, in much better agreement with the experiment. The discrepancy has been reduced from $88 \%$ to $15 \%$.

We have used neutron diffraction at LANSCE and at the Intense Pulsed Neutron Source to measure the temperature dependence of $\Theta_{\text {Debye }}$ for all the light actinides (Figure 1). The sort of data that we get appear in Figure 2, which shows the mean-square atomic vibration amplitude plotted versus temperature. It is immediately apparent that both the slope and the curvature of the plutonium data are much larger that those of thorium. The larger slope means that the $\Theta_{\text {Debye }}$ (or $\kappa_{\text {atom }}$ ) is smaller. The larger curvature means that the temperature dependence is larger. We have data like these for all the light actinides. $4,5,6$

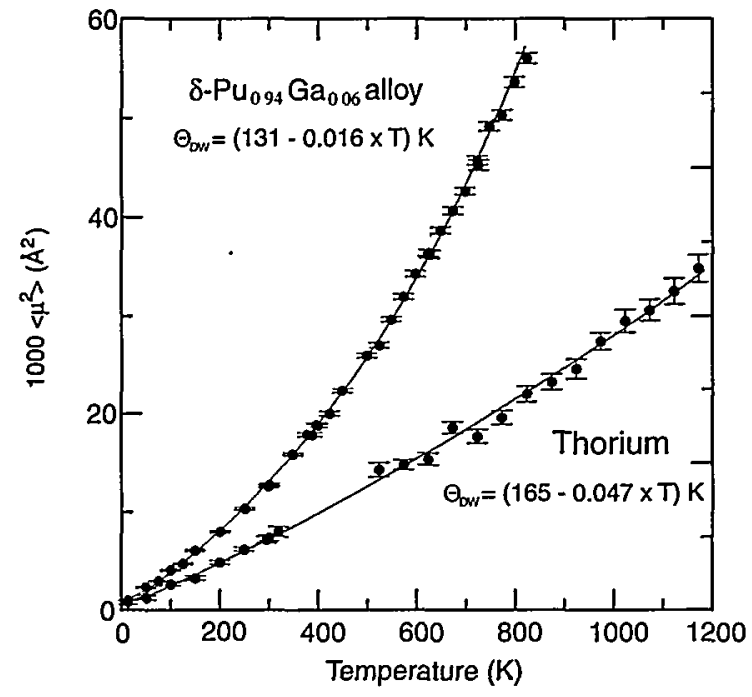

$\Delta$ FIGURE 2. Meonsquare thermol vibration amplitudes for thorium metal and for a dphose PuGa olloy. The two materials hove the same crystol structure, but their behovior is quite different. The atomic force constants for Pu are smoller and more femperaturedependent than those for $T h$.

Figure 3 shows the melting points for the light actinides together with two estimates of the melting point based on the Lindemann rule. The black bars show the measured melting points. The red bars show the Lindemann estimate of the melting point based on the value of $\kappa_{\text {atom }}$ uncorrected for its temperature dependence. The blue bars show the Lindemann estimate with $\kappa_{\text {atom }}$ corrected for its temperature dependence with data like that shown in Figure 2. The corrected data provide a good estimate of the melting point.

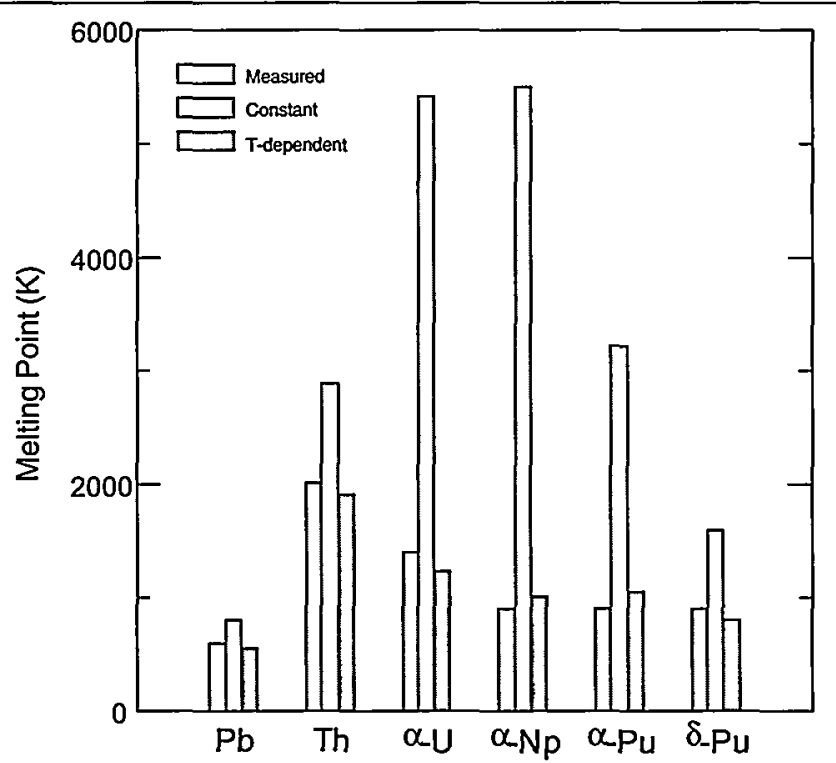

$\Delta$ FIGURE 3. Melfing points for the light actinides. Three values are shown for each material: the measured value, the value colculated from the low femperature atomic force constants, and the volue colculated from the force constont evaluated ot the meling point. Values for lead are shown for comparison.

Even though the physical basis of the Lindemann rule is not very well established, it is quite satisfactory to bring the light actinides into the group of materials that are described by this rule. The physical origins of the low melting points for the light actinides are now to be sought in the temperature dependencies of the elastic propertiesand these are now amenable to calculation.

As far as applications of plutonium are concerned, variations of the melting point of even $10 \%$ would mean profound differences in both performance and processing techniques. The melting point is well worth understanding.

\section{References}

1. F. A. Lindemann, "Über die Berechnung Molecularer Eigenfrequenzen," Phys. Z. 11, 609-612 (1910).

2. J. J. Gilvarry, "The Lindemann and Gruïneisen Laws," Phys. Rev. 102, 308-316 (1956).

3. B. T. Matthias, W. H. Zachariasen, G. W. Webb, and J. J. Engelhardt, "Melting Point Anomalies," Phys. Rev. Lett. 18, 781 (1967).

4. A. C. Lawson, J. A. Goldstone, B. Cort, R. I. Sheldon, and E. M. Foltyn, “Atomic Thermal Vibrations of the Light Actinide Elements," J. Alloys Compd. 213/214, 426-428 (1994).

5. A. C. Lawson, J. A. Goldstone, B. Cort, R. I. Sheldon, and E. M. Foltyn, "Debye-Waller Factors of the Light Actinide Elements," in Actinide Processing: Methods and Materials, B. Mishra and W. A. Averill, Eds. (TMS, Warrendale, Pennsylvania, 1994) pp. 31-44.

6. A. C. Lawson et al., "Debye-Waller Factors of Thorium Metal," IPNS Experiment Report, Argonne National Laboratory (1996) (submitted). 


\section{Correlated-Electron Physics}

\section{N. Argyriou (LANSCE Division), H. Nakotte (New Mexico State University and LANSCE Division), R. A. Robinson (LANSCE Division)}

Correlated-electron materials are those whose properties cannot be covered fully by one-electron bandstructure calculations, or stated another way, are materials in which many-body electron physics is crucial to their properties. They include heavyfermion (f-electron) systems, high- $T_{c}$ and other unconventional superconductors, and colossal-magnetoresistance (CMR) manganites. LANSCE and its users have considerable activity in this area, and we give three examples of work performed using HIPD, $S C D$, and PHAROS: the magnetic and crystallographic structures of $U P d_{2} P b$, the quadrupolar Kondo system PrAg In, and the naturally layered CMR compounds $\mathrm{La}_{2-2 x} \mathrm{Sr}_{1+2 x} \mathrm{Mn}_{2} \mathrm{O}_{7}$.

The condensed-matter physics community at large has an intense interest in correlated-electron materials, and Los Alamos has played a leading role in this field from the beginning. This class of materials covers heavy-fermion materials, which have strongly enhanced low-temperature thermodynamic properties, high-temperature superconductors, manganites exhibiting magnetically driven metal-insulator transitions (so-called colossal-magnetoresistance or CMR materials), many other transition-metal oxide systems, and other exotic superconductors. At LANSCE, we have contributed to the basic research on a number of the materials of current interest, and we briefly describe our work on three of them.

We have studied the heavy-fermion Heusler alloy $\mathrm{UPd}_{2} \mathrm{~Pb}$ in collaboration with colleagues at the University of California at San Diego and the National Institute of Standards and Technology (NIST). ${ }^{1}$ Our main result is that there is a previously undiscovered martensitic structural transition at $\sim 210 \mathrm{~K}$, where the cubic crystal distorts to form a sheared monoclinic structure, as shown in Figure 1(b). At the lower temperature of $40 \mathrm{~K}$, the material becomes antiferromagnetic, with magnetic moments alternating in

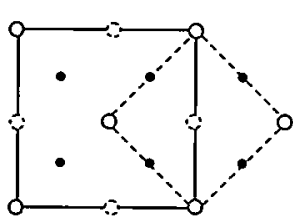

(a)

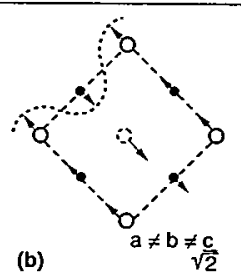

(b)

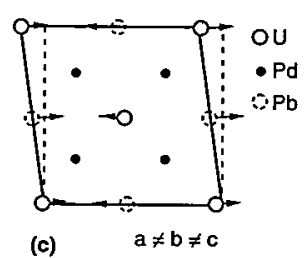

(c) direction as shown in Figure 1(c). We were even able to determine the moment direction within the sheared monoclinic cell of the martensitic phase. These structures were determined on HIPD, while the transition temperatures were determined using selected reflections at NIST.

The second example is another heavy-fermion Heusler alloy $\operatorname{PrAg}_{2} \mathrm{In}$, which has one of the largest reported electronic specific heats $\left(\gamma=6.5 \mathrm{Jmol}^{-1} \mathrm{~K}^{-2}\right)$, and which we have studied on PHAROS in collaboration with University of California at Riverside, Ames Laboratory, and Argonne National Laboratory. ${ }^{2}$ There is good reason to believe that the correlated state develops from a nonmagnetic crystalfield doublet ground state, and the data shown in Figure 2 confirm this definitively. In the level diagram, all allowed transitions are shown, and the $\Gamma_{3}-\Gamma_{4}$ matrix element (transition intensity) has roughly twice the strength of the $\Gamma_{3}-\Gamma_{5}$ matrix element, in accordance with the energy spectrum shown in Figure 2. The importance of this result is that one normally thinks of heavy-fermion behavior in terms of the Kondo effect (an interaction between a local magnetic impurity and the conduction electrons). This effect cannot apply to a nonmagnetic ground state, and therefore the prospect exists of the more exotic quadrupolar Kondo effect in this system.

Our third example is a study of the naturally layered CMR materials $\mathrm{La}_{2-2 \mathrm{x}} \mathrm{Sr}_{1+2 \mathrm{x}} \mathrm{Mn}_{2} \mathrm{O}_{7}$ ( $\mathrm{x}=0.4,0.36$ ), which exhibit

\begin{tabular}{|c|c|}
\hline \multicolumn{2}{|c|}{ Energy Levels } \\
\hline$\Gamma_{1}$ & 15 \\
\hline$\Gamma_{5}$ & -8 \\
\hline$\Gamma_{4}$ & -6 \\
\hline$\Gamma_{3}$ & $\begin{array}{c}0 \\
\mathrm{MeV}\end{array}$ \\
\hline
\end{tabular}

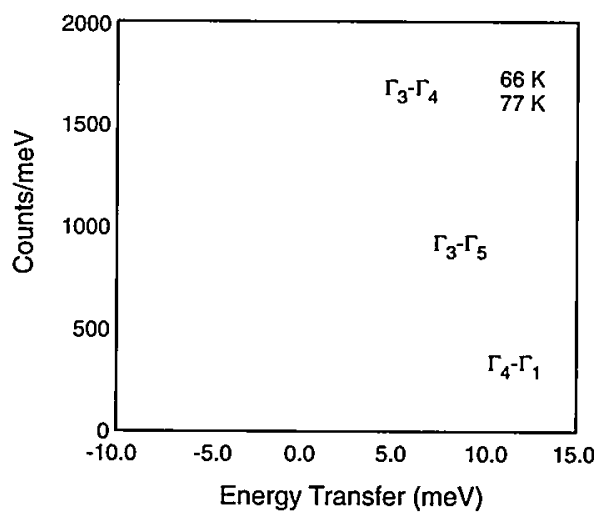

$\triangle$ FIGURE 2. Cnstalfield level diagram for the heowyfermion compound PrAg 2 In, os measured on PHAROS. PrAg 2 In hos the some face-centered cubic crystollographic structure as shown in figure $l(0)$ for UPd, Pb. At low temperature, transitions are allowed from the doublet crystaffield ground stote $\Gamma_{3}$ to both triplet excited states $\left(\Gamma_{4}\right.$ and $\left.\Gamma_{5}\right)$, as shown in red in the energy spectrum. The tronsition to the $\Gamma_{1}$ singlet is forbidden by symmetry. When the femperoture is roised to $77 \mathrm{~K}(-7 \mathrm{meV})$, both tiplets are partially occupied and can be deexcited bock to the ground state (see peaks of -6 and $-8 \mathrm{meV}$ ), and the $\Gamma_{4}-\Gamma_{5}$ and $\Gamma_{4}-\Gamma_{1}$ excitotions can also be seen of $2 \mathrm{meV}$ and $9 \mathrm{meV}$, respecfively. This information confims a picture in which the ground stote is a nonmognetic doublet $\left(\Gamma_{3}\right)$, which cannof be Zeeman split by a magnetic field, and the heay-femion properties cannot therefore be understood in terms of the normal dipolar Kondo effect. 

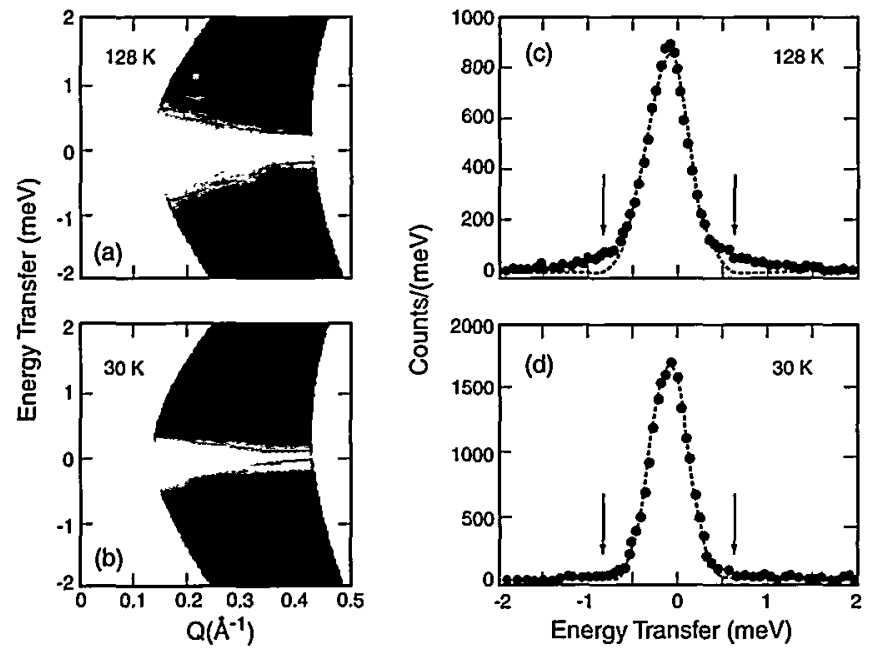

A FIGURE 3. Inelastic neutron scattering dofa from $\mathrm{LO}_{222 x} \mathrm{Sr}_{1+2 x} \mathrm{Mn}_{2} \mathrm{O}_{7}$, as meosured on PHAROS at (o) $128 \mathrm{~K}$ and (b) $30 \mathrm{~K}$, obove and below the ferromognefic transition femperature $T_{c}$ of $\sim 175 \mathrm{~K}$. Panels (c) and (d) show the same data plotted as a function of energy transfer. Note the presence of the vings in panel (c).

huge changes in resistivity (over four orders of magnitude) in the vicinity of the ferromagnetic Curie temperature $T_{c}$ with applied magnetic field. The underlying physics of the CMR materials is widely thought to involve polaronic degrees of freedom, at least above $T_{c}$. Theoretical models predict the existence of unusual spin dynamics associated with the hopping of Jahn-Teller polarons-i.e., mobile lattice distortions carrying spin. Recent experiments at LANSCE have demonstrated that two-dimensional ferromagnetic correlations exist in these layered CMR materials, as high as $2.8 \mathrm{~T}_{\mathrm{c}}$. These correlations grow in spatial extent as $\mathrm{T} \rightarrow \mathrm{T}_{\mathrm{c}}$, reaching a size of $\sim 10 \AA$ at the transition. Figure 3 shows some quasi-elastic neutron scattering data, taken on PHAROS, which probes their dynamics. ${ }^{3}$ The additional ferromagnetic scattering is seen as broad tails to the elastic peak above $T_{c}$, while the tails disappear below $T_{c}$. From these data, we can also determine the Q-dependent lifetimes, which are consistent with a spin-diffusion model, which in turn lends support to the polaronic model. On $\mathrm{SCD}$, we looked more closely at the Q-dependence and anisotropy of the same ferromagnetic correlations in a

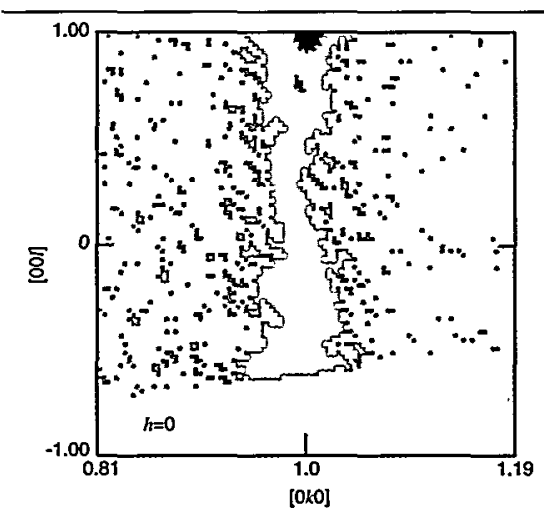

(a)

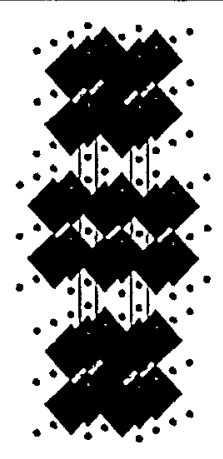

(b) $\triangle$ FIGURE 4. (a) A portion of the time-offlight neutron diffraction data token on SCD from a single crystal of $\mathrm{LO}_{2.2 \mathrm{~S}} \mathrm{Sr}_{7+2 \mathrm{x}} \mathrm{Mn}_{2} \mathrm{O}_{7} \mathrm{x}=0.36$ at $720 \mathrm{~K}$. The vertical strip extends along the $c^{*}$-ditection and is indicative of two-dimensionol fenomognetic mognetic conelations within the Mn-O bilayers. (b) The structure of these layered CMR moterials consists of perouskite bilayers (purple octahedro), separoted by a rocksalt $($ La, Sr) O layer.

single crystal. Figure 4 shows rod-like diffuse scattering, 3,4 which is indicative of two-dimensional ferromagnetic correlations. This work was performed in collaboration with colleagues at Argonne National Laboratory and NIST.

\section{References}

1. R. A. Robinson, H. Nakotte, N. R. Dilley, M. B. Maple, J. W. Lynn, and S. Skanthakumar, "Structural and Magnetic Transitions in UPd 2 Pb" (submitted to Phys. Rev. B, 1998).

2. T. M. Kelley, R. A. Robinson, F. Trouw, H. Nakotte, P. C. Canfield, and W. P. Beyermann, "Inelastic Neutron Scattering in PrInAge" (submitted to Phys. Rev. B, 1998).

3. D. N. Argyriou, T. M. Kelley, J. F. Mitchell, R. A. Robinson, R. Osborn, S. Rozenkranz, and J. D. Jorgensen, "Two-Dimensional Ferromagnetic Spin Correlations above $\mathrm{T}_{\mathrm{c}}$ in the Layered CMR Manganite $\mathrm{La}_{2-2 \mathrm{x}} \mathrm{Sr}_{1+2 \mathrm{x}} \mathrm{Mn}_{2} \mathrm{O}_{7}(\mathrm{x}=0.3-0.4)$," J. Appl. Phys. 83, 6374 (1998).

4. R. Osborn, S. Rosenkranz, D. N. Argyriou, L. Vasiliu-Doloc, J. W. Lynn, S. K. Sinha, J. F. Mitchell, K. E. Gray, and S. D. Bader, "Neutron Scattering Investigation of Magnetic Bilayer Correlations in $\mathrm{La}_{1.2} \mathrm{Sr}_{1.8} \mathrm{Mn}_{2} \mathrm{O}_{7}$ : Evidence of Canting above $\mathrm{T}_{\mathrm{c}}$ " (Phys. Rev. Letters, in press, 1998). 


\title{
Is Magnetic Order Possible in a Nonmagnetic Material?
}

\author{
A. L. Shapiro, F. Hellman (University of California at San Diego), M. R. Fitzsimmons (LANSCE Division)
}

A magnetic moment can be induced in a material that is not normally magnetic, but is easily polarizable, or it can be enhanced in a material that is in close proximity to a strongly magnetic material. ${ }^{1}$ The polarization of the nonmagnetic material by a magnetic material is called the proximity effect. Recently, we investigated the proximity effect in a thin single crystal film of platinum (Pt) containing 10 atomic percent cobalt $(\mathrm{Co})$ above the Curie temperature of this material in the bulk.

For most research in magnetic thin films, and in fact for many modern bulk magnetic materials, the crucial questions are not connected with bulk magnetic properties but with structures on length scales comparable to the fundamental length scales of magnetism. Technological advances in sample preparation and characterization (e.g., multilayers with well-defined interfaces or ultra-thin single crystal films of $\mathrm{Fe}$ ) and processing (e.g., electron beam lithographically defined dots showing mesoscopic phenomena) have opened up new areas of physics in which structural length scales can play an important role in determining the magnetic properties of materials. The importance and rationale for studies of magnetic materials with reduced dimensionality is that the understanding of many (if not all) of the interesting magnetic phenomena in microstructures relies on a detailed knowledge of the magnetic structure.

Our motivation was to explore the proximity effect in alloys of $\mathrm{Co}$ and $\mathrm{Pt}$-here, $\mathrm{Pt}$ is the easily polarizable material. We chose a low Curie temperature thin film, $\mathrm{Co}_{0.1} \mathrm{Pt}_{0.9}$, which was epitaxially deposited ${ }^{2}$ onto a single crystal film of $\mathrm{CoPt}_{3}$ with a high Curie temperature to form a bilayer sample (Figure 1). Two issues are posed by this study. First, what is the magnetic moment of $\mathrm{Pt}$ in the two Co-Pt alloys? Second, could ferromagnetic ordering in the $\mathrm{Co}_{0.1} \mathrm{Pt}_{0.9}$ overlayer be induced above its Curie temperature in the bulk by the strongly ferromagnetic $\mathrm{CoPt}_{3}$

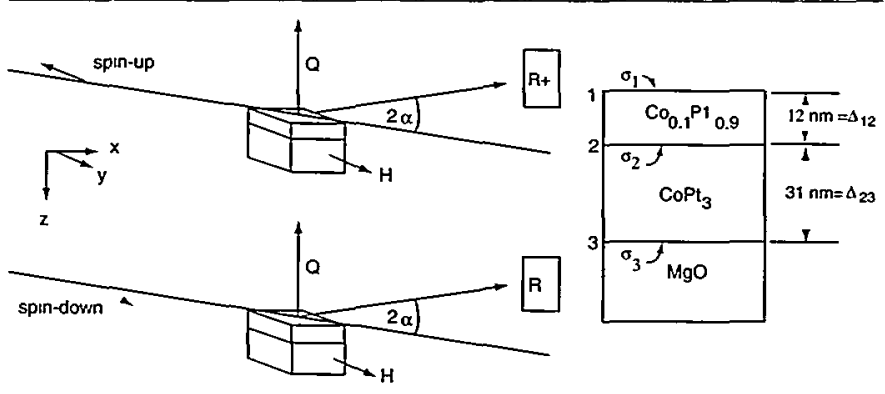

A FIGURE 1. Diagram of a scaftering experiment using o beom of (xfoys) neutrons with spins aligned ontiparollel (spinev) or parallel (spindown) to the field, $\boldsymbol{H}$, opplied to the sample. Inset: diagram of the $\mathrm{CO}_{0.1} \mathrm{Pt}_{0.9} \mathrm{C}_{\mathrm{O}} \mathrm{Pt}_{3}$ bilayer sample. underlayer? To address these issues, we used polarized neutron reflectometry - a technique well suited to studies of ferromagnetism across interfaces and in thin films. ${ }^{3}$

Polarized neutron reflectometry involves measuring the intensity of a polarized neutron beam reflected by the sample for polarization states aligned parallel (spin-down) and anti-parallel (spin-up) to the magnetic field applied to the sample. The reflected beam's intensity is measured as a function of momentum transfer, $Q=4 \pi \sin (\theta) / \lambda$. $Q$ is varied by changing neutron wavelength, $\lambda$, and the sample angle, $\theta$. From the difference between the spin-up and spindown reflectivities (Figure 2), we can deduce the magnetization depth profile, i.e., the variation of the sample magnetization with depth into the sample, averaged over the lateral dimensions of the sample.

The quality of data in Figure 2 is too poor to determine reliably both the chemical and magnetic variation of the sample depth profile; therefore, we took additional data using a cryostat and the rotating anode $\mathrm{x}$-ray generator at LANSCE (Figure 3). We used the sample's $x$-ray reflectivity profiles at the different temperatures to determine its chemical variation (Figure 3 inset), e.g., the thicknesses of the $\mathrm{Co}_{0.1} \mathrm{Pt}_{0.9}$ and $\mathrm{CoPt}_{3}$ films, with a high degree of certainty. We used this information to constrain the sample's chemical profile in the refinement of a model, which

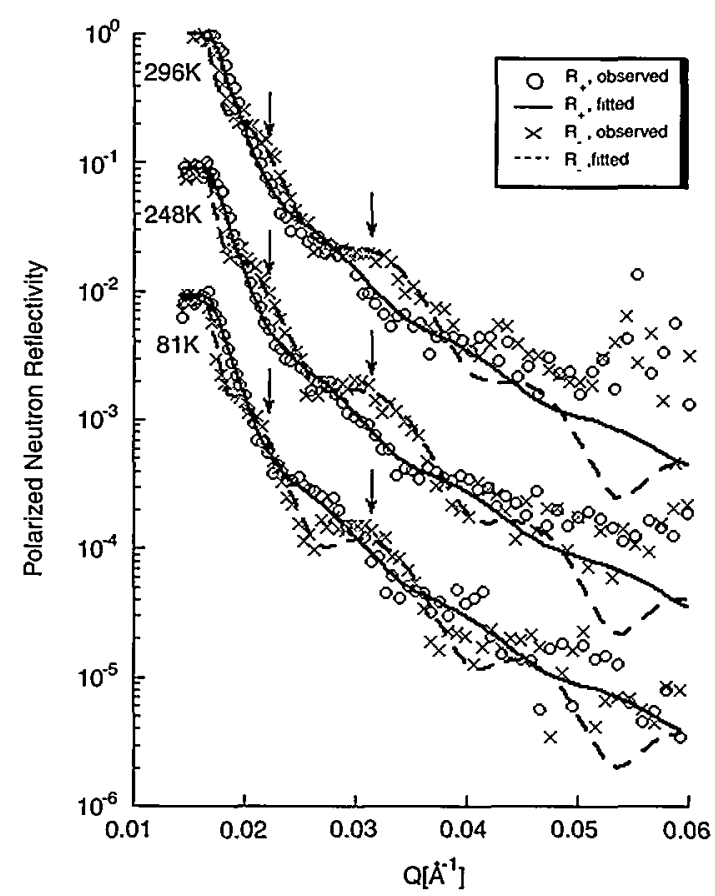

$\triangle$ FIGURE 2. Polarized neutron reflectivity profiles for the $\mathrm{CO}_{0 .} \mathrm{Pt}_{0.9} \mathrm{CO} \mathrm{Pt}_{3}$ somple measured of 81 , 248 , and $296 \mathrm{~K}$ (profiles ore displaced for the soke of clarity). Differences with semperofure were most pronounced for the spindown profile in the region $0.02 \dot{A}^{-1}<0<0.03 \dot{A}^{-1}$ (orrows). 


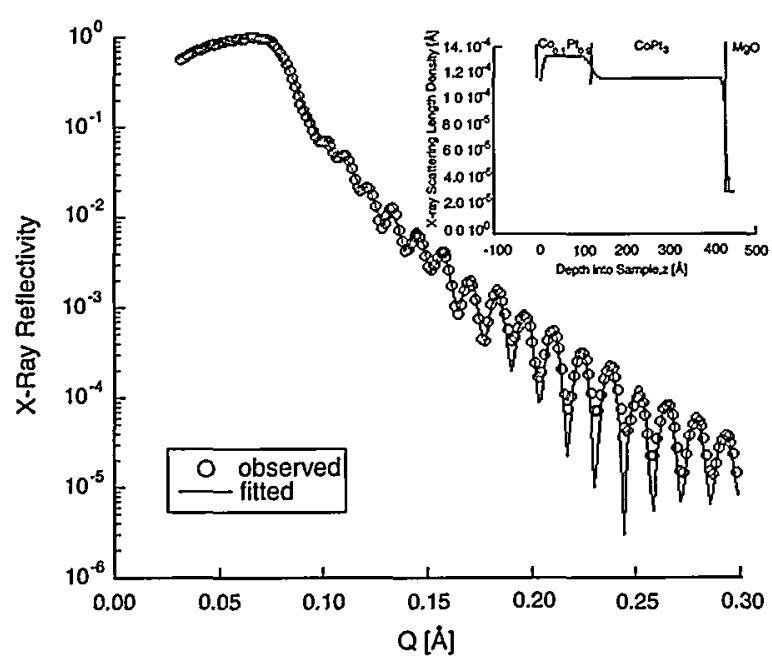

$\triangle$ FIGURE 3. Reflectivity of the bilayer somple measured with X-rays (0) of $296 \mathrm{~K}$. The solid cunve is the profile colculated for o model structure whose xray scattering length density varies with depth into the sample (inset).

included the possibility for magnetism in the Pt alloy films, of the polarized neutron data.

The magnetization density profile that best fitted the neutron data treated each Pt-alloy film as a film with uniform magnetization. The degree to which the films were ferromagnetic can be discerned from the splitting of the curves (Figure 4), which represent the neutron scattering potential for spin-up and spin-down neutrons.
From the splitting of the curves in Figure 4, we can determine the magnetic moments of $\mathrm{Co}_{0.1} \mathrm{Pt}_{0.9}$ and $\mathrm{CoPt}_{3}$. At $81 \mathrm{~K}, \mu\left(\mathrm{Co}_{0.1} \mathrm{Pt}_{0.9}\right)=0.25(2) \mu_{\mathrm{B}}$, and $\mu\left(\mathrm{CoPt}_{3}\right)=0.46(1) \mu_{\mathrm{B}}$ (Figure 4a). At $248 \mathrm{~K}(\sim 8 \mathrm{~K}$ above the Curie temperature for $\mathrm{Co}_{0.1} \mathrm{Pt}_{0.9}$ ), the mean moment for the $\mathrm{Co}_{0.1} \mathrm{Pt}_{0.9}$ overlayer decreased to $\mu\left(\mathrm{Co}_{0.1} \mathrm{Pt}_{0.9}\right)=0.04(2) \mu_{\mathrm{B}}$ (Figure $4 \mathrm{~b}$ ), and at $296 \mathrm{~K}$, the overlayer had no ferromagnetic component in the direction of the applied field at room temperature (Figure 4c).

In contrast to magnetometry measurements, we observed evidence for some ferromagnetic order of $\mathrm{Co}_{0.1} \mathrm{Pt}_{0.9}$ in a 12-nm-thick film $8 \mathrm{~K}$ above $\mathrm{T}_{\mathrm{c}}$ for $\mathrm{Co}_{0.1} \mathrm{Pt}_{0.9}$ in the bulk. While the magnetometry and neutron studies used different thermometers, both were calibrated thermometers and suitable for use in high magnetic fields; therefore, the discrepancy was not likely due to errors in thermometry. A more plausible explanation is one attributing the increase of $T_{c}$ to a higher degree of sensitivity of polarized neutron reflectometry than of magnetometry in detecting ferromagnetism in thin films.

Finally, we calculated the magnetic moment induced in $\mathrm{Pt}$ when it is in close proximity to Co at $81 \mathrm{~K}$ for the two film compositions. If the magnetic moment of $\mathrm{Co}$ in the films were that of $\mathrm{Co}$ in the bulk, $\mu_{\mathrm{Co}}=1.715 \mu_{\mathrm{B}},{ }^{4}$ and the moment of Co were parallel to the applied field, then we establish lower limits for $\mu_{\mathrm{Pt}}$. In the $\mathrm{Co}_{0.1} \mathrm{Pt}_{0.9}$ overlayer, $\mu_{\mathrm{Pt}}$ is at least $0.09(1) \mu_{\mathrm{B}}$, and in the $\mathrm{CoPt}_{3}$ underlayer, $\mu_{\mathrm{Pt}}$ is at least $0.04(1) \mu_{B}$; thus, we found evidence for an induced magnetic moment in $\mathrm{Pt}$ when it is in proximity to Co.

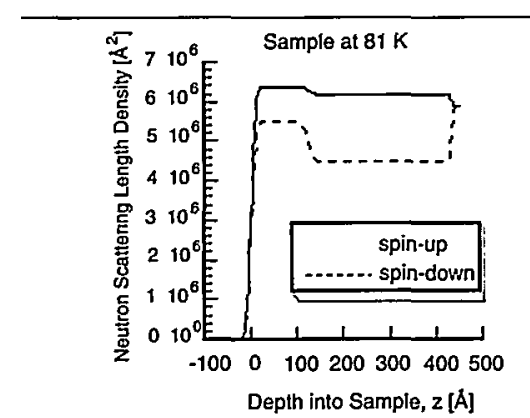

(a)

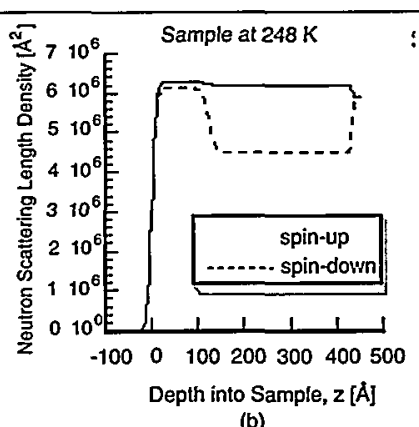

(b)

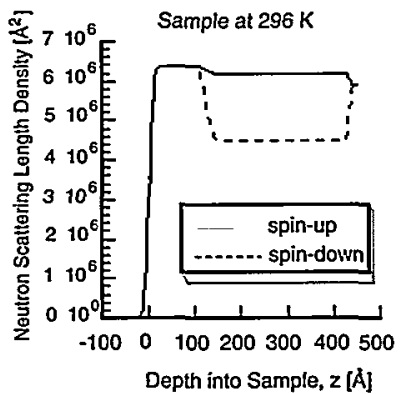

(c)

A FIGURE 4. Splitting between the spin-up and spin-down neutron scattering length density profiles is indicative of the film magnefization corresponding to a particular depth into the sample.

\section{References}

I. L. M. Falicov et al., "Surface, Interface, and Thin-Film Magnetism," J. Mater. Res. 5 (6), 1299 (1990).

2. P. W. Rooney, A. L. Shapiro, M. Q. Tran, and F. Hellman, "Evidence of a Surface-Mediated Magnetically Induced Miscibility Gap in Co-Pt Alloy Thin Films," Phys. Rev. Lett. 75 (9), 1843 (1995).

3. G. P. Felcher et al., "Polarized Neutron Reflectometer: A New Instrument to Measure Magnetic Depth Profiles," Rev. Sci. Instrum. 58, 609 (1987).

4. C. -W. Chen, Magnetism and Metallurgy of Soft Magnetic Materials (Dover Publications, New York, 1986), p. 38. 


\title{
Studies Relating to Adhesion at Polymer/Solid Interfaces
}

\author{
M. S. Kent, W. F. McNamara, D. F. Fein (Sandia National Laboratories), J. Majewski, G. S. Smith \\ (LANSCE Division), S. Satija (National Institute of Standards and Technology)
}

\begin{abstract}
Neutron reflection is used to address several problems of fundamental importance to adhesion and aging of organic/inorganic interfaces. This technique can provide valuable information regarding gradients in density, composition, and cross-link density normal to a surface. We focus on water adsorption, the segregation of low molecular weight species to interfaces, and the density and cross-link density of an epoxy near a substrate surface.
\end{abstract}

The phenomenon of adhesion and the loss of strength over time for organic adhesive joints is influenced by many factors. $1,2,3$ Most research in this field has focused on the extent and nature of the chemical bonds formed, continuum stress analysis, and fracture mechanics. Chemical bonds clearly play an important role and can be investigated by various spectroscopic methods. However, interphase structure also plays an important role for many phenomena, yet this aspect has not received as much attention due perhaps to the experimental difficulties involved. ${ }^{4}$

In this work we used neutron reflection to study interphase structure. Neutron reflection is ideal for such a study because (a) the resolution of 5 to $10 \AA$ normal to the surface is sufficient to map out detailed gradients within the interphase, which ranges from tens of angstroms to hundreds of nanometers; (b) the interphase can be probed in situ since the neutron beam can pass through a silicon substrate or a thin organic film with low loss of intensity; and (c) hydrogen/deuterium substitution can be used to optimize contrast within the organic adhesive allowing the determination of composition gradients. The present work is concerned with thermosetting adhesives such as silicones and epoxies.

Several important aspects of interphase structure, which impact adhesion and the aging of joints, can be addressed with neutron reflection. Interfacial chemical bonds are often reversible over the required range of service temperatures. The state of chemical equilibrium can be affected by changes in local concentrations arising from preferential segregation of low molecular weight components to the interface or the adsorption of contaminants such as water. Also, the stress distribution through the interphase, or at a flaw or crack tip, plays a critical role in fracture. Gradients in composition, density, and cross-link density can affect stress distribution and energy dissipation mechanisms. Such gradients are all amenable to study by this method.
Our work has focused on the preferential segregation of non-bonded, low molecular weight components to an interface (silicones), variations in density and cross-link density normal to an interface (epoxies), and the accumulation of moisture at interfaces (silicones and epoxies). The latter is discussed as an example.

The detrimental affects of water at organic/inorganic solid interfaces are well known. ${ }^{1,5}$ However, the conditions under which water will accumulate at an interface, and how rapidly water is removed upon desiccation or evacuation, are not well established. These questions have relevance to items that are fabricated in ambient conditions and then hermetically sealed in the presence of desiccant. We have addressed these questions for silicone (polydimethylsiloxane or PDMS) films coated onto copper, nickel, aluminum, and silicon oxide surfaces.

Thin $~ 300-\AA$ films of PDMS were spun onto bare silicon wafers and also onto wafers containing sputtered films of copper, nickel, and aluminum. The reflectivity was initially measured for the samples in the desiccated state. The samples were then exposed to air saturated with $\mathrm{D}_{2} \mathrm{O}$ at room temperature, and the reflectivity was obtained as a function of time until steady state was achieved. The presence of moisture at the interface was easily detectable for PDMS on nickel, copper, and aluminum. The result for copper is shown in Figure 1(a). The volume fraction of $\mathrm{D}_{2} \mathrm{O}$, obtained from the fitted scattering length density profiles before and after exposure, is shown in Figure 1(b). The data indicate a thin water-rich layer adjacent to the copper oxide surface. This result contrasts with results obtained for aluminum films (not shown), where water penetrates into the oxide layer rather than forming a layer at the interface.

The variation in reflectivity before and after $\mathrm{D}_{2} \mathrm{O}$ exposure for PDMS on silicon oxide, shown in Figure 2, was near the uncertainty in the measurement. Thus, a much greater quantity of moisture accumulates at the interfaces of PDMS with the metal films than for the interface of PDMS with silicon oxide. This is likely due to the stronger interaction of PDMS with the silicon substrate. PDMS interacts strongly with silicon oxide through hydrogen bonds, whereas PDMS interacts more weakly with the metal oxides. This direct measurement of the displacement of silicone from a metal interface by water has implications for corrosion resistance. We also addressed the question of whether interfacial moisture is removed upon desiccation. This indeed is the case for water adsorbed to the native oxide of a sputtered aluminum film. 

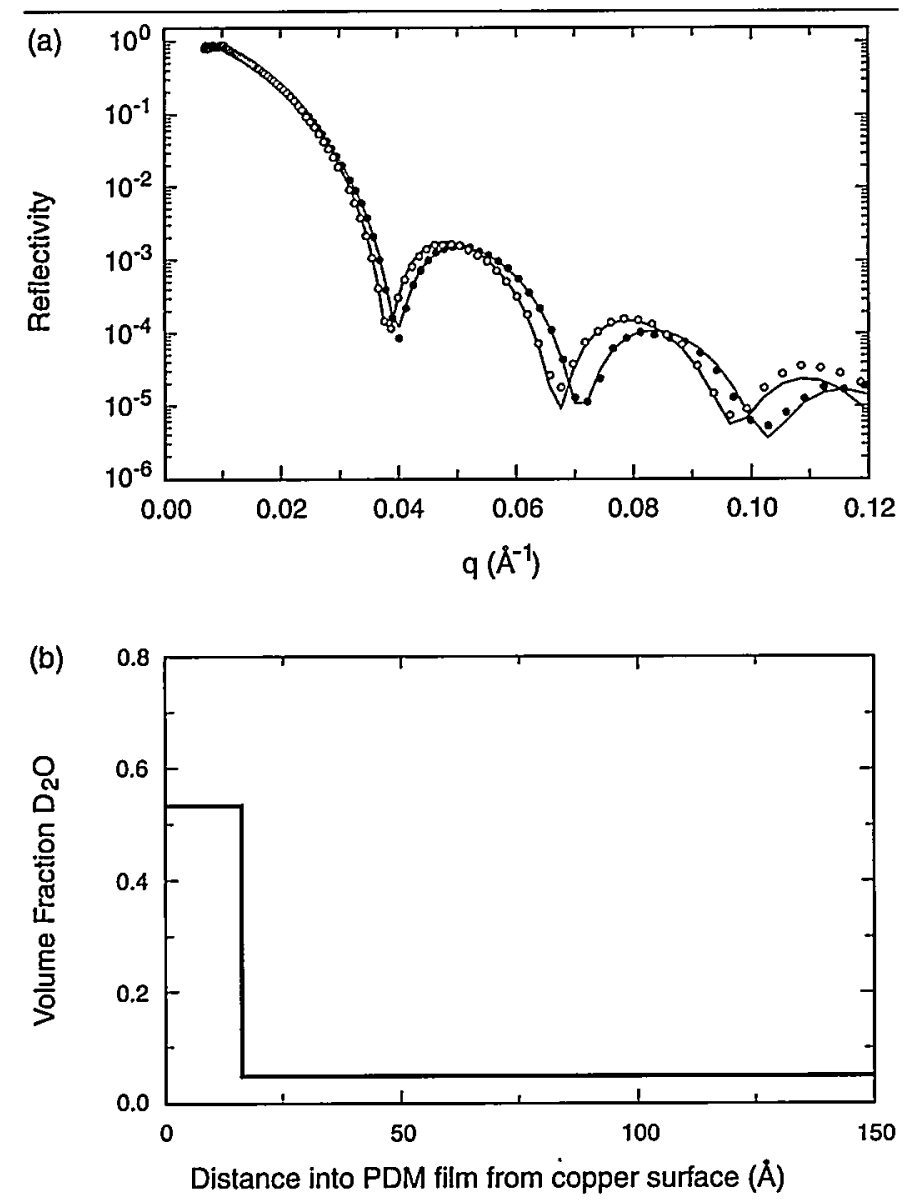

$\triangle$ FIGURE 1. (a) Reflectivity for a PDMS-coated copper film on a silicone wafer in the desiccoted state (red line) and in o sofuroted $\mathrm{O}_{2} \mathrm{O}$ atmosphere of $22^{\circ} \mathrm{C}$ (blue line). (b) $\mathrm{O}_{2} \mathrm{O}$ volume fraction profile determined from best-fit scattering length density profiles. These dato indicate a $16-\mathrm{A} \mathrm{O}_{2} \mathrm{O}$-rich loyer at the interface under these conditions.

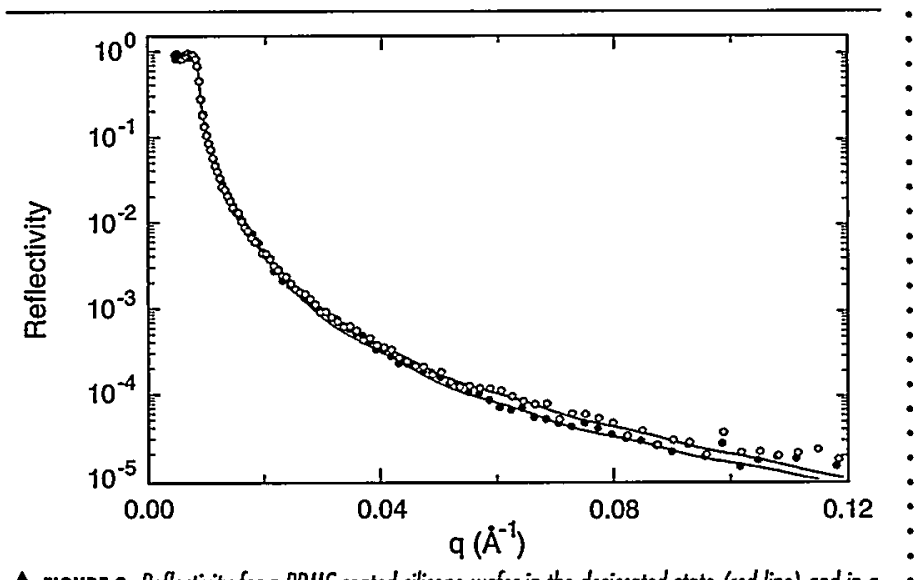

$\triangle$ FIGURE 2. Reflectivity for a PDMS-coated silicone wofer in the desiccated state (red line) ond in a: saturated $\mathrm{O}_{2} \mathrm{O}$ atmosphere of $22^{\circ} \mathrm{C}$ (blue line). Little $(<5 \AA)$, if ony, $\mathrm{D}_{2} \mathrm{O}$ adsorbs onto silicon oxide under these conditions.

\section{References}

1. L. H. Lee, Fundamentals of Adhesion (Plenum Press, New York, 1991).

2. A. J. Kinloch. "The Science of Adhesion, Part 1. Interfacial Aspects," J. Mater. Sci. 15, 2141-2166 (1980).

3. A. J. Kinloch. "The Science of Adhesion, Part 2. Mechanics and Mechanisms of Failure," J. Mater. Sci. 17, 617-651 (1982).

4. E. K. Drown, H. A. Moussawi, and L. T. Drzal, "Glass Fiber 'Sizings' and Their Role in Fiber-Matrix Adhesion," J. Adh. Sci. Technol. 5 (10), 865-881 (1991).

5. C. A. J. Hoeve, "The Structure of Water in Polymers," ACS Symp. Ser., S. P. Rowland, Ed. (American Chemical Society, Washington, D.C., 1980), Vol. 127, pp. 135-146. 


\title{
Accelerated Aging of Tantalum Nitride Thin Film Resistive Coatings
}

\author{
T. Rieker, P. Hubbard (University of New Mexico), J. Majewski, G. Smith (LANSCE Division), N. Moody \\ (Sandia National Laboratories, California)
}

We use neutron reflectivity to investigate the effects of accelerated aging on tantalum nitride $\left(\mathrm{Ta}_{2} \mathrm{~N}\right)$ thin films. The long-term stability of $\mathrm{Ta}_{2} \mathrm{~N}$ thin films used as resistors in integrated circuits in weapons systems becomes important when the design lifetimes are exceeded. Nanoscratch and nanoindent testing of $\mathrm{Ta}_{2} \mathrm{~N}$ thin films processed under extreme conditions in hydrogen and oxygen environments demonstrate degradation of such film at the film/substrate and film/air interfaces, respectively. The ability of the neutron reflectivity experiments to probe buried interfaces complements the mechanical tests.

Tantalum nitride $\left(\mathrm{Ta}_{2} \mathrm{~N}\right)$ thin film resistors, first produced by Bell Laboratories, demonstrate excellent chemical, thermal, and mechanical properties. ${ }^{1}$ The negative thermal coefficient of resistivity of $\mathrm{Ta}_{2} \mathrm{~N}$ makes it ideal for highpower density applications. Used routinely in hybrid and multichip modules, it is of particular interest for use in weapons systems that require long-term (30 years or more) electronics stability.

Typically, thin films of $\mathrm{Ta}_{2} \mathrm{~N}$ are sputter-deposited on ceramic substrates for their excellent thermal conductivity. Sputter deposition creates large numbers of structural defects and high compressive residual stresses. ${ }^{2,3} \mathrm{Ta}_{2} \mathrm{~N}$ films are air-annealed to improve stability. ${ }^{4,5,6}$ There are also proposals to add hydrogen during the deposition process to control the microstructure motivated by observations that it suppresses crystalline growth in tantalum films.

Nanoscratch and nanoindentation tests on $\mathrm{Ta}_{2} \mathrm{~N}$ thin films reveal differences in susceptibility to fracture, depending on annealing conditions. ${ }^{7,8,9}$ When annealed in an oxygen atmosphere, a thick tantalum oxide layer forms on $\mathrm{Ta}_{2} \mathrm{~N}$ film. This layer is prone to spallation both within the oxide and along the oxide substrate interface (Figure 1a). Thin films of $\mathrm{Ta}_{2} \mathrm{~N}$ annealed in hydrogen also are prone to fracture along the $\mathrm{Ta}_{2} \mathrm{~N} /$ substrate interface (Figure $1 \mathrm{~b}$ ). The enhanced susceptibility to interfacial fracture is attributed to the segregation of hydrogen to grain boundaries and other interfaces. ${ }^{9,10}$

Neutron reflectivity is an ideal technique to study multilayered thin films and buried interfaces. It is used to determine film thickness $(t)$, coherent neutron scattering length density (b), interfacial roughness (s), and interdiffusion of multilayered thin films. Neutron reflectometry is one of the few techniques that can detect hydrogen at interfaces. The technique is particularly useful for understanding the effects of

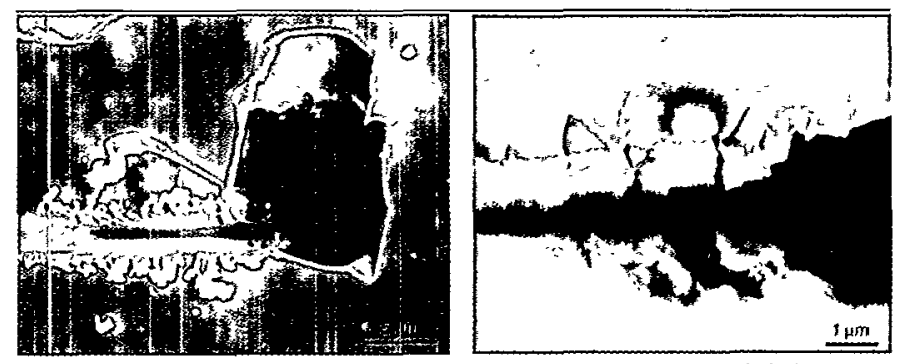

$\triangle$ FIGURE 1. Optical microgrophs of microscroftch tests on (o) oir-onneded To 2 N, which shows frocture occurred within the oxide and along the oxide substrate inferface, and (b) hydrogen-chorged $\mathrm{Ta}_{2} \mathrm{~N}$, which shows fractures occurred along the film substrate interface.

aging or corrosion at buried interfaces, where we use it to investigate the formation of a tantalum oxide layer and the possible formation of a hydrogen-rich layer at the $\mathrm{Ta}_{2} \mathrm{~N} /$ substrate interface. The sensitivity of neutron reflectivity to hydrogen enrichment at an interface is materialdependent. ${ }^{11}$ Model calculations for the $\mathrm{Ta}_{2} \mathrm{~N} / \mathrm{SiO}_{2}$ interface demonstrate a minimum sensitivity to an $\sim 20-\AA$-thick layer with one hydrogen atom per $\mathrm{Ta}_{2} \mathrm{~N} .{ }^{12,13}$

We prepared samples for neutron reflectivity on 0.2 -in.thick silicon (111) wafers. Initial experiments proved that highly polished aluminum oxide and aluminum nitride ceramic substrates are too rough. We then cleaned the silicon wafers by sputter etching, then passivated them with a thermally grown silicon oxide layer. After sputter coating the substrates with a nominally 700 - $\AA$-thick $\mathrm{Ta}_{2} \mathrm{~N}$ layer, we treated three $\mathrm{Ta}_{2} \mathrm{~N}$ samples separately under different atmospheres. The first, a control sample, was annealed in vacuum at $300^{\circ} \mathrm{C}$ for $100 \mathrm{~h}$. The second was annealed in an ultra-pure hydrogen atmosphere at $300^{\circ} \mathrm{C}, 1000 \mathrm{psi}$, for $100 \mathrm{~h}$. The third was annealed in a furnace open to air at $600^{\circ} \mathrm{C}, 1 \mathrm{~atm}$, for $100 \mathrm{~h}$.

The neutron measurements were made on the time-of-flight SPEAR reflectometer at the Lujan Center. Typical data collection times were 3 to $4 \mathrm{~h}$.

Data analysis consisted of building a reasonable structural model for which a theoretical reflectivity curve is calculated using the iterative, dynamical method. ${ }^{14}$ Each layer in this model was defined by a density, a thickness, and a root mean square roughness. The substrate was treated as a layer of semi-infinite thickness with the top surface defined as $z=0$. We modeled sharp interfaces or interfaces with diffusion profiles between regions of different density and refined calculated curves that compared favorably with the experimental data using a Marquardt-Levenberg nonlinear, least-squares fitting routine to obtain the best fitting parameters. The fits included an additional parameter to normalize the calculated reflectivity to the data. 


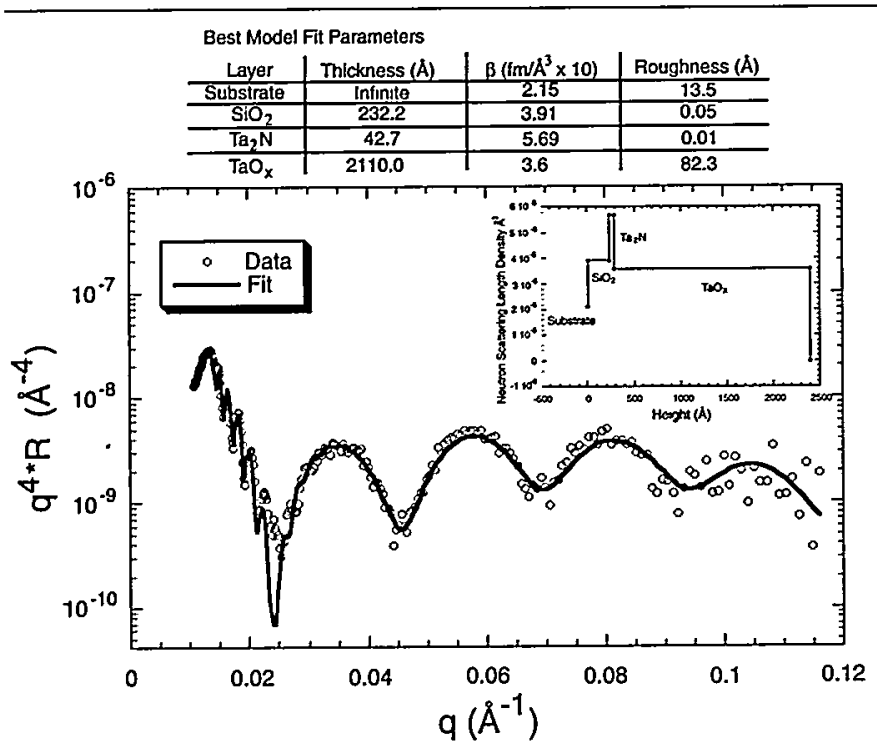

$\triangle$ FIGURE 2. Neutron reflectivity data $(0)$ for the $\mathrm{Ta}_{2} \mathrm{~N}$ sample annealed in air and fit $(\rightarrow)$ to the dato that yields the neutron scottering length profile displayed in the inset; the table lists best model fit porometers.

Figure 2 shows the reflectivity curve for the oxygenannealed sample with a curve fitted to the data that yields the layer model shown in the inset. Note the formation of a $2100-\AA$-thick tantalum oxide $\left(\mathrm{Ta}_{2} \mathrm{O}_{5}\right)$ layer at the air $/ \mathrm{Ta}_{2} \mathrm{~N}$ interface. This layer is five times thicker than the $\mathrm{Ta}_{2} \mathrm{~N}$ layer consumed in its formation. This swelling generates cracks in the thin film that are visible in reflected light.

Figure 3 shows neutron reflectivity data for the hydrogenannealed sample, and a fit to the scattering data that yields the layer model shown in the inset. Analysis shows the presence of a $90-\AA$-thick, negative scattering length density layer on top of the $\mathrm{Ta}_{2} \mathrm{~N}$ film. All atomic species in this study, except hydrogen, have a positive scattering length. Therefore, the overlayer is rich in hydrogen and most likely a mixture of tantalum hydride and nitride. Models that introduced layers at the $\mathrm{Ta}_{2} \mathrm{~N} / \mathrm{SiO}_{2}$ interface, or that model diffusion profiles, were inconsistent with the data.

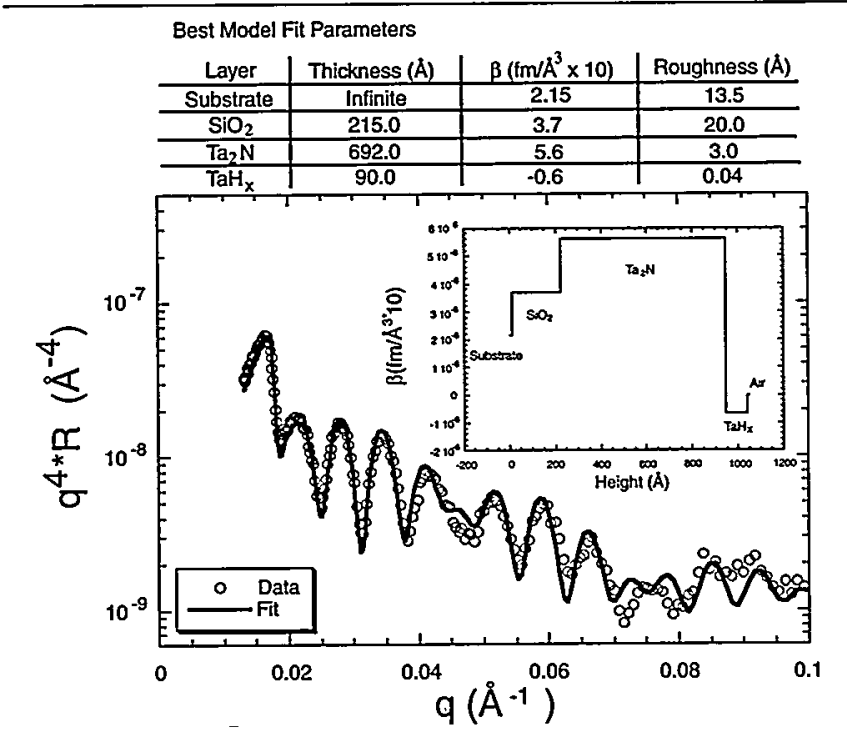

$\Delta$ FIGURE 3. Neutron reflectivity dofo (0) for the $T_{0} \mathrm{~N}$ somple annealed in hydrogen ond fit $(\rightarrow)$ to the dato thot yields the neutron scattering length profile displayed in the inset, the foble lists best model fit parameters.

Therefore, we see no evidence of a hydrogen-rich layer at the $\mathrm{Ta}_{2} \mathrm{~N} / \mathrm{SiO}_{2}$ interface. The expected hydrogen-rich layer may be below our detection limits or may be obscured by the hydride layer at the gas $/ \mathrm{Ta}_{2} \mathrm{~N}$ interface.

In conclusion, both samples demonstrate the formation of surface-reaction layers. The oxygen-annealed sample demonstrates the formation of a thick tantalum oxide layer at the air $/ \mathrm{Ta}_{2} \mathrm{~N}$ interface. The tantalum oxide layer is five times thicker than the consumed fraction of the $\mathrm{Ta}_{2} \mathrm{~N}$ layer, producing visible (micron sized) cracks in the sample that weaken the film. Scattering results from the hydrogenannealed sample show the formation of a tantalum-hydride surface layer. Fits to the scattering data are inconsistent with hydrogen enrichment at the $\mathrm{Ta}_{2} \mathrm{~N} /$ substrate interface. We assume from microscratch tests that hydrogen enrichment does indeed occur, but is below our detection limit or is obscured by the formation of a surface hydride layer.

\section{References}

1. R. W. Berry, P. M. Hall, and M. T. Harris, Thin Film Technology (Litton Educational Publishing, Inc., VanNostrand Reinhold Co., New York, 1968).

2. R. C. Sun, T. C. Tisone, and P. D. Cruzan, J. Appl. Phys. 46, 112 (1975).

3. E. Klokholm, J. Vac. Sci. Technol. 8, 148 (1971).

4. D. P. Brady, F. N. Fuss, and D. Gertenberg, Thin Solid Films 66, 287 (1980).

5. J. R. Adams and D. K. Kramer, Surface Science 56, 482 (1976).

6. S. Ohfuji and C. Hashimot, "Oxidation of Hydrogen Doped Tantalum Films on Silicon," J. Vac. Sci. Technol. B4, 714 (1986).

7. N. R. Moody, R. Q. Hwang, S. Venkataraman, J. E. Angelo, et al., Acta Materialia 46, 585 (1998).

8. N. R. Moody, S. V. Venkataraman, J. C. Nelson, et al., Polycrystalline Thin Films: Structure, Texture Properties, and Applications, K. Barmak, M. A. Parker, J. A. Floro, R. Sinclair, and D. A. Smith, Eds. (Materials Research Society, Pittsburgh, Pennsylvania, 1994), Vol. 343 , p. 603.

9. N. R. Moody, S. K. Venkataraman, R. Q. Hwang, J. E. Angelo, and W. W. Gerberich, in Second International Conference on Corrosion-Deformation Interactions, CDI '96, T. Magnin, Ed. (European Federation of Corrosion Publications, Number 21, Institute of Materials, London, 1997$)$ p. 227.

10. S. M. Myers, M. I. Baskes, H. K. Birnbaum, J. W. Corbett, G. G. De Leo, et al., Rev. Mod. Phys. 64, 559 (1992).

11. G. P. Felcher, W. Dozier, and A. D. Marwick, Sth Surface X-Ray and Neutron Scattering Conference, Oxford, July 18, 1997 (to be published in Physica B).

12. Neutron reflectivity code BRUFF, written by W. Hamilton while at the Manuel Lujan Jr. Neutron Scattering Center.

13. W. Nistler and R. Scherm, Praktische Physik, Kohlrausch Ed., 3, 275 (1983).

14. T. P. Russell, Materials Science Reports 5, 171 (1990). 


\title{
Polymer-Supported Bilayer Films for Biosensors
}

\author{
J. Wong,* C. Park, M. Seitz, J. Israelachvili (University of California at Santa Barbara), J. Majewski, \\ G. S. Smith (LANSCE Division)
}

\begin{abstract}
Supported lipid bilayers containing proteins on solid substrates are novel materials for potential biosensor applications. When transmembrane proteins are inserted into supported lipid bilayers, they are generally nonfunctional. This effect is because of the unfavorable interactions of the protein with the underlying substrate. This highlight presents results of neutron-reflectivity experiments for a system that uses a soft polymer to serve as a cushion for the lipid bilayer-separating it from the underlying substrate. Moreover, the floating bilayer retains fluidity-more closely mimicking an actual biomembrane.
\end{abstract}

Solid-supported biomembranes have been used as simple systems to enhance our understanding of the structurefunction relationships of cell membranes. The solid support enables one to probe the structure of biomembranes using a number of techniques: atomic force, fluorescence and reflection interference contrast microscopy, surface forces apparatus, Fourier transform infrared and surface plasmon spectroscopy, ellipsometry, and neutron reflectivity. The membrane usually is separated from the substrate by a 10 to 20 - $\AA$-thick layer of water. While lipid molecules are able to freely diffuse laterally within the membrane, protrusions normal to the surface are dampened by the substrate. Furthermore, transmembrane proteins containing extracellular domains with dimensions larger than the $20 \AA$ can be immobilized due to direct interaction with the solid support. $1,2,3$

One strategy for decoupling the membrane from the underlying surface is to rest it on a soft, hydrated polymer film, like the cytoskeletal support found in a mammalian cell. Sackmann reviewed various approaches to create such polymer-lipid composite films. ${ }^{4}$ In this study, we used neutron reflectivity to probe the structure of lipid bilayers physisorbed onto a polymer thin film (Figure 1). The polymer was polyethyleneimine (PEI), which is highly swollen and positively charged in aqueous environments.

The common methods used to form biomembranes onto surfaces are vesicle fusion ${ }^{5,6,7}$ and the Langmuir-Blodgett (LB) technique. We evaluated these techniques by neutron reflectivity. The first method we chose involves polymer adsorption and vesicle fusion. We prepared small unilamellar vesicles of dimyristoylphosphatidylcholine (DMPC), which have been shown to form bilayers on quartz substrates. $^{8}$

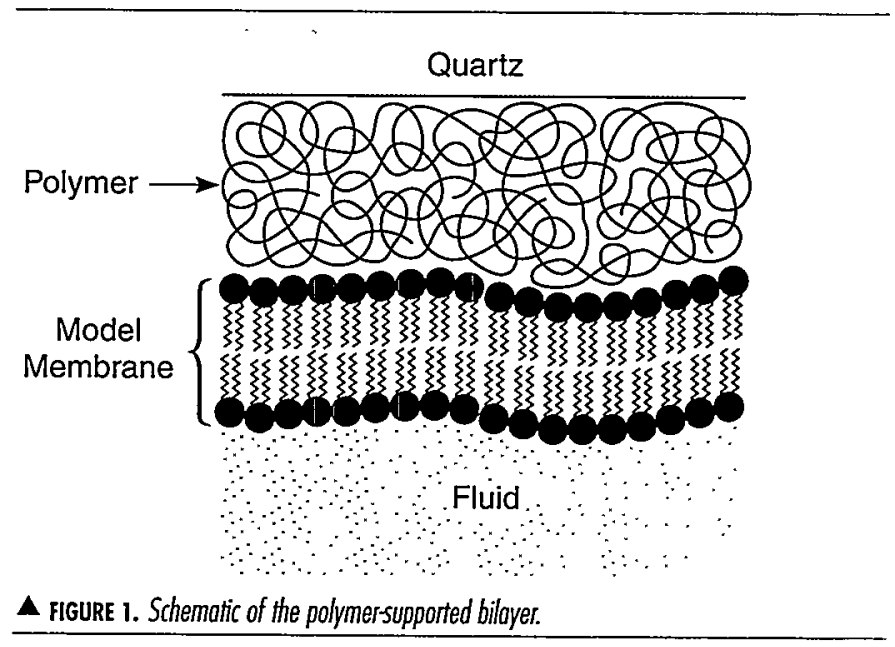

When vesicles were added to a polymer film that was preadsorbed on quartz, a very inhomogeneous and complex surface structure was formed, as indicated in Figure 2(a).
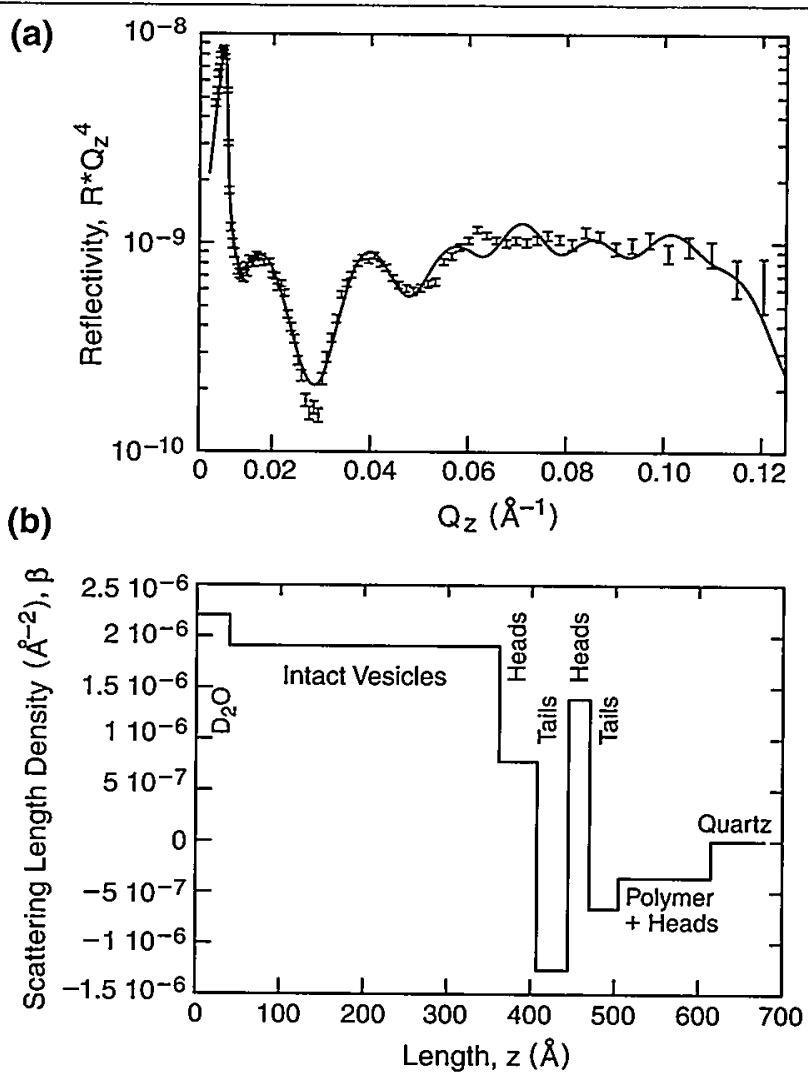

$\triangle$ FIGURE 2. (a) Neutron reflectivity cunve of the $D_{2}$ O-quartz interfoce, after vesides were adsorbed onto a PEl-coated quartz substrate. The multiple peaks at low $Q_{z}$ values indicate that $500-600 \dot{A}$ structures are present. The solid curve is the fit using the box model. (b) The resulting scattering length density is not due to a single bilayer above the PEI cushion. 
In contrast, we found that DMPC vesicles adsorbed to bare quartz to form an almost perfect bilayer. We saw significant changes in the neutron scattering profile compared to DMPC on bare quartz after we added PEI to the system (Figure 3). To account for the change, we tried to accommodate the location of the polymer at the physically allowable positions: above the outer lipid layer, below the inner lipid layer, and both above and below the lipid layer. This procedure showed that the only reasonable fit occurs when

(a)

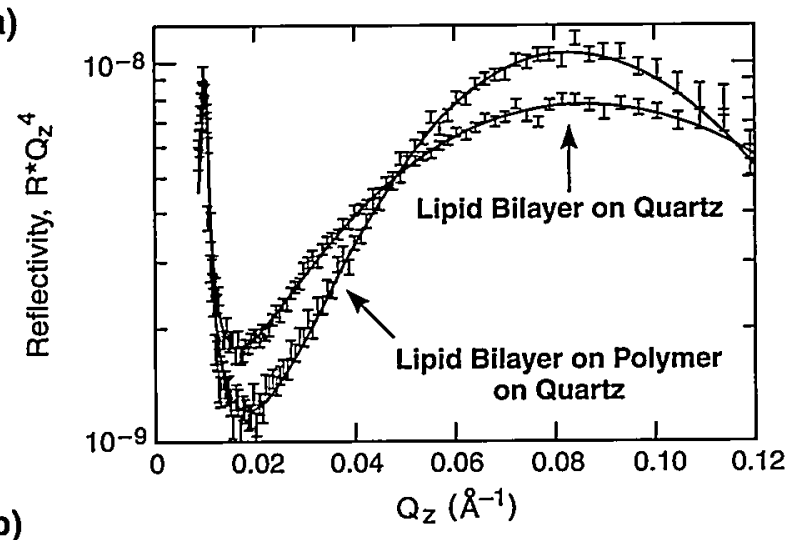

(b)

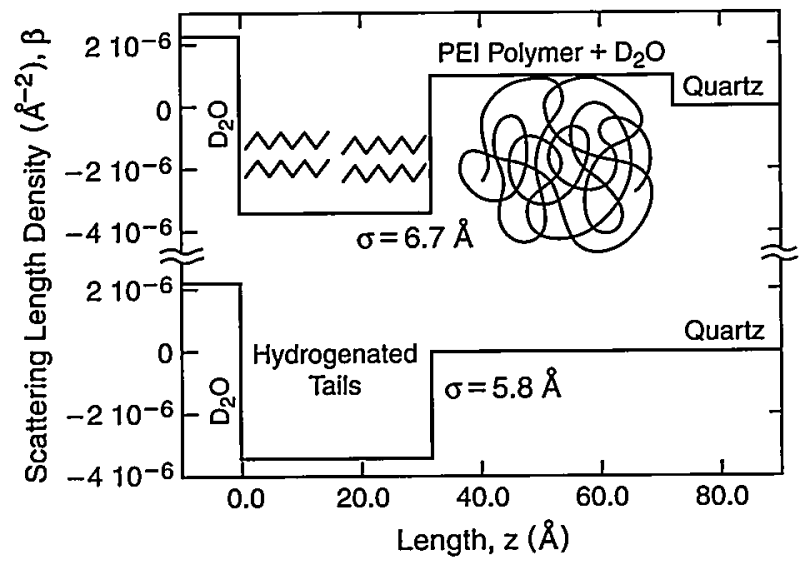

$\triangle$ FIGURE 3. (o) Neutron reflectivity profile of o DMPC billoyer of the $\mathrm{D}_{2}$ Oquartz interfoce ofter addF fion of PEI. Solid arve is a fit using box models. (b) Corresponding scoltening length density profile. the $\mathrm{PEI} / \mathrm{D}_{2} \mathrm{O}$ is located between the quartz substrate and the inner phospholipid monolayer. The notion that PEI could crawl underneath the lipid bilayer and lift it up may sound implausible. However, PEI is positively charged and would rather sit next to the quartz. Further, the PEI-DMPC interaction is weak and allows the energetically favored PEI to displace the bilayer.

The second method of preparation involves both the LB technique and vesicle fusion. We can deposit a monolayer of lipid molecules onto a solid substrate by raising the sample through the air-monolayer-water interface. For our polymer-supported bilayers, we added a polymer solution to the subphase, allowing the polymer to adsorb strongly to the negatively-charged quartz substrate before depositing the lipid monolayer. Although the PEI-monolayer adsorbs well, when this surface is passed through the lipid monolayer a second time, the first monolayer peels off due to weak interaction between the DMPC and the PEI. To avoid this peeling and create the second layer, we used vesicle fusion. In this case we found similar reflectivity curves as the single DMPC bilayer on the polymer cushion (data not shown here) indicating this is another method to form polymer-supported bilayers.

In conclusion, neutron reflectivity has allowed us to investigate biologically relevant bilayer structures at the solidliquid interface. We found that DMPC vesicles added to PEI-coated quartz substrates lead to a nonhomogeneous structure consisting of a mixture of vesicles and lipid multilayers. In contrast, PEI appears to diffuse underneath a preformed DMPC bilayer, cushioning the bilayer from the underlying quartz substrate. Thus, this method may be a simple way to prepare softly supported biomembranes that can be used for biosensor applications.

\footnotetext{
*J. Wong is currently at Boston University, Department of Biomedical Engineering.
}

References

1. H. M. McConnell et al., "Supported Planar Membranes in Studies of Cell-Cell Recognition in the Immune System," Biochim. Biophys. Acta 864, 95-106 (1986).

2. E. Kalb and L. K. Tamm, "Incorporation of Cytochrome $b_{5}$ into Supported Phospholipid Bilayers by Vesicle Fusion to Supported Monolayers," Thin Solid Films 210/211, 763-765 (1992).

3. J. Salafsky, J. T. Groves, and S. G. Boxer, "Architecture and Function of Membrane Proteins in Planar Supported Bilayers-A Study with Photosynthetic Reaction Centers," Biochemistry 35(47), 14773-14781 (1996).

4. E. Sackmann, "Supported Membranes: Scientific and Practical Applications," Science 271, 43-48 (1996).

5. L. K. Tamm and H. M. McConnell, "Supported Phospholipid Bilayer," Biophys. J. 47, 105-113 (1985).

6. R. G. Horn, "Direct Measurement of the Force Between Two Lipid Bilayers and Observation of their Fusion," Biochim. Biophys. Acta 778, 224-228 (1984).

7. T. M. Bayerl and M. Bloom, "Physical Properties of Single Phospholipid Bilayers Adsorbed to Micro Glass Beads -A New Vesicular Model System Studied by h-2-Nuclear Magnetic Resonance," Biophys. J. 58(2), 357-362 (1990).

8. S. J. Johnson et al., "Structure of an Adsorbed Dimyristoylphosphatidylcholine Bilayer Measured with Specular Reflection of Neutrons," Biophys. J. 59, 289-294 (1991). 


\title{
Understanding the Activity of the Titanium Silicalite Catalyst TS-1
}

\author{
K. C. Ott, C. Hijar (Chemical Science and Technology Division), J. Eckert (LANSCE Division)
}

The discovery of the framework substituted microporous materials titanium silicalite-1 (TS-1) and iron silicalite-1 (FeS-1) was one of the most important developments in heterogeneous catalysis within the last decade. These materials exhibit extremely high selectivity in oxidation reactions using hydrogen peroxide, with water as the major byproduct. A full understanding of the remarkable catalytic properties of these materials requires a detailed picture of the local structure at the active site. We have used neutron diffraction and inelastic neutron scattering studies to determine the location of the substituted titanium atoms in the zeolite framework and to identify intermediate species in the oxidation of propylene by hydrogen peroxide at the titanium site.

Catalysis provides economic advantages by allowing chemical reactions to occur more rapidly, which results in reduced capital outlay, reduced energy costs, and reduced waste generation. Catalysis cuts across all U.S. industries; products from catalysis account for $20 \%$ to $30 \%$ of the gross national product. For this reason, the development of novel catalysts is an ongoing effort of major importance. Innovations are needed with respect to the efficiency of the catalyst, selectivity of the reaction products, separation efficiency of these products, and improved environmental properties of the byproducts of the reactions.

A number of oxidative transformations of alkanes and arenes represent a great scientific challenge and technological opportunity. Many of these transformations (Figure 1) are known and practiced commercially but at low efficiencies. The occurrence of other undesirable byproducts is a common problem that many current catalysts exhibit. A significant advance in the field of oxidation catalysis was

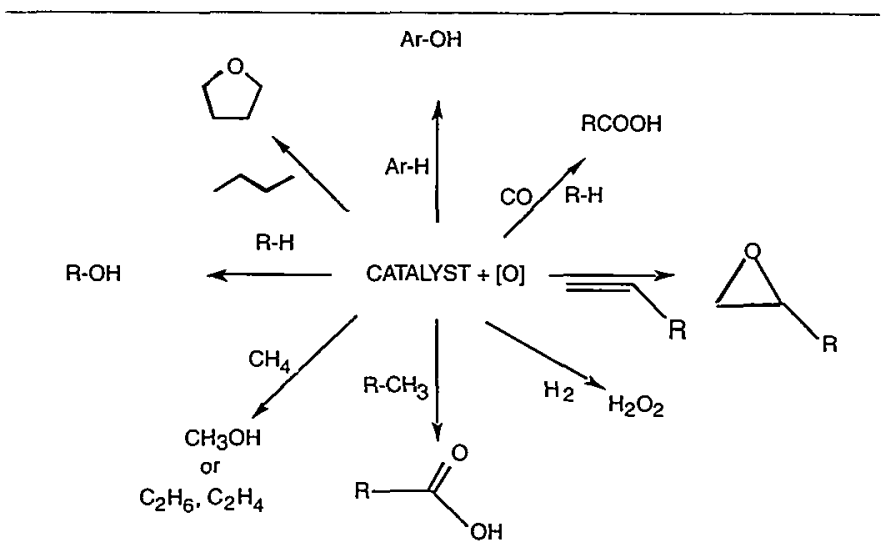

$\triangle$ FIGURE 1. Oxidative transformations of common orgonic substrates and reagents to compounds of higher value and utility. realized when an Italian group synthesized ${ }^{1}$ the first member of a family of titanosilicate zeolites called TS-1. The interest in these titanium-containing microporous materials lies in their catalytic properties. ${ }^{2}$ In the presence of hydrogen peroxide, these materials selectively oxidize alkanes to alcohols or aldehydes, alkenes to epoxides, alcohols to aldehydes or ketones, and phenol to catechol and hydroquinone under moderate conditions $\left(0-100^{\circ} \mathrm{C}, 1 \mathrm{~atm}\right)$. The byproduct of TS-1 catalyzed oxidation is water.

The titanosilicate zeolites (TSZs) are the most exciting catalytic systems discovered in years because of their catalytic properties and their ability to form an environmentally benign byproduct (water). Oxidation reactions in TSZs occur in the sterically restricted channels of a zeolite, resulting in unusual selectivity. Zeolite catalysts for other chemical processes are extremely important because of the selectivity and high yields that provide economic advantages inherent in processes that avoid waste generation. It is also important to note that non-zeolitic titanosilicates and anatase $\left(\mathrm{TiO}_{2}\right)$ are completely inactive for these reactions, and it is only when titanium is site-isolated in the zeolite framework that active, selective oxidation catalysis is observed.

Many catalysts are solid materials that have a low concentration of active sites-low enough to consider them as having active sites that are isolated from one another. This site-isolation is thought to give rise to catalytic activity and selectivity. However, the low concentration of active sites presents challenges to scientists attempting to study the structure and mechanism of these catalytic reactions. A dearth of techniques is available to address these issues. This lack of knowledge of details at the atomic level is a significant barrier to the ability to design and synthesize more active and selective catalysts for a desired chemical reaction.

We have concentrated on using neutron diffraction and scattering techniques to determine short- and long-range order in these systems, using nuclear magnetic resonance techniques to develop probes for short-range ordering of active sites and for fundamental physical chemistry of porous systems, and using theory to help interpret experiments and to develop new theoretical methodologies to deal with these large, complex systems. Much of our work has focused on the TS-1 catalyst.

Site-isolated metal species are often critical for catalytic activity and selectivity. The siting of the active metal ion species will determine the local coordination environment around the active species and is thought to be important for determining the catalytic properties. To date, very little definitive information is available to address these issues. The very nature of site-isolated metal species requires that they be 
dilute within the host, which implies that these species may be disordered or partially ordered within the host.

Because of the low concentrations and the small differences in $\mathrm{x}$-ray scattering between silicon and the transition elements of interest (titanium, vanadium, iron, etc.), x-ray diffraction cannot be used to determine the location or site occupancies of the minority metal species in the zeolite host. For some elements, titanium being one of them, differences in neutron scattering properties can give rise to adequate contrast in the neutron diffraction experiment to determine the locations and site populations of dilute species. This is the case for TS-1, where x-ray diffraction has failed to yield any useful information about the location and site populations of the titanium ions in the framework. We have, therefore, collected neutron diffraction data at the Lujan Center on a number of samples of TS-1 in an attempt to define the locations of the 2-3 at.\% titanium that may be distributed among the 12 crystallographically distinct silicon sites present within this particular zeolite structure type (Figure 2). Rietveld analysis of our data clearly indicates that titanium is sited preferentially at only 4 or 5 of the 12 possible sites. Moreover, all of these sites are located at the intersection of the channels in the structure and thus may more readily accommodate the titanium atoms with the attendant larger Ti-O distance. This siting may also explain the exceptional acitivity of TS-1, as these sites are more accessible to the reactant molecules. Further support for this conclusion comes from the fact that titanium-substituted ALPO-type zeolites do not show any activity compared with TS-1, as there are no intersecting channels for siting the titanium atoms.

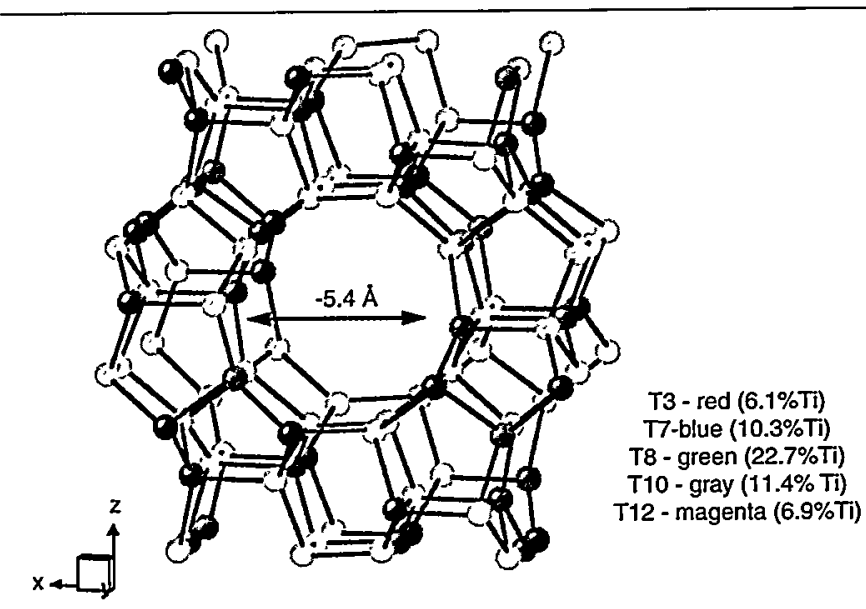

$\Delta$ FIGURE 2. Stucture of IS.1 defermined by neution diffraction. Silicon sites are shown in yellow, and sites with high likelihood of fitanium occupancy instead of silicon are shown in color occording to the key.
Given the new structural information about the active sites in TS-1, we can now address the question of how reactants interact at this site and how the oxidation reaction proceeds. We have employed inelastic neutron scattering (INS) vibrational spectroscopy to obtain details of the interactions between $\mathrm{H}_{2} \mathrm{O}_{2}$ and the zeolite TS-1 host before, during, and after the oxidation of propylene to propylene oxide. The initial steps of this process are shown schematically in Figure $3 .^{3}$ INS spectroscopy has a distinct advantage over the analogous optical techniques (IR absorption, Raman

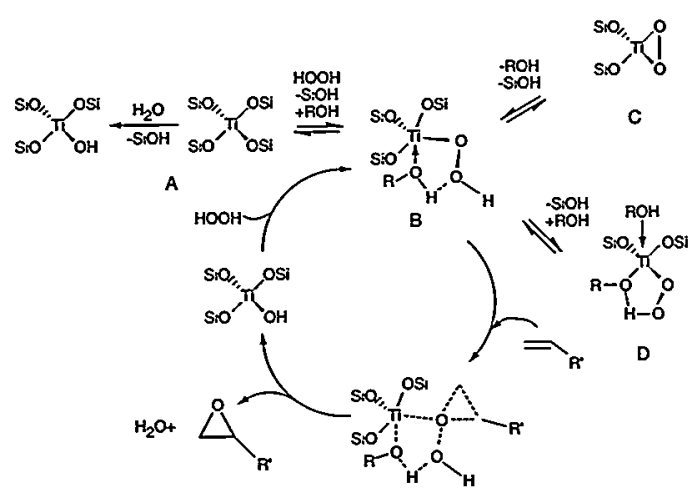

$\Delta$ FIGURE 3. Interaction of water with the fitanium site in TS-I (left) and woter/hydrogen peroxida (right). INS spectroscopy has provided direct evidence for the first steps in both of these reactions.

scattering) in that the vibrational modes of any $\mathrm{H}$-containing adsorbate molecule will dominate the spectrum relative to that of the zeolite framework, whereas the reverse is true for the optical techniques.

The experiments were carried out in situ on the FDS instrument at the Lujan Center. INS spectra of TS-1, with one molecule $\mathrm{H}_{2} \mathrm{O}$ per titanium, show evidence for the presumed hydrolysis of a Ti-O-Si bond by way of the observation of Ti-OH bending modes. TS- 1 was then loaded with a coverage of one molecule $\mathrm{H}_{2} \mathrm{O}_{2}$ per titanium atom, which resulted in a spectrum characteristic of a $\left(\mathrm{H}_{2} \mathrm{O}\right) \mathrm{TiOOH}$ complex. Subsequent addition (at $\mathrm{T}=250,000$ ) of perdeuterated propylene (which is nearly "invisible" in the INS) shows the effects of the formation of a propylene complex at the titanium site by the "disappearance" and/or shifting of the bands related to the water/hydrogen peroxide complex. These results are being interpreted by comparison with theoretical calculations of INS spectra. This type of analysis is very powerful and is expected to provide more detailed information on the precise nature of the above intermediates and (upon repeat of the experiment with normal propylene and deuterated hydrogen peroxide) the nature of the intermediate propylene complex as well.

\section{References}

1. M. Taramasso, G. Perego, and B. Notari, U.S. Patent 4410501, 1983.

2. See Davis et al., "Selective Catalytic Oxidation," ACS Symposium Series 523, 273 (1993) for an overview of TS-1 catalysis.

3. W. Adams, A. Corma, T. I. Reddy, and M. Renz, J. Org. Chem. 62, 3631 (1997). 


\section{Accelerated Neutron Testing of Semiconductor Devices}

\section{S. Wender (LANSCE Division)}

The high-energy neutron source at LANSCE provides a capability for accelerated neutron testing of semiconductor devices. This testing is important because neutrons are thought to be a significant threat to semiconductor devices at aircraft altitudes and below. The shape of the neutron spectrum produced at LANSCE is very similar to the spectrum of neutrons produced in the atmosphere by cosmic rays but is greater than 5 orders of magnitude more intense. Over the past 5 years, many companies have used the LANSCE beam to study various failure modes caused by neutron radiation. These failure modes include single-event upsets, multiple-event upsets, latchup, gate rupture, and so forth.

Since 1992 many companies, including Texas Instruments, the Boeing Company, Hewlett Packard, Lockheed Martin, Digital Equipment, Intel, and so forth, have used the highenergy neutron source at LANSCE to study the effect of neutron radiation on semiconductor devices. Neutron radiation has become a concern since it was recognized that neutrons can induce failures in semiconductor devices. Neutrons are produced by galactic and solar cosmic rays reacting with the nuclei in the atmosphere. Although most of the primary cosmic rays and the charged particle component of the cosmic-ray shower are absorbed by the atmosphere, neutrons, because they have long mean-free paths, reach aircraft altitudes and below. These neutrons can interact with the material in the semiconductor device and cause charged recoils and secondary particles that deposit charge and energy in the devices. This charge deposition can initiate so-called single-event effects in semiconductor devices.

Single-event effects include single-event upset (SEU), multiple-event upset (MEU), single-event latchup, single-event burnout, gate rupture, etc. Single-event upsets occur when a memory location changes its state because of charge deposited by an energetic particle. SEUs typically occur at a rate of approximately $10^{-9}$ upsets per bit per hour at aircraft altitudes. Although this type of failure may be corrected by error checking, increased costs and a loss of performance are associated with this solution. MEUs, where several memory locations are altered, have been measured to be a few percent of the single-event rate. MEU failures may be more difficult to correct than SEUs. Failures such as latchup, which are significantly less frequent, may be more serious because they cannot be corrected and usually require turning off the device to eliminate the latchup. Other failure modes occur in high-power devices in which the neutron initiates a charge particle cascade that causes the device to draw large currents. In these devices, the failure rate strongly depends on the applied voltage. It is observed that the failure rate increases dramatically above a critical threshold voltage that may be significantly less than the rated voltage of the device.

An experimental area that is dedicated to accelerated neutron testing is located on the $30^{\circ} \mathrm{L}$ (left of the incident proton beam) flight path at the high-energy neutron source at LANSCE. The shape of the neutron spectrum on the $30^{\circ} \mathrm{L}$ flight path along with the cosmic-ray flux (multiplied by $3 \times 10^{5}$ ) is shown in Figure 1. As shown in the plot, the shape of the LANSCE spectrum is very similar to the cosmic-ray induced neutron spectrum but more than 5 orders of magnitude more intense. The integrated neutron flux above $1 \mathrm{MeV}$ is approximately $10^{6}$ neutrons per $\mathrm{cm}^{2}$ per sec. This large intensity allows testing of semiconductor devices at a greatly accelerated rate in which 1 hour of exposure at LANSCE is equivalent to more than 30 years of flight experience.

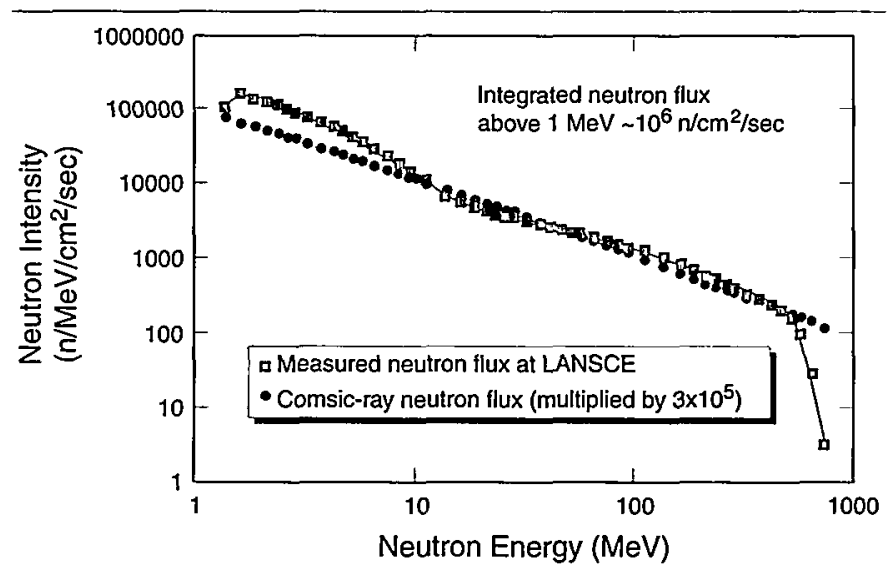

$\triangle$ FIGURE 1. The energy dependence of the cosmictay neution flux and the meosured flux of LANSCE. The cosmictoy neutron flux is multiplied by $3 \times 10^{5}$.

The shape of the neutron spectrum may be altered by placing absorbing material in the beam to reduce the low-energy intensity relative to the high-energy part. Figure 2 shows the effect on the shape of the neutron spectrum obtained by adding various amounts of polyethylene absorbers in the neutron beam.

The devices under test may be placed in air in the neutron beam, which has a spot-size diameter of approximately $10 \mathrm{~cm}$. Because the neutrons are not strongly absorbed by the device and the supporting boards, several devices may be placed in the neutron beam, one behind the other. The irradiation space is in a building that cannot be occupied while the beam is on. A second building, which is located 


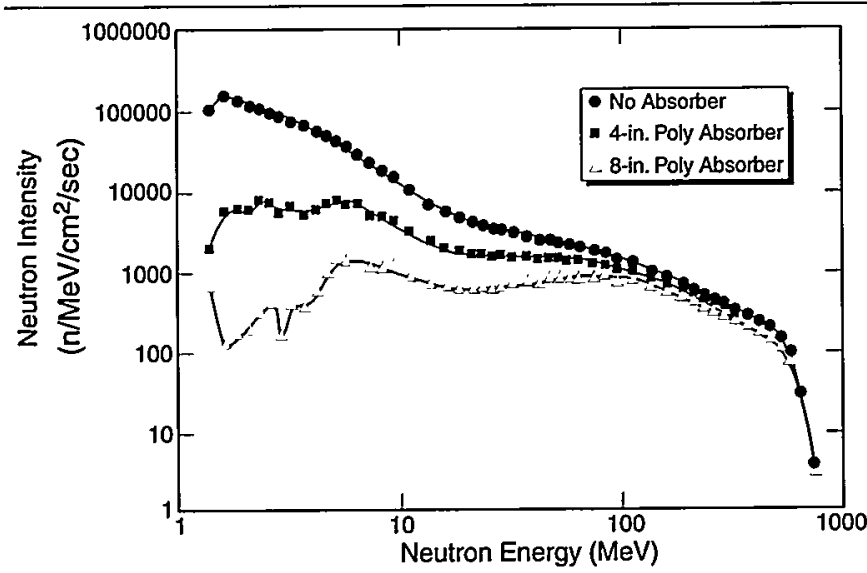

$\triangle$ FIGURE 2. The meosured energy dependence of the neutron flux of LANSCE for three absorber situations: no absorbers, 4 in. of polyethylene, and 8 in. of polyethylene.

approximately $20 \mathrm{ft}$ away, is where the control instrumentation is placed and can be occupied while the beam is on.

On April 20,1998, the LANSCE User Group sponsored a workshop to discuss high-energy neutron irradiation of semiconductor devices. The workshop was held in conjunction with the Single-Event Effects Symposium held in Manhattan Beach, California. Continued use of LANSCE as an irradiation test facility was strongly endorsed by the participants of this workshop. 


\section{Microwave Beams for Material Processing}

\section{T. W. Hardek, D. E. Rees (LANSCE Division), J. D. Katz (Materials Science and Technology Division)}

\section{We have combined our expertise in assembling and operating microwave power sources with the materi- als science expertise of Materials Science and Technology Division to provide a unique millimeter- wave materials processing facility. The facility pro- vides both quasi-optical and multi-mode microwave beams over the frequency range of $30 \mathrm{GHz}$ to 84 $\mathrm{GHz}$ to demonstrate unique materials processing features not possible at the normal processing fre- quencies. ${ }^{I}$}

Microwave processing of materials has traditionally used frequencies in the 0.915 - and $2.45-\mathrm{GHz}$ frequency regions. Microwave power sources are readily available at these frequencies, but the relatively long wavelengths can present challenges in uniformly heating materials. An additional difficulty is the poor coupling of ceramic-based materials to the microwave energy. Working with the National Center for Manufacturing Sciences, we have assembled a high-frequency demonstration processing facility using gyrotronbased radio-frequency (rf) sources. The facility is primarily intended to demonstrate the unique features available at frequencies as high as $84 \mathrm{GHz}$. We are preparing a new facility to demonstrate the sintering of ceramics at $30 \mathrm{GHz}$.

Figure 1 shows the gyrotron equipment and processing chamber associated with the $37 / 84-\mathrm{GHz}$ quasi-optical material processing system. The system was provided by the Paton Welding Institute, Kiev, Ukraine. The gyrotron tubes were manufactured by the Sayute company in Russia. Replacement tubes now come from GYCOM of Nizhny

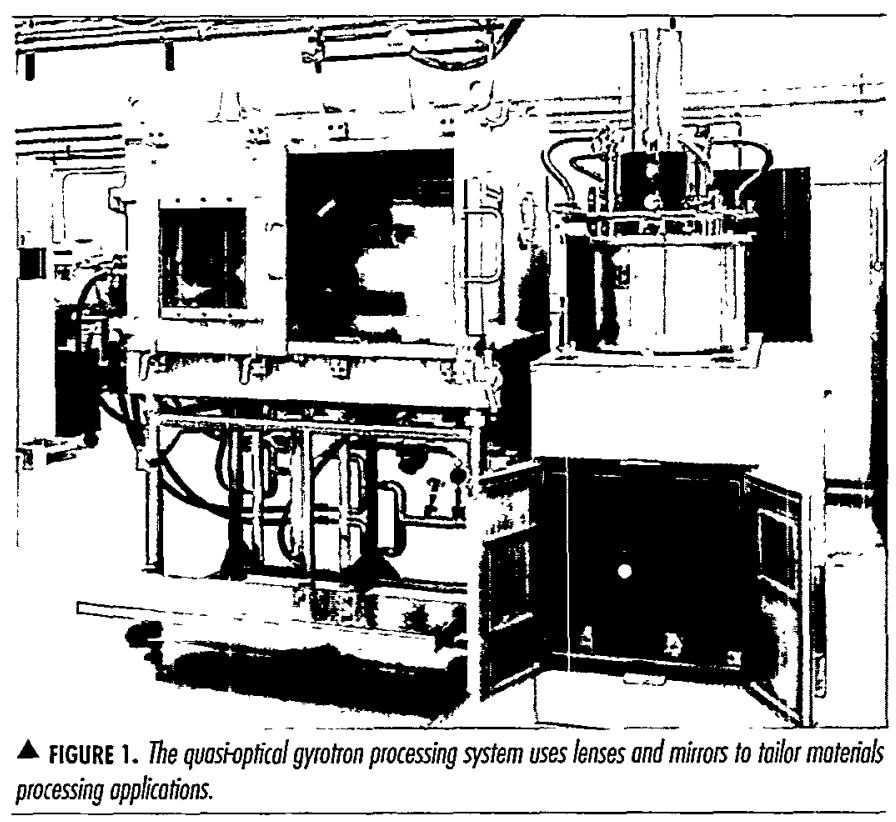

Novgorod, Russia. The gyrotrons for this system require a superconducting magnet, which is the cylindrical structure above the cabinet to the right in Figure 1. The entire system includes high-voltage power supplies and a control console not shown. This system can be operated with an 84-GHz tube or a 37-GHz tube at power levels from a few hundred watts to $10-\mathrm{kW}$ continuous wave (cw). The system may also be operated in a pulsed mode.

The system features a quasi-optical beam which may be tailored to the processing application by combinations of dielectric lenses and metal mirrors. In addition to adjusting the beam distribution, the mirrors may be scanned to sweep the beam across a large area sample. The beam may be focused to a spot or line distribution or metal masking may be used to provide more precise patterns.

Processing is done primarily in an air environment, although we sometimes insert the sample within a quartz tube and back fill the tube with an inert gas. A blower system fitted with a HEPA filter draws gas byproducts clear of the processing environment. The process may be viewed through water-filled, microwave-screened windows. We have a thermal-imaging camera and other thermal-detecting equipment available to document the process.

A major advantage of this kind of quasi-optical beam is for dedicated zone heating applications in which adjacent materials are not heated.

Figure 2 shows the $30-\mathrm{GHz}$ ceramic sintering system. The gyrotron is located in the left bay. The housing that fills the bottom of both bays is an optical transmission line.

Gyrotron output is converted from a TE02 mode to a quasioptical pseudo-Gaussian mode to transport it to the processing chamber. The bottom surface of the transmission line is covered by microwave lossy tiles to absorb energy reflected from the processing chamber. The processing chamber fills most of the right-hand bay.

The 30-GHz multi-mode cavity processing system produced by the Initiative of Applied Science, Nizhny Novgorod, Russia, was originally intended for the sintering of ceramics. ${ }^{2}$ The processing chamber has a uniform field region of about 1 cubic foot allowing bulk heating of samples in a controlled environment. A computer-based control capability offers the unique ability to program a variety of processing cycles with repeatable characteristics. In Figure 3, a sample temperature profile shows the actual temperature of a processed part as it follows a programmed function. 


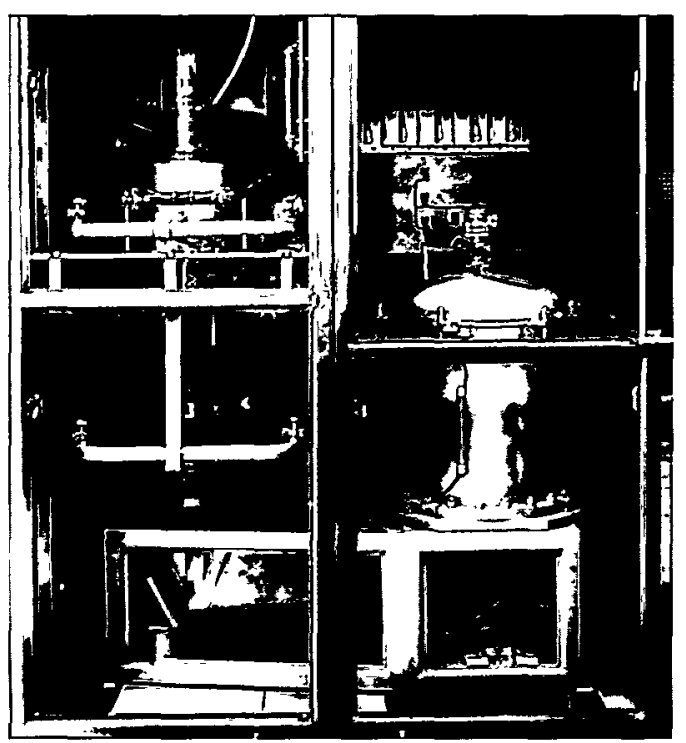

A FIGURE 2. The gyrotron sintering system allows for bulk heating of samples in o controlled environment.

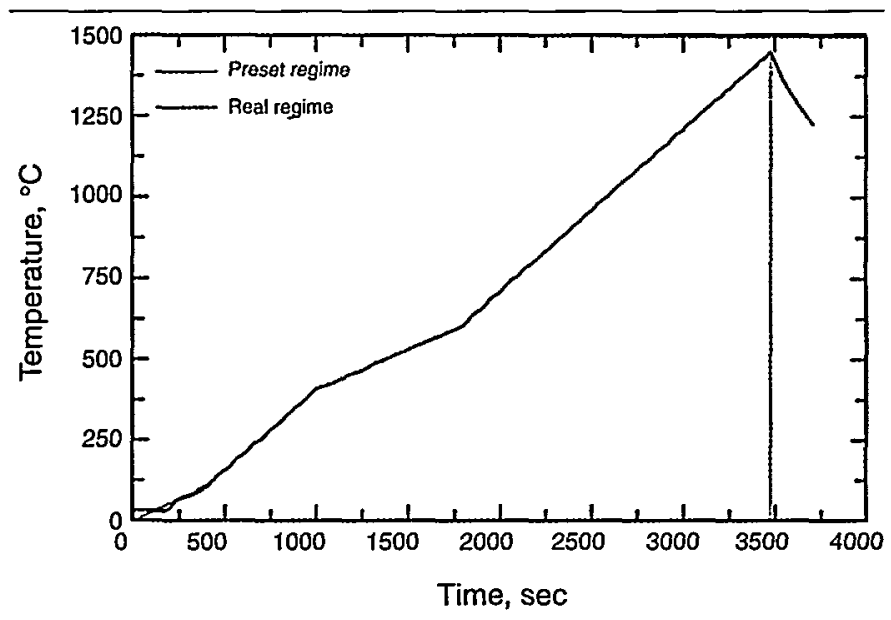

$\triangle$ FIGURE 3. This sample femperoture curve demonstrotes the unique ability of the gyrotion sintering system to repeat characteristics of processing cycles.

Figure 4 shows a typical material-processing fixture. ${ }^{3}$ This particular fixture was designed for a silicon carbide joining experiment. The fixture holds two cylindrical pieces of silicon carbide under tension. The motor to the right rotates the disks allowing the gyrotron beam access to all sides of

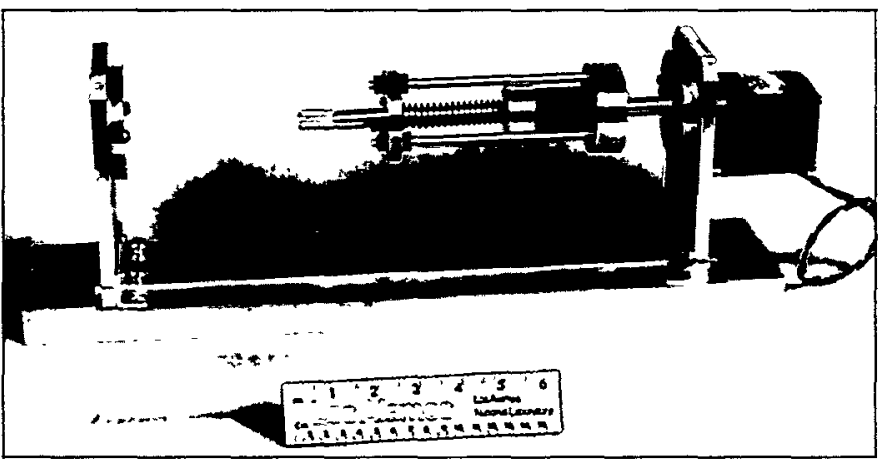

A FIGURE 4. A silicon carbide joining fixture used in the quasioptical gyrotton processing system.

the material. The video image in Figure 5 shows the material being heated by the gyrotron beam. The beam was focused to a spot of about $1 \mathrm{~cm}$ in diameter and directed at the joint region between the two cylindrical samples.

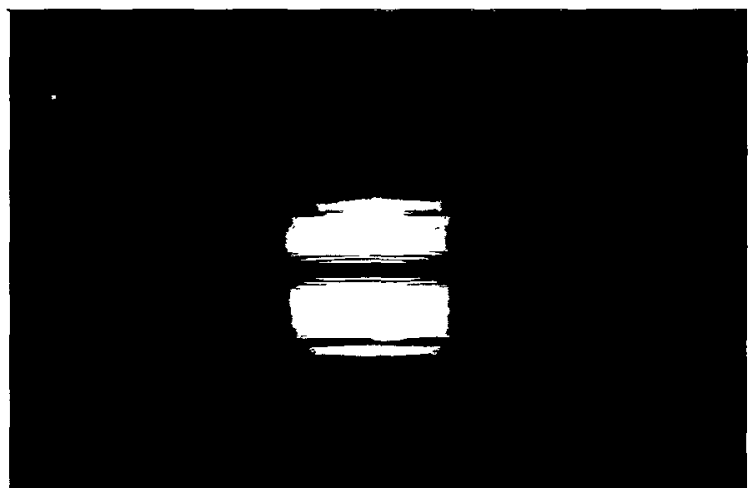

$\Delta$ FIGURE 5. Video frome shoving the heoting pottern produced by a $37-6 \mathrm{~Hz}$ beam spot focused onto the silicon carbide samples shown in Figure 4.

Figure 6 shows another processing fixture. ${ }^{3}$ Here a hairpin spring is used to hold the sample in place. The sample is metallic and is indirectly heated by directing the beam onto a microwave lossy material surrounding the sample.

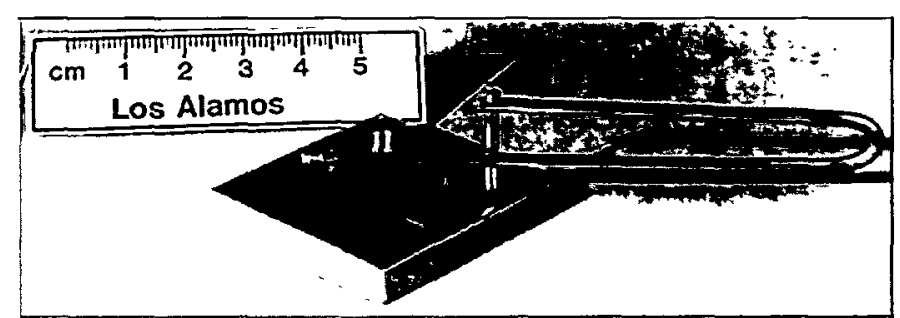

$\Delta$ FIGURE 6. A moterial joining fixture used in the quasiopticol gyrotron processing system.

\section{References}

1. J. D. Katz and D. E. Rees, "Quasi-Optical Gyrotron Materials Processing at Los Alamos," Ceramic Transactions 59, 141-147 (1995).

2. Y. Bykov, A. Eremeev, and V. Holoptsev, "Comparative Study of $\mathrm{Si}_{3} \mathrm{~N}_{4}$-Based Ceramics Sintering at Frequencies 30 and $83 \mathrm{GHz}$," in Microwave Processing of Materials V, M. F. Iskander, E. R. Peterson, J. O. Kiggans, and J. Ch. Bolomey, Eds. (MRS, Pittsburgh, Pennsylvania, 1996), pp. 613-618.

3. V. Skliarevich, consultant for NCMS, and M. Shevelev and P. Syrovets, Paton Welding Institute, Kiev, Ukraine, 


\title{
Parameterization of Structures in Energetic Materials
}

\author{
R. P. Hjelm (LANSCE Division), J. T. Mang, C. B. Skidmore, P. M. Howe (Dynamic \\ Experimentation Division)
}

\begin{abstract}
We have developed small-angle neutron scattering (SANS) as a nondestructive method to measure the external surface area per gram and internal void size distributions in crystalline high explosives. In this work, we highlighted the surface of the crystallites or the internal voids by immersing the sample in a uniform fluid of known neutron scattering length density; thus changing the contrast of the crystals against the background. This approach is known as the method of contrast variation. The SANS measurements were done on the high explosive, $H M X$, in both fine and coarse crystalline powders and in pressed pellets. Detailed analysis showed that the external surface area per gram is larger in fine versus coarse crystalline powders, and that the fabrication of pellets by pressing has little or no effect on the measured surface area. The analysis also showed that fine material had considerably smaller internal voids than coarse and that pressing reduced the mean size of both to about the same absolute value.
\end{abstract}

High explosive (HE) materials are composites, consisting of a crystalline $\mathrm{HE}$ and a polymeric binder. These materials possess both naturally occurring and process-related defects that can produce locally heated regions-hot spots - under conditions of impact or shock, for example. Hot spots are thought to occur by conversion of mechanical work into thermal energy through viscous void collapse. ${ }^{1}$ The critical hot spot temperature is known to be dependent upon both the size and shape of the defect, so shifts in the pore size distribution in an explosive can markedly change the explosive response. ${ }^{1}$ Though larger pores $(>1 \mathrm{~mm}$ diameter) are generally thought to be of more consequence in generating hot spots, recent molecular dynamics simulations ${ }^{2}$ have shown that nanometer-sized defects can have profound effects on the shock to detonation transition. Prediction of an explosive's response requires accurate measurements of a number of parameters for the HE composite structure, particularly surface area, porosity, and pore size distributions. We used SANS as a nondestructive measurement of these parameters on the nanometer to tens of nanometers scales to complement measurements by other structural probes such as optical microscopy and scanning electron microscopy. ${ }^{3}$

In these studies, we used SANS to measure the structure of coarse (C-HMX) and fine (F-HMX) grade powders of the $\mathrm{HE}, \mathrm{HMX}$, as well as to measure pellets of each pressed to
93\% (C-93) and 90\% (F-90) theoretical maximum density (TMD), respectively.

Scattering arises from the average squared scattering length density fluctuations in the sample, $\left\langle\Delta \rho^{2}\right\rangle$. Scattering length density, $\rho(\mathbf{r})$, is defined as the sum of the neutron scattering lengths for all the nuclei within a small sample volume at position $\mathbf{r}$ in the sample, divided by that volume. For HMX in air, the scattering is dominated by differences in $\rho$ between the voids and inter-crystal spaces, where $\rho \approx 0$, and the HMX crystal, where $\rho=4.5 \times 10^{10} \mathrm{~cm}^{-2}$. Our problem in measuring external surface and porosity is to differentiate between these two contributions to the scattering. Suppose we immerse the HMX crystals in a fluid with the same scattering length density as HMX. Then the contributions to $\left\langle\Delta \rho^{2}\right\rangle$ from the intercrystal spaces can be suppressed, leaving the dominant contribution to the scattering from the voids. In contrast to this case, suppose the fluid has a very different $\rho$ from HMX; then the scattering from the $\left\langle\Delta \rho^{2}\right\rangle$ between the intercrystal spaces and the HMX crystals will predominate. We can make such a fluid with adjustable $\rho$ by mixing cyclohexane, $\mathrm{C}_{6} \mathrm{H}_{12}$, with its deuterated counterpart, $\mathrm{C}_{6} \mathrm{D}_{12}$. In this case, we use the large difference in $\rho$ between hydrogen and deuterium to adjust the scattering length density of the fluid, $\rho_{\mathrm{f}}$, from $-0.28 \mathrm{x}$ $10^{10} \mathrm{~cm}^{-2}$ to $6.68 \times 10^{10} \mathrm{~cm}^{-2}$. In this, the method of contrast variation, we measure the scattering at several $\rho_{\mathrm{f}}$, then use the fact the intensity will be parabolic with $\rho_{f}, 3-5$

$I(Q, \Delta \rho)=\Delta \rho^{2} I_{\Omega}(Q)+\Delta \rho I_{\Omega, \zeta}(Q)+I_{\zeta}(Q)$.

Thus, we find the scattering at the zero contrast point, $I_{\zeta}(Q)$, by interpolating to $\Delta p=\left(\rho-p_{f}\right)=0$, the zero contrast point. The crystallite shape scattering, $\mathrm{I}_{\Omega}(\mathrm{Q})$, is determined by extrapolating to $\Delta \rho=\infty$, the infinite contrast point. With the scattering functions thus separated, we can determine the crystal external surface area from the Porod Law for the shape scattering, ${ }^{6}$

$$
\lim _{Q R>1} I_{\Omega}(Q)=\frac{2 \pi}{Q^{4}} S
$$

where $S$ is the surface area. The zero contrast scattering function is modeled by assuming Guassian distributions of spheres to give a measure of the void size distributions in the crystals.

The crystallite shape scattering functions, $\mathrm{I}_{\Omega}(\mathrm{Q})$, for the coarse and fine powders and pressed samples are shown in Figure 1. All samples displayed scattering that can be modeled using Equation (2). The results (Table 1) show that the fine crystals have significantly higher surface area than the 


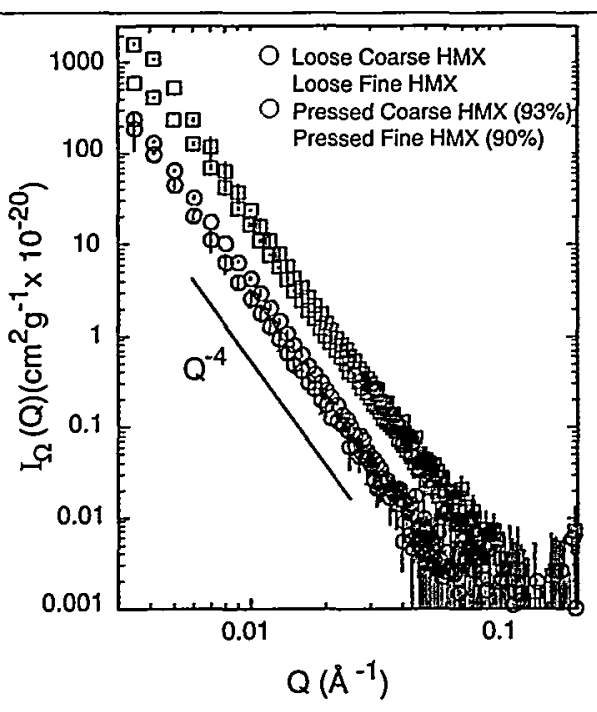

$\triangle$ FIGURE 1. Shape scattering functions, $I_{\Omega}(Q)$, for HMX. Line shows $Q^{-4}$ trend.

TABLE 1. Porameters Extracted from SANS Meosurements of HMX

\begin{tabular}{l|c|c|c|c}
\hline Sample & $\begin{array}{c}\text { Surface } \\
\text { area }\left(\mathbf{m}^{\mathbf{2}} \mathbf{g}^{-1}\right)\end{array}$ & $\begin{array}{c}\text { Internal Void } \\
\text { fraction, } \phi_{V}\end{array}$ & $\begin{array}{c}\text { Mean Pore } \\
\text { Diameter }(\dot{A})\end{array}$ & $\begin{array}{c}\text { Diameter } \\
\text { RMS }(\dot{A})\end{array}$ \\
\hline HMX-C & 0.45 & - & 560 & 320 \\
\hline HMX-F & 3.61 & 0.08 & 112 & 104 \\
\hline C93 & 0.65 & 0.04 & 98 & 46 \\
\hline F90 & 2.64 & 0.05 & 34 & 10 \\
\hline
\end{tabular}

coarse, as expected. Pressing induces a slight increase in $\mathrm{S}$ of the coarse material, probably due to breaking of the crystals. There is a decrease in $\mathrm{S}$ for the fine material. The scattering at zero contrast, or internal shape functions, $I_{\zeta}(Q)$, are shown in Figures 2(a) and 2(b). The void size means and the root mean deviations (RMS) from the sphere probe analysis (Table 1) show that, as expected, the voids are significantly larger in the coarse than in the fine material. Pressing reduces the dimensions of the pores significantly in both cases, the likely cause of which is void collapse and internal cracking.

Our results demonstrate that SANS is a powerful tool for use in the characterization and surveillance of $\mathrm{HE}$ materials.
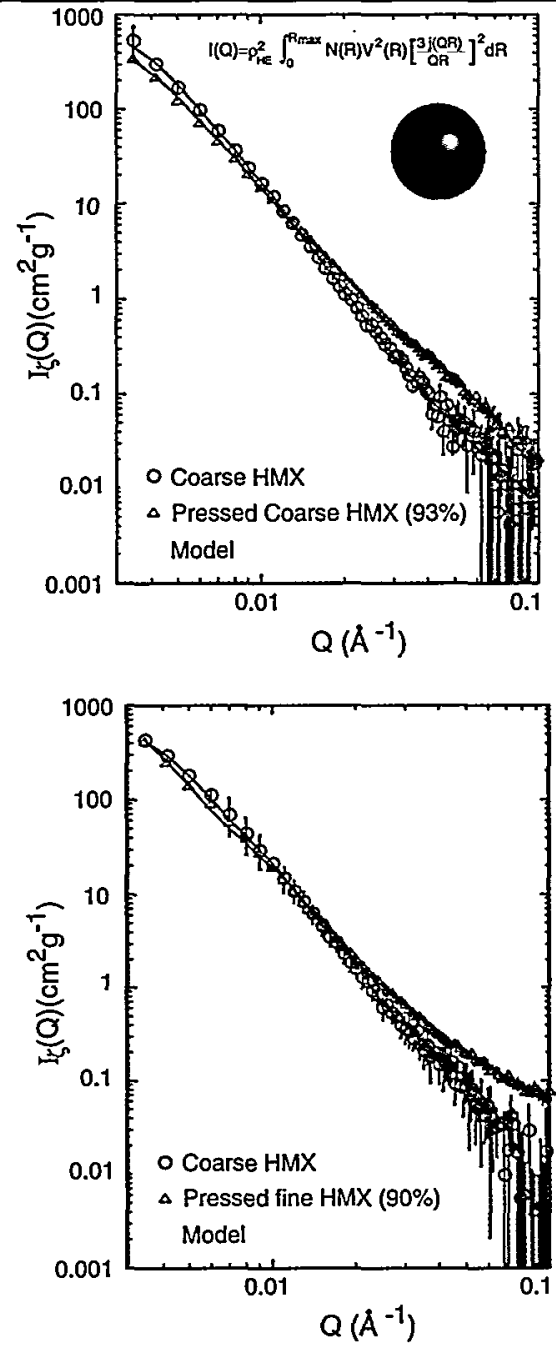

$\triangle$ FIGURE 2. Intemal structure scottering functions, $I_{\zeta}(Q)$ for HMX. (o) Coarse HMX. (b) Fine HMX. Lines are fits to the distributions for the pore diameters with mean and RMIS volues given in Toble I.

With SANS, we can obtain in situ measurements of surface area and quantitative measurements of internal void populations as well as changes in these quantities dependent upon external insult. These results indicate that there is significant structure in HMX in the range of 10 to $1000 \AA$, which may influence the shock-to-detonation transition. ${ }^{2}$

\section{References}

1. C. Tarver, S. Chidester, and A. Nichols, "Critical Conditions for Impact- and Shock-Induced Hot Spots in Solid Explosives," J. Phys. Chem. 100, 5794 (1996).

2. C. White, J. Barrett, J. Mintmire, M. Elert, and D. Robertson, "Effects of Nanoscale Voids on the Sensitivity of Model Energetic Materials," Decomposition, Combustion, and Detonation Chemistry of Energetic Materials (Materials Research Society, Pittsburgh, Pennsylvania, 1996), Vol. 418.

3. J. T. Mang, C. B. Skidmore, R. P. Hjelm, and P. M. Howe, "Quantification of Microstructural Features in HMX Using Small-angle Neutron Scattering Techniques," in Proceedings of 29th International Institut Chemische Technologie Conference (in press).

4. R. Hjelm, W. Wampler, P. Seeger, and M. Gerspacher, "The Microstructure and Morphology of Carbon Black: A Study Using Small-angle Neutron Scattering and Contrast Variation," J. Mater. Res. 9, 3210-3222 (1994).

5. R. Hjelm, J. Mang, C. Skidmore, and M. Gerspacher, "Probing the Structure of Composite Materials with Small-angle Neutron Scattering," in Proceedings of the Materials Research Using Cold Neutrons at Pulsed Sources Conference (World Scientific Publishing, in press).

6. O. Glatter and O. Kratky, Small Angle X-ray Scattering (Academic Press, London, 1982). 

Proton Storage Ring Béam Dynamics: Beam Instability and'Space-Charge Compensóting Inductor Experiments

:

LANSCE Accelerator Performance in the Long-Pulse Spallation Source Tests

Self-Amplified Spontaneous Emission and Régenérative Amplifier Free-Electron Laser

Intense, Sub-Picosecond Electron Beams

Explosive Pulsed-Power Developments and Modeling:

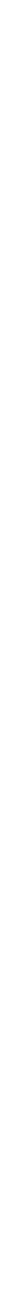




\section{EPICS Developments and the EPICS Collaboration}

\section{P. Gurd (LANSCE Division)}

\section{The Experimental Physics and Industrial Control System (EPICS) is a widely used control-system- development tool kit that originated in the Accelerator Controls, Automation, and Computer Networking Group (LANSCE-8). In the past 2 years, both the technological and the sociological (collabo- rative) aspects of EPICS have grown significantly. The collaboration now has just under 100 partici- pants, and new technological developments are being applied to LEDA experiments at LANSCE.}

\section{EPICS: The Collaboration}

EPICS is a control-system tool kit based on the groundtest-accelerator control system developed initially by LANSCE-8 at Los Alamos National Laboratory, and later jointly with the Advanced Photon Source (APS) Controls Group at Argonne National Laboratory. Starting in 1993, other laboratories joined this collaboration, and by early 1998 ninety-five laboratories worldwide were participating, with more applications pending (Figure 1.) The tool kit is distributed free of charge to not-for-profit organizations, universities, and national laboratories. In addition, two commercial licensees have installed the tool kit for various large industrial applications, ranging from wastewater treatment to wind tunnels.

The EPICS tool kit has become the method of choice for accelerator and telescope control systems. Besides the APS and the Continuous Electron Beam Accelerator Facility at the Thomas Jefferson National Accelerator Facility (TJNAF), new accelerators under construction or proposed for construction have also selected EPICS. These include the Swiss Light Source, the Japanese B-Factory, the Spallation Neutron Source at Oak Ridge National Laboratory, and the Next Linear Collider, a 30-km-long behemoth proposed by the Stanford Linear Accelerator Center. The largest new telescopes, KECK II and Gemini, also use EPICS, as do many of the detectors planned for new accelerator facilities, such as the relativistic heavy-ion collider at Brookhaven National Laboratory.

Although the core parts of EPICS are still maintained at Los Alamos and APS, a large fraction of EPICS collaborators contribute significantly to both system improvements and new applications. Mutual assistance is provided with an internet "exploder," and the collaborators meet two to three times a year to share experiences and plan direction. A recent (April 1998) collaboration meeting at Los Alamos drew over 60 participants from 25 institutions in Europe, Asia, and the U.S.

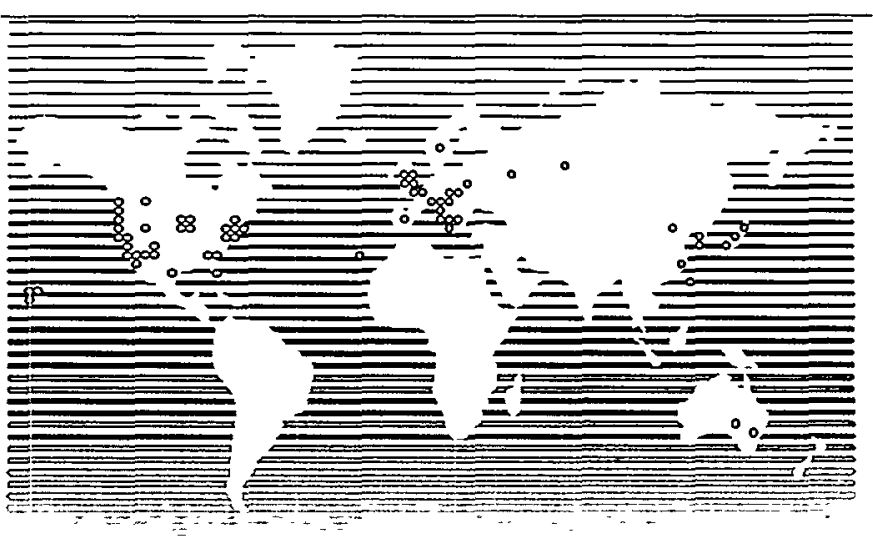

A fIGURE 1. Worldwide EPICS distibution-95 sites.

Previously, LANSCE personnel frequently visited other sites to provide training and help with the installation of EPICS systems. In the past 18 months, however, we have held a series of week-long workshops (one every few months) at which hands-on training is provided to a maximum of 8 new EPICS users. These workshops have been extremely successful, and each one has drawn nearly twice as many applicants as space allows. Participation has been worldwide.

\section{Development of the EPICS Tool Kit and Its Applications to LEDA}

EPICS is a distributed system that uses the popular clientserver architecture. Input/output controllers (IOCs) with local processors are distributed close to sensors and actuators, and workstation-based consoles are located at local control stations and in a central control room. The most common implementations use UNIX workstations as clients, and VME and VXI chassis with Motorola series single-board computers as local processors. (VME is an electrical and mechanical standard for housing elec-tronics modules, and VXI is a similar standard that is used when a much-improved electromagnetic interference environment is required.)

The IOCs and clients are linked on a standard transmission-control-protocol/internet protocol network (TCP/IP), generally but not necessarily implemented with Ethernet. The higher level protocol used by EPICS on this network is known as channel access (CA), developed at Los Alamos and optimized for performance. The database, which describes the complete configuration of $\mathrm{I} / \mathrm{O}$ signals and devices and their interrelationships, is distributed among the IOCs. Unlike conventional accelerator control system databases, the EPICS database contains links between database elements, or records, which can then be executed in 
"linked lists" without explicit commands from clients.

These two components, CA and the active, fully distributed database, characterize and distinguish EPICS.

Built on these core components is a host of other tools and applications. These include the following:

- configuration management tools,

- display editors and user interfaces,

- a state notation language,

- an alarm handler,

- a choice of archivers, and

- a long list of specialized applications and

device and field bus interfaces.

Change is quickest and collaborators make the largest contribution in this area of the so-called "extensions" to EPICS. LANSCE and the APS control groups together manage these changes and their integration and redistribution in the form of new EPICS releases.

The LEDA injector test stand, in use at TA-53 for several years, runs on the EPICS release numbered "EPICS version 3.11." A major change undertaken for the LEDA injector itself was to upgrade this control system to the current release of EPICS, version 3.13. This version includes a number of improvements incorporated over the past few years:

- access control to limit access by unauthorized users;

- synchronous "puts"- the ability to synchronize state changes;

- source release under the commercial Concurrent Version System (CVS) configuration control tool;

- portable CA server for use outside the IOC controller, allowing easier integration of legacy control and data acquisition systems;

- database changed to ASCII (text) encoding, making cross-platform development easier;

- ability to modify I/O addresses while control process is running;

- a gateway function for secondary user access with minimal performance impact;

- the capability to build IOC software under windows NT (in earlier versions it could be built only on UNIX systems);

- EPICS core on Power PC, Pentium, and Alpha platforms in addition to UNIX platforms;

- new clients, including the object-oriented device support layer (CDEV) developed at TJNAF; and

- new features added to display managers: state notation language and device support.
These new features are all available in the LEDA control system currently under development at LANSCE, and many are being used. The control-system layout is pictured in Figure 2. The diagram shows three varieties of IOC: Pentium-based PC IOCs in addition to the traditional VME- and VXI-based IOCs. These PC IOCs were introduced and commissioned on the LEDA if window test and are being incorporated in the high-power if control system. It appears possible to configure a PC IOC for under $\$ 1000$, as compared to approximately $\$ 5000$ for the VME-based IOC it replaces. We are minimizing the risk in using this new technology by limiting the number and types of $\mathrm{I} / \mathrm{O}$ devices driven by PC IOCs. In particular, the high-power if systems are being interfaced exclusively through vendorsupplied, commercial, programmable-logic-controller interfaces, for which a new EPICS software driver was written.

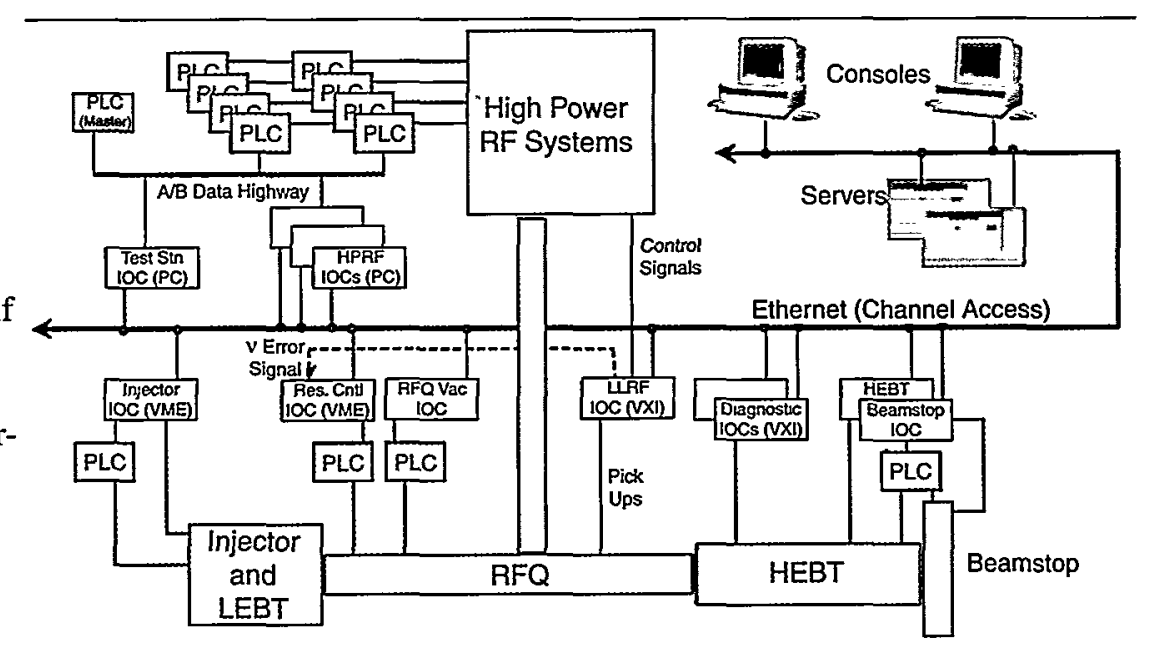

A FIGURE 2. The LEDA control system.

Software I/O drivers for "industry pack" (IP) modules have also been developed in association with the PC IOC development project. These inexpensive commodity modules can be mounted in both PC and VME formats, and so bring another level of commonality to the various form factors and buses that can be used with EPICS. A new archi-ver client suitable for the long-term production running required at LEDA was also developed for this project and is being commissioned on the rf window test.

\section{Conclusion}

The EPICS collaboration is healthy and continues to grow. Technically, the capability of EPICS is expanding, and many of the new features are being applied to LEDA at LANSCE. 


\title{
The DOE Grand Challenge in Computational Accelerator Physics
}

\author{
R. Ryne (LANSCE Division), S. Habib (Theoretical Division), J. Qiang (LANSCE Divison), K. Ko, Z. Li, \\ B. McCandless, W. Mi, C. Ng, M. Saparov, V. Srinivas, Y. Sun, X. Zhan (Stanford Linear Accelerator \\ Center)
}

\begin{abstract}
Particle accelerators are playing an increasingly important role in basic science, applied science, and solving problems of national importance. However, the technological challenges associated with future accelerators will require a numerical modeling capability far beyond that which currently exists in the accelerator community. Such modeling is, in fact, essential to the success of many near-term and future accelerator projects. In 1997, the U.S.

Department of Energy initiated a "Grand Challenge" in Computational Accelerator Physics. The primary goal of this project is to develop a new generation of accelerator modeling tools for high performance computing platforms and to apply them to problems of national importance. This report describes the Grand Challenge and the progress-todate in performing very large-scale beam dynamics simulations on parallel computers.
\end{abstract}

Particle accelerators are playing an increasingly important role in basic and applied science. For example, many countries are now involved in efforts aimed at developing accelerator-driven technologies for transmutation of radioactive waste, disposal of plutonium, tritium production, and energy production. Additionally, next-generation spallation neutron sources based on similar technology will play a major role in materials science and biological science research. Finally, other types of accelerators such as the Large Hadron Collider, Next Linear Collider, and fourth-generation light sources will have a significant impact on basic and applied scientific research.

For all of these projects, high-resolution modeling far beyond that which has ever been performed by the accelerator community is required to reduce cost and technological risk and to improve accelerator efficiency, performance, and reliability. Indeed, such modeling is essential to the success of many of these efforts. For example, highaverage-power linear accelerators must operate with extremely low beam loss (of order $0.1 \mathrm{nA} / \mathrm{m}$ ) to prevent unacceptably high levels of radioactivity. To ensure that this requirement will be met, it is necessary to perform very high-resolution simulations using on the order of 100 million particles in which the beam propagates through kilometers of complicated accelerating structures. These simulations can only be performed on the most advanced high performance computing platforms using software and algorithms targeted to parallel and distributed computing environments. The calculations require performance of hundreds of GFLOPS to TFLOPS and core memory requirements of hundreds of Gbytes. An equally challenging issue is modeling the fields in electromagnetic structures, especially those having a complex three-dimensional nature. An example is provided by the NLC, for which the geometry of the accelerating structures varies on the order of microns from cell to cell, with the dimensions chosen to minimize wakefields effects. Modeling an entire 206-cell structure can only be accomplished on state-of-the-art, highly parallel computer systems.

In 1997 the U.S. Department of Energy initiated a Grand Challenge in Computational Accelerator Physics. The primary goal of this project is to develop a new generation of accelerator modeling tools for high performance computing platforms and to apply them to projects of national importance, including those mentioned above. The new tools will enable the simulation of problems 3 to 4 orders of magnitude larger than has ever been done before; additionally, the use of algorithms and software optimized for the high performance computing environment will make it possible to obtain results quickly and with very high accuracy. Significant progress has already been made in regard to parallel beam dynamics calculations for proton linac design and parallel wakefield calculations for linear collider design. 1,2 The tools are being developed by a collaboration involving U.S. national laboratories (Los Alamos and Stanford Linear Accelerator Center), universities (University of California at Los Angeles and Stanford University), and high performance computing and communications research centers (Advanced Computing Laboratory and National Energy Research Scientific Computing Center). The remainder of this report focuses on our progress in performing very large-scale beam dynamics simulations of high-current proton linacs.

As mentioned previously, future high-average-power linacs will have to operate with extremely low beam loss in order to prevent unacceptably high levels of radioactivity. Very small losses are known to be associated with the beam halo, a very low-density region far from the beam core. Understanding, predicting, and minimizing beam halo has become a major issue for projects such as the APT project. As of this time, we have performed the largest linac simulations to date for the APT project, using up to 10 million particles. This was accomplished by developing parallel versions of the codes PARMILA and LINAC. In addition, a new code called HALO has been developed specifically for modeling beam halo. This code was originally developed 
in collaboration with the University of Maryland, ${ }^{3}$ and has since been extended to treat nonlinear of focusing fields as part of a collaboration involving Lawrence Livermore National Laboratory. ${ }^{4}$

The huge amount of data in a high-resolution beam dynamics simulation, coupled with the fact that we are often interested in the very small fraction of the particles in the halo, necessitates the use of internal data analysis in our codes prior to storing simulation results. We have developed parallel algorithms that drastically reduce the amount of data needed to visualize the halo. An example is shown in Figures 1 and 2, which are the result of performing a HALO simulation with an initially mismatched Gaussian beam using 25 million particles. Figure 1 shows the result of the simulation by plotting 32,000 particles at random. In this figure, the details of the beam halo are nearly invisible. In contrast, Figure 2 also shows 32,000 particles, but the data was pruned during the execution of our parallel code using.a technique that we call a "low-density cut." In this figure, the details of the halo are clearly visible. Lastly, Figure 3 shows the beam density in $(x-y)$ space from a 10 million particle simulation of an initially mismatched beam using a parallel version of PARMILA. Unlike the usual (nonparallel) version of the code that uses a $2 \mathrm{D}(\mathrm{r}-\mathrm{z})$ mesh, we developed a parallel, 3D space charge routine for this version of the code. This plot demonstrates that we are currently able to perform simulations with a dynamic range of approximately four decades in beam density.

In conclusion, high performance computers are enabling us to perform accelerator simulations of unprecedented size and resolution. We have ported existing codes to parallel platforms and we have developed new codes, in order to improve our capability to design next-generation accelerators and to explore the physics of intense charged particle beams. Considering the advances that we have already made and the expected increase in computational power in the next few years, it is likely that we will soon be able to model high-current linacs with 1 billion particles, a number roughly equal to the actual number of particles in a beam bunch.

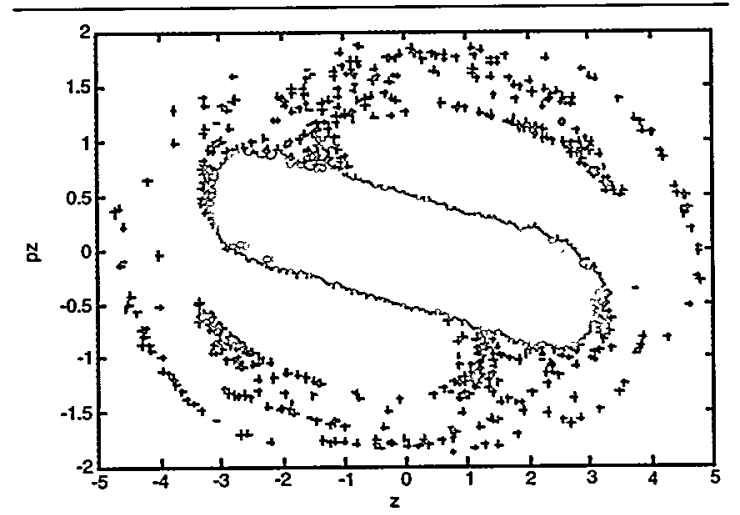

$\triangle$ FIGURE 1. The result of a HALO simulation that plotted 32,000 particles of rondom.

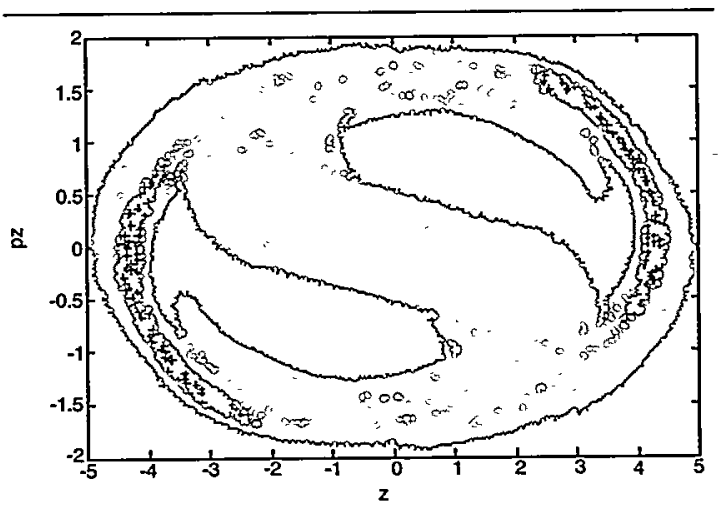

$\Delta$ FIGURE 2. The result of a HALO simulation that plotted 32,000 particles using a fechnique colled "low-density cut."

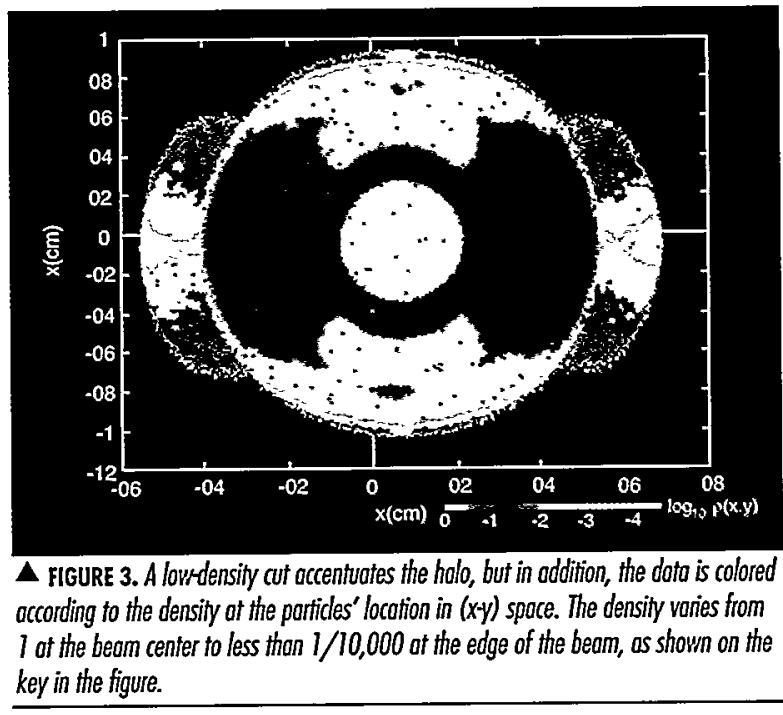

\section{References}

1. R. Ryne and S. Habib, "Parallel Beam Dynamics Calculations on High Performance Computers," in AlP Conf. Proceedings, J. Bisognano and A. Mondelli, Eds. (AIP Press, Woodbury, NY, 1997), Vol. 391, p. 377.

2. X. Zhan and K. Ko, "Parallel Computation of Transverse Wakes in Linear Colliders," in AIP Conf. Proceedings, J. Bisognano and A. Mondelli, Eds. (AIP Press, Woodbury, NY, 1997) Vol. 391, p. 389.

3. R. Gluckstern, A. Fedotov, S. Kurennoy, and R. Ryne, "Halo Formation in 3D Bunches," Los Alamos National Laboratory document (submitted to Phys. Rev. E).

4. J. Barnard, S. Lund, and R. Ryne, "Self-Consistent 3D Simulations of Beams in rf-Linacs," 1997 Linac Conference, Lawrence Livermore National Laboratory document. 


\title{
Proton Storage Ring Beam Dynamics: Beam Instability and Space-Charge Compensating Inductor Experiments
}

\author{
M. A. Plum, D. Fitzgerald, R. J. Macek, F. E. Merrill, F. Neri, O. R. Sander, H. A. Theissen, \\ J. R. Langenbrunner, P. L. Walstrom, C. A. Wilkinson (LANSCE Division), D. Johnson, B. Prichard \\ (Scientific Applications International Corp.)
}

\section{The Proton Storage Ring (PSR) beam instability must be controlled to reach the Short-Pulse Spallation Source Enhancement Project (SPSS-EP) design goal of $200 \mu \mathrm{A}$ at $30 \mathrm{~Hz}$. In 1997, experi- ments were conducted to explore beam parameters that affect the instability threshold, to test theoretical models of the instability, and to test the idea of using a space-charge compensating inductor to augment the rf system in the PSR.}

A primary goal of the SPSS-EP is to double the beam current of the PSR from $100 \mu \mathrm{A}$ at $20 \mathrm{~Hz}$ to $200 \mu \mathrm{A}$ at $30 \mathrm{~Hz}$. To achieve this goal we must raise the threshold of the PSR beam instability and we must counteract the increased longitudinal space-charge forces. Both results are typically achieved by increasing the of buncher voltage. This is effective up to a point, but if we extrapolate to the buncher voltage needed to reach our design intensity, the resultant momentum spread will increase the beam size and, therefore, the beam loss. Also, buncher systems are large and expensive, and there may be a more efficient method of controlling the instability.

To investigate these issues, we conducted experiments in 1997 that can be divided into two categories: (1) measurements related to the beam dynamics of the instability and (2) experiments with a space-charge compensating inductor.

Past experiments indicate that the PSR beam instability is most likely an electron-proton (e-p) instability. In this type of instability, the circulating protons interact with background, low-energy electrons to develop coupled oscillations. When the beam intensity reaches a certain level, the transverse oscillations can increase in amplitude until the beam hits the beam pipe walls and is lost. Simple theoretical models ${ }^{1}$ predict that an increase in the rf buncher voltage will increase the instability threshold, and this is, in fact, what we have observed in the past, as shown in Figure 1. In 1997, to learn more about the instability, we tested more detailed predictions. ${ }^{2}$ For example, the models predict that the threshold is proportional to the amount of beam that leaks into the gap between the head and the tail of the beam bunch (due to longitudinal space-charge forces). By modifying the chopper control circuitry in the low-energy transport region of the linear accelerator, we purposely injected beam into the gap. The results, shown in Figure 2, confirmed that the threshold is, in fact, directly

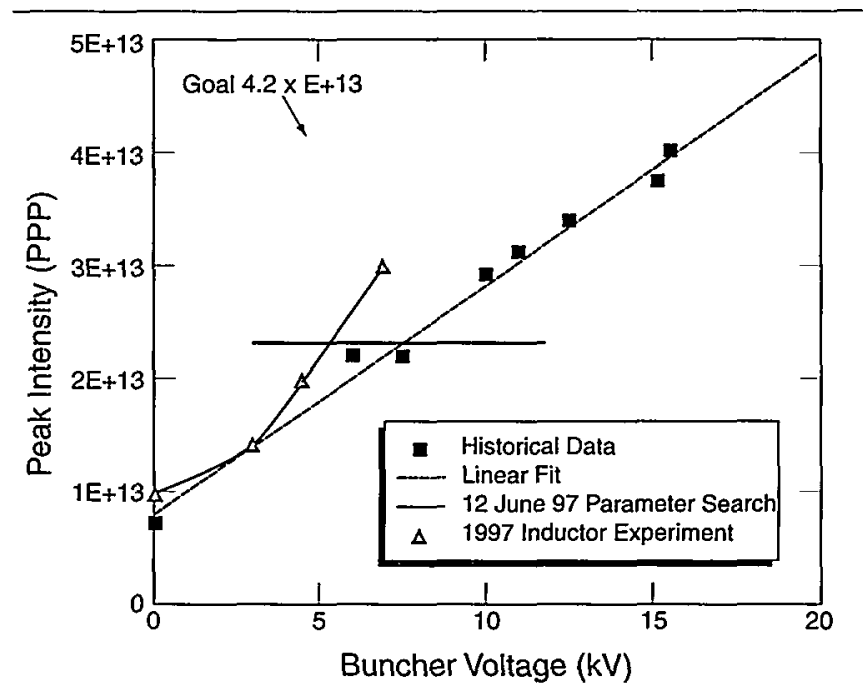

A FIGURE 1. Moximum stoble peok beam current as a function of buncher voltage (squares and dashed line), threshold variation os a function of beam ond PSR lattice parameters (horizontal thick solid line), and the improvement in the threshold due to the inductor (triangles ond thin solid line).

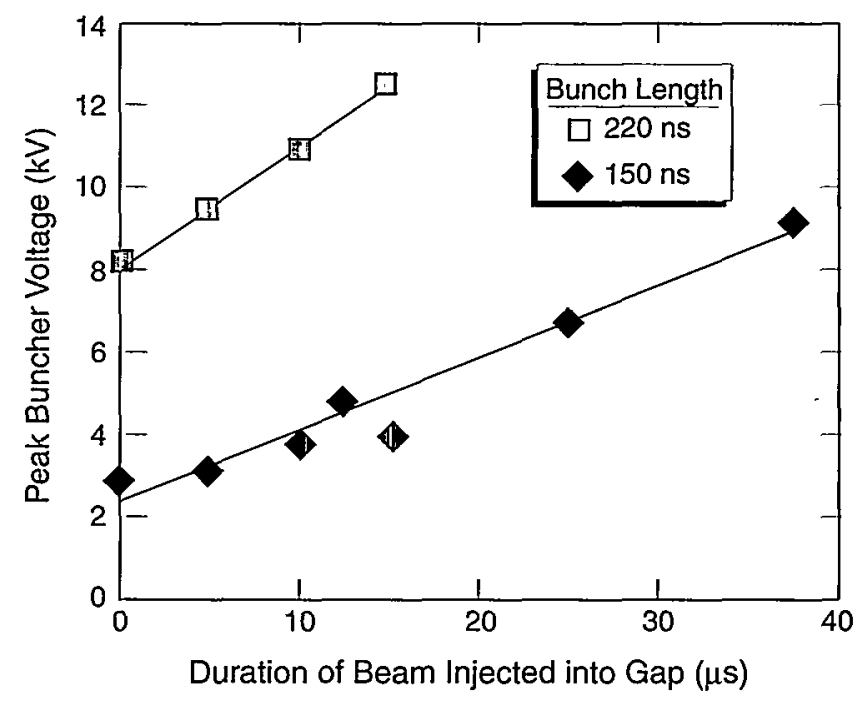

A FIGURE 2. Buncher voltoge needed to stabilize the beam, os a function of beam injected into the gap, for two different bunch lengths.

proportional to the beam injected into the gap. In another experiment, we varied beam parameters to purposely mismatch the beam injected into the longitudinal phase space of the PSR. The data show a variation of the instability threshold by a factor of more than 2 , as indicated by the horizontal bar in Figure 1. This result is also consistent with model predictions. 
Another model prediction is that the frequency of the transverse oscillations will be proportional to the square root of the beam intensity. We examined the frequency spectra of the oscillations for two different beam intensities, and we found that both the magnitude of the oscillation frequency and the scaling of the frequency with beam intensity are consistent with model calculations.

The second category of experiments involved inserting an inductor into section 3 of the PSR. The idea is to increase the natural inductance of the PSR enough to offset the effect of the space-charge forces that tend to spread the beam longitudinally. (The beam bunch has a triangular shape in the longitudinal dimension, and the inductor will create an induced voltage proportional to the slope of the line-charge distribution. This voltage will tend to push particles back toward the center of the bunch, thus counteracting the effects of space charge.)

The inductor, built at Fermilab, is $2.4 \mathrm{~m}$ long and has an inductance of about $8 \mu \mathrm{H}$. Solenoidal coils wrapped around the inductor allowed us to reduce the inductance by saturating the ferrite rings inside the vacuum chamber. If the inductor acts as expected, the bunch shape should be more diffuse when the solenoidal coils are energized. This is, in fact, what we observed, as shown in Figure 3. Also, if the inductor improves the beam stability, we expect that lower buncher voltages will be needed to control the instability. We measured the maximum stable beam intensity that could be stored as a function of buncher voltage. These measurements are compared with the historical data in Figure 1. From this figure, we conclude that the inductor appears to raise the instability threshold. However, more work is needed to draw more definitive conclusions.

In summary, all of our instability tests to date are consistent with e-p instability models. The new data we obtained in 1997 increases our confidence that we will be able to effectively control the instability at the design intensity. Preliminary tests using an inductor to augment the if system are encouraging, and we are now planning further inductor-based experiments.

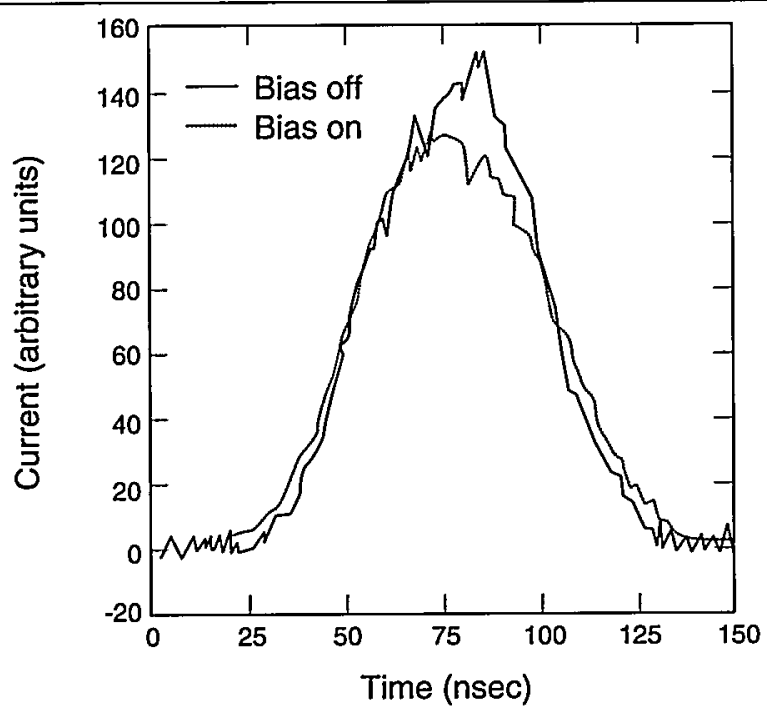

$\Delta$ FIGURE 3. Measured pulse shopes ofter $625 \mu \mathrm{s}$, for an injected bunch length of $50 \mathrm{~ns}$, with and vithout the solenoidal bios field. Note thot the pulse is shoper for the bios off (maximum inductance) case.

\section{References}

1. D. Neuffer et. al., "Observations of a Fast Transverse Instability in the PSR," Nucl. Inst. and Meth. A321, 1-12 (1992).

2. M. Plum et al., "Recent Experimental Evidence for the Los Alamos Proton Storage Ring Beam Instability," Proceedings of the IEEE Particle Accelerator Conference, Vancouver, Canada, May 12-16, 1997, Los Alamos National Laboratory report LA-UR-97-1741. 


\section{LANSCE Accelerator Performance in the Long-Pulse Spallation Source Tests}

\section{J. Rybarcyk, J. T. M. Lyles (LANSCE Division)}

\section{A new neutron source that would complement the exist- ing Lujan Center source has been proposed for the LANSCE facility. We led a series of tests to examine the capabilities of the LANSCE accelerator in the context of this long-pulse spallation source (LPSS) upgrade.}

The LPSS is an upgrade path recently proposed for the LANSCE accelerator facility. The LPSS design consists of a 1-MW neutron spallation target fed directly by a pulsed $\mathrm{H}^{+}$beam from the LANSCE linear accelerator (linac). The spallation target and experimental hall would reside in the former meson physics hall, Area A. Experimental considerations dictate a proton-beam repetition rate and pulse length of $60 \mathrm{~Hz}$ and $1 \mathrm{~ms}$, respectively, which equal a duty factor of $6 \%$. To achieve this beam power level at this duty factor, an average (peak) current of $1.25 \mathrm{~mA}(21 \mathrm{~mA})$ would be required for the $800-\mathrm{MeV}$ proton beam. The LANSCE accelerator would also be required to simultaneously deliver $\mathrm{H}^{-}$beams to the short-pulse spallation source (SPSS) at the Lujan Center and to WNR at the requisite duty factors and currents. This scenario, however, places more stringent operating conditions on the linac and its associated radio-frequency (rf) systems. For this reason, we performed tests to examine the response of the rf systems to conditions that mimic aspects of this scenario.

The main portion of the accelerator comprises a $100-\mathrm{MeV}$ drift-tube linac (DTL) followed by an $800-\mathrm{MeV}$ side-coupled linac (SCL). The DTL is made up of four accelerating cavities, each powered by separate 201.25-MHz (201) amplifiers. The SCL comprises forty-four $805-\mathrm{MHz}(805)$ accelerating modules, each powered by separate klystron amplifiers. Duty factors and beam currents that are typical of recent three-beam operations are summarized in Table 1.

\begin{tabular}{|c|c|c|}
\hline System or Beam & Duty Factor & Avg. Current \\
\hline 201 rf (DTL) & $10.0 \%, 120 \mathrm{~Hz} \times 835 \mu \mathrm{s}$ & \\
\hline 805 rf (SCL) & $9.4 \%, 120 \mathrm{~Hz} \times 785 \mu \mathrm{s}$ & \\
\hline Area $\mathrm{A}\left(\mathrm{H}^{+}\right)$ & $6.2 \%, 100 \mathrm{~Hz} \times 625 \mu \mathrm{s}$ & $1 \mathrm{~mA}$ \\
\hline Lujan Center $\left(\mathrm{H}^{-}\right)$ & $1.2 \%, \quad 20 \mathrm{~Hz} \times 600 \mu \mathrm{s}$ & $70 \mu \mathrm{A}$ \\
\hline WNR $\left(\mathrm{H}^{-}\right)$ & $6.2 \%, 100 \mathrm{~Hz} \times 625 \mu \mathrm{s}$ & $2 \mu \mathrm{A}$ \\
\hline
\end{tabular}

As shown in Table 1, the if pulses are somewhat longer than the beam pulses because of the time required to establish stable accelerating fields in the structures. A single test of the complete LPSS scenario was not possible because of beam current limitations in Area $\mathrm{A}$ and average power dissipation restrictions in the final power amplifiers (FPAs) used in the DTL if system.
We performed the tests during two separate beam development periods. We used the first test to evaluate the accelerator if systems under an operating scenario with LPSS-like pulse patterns and duty factors. The objective of this experiment was to simultaneously deliver an evenly spaced $60-\mathrm{Hz} \times 1-\mathrm{ms}, 1-\mathrm{mA}, 800-\mathrm{MeV} \mathrm{H}^{+}$beam to Area A ( $800 \mathrm{~kW}$ or $80 \%$ of LPSS design), an evenly spaced $20-\mathrm{Hz} \times 600-\mu \mathrm{s}, 70-\mu \mathrm{A} \mathrm{H}-$ beam to the Lujan Center, and $60-\mathrm{Hz} \times 600-\mu \mathrm{s}, 1-\mu \mathrm{A} \mathrm{H} \mathrm{H}^{-}$beam to WNR. We performed the test with an $\mathrm{H}^{+}$peak current $(16.5 \mathrm{~mA})$ that is a large fraction of the LPSS design current and is typical of our most recent reliable high-peak operation. Achieving the 1-ms-long $\mathrm{H}^{+}$beam pulse required increasing the rf pulse lengths by an additional $400 \mu$ s over nominal values. To maintain the same rf duty factor, without increasing the plate dissipation on any of the 201 FPAs used for the DTL, we reduced the repetition rate for the rf pulses to $80 \mathrm{~Hz}$ from $120 \mathrm{~Hz}$. The duty factors used during the test are shown in Table 2.

\begin{tabular}{|c|c|}
\hline System or Beam & Duty Factor \\
\hline $201 \mathrm{rf}$ (DTL) & $9.9 \%, 80 \mathrm{~Hz} \times 1235 \mu \mathrm{s}$ \\
\hline $805 \mathrm{rf}(\mathrm{SCL})$ & $9.5 \%, 80 \mathrm{~Hz} \times 1185 \mu \mathrm{s}$ \\
\hline Area A $\left(\mathrm{H}^{+}\right)$ & $6.1 \%, 60 \mathrm{~Hz} \times 1015 \mu \mathrm{s}$ \\
\hline Lujan Center $\left(\mathrm{H}^{-}\right)$ & $1.2 \%, 20 \mathrm{~Hz} \times 600 \mu \mathrm{s}$ \\
\hline WNR $\left(\mathrm{H}^{-}\right)$ & $3.8 \%, 60 \mathrm{~Hz} \times 625 \mu \mathrm{s}$ \\
\hline
\end{tabular}

The results of this demonstration ${ }^{1}$ were very encouraging. They showed that the present linac is capable of delivering $80 \%$ of the LPSS design current at the requisite duty factor while simultaneously providing the standard production beams to the Lujan Center and WNR facilities. Although the duration of the test was short ( 2 days), the final availability for all beams during the last 32 hours was greater than $87 \%$, and beam losses along the linac were slightly lower than normal. The DTL's 201 rf systems performed well under the LPSS test conditions. Only minor adjustments were required to maintain proper operation. Although the $805 \mathrm{rf}$ systems required more attention and resulted in the largest source of downtime, they generally performed well. Over half of the total amount of downtime attributed to these systems was associated with faults that were unrelated to the long-pulse operation. The combination of irregular pulse spacing and longer pulse length resulted in appreciable voltage droop on the SCL rf system high-voltage power supplies (HVPSs). This resulted in larger pulse-to-pulse variations in klystron gain, which required the feedback control loops to operate over a larger dynamic range than usual. Improvements to the HVPS capacitor banks would help mitigate this problem. 
We performed a follow-up test more recently to evaluate the accelerator if systems under 21-mA peak beam current operating conditions. The objective of this test was to deliver sufficient average current at $21-\mathrm{mA}$ peak to examine the rf system performance under these conditions. For this test, the linac rf duty factors were not modified but remained at the values shown in Table 1. Also, the Lujan Center and WNR $\mathrm{H}^{-}$beams were not delivered during this test. The average beam current delivered to Area $A$ was limited by restrictions from experimental hardware in that beam line.

The results of this test were also encouraging. For a period of about 12 hours, $315 \mu$ As of $21-\mathrm{mA}$ peak $\mathrm{H}^{+}$beam was successfully delivered to Area A with greater than $88 \%$ beam availability. Once again, the 201- and 805-rf systems performed well, although the SCL systems were more problematic. Large beam turn-on transients observed in the error signals of several klystron low-level controls exceeded the limits allowed for normal beam operation. Temporary modifications to these systems were required to allow beam operations to proceed. This deficiency could be corrected with improvements to the existing feed-forward portion of the low-level control system. Although beam losses were significantly higher than usual in the linac, this was not unexpected and was due in part to the limited amount of beam matching performed during the short duration test.

The response of the rf systems to the simulated LPSS beam conditions in these two experiments has helped us identify shortcomings of the present linac in meeting LPSS performance goals. We are investigating a new 201-rf amplifier system capable of higher power operation to replace the present one. Power supplies and other supporting infrastructure (cooling, transmission lines, rf windows, diagnostics, etc.) are being incrementally upgraded for higher reliability and efficiency. ${ }^{2}$ We are also evaluating the HVPS capacitor banks and low-level control systems of the 805-rf systems to develop a plan to continually improve performance and meet future LANSCE needs.

\section{References}

1. L. J. Rybarcyk, J. T. M. Lyles, et al., "LPSS Demonstration Using the LANSCE Linear Accelerator," AOT-6 internal report AOT-6-96-44-TR (March 1996).

2. J. T. M. Lyles and C. C. Friedrichs, Jr., "LANSCE 201.25 MHz Drift Tube Linac RF Power Status," in Proceedings of the XVIII International Linac Conference, CERN report 96-07 (August 1996), p. 698. 


\title{
Self-Amplified Spontaneous Emission and Regenerative Amplifier Free-Electron Laser
}

\author{
D. C. Nguyen, R. L. Sheffield, N. A. Ebrahim, C. M. Fortgang, R. F. Harrison, J. M. Kinross-Wright, \\ S. K. Volz, G. E. Faulkner (LANSCE Division)
}

We report the observation of very high free-electron laser (FEL) gains, greater than $10^{5}$ in a single pass, in recent self-amplified spontaneous emission (SASE) experiments performed with the advanced FEL high-brightness, 17-MeV electron beam. Our experiments confirm theoretical predictions of FEL operating in the high-gain, single-pass regime. By introducing a small amount of optical feedback, we also achieved saturated operation of a new FEL design called the regenerative-amplifier $F E L$.

\section{High-Gain, Self-Amplified Spontaneous Emission} A FEL approach called SASE has recently received considerable interest. This approach relies on a very long wiggler in which an electron beam amplifies its own spontaneous emission and the radiation grows exponentially with distance in the wiggler. The interest in SASE results from a strong possibility of producing a picosecond, high-intensity beam of coherent $x$-rays needed for the fourth-generation light source. Although high-gain SASE has been demonstrated in the millimeter-wave region, experiments at shorter wavelengths have up to now produced single-pass gains less than 10 .

We report the observation of SASE gains larger than $10^{5}$ in the infrared with use of high-current, low-emittance electron beams from the Advanced FEL photoinjector. We show that the infrared radiation power measured at a distance from the wiggler evolved from spontaneous emission to SASE as we varied the beam current. By fitting the measured infrared energy versus current to an analytic model, we obtain the single-pass gain versus current. At a beam current of $260 \mathrm{~A}$, the first wiggler with a 1-m uniform section has a single-pass gain of 300 . The second wiggler, which is $2 \mathrm{~m}$ long, has a gain greater than $10^{5}$, the largest single-pass gain ever observed in the infrared region.

The SASE experimental setup consists of a high-brightness photoinjector integrated into an L-band linac, a 2-m-long wiggler, electron beam position and temporal diagnostics, and infrared radiation detectors. The $1300-\mathrm{MHz}$ linac consists of 10.5 cells, with a $\mathrm{Cs}_{2} \mathrm{Te}$ photocathode in the first half-cell. In operation, the photocathode is illuminated with the quadrupled-frequency, 8-ps Gaussian pulses of a modelocked Nd:YLF laser. Two solenoids, one at the photoinjector and the other in front of the wiggler, are used to focus the electron beam into the wiggler. The plane-polarized wigglers have a $2-\mathrm{cm}$ period in a modified Halbach design.
The first wiggler, which we called the RAFEL (for regenerative-amplifier FEL) wiggler, consists of a 1-m uniform section with an on-axis magnetic field of $0.7 \mathrm{~T}$ and a $1-\mathrm{m}$ tapered section. This wiggler has notched magnets to provide equal two-plane focusing such that a matched electron beam propagates through the wiggler as a round beam with a constant rms radius. The second wiggler, which we called the UCLA (for University of California at Los Angeles) wiggler, is a 2-m uniform wiggler with external quadrupole magnets to provide nearly equal two-plane focusing. This wiggler was built by researchers from UCLA and the Russian Kurchatov Institute from magnets provided by Los Alamos.

To determine the dependence of the SASE signal on electron beam current, we varied the charge in each electron bunch and measured the infrared energy in each micropulse with an infrared detector. At the highest charge, we measured an infrared energy in each 10-ps micropulse of $0.2 \mathrm{~nJ}$ from the RAFEL wiggler and $30 \mathrm{~nJ}$ from the UCLA wiggler. The micropulse energies are plotted versus beam currents in Figures 1a (RAFEL wiggler) and $1 \mathrm{~b}$ (UCLA wiggler). The infrared pulse energy, measured at the wiggler exit as a function of current, can be derived from an analytic model that we developed. Based on that model, we deduced a single-pass of 300 for the RAFEL wiggler and greater than $10^{5}$ for the UCLA wiggler. These two measurements are also in agreement with the exponential dependence of SASE power on the wiggler length, as the 2-m wiggler should have a gain that is the square of that of the $1-\mathrm{m}$ wiggler.

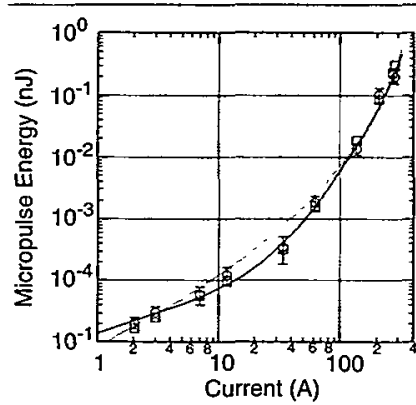

$\Delta$ FIGURE la. Loglog plot of SASE pulse energy versus current for the RAFEL viggler.

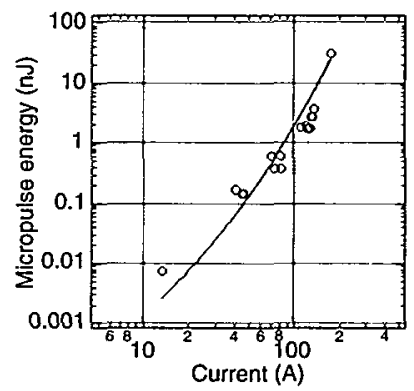

$\triangle$ FIGURE lb. Log log plot of SASE pulse energy versus current for the UCLA viggler.
The Regenerative Amplifier FEL Approach

These SASE experiments will reach saturation if the wiggler length is increased to about 3 to $4 \mathrm{~m}$. To reduce the wiggler length needed to reach saturation, we modified the standard SASE approach by reflecting a small amount of 
light exiting the wiggler back to the wiggler entrance via an optical feedback loop. The purpose of the optical feedback loop is to provide a small amount of optical power to the wiggler entrance so that the amplification process starts from a coherent signal. Essentially, by providing a small amount of optical feedback, we reuse the wiggler several times. The simplest way to provide a small optical feedback is to employ four mirrors-two flat annular mirrors and two parabolic mirrors-forming a rectangular ring (Figure 2). Light exciting the wiggler is captured by the downstream annular mirror and collimated by the righthand parabolic mirror. The collimated light is then focused by the left-hand parabolic mirror to the wiggler entrance. Because of its similarity to conventional laser regenerative amplifiers, we called this approach RAFEL.

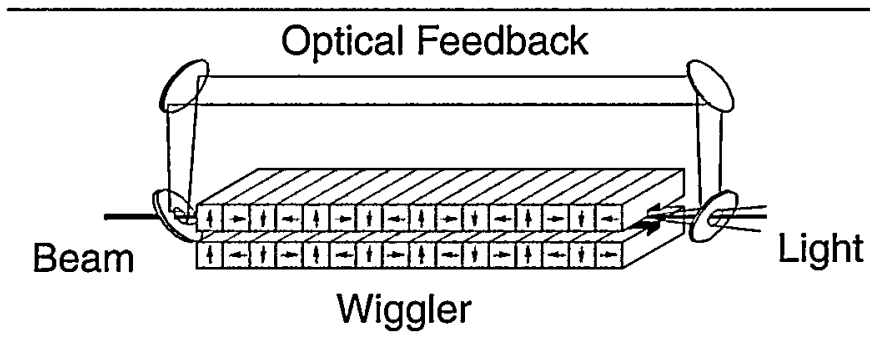

$\Delta$ FIGURE 2. Illustration of the RAFEl idea. The optical feedback loop consists of tyro onnulor mirrors and iwo paraboloids. The electron beam enters the high-goin viggler from the left through a small hole in the upstream minor. The fEL beam exits the feedback cavity through o lorge hole in the downstieam mirror.

After installing the feedback loop, we observed an optical power that exceeded the SASE power by more than six orders of magnitude. The measured micropulse energy was $1.5 \mathrm{~mJ}$, corresponding to a peak power of $\sim 100 \mathrm{MW}$. Since these results were obtained with $4 \mathrm{nC}$ of charge at $17 \mathrm{MeV}$, corresponding to $70 \mathrm{~mJ}$ of energy in each electron micropulse, we deduced $2 \%$ of the beam power was converted to FEL light exiting the cavity. The optical buildup to saturation was recorded with a high-speed copper-doped germanium detector that integrated the optical energy over each micropulse and yielded the pulse energies of individual micropulses. As there are two optical pulses in the feedback cavity, two sets of optical micropulses build up from intrinsically different gain conditions and achieve saturation at different times (Figure 3a). Unlike the output of a

SASE FEL, which has significant pulse-to-pulse fluctuations, the RAFEL output is relatively constant in pulse energy (Figure $3 b$ ) until the electron beam is turned off. When that happens, the infrared pulse energy decays exponentially with a loss per pass of $66 \%$. The high out-coupling loss is also characteristic of this new FEL concept.

\section{Future Prospects}

The success of this project already has significant impact on the international effort toward short-wavelength FELs. First, the observation of large single-pass gains demonstrates unequivocally the SASE principle and forms an important milestone in the collaboration to extend SASE toward the X-ray region. Secondly, the successful demonstration of RAFEL enables us to improve on the design of the fourth-generation light sources. Los Alamos is presently collaborating with the Stanford Linear Accelerator Center, UCLA, Brookhaven National Laboratory, and Lawrence Livermore National Laboratory on a project known as the Linac Coherent Light Source. Our next step is to demonstrate SASE in the visible, ultraviolet, and eventually the $x$-ray region. The generation of a beam of picosecond coherent $\mathrm{x}$-rays will benefit many areas of basic research such as high-field atomic and molecular physics, the study of high-energy-density phenomena, and protein dymanics. Likewise, the RAFEL concept can also enable the demonstration of a high average power from a compact FEL. Such a device will be useful for many military and commercial applications.

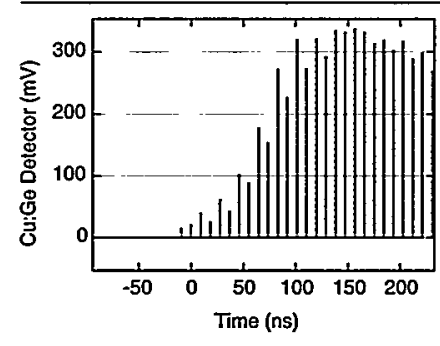

$\triangle$ FIGURE 3a. Opfical buildup shows two sets of opticol micropulses reaching saturotion at different times.

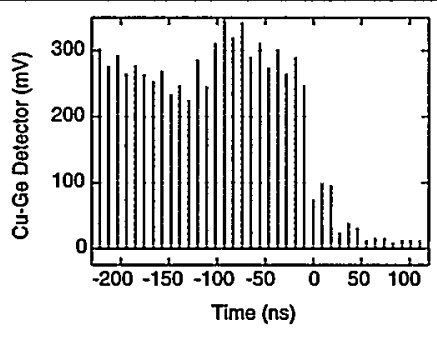

$\triangle$ FIGURE 3b. RAFEL ringdown shows optical power decoying by a foctor of 3 in successive posses.

\section{References}

1. D. C. Nguyen, R. L. Sheffield, C. M. Fortgang, J. C. Goldstein, J. M. Kinross-Wright, and N. A. Ebrahim, "Self-Amplified Spontaneous Emission Driven by a High-Brightness Electron Beam" Phys. Rev. Lett. 81 (4), 810 (1998).

2. D. C. Nguyen et al., "Initial Results of the Infrared Regenerative Amplifier FEL," in Coherent Electron-Beam X-Ray Sources: Techniques and Applications, A. K. Freund, H. P. Freund, M. R. Howells, Eds., (SPIE, 1997), Vol. 3154, p. 39.

3. R. L. Sheffield, "Electron Beamline Design for the Los Alamos National Laboratory 1-kW FEL," Nucl. Instrum. Methods Phys. Res., Sect. A393, 204 (1997).

4. C.M. Fortgang, "A Pure Permanent Magnet, Two-Plane Focusing, Tapered Wiggler for a High Average-Power FEL," Nucl. Instrum. Methods Phys. Res., Sect. A393, 385 (1997). 


\section{Intense, Sub-Picosecond Electron Beams}

\section{S. J. Russell, B. E. Carlsten (LANSCE Division)}

Many devices that use electron accelerators require high-quality, short-pulse electron beams. At LANSCE, we have constructed the Sub-Picosecond Accelerator (SPA) to study these advanced beams. The SPA accelerates a beam of high-charge electron bunches to nearly the speed of light and then compresses them to less than $0.3 \mathrm{~mm}$ in length. If directed onto a target, the tail of the electron bunch will strike $1 \times 10^{-12} s$ (one trillionth of a second) after the front. This intense and very short burst of electric charge is desirable for many applications.

The SPA facility is the first capable of this extreme bunch compression, and with it we intend to study the properties of these advanced beams and determine how they can be improved. In addition, we will develop new diagnostic techniques able to operate at these very short time scales and accommodate other unique properties of electron beams in this regime.

Next-generation colliders and free-electron lasers are examples of electron beam applications that demand ultrashort ( $<1 \mathrm{ps}$ ), high-charge $(>1 \mathrm{nC}$ ), high-quality (normalized rms emittance $\leq 1 \mathrm{n} \mathrm{mm}$, mrad) electron beams. At present, there is no electron beam facility that can meet all of these requirements simultaneously. However, progress toward this goal is rapid, and much of the work is being done on the SPA at LANSCE.

The SPA facility is an 8-MeV radio-frequency ( $\mathrm{rf}$ ) photoinjector operating at a frequency of 1,300 MHz. ${ }^{1}$ The SPA compresses electron pulses containing more than $1 \mathrm{nC}$ of charge to sub-picosecond lengths with a magnetic chicane. ${ }^{2}$ Its primary mission is to explore the dynamics and uses of ultra-short electron beams and to develop new diagnostic techniques capable of operating in the regime of these advanced beams.

In photoinjectors, which were developed at Los Alamos, $, 3,4$ a photocathode located inside the accelerator is struck by a short-pulse, high-energy drive laser to form the electron beam. By injecting the drive laser pulse at an appropriate phase in the accelerator's if cycle, an energy versus phase correlation (Figure 1a) is introduced. When the electron beam passes through the chicane (Figure 1b), the lower-energy electrons at the front of the beam bunch travel a longer path than those at the back. If the energy slope, initial bunch length, and magnetic field strength in the chicane are chosen correctly, the electrons at the back of the bunch catch those at the front, compressing the beam. (a)

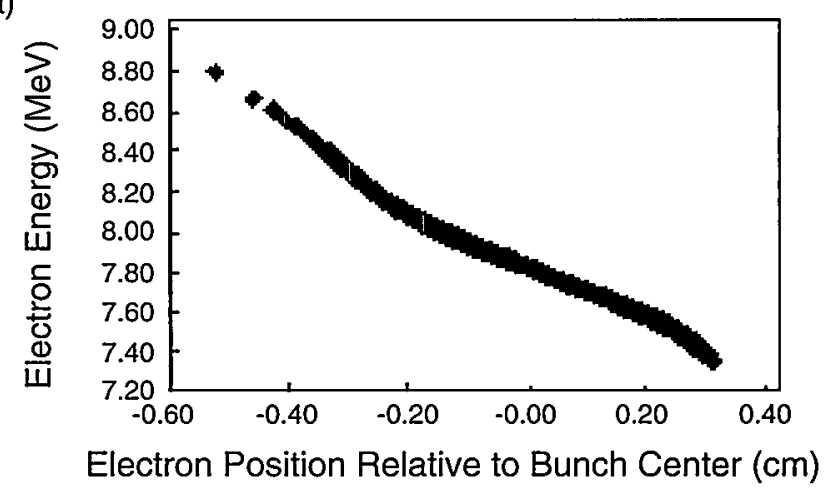

(b)

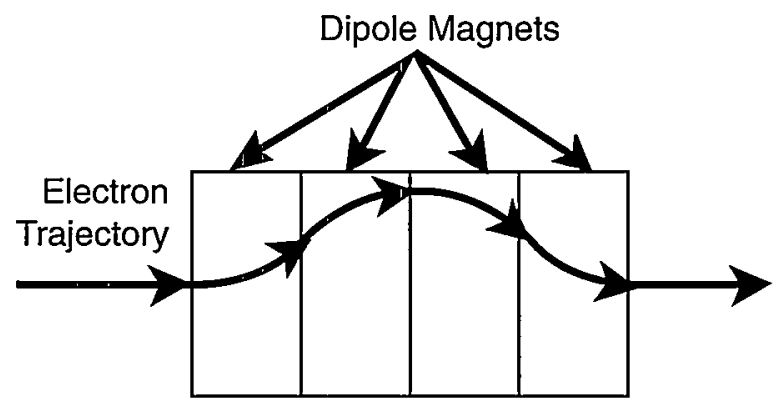

A FIGURE 1. (a) Electron energy versus position in bunch from simulation. (b) Schematic of mognefic chicone.

Because they degrade beam quality, space-charge forces within an electron beam are a major consideration in accelerator design. In a beam that is not being accelerated, these forces scale inversely with the square of the relativistic factor $\gamma$. However, recent work ${ }^{5}$ has identified two spacecharge induced forces for beams in circular motion that are mostly independent of energy. The first is a space-charge curvature effect and is known as the noninertial spacecharge force. The second is the coherent synchrotron radiation force. Both effects lead to a redistribution of the energy of electrons that are within an achromatic bend, such as the chicane on the SPA. This redistribution can lead to a large increase in the beam's emittance that is roughly independent of beam energy. So, as the chicane compresses the beam, it is likely to destroy the beam quality. One of the main concerns of the SPA is to understand these effects and find ways to mitigate them.

The transverse beam quality is summarized by the emittance. Standard electron beams thermalize, usually evolving into a Gaussian shape. This apriori knowledge of the 
beam distribution is a great advantage and is used in many emittance measurements. Unfortunately, photoinjector beams do not possess this property, and Gaussian assumptions are not valid. For this and other reasons, care must be taken in choosing an emittance measurement. At LANSCE, we have worked to perfect a new technique first proposed at the Stanford Linear Accelerator Center ${ }^{6}$ that uses beam position monitors (BPMs) as emittance probes. Normally used to measure the position of the beam center (first moment of the beam image charge), BPMs can also measure the emittance of the beam, independent of the beam distribution, by looking at the second moment of the image charge. We have recently demonstrated this new technique. ${ }^{7}$

Measuring the length of sub-picosecond electron beams is a great challenge, and we are working on two novel diagnostics for this task. The first uses a cylindrical cavity and a $\mathrm{BPM}^{8}$ (Figure 2). The beam is passed through the fast deflector cavity, which is operating in its $\mathrm{TM}_{110}$ mode, at an appropriate time in the rf cycle so that the trajectory of the beam center is unchanged. However, the cavity fields do deflect the front and back of the beam bunch in opposite directions. As the beam drifts, it spreads in one plane in an amount dependent upon the length of the beam bunch. This spread can be measured by a BPM.

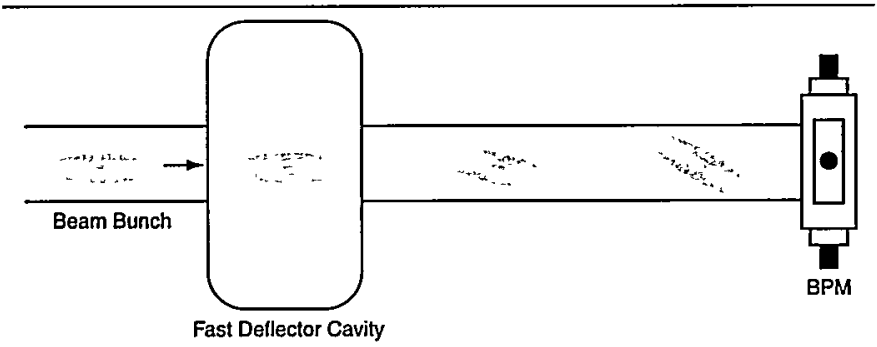

A FIGURE 2. Illustrotion of fost deflector covity streaking on electron beam bunch.
The second length measurement exploits Smith-Purcell radiation as a bunch-length diagnostic. Smith-Purcell radiation is generated when a charged particle beam passes over a metal grating. It is possible to determine the length of the beam by measuring the angular distribution of this radiation.

In conclusion, LANSCE is in a good position to make significant contributions to electron beam development. The SPA facility is unique and will enable us to do very good science over the next several years.

\section{References}

1. B. E. Carlsten, S. J. Russell, J. M. Kinross-Wright, M. Weber, J. G. Plato, et al., "Subpicosecond Compression Experiments at Los Alamos National Laboratory," Proc. Micro Bunches Workshop (Upton, New York, 1995), AIP Conf. Proc. Vol. 367, p. 21.

2. B. E. Carlsten and S. J. Russell, "Subpicosecond Compression of 0.1-1 nC Electron Bunches with a Magnetic Chicane at 8 MeV," Phys. Rev. E 53, 2072 (1996).

3. J. S. Fraser, R. L. Sheffield, E. R. Gray, P. M. Giles, R. W. Springer, and V. A.Loebs, "Photocathodes in Accelerator Applications," Proc. 1987 Part. Accel. Conf. (New York, 1987), IEEE catalog no. 87CH2387-9, p. 1705.

4. J. S. Fraser and Richard L. Sheffield, "High-Brightness Injectors for RF-Driven Free-Electron Lasers," IEEE J. Quantum Electron. 23, 1489 (1987).

5. B. E. Carlsten, "Calculation of the Noninertial Space-Charge Force and the Coherent Synchrotron Radiation Force for Short Electron Bunches in Circular Motion Using the Retarded Green's Function Technique," Phys. Rev. E 54, 838 (1996).

6. R. H. Miller, J. E. Clendenin, M. B. James, and J. C. Sheppard, "Non-Intercepting Emittance Monitor," Proc. 12 Int. Conf. High Energy Accel. (Fermilab, 1983), Vol. 602.

7. S. J. Russell, "Emittance Measurements of the Sub-Picosecond Accelerator Electron Beam Using Beam Position Monitors" (submitted to Rev. Sci. Instrum.).

8. S. J. Russell, "Measuring the RMS Length of Short Electron Pulses with an RF Cavity and a Beam Position Monitor" (to be published in Proc. 1997 Part. Accel. Conf.). 


\section{Explosive Pulsed-Power Developments and Modeling}

\section{M. Fortgang (LANSCE Division)}

\section{LANSCE is advancing the technology of explosive pulsed-power by making systems more compact and by optimizing them for more specific applications. Flux-compression generators (FCGs) are the prima- ry explosive pulsed-power sources we use. Over the past 2 years we have concentrated on three areas of explosively-driven FCGs: replacing the external capacitor bank that provides the initial seed current (and magnetic) flux with permanent magnets, devel- oping and testing high-voltage air-core transformers used to couple electrical loads of various impedance to the FCG, and developing better computer models that help us design and optimize the performance of these devices.}

Los Alamos has a more than a 40-year history of developing and using explosively-driven pulsed-power sources and related power-conditioning technology. LANSCE is advancing the technology of explosive pulsed-power by making systems more compact and by optimizing them for more specific applications. The primary explosive pulsedpower sources we use are FCGs, which operate on the principle of magnetic-flux conservation. They work in the following manner.

In an electrical circuit, current generates a magnetic field and thus magnetic flux (proportional to the magnetic-field strength times the cross-sectional area through which the magnetic-field lines cross). A simple example of such a system is a magnetic solenoid. In electrical engineering terms the magnetic flux is given by the product of the circuit inductance and the electrical current. The inductance of a circuit depends solely on its geometry. If magnetic flux is conserved, the current must increase when the system inductance decreases. However, magnetic energy scales proportionately to the inductance times the square of the current, producing a net increase in energy.

In an explosively driven FCG, high explosives are used to decrease circuit inductance. Therefore, FCGs are singleshot devices. Their appeal, however, lies in the very high magnetic fields and energy densities they can achieve. The increase in electromagnetic energy actually comes from the high explosive, which is doing work (via chemical energy) on the circuit as it compresses the magnetic flux. Figure 1 is a schematic of one important type of FCG, called a helical flux compressor. Here the magnetic flux that is being compressed lies in the volume between the aluminum tube (or armature) and copper helix (or stator). The armature is filled with explosive and is detonated from one end. As the

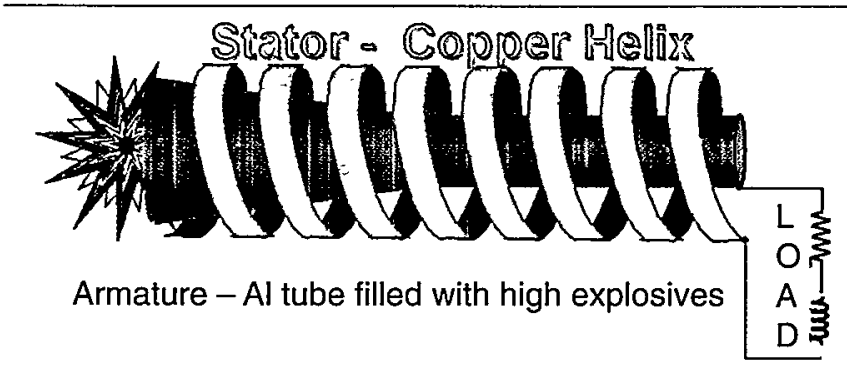

A FIGURE I. Schematic of one important type of $F C G$, colled o helicol flux compressor.

armature expands radially it forms a cone whose apex travels along the axis, compressing but conserving the flux while the inductance of the circuit element formed by the armature and stator decreases. The current increases exponentially as the generator inductance approaches zero. Figure 2 is a cutaway view of a flux compressor. The aluminum tube is detonated at the end extending out and beyond the copper-wire helix. On the other end a transformer enables the generator to work more efficiently into the electrical load.

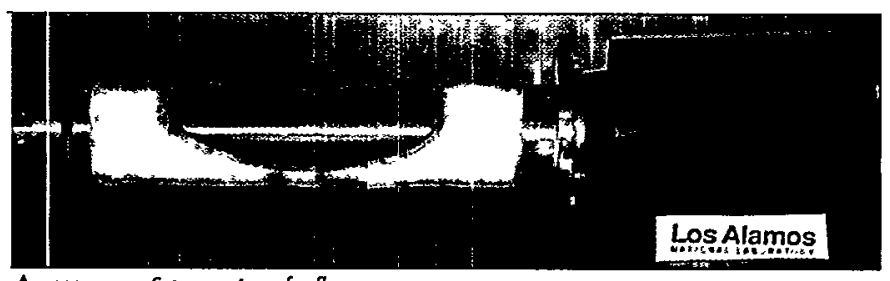

$\triangle$ FIGURE 2. Cutawoy view of a flux compressor.

For the past 2 years, we have concentrated on three main areas of explosively driven FCGs: replacing the external capacitor bank that provides the initial seed current (and magnetic flux) with permanent magnets; developing and testing high-voltage, air-core transformers used to couple electrical loads of various impedance to the FCG; and developing better computer models that help us design and optimize the performance of these devices.

\section{Permanent-Magnet Seeded Flux Compressors}

All flux compressors need a seed source of magnetic flux. Conventionally, this flux has been supplied by capacitor banks discharged into the circuit just before the high explosive is ignited. However, capacitor banks are bulky, not easily transported, and require special high-voltage switches that need maintenance and are sometimes unreliable. We are working on designs that eliminate the capacitors and switches by using permanent magnets to supply the seed 


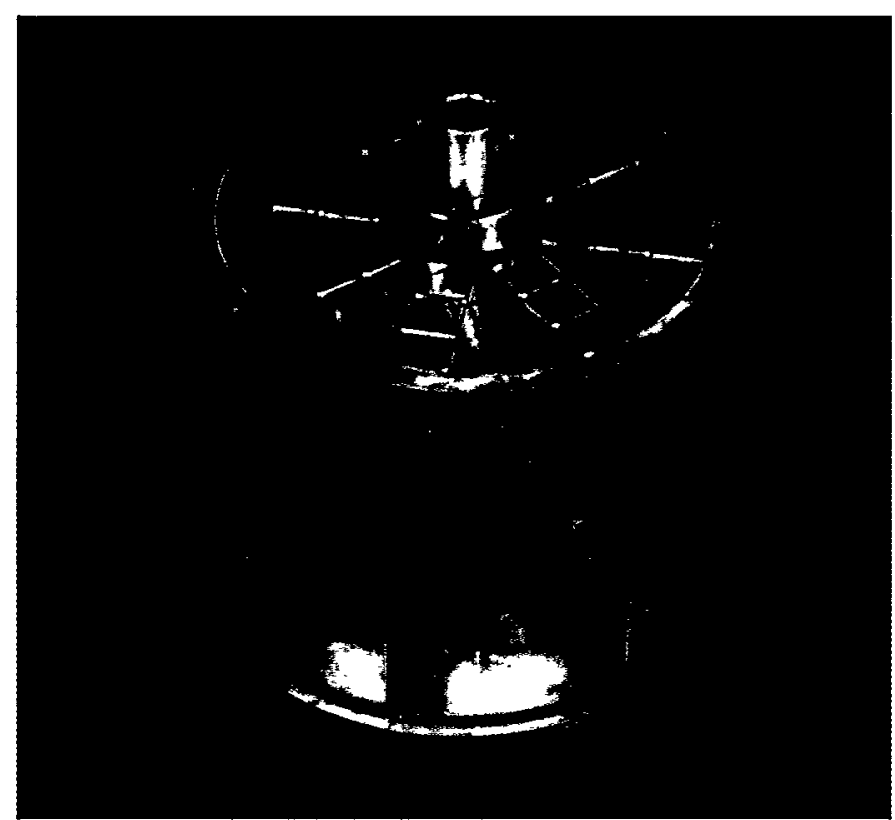

$\Delta$ FIGURE 3. This smoll generator has an energy goin of obout 100, vielding on output energy of several hundred joules. That energy and current then can be fed cascade foshion into a subsequent generator and amplified further.

magnetic flux. The permanent magnets are arranged around the outside of a small flux compressor so as to generate a magnetic field inside, between the aluminum tube and copper helix. Although the initial magnetic energy is only a few joules, a small generator such as the one shown in Figure 3 has an energy gain of about 100 , yielding an output energy of several hundred joules. That energy and current then can be fed cascade fashion into a subsequent generator and amplified further. We have tested two such devices and have demonstrated output currents of about 25 $\mathrm{kA}$. We presently are working toward our goal of an energy gain of 100 .

\section{High-Voltage Pulse Transformers}

Magnetic flux compressors are low-impedance sources. That is, they work best at high current ( $\left.10^{6} \mathrm{amps}\right)$ and relatively low voltage (10s of kilovolts). Typically, the load is an inductor in the range of 20 to $500 \mathrm{nH}$. However, there are applications with higher load impedance that, if directly connected to the flux compressor, would significantly degrade performance. By using a transformer, one can make a high-impedance load appear to the generator as a low-impedance load. When designing high-voltage pulse transformers there is a trade-off between good coupling (which requires a tight spacing between primary and secondary windings) and high-voltage standoff (which requires insulation and thus more spacing between primary and secondary windings). Iron-core transformers are not an option due to saturation effects. We have developed and used air-core transformers that have coupling constants of about 0.94 and output voltages of about $300 \mathrm{kV}$. These transformers are of a coaxial geometry and have 12 turns of several parallel wires in the secondary around a singleturn primary made of aluminum tubing. These transformers perform better than those used in the past. We still are testing these newly designed transformers to find their ultimate voltage limit.

\section{Flux Compressor Modeling}

Accurate modeling of flux compressors is difficult. Some three-dimensional (3-D) effects make simple modeling using symmetry inaccurate. The problems of magnetic diffusion and unwanted resistive losses result in magnetic flux being only approximately conserved. In addition, there are usually stray inductance and resistance that should be accounted for and included in a model. The problem usually is solved by coupling two systems of equations. One system calculates how the circuit inductance and resistance are changing in time; it entails keeping track of the changing geometry and its inductance, the diffusion of magnetic flux, and heating and resistance of the current-carrying conductors. At each time step, these results are fed into a circuit equation solver, which then calculates the new currents and voltages. This is a work in progress but we already have improved our modeling of the 3-D aspects of the helix by including the pitch effect. Also, the azimuthal currents induced on the armature as it expands are now self-consistently calculated. We are working on including the effects of magnetic diffusion and resistive heating of the copper wires. Figure 4 compares our most recent computer modeling and an actual flux compressor.

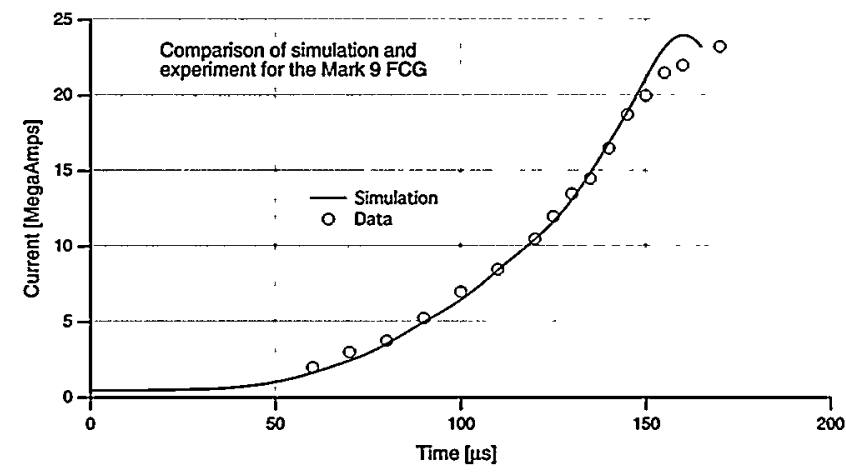

A FIGURE 4. A comparison of our most recent computer modeling with an actual fux compressor. 


\title{
Ion Implantation Linear Accelerator Studies For Eaton Corporation
}

\author{
W. P. Lysenko, E. A. Wadlinger, B. Rusnak, F. Krawczyk (LANSCE Division), K. Saadatmand, Z. Wan \\ (Eaton Corporation)
}

\section{We studied the possibility of improving the perfor- mance of high-energy ion implanters made by Eaton Corporation for semiconductor fabrication. We ana- lyzed the present design, studied the feasibility of new approaches, and developed a systematic approach to designing improved radio-frequency ( $f f$ ) ion linear accelerators. We found that substantially improved performance is possible using existing technology proven at Eaton Corporation.}

Eaton Corporation builds high-energy ion implanters for semiconductor device fabrication. These machines are if linear accelerators that accelerate ions to energies up to about 1 $\mathrm{MeV}$. Recently, Eaton has been working with Los Alamos National Laboratory (LANL) to study ways to improve their machines to meet the future needs of Eaton's customers. The main requirements for a near-future ion implanter linac are

- Various ion species. From ${ }^{11} \mathrm{~B}^{+}$to ${ }^{75} \mathrm{As}^{+}$.

- Variable energies. Up to several MeV.

- High current. Several mA.

- Short length. Not more than about $2 \mathrm{~m}$ long.

Additional requirements are low power consumption and low energy spread. The Eaton project consisted of analyzing the present Eaton linac, studying new approaches for improved performance, and determining the best design for a machine based on the present technology used at Eaton.

\section{Study of Present Eaton Machine}

The Eaton linac uses 13.56-MHz rf cells to accelerate and provide longitudinal focusing. Each cell consists of a drift tube, fed by an external helical resonator, in a grounded cavity. This provides two of gaps per cell and is a practical way to get a compact and efficient structure at low frequencies. Electrostatic quadrupole lenses, in an alternating-gradient arrangement, provide transverse focusing. In general, accelerator performance can be limited by weak focusing, poor matching, or poor bunching.

We found that the present machine has adequate focusing for accelerating ${ }^{11} \mathrm{~B}^{+}$at $3 \mathrm{~mA}$ but transmits only about a third of the particles injected. The losses are caused mainly by emittance growth in the rf gaps. This is a nonlinear effect caused by different particles seeing different rf phases as they cross the rf gaps. Improved bunching and matching could double the output current. To further increase the current or to get good performance for heavier ions, we need to improve the transverse focusing.

\section{Study of New Approaches}

New structures and focusing

A single-gap rf structure (resonant cavity) has the advantage that we can phase each gap independently. We investigated the feasibility of resonant cavities at low frequencies ( $40 \mathrm{MHz}$ ). While it is possible to get a cavity of reasonable size at this low frequency, the efficiency is very low.

We studied variable-frequency radio-frequency quadrupole (RFQ) structures. The variable frequency is necessary to accelerate particles of different masses. The high cost of variable-frequency if power is a problem. The high transmission of most RFQ linacs is the result of adiabatic bunching. However, this bunching is not practical for an ion implanter because of the length constraint. A segmented RFQ or an RFQ with a separate buncher may turn out to be feasible.

We have also studied modifications to the drift-tube noses in a gap structure to introduce quadrupole fields that would contribute to transverse focusing.

We can use the substantial transverse components of the fields in the if gaps to provide transverse focusing. We can do this by alternating the synchronous phases in adjacent if gaps. With alternating phase focusing, there would be no need for the focusing quadrupoles. This idea has been investigated at LANL and elsewhere for other applications. As before, we found that the phase-space acceptance of such machines is low.

\section{Improved bunching}

A well-designed linac with a conventional (single frequency) buncher can accelerate about $75 \%$ of the particles injected. While multiple-frequency bunchers are probably not worthwhile, two separated cavities are desirable even for a singlefrequency approach because of the range of particle velocities associated with different particle masses.

\section{Scaling studies}

We have determined optimum quadrupole strengths and period lengths required for an alternating gradient focusing system, taking into account the defocusing effect of the rf gaps. We have also verified that we can reduce the length of the linac by raising the frequency while maintaining the electrostatic and rf voltages. This approach works when the quadrupoles and the rf gaps are voltage (not field) limited.

\section{Improved Double-Gap Design}

We have developed a new theory (Reference 2 describes an earlier version of the theory) and design procedure for 
linacs having double-gap resonators and electrostatic quadrupoles. Our goal was to determine the maximum performance using technology that has been proven at Eaton. The basic idea is that we design the linac for a certain design-reference particle. Then we scale the design-reference tune (voltages and phases) to new particles.

\section{Theory for double-gap structures}

We can set the synchronous phase at the first gap of a double-gap resonator to any value. We obtain the desired synchronous phase at the second gap for the design-reference particle by specifying the distance between the gaps. Once we design and build the machine, we can no longer change any gap positions, but we are free to reset voltages and phases.

We define ratios for various quantities, all relative to the design-reference particle. Our theory determines the new tune that corresponds to specified values of the various ratios. For the case of the Eaton resonators, which are voltage limited, it is useful to fix the voltage ratio. Figure 1 shows the energies that can be achieved for different particle masses. We see, for example, that if the synchronous phases on the two gaps are $5^{\circ}$ and $-35^{\circ}$, we get equivalent performance for masses over a range of about a factor of three. We can accelerate particles of even lower or higher mass, but only to lower energies.

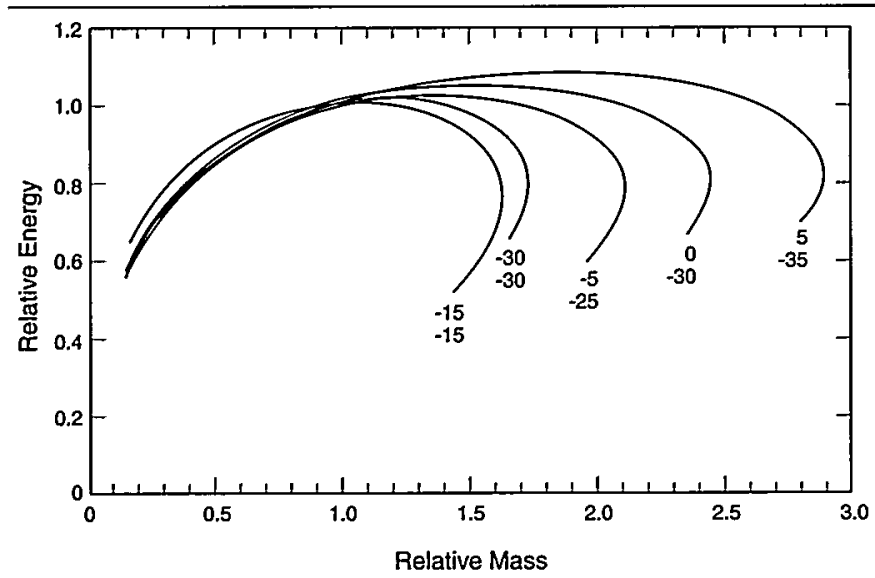

A FIGURE I. This groph shows how the energy chonges when we scale a tune fo chonge the parti cle moss (both quantities are relative to the values of the design-reference porticle). The curves correspond to a fixed focusing strength ond if gap voltage and are labeled by the values of the synchronous phoses on the two gaps.

\section{New design procedure}

We start with the TRACE three-dimensional (3-D) code, with user-defined elements for electrostatic quadrupoles and double-gap if cells. These elements operate in two modes. In the design mode, the code sets the quadrupole lengths and if gap separations automatically. In the retune mode, an existing design is retuned to a new particle according to our theory. Once we have a TRACE 3-D design, we use the PARMELA (Phase and Radial Motion in Electron Linear Accelerators) particle-tracking code to determine the fraction of the beam transmitted by the machine. We use an optimizing version of PARMELA to fine tune the quadrupole settings. We found that performance can be substantially improved compared with performance of existing machines while using proven technology.

\section{Conclusion}

The new approaches need further study. However, we found that the present approach of using double-gap resonators and electrostatic quadrupoles can provide ion implanters of substantially improved performance over a wide range of mass/charge values. The design procedure we developed ensures we get the good bunching and matching and the maximum transverse focusing. Further work on improving the $\mathrm{ff}$ resonators would greatly benefit this project. The rf gap voltage limitation makes it impossible to obtain significantly higher energies within the length constraint.

\section{References}

1. Z. Wan, K. Saadatmand, F. Sinclair, W. Lysenko, B. Rusnak, and L. Young, "Linac Simulation for High Energy Ion Implantation," in 11th International Conference on Ion Implantation Technology, Austin, Texas, June 16-21, 1996 (IEEE, New York, New York, 1997), pp. 371-374.

2. E. A. Wadlinger, W. P. Lysenko, B. Rusnak, and K. Saadatmand, "Beam Dynamics of a Double-Gap Acceleration Cell for Ion Implantation with Multiple Atomic Species," in Applications of Accelerators in Research and Industry, J. L. Duggan and I. L. Morgan, Eds. (AIP Press, New York, New York, 1997), AIP Conference Proceedings 392, pp. 941-944. 


\section{Linac Coherent Light Source}

\section{R. L. Sheffield (LANSCE Division)}

The growing use of synchrotron light sources and the unique results obtained from experiments with these sources over the last 20 years have led to great interest in a fourth-generation light source. $A$ fourth-generation machine would enhance by one or more orders of magnitude some or all of the performance parameters of the third-generation sources used now. Los Alamos National Laboratory is collaborating in the proposal to build the Linac Coherent Light Source (LCLS) at the Stanford Linear Accelerator Center (SLAC). The LCLS is a single-pass, $x$-ray free-electron laser (FEL) operating in the very short $x$-ray ( 1 to $2 A$ ) wavelength region, using 15-GeV electron beams from the Stanford linac.

$\mathrm{X}$-rays are invisible, highly penetrating electromagnetic radiations of much shorter wavelength (higher frequency) than visible light. Discovered by Wilhelm Roentgen, $\mathrm{x}$-rays could be produced by the impact of high-energy electrons (several thousand volts on a metal anode in a highly evacuated glass bulb called a Roentgen tube). From 1895, when $\mathrm{x}$-rays were discovered, to the late $1960 \mathrm{~s}$, the main source of $x$-rays for research was the Roentgen tube. After the first observation of synchrotron radiation ${ }^{1}$ and the evolution of the cyclic electron synchrotron, followed by the development of storage rings for high-energy physics applications, physicists realized that these accelerators could be used as more intense $x$-ray sources than the previous sources. Since then, synchrotron light sources have evolved through three generations. First-generation systems were constructed in the U.S., Germany, Japan, and the Soviet Union. These storage-ring-based light sources initially used the radiation generated by an electron beam being turned by the large magnets that kept the electron beam in circular motion. These first-generation light sources operated parasitically on machines devoted mainly to high-energy physics studies.

The second-generation machines were specifically built to be light sources, but still used, initially, the radiation from single bending magnets. Eventually, these facilities added straight magnetic components with periodic transverse magnetic fields (called "wigglers" or "undulators") that forced electrons to undergo many transverse oscillations instead of following a single smooth orbit around the storage ring, thus generating more $\mathrm{x}$-rays. However, since these storage rings did not include a large number of straight sections, the number of wigglers that could be used was limited. Around 1980, new second-generation facilities were completed in the People's Republic of China, England, France, Germany, Japan, the U.S., and the Russian republics.

The third-generation facilities (such as the Advanced Photon Source at Argonne National Laboratory, the Advanced Light Source at Lawrence Berkeley National Laboratory, and similar facilities around the world) were also built specifically for light-source applications. These machines incorporated more straight sections for the placement of wigglers and undulators, allowing more people to use the light source. Also, the storage rings were designed to have a very-smalldiameter electron beam, resulting in a smaller-diameter $\mathrm{x}$-ray beam with increased brightness. The typical electron-beam quality (quality in this sense is the ability to maintain a small electron beam spot over some fixed distance, in units of amps $/ \mathrm{m}^{2} / \mathrm{rad}^{2}$ ) in third-generation sources was 50 times larger than it was in earlier sources. These facilities increased $x$-ray-beam brightness by several orders of magnitude in comparison to previous generation sources. However, for $\mathrm{x}$-rays shorter than a few hundred angstroms, these devices produced an $\mathrm{x}$-ray beam with very little optical coherence across the beam. This property is called transverse coherence.

The growing use of synchrotron light sources and the unique results obtained from experiments with these sources over the last 20 years have led to great interest in a fourth-generation light source. A fourth-generation machine would enhance by one or more orders of magnitude some or all of the performance parameters of the third-generation sources used now. Although the exact characteristics of a facility depend on its scientific applications, scientists agree that high brightness and transverse coherence are very important. Another necessary characteristic is the generation of shorter $\mathrm{x}$-ray pulses than those produced now by third-generation sources.

Stimulated by the Workshop on Fourth-Generation Light Sources (Stanford, 1992), we began discussing the possibility of using the SLAC linac to drive a fourth-generation light source. The approach selected to generate the $\mathrm{x}$-rays is different from the previous generations of light sources. Instead of using a ring that circulates the same electron beam through the bending magnets many times, this design uses a single pass of an electron pulse through a long undulator. During the transit of the electron bunch through the long undulator, the $\mathrm{x}$-ray pulse starts up from noise and builds up to very high powers using the self-amplified spontaneous-emission (SASE) mode of operating the FEL. As with many lasers, the resulting light has a narrow bandwidth and is coherent, compared to light-source generated light. 
The proposed machine is named the Linac Coherent Light Source (LCLS), and a schematic of the LCLS is shown in Figure 1. The LCLS is a multi-institutional proposal for a single-pass, $x$-ray FEL operating in the 1 to $2 \AA$ wavelength region, using electron beams from the SLAC linac at $\sim 15 \mathrm{GeV}$ of energy.

An LCLS type of fourth-generation light source can now be designed because of (1) the development of the photoinjector (invented at the Laboratory), (2) our ability to construct precision undulators, and (3) our improved understanding of linac beam dynamics and pulse compression. To propose building the LCLS, we still had to demonstrate the very high gains predicted by SASE theory.

Our collaboration with UCLA on the advanced free-electron laser (AFEL) system at Los Alamos demonstrated our understanding of the scientific basis of SASE. This collaboration has validated the SASE theory. The Los AlamosUCLA team measured FEL laser gains of greater than 500,000 in the infrared optical region. This measurement can be compared with the gains of less than 2 typically measured in infrared FELs. The gain was due to the excellent electron-beam characteristics provided by the AFEL accelerator and the unique wiggler provided by UCLA. The experimental results are also close to the predicted performance based on SASE theory. Our experiment is the first, clear confirmation supporting the SASE theoretical foundation; it justifies using this theory as a basis for designing higher-energy, shorter-wavelength light sources.

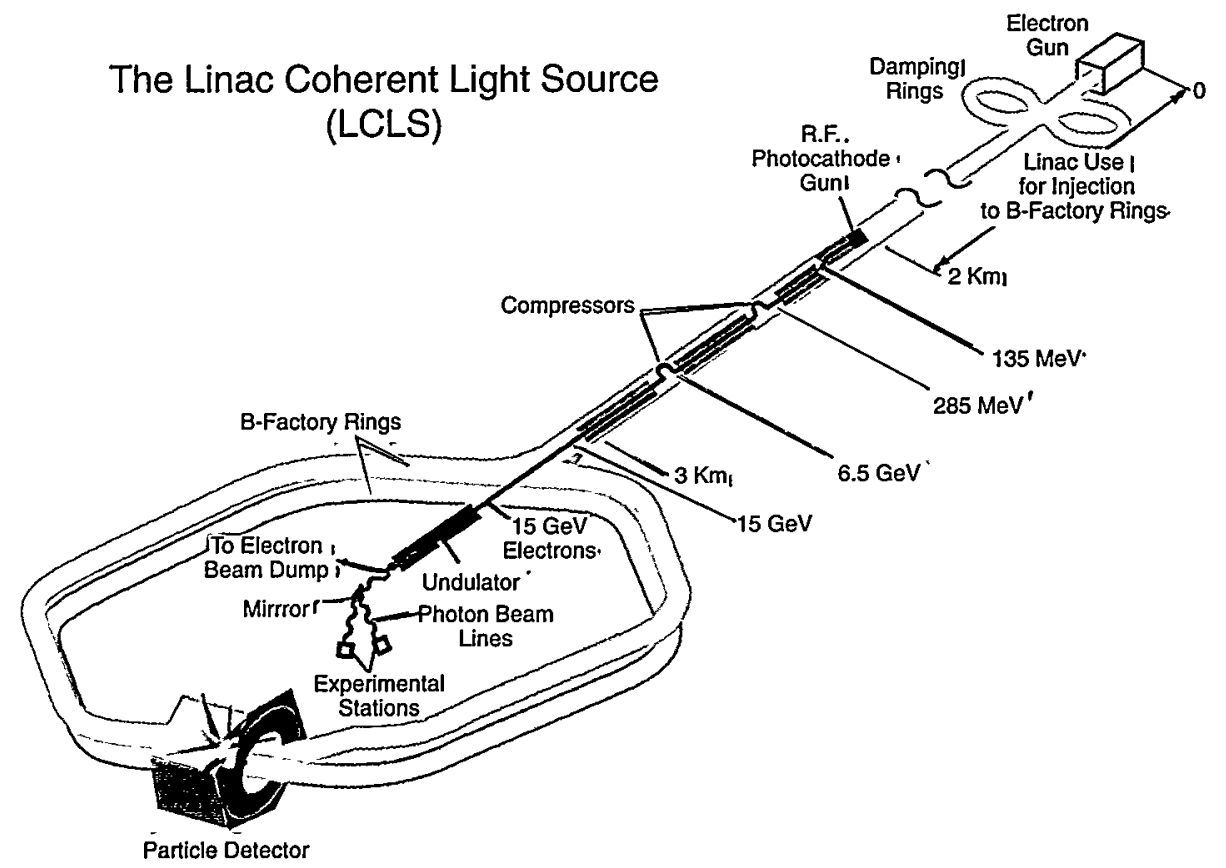

$\triangle$ fIGURE I. Schematic of the proposed $x$ fay light source to be built of SLAC. The project would use one-third of the existing accelerntor. The compressors shorten the time length of the electron bunch to get a higher instantaneous current. An $80 \mathrm{~m}$ viggler follows the accelerator.

\section{Reference}

1. J. P. Blewett, "Radiation Losses in the Induction Electron Accelerator," Phys. Rev. 69, 87 (1946). 


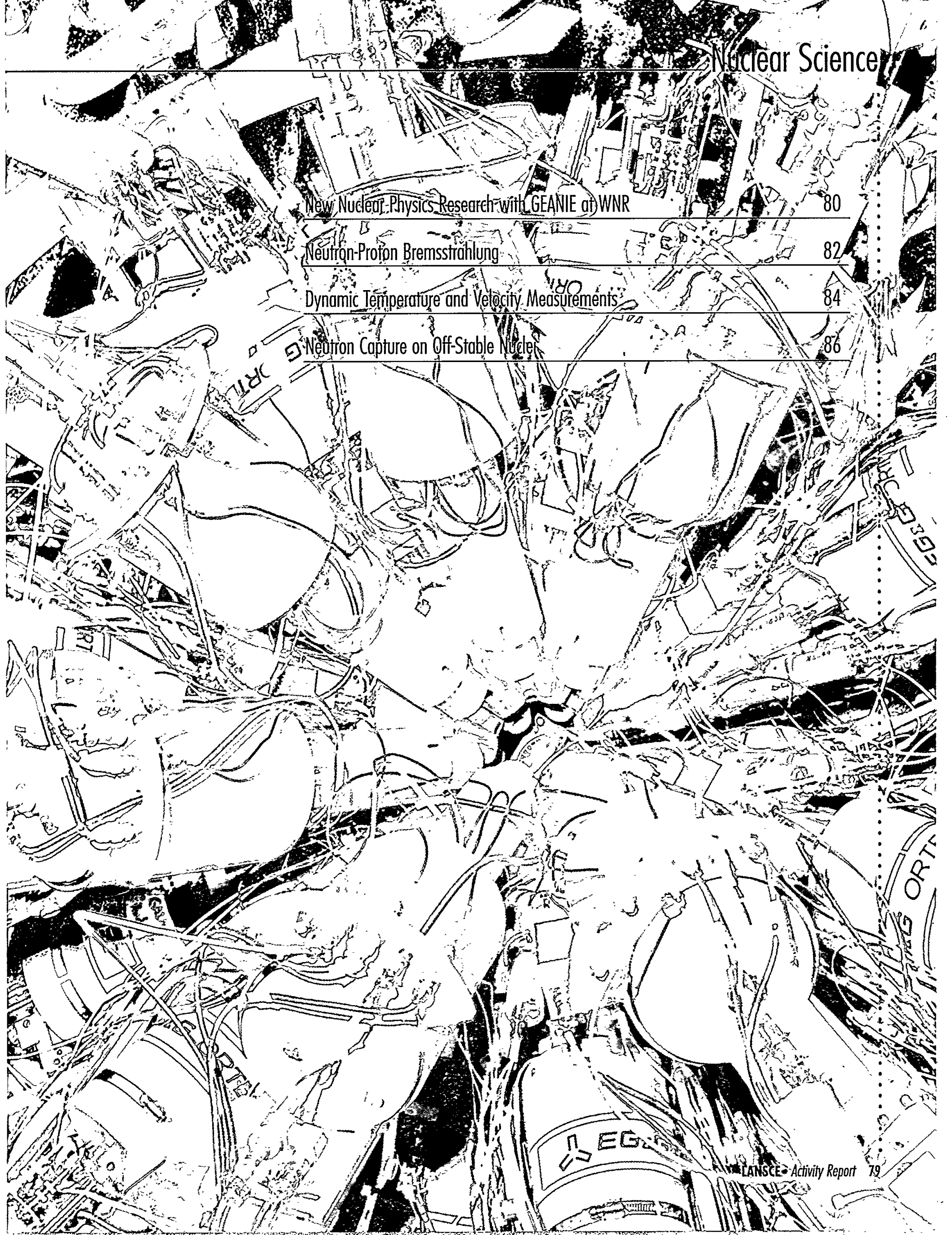




\title{
New Nuclear Physics Research with GEANIE at WNR
}

\author{
R. O. Nelson, G. D. Johns (LANSCE Division), W. S. Wilburn (Physics Division), D. M. Drake (Amparo \\ Corp. and LANSCE Division), D. E. Archer, J. A. Becker, L. A. Bernstein, W. Younes (Lawrence \\ Livermore National Laboratory), S. W. Yates, P. Garrett (University of Kentucky)
}

\section{The large, high-resolution gamma-ray detector array, GEANIE, is now being used to perform inno- vative research using the high-energy white spec- trum WNR neutron source at LANSCE. Current experiments are driven by the need for improved data for the stockpile stewardship program, APT, and opportunities to explore new regions of excita- tion in basic nuclear physics. In operation for a rel- atively short time, exciting new results are being obtained.}

The Germanium Array for Neutron-Induced Excitations, GEANIE, is a large, high-resolution gamma-ray detector array at LANSCE. GEANIE, sited at WNR - an intense, pulsed, broad-spectrum neutron source, ${ }^{1}$ provides new and powerful capabilities for Science-Based Stockpile Stewardship and nuclear science. The acquisition, installation, operation, and use of the array is a major collaborative effort between researchers from Lawrence Livermore National Laboratory and Los Alamos National Laboratory. A primary goal of the collaboration is to accurately determine the ${ }^{239} \mathrm{Pu}(n, 2 n)$ cross section and to address important nuclear data needs. Innovative research in nuclear physics using the unique combination of a high-resolution gammaray $(\gamma$-ray) spectrometer with a high-energy neutron source is also a priority.

The development and use of GEANIE creates a powerful new tool for nuclear physics research. At full power, the WNR spallation source is the most intense high-energy neutron source in the world. This is the first time that a large $\gamma$-ray detector array has been used at a spallation neutron source, which makes it possible to measure excitation functions over a wide energy range simultaneously. This unique combination opens up new possibilities for research in areas of nuclear excitation that previously have been difficult to access.

The important characteristics of GEANIE for nuclear spectroscopy are high-energy resolution $(\sim 0.2 \%)$, good efficiency, escape suppression for background reduction, and high granularity. Measuring characteristic $\gamma$ rays that follow neutron-induced reactions usually allows the determination of both the reaction channel-for example, $(n, 2 n)$ or $(n, 3 n)$ and the particular level excited in the product nucleus. In addition, the reaction thresholds and cross-section peaks observed in excitation functions provide information for the identification and study of the various reaction products. Because of the high neutron energies available, many different reactions are possible. Typical reactions include: inelastic scattering, multiple-neutron emission, proton emission, alpha emission, combinations such as two-proton plus three-neutron emission, and fission.

GEANIE presently consists of 20 bismuth germanate escape-suppression shields surrounding 20 germanium (Ge) detectors. GEANIE was built using elements of the High-Energy Resolution Array (HERA) that was developed by the Nuclear Structure group at Lawrence Berkeley National Laboratory. ${ }^{2}$ The original coaxial Ge detectors have been augmented by the addition of planar Ge detectors. The combination of both coaxial and planar germaniun detectors provides a powerful spectrometer for a wide range of experiments. The coaxial detectors have a useful gamma-ray detection range to several $\mathrm{MeV}$. The planars are especially useful at lower gamma-ray energies and have extremely good energy resolution. Six more Ge detectors are being added to the array for the 1998 beam time. Incident neutron energies are determined using time-offlight techniques. GEANIE (Figure 1) is located $20 \mathrm{~m}$ from the neutron production target.

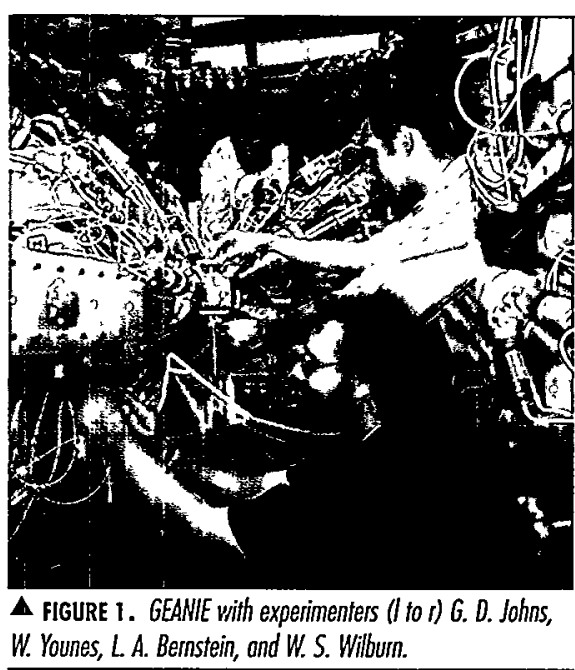

GEANIE is being used to obtain an accurate measurement of the ${ }^{239} \mathrm{Pu}(n, 2 n)^{238} \mathrm{Pu}$ reaction cross section needed to better understand the radiochemical data from past Nevada Test Site experiments. From measured $\gamma$-ray production cross sections, we obtain an estimate of total reaction cross sections as a function of incident neutron energy. By combining our results with calculations from the coupled preequilibrium and Hauser-Feshbach computer program GNASH, ${ }^{3}$ we can use theory to account for the fraction of the cross section that is not measured with our technique. 
Figure 2 shows excitation functions of one of the $\gamma$ rays in ${ }^{238} \mathrm{Pu}$ resulting from the ${ }^{239} \mathrm{Pu}(n, 2 n)$ reaction. The ability to simultaneously measure excitation functions for many $\gamma$ ray transitions provides numerous checks for validation of the data and models used in the GNASH code.

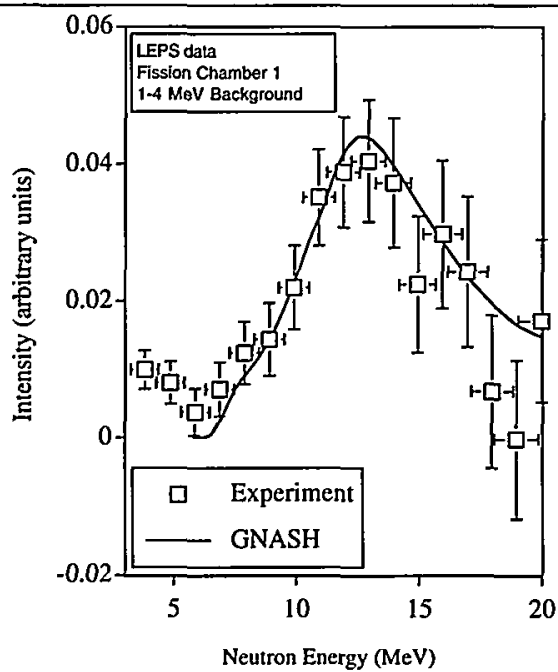

$\triangle$ FIGURE 2. Excitation function for the $210 \mathrm{keV} / 8^{+}$to $6^{+}$transition in the $239 \mathrm{PU}(\mathrm{n}, 2 \mathrm{n}) 238 \mathrm{Pu}$ reaction measured with GEANIE in 1997. The GNASH colculations hove been normolized to the data pending further work on the efficiencies, offenuation factors, ond other corrections. Relatively good ogreement between the colculated and meosured excitotion function shopes is obtained.

The use of $\gamma$ rays is an important tool for $(n, x n)$ measurements on actinide nuclei because the production of neutrons from fission interferes with a direct measurement of the $(n, x n)$ neutrons. The good peak-to-total ratio of the GEANIE detectors allows the resolution of individual $\gamma$-ray lines above the background from fission and other $\gamma$ rays. The planar detectors play an especially important role in these measurements because they have excellent energy resolution and are relatively insensitive to neutrons. This is the first time that an array of planar germanium detectors has been used to study actinide nuclei. Figure 3 shows a three-dimensional plot of data from the planar detectors taken with a ${ }^{238} \mathrm{U}$ sample. The plot shows the separation of gamma-rays from inelastic scattering and $(n, 3 n)$ reactions that differ in energy by less than $1.3 \mathrm{keV}$.

Neutron-induced gamma-ray measurements provide a means to study nuclei via $(n, x n)$ reactions (where $x=1,2$, 3 ...), probing nuclei that are otherwise difficult to access.

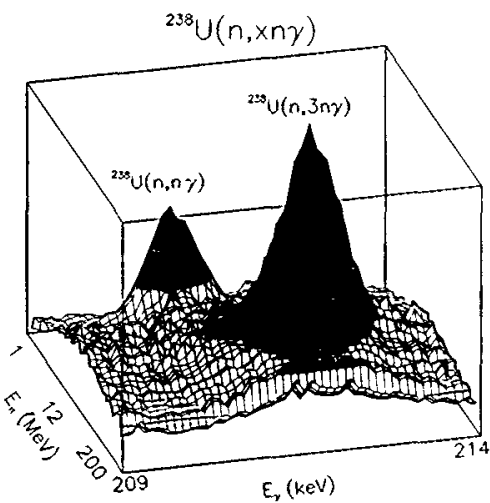

$\Delta$ FIGURE 3. Threedimensionol spectum from the planor germanium detectors showing the measured $8^{+}+10-6+$ transitions in $238 \mathrm{U}$ and $236 \mathrm{U}$ near $E \mathrm{~F}=212 \mathrm{keV}$. The two gammorays differ in energy by less thon $1.3 \mathrm{keV}$. The plot covers the neutron energy ronge from $1 \mathrm{MeV}$ to $200 \mathrm{MeV}$. The different thresholds for the two reactions (inelastic ond $(n, 3 n)$ ) are evident in the plot.

Our previous experiments ${ }^{4}$ with a single unsuppressed germanium detector in which we observed reactions on ${ }^{208} \mathrm{~Pb}$ up to $(n, 9 n)$ laid some of the groundwork for current studies. But with GEANIE the ability to measure $\gamma-\gamma$ coincidences opens up a much wider range of possible research. A sampling of the proposed physics being pursued with GEANIE includes a search for rigid triaxial motion, an investigation of vibrational excitations in rareearth nuclei, level-density studies, and exploration of the structure of exotic neutron-deficient nuclei. Other proposals are to measure the $\mathrm{Lu}(n, x n)$ reaction cross sections for use as a diagnostic in experiments related to APT and to determine neutron-induced reaction cross sections on $\mathrm{W}$, also of importance to APT. Off-line experiments using radioactive samples are planned for times when the accelerator is not in operation.

Recent results of GEANIE research include the observation of structure-dependent reaction effects in ${ }^{196} \mathrm{Pt}(n, x n)$ reactions, ${ }^{5}$ studies of neutron-deficient nuclei using, for example, ${ }^{112} \mathrm{Sn}(n, \alpha \times n)$ reactions, and identification of a candidate for a two-phonon octupole state in ${ }^{170} \mathrm{Er}$.

At present over 20 researchers are involved with GEANIE. These researchers include professors and postdoctoral fellows from universities as well as researchers from laboratories around the world. GEANIE is an important asset for nuclear physics and is attracting outstanding researchers because of the new opportunities available.

\section{References}

1. P. W. Lisowski et al., "The Los Alamos National Laboratory Spallation Neutron Sources," Nuclear Science and Engineering 106, 208 (1990).

2. F. S. Stephens, "Spectroscopy of the Highest-Spin States," in Proceedings of the International Symposium on In-Beam Nuclear Spectroscopy (Debrecen, Hungary, May 14-18, 1984), Akadémiai Kiadó, Budapest, p. 205.

3. P. G. Young, E. D. Arthur, and M. B. Chadwick, "Comprehensive Nuclear Model Calculations: Introduction to the Theory and Use of the GNASH Code," Los Alamos National Laboratory report LA-12343-MS (July 1992).

4. H. Vonach et al., "207,208 $\mathrm{Pb}(n, x n \eta)$ Reactions for Neutron Energies from 3 to $200 \mathrm{MeV}$," Physical Review C 50, 1952 (1994) and A. Pavlik, et al., "27 Al $(n, x g)$ Reactions for Neutron Energies from 3 to $400 \mathrm{MeV}$," Physical Review C 57, 2416 (1998).

5. L. A. Bernstein, D. E. Archer, J. A. Becker, W. Younes, D. M. Drake, G. D. Johns, R. O. Nelson, and W. S. Wilburn, "Probing Reaction Dynamics with the ${ }^{196} \mathrm{Pt}(n, x n \gamma)$ Reactions for $\mathrm{x}<15$," Physical Review C 57, R2799 (1998). 


\title{
Neutron-Proton Bremsstrahlung
}

\author{
J. L. Matthews, Y. Safkan, W. M. Schmitt, V. V. Zelevinsky, J. da Graça, M. A. Greene, L. W. Kwok \\ (Massachusetts Institute of Technology), S. A. Wender, P. A. M. Gram, T. N. Taddeucci (LANSCE \\ Division), S. F. Pate (New Mexico State University)
}

\section{A long-standing goal of nuclear physics has been to understand the fundamental neutron-proton interac- tion. While many experiments have been performed on elastic scattering, inelastic processes, such as those in which a photon or meson is produced, are far less well known. This highlight describes an ongoing investigation of these processes, including work done in 1997.}

In inelastic scattering of neutrons and protons (generically nucleons), some of the nucleons' energy is given up to produce other particles, such as $\gamma$-ray photons and $\pi$-mesons. The photon emission process is known as neutron-proton bremsstrahlung. This reaction is similar to the familiar electron bremsstrahlung process where a photon is radiated when an accelerated electron interacts with an atom's electromagnetic field.

In the nucleon-nucleon case, a bremsstrahlung photon can be radiated when the nucleons interact via the strong nuclear force. The four diagrams in Figure 1 (L) illustrate this process (an oval represents the nucleon-nucleon interaction). This interaction can be described as arising from the exchange of virtual mesons. In the neutron-proton interaction the mesons can be either electrically neutral (such as the $\left.\pi^{\circ}\right)$ or charged $\left(\pi^{+}\right.$or $\left.\pi^{-}\right)$. A charged meson also can emit a bremsstrahlung photon (Figure 1, R).
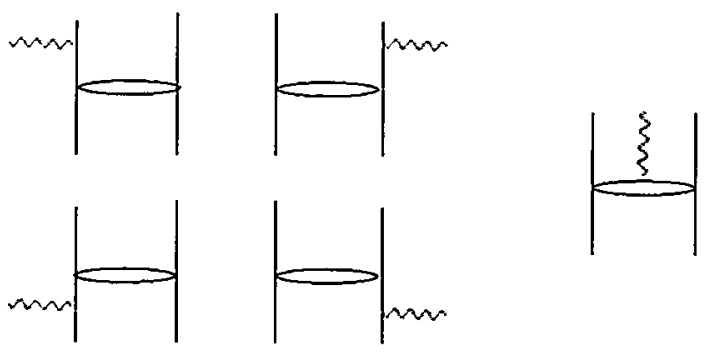

$$
\begin{array}{ll}
\text { External } & \text { Exchange } \\
\text { Diagrams } & \text { Diagram }
\end{array}
$$

$\Delta$ FIGuRE 1. Diogroms of mechonisms for nudeonnudeon bremsstrohlung. Verfical lines represent nudeons propagating through spoce-time; ovols symbolize the nudeonnudeon interaction. Photons (wow lines) con be emitted either by a nucleon (four diograms, $L$ ) or by a charged meson in the exchonge process (diogrom, $R$ ).

A nucleon cannot emit a single photon and conserve momentum and energy. However, energy conservation can be violated for the short time interval allowed by Heisenberg's uncertainty principle, during which the nucleon is said to be "off-shell." Then a second interaction must occur to restore conservation of energy.

Figure 1 shows that a study of the neutron-proton bremsstrahlung process affords the possibility of learning about both the off-shell and meson-exchange aspects of the nucleon-nucleon interaction. Figure 2 shows a theoretical calculation $^{1}$ of the angular distribution of the photons emitted in neutron-proton bremsstrahlung, for incident neutron energy $E=200 \mathrm{MeV}$ and outgoing neutron and proton angles $\theta_{n}=\theta_{p}=30^{\circ}$. The top panel shows the effect of changing the form of the nuclear force. The bottom panel illustrates the contributions of the external (off-shell) and exchange processes. The former process is seen to produce an angular distribution that resembles a classical electric dipole radiation pattern. The meson exchange contribution is asymmetric, favoring the negative-angle (neutron) side over the positive-angle (proton) side of the incident neutron direction. The two contributions are of comparable magnitude. Neutron-proton bremsstrahlung can thus provide a simple, direct test of models of meson exchange currents, which are important in describing many other phenomena in nuclear and particle physics.

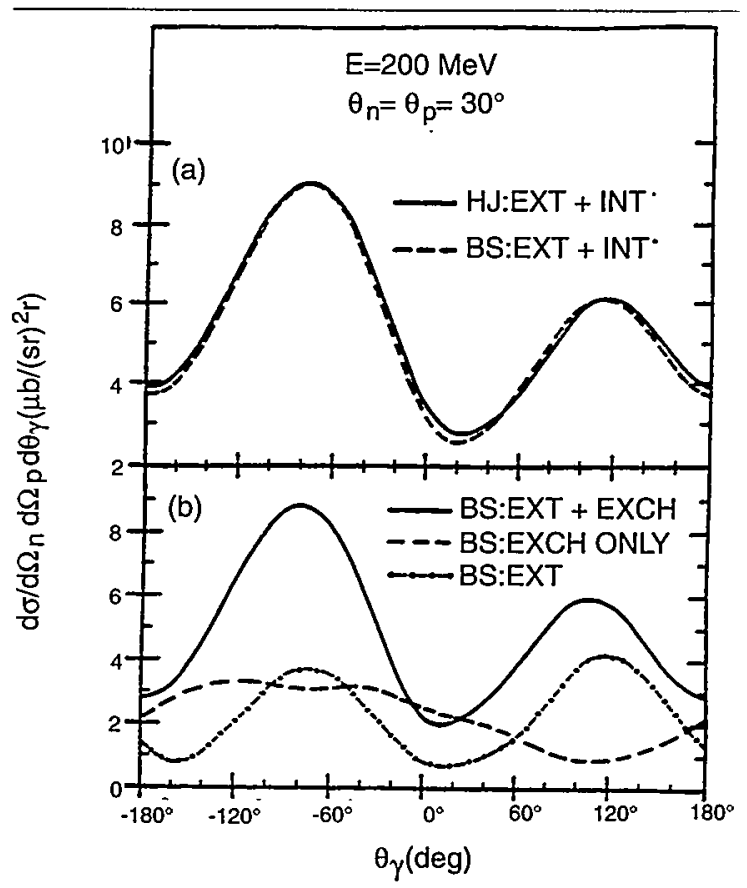

$\triangle$ FIGURE 2. Theorefical calculation of the photon angular distribution in neutronproton bremsstrohtung, for incident neutron energy $E=200 \mathrm{MeV}$ and neutron and proton ongles $\theta_{n}=\theta_{p}=300$ : (a) comparison of results using two forms for the nucleon-nudeon potential; (b) contributions of external (EXT) and exchange (EXCH) processes. 
The intense high-energy neutron beam at WNR, with its continuous spectrum of energies up to nearly $700 \mathrm{MeV}$, presents a unique opportunity to observe neutron-proton bremsstrahlung and also, for energies above around 300 $\mathrm{MeV}, \pi$-meson production. The beam impinges on a liquid hydrogen target; scattered neutrons and recoil protons are

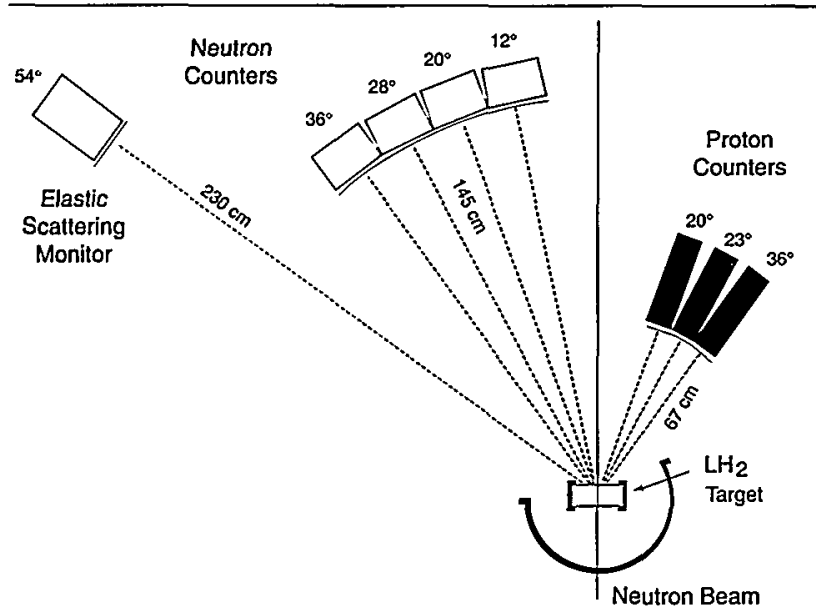

A FIGURE 3. Apparatus for observing neuhon-profon bremsstrahlung.

detected in coincidence by the array of counters (Figure 3). The neutron counter at $54^{\circ}$ continuously monitors neutronproton elastic scattering. Since the angle between the neutron and the proton in elastic scattering is $90^{\circ}$, independent of energy, the detectors at $20^{\circ}, 28^{\circ}$, and $36^{\circ}$ will see only inelastic processes. Below the $\pi$-production threshold, the only possible inelastic process is neutron-proton bremsstrahlung. Knowing the detector angles and measuring the energies of the detected particles, one can deduce the emission angle of the undetected photon. The neutron energies are determined by time-of-flight and the proton energies by the pulse height information from the CsI proton counters. The time-of-flight "clock" is started when the LANSCE proton beam strikes the spallation source; i.e., when the neutrons are produced, "stop" signals are produced by scattered neutrons and recoil protons reaching their respective detectors.
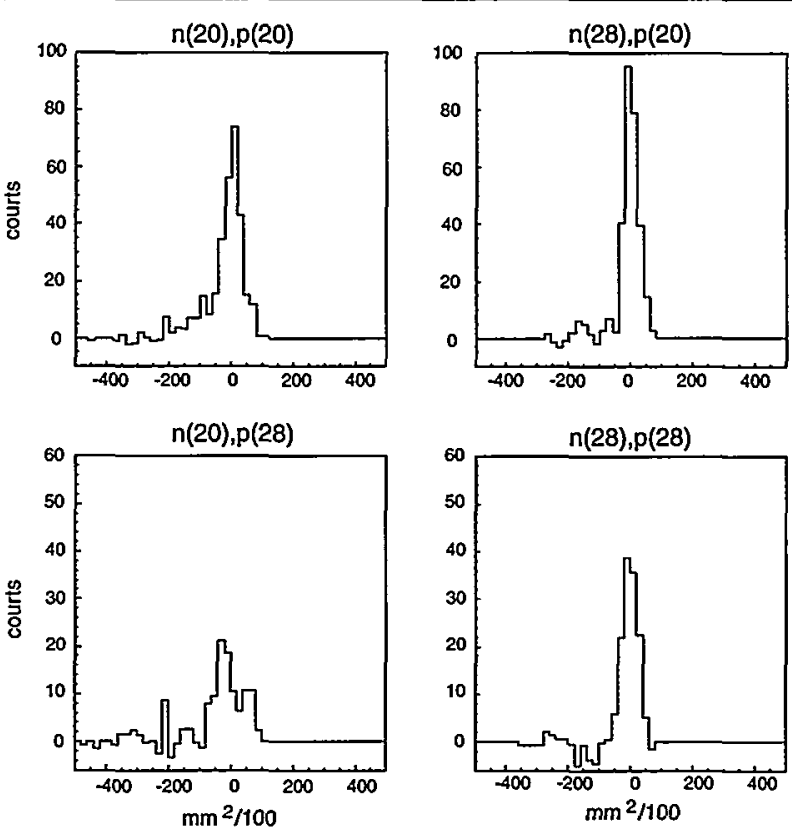

$\triangle$ FIGURE 4. Plots of the squore of the missing mass (in [Mel]2) in the finol state in inelastic neutron-proton scattering, for incident neutron energy $225 \mathrm{MeV}$. Emptytarget background data were subtracted from dato obtained with the hydrogen farget. The neutron and proton detector ongles (in degrees) are given of the top of each plot. The peaks at zero missing mass are due to neuton-proton bremsstrohtung.

For incident energies above $280 \mathrm{MeV}$, bremsstrahlung events can be distinguished from $\pi$-production events by using the measured quantities to calculate the "missing mass" in the final state. Figure 4 shows examples of missing-mass plots for neutron and proton detectors at $\left(20^{\circ}, 20^{\circ}\right),\left(28^{\circ}, 20^{\circ}\right),\left(20^{\circ}, 28^{\circ}\right)$, and $\left(28^{\circ}, 28^{\circ}\right)$ for incident neutron energy $225 \mathrm{MeV}$. Since this energy is below the $\pi$ production threshold, one sees a peak only at $0 \mathrm{MeV}$, corresponding to the zero-mass photon emitted in neutronproton bremsstrahlung.

\section{Reference}

1. V. R. Brown and J. Franklin, "Meson-Exchange Effects in Neutron-Protron Bremsstrahlung," Physical Review C 8, 1706 (1973). 


\title{
Dynamic Temperature and Velocity Measurements
}

\author{
V. W. Yuan, J. D. Bowman, G. L. Morgan (Physics Division), C. Forest, R. S. Hixson B. W. Asay, \\ D. J. Funk, D. Idar, R. L. Rabie, R. M. Boat, L. M. Hull, R. K. London (Dynamic Experimentation \\ Division), B. I. Bennett, C. E. Ragan (Applied Theoretical and Computational Physics Division)
}

The broadening of neutron resonances can provide information on the temperatures of materials being traversed by neutrons. We are developing the capability to use LANSCE beams to measure the temperatures in dynamically loaded systems of interest to science-based stockpile stewardship (SBSS), where a snapshot of temperature must be made on fast time scales of a microsecond or less. Experiments have begun to study the temperature of explosively driven metal jets and the temperature in a shocked metal.

How does one measure the internal temperature of a system undergoing dynamic loading-such as a metal being shocked? This challenge is one whose successful solution is of great interest to the shock physics, weapons physics, and industrial communities. Existing methods of temperature measurement in dynamic systems have various inherent limitations. Optical methods interact with sample surfaces, and during dynamic events the surface may be obscured from view by reaction products. Even if the surface can be clearly viewed, temperatures at the surface may not equal those present in the interior. Probes placed in the interior of the sample are short-lived and often destroyed during the dynamic event. Finally, the presence of invasive diagnostics may alter the dynamics of the very interaction one desires to study.

Neutrons are temperature probes that can penetrate a sample to view its interior. At the same time products that may optically shield a sample are usually not opaque to neutrons so that temperature measurements are possible in their presence. When epithermal neutrons are attenuated by a sample material, the time-of-flight (TOF) spectrum of the transmitted neutrons exhibits a series of characteristic dips or resonances. These dips result from neutrons that are removed from the beam during the formation of excited states in the compound, $A+1$ nucleus. The locations and line shapes of resonances that appear in the TOF spectrum are unique to each isotopic element and therefore can pinpoint temperature determinations to the location of the resonant material.

In neutron resonance spectroscopy (NRS), the temperature of an irradiated sample is determined from temperaturedependent changes to the Lorentzian line shape of a resonance. The energy available when neutron and nucleus interact is dependent on the relative velocity of the two bodies. As a result, the observed line shape becomes a convolution between a Lorentzian and a Gaussian of width proportional to the square root of sample temperature. One must be aware, however, that other undesired factors may also influence the resonance line shape. Two such factors are: 1) the spread in times to slow fast neutrons to the energy of the resonance being studied, and 2) phosphorescence in the light emitted by scintillation media (such as ${ }^{6} \mathrm{Li}$-loaded glass) used for neutron detection.

The neutrons for NRS measurements are produced either at the neutron production target of the Lujan Center or in a special target/moderator assembly located in the Blue Room at the WNR facility. The former site offers a $20-\mathrm{Hz}$ beam that is advantageous for multiple-pulse measurements. Most dynamic measurements require only singlebeam pulses, and the Blue Room setup allows for more intense neutron pulses as well as permitting the sample to be closer to the neutron source, which results in a six-fold better time resolution of a dynamic event.

What is the time scale for a dynamic temperature measurement using NRS? The answer to this question depends on the transit time for neutrons of the studied resonance to pass through the sample. For the temperature measurement to be meaningful, the dynamic system being studied must remain quasi-stable during this time of passage. Figure 1 shows the resonance profile for the $21.1-\mathrm{eV}$ resonance in ${ }^{182} \mathrm{~W}$ as observed $1 \mathrm{~m}$ from the neutron source. The width of the resonance is $0.17 \mu \mathrm{s}$-narrow enough for studies on the submicrosecond time scale. Figure 2 clearly shows the difference in resonance line shapes for a tungsten sample heated to various temperatures.

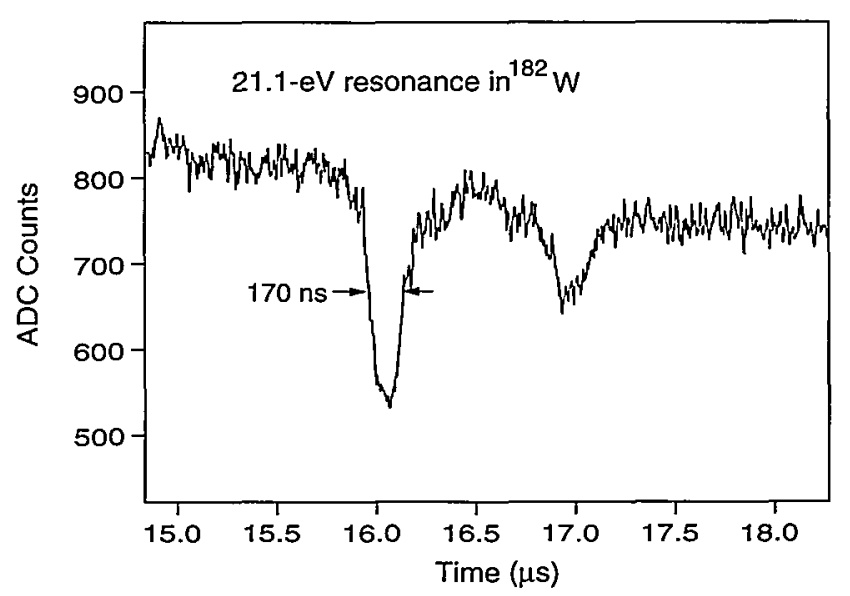

$\Delta$ FIGURE 1. Time scale for the 21. IeV resonance in tungster- 182 when viewed at $1 \mathrm{~m}$ distance from the neutron source. The $170 \mathrm{~ns}$ width permits submicrosecond resolution of dynomic events. 


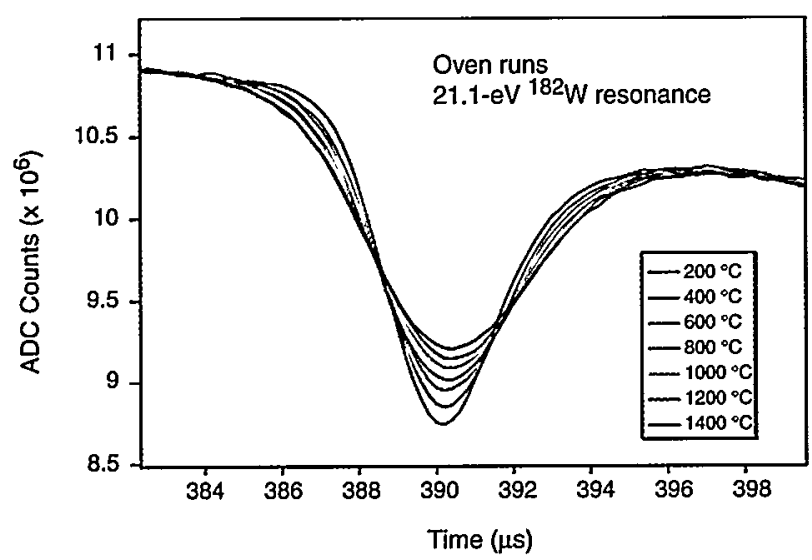

A FIGURE 2. Changes in resononce profile accompanying different sample temperatures. The tungster-182 somple wos heated in on oven.

In 1996, we commenced a program of experiments to measure temperatures in an explosively formed metal jet. In the comparison between a silver sample at room temperature and the heated silver in a jet (Figure 3), it is easy to see the resonance broadening resulting from the large temperature difference. Radiographs taken at PHERMEX provide additional information on variations in jet thickness and jet velocity as a function of time, and we include their effects in our analysis of the data.

$\Delta$ FIGURE 3. Comparison of $30.4 \mathrm{eV}$ resononce in silver as observed before and during the formotion of a metal jet. The broadening resuling from the higher temperature of the jet is dearly evident.

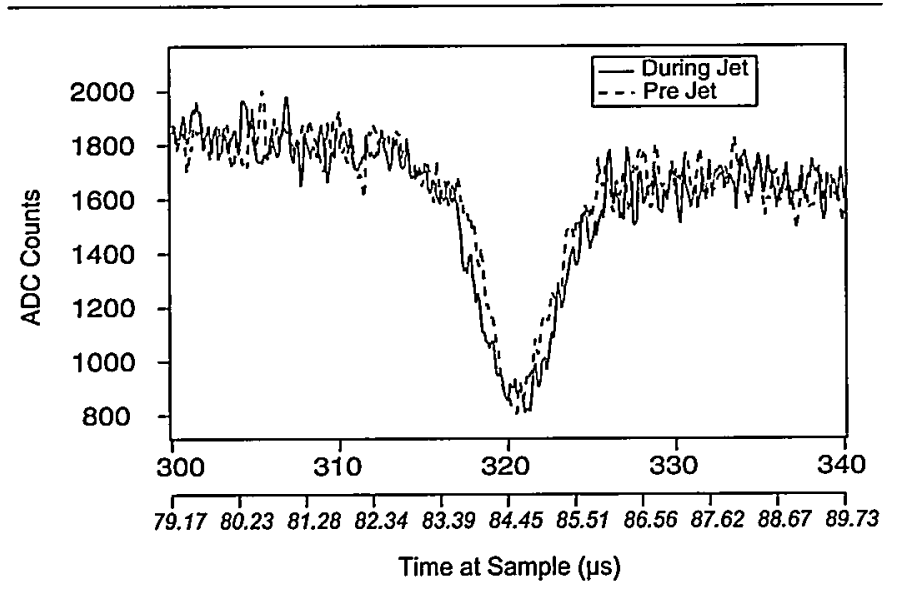

In 1997, a second set of experiments was initiated-this time in the Blue Room-to measure temperatures in a metal after passage of a shock wave. Measurements of this type did not previously exist, and temperature data was sought to improve understanding of the shocked material. In similar fashion to the metal-jet experiment, our data clearly show that the resonance profile after passage of the shock is both broadened and Doppler-shifted to an earlier time when compared with the resonance profile before passage of the shock. The broadening is the result of a higher temperature, and the centroid shift is due to the relative motion of the shocked sample towards the neutron source. 


\section{Neutron Capture on Off-Stable Nuclei}

\section{R. S. Rundberg, J. B. Wilhelmy, M. M. Fowler, G. G. Miller (Chemical Science and Technology Division), J. L. Ullmann, R. C. Haight (LANSCE Division)}

An accurate knowledge of the probability for the capture of a neutron by a nucleus, subsequently followed by gamma ray emission, is important to understanding the results of historical nuclear weapons tests, as well as to understanding the synthesis of the chemical elements in stars. Although many such experiments have been done on stable nuclei, very few have been done using radioactive nuclei. We have measured this process on the radioactive isotope ${ }^{171}$ Tm, on Flight Path 4 at the Lujan Center. The results indicate that the parameters used in the theoretical calculation of the reaction may be inadequate for radioactive nuclei.

The capture of a neutron by a nucleus and subsequent gamma ray emission is one of the simplest nuclear reactions. Experiments measuring it are rather easy in concept and have been done for a wide range of stable isotopes. However, there are very few measurements on rare isotopes or on radioactive targets. Current applications require accurate estimates of the probability of this reaction for these targets. The very intense neutron beam from the Proton Storage Ring at the Lujan Center enables us to measure target material as small as $1 \mathrm{mg}$, opening the way for experiments on radioactive targets.

The weapons interest centers around the use of small amounts of various stable isotopes as tracers in nuclear weapons tests. In the extreme environment of a nuclear explosion, there can be multiple nuclear reactions on these tracers, leading to various unstable nuclei that can in turn undergo further reactions. The energy range of interest is about 1 to $100 \mathrm{keV}$. In order to model the archived results of nuclear tests, the probabilities for the various reactions must be accurately known.

A different area of interest is in studying the production of chemical elements in the stars. One of the main processes in nucleosynthesis is the slow ("s-") process neutron capture, in which stable or nearly stable nuclei are formed by neutron capture on the next lighter nucleus. For unstable isotopes, there is a competition between radioactive decay and neutron capture. The rate of capture depends on the capture probability and the density and energy of the neutrons at the s-process site. Thus, the branchings can provide insights to the stellar neutron environment. ${ }^{1}$ Accurate estimates of the capture probability are required for calculations to predict the observed cosmic abundances of the isotopes. The energy range of interest depends on the stellar model but varies from 1 to $200 \mathrm{keV}$. In yet another application, neutron capture on unstable targets is of growing interest for studies of radioactive waste transmutation.

The experiment was done on Flight Path 4 at the Lujan Center, at $8 \mathrm{~m}$ from the "high-intensity" water moderator. The setup was the same as used by Koehler ${ }^{2}$ in earlier measurements. The neutron flux was determined by simultaneously measuring the ${ }^{6} \mathrm{Li}(\mathrm{n}, \alpha)$ reaction using a target consisting of a thin deposit of ${ }^{6} \mathrm{LiF}$ on an aluminum backing. The shape of the measured neutron flux is in good agreement with the flux calculated by Russell, ${ }^{3}$ as well as an earlier flux measurement by Koehler, ${ }^{4}$ which used a different set of collimators. The magnitude of the measured fluxes appears to be slightly less than the calculated value in both measurements.

The experiment in 1997 served as a "warm up" experiment to test the feasibility of using radioactive targets. The targets studied were ${ }^{171} \mathrm{Tm}$, which has a 1.92-year half-life, and the stable isotope ${ }^{169} \mathrm{Tm}$. We chose ${ }^{171} \mathrm{Tm}$ because thulium is one of the principle elements used as a weapons tracer. In addition, ${ }^{171} \mathrm{Tm}$ is relatively easy to produce and presents a relatively benign radiological handling hazard. We measured ${ }^{169} \mathrm{Tm}$ because it occurred as a contaminant in the ${ }^{171} \mathrm{Tm}$, but also because it has been well studied and provided a mechanism of validating our experimental ability to perform these types of measurements. The ${ }^{171} \mathrm{Tm}$ target was made by irradiating ${ }^{170} \mathrm{Er}$ in a reactor at the Idaho National Engineering and Environmental Laboratory, followed by chemical separation and processing in a hot cell at TA-48 in Los Alamos. The ${ }^{171} \mathrm{Tm}$ was separated from the erbium target using high-performance liquid chromatography about 2 weeks before the experiment. The targets were made by electroplating the ${ }^{171} \mathrm{Tm}$ on a 0.5 -mil beryllium foil flashed with a 700- $\AA$ layer of titanium and then covered with a second layer of beryllium foil. The induced activity was as expected, with few impurities.

Figure 1 shows the data from ${ }^{169} \mathrm{Tm}$. The $\mathrm{x}$-axis shows the neutron energy, and the $y$-axis is the reaction probability, or cross section, expressed in units of barns. The peaks at low energy, especially obvious at $4.92 \mathrm{eV}$, are "resonances" that correspond to neutron capture into distinct nuclear states. At the higher energies, these states wash out and overlap until finally the spectrum becomes a featureless straight line. Also shown in the figure is a theoretical calculation using the GNASH program. ${ }^{5}$ For ${ }^{169} \mathrm{Tm}$, the calculation is in good agreement with the measured data. However, as shown in Figure 2 for ${ }^{171} \mathrm{Tm}$, the calculated cross sections are as much as a factor of five above the measurement. 


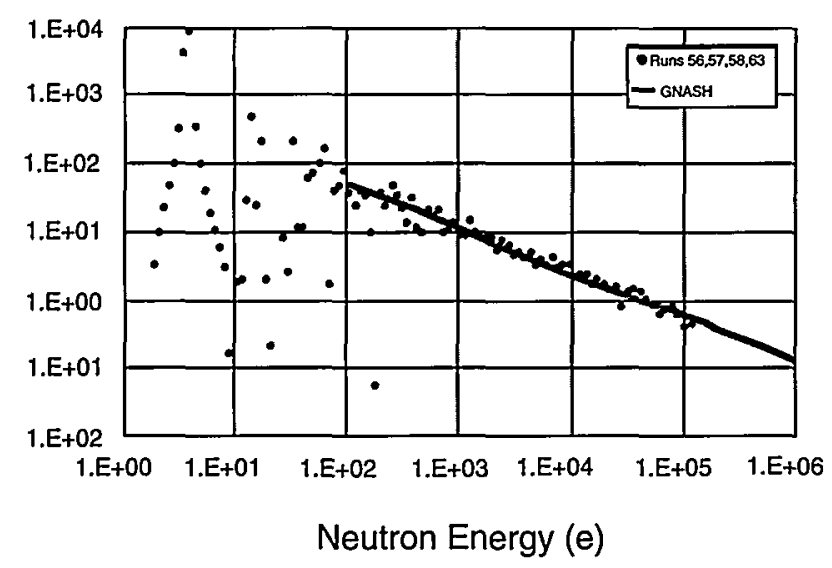

$\triangle$ FIGURE 1. Cross section of $169 \mathrm{Im}$ meosured in this experiment compored to a theoretical collulat tion using the GNASH program. ${ }^{5}$

This calculation represents the first neutron capture measurement of this isotope. The discrepancy is not entirely surprising because GNASH relies on parameters, such as the number of nuclear states per unit excitation energy in the final nucleus, that must be fit to data. This parameter could be different for stable and unstable target nuclei. The results of this experiment not only will provide a measurement of the cross sections for ${ }^{171} \mathrm{Tm}$ but also will increase our knowledge of these crucial parameters.

A serious problem in the experiment was the background rate caused by scattered neutrons that produced gamma rays in or near the detectors. These neutrons came from the beryllium backing foil in the experiment as well as from adjacent flight paths. In 1998, we are planning on using Flight Path 2 at the Lujan Center for this experiment. This will give us a longer flight path as well as better isolation from adjacent experiments. We will also try a different set of collimators, which will give a higher neutron flux.

However, in order to study other more radioactive targets, a highly segmented detector array will be required to minimize the number of decays counted in each detector. A proposal has been written to use Flight Path 14 for continuation of these experiments.

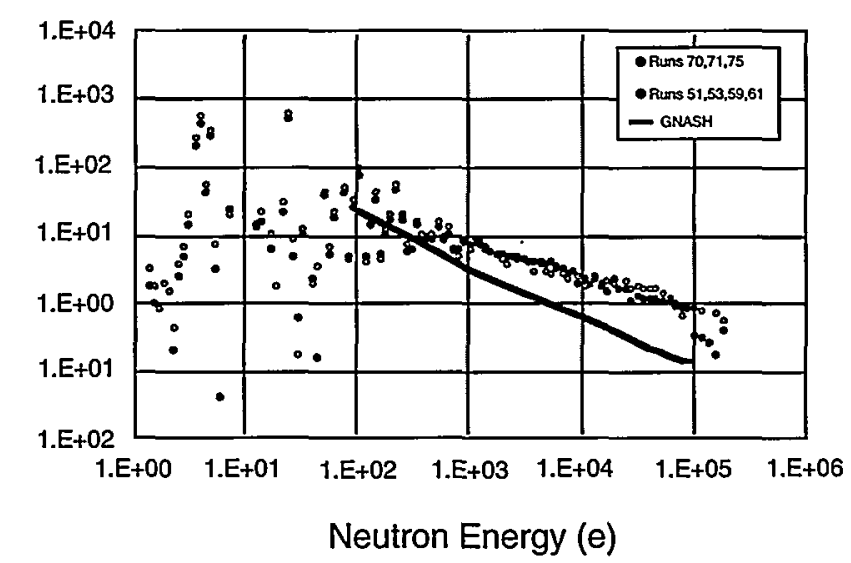

A FIGURE 2. Cross section of ${ }^{17 I} I_{m}$ meosured in this experiment compared to a theoretical calculafion using the GNASH progrom. ${ }^{5}$ Dato from two different sets of runs are shown. This represents the first meosurement of neution copture on this rodioactive forget.

\section{References}

1. F. Käppeler, "New Experimental Data for the S-Process: Problems and Consequences," in Nuclei in the Cosmos III (AIP 1995) AIP Conf. 327, p. 101. G. Wallerstein et al., "Synthesis of the Elements in the Stars: Forty Years of Progress," Reviews of Modern Physics 69, 995 (1997).

2. P. E. Koehler and F. Käppeler, "Measurements of $\gamma$ Cross Sections for Very Small Stable and Radioactive Samples of Interest to the S- and P-Process," in Proceedings of the International Conference on Nuclear Data for Science and Technology, J. K. Dickens, Ed. (Gatlinburg, Tennessee, 1994) p. 179.

3 G. J. Russell, private communication $(1989,1997)$. Calculations of the neutron brightness for the 1998 upgrade are available at www.lansce.lanl.gov/mlnsc.

4. P. E. Koehler, "Measurements of the LANSCE Neutron Flux from 0,025 ev to $100 \mathrm{keV}$," Nucl. Inst. Meth. 292, $541-545$ (1990).

5. P. G. Young, private communication (1997). 




\title{
Fast Neutron Radiography and Imaging
}

\author{
T. N. Taddeucci (LANSCE Division), C. L. Morris, K. B. Morley, J. Selden (Physics Division)
}

\section{Storage-phosphor image plates have been used to explore techniques for performing high-energy $(>50-\mathrm{MeV})$ neutron radiography with the WNR spallation source. Results from test measurements performed in July 1997 show great promise for applications ranging from beam diagnostics to detailed tomography of complex abjects.}

Neutron radiography is a technique for imaging the internal structure of an object by measuring intensity variations in a beam of neutrons transmitted through the object. The amount of detail that can be resolved and the type of objects that can be imaged depend on the energy of the neutron beam, the size of the neutron source, and the spatial resolution of the detector used to measure the intensity of the transmitted neutrons.

Neutrons are electrically neutral particles that interact with matter by scattering from atomic nuclei. The parameter that describes the probability of such an interaction is called the neutron total cross-section. Neutrons have the greatest penetrability, i.e., the ability to probe deep inside massive objects, when the neutron total cross-section is lowest. The neutron total cross-section for all atomic nuclei from hydrogen to uranium reaches a broad minimum for neutron energies centered about $300 \mathrm{MeV}$. The WNR Target-4 source is an unmoderated spallation neutron source that produces a broad spectrum of neutron energies reaching up to almost $800 \mathrm{MeV} .^{1}$ This source is therefore well suited for neutron radiography applications where maximum penetrability is desired. In July 1997 we performed a set of neutron radiography measurements designed to demonstrate the ability to image the interior of a massive object with good spatial resolution. The measurements were performed on WNR Target-4 flight path 4FP30R.

The test object consisted of a depleted uranium cylinder 6 in. in diameter with a central cavity $2-13 / 16$ in. in diameter. This cavity was filled with a polyethylene plug in which three holes were drilled parallel to the cylinder axis. The hole diameters were $1 / 2$ in., 1/4 in., and 1/8 in.

We employed a modified version of an imaging system that has been successfully applied to proton radiography: storage-phosphor image plates. These plates consist of a $\mathrm{BaFBr}: \mathrm{Eu}^{2+}$ phosphor bonded onto a polyester backing. While designed primarily for medical $x$-ray imaging purposes, the storage phosphors will also form latent images when exposed to charged particles such as protons. Neutrons are detectable by using a thin copper converter layer in front of the image plate. A neutron that interacts inelastically in the copper layer can eject one or more protons that stimulate the phosphor and form the latent image. To increase the efficiency of the detection process, we used ten alternating layers of copper and storage phosphor, forming a sandwich about one inch thick.

Before radiographing the test object, the image-plate detector system was tested by producing pinhole images of the Target- 4 source. A shielded brass pinhole was placed in the neutron beamline $10 \mathrm{~m}$ from Target-4. A 10-layer stack of image plates was positioned $10 \mathrm{~m}$ downstream from the pinhole. This arrangement thus produced images with a magnification of 1 . The pinhole consisted of a 4 in. $\times 4$ in. $x 10.5$ in. brass brick with a central bore that tapered to $1 / 16$ in. at the center.

There are two spallation targets available at WNR. The standard target is a water-cooled tungsten cylinder $3.0 \mathrm{~cm}$ diameter by $7.5 \mathrm{~cm}$ long. This target yields a large neutron flux but is unsuitable for detailed radiography measurements because of image blurring produced by its large angular size. A second target constructed specifically for the radiography measurements is $1 \mathrm{~cm}$ diameter by $2.5 \mathrm{~cm}$ long. This smaller target produces a much sharper image at the expense of reduced neutron flux (about a factor of 10). Neutron pinhole images of the two WNR spallation targets are shown in false color in Figure 1. The top picture shows the standard target. The viewing angle of $30^{\circ}$ foreshortens the apparent length by a factor of two. In this picture the proton beam enters from the left at an upward angle of $8.5^{\circ}$, which is doubled in this image by the $30^{\circ}$ perspective. The bottom image shows the smaller radiography target. The rectangular outline surrounding each target image is produced by unfocused neutrons filling a $3.2-\mathrm{cm} \times 4.3-\mathrm{cm}$ collimator aperture upstream and downstream of the pinhole.

Neutron radiographs of the cylindrical test object were obtained with the object at a distance of $18 \mathrm{~m}$ from Target4 , with the image plates $1 \mathrm{~m}$ farther downstream. The cylinder was oriented with its axis perpendicular to the incident beam. Nine exposures were made, with the object rotated $20^{\circ}$ about its axis between each exposure. This set of images allowed us to make a tomographic reconstruction of the interior of the cylinder. The raw image-plate data was scanned at a resolution of $150 \mathrm{dpi}$ and was subsequently rebinned into $1-\mathrm{mm}$ pixels to improve statistics.

As a basis for comparison, some theoretical radiographs of the cylindrical test object were also calculated and then processed in a manner identical to the data. The calculations did not include the effects of small-angle elastic scat- 

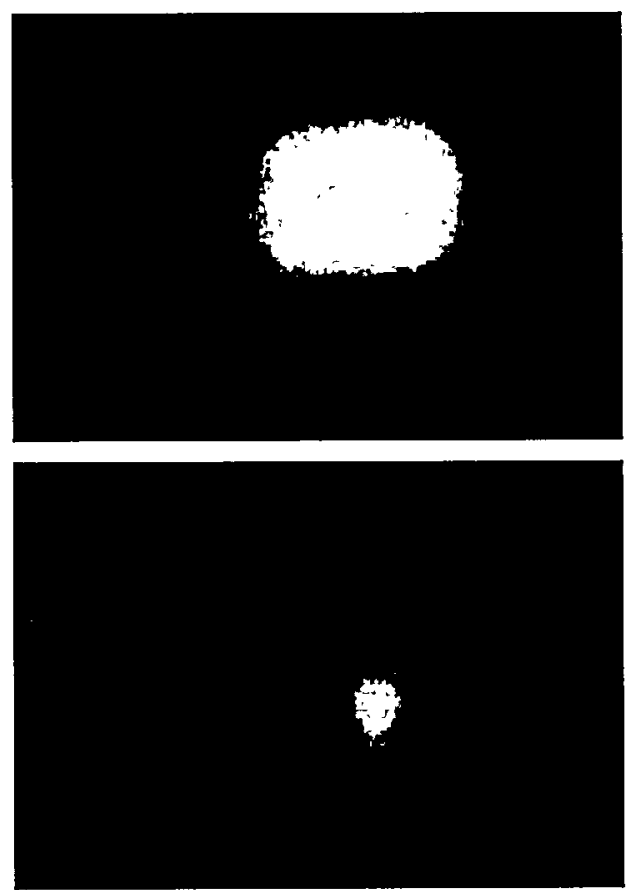

$\triangle$ FIGURE I. Neutron pinhole imoges of the WWR Torget-4 source. the top imoge is the standard source. The bottom image is a smoller source designed for radiography opplications.

tering or detection statistics. These calculations thus represent the best possible image that can be obtained for this particular geometry.

Tomographic reconstructions were done using filtered back projection. Two types of images were produced: differential and full. In the differential image the cylindrical symmetry of the object was used to subtract the average neutron intensity before reconstruction. This method enhances the visibility of "non-average" features such as the holes in the polyethylene. In the full images no information was subtracted and the derived image intensity is linearly related to the actual "density" (inverse attenuation length) at each point.

Results are shown in Figure 2. The left-hand images in this figure are a product of the model calculations, and the right-hand figures are based on the real data. The top figures are differential reconstructions; the bottom figures are full reconstructions. In the data reconstructions only the region of the central polyethylene cylinder (colored region) is valid. Data outside this region were not usable because of some uncorrectable response variations in the image plates. Inside this region agreement with the model calculations is very good.
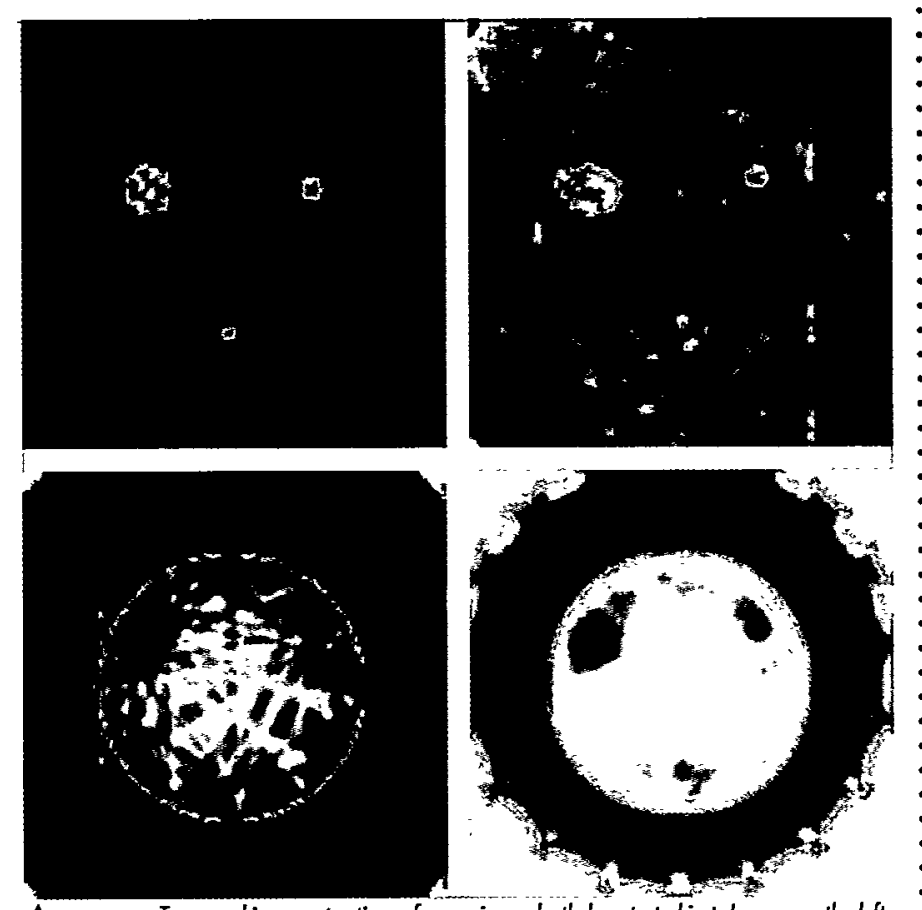

$\Delta$ FIGURE 2. Tomographic reconstuctions of a uranium-polyethylene test object. Images on the left are theoretical colculations. Images on the right are actual doto.

\section{References}

I. P. W. Lisowski, C. D. Bowman, G. J. Russell, and S. A. Wender, Nuclear Science and Engineering 106, 208 (1990). 


\title{
Cold Neutron Radiography Allows for Energy-Selected and Conventional Radiography
}

\author{
T. E. McDonald, Jr. (Physics Division), T. O. Brun (LANSCE Division), T. N. Claytor (Engineering \\ Science and Applications Division), E. H. Farnum (Materials Science and Technology Division), \\ R. A. Gallegos (Physics Division), G. L. Greene, L. F. Hunt (LANSCE Division), S. A. Jaramillo, \\ R. N. Mortensen, C. Morris, P. D. Pazuchanics, S. J. Seestrom (Physics Division), M. J. Schwab, \\ D. M. Stupin (Engineering Science and Applications Division), W. L. Sievers, M. J. Sheats (Mason \& \\ Hanger Corp., Pantex Plant, Amarillo, Texas), W. C. Moffatt (Sandia National Laboratories)
}

\begin{abstract}
We have established a neutron radiography capability on Flight Path 11a at the Lujan Center. The neutron beam at the Lujan Center is attractive for neutron radiography because it is pulsed and because it has low gamma content. The pulsed characteristic of the beam allows us to use a time-gated imaging technique that we have recently developed to obtain radiography at specific neutron energies. This capability can be combined with the Bragg cutoff phenomenon of selected materials to achieve materials discrimination. We have carried out radiography on test components using three imaging techniques: time-gated imaging, an amorphous silicon detector, and conventional $x$-ray film.
\end{abstract}

Neutron radiography is an attractive technique in nondestructive testing and evaluation because of the strong energy variation of neutron cross-section characteristics of nearly all the nuclides. As a result of this strong variation, measurement of the neutron transmission as a function of energy and position over a broad range of neutron energies can be used to give elemental and spatial information about an object. Neutron radiography is generally carried out at reactors where a steady-state source of neutrons covering a broad energy range is available. Spatial resolutions of a few tens of microns are readily achievable in such radiographs, and resoIutions as high as 1 micron have been reported by the Phoenix Memorial Laboratory, University of Michigan. ${ }^{1}$

Over the past 2 years, we have used three imaging techniques at the Lujan Center: time-gated cold neutron radiography, radiography using an amorphous silicon detector, and radiography with $\mathrm{x}$-ray film. These techniques are applied to the Science-Based Stockpile Stewardship program and industrial research.

\section{Time-Gated Cold Neutron Radiography}

Neutron radiography with cold neutrons using time-gating is a technique that was first demonstrated at LANSCE. This technique is of interest because of an important behavior of crystalline materials at cold neutron energies. Below an energy of a few meV (the exact energy depends on the material), there is an abrupt drop in the scattering cross section when the wavelength reaches twice the largest d-spacing of the material. The energy level at which this abrupt drop occurs is referred to as the Bragg cutoff. This behavior is not exploited in a reactor environment because the steady-state nature of the neutron source is not favorable to separating neutrons of different energies. However, a pulsed neutron source, such as at the Lujan Center, can employ time-of-flight to obtain radiographs at specific neutron energies (or in a narrow range of neutron energies). By recording image data at different times during a neutron pulse, radiographs can be made at neutron energies of interest, for example, just above and just below the Bragg cutoff of a given material.

Consider a sample or device consisting of components of various materials. A radiograph taken at an energy above the Bragg cutoff of all the components in the device would show the entire device. However, choosing an energy below the Bragg cutoff of some materials and above the Bragg cutoff of others produces a radiograph having the "below the Bragg cutoff" components much less absorbing than the remaining components. Therefore, it is possible to separate components and materials in a sample or device by judiciously choosing the neutron energy at which a radiograph is made. With this technique, it is possible to obtain multiple radiographs emphasizing some components or constituents and de-emphasizing others, without modifications to the object. These component images can likely be separated through signal processing. In addition, imaging in three dimensions can be obtained by using tomographic image reconstruction techniques.

This phenomenon was demonstrated on Flight Path 11a during the 1997 run cycle. A lithium zinc sulfide scintillator (Bicron 704) $)^{2}$ was used as a neutron-to-light converter, and the image on the scintillator was relayed by a mirror to a gated, intensified, cooled charged-coupled device (CCD) camera. The demonstration was carried out using two materials that have a well-defined Bragg cutoff: beryllium, which has a cutoff at approximately $6 \mathrm{meV}$, and carbon, which has a cutoff at approximately $1.9 \mathrm{meV}$. A 25-mmthick beryllium block was obtained into which a 1/2-in. diameter threaded hole ( 20 threads per inch) was cut. 


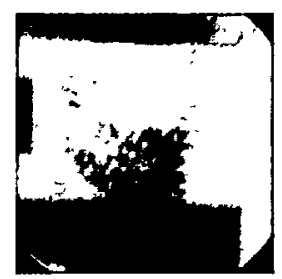

FIGURE la.

Neution Energy: $7.5 \mathrm{meV}$ Above Cutoff of $\mathrm{Be}(6 \mathrm{mel})$ Above Cutoff of $(2 \mathrm{meV})$ Both Be and C Dark

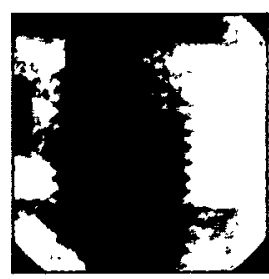

FIGURE $\mathrm{Ib}$.

Neutron Energy: $2.9 \mathrm{meV}$ Below Cutoff of $B e$ Above Cutoff of $C$ Be light, C Dork

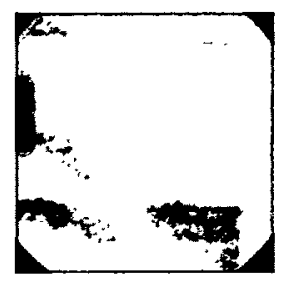

FIGURE ]C. Neutron Energy: $1.5 \mathrm{meV}$ Below Cutoff of both Be and C Both Be and C Light

$\Delta$ FIGURE 1. Demonstrotion of cold neutron radiogrophy using time-offlight and goted imaging to obtain rodiogrophs of specific neutron energies. These imoges demonstrate materials discrimination using the Brogg cutoff phenomenon. The imoges are a series of radiogrophs of a carbon bolt threaded into a beryllium block. Image (a) is a rodiograph token ot approximately $7.5 \mathrm{meV}$, which is above the Bragg cutoffs of both beryllium (6 meV) and carbon (2 meV) ond shows both the beryllium and corbon as relotively dark. Image (b) is a radiograph taken at opproximately 2.9 meV, which is between the Brogg cutoffs of beryllium ond carbon; the beryllium has become lighter becouse of the reduction in the scaltering cross section ocross the Brogg cutoff, and the corbon bolt inside the beryllit um hos become visible. Imoge (c) is a rodiograph taken at a neuton energy of opproximately $1.5 \mathrm{moV}$, which is below the Brogg cutoff of both beryllium and carbon, ond both the beryllium and carbon have become lightened. Note that if any denser material had been obscured by the beryllium and corbon moterials, it would hove been seen in Imoge (c).

A $1 / 2$ by 20 carbon screw was inserted into the hole. Radiographs were made above, between, and below the two Bragg cutoffs. The results of the radiography are shown in Figure 1. The radiographs demonstrate that material discrimination among various materials is possible. Although this phenomena is material dependent and is not exhibited for all materials, we believe there are enough materials, such as carbon and iron, for which an abrupt change is exhibited so that the approach can be exploited in programs of interest.

\section{Radiography Using an Amorphous Silicon Detector} Use of an amorphous silicon detector was also demonstrated on the 11a beam line. The advantage of the amorphous silicon detector is that, unlike a CCD silicon detector, it can be placed directly in the neutron beam path with effectively no damage if the beam is not left on the detector for long periods of time. Thus, there is no need to relay the image using optics, and a good quality image can be obtained relatively quickly (within a few seconds). Unfortunately, the amorphous silicon detector cannot be gated and, thus, cannot be used for gated imaging. Figure 2 shows an example of an image we obtained with a Reticon amorphous silicon detector.

\section{Radiographs with X-Ray Film}

The highest quality and resolution radiographs we have obtained have been with $\mathrm{x}$-ray film. A thin gadolinium foil is placed next to the film and produces $\mathrm{x}$-rays proportional to the local neutron flux. The x-rays expose the film. Figure 3 shows a radiograph of a cracked fire-set sample provided by Sandia. We estimate the resolution of this radiograph to be in the neighborhood of 25 microns. As with the amorphous silicon detector, gated imaging cannot be obtained with the film technique.

During this run cycle, we will be examining samples and components from Los Alamos, Sandia, and Oak Ridge ( $Y$ 12). We are also examining possible applications for the newly developed technique of time-gated radiography.

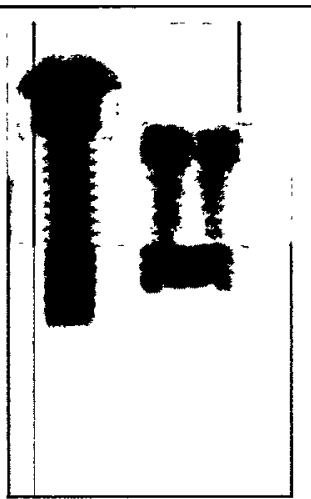

$\Delta$ FIGURE 2. Neutron radiograph taken with a Reticon amorphous silicon detector. The sample is o 25 -mm-thick beryllium block vith threaded holes (20 threads per inch) into which ore screwed a nylon screw (left) and a brass fitting (right). An oluminum nut hos been fully screwed onto the nylon screw and can be seen abuting the screw head. Also seen in the image is a nylon Oring inside the bross fitting. Such on Oring would not be visible in an X-ray radiogroph. The resolution of this neutron rodiograph is approximately 150 microns. The dark vertical lines in the image are columns of defective pixels in the detector.

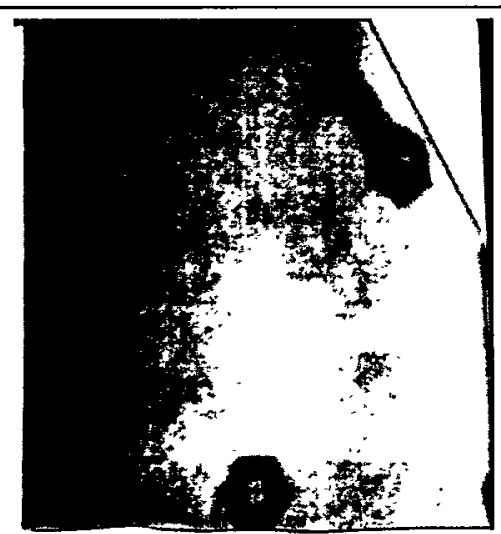

$\Delta$ FIGURE 3. This image is an example of the highest resolution radiogrophy that has been achieved on the LANSCF cold neutron radiography facility. The image is a radiograph of a cracked fireset case sample provided by Sandia National Laboratories. The radiograph is an experiment to determine if such a crack could be imoged by neutron radiography. The crack is well defined in the radi ogroph with a resolution estimated to be in the neighborhood of 25 microns. The rodiograph wos obtoined with xray film; a thin foil of gadolinium wos ploced next to the film to serve as a neutronto-xray converter.

\section{References}

1. J. Lindsay, Phoenix Memorial Laboratory, University of Michigan, personal communication (August 1997).

2. A. R. Spowart, "Optimizing Neutron Scintillators for Neutron Radiography," Br. J. Non-Destr. Test. 11 (1), 2-11 (March 1969). 


\section{Proton Radiography}

\section{J. B. McClelland (Physics Division) and the Proton Radiography Team}

Many modern nuclear weapons incorporate insensitive high explosives (IHEs) to greatly reduce accidental detonation during transportation or handling. Because of the reduced sensitivity of the $I H E$, its initiation and detonation are much more difficult to accurately model in our computer codes. Therefore reliable detonation is more difficult to guarantee under a wide range of conditions. The proton radiography experiments at LANSCE provided an extensive set of data for IHE detonation for various initiation conditions and temperatures. Such data show that our present calculational models have shortcomings; this data also can be used to help us develop and validate better models. Better understanding will help us maintain our confidence in these weapons into the future.

Flash x-ray radiography has been used for over 40 years to image dynamic processes. Proton radiography is a sharp departure that draws on the skills and techniques developed in nuclear and particle physics. In the past, protons were used to image thin systems. However, the technique was limited by image blurring, which resulted from multiple scattering of the protons in the object being radiographed, due to the proton's charge. Recent experiments have demonstrated a new magnetic-lens system, which removes much of this blur even for thick objects. This technique can be extended to gain information on the material composition of an object in addition to its density by cascading two lenses with different angular apertures. This is a unique feature of protons over conventional $x$-ray radiography.

In proton radiography, a beam of protons impinges directly on the object to be radiographed. There is no need for a bremsstrahlung converter (which is needed to produce $\mathrm{x}$-rays) or its equivalent, since the proton beam directly illuminates the radiographed object. Ultimately, an accelerator facility capable of providing $50-\mathrm{GeV}$ proton beams will be needed for hydrotesting purposes. This capability is crucial to Science-Based Stockpile Stewardship, Los Alamos's effort to scientifically verify the reliability of its nuclear stockpile without nuclear testing.

The principle radiographic advantages of protons over $\mathrm{x}$ rays are (1) the long mean-free-paths of protons, well matched for imaging thick-dense objects; (2) existing proton accelerator technology to provide the required beam; (3) significantly better signal to noise over x-rays in the final image; (4) sensitivity to both material density and composition; (5) direct utilization of the proton beam as the radiographic probe, thereby eliminating the need for an intermediate bremsstrahlung converter; and (6) high detection efficiency, allowing a "motion picture" of the explosion with many frames and simultaneous viewing directions. LANSCE has been one of the most important facilities in demonstrating this new technique.

Los Alamos is leading a multilaboratory effort to demonstrate protons as a viable new radiographic probe to image imploding or exploding objects with high spatial and temporal resolution. The proton radiography team also includes scientists, engineers, and technicians from Lawrence Livermore National Laboratory, Lawrence Berkeley National Laboratory, Brookhaven National Laboratory, Indiana University, and Bechtel Nevada.

LANSCE is capable of providing $800-\mathrm{MeV}$ protons. This energy is well suited for examining shock-wave propagation in small-scale, high-explosive systems. The limitations to thin, low-Z systems at $800 \mathrm{MeV}$ come primarily from multiple scattering and energy loss within the object and aberrations in the lens system. These effects become less important as the beam energy increases. LANSCE experiments addressed some specific problems involving detonation wave propagation inside a high-explosive assembly as a function of temperature and other high-explosive properties. In addition, high-energy static demonstrations are being performed using high-energy protons (up to $25 \mathrm{GeV}$ ) from the AGS at Brookhaven National Laboratory.

New beam line diagnostics, a containment vessel, and lens system were installed in the Line B area at LANSCE in preparation for the FY97 running period. These improvements provided the first demonstration of a proton radiograph on a dynamic system, using high-explosive charges of 100 to $700 \mathrm{~g}$.

Twenty-five dynamic shots were taken from April to August 1997 at LANSCE. These shots investigated the characteristics of shock propagation in different lots of high explosives over a range of temperatures. Typically, four to six frames were taken of each explosion.

Many modern nuclear weapons incorporate IHEs to greatly reduce the chance of an accidental detonation during transportation or handling. Because of the reduced sensitivity of the IHE, its initiation and detonation is much more difficult to accurately model in computer codes, making reliable weapons detonation more difficult to guarantee under a wide range of conditions. The proton radiography at LANSCE provided an extensive set of data for IHE detonation for various initiation conditions and temperatures. 
Such data show that our present calculational models have shortcomings and will help us develop and validate better models. Better understanding will help us maintain our confidence in these weapons into the future.

Figure 1 shows a detonation wave at four different times in a high-explosive assembly. These exposures correspond to $0.99,1.90,2.50$, and 3.25 microseconds (top to bottom) after detonation. The detonation wave is clearly evident in the radiographs as it propagates from the detonator to the outer surface of the explosive materials. The images were recorded on a phosphor image plate that allows one image per shot. An active camera system has now been installed and used to capture up to six frames in the time of a single high-explosive detonation. Future detector development is expected to provide the ability to take thousands of frames during the explosion to produce a "motion picture" of the event.

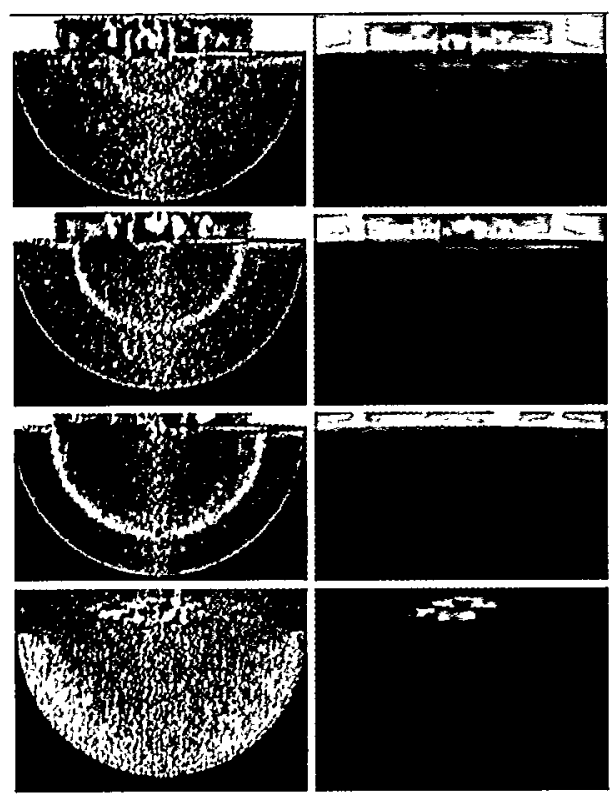

$\Delta$ FIGURE 1. Results from the ondysis of proton rodiographs of the defonation wove in a highexplosive assembly of four different times. Areol densities (L) and reconstructed volume densities (R), extracted under the assumption of axial symmetry, are shown.

Figure 2 shows a schematic of the Line B area used for the FY97 shots as well as an upgraded facility currently being developed in Line $C$. This new Line $C$ facility will have a three-lens system, permitting beam, density, and material identification measurements. It also will be capable of handling larger explosive charges, due to a larger containment vessel. This new facility should be ready for first beam in June 1998.

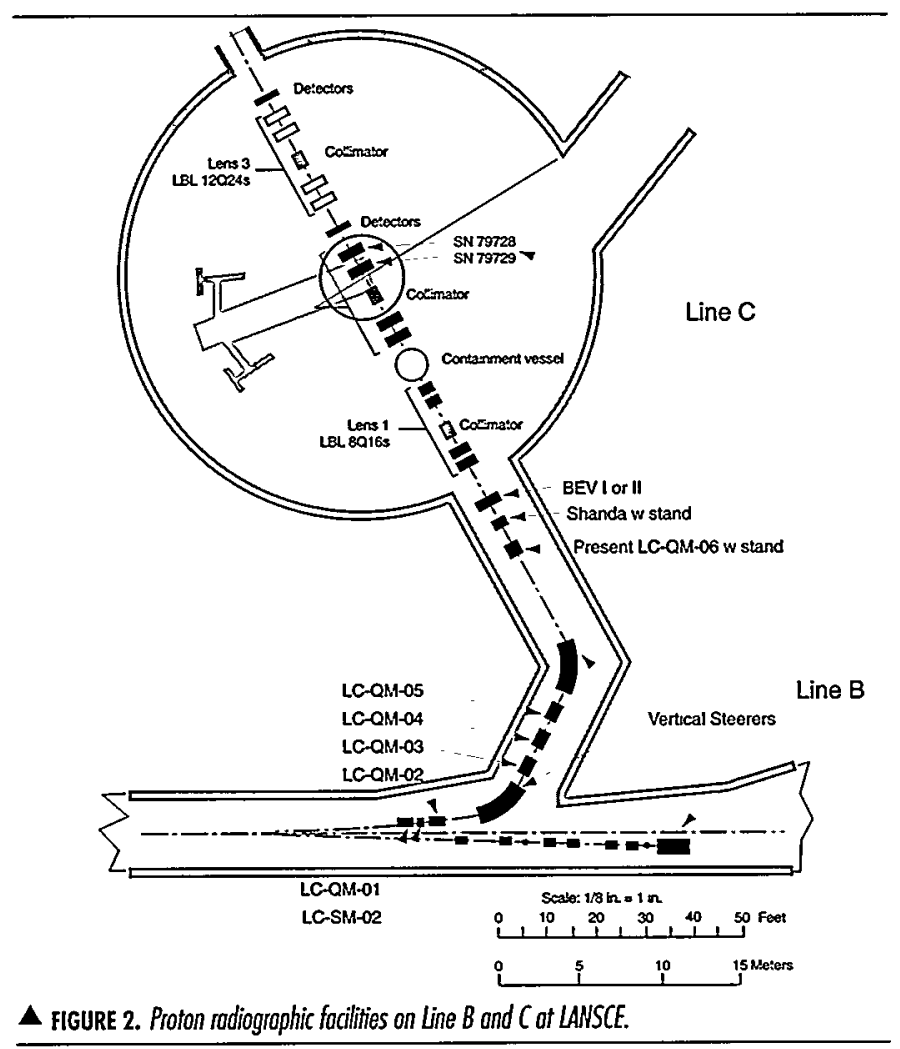

$\triangle$ fIGURE 2. Proton rodiogrophic facilities on Line $B$ and $C$ at LANSCE. 



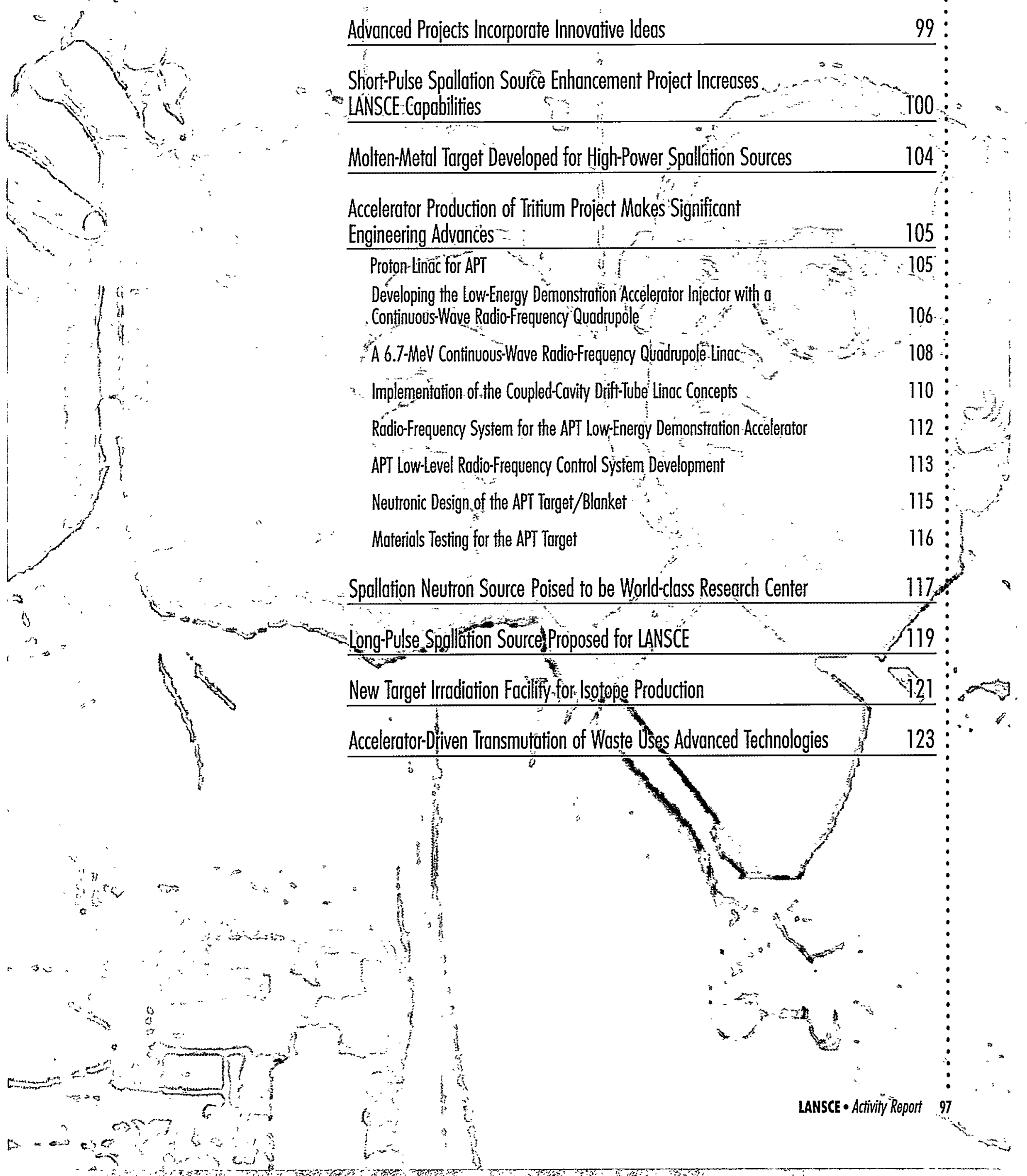


$\ldots$ 


\section{Advanced Projects Incorporate Innovative Ideas}

LANSCE has a number of ongoing projects that are important to future defense and civilian activities. One of these, the Short-Pulse Spallation Source (SPSS) Enhancement Project, jointly funded by DOE Offices of Defense Programs and Basic Energy Sciences, will increase the peak neutron flux available at the Lujan Center to a level that exceeds the level currently available worldwide. In addition, this project will fund the construction of five new neutron scattering spectrometers over the next 3 years. These upgrades will provide the U.S. defense and civilian research communities with neutron scattering capabilities that can compete with any facility in the world.

The spectrometer development project at the Lujan Center uses a model that has not been tried before by the U.S. neutron scattering community. Proposals for new spectrometers were solicited from the scientific community, and an external panel of experts was convened to determine which spectrometers would best serve the needs of defense, civilian, and industrial scientists. The teams that are building the spectrometers will be able to use $35 \%$ or more of the available beam time during the first 3 years after construction, as recompense for their efforts in designing and building the spectrometers. The remainder of the time will be distributed to general users on the basis of proposals that are peer reviewed by the LANSCE Program Advisory Committee.

Although the SPSS Enhancement Project will place the Lujan Center in a leadership position for several years, part of the reason for this upgrade is to allow the community to prepare for neutron scattering research at the Oak Ridge Spallation Neutron Source (SNS) when it comes on-line in 2005. In addition to this involvement with the SNS, Los Alamos is one of the five DOE laboratories that will collaborate to build that facility. LANSCE, with a great deal of help from the Engineering Sciences and Applications (ESA) Division and an industrial-support contractor yet to be chosen, will design and build the linac and the overall control system for the SNS.

The linac design activity for the SNS builds on the experience that LANSCE has had over the past 4 years helping to design a much more powerful continuous-wave, superconducting linac for the Accelerator Production of Tritium (APT) project. Both the SNS and APT projects exercise and build the Division's core competency in the design and construction of high-intensity accelerators. In addition, these projects will allow the Laboratory to attract key individuals with expertise in this area.

Although the SNS will be similar to the Lujan Center in that it will be a short-pulsed source, the technology that is being developed for the APT allows one to consider an even more powerful source of neutrons for scattering experiments-a long-pulsed spallation source (LPSS) with an average power of 5-10 MW and a duty factor of 5\% to 10\%. As Ferenc Mezei, currently the Los Alamos John Wheatley Scholar, showed several years ago, such a source would have a capability more than 25 times that of the Institut Laue-Langevin for cold-neutron research.

To fully exploit an LPSS will require the use of a liquidmetal spallation target because it would be impossible to cool a compact, solid target subjected to a proton beam power of more than $5 \mathrm{MW}$. Similar liquid spallation targets will also be required by the Accelerator-Driven Transmutation of Waste (ATW) project. As a step toward developing this technology, LANSCE has been working with Russian scientists from Obninsk who are designing and building a liquid lead-bismuth eutectic target that will be placed in the LANSCE beam early in the next century. The technology for this target is based on that used by the former Soviet Union for cooling nuclear reactors in its submarines.

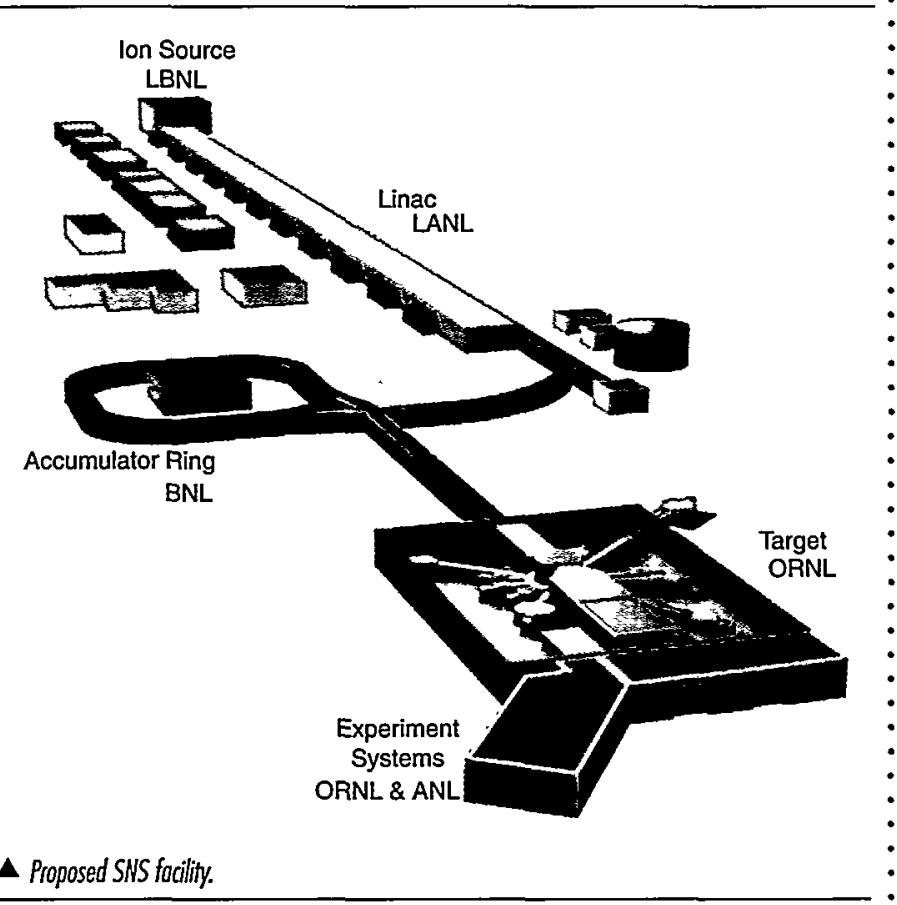

LANSCE - Activity Report 


\section{Short-Pulse Spallation Source Enhancement Project Increases LANSCE Capabilities}

The goal of the Short-Pulse Spallation Source (SPSS)

Enhancement Project is to significantly upgrade LANSCE capabilities by increasing the neutron source intensity and by constructing additional neutron scattering spectrometers. Because the enhanced LANSCE SPSS facility will support both the defense and basic research communities, this project is jointly funded by the DOE Offices of Defense Programs and Basic Energy Sciences. The Office of Defense Programs is supporting the accelerator improvements, which will allow an increase in the proton beam power delivered to the Lujan Center target to $160 \mathrm{~kW}$. The Office of Basic Energy Sciences (OBES) is supporting the design, development, and fabrication of a new suite of neutron scattering spectrometers. Figure 1 shows the locations of the components of this project.

\section{SPSS Accelerator Enhancements}

The accelerator enhancement portion of the project is an upgrade to existing LANSCE systems and components. The overall performance goals of the accelerator upgrade are

- to increase the average proton current delivered to the neutron spallation target to $200 \mu \mathrm{A}$ at a $30-\mathrm{Hz}$ repetition rate, in order to provide $160 \mathrm{~kW}$ of power to the target

- to maintain beam losses in the Proton Storage Ring (PSR) at levels less than or equal to $0.3 \mu \mathrm{A}$ in order to permit "hands-on" maintenance

- to maintain beam reliability and availability at greater than $85 \%$

To achieve these performance goals, three major modifications to the accelerator facilities are being carried out:

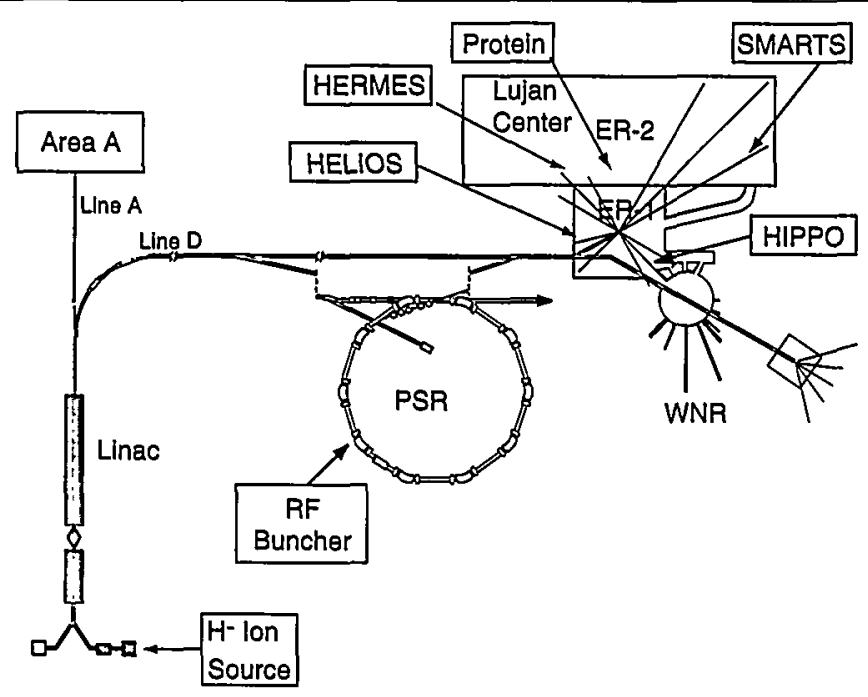

FIGURE 1. Locations of the major elements of the SPSS Enhancement Project.
- A refurbishment of the existing radio-frequency (rf) buncher in the PSR to increase the rf voltage from 12 to $18 \mathrm{kV}$ and a utilities upgrade to increase the capacities of the electrical and cooling water systems. These upgrades began in 1997 and were completed in 1998.

- The construction of a second if buncher in the PSR to add a $12-\mathrm{kV}$ second harmonic to the rf waveform. Work on this upgrade will begin in late 1998 and be completed in late 2000 .

- The development of a new $\mathrm{H}^{-}$ion source and redesigned injector for the accelerator to increase the peak intensity of the injected beam from 16 to $40 \mathrm{~mA}$. This upgrade began in 1997 and will be completed in early 2000 .

The objective of the buncher refurbishment was to increase the peak rf voltage and to eliminate rf instabilities in the existing system. Major elements of the buncher refurbishment were upgrades to the if amplifiers, including the development of a new intermediate power amplifier (IPA); relocation of the IPA from the ring equipment building to the PSR; modification of the buncher gap, ferrite assembly, and bias circuit; and upgrades to the electrical and coolingwater utilities. The refurbished buncher was installed in July 1998 and commissioning was completed in September. The system has met or exceeded all of its design goals and is now $100 \%$ operational. In particular, testing has shown that the buncher can reliably provide the required $18 \mathrm{kV}$ at $2.8 \mathrm{MHz}$; the effective output impedance is very low, resulting in minimal beam loading; and the if instabilities have been eliminated.

\section{SPSS Project - Key Participants}

$\begin{array}{ll}\text { Project Office } & \\ \text { Paul Lewis } & \text { SPSS Project Leader } \\ \text { Michael Klein } & \text { Project Controls }\end{array}$

LANSCE-DO FE-6/Fluor-Daniel

\section{Accelerator Enhancement}

Robert Macek

John Lyles

Benjamin Prichard

Technical Design Authority Buncher Engineering Task Leader Ion Source Tosk Leader \& Uitilities Upgrade Task Leader

Michael Plum Risk Reduction Tosk Leader \& Physics Integration Task Leader

LANSCEDDO

LANSCE-5 LANSCE-DO/SAIC

LANSCE-2

Spectrometer Development

$\begin{array}{lll}\text { Geoff Greene } & \text { Spectrometer Dev. Project Leader } & \text { LANSCE-DO } \\ \text { Jon Kapustinsky } & \text { Spectrometer Dev. Project Leader } & \text { LANSCE-DO } \\ \text { Benno Schoenborn } & \text { Protein Principal Investigator } & \text { LS.DO } \\ \text { Rudy Wenk } & \text { HIPPO Principal Investigator } & \text { UC Berkeley } \\ \text { Mark Bourke } & \text { SMMARTS Principal Investigator } & \text { MST.8/LANSCE-12 }\end{array}$




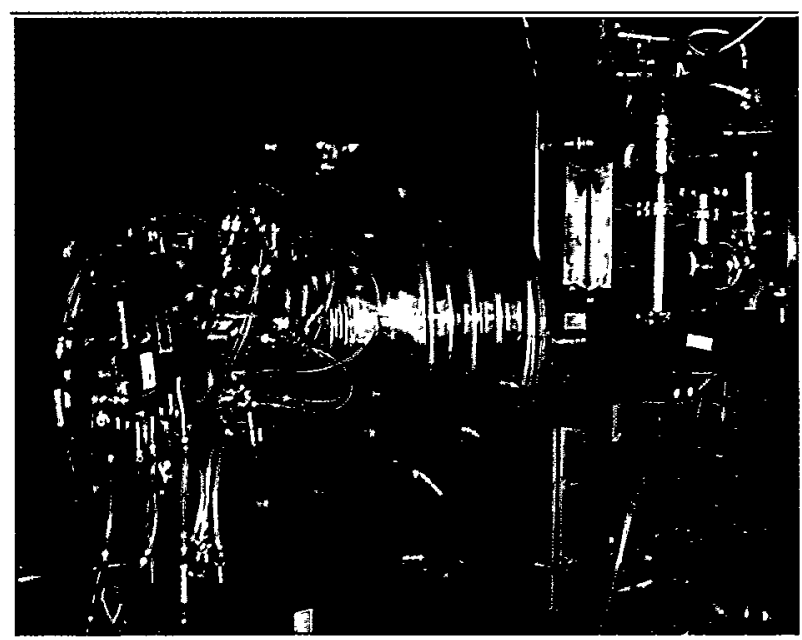

A FIGURE 2. The first prototype ion source; the $80 \mathrm{kV}$ column is mounted on the ion source test stand.

Operation of the PSR at $200 \mu \mathrm{A}$ will require a two-harmonic If bunching waveform, with up to $18 \mathrm{kV}$ of first harmonic $(2.8 \mathrm{MHz})$ and up to $12 \mathrm{kV}$ of second harmonic $(5.6 \mathrm{MHz})$. The second harmonic provided by the second buncher will improve the bunching factor (ratio of average to peak current) of the circulating beam from 0.35 to 0.50 and permit higher average current without a corresponding increase in peak current.

The ion source and injector upgrade is a collaborative effort with Lawrence Berkeley National Laboratory (LBNL). LBNL is designing and fabricating the new source, and LANL is testing the source and redesigning the injector and support equipment. An ion source test stand has been built at LANL to allow thorough testing and characterization of the new source and injector modifications before they are installed on the accelerator. As of October 1998, two prototype sources have been built (Figure 2) and successfully tested at $40 \mathrm{~mA}$, and construction of the final production source will be completed before the end of the year. The $80-\mathrm{kV}$ column in the injector has been redesigned and is being fabricated. Redesign of the remainder of the injector will be completed in early 1999 .

\section{Spectrometer Development}

The spectrometer development portion of the project will add neutron scattering instruments to the Manuel Lujan Jr. Neutron Scattering Center (Lujan Center) at LANSCE. The individual instruments will be designed and constructed by collaborative Spectrometer Development Teams (SDTs). One of the instruments is a structural biology spectrometer funded by the DOE Office of Biological and Environmental Research (OBER); OBES is funding the remaining four instruments.
Proposal Evaluation Committee Membership

John Carpenter

Anthony Cheetham

Patrick Gallogher

Tonya Kuhl

Terry Lowe

Thom Mason

Herbert Mook

Cevdet Noyan

Lourence Passell (retired)

George Samara, Chair

Gopal Shenoy

Greg Smith, Executive Secretary

W. Gavin Williams
Argonne National Laboratory

University of Californio at Santa Barbara

National Inst. of Standards and Technology

University of Californio ot Santa Barbara

Los Alamos National Laboratory

Oak Ridge National Laboratory

Oak Ridge National Laboratory

International Business Machines

Brookhaven National Laboratory

Sandia Nationol Laboratories

Argonne National Laboratory

Los Alamos Notional Laboratory

Rutherford-Appleton Laboratory, ISIS
A key initial goal of the project was to select a suite of OBES spectrometers that will maximize the overall scientific benefit to the U.S. neutron scattering community. As a result, competitive selection of the instruments is an integral part of the project. During 1997, members of the neutron scattering community were invited to organize SDTs and submit letters of intent proposing the construction of specific instruments. Nineteen letters were submitted to LANSCE from a broad spectrum of the community, including academia, industry, national laboratories, and foreign institutions.

To assist LANSCE in the evaluation of the proposed instruments, an external Proposal Evaluation Committee (PEC) was established to represent the broad neutron scattering community, including both defense and civilian research interests. After reviewing the letters, the PEC recommended that nine SDTs submit full proposals. Following extensive review of the submitted proposals, in October 1997 and March 1998, the PEC recommended that four instruments be included in the OBES spectrometer suite:

- Spectrometer for Materials Research at Temperature and Stress (SMARTS)

- High-Pressure and Preferred Orientation (HIPPO) spectrometer

- High-Intensity Chopper Spectrometer (HELIOS)

- High-Resolution Crystal Backscattering Instrument (HERMES)

In addition to the OBES instruments, the project also includes the OBER-funded protein crystallography spectrometer.

As of October 1998, HIPPO, SMARTS, and protein crystallography have been formally incorporated into the project through memoranda of understanding between LANSCE and the individual SDTs. Work has begun on all 
Spectrometer Development Team Memberships

\begin{tabular}{|c|c|}
\hline HIPPO & \\
\hline Rudy Wenk, Principal Investigator & University of Colifornia af Berkeley \\
\hline Bob Von Dreele & Los Alamos Neutron Science Center \\
\hline Kristin Bennett & Los Alamos Neutron Science Center \\
\hline Poul Alivisotos & University of Colifornia of Berkeley \\
\hline Alon Ardell & University of Collfornia of Los Angeles \\
\hline Bob Asaro & University of California of San Diego \\
\hline David Belanger & University of Colifornia at Santa Cruz \\
\hline George Brown & University of California at Santa Cruz \\
\hline Tony Cheetham & University of California at Santa Barbara \\
\hline David Clarke & University of Californio of Santa Barbara \\
\hline Ray Jeanloz & University of California ot Berkeley \\
\hline George Johnson & University of Californio at Berkeley \\
\hline Alex Zettl & University of California of Berkeley \\
\hline David Londono & Ook Ridge National Laboratory \\
\hline B. K. Annis & Oak Ridge National Laboratory \\
\hline Mike Entough & Sandia National Laboratories \\
\hline Bruno Morosin & Sandia National Loboratories \\
\hline Mark Rodriguez & Sandia National Laboratories \\
\hline Ralph Tissot & Sandia National Laboratories \\
\hline Choong-Shik Yoo & Lawrence Livermore National Laboratory \\
\hline John Bingert & Los Alamos National Laboratory-MST \\
\hline Rusty Gray & Los Alamos National Laboratory-MST \\
\hline Yusheng Zhroo & Los Alamos Neutron Science Center \\
\hline SMARTS & \\
\hline Mark Bourke, Principal Investigator & \\
\hline and Spokesperson & Los Alamos National Laboratory-MST \\
\hline Ersan Üstündag, Principal Investigator & Californio Institute of Technology \\
\hline David Dunand, Principal Investigator & Northwestern University \\
\hline Bob Asaro & University of California at San Diego \\
\hline Bimal Kad & University of Californic at San Diego \\
\hline Aaron Krawizz & University of Missouri \\
\hline Phillip Nosh & Illinois Institute of Technology \\
\hline Andy Winholtz & University of Missouri \\
\hline Tom Holden & Atomic Energy of Canada Limited \\
\hline Cev Noyan & IBM \\
\hline Ken Wright & General Electric \\
\hline
\end{tabular}

SMARTS, continued

Jim Richardson

Bjorn Clausen

Mark Daymond

Luke Daemen

Ray Green

Mike Prime

Partho Rongoswamy

Ravi Vormo

HELIOS

Collin Broholm, Spokesperson

Gabe Aeppli

Brent Fultz

Bernhard Keimer

Sanford Kern

Thom Mason

Herb Mook

Stephen Nagler

Ray Osborn

Robert Robinson

HERMES

John Larese, Principal Investigator Brookhaven National Laboratory

Torben Brun (retired)

Juergen Eckert

Luke Daemen

Colin Carlile

Jack Carpenter

Frans Trouw

Anthony Cheetham

Henry Glyde

Larry Passell

K. MeCall

M. Popovici

Paul Sokol

H. Taub

0 . Vilches
Argonne Nationol Laboratory, IPNS Los Alamos Neutron Science Center Rutherford-Appleton Laboratory, ISIS Los Alamos Neutron Science Center Los Alamos National Laboratory-ESA Los Alamos National Laboratory-ESA Los Alamos National Laboratory-MST Los Alamos National Laboratory-MST

\author{
Johns Hopkins University \\ NEC Research \\ California Institute of Technology \\ Princeton University \\ Colorado State University \\ Ook Ridge National Loboratory \\ Oak Ridge National Laboratory \\ Oak Ridge Notionol Laboratory \\ Argonne National Laboratory \\ Los Alamos Neutron Science Center
}

Los Alamos Neutron Science Center

Los Alamos Neutron Science Center

Los Alamos Neutron Science Center

Rutherford-Appleton Laboratory, ISIS

Argonne National Laboratory

Argonne National Laboratory

University of California at Santa Barbara

University of Delaware

Brookhaven National Laboratory

University of Nevada, Reno

MURR

Pennsylvania State University

University of Missouri

University of Woshington three instruments. LANSCE is still negotiating with the HELIOS and HERMES SDTs. Work on HELIOS and HERMES is expected to begin in 1999.

SMARTS is a new neutron powder diffractometer instrument (Figure 3) that is optimized for strain measurements and engineering studies. SMARTS will use flight path 2 at the Lujan Center and will be located on the end of a neutron guide in Experimental Room (ER) 2. The use of the neutron guide and an optimized detector geometry will allow SMARTS to have a 30 times increase in count rate for comparable measurements on NPD, which is the current venue for strain measurements at LANSCE. SMARTS will also have a extensive array of in situ capabilities for sample environments, the ability to make measurements on both small and very large samples, and easy access to and control of the sample position. Included in the SMARTS baseline design is a flexible high-temperature and high-stress furnace/load frame, which will conveniently allow a wide variety of studies of materials in extreme environments.

Like SMARTS, HIPPO is a new powder diffraction instrument (Figure 4). However, HIPPO is optimized for the very highest intensity and will be devoted primarily for studies 


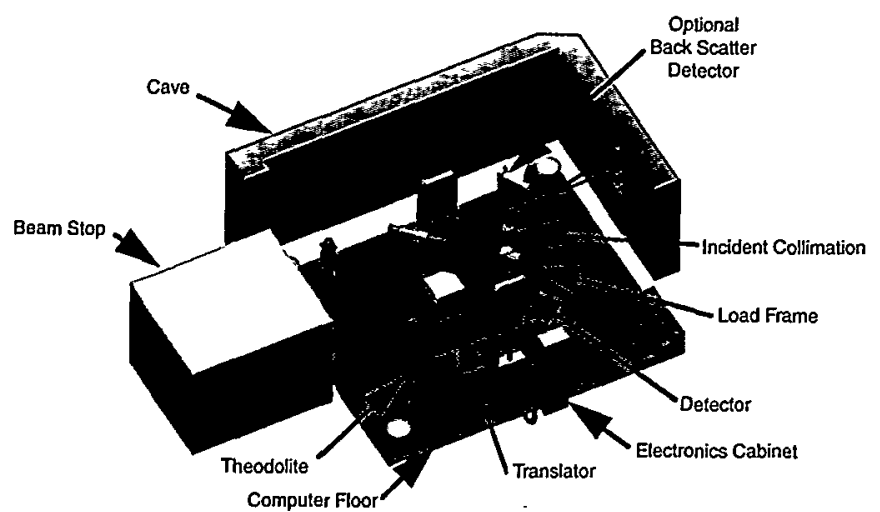

A FIGURE 3. SMARTS vill enable scientists to observe a stronger neutron signal.

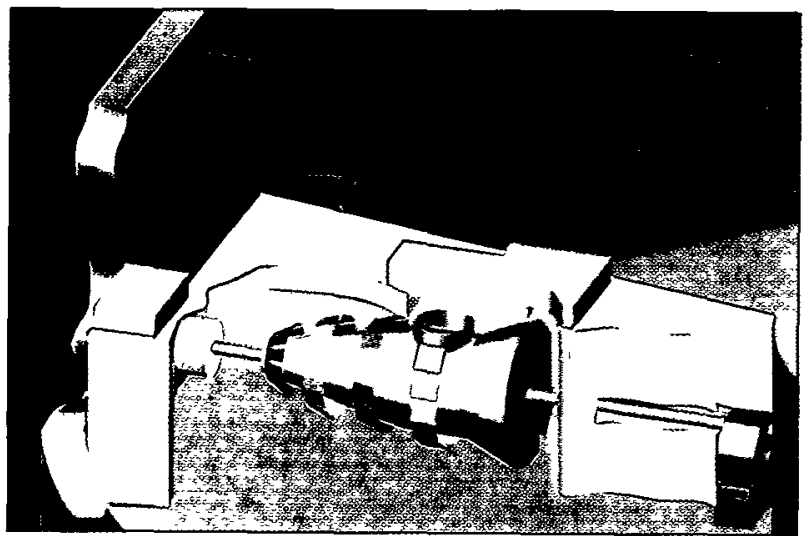

$\triangle$ FIGURE 4. Conceptual design of the HIPPO instrument.

involving preferred orientation, or texture. HIPPO will be located in ER-1 on flight path 4. An increased number of detectors, covering a very large solid angle (including backscattering detectors) will provide an estimated 20 times increase in detection efficiency compared with HIPD, the Lujan Center's current high intensity powder diffractometer. When this improved efficiency is combined with the increased intensity of the upgraded Lujan Center target, an overall increase in experimental capability of 60 times over HIPD is expected. HIPPO will also include an improved sample environment and an order of magnitude increase in sample throughput through the use of a high-capacity automated sample changer. HIPPO has benefited from a very strong commitment by the University of California (UC), which includes not only the involvement of multiple UC campuses but also significant investment of UC funds to help defray some of the costs of building this spectrometer.

The new protein crystallography spectrometer is a singlecrystal diffractometer designed for structure determinations of large biological molecules. This instrument will be the first dedicated instrument to exploit a spallation neutron source for the determination of the structures of important biological molecules. This spectrometer will be installed in ER-2 and will use flight path 15.
The HELIOS spectrometer is a high-intensity, direct-geometry, time-of-flight spectrometer, which will be located in ER 1. HELIOS will be optimized to provide the highest possible neutron flux at the sample, high detection efficiency, and sufficient energy resolution to study dynamical processes in a wide variety of materials. The spectrometer will use the full-energy spectrum of neutrons provided by the water moderator that serves flight path 8 . It is anticipated that HELIOS will be effective for studies of excitations from a few $\mathrm{meV}$ to several hundred $\mathrm{meV}$. The HELIOS SDT has strong participation and support from the Spallation Neutron Source project at Oak Ridge National Laboratory.

The HERMES inelastic spectrometer is a high-resolution crystal backscattering instrument, which will be located in ER 2. HERMES will "view" a liquid hydrogen moderator (flight path 11) and will use a neutron guide to maximize intensity. HERMES is expected to provide an important tool for a broadly based scientific program that will include investigations of topics in biology, chemistry, geology, materials science, and physics. The HERMES SDT is lead by Brookhaven National Laboratory.

-Paul Lewis (LANSCE Division) 


\section{Molten-Metal Target Developed for High-Power Spallation Sources}

The development of compact, high-power neutron production targets is crucial for the implementation of the next generation of spallation neutron sources. In these neutron sources, neutrons are produced via spallation reactions in the target material following high-energy proton bombardment. Typically, tens of neutrons are produced per incident proton depending on the energy of the incident proton beam.

Accelerators of several megawatts are presently contemplated for neutron production sources such as the Oak Ridge Spallation Neutron Source (SNS) and the European Spallation Source (ESS). These sources are planned to have an ultimate power level of $5 \mathrm{MW}$. Currently, only solid neutron production targets are in operation. It is generally believed that solid targets will function up to approximately $1 \mathrm{MW}$ of beam power. Above that power level, the ratio of solid material to cooling fluid decreases to the point where solid targets lose neutron production efficiency. Flowing molten-metal targets of high- $\mathrm{Z}$ material provide a solution to this problem by eliminating the thermal conduction path that exists with solid targets. The high- $Z$ and density of these fluids result in high neutron production efficiency. In molten-metal targets, heat is removed by circulating the fluid from the irradiation region through heat exchangers.

Mercury has been chosen as the neutron production fluid for the SNS because of its high density, high atomic number, and low melting point. Unfortunately, mercury has a large thermal absorption cross section $(\sim 370 \mathrm{~b})$ and low boiling point $\left(357^{\circ} \mathrm{C}\right)$. Lead-bismuth eutectic (LBE) is another fluid that has the advantage of very low absorption cross section $(0.09 \mathrm{~b})$ and a high melting point $\left(1670^{\circ} \mathrm{C}\right)$. It seems that mercury may work in short-pulse sources where its large absorption cross section may not be a serious detriment. For continuous neutron sources or long-pulse sources, LBE seems like a much better choice.

In another application, the current design for the Los Alamos version of an accelerator-driven system to burn radioactive waste uses LBE as a coolant as well as a neutron production fluid. It will be necessary to develop LBE technology so that it can be applied in this situation. As a step toward developing LBE technology, we have designed an LBE test loop. We are developing this loop in collaboration with scientists at the Institute of Physics and Power Engineering (IPPE) in Obninsk, Russia. Scientists from IPPE have considerable experience in LBE because they used LBE for naval submarine reactors.

The test loop shown in Figure 1 will allow us to address important issues in LBE technology. In particular, materials compatibility and corrosion can be studied in both isothermal and non-isothermal regimes.

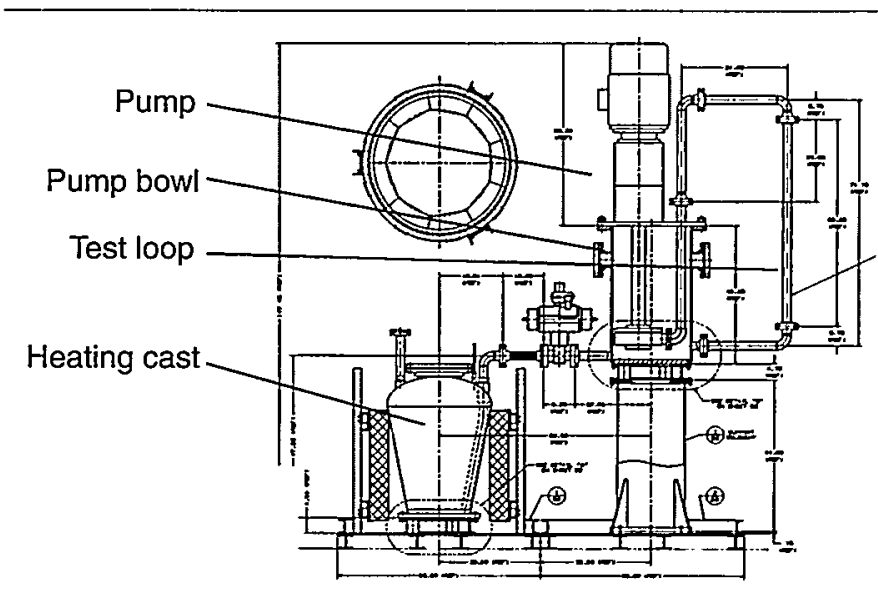

$\triangle$ FIGURE 1. The molten-metal test loop.

- S. A. Wender, F. Venneri (LANSCE Division), $N$. Li (Materials Science and Technology Division), $K$. Woloshun (Engineering Sciences and Applications Division), J. D. King (Chemical Science and Technology Division) 


\section{Accelerator Production of Tritium Project Makes Significant Engineering Advances}

Approximately a decade ago, Los Alamos initiated a study to determine the effectiveness of producing the tritium required for the nation's stockpile based on a high-intensity proton accelerator driving an appropriate spallation target. Initial results of the study showed that this acceleratordriven target method had merit and should be studied in more depth. With more information on aspects of the needed proton injector, linear accelerator (linac), radio-frequency (rf) system, controls, diagnostics, spallation target, tritium extraction, and materials, it became apparent that the accelerator-based method was competitive with standard reactor methods for producing tritium. In fact, the accelerator-based method had a few advantages in terms of not producing fission products and being able to shut down quickly. These studies were completed in collaboration with other national laboratories, following agreements to work together. At the time, these organizations included Brookhaven and Hanford national laboratories.

As time passed, more investments were made in the spallation target, neutronics, and accelerator development. For the development of the accelerator, we acquired a continuous-wave (CW) injector and if quadrupole (RFQ) from Chalk River Laboratories (CRL) when their high-intensity proton accelerator development program was canceled. This device, known as the Chalk River Injector Test Stand (CRITS), provided us with a front-end demonstrator that led to invaluable experience with $\mathrm{CW}$ proton beams of the type required for an Accelerator Production of Tritium (APT) system. At the same time, the capabilities and tools developed during the strategic defense initiative studies of neutral particle beam systems allowed major advances in linear accelerator design and fabrication.

With Department of Energy (DOE) support, we initiated an APT program for investigating the production of tritium from an intense proton accelerator $(100 \mathrm{MW}$ of $\mathrm{CW}$ proton beam) in 1992. This concept uses a high-power proton linear accelerator to drive a tungsten spallation target, from which copious neutrons are produced that interact with ${ }^{3} \mathrm{He}$ to produce the required tritium. We are investigating all aspects of the system, including siting and operations of a production facility at the Savannah River Site (SRS). Los Alamos is responsible for the national program with $\mathrm{DOE}$, and LANSCE participates in the accelerator and target developments. The APT project integrates national laboratories (Los Alamos, Livermore, Savannah River, Brookhaven, Thomas Jefferson, and Sandia) and industrial firms (Burns and Roe, Inc., and General Atomics) with other contractors in a single project office to carry the APT concept from its preliminary conceptual stage through to completion as an operational facility as early as 2008 .
LANSCE provides much of the technical staff, expertise, and facilities to the APT project.

To be prepared for construction of the production facility at the Savannah River site, a number of engineering design and development activities have been underway at Los Alamos, some of them representing plant prototypes. A full-power, low-energy demonstration accelerator (LEDA) is being built and commissioned in stages to provide operational experience, design verification, and assembly checks for the front end of an APT system. LEDA has provided invaluable information as a prototype in design, construction, assembly, alignment, and low-power measurements. It will continue to provide needed information as more components are installed and as the system begins high-power commissioning and operations. LEDA has already demonstrated an injector with beam performance exceeding the $100-\mathrm{mA} \mathrm{CW}$ requirement for the APT accelerator. LEDA will demonstrate operations with an injector, RFQ to $6.7 \mathrm{MeV}$, and a new structure, the coupled-cavity drift-tube linac (CCDTL), to $12 \mathrm{MeV}$ initially. Later upgrades will take the output energy to $20 \mathrm{MeV}$ and then to $40 \mathrm{MeV}$, if funding is available for these upgrades.

The following sections provide information on advances and developments by LANSCE staff in the areas of the accelerator design, injector, RFQ, CCDTL, if systems, low-level if control, neutronic design, and materials testing.

\section{Proton Linac for APT}

The proton linac designed for the APT project ${ }^{1}$ is unique in two respects. First, APT will extend the frontier of average beam intensity by a factor of 100 and will deliver the world's most powerful beam (170 MW) to a target for tritium production. Second, APT will become the world's first superconducting proton linac. This section describes the basic features of the design.

The APT accelerator will consist of a superconducting (SC) high-velocity proton linac with a $1.7-\mathrm{GeV}$ final energy, which accepts an input beam from a normal-conducting (NC) low-velocity linac at $211 \mathrm{MeV}$. Both linacs will accelerate a $100-\mathrm{mA}$ beam using a continuous rf wave to produce a final beam power of $170 \mathrm{MW}$ that is delivered to the tritium production target. The SC linac design provides significant power savings and lower operating and capital costs. It allows a much larger aperture at high energies and permits greater operational flexibility. The design has been approved by high-level technical panels and is published in a conceptual design report. ${ }^{2}$ The SC linac employs elliptical-type niobium cavities, while the NC linac is constructed from copper cavities. The overall result is an accelerator 
design that makes optimum use of the two technologies in their appropriate regions of application.

The system architecture is displayed in Figure 1. A 75-keV injector housing a microwave-driven ion source generates a continuous $110-\mathrm{mA}$ proton beam. From this input, a 350-MHz, 8-m-long RFQ linac produces a 100-mA beam at $7 \mathrm{MeV}$. The output beam is matched into a $700-\mathrm{MHz}$ CCDTL that accelerates it to $100 \mathrm{MeV}$. The CCDTL is a coupled sequence of 2-gap and 3-gap short drift-tube linacs (DTLs), embedded in a singlet focusing lattice in which quadrupole magnets with alternating polarity are external to the accelerating structure with a spacing that increases slowly in proportion to the beam velocity. Acceleration continues to $211 \mathrm{MeV}$ in a $700-\mathrm{MHz}$ side-coupled linac, similar to the LANSCE linac.

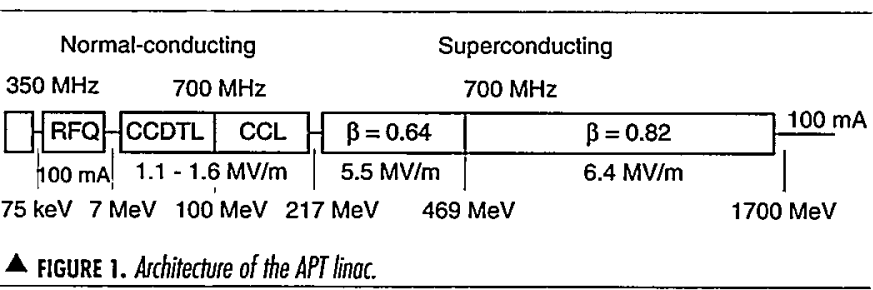

The SC high-energy linac consists of a string of cryomodules maintained at an operating temperature of $2 \mathrm{~K}$ by a liquid helium refrigerator, and each contains two, three, or four 5-cell $700-\mathrm{MHz}$ niobium accelerating cavities. There are two kinds of SC cavities, each type designed for efficient acceleration in a different velocity range. Cavity-cell shapes in the medium-beta section are optimized at velocity $\beta=0.64$ times the speed of light, and in the high-beta section at $\beta=0.82$ times the speed of light. The cell shapes are similar to the well-established designs for electron linacs and storage rings but are compressed longitudinally in proportion to beta. Because the cavities are relatively short and are driven independently, each section of the SC linac has a broad velocity bandwidth, which allows the cavities to accelerate the beam over a wide range of velocities. Each cavity is supplied rf power by two antenna-type coaxial couplers mounted on opposite sides of the beam tube. Figure 2 shows a single period of the $\beta=0.82$ section. Each cryomodule contains four 5-cell cavities, with a single klystron driving each cavity pair.

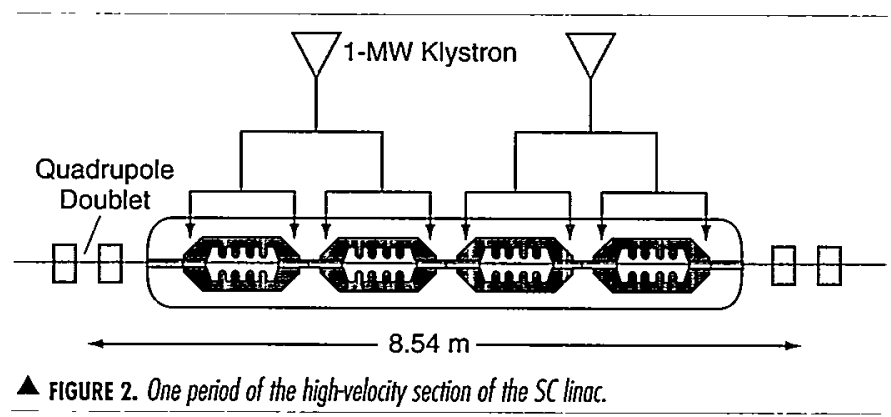

Transverse focusing is provided by conventional quadrupole doublets located in the warm regions between cryomodules. Figure 3 shows a drawing of the $\beta=0.64$ prototype cavity assembly.

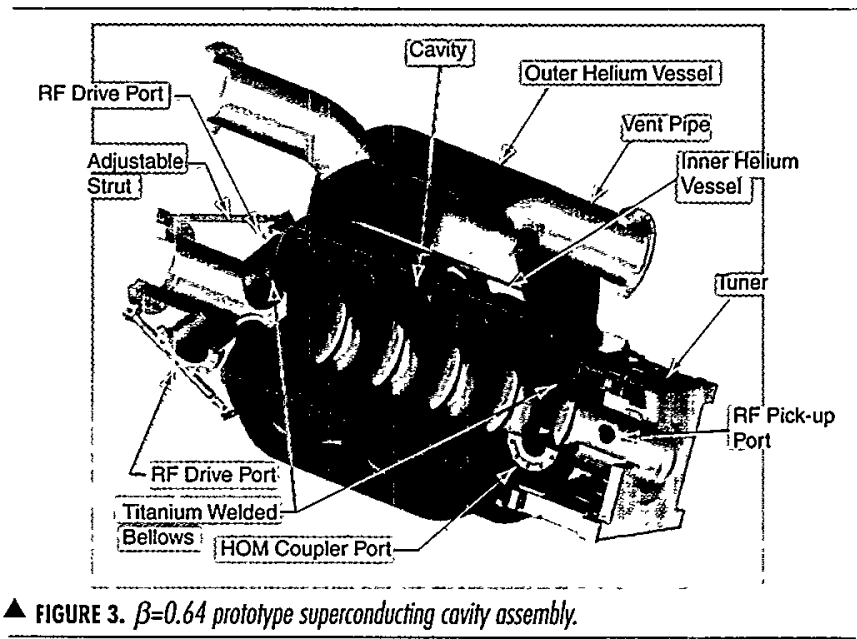

The main beam-dynamics goal for the accelerator is to achieve very low beam losses in order to assure unrestricted hands-on maintenance. The linac design provides apertures that are much larger than the rms beam size, with the largest apertures at high energies where the activation per lost proton is greatest. The SC linac is expected to be insensitive to a broad range of construction or operating errors. Operational flexibility is enhanced by the retunability of the high-beta section of the SC linac and the adjustability of the cavity fields. The APT linac design will also serve as a starting point for important future applications such as accelerator-driven nuclear waste transmutation and accelerator-driven fission reactors.

\section{References}

1. P. W. Lisowski, "The Accelerator Production of Tritium (APT) Project," 1997 Particle Accelerator Conference, Vancouver, Canada (May 1997).

2. APT Conceptual Design Report, Los Alamos National Laboratory report LA-UR-97-1329, April 15, 1997.

\section{-T. P. Wangler (LANSCE Division)}

\section{Developing the Low-Energy Demonstration Accelerator Injector with a Continuous-Wave Radio-Frequency Quadrupole}

We have developed a versatile and reliable dc proton injector for testing $100-\mathrm{mA} \mathrm{CW}$ linacs. To inject a $1.25-\mathrm{MeV}$ CW RFQ, we configured the 75-keV LEDA proton injector to operate at $50-\mathrm{keV}$ beam energy. We obtained RFQ currents up to $100 \mathrm{~mA}$ and measured beam transmissions of $80 \%$ to $85 \%$ at the $75-\mathrm{mA}$ RFQ design current. Our results 
are in accord with RFQ design codes and provide basic knowledge and experience for testing the $6.7-\mathrm{MeV}, 100-\mathrm{mA}$ LEDA RFQ.

We will use LEDA to demonstrate the technology required for the low-energy end of an APT machine. ${ }^{1}$ The hardware for developing the LEDA injector originated in our collaboration with the CRL in Ontario, Canada, ${ }^{2}$ when a microwave proton source and a $1.25-\mathrm{MeV} C W \mathrm{RFQ}$ were transferred to Los Alamos. Subsequent development of the microwave proton source led to the demonstration of a $75-\mathrm{keV}$ prototype injector with parameters suitable for injecting the 6.7-MeV LEDA $\mathrm{RFQ}^{3}$ (Table 1).

TABLE 1. Demonstrated Beam Porometers for a Prototype Version of the LFDA Injector

\begin{tabular}{|c|c|}
\hline Injector Parameter & Status \\
\hline Proton beam current (mA) & 117 \\
\hline Proton fraction (\%) & 90 \\
\hline Beam energy (keV) & 75 \\
\hline Discharge power (W), frequency (GHz) & 600 to $800,2.45$ \\
\hline Axial magnetic field $(\mathrm{G})$ & 875 to 960 \\
\hline Injector reliability & $96 \%$ to $98 \%$ \\
\hline Duty factor $(\%)$ & $100(\mathrm{dc})$ \\
\hline Gas flow (sccm) & 2 to 5 \\
\hline Emission aperture radius (mm) & 4.3 \\
\hline Extraction gap (mm) & 13.2 \\
\hline Beam noise (\%) & \pm 1 \\
\hline Ion source emittance ( $\pi \mathrm{mm}-\mathrm{mrad})$ & 0.13 (rms, normalized) \\
\hline RFQ match point emittance ( $\pi \mathrm{mm}-\mathrm{mrad})$ & 0.20 (rms, normalized) \\
\hline$\alpha$ RFQ (LEDA RFQ) & 1.944 \\
\hline$\beta R F Q(\mathrm{~mm} / \mathrm{mrad})(\mathrm{LEDA} \mathrm{RFQ})$ & 0.1193 \\
\hline
\end{tabular}

By the fall of 1997, we realized that a version of the LEDA injector incorporating split solenoids, a pair of steering magnets, a dc beam stop, and a suitable array of noninterceptive video diagnostics could be quickly assembled and installed on the $1.25-\mathrm{MeV}$ RFQ (commonly referred to at Los Alamos as CRITS) brought from CRL (Figure 4). This version of the LEDA injector low-energy beam transport (LEBT) system transports the beam $2.5 \mathrm{~m}$ from the ionsource extraction system to the CRITS RFQ match point. This test helped us achieve the following objectives: - This early beam test of the injector design concepts allowed us to get results on a state-of-the-art CW RFQ. The tests included measuring beam-transmission characteristics through the LEBT and beam-matching into the RFQ.

- By the fall of 1997 it was also clear that collaborators from SRS and General Atomics (GA) would be primary participants in the APT accelerator development. By operating the CRITS RFQ on the site, SRS and GA personnel gained experience in operating an integrated

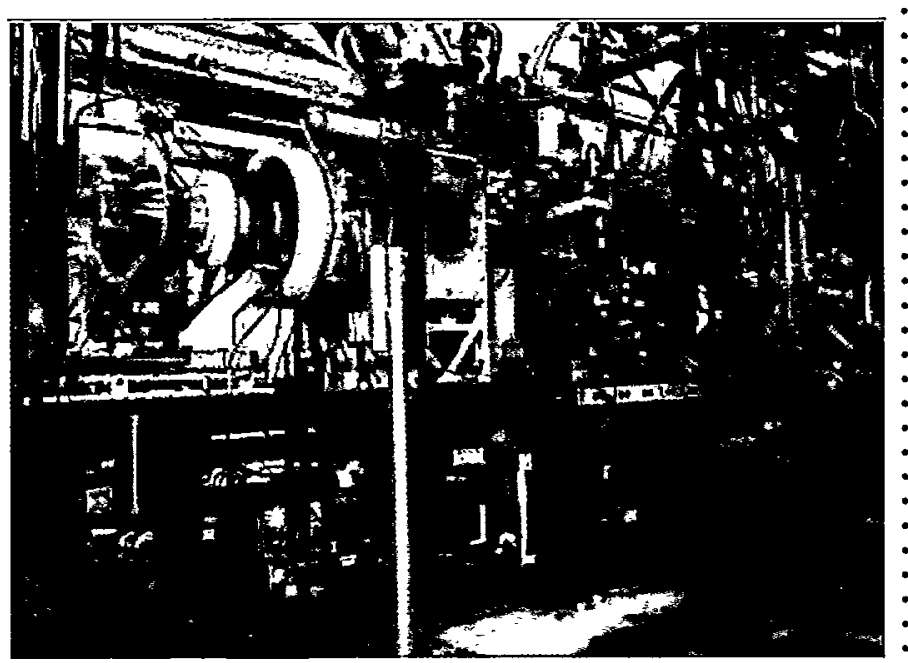

$\triangle$ FIGURE 4. The 50keV version of the LFDA injector installed on the CRITS RFQ.

accelerator. Figure 5 shows the RFQ beam stop fabricated by GA and is an example of the collaborative work. This RFQ beam stop successfully removed $125-\mathrm{kW}$ beam power.

- The CRITS RFQ had a vane replaced in 1991 at CRL $^{4}$ using design codes equivalent to those used for the LEDA RFQ. Beam transmission studies on the CRITS RFQ are thus useful for comparison with codes such as PARMTE QM and RFQTRAK.

The injection energy for the CRITS RFQ is $50 \mathrm{keV}$, and the LEDA injector was adapted to a $50-\mathrm{keV}$ triode extraction system. Details of the injector modifications required for the $50-\mathrm{keV}$ operation will be given in the 1998 Linear

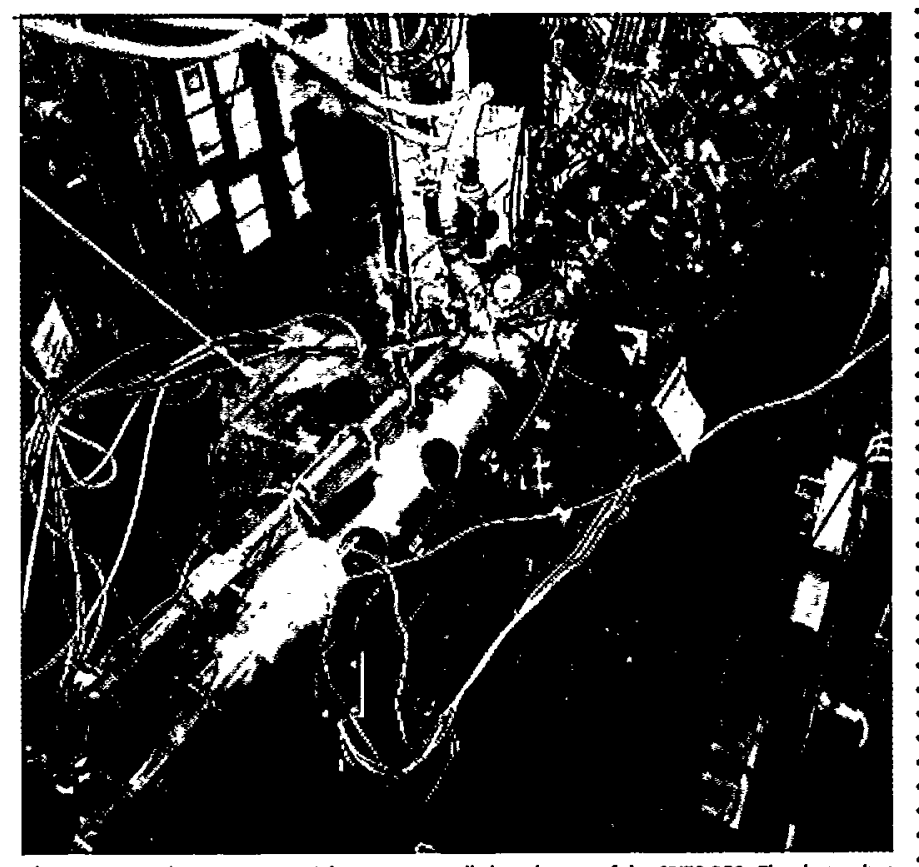

$\triangle$ FIGURE 5. The 1.25-MeV RFQ beam stop installed on the exit of the CRITS RFQ. This device dissi poted up to $125 \mathrm{~kW}$ of beam power, and it olso confirmed the RFQ beom current measurements through calorimetry. 
Accelerator Conference Proceedings. ${ }^{5}$ One interesting result demonstrating the injector-RFQ integrated performance is shown in Figure 6. The contours show the beamcurrent transmission from the injector through the CRITS RFQ as a function of the LEBT solenoid magnet settings. These measurements were performed on several occasions; the beam transmission is $80 \%$ to $85 \%$ at the $75-\mathrm{mA}$ RFQ design current.

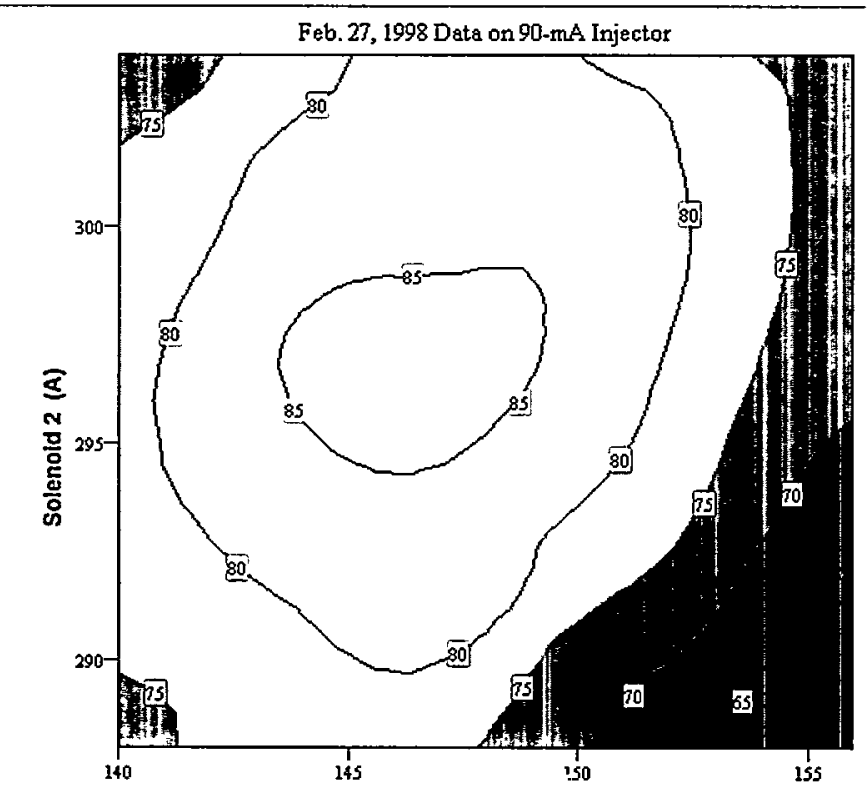

$\triangle$ figuRE 6. Februor 27, 1998, dato on the 90-mA injector. The contour lines represent the CRITS RFQ beam tronsmission in percentage versus LEBT solenoid I and LFBT solenoid 2 currents.

One important injector-RFQ subject is the beam current limit. On one occasion, the injector was tuned for maximum current, and as much rf power as possible was obtained from the 268-MHz klystrode tube. For a few moments, as much as a $100-\mathrm{mA}$ current was accelerated through the RFQ, indicating that the RFQ current limit is at least this magnitude. The measured RFQ beam current was checked against a beam calorimetric measurement made with the beam stop shown in Figure 5. The two measurements agreed to an order of $10 \%$. Thus, we have successfully tested the LEDA injector concepts and obtained useful RFQ results that will help us benchmark design codes. We presented the results in detail at the August 1998 Linear Accelerator Conference.

\section{References}

1. J. D. Schneider, "APT Accelerator Technology," in Proceedings of the XVIII International Linear Accelerator Conference, European Center for Nuclear Research report CERN 96-07 (August 1996), p. 22.

2. J. D. Schneider, G. O. Bolme, V. Brown, M. L. Milder, G. Neuschaefer et al., "Installation of a CW Radio Frequency Quadrupole Accelerator at Los Alamos National Laboratory," in Proceedings of the 1994 International Linac Conference (Tsukuba, Japan, August 1994), p. 149.
3. D. L. Schrage, P. L. Roybal, L. M. Young, W. L. Clark, R. F. Depaula, et al., "A New RFQ Linac Fabrication Technique," in Proceedings of the 1994 International Linac Conference (Tsukuba, Japan, August 1994), p. 152.

4. N. J. Diserens, "Space and Image Charge Calculations for the Chalk River RFQ1-1250 Accelerator Using the RFQTRAK Beam Simulation Program," in Proceedings of the 1992 Linear Accelerator Conference, Atomic Energy of Canada, Ltd., report AECL-10728 (August 1992), p. 214.

5. J. D. Sherman, G. O. Bolme, L. D. Hansborough, D. J. Hodgkins, E. A. Meyer, et al., "Development and Test Results of the LowEnergy Demonstration Accelerator (LEDA) Proton Injector on a 1.25-MeV cw Radio Frequency Quadrupole," Los Alamos National Laboratory report LA-UR-98-1545 (April 1998).

-G. O. Bolme, L. D. Hansborough, T. W. Hardek, D. J. Hodgkins, E. A. Meyer, C. R. Rose, J. D. Schneider, J. D. Sherman, H. V. Smith, Jr., M. W. Stettler, R. R. Stevens, Jr, M. E. Thuot, T. J. Zaugg (LANSCE Division), A. H. Arvin, A. S. Bolt, M. C. Richards (Savannah River Site), J. H. Kamperschroer (General Atomics), P. P. Balleyguier (CEA-Bruyeres le Chatel, France)

\section{A 6.7-MeV Continuous-Wave Radio-Frequency Quadrupole Linac}

A proton linac that will accelerate a $100-\mathrm{mA}$ beam from $75 \mathrm{KeV}$ to $6.7 \mathrm{MeV}$ has been designed and fabricated for the APT project at Los Alamos. This 8-m-long RFQ structure consists of four resonantly coupled segments and is being fabricated using hydrogen furnace brazing as a joining technology.

The linear accelerator for the APT project ${ }^{1}$ will include a 6.7-MeV RFQ linac. The first phase of the project, LEDA, ${ }^{2}$ is to demonstrate performance of the RFQ plus a $\mathrm{CCDTL}^{3}$ to $20 \mathrm{MeV}$. The technical specifications for the APT/LEDA RFQ are given in Table 2. The design and construction of an RFQ to deliver an average proton current of $100 \mathrm{~mA}$ at 6.7 $\mathrm{MeV}$ is a significant challenge for the beam dynamics and thermal management. The cavity cross section is the "conventional" triangular shape with a significant longitudinal variation in the width of the vane skirt. The skirt profile is shown in Figure 7. This profile minimizes the power deposited on the cavity walls.

The 8-m-long structure is designed as four resonantly coupled 2-m-long segments ${ }^{4}$ to ensure longitudinal stabilization. Stabilizer rods $s^{5}$ on the intersegment coupling plates and end walls provide azimuthal stabilization without the scalloping of the on-axis fields associated with vane-coupling rings ${ }^{6}$ or pi-mode stabilizers. ${ }^{7}$

The cavity is fabricated as eight 1-m-long sections each consisting of two major and two minor vanes. The 24 longitudi- 


\begin{tabular}{l|l}
\hline TABLE 2. APT/LEDA RFQ Specificalions \\
\hline Parameter & Value \\
\hline Frequency & $350.00 \mathrm{MHz}$ \\
\hline Particle & $\mathrm{H}^{+}$ \\
\hline Input Energy & $75 \mathrm{keV}$ \\
\hline Input Current & $105 \mathrm{~mA}$ \\
\hline Input Emittance, trans./norm. & $0.020 \pi$-cm-mrad rms \\
\hline Output Energy & $6.7-\mathrm{MeV}$ \\
\hline Output Current & $100 \mathrm{~mA}$ \\
\hline Output Emittance, trans./norm. & $0.022 \pi-\mathrm{cm}-\mathrm{mrad} \mathrm{rms}$ \\
\hline Transmission & 0.174 deg-MeV \\
\hline Duty Factor & $95 \%$ \\
\hline Peak Surface Field & $100 \%$ \\
\hline Average Structure Power & $1.8 \mathrm{Kilpatrick}$ \\
\hline Average Beam Power & $1.2 \mathrm{MW}$ \\
\hline Average Total Power & $0.7 \mathrm{MW}$ \\
\hline RF Feeds & $1.9 \mathrm{MW}$ \\
\hline Average Heat Flux & $12 \mathrm{Waveguide} \mathrm{Irises}$ \\
\hline Maximum Local Heat Flux & $11 \mathrm{~W} / \mathrm{cm}^{2}$ \\
\hline Resonant Segments & $65 \mathrm{~W} / \mathrm{cm}{ }^{2}$ \\
\hline Brazed Sections & 4 @ $2.0 \mathrm{~m}$ each \\
\hline Slug Tuners & $8 @ 1.0 \mathrm{~m}$ each \\
\hline Length & 128 total \\
\hline Weight & $8.0 \mathrm{~m}$ \\
\hline Inlet Coolant Temperature & $5000 \mathrm{lb}$ \\
\hline Operating Temperature & $50^{\circ} \mathrm{F}$ \\
\hline & $85^{\circ} \mathrm{F}$ \\
\hline
\end{tabular}

nal coolant passages in each of the sections remove the 1.2 MW of average structure power. These passages are machined into the oxygen-free-electron-copper substrate and then plugs are brazed on. To provide coolant passages as near as possible to the vane tips, the vane tips are fabricated separately and brazed onto the vane bases. These are the only water-to-vacuum braze joints, and they are a double joint of nearly 1-in. width (Figure 8).

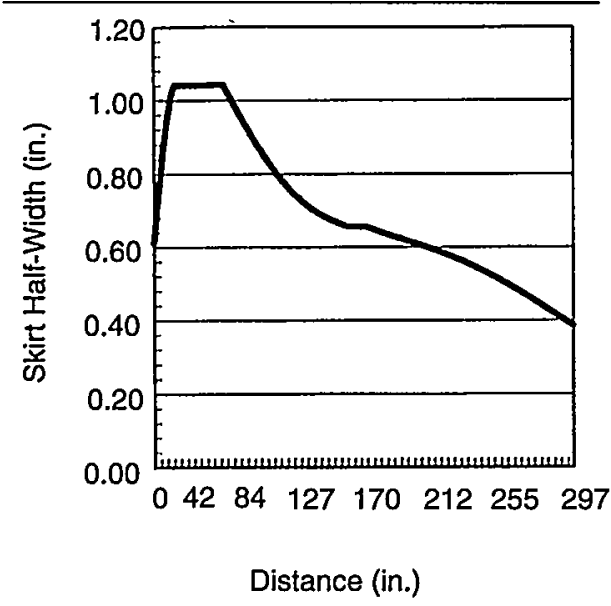

A FIGURE 7. Vane skit width variotion.

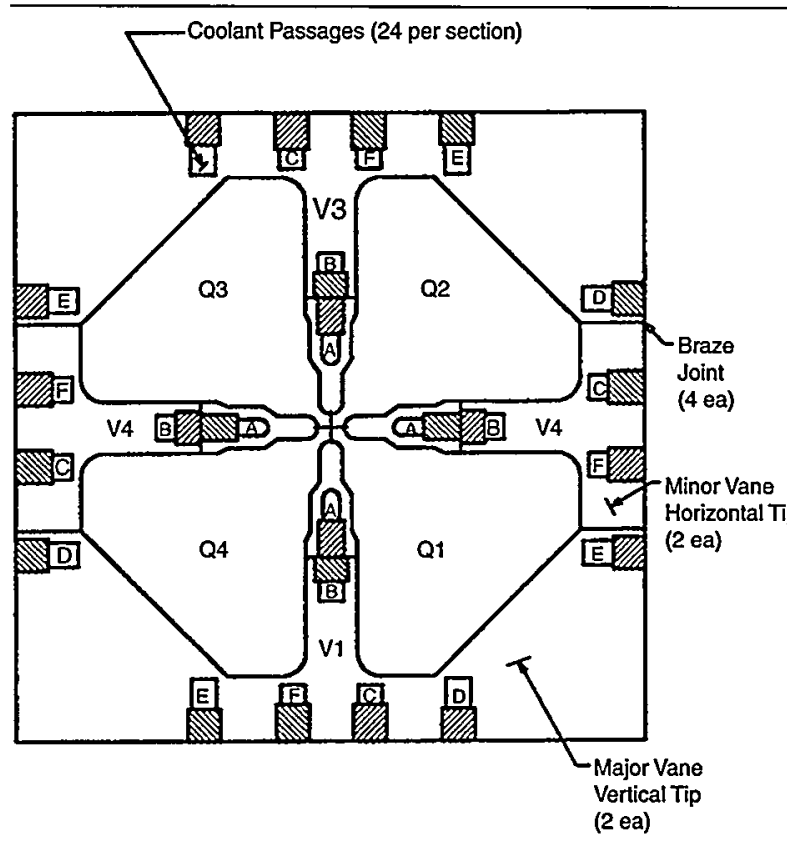

$\triangle$ FIGURE 8. RFQ cross section.

For resonance control, the tip coolant passages ( $\mathrm{A}$ and $\mathrm{B}$ ) are operated with $50^{\circ} \mathrm{F}$ coolant while the temperature of the coolant in the outer passages (C, D, E, and F) is modulated to maintain the cavity on resonance. The rf power in each of the four resonant segments is significantly different $(\mathrm{A}=188 \mathrm{KW}, \mathrm{B}=318 \mathrm{KW}, \mathrm{C}=361 \mathrm{KW}$, and $\mathrm{D}=398 \mathrm{~K}$ ), and the inlet temperature of the coolant is varied accordingly. The error signal for the resonance control system is derived from the reflected power. A compromise between longitudinal temperature variation in the sections and flowerosion considerations led to a decision for a maximum bulk velocity of $15 \mathrm{ft} / \mathrm{s}$. The total flow through the cavity is 1190 GPM. The peak surface heat flux on the cavity walls is $13 \mathrm{~W} / \mathrm{cm}^{2}$ at the high-energy end. The peak temperature on the cavity wall surfaces is predicted to be $100^{\circ} \mathrm{F}$.

The peak surface heat flux in the undercut region at the high-energy end is predicted to be $65 \mathrm{~W} / \mathrm{cm}^{2}$ with the peak temperature in this region predicted to be $130^{\circ} \mathrm{F}$.

The $50^{\circ} \mathrm{F}$ inlet coolant temperature requires a refrigeration system instead of the cooling tower more commonly used for linacs. The cooling tower would provide an inlet coolant temperature of about $105^{\circ} \mathrm{F}$ with correspondingly higher peak surface temperatures on the cavity walls and end undercut regions. The higher temperatures on these surfaces would have higher thermal loads due to increased surface electrical resistance. Additional if power would also have been required with the higher coolant inlet temperature.

The beam loss will be approximately $5 \mathrm{~mA}$ of $\mathrm{H}^{+}$and will occur in the first section of the RFQ. The vacuum system ${ }^{8}$ 
will have five 8-in. cryopumps, which includes installed redundancy to allow regeneration while the linac operates.

Power is supplied to the cavity through 12 waveguide irises. Three 1-MW klystrons will normally operate at 2/3-rated capacity. This design will extend both rf window and klystron lifetimes. In the event of a klystron failure, the RFQ can operate with the remaining two klystrons. ${ }^{9}$

Detail design of the APT/LEDA RFQ began in October 1995. The RFQ cavity was fabricated and brazed in Los Alamos National Laboratory shops. Section A2 is shown in Figure 9. The eight sections have been completed and final if tuning is underway. The complete eight section RFQ assembled for pre-installation of tuning is shown in Figure 10.
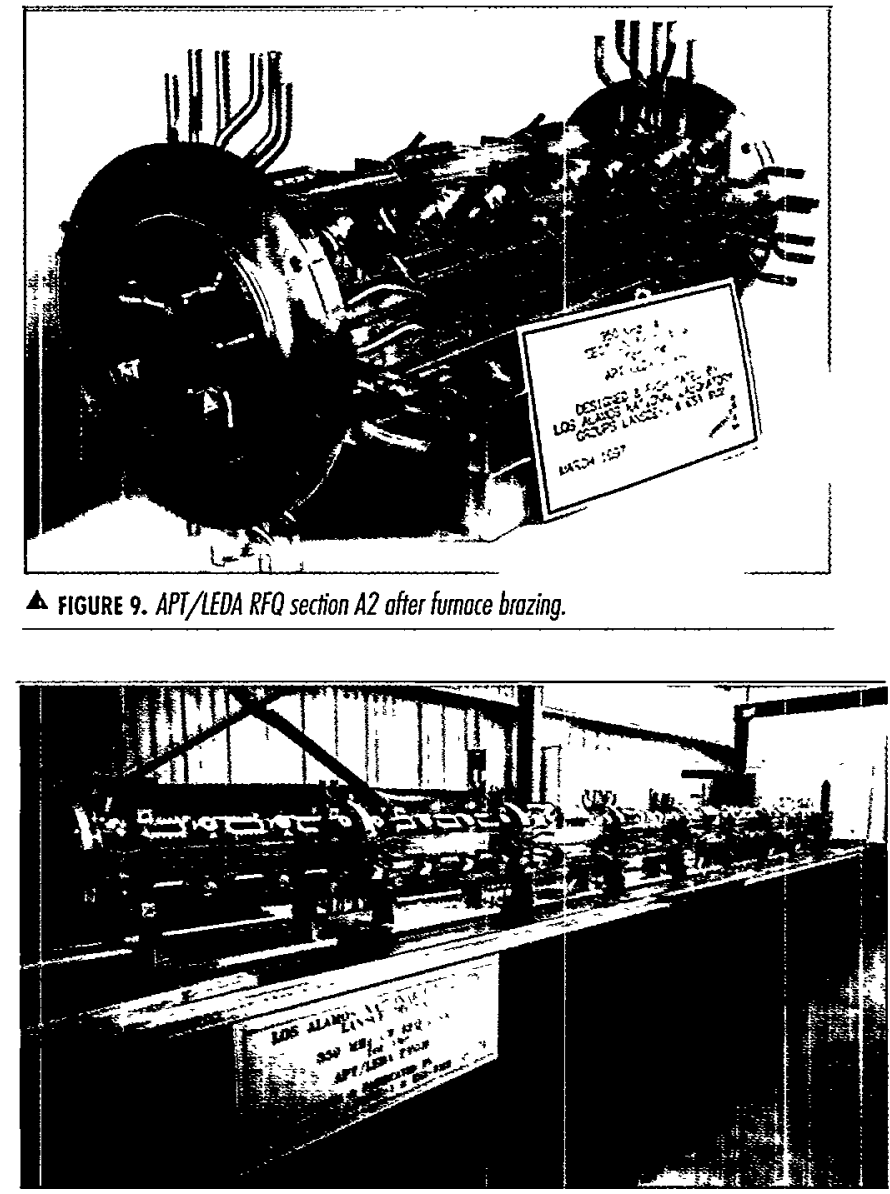

$\triangle$ FIGURE 10. APT/LEDA RFQ assembled for pre-instollotion if tuning.

Other institutions participating in the RFQ project include Lawrence Livermore National Laboratory (vacuum system), Allied Signal (resonance control cooling system), and Northrop-Grumman (engineering support).

\section{References}

I. T. P. Wangler, "Proton Linac for Accelerator Production of Tritium," published in this LANSCE Activity Report.
2. J. D. Schneider and K. C. Chan, "Progress Update on the LowEnergy Demonstration Accelerator (LEDA)," in Proceedings of the 1997 Particle Accelerator Conference (Vancouver, British Columbia).

3. J. H. Billen et al., "A New RF Structure for Intermediate-Velocity Particles," in Proceedings of the 1994 Linear Accelerator Conference (Tsukuba, Japan).

4. L. Young, "Segmented Resonantly-Coupled Radio-Frequency Quadrupole (RFQ)," in Proceedings of the 1993 Particle Accelerator Conference (Washington, DC).

5. M. Vretenar, "RFQ Field Stabilization Use Dipole Suppressers," in Proceedings of the First Workshop on INFN Eliosatron Project (Rice, Sicily, 1986).

6. H. Schneider and H. Lancaster, "Improved Field Stability in RFQ Structures with Vane Coupling Rings," IEEE Trans. Nucl. Sci. NS-30 (4), (1983).

7. A. Ueno et al., "The $\pi$-Mode Stabilizing Loop for Four-Vane Type RFQ's," in Proceedings of the 1990 Linear Accelerator Conference (Albuquerque, New Mexico).

8. S. Shen et al., "APT/LEDA RFQ Vacuum Pumping System," in Proceedings of the 1997 Particle Accelerator Conference (Vancouver, British Columbia).

9. J. Bradley et al., "An Overview of the Low Energy Demonstration Accelerator (LEDA) Project RF Systems," in Proceedings of the 1997 Particle Accelerator Conference (Vancouver, British Columbia).

- D. Schrage, L. Young, W. Clark, T. Davis, F. Martinez, A. Naranjo, P. Roybal (LANSCE Division)

\section{Implementation of the Coupled-Cavity Drift-Tube Linac Concepts}

The CCDTL is a new if structure that plays a major role in some proposed accelerator designs. Its segmented architecture allows room for frequent beam-focusing magnets on rigid mounts, a necessity for these high-current applications. The LEDA will be the first working accelerator of this type. In 2001, the LEDA beam of $100 \mathrm{~mA}$ of continuous current at an energy of $10.05 \mathrm{MeV}$ should be the world's most powerful proton beam. This section compares the CCDTL with the conventional DTL.

In July 1993, J. Billen first presented the CCDTL concept. ${ }^{1}$ In 1995, we proposed using the CCDTL in the low-energy portion of the APT. ${ }^{2}$ In December 1996, the U.S. Patent and Trademark Office issued patent number 5,578,909 on the CCDTL, which now is part of the official design for both the APT plant and the Oak Ridge Spallation Neutron Source (SNS) linacs.

\section{CCDTL Versus DTL}

The CCDTL has certain advantages over the conventional DTL. Placing quadrupole magnets inside the DTL drift tubes makes efficient use of the longitudinal space. However, the DTL must operate at a relatively low frequency to provide enough transverse space for the magnets. These drift-tube magnets are difficult and costly to 
service and replace, and their required precise alignment can be hard to achieve. In contrast, the segmented architecture of the CCDTL has frequent focusing magnets on rigid external mounts. Without the internal magnets, the designer can optimize the drift-tube shape for high efficiency and higher frequency, which also improves structure efficiency.

The DTL and CCDTL also differ in methods used to achieve stability of the rf field distribution. The post couplers that stabilize the DTL fields are immersed in the cavity rf fields, making them hard to cool in a high-power application. The CCDTL has the inherent excellent stability of the coupled-cavity linac (CCL) operating in the $\pi / 2$ structure mode. This nomenclature refers to the resonant mode in which every other cavity in the chain is empty of rf fields. Researchers designing the Clinton P. Anderson Meson Physics Facility (now LANSCE) CCL described the advantages of the $\pi / 2$ mode in a now-famous paper ${ }^{3}$ published in 1967.

\section{Construction}

Building a CCDTL is similar in many ways to building a CCL. However, the CCDTL has some unique features that require new design, fabrication, and tuning tools and procedures. LANSCE physicists have added new capabilities to the computer codes SUPERFISH (for rf cavity design) and PARMILA (for beam-dynamics design and simulation). We built low-power aluminum models to measure the cavity if fields and the coupling between cavities. From the resulting data, we are developing procedures for designing, building, and tuning the copper linac structures.

\section{Design}

One design challenge is the very high power associated with CW operation of the APT linac. For example, the drift tube in the 96-MeV APT CCDTL cavity is slightly smaller than an aluminum beverage can, and will dissipate $5 \mathrm{~kW}$ of rf power. Removing this much power with minimal thermal distortion requires a dense array of internal cooling passages. To achieve highest cavity efficiency, the drift-tube noses should be sharply pointed. However, that shape makes cooling the noses difficult. Making the noses a little more blunt is a compromise between efficiency and our ability to cool the drift tube.

Preparing to fabricate these drift tubes with their maze of internal coolant passages led to other new developments. For example, in our new concentric braze technique, we form most passages with simple lathe processes, and only a few milled cross connections (Figure 11). We place the nested copper cylinders in a molybdenum ring with $0.002-$ in.-thick gold-copper braze alloy foil in the gaps between the cylinders. The thermal expansion of the copper exceeds that of the molybdenum. Thus, at the brazing temperature, the outer copper pieces will compress plastically onto the

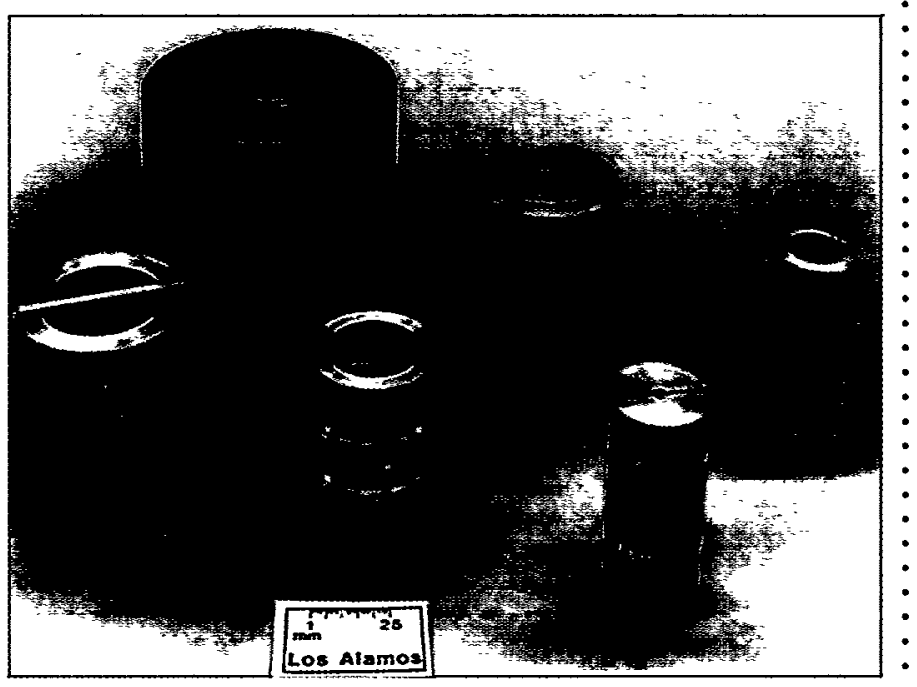

$\Delta$ FIGURE 11. Driff tubes fabricoted using a new concentric braze technique.

innermost piece, closing the gaps. This action produces a slug with leak-tight joints, which then is machined into the finished drift tube shape.

Little data has been published on heat transfer in short, curved, rectangular coolant passages like those in these drift tubes. We are improving our data by conducting heat transfer experiments on drift-tube prototypes, using electric heaters. The measurements suggest that the heat transfer in these passages will be 1.5 to 2.5 times better than predicted for fully developed turbulent flow. Presumably, the abrupt geometry of these passages prevents building a stable boundary layer.

The ultimate heat transfer test will be on a $700-\mathrm{MHz}$ CCDTL high-power model currently being fabricated. This 1-m-long, 30-kW, brazed copper structure will operate in late 1998. Figure 12 shows that the model has five $700-\mathrm{MHz}$ accelerating cavities similar to those used in APT between $7 \mathrm{MeV}$ and $20 \mathrm{MeV}$. We will control the resonant frequency with a scale model of the cooling system planned for the LEDA CCDTL. The cooling control system modulates the temperature of the recirculating coolant water in response to a resonant frequency error signal. At full if power, the coolant water temperature set by the control system will provide information about the effective heat transfer rate.

High water velocity can erode copper. For a conservative assumption of the heat transfer coefficient, the water velocity in the APT 96-MeV drift tubes must be about $5 \mathrm{~m} / \mathrm{s}$. If experiments on the high-power model confirms that the heat transfer rates are better than we assumed, we will lower the LEDA flow velocities rather than raise the coolant temperature. 


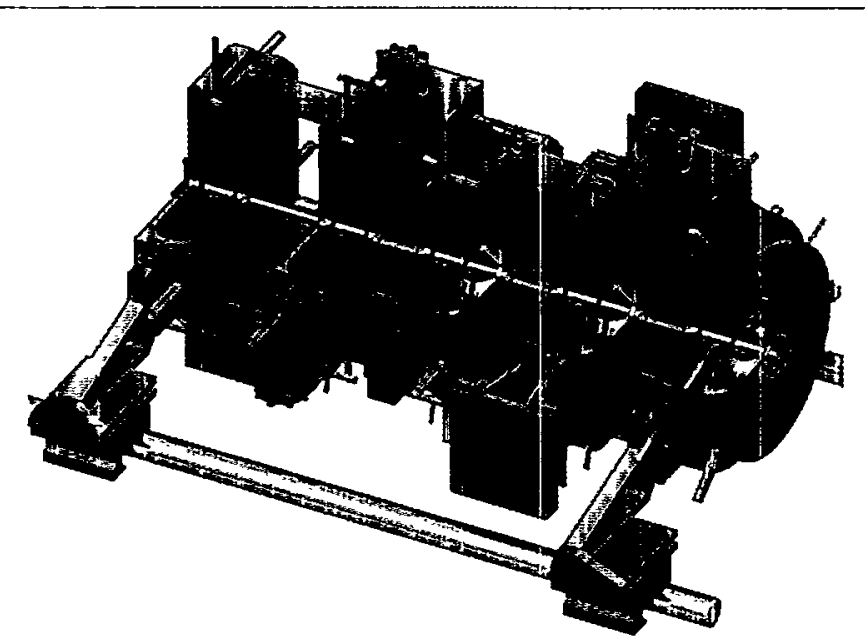

A FIGURE 12. Rendering of a CCDTL high-power model with five 700-MHz acceleroting covifies.

Another major activity at Los Alamos National Laboratory in 1997 was the generation of UNIGRAPHICS parametric solid models of CCDTL assemblies. This allows us to get a big head start on producing the LEDA and APT linac fabrication drawings, although we do not have final cavity dimensions. As these dimensions are made available, the UNIGRAPHICS automatically updates all pertinent drawing files to match the new values.

\section{References}

1. J. H. Billen, "An Efficient Accelerating Cavity for IntermediateVelocity Particles," Los Alamos National Laboratory memorandum AT-1:93-192 (July 28, 1993).

2. J. H. Billen et al., "A Compact High-Power Proton Linac for Radioisotope Production," in Proceedings of the 1995 Particle Accelerator Conference 2, pp. 1137-9 (May 1-5, 1995).

3. D. E. Nagle, E. A. Knapp, and B. C. Knapp, "Coupled Resonator Model for Standing Wave Accelerator Tanks," Review of Scientific Instruments 38, No. 11, pp. 1583-1587 (1967).

4. R. L. Wood, W. L. Clark, F. Martinez, and F. E. Sigler, "Thermal/Structural Design and Fabrication Development of High Power CCDTL and CCL Structures," THP41, in Proceedings of the XVIII International Linear Accelerator Conference (August 26-30, 1996).

\section{-R. L. Wood, J. H. Billen (LANSCE Division)}

\section{Radio-Frequency System for the APT Low-Energy Demonstration Accelerator}

The LEDA being constructed at Los Alamos National Laboratory will serve as the prototype for the low-energy section of the APT accelerator. The APT accelerator requires over 244 rf systems, each with a CW output power of $1 \mathrm{MW}$. The efficiency, reliability, and availability of these if systems is critical to the successful operation of the APT plant, and prototypes of these systems are being developed and demonstrated on LEDA. The rf system designs for LEDA include three 1.2-MW, 350-MHz, CW rf systems driving a RFQ; one 1.0-MW, 700-MHz, CW rf system driving a CCDTL; one 1.0-MW, 700-MHz CW rf system for superconducting cavity and coupler tests; and a full-power test stand at each frequency for the test of $\mathrm{rf}$ components, including the waveguide, if vacuum windows, circulators, and loads. When complete, the rf system will be able to provide a maximum of $7.8 \mathrm{MW}$ of $\mathrm{CW}$ rf power and will require $12 \mathrm{MW}$ of ac power from the grid.

The if system requirements for the $1,700-\mathrm{MeV}, 100-\mathrm{mA}$ APT accelerator are summarized in Table 3, which illustrates two of the important challenges for the APT rf system. The large number of rf systems requires that the individual systems be very reliable in order to achieve an $\mathrm{rf}$ system availability of $95 \%$. Also, the magnitude of the required rf power means that the dc-to-rf conversion efficiency is very important to minimize the operating cost.

\begin{tabular}{lcccc}
\hline TABLE 3. APT f System Description & & & \\
\hline Structure & $\begin{array}{c}\text { Frequency } \\
\text { (MHz) }\end{array}$ & $\begin{array}{c}\text { Number of } \\
\text { rf Systems }\end{array}$ & $\begin{array}{c}\text { Total rf } \\
\text { Required } \\
\text { (MW) }\end{array}$ & $\begin{array}{c}\text { HVDC Power } \\
\text { Installed } \\
\text { (MW) }\end{array}$ \\
\hline RFQ & 350 & 3 & 2.3 & 5.5 \\
\hline CCDTL & 700 & 22 & 17 & 34 \\
\hline CCL & 700 & 29 & 24.6 & 45 \\
\hline SC $-\beta=0.64$ & 700 & 36 & 33 & 55 \\
\hline SC $-\beta=0.82$ & 700 & 154 & 154 & 237 \\
\hline Totals & & 244 & 231 & 377 \\
\hline
\end{tabular}

The demonstration of the APT rf systems under the LEDA project is progressing nicely. The $350-\mathrm{MHz}$ if systems have already been completed and tested, and the installation of the $700-\mathrm{MHz}$ systems is in progress. Figure 13 shows the LEDA rf systems that are installed in building MPF-365. Not shown in the figure are the high-voltage direct current (HVDC) power supply installations, which are external to the building. The klystrons are housed in lead garages for radiation protection. The four if systems to the left of the figure are the $350-\mathrm{MHz}$ systems, and they are connected through a WR2300 waveguide and a circulator to the RFQ. The two if systems to the right of the figure are the $700-\mathrm{MHz}$ systems. They are connected through a WR1500 waveguide and circulator to the first section of the CCDTL and to a waveguide run under the mezzanine. The waveguide runs from right to left beneath the floor and will be used to test superconducting components and cavities.

\section{The 350-MHz if systems use a 1.2-MW klystron} (Figure 14) and a 95-kV, 21-A silicon-controlled rectifier (SCR)-regulated dc power supply. These rf systems have been tested and have demonstrated $1.2 \mathrm{MW}$ of if power delivered to a load with a system efficiency of $65 \%$ for the klystron and 95\% for the HVDC power supply. The control 
racks for the SCR power supply and the klystron electronics are shown in Figure 15.

The $350-\mathrm{MHz}$ RFQ rf systems have some unique features. The power from three klystrons is being combined in the $\mathrm{RFQ}$, with the RFQ acting as the power combiner. Only two of the three klystrons attached to the RFQ are required for operation. If one of the If systems fails, it can be isolated from the RFQ by a waveguide switch, and the remaining two klystrons can provide the required power to the $\mathrm{RFQ}$. This architecture is used to increase the if system

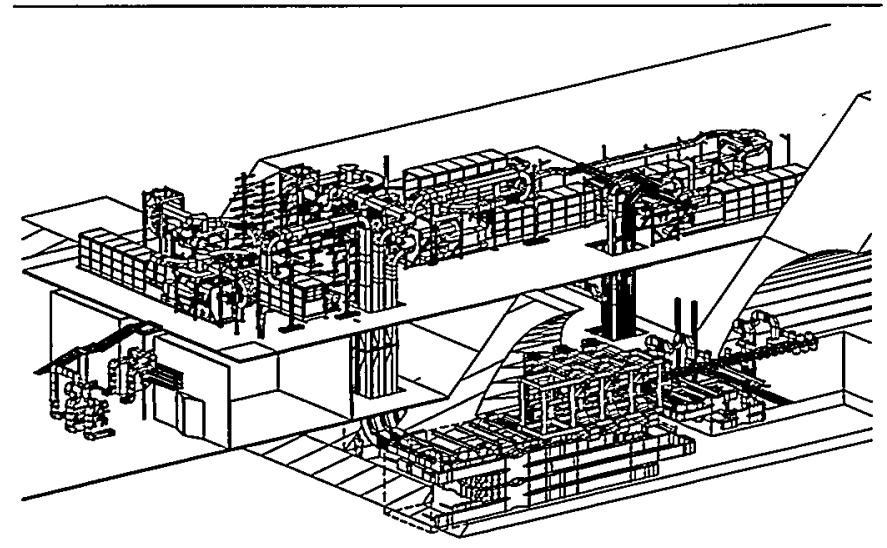

$\triangle$ FIGURE 13. The if system for LEDA.

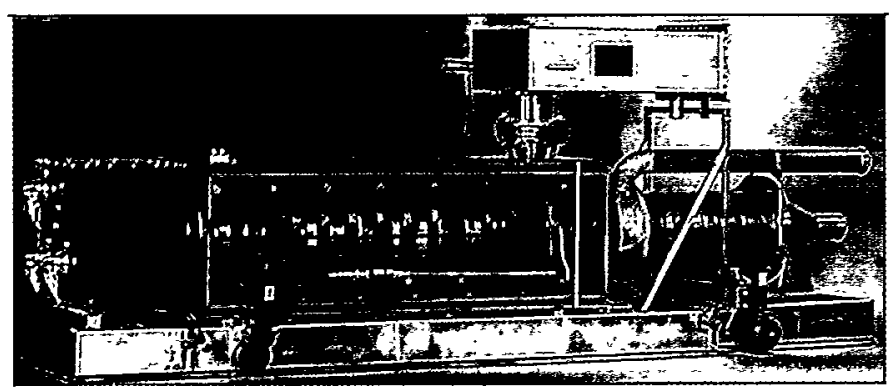

$\triangle$ FIGURE 14. The 350-MHz klystron amplifier is manufactured and provided by $\mathrm{EEV}$.

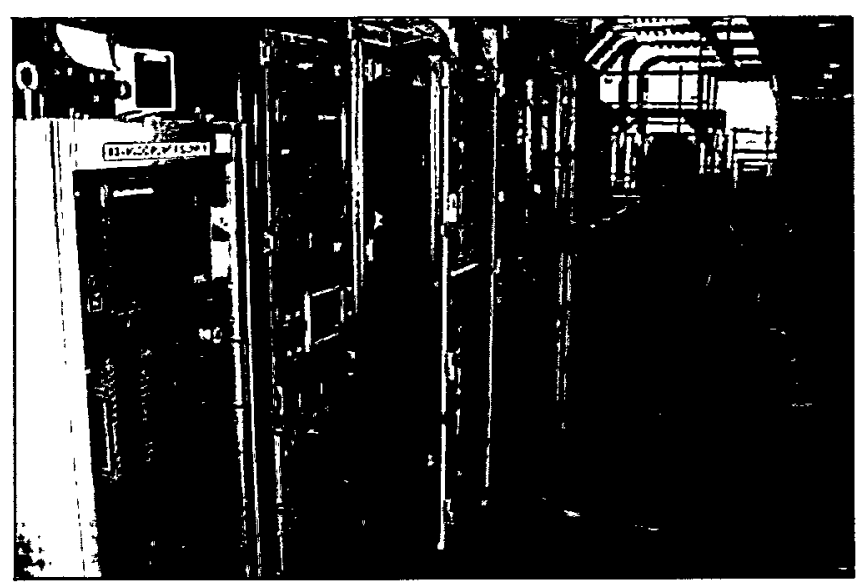

$\triangle$ FIGURE 15. The SCR povier supply and klystron electronics control rocks. availability of the entire low-energy APT linac that is being demonstrated on LEDA. Because of the very high average power capacity of the klystrons, the output of a single klystron is divided into four equal parts by hybrid power dividers. The waveguide then transitions to half-height WR2300, and the power enters the RFQ through four if vacuum windows per klystron. The power division is intended to lower the power per if vacuum window to a level felt to be compatible with a very low failure rate for the rf vacuum windows, but it significantly complicates the waveguide installation and requires very careful phase matching of the waveguide runs. The complexity of the waveguide installation can be seen in Figure 13.

The 700-MHz rf system is currently in the process of being installed. Two vendors, English Electric Valve and Communication and Power Industries, are providing the 700-MHz klystrons. The $700-\mathrm{MHz}$ systems will use a stacked inverter, insulated-gate bipolar-transistor power supply to provide $95 \mathrm{kV}$ and $17 \mathrm{~A}$ with an efficiency of $97 \%$. The $700-\mathrm{MHz}$ klystrons have been tested and have demonstrated that they can meet the required performance of providing 1.0 MW CW at a dc-to-rf conversion efficiency of $65 \%$.

-D. E. Rees, J. T. Bradley, K. A. Cumming, T. W. Hardek, M. T. Lynch, W. T. Roybal (LANSCE Division)

\section{APT Low-Level Radio-Frequency Control System Development}

The low-level radio-frequency (LLRF) control system for APT has been designed to perform various functions.

Foremost is the feedback control of the accelerating fields within the cavity in order to maintain field stability within $\pm 1 \%$ amplitude and $\pm 1^{\circ}$ phase. The feedback control system requires a phase-stable if reference subsystem signal to correctly phase each cavity. Also, instead of a single klystron If source for individual accelerating cavities as has been done in the past, multiple klystrons will drive a string of resonantly coupled cavities, based on input from a single LLRF feedback control system. To achieve maximum source efficiency, we will be employing feedback controls around individual klystrons such that their phase characteristics will be "identical." In addition, the resonance condition of the cavities is monitored and maintained. To quickly respond to if shutdowns, and hence, rapid accelerating cavity cool-down due to rf fault conditions, drive frequency agility in the main feedback control subsystem will be incorporated. Top-level block diagrams are presented and described as they are developed and will be demonstrated on LEDA.

The design of the LLRF control system is complete for LEDA to be built at Los Alamos National Laboratory. This 
system is designed to be easily extended to meet the APT plant requirements. A single control system is used for the supermodule set of klystrons in the normal conducting portion of the linac, whereas for the superconducting portion we will be implementing one LLRF control system per klystron. Either way, implementation is basically the same. A new scheme for field control will be implemented. Rather than a single analog proportional-integral-differential algorithm, we will be using a combination of analog and digital circuitry that divides this control algorithm into two separate compensators working in parallel. Each controller provides both field feedback and beam feedforward control and takes advantage of its particular components, allowing the control bandwidth of the system to be covered by the best techniques available. This methodology is shown in Figure 16.

\begin{tabular}{l|ll|l}
\multicolumn{2}{c}{ Precision Digital } & \multicolumn{2}{c}{ Fast Analog } \\
\hline DC & $10 \mathrm{kHz}$ & $>100 \mathrm{kHz}$ \\
A FIGURE 16. URF field confrol system methodology. & \\
\hline
\end{tabular}

The precision digital compensator provides extremely accurate $\mathrm{dc}$ and low-frequency measurements by employing quadrature sampling and digital signal processing (DSP) techniques. Its bandwidth is limited to about $10 \mathrm{kHz}$ by the digital throughput of the analog-to-digital converters and digital signal processor. The fast analog compensator is implemented in high-bandwidth if and analog circuitry to maximize the closed-loop bandwidth. (It is limited to a little over $100 \mathrm{kHz}$ by the group delay through the other components of the rf system. Transmission delay of up to $700 \mathrm{~ns}$ precludes feedback compensation for more than a couple hundred kilohertz.) This type of fast analog electronics is susceptible to dc offsets and drifts and will have its low-frequency gain reduced for those frequencies where the precision digital compensator is more effective. Figure 17 describes these control techniques, as well as other LLRF system functions, in block-diagram format.

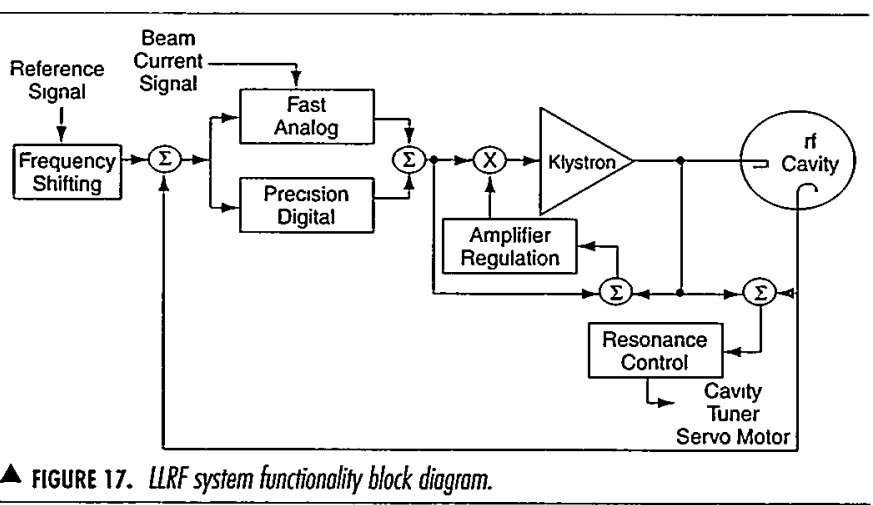

In addition to field control, we provide the rf reference system for the entire accelerator, as well as resonance control of the accelerating cavities. The LLRF control system physically consists of a five-module VXIbus set: a clock distribution module, a field control module, an amplifier control module, a resonance control module, and a highpower radio-frequency (HPRF) protect module. All if and intermediate-frequency signals will be transmitted between modules using front-panel coaxial connectors. All of the baseband and digital signals will be transmitted over the VXIbus backplane. The clock module receives a $10-\mathrm{MHz}$ reference from our reference system and produces the various synchronizing frequencies needed for downconversion and I/Q sampling. The field control module contains the analog and digital electronics described above, which perform field control. The amplifier control module performs the phase equalization needed to balance the three klystrons driving the radio-frequency quadrupole. The resonance control module is primarily a digital module that determines the resonance condition of the cavity.

Depending on how far from the fundamental frequency the cavity is, the resonance control module either provides a control signal to the cavity cooling water system or changes the drive frequency of the klystrons to match the cavity frequency and bring the cavity back to the fundamental frequency through if heating. The HPRF protect module provides an rf shutdown signal, should any operator-defined fault condition occur within the HPRF transport path (waveguides, high-power splitters, etc.). An example of one of these VXIbus modules (the resonance control module) is shown in Figure 18.

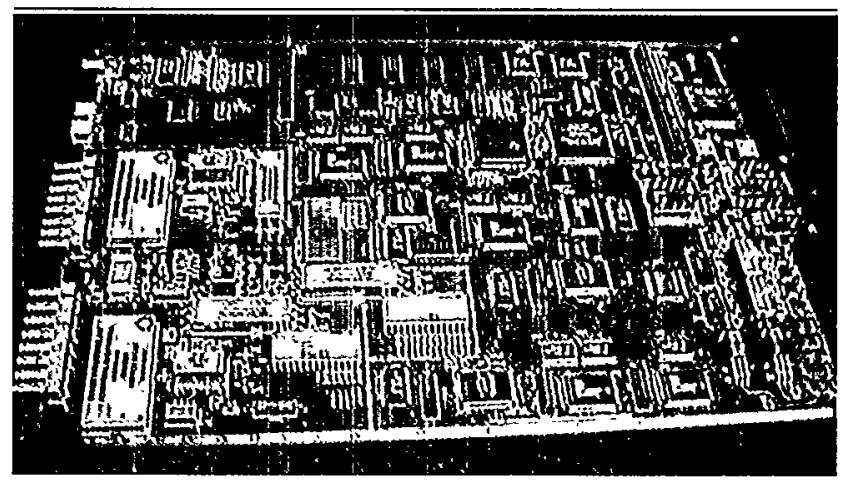

$\Delta$ FIGURE 18. Resononce control VXlbus module.

More detailed descriptions of this system and the details of its design can be found in References $1-4$.

\section{References}

1. A. H. Regan and C. D. Ziomek, "APT LLRF Control System Model Results," Particle Accelerator Conference, Vancouver, British Columbia, Canada, May 12-16, 1997, Los Alamos National Laboratory report LA-UR-97-1652.

2. Y. M. Wang and A. H. Regan, "Algorithms and Implementations of APT Resonant Control System," Particle Accelerator Conference, Vancouver, British Columbia, Canada, May 12-16, 1997, Los Alamos National Laboratory report LA-UR-97-1772. 
3. A. H. Regan et al., "APT LLRF Control System Functionality and Architecture," International Linac Proceedings, 1996, available on CD, ISBN 92-9083-098-0, C. Hill and M. Vretnar, Eds., (CERN Scientific Information Service, CH 1211, Geneva 23, Switzerland), pp. 225-227.

4. M. T. Lynch et al., "The RF System for the Accelerator Production of Tritium (APT) Low Energy Demonstration Accelerator (LEDA) at Los Alamos," International Linac Proceedings, 1996, available on CD, ISBN 92-9083-098-0, C. Hill and M. Vretnar, Eds., (CERN Scientific Information Service, CH 1211, Geneva 23, Switzerland), pp. 692-694.

\section{-A. H. Regan, J. A. Latino, A. S. Rohlev, Y. M. Wang (LANSCE Division), C. D. Ziomek (ZTEC, Inc., Albuquerque)}

\section{Neutronic Design of the APT Target/Blanket}

The APT project has as its goal the design of an accelerator-driven neutron source for the production of tritium. We have optimized this source for maximum tritium production within the constraints imposed by engineering factors such as heat flux limits, structural integrity, fabricability, and safe and reliable operation. For a 1.7-GeV, 100-mA proton beam delivered with $75 \%$ availability, the APT target/blanket produces $3 \mathrm{~kg}$ of tritium per year.

The APT plant has three main components: the accelerator, the target/blanket (T/B), and the tritium separation facility. The accelerator delivers a $1700-\mathrm{MeV}, 100-\mathrm{mA}$ proton beam to the $T / B$, which uses this beam to produce neutrons through a nuclear process termed "spallation." The neutrons released by the spallation process are slowed and then captured by helium-3 nuclei to produce tritium. Gaseous helium-3 flows from the blanket to the tritium separation facility, where the tritium product is extracted from the gas before it is returned to the blanket.

The $\mathrm{T} / \mathrm{B}$, shown in Figure 19, is composed of a centrally located tungsten neutron source (TNS), surrounded by a lead blanket. Tungsten and lead are desirable spallation source materials because large numbers of neutrons are released in the spallation process because of their high atomic masses. Most of these neutrons are born in the "evaporation" stage of the spallation process, and so are born with a broad spectrum of energies centered on a few MeV.

The tungsten, cooled by heavy water, is housed in the rungs of the thirteen ladders that comprise the TNS. The peak tungsten power density of $1.8 \mathrm{MW} / \mathrm{liter}$ occurs in the second ladder, which has a tungsten volume fraction of about $59 \%$. In the thirteenth ladder, where power densities are an order of magnitude lower, the tungsten volume fraction is $77 \%$. The tungsten volume fraction is maximized, within the constraints imposed by safety and thermal-hydraulic considerations, so as to produce the most neutrons possible.

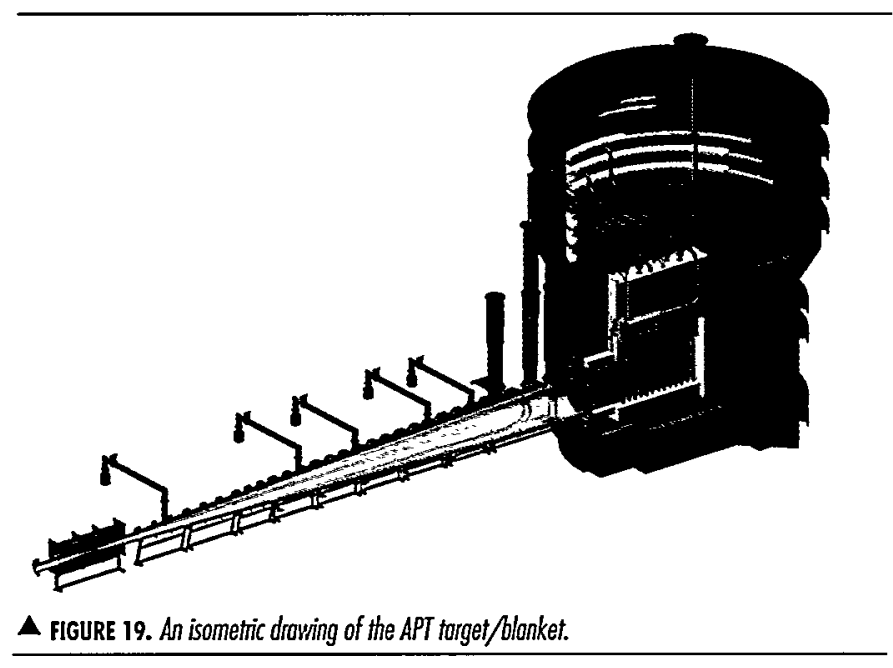

The lead blanket is contained in aluminum extrusions that are designed to minimize aluminum content and provide conduction paths to cavity floodwater during off-normal events. These extrusions have flow channels for light-water coolant and holes where helium-3-filled aluminum tubes are inserted. These tubes are arranged on a $3-$ to $5-\mathrm{cm}$ pitch that minimizes parasitic capture of neutrons by lead. The lead volume fraction, also maximized to enhance neutron production, ranges from $55 \%$ in the high-power regions to $73 \%$ in the low-power regions.

The proton flux distribution within the T/B is shown in Figure 20(a). The black area shows the unperturbed proton beam striking the first tungsten ladder. As the proton beam traverses the TNS region, it disperses, and a portion of it strikes the lead blanket region located to the sides of the TNS. The lead blanket quickly attenuates this scattered proton beam. A plot of the neutron flux through the horizontal
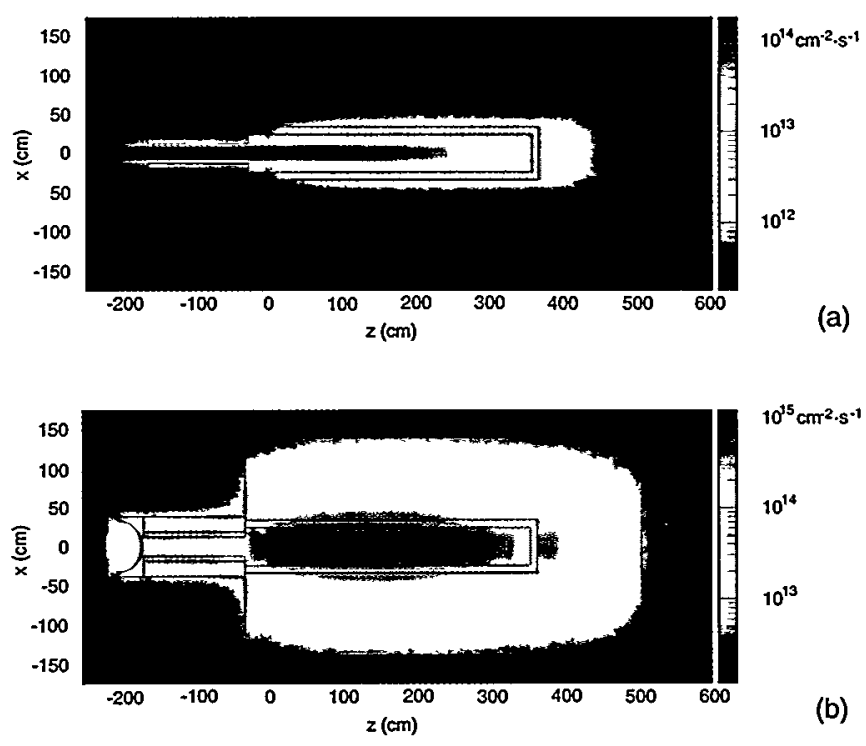

$\triangle$ FIGURE 20. (o) Proton and (b) neutron flux mops within the torget/blanket. 
mid-section of the T/B is shown in Figure 20(b). The peak neutron flux of $1.5 \times 10^{15} \mathrm{n}^{\bullet} \mathrm{cm}^{-2} \cdot \mathrm{s}^{-1}$, comparable to the peak flux found in the world's most powerful research reactors, occurs in the tungsten of the central ladders.

On average, each $1700-\mathrm{MeV}$ proton that strikes the T/B produces 48 neutrons. Of these, 40.5 neutrons are captured by helium-3 to produce tritium. If the plant operates for 274 days each year (corresponding to $75 \%$ availability), then the plant will produce $3 \mathrm{~kg}$ of tritium per year.

\section{-E. J. Pitcher, G. J. Russell, P. D. Ferguson (LANSCE Division)}

\section{Materials Testing for the APT Target}

The APT facility will contain a high-power T/B system at the end of a $100-\mathrm{mA}, 1.7-\mathrm{GeV}$ proton accelerator. The beam will be expanded to a size of $19 \times 190 \mathrm{~cm}^{2}$ before entering the target; the specific power is $47 \mathrm{~kW} / \mathrm{cm}^{2}$. The current expander design is a rastering system; however, a preliminary system composed of vertical and horizontal nonlinear expander magnets was tested in LANSCE Area C in 1997.

The target consists of heavy-water-cooled Inconel-clad tungsten in the form of concentric cylinders arranged in several ladders along the path of the beam. Surrounding the target is a "decoupler" region consisting of light-watercooled aluminum-alloy-6061 tubes containing helium-3 for tritium production. These in turn are surrounded by lightwater-cooled aluminum-clad lead plates, which also hold aluminum-alloy-6061 helium-3 tubes. The APT T/B and Materials Engineering Development and Demonstration Project has the responsibility for characterizing the behavior of plant structural materials in the high-energy particle radiation environment.

In the past, materials irradiation information has been largely generated at reactors where information is needed for neutron radiation damage effects in the energy range from thermal up to $\sim 1 \mathrm{MeV}$. Very low dose investigations have also been done for potential fusion reactor applications using neutrons with an energy of $\sim 14 \mathrm{MeV}$.

Information at higher energies and for other particles such as protons is lacking, although phenomenological data from the many years of LANSCE operations gives us confidence in the integrity of many systems. One particular question involves the commonly used quantity "dpa" (displacements per atom), which is a scaling parameter meant to account for different particle energy spectra. It is not yet clear if such scaling will hold at high particle energy (up to 1.7 $\mathrm{GeV}$ ) and as copious impurities are introduced into the materials through spallation reactions.
In order to obtain data for APT, the Los Alamos Spallation Radiation Effects Facility (LASREF) at LANSCE Target Station A-6 was used for a major irradiation in 1996 and 1997. Further irradiations will be done in 1998 and 1999. Over 5,000 mechanical test samples were included directly in the beam, with maximum proton fluence of $\sim 5 \times 1021$ $\mathrm{p} / \mathrm{cm}^{2}$. Analysis of the samples is being done at the Los Alamos, Oak Ridge, Brookhaven, and Pacific Northwest national laboratories and at SRS; the results will be included in an APT Materials Handbook.

An additional test was included in Area $\mathrm{A}$ to measure online corrosion rates of Inconel using electrochemical impedance spectroscopy (EIS) techniques. Follow-up corrosion rate measurements on additional materials will be done in the 1998 and 1999 irradiation. Transient analysis techniques to measure the buildup of corrosion products directly in water was also done at the LANSCE Blue Room, employing EIS as well as Raman spectroscopy.

Model components of the tungsten neutron source and the blanket were included in the irradiation. The large volume available at LASREF was used to good advantage in this application (Figure 21).

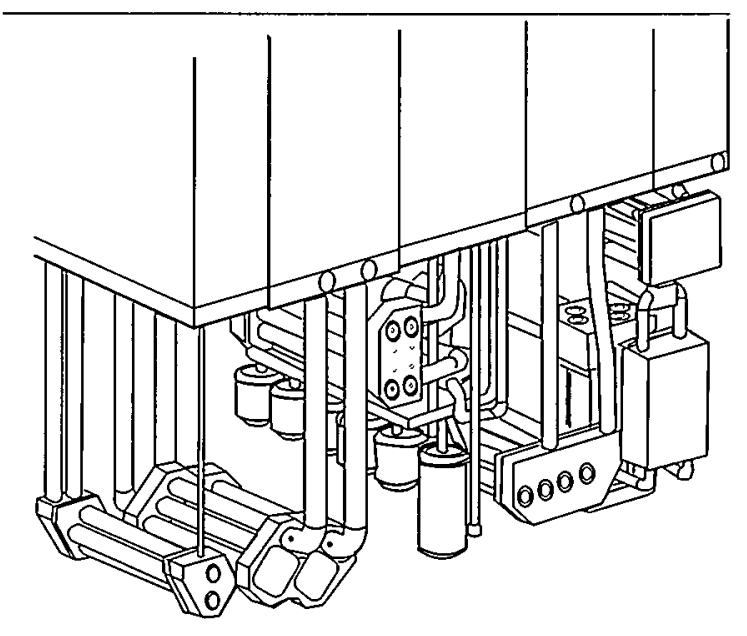

$\triangle$ FIGURE 21. Schematic diagram of the five intheam inserts irradiated of LASREF in FYs 1996-97. A second inodiation with a new set of experiments is planned for FYs 1998-99.

Overall, the APT work is the largest high-power, highenergy irradiation specifically focused on accelerator materials ever done, and it takes full advantage of the unique LASREF capabilities.

\section{- W. F. Sommer, L. S. Waters (APT Technical Project Office)}




\section{Spallation Neutron Source Poised to be World-class Research Center}

The Spallation Neutron Source (SNS) is a construction line-item project sponsored by the Department of Energy (DOE) Office of Basic Energy Sciences. The SNS will be a world-class center for neutron scattering research and will produce the most intense pulsed neutron beam in the world. The facility will include an accelerator-based pulsed neutron source, research facilities, and user support facilities needed to create a fully operational research center. When operational, it will be used for a wide range of materials research and neutron scattering experiments.

Oak Ridge National Laboratory (ORNL) has been charged by DOE with the responsibility for coordinating the SNS in collaboration with other DOE laboratories. Collaborating laboratories are Argonne National Laboratory (ANL), Brookhaven National Laboratory (BNL), Lawrence Berkeley National Laboratory (LBNL), and Los Alamos National Laboratory (LANL). Each Laboratory is responsible for a major SNS system. Although the final National Environmental Policy Act determination is pending, Tennessee is the preferred site for the SNS.

The SNS will have an ion source that produces negative hydrogen ions, accelerates them to 2.5 million electron volts, and delivers them to the linear accelerator, or linac. The linac will then accelerate the hydrogen ions to one billion electron volts for injection into an accumulator ring. In this ring, the hydrogen ions will be bunched to form an intense, less than one microsecond pulse. The pulse is then delivered onto a mercury target that produces the neutron

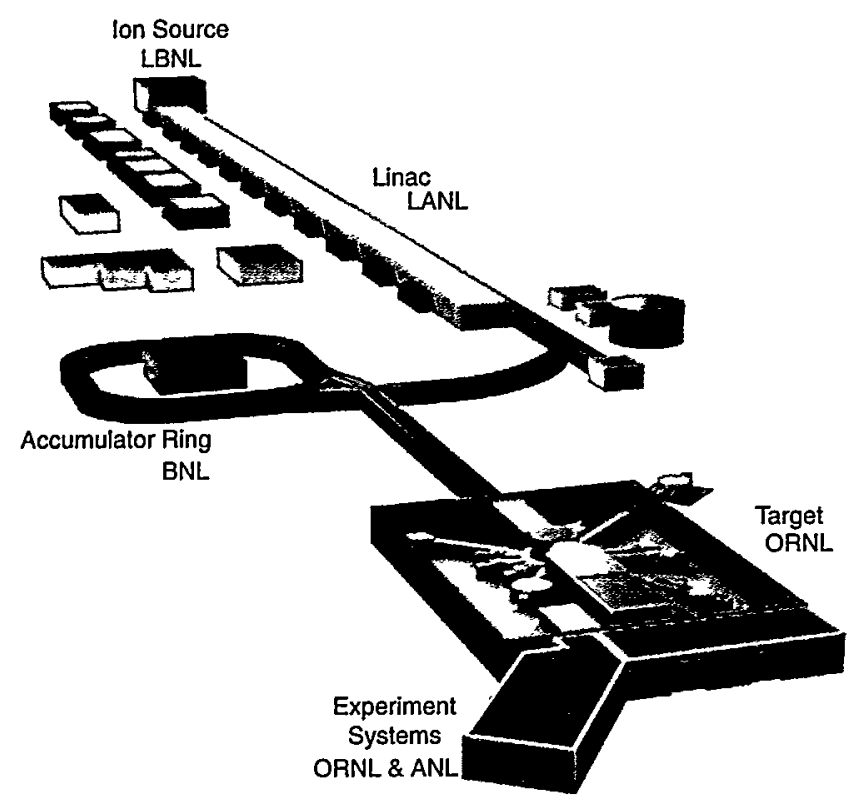

A FIGURE 1. Artist's conception of the SNS focility. burst, which is aimed at the target samples. Figure 1 shows a sketch of the SNS configuration.

Los Alamos is responsible for the linac capable of delivering a pulsed 1-MW negative hydrogen ion beam. This responsibility includes the design, procurement, fabrication, equipment delivery to the site, oversight of equipment installation, technical oversight and assistance for linac activities performed at the site, system testing, commissioning support, and turnover to ORNL. The Laboratory also will build control and diagnostic systems for beam handling and optimization.

Initially, the linac (Figure 2) will produce a beam of 1-MW total power, although it will be upgradable to either 2 or $4 \mathrm{MW}$. The linac is sequentially composed of a drift-tube linac (DTL), a coupled-cavity drift-tube linac (CCDTL), and a coupled-cavity linac (CCL); i.e., a linear series of radio-frequency (rf) accelerating structures specifically tailored to the beam energy attained. The 1-MW design will accelerate a peak current of $27.7 \mathrm{~mA}$ with a duty factor of $5.84 \%$. The conceptual design review (CDR) for the system was held in June 1997. Line-item funding and authorization to proceed into the construction phase was approved to start in October 1998.

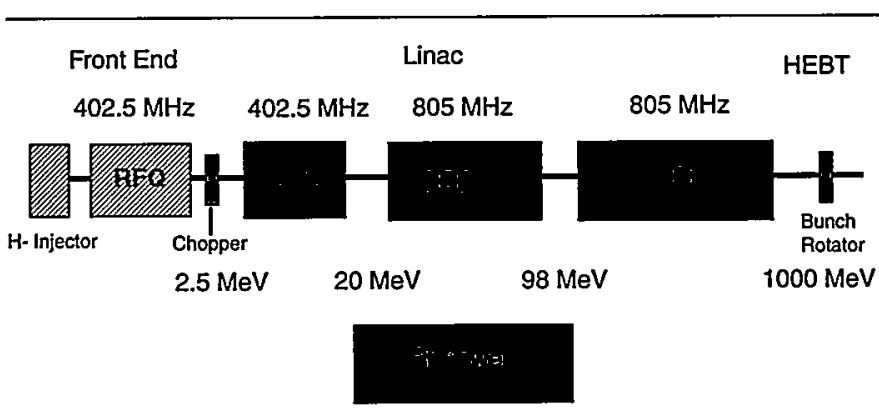

A FIGURE 2. Block diagram of the SNS linac.

The Laboratory is scheduled to receive $\$ 30.4 \mathrm{M}$ in funding for the project during FY 1999 and about $\$ 350 \mathrm{M}$ over the 7-year life of the design and construction phases. Laboratory activities to date include physics support, accelerator engineering, If engineering, controls support, and technical support.

Physics support encompasses overall system architecture as well as quantitative beam dynamics modeling. Physics design has been especially directed toward minimizing beam loss, a critical factor in facility availability. Los Alamos has refined the initial architecture presented at the CDR to simplify the design and manufacturing processes. Beam dynamics simulations and error analyses have determined the design feasibility. Work is underway to establish the 
many system parameters needed for detailed power systems and mechanical engineering final design. The work will also define commissioning procedures and complete specification of the beam diagnostics system. Efforts to establish this important beam system, though small to date, have extended the results of other projects to SNS. The diagnostics include devices that measure the beam profile, longitudinal extent, transverse position, current, and energy, as well as other parameters needed for linac tuning and control. Several innovative concepts will be used in the diagnostic-devices design and in the associated electronics processing.

Accelerator engineering activities include optimizing mechanical systems in conjunction with the physics effort. The 467 meters of rf-driven accelerating structure that constitutes the linac must dissipate nearly $5 \mathrm{MW}$ of thermal energy to produce the electromagnetic fields that accelerate the beam. Additionally, the structure must be accurately tuned to its correct resonant frequency. Regarding this last effort, cold models (a low-power structure that allows empirical specification of critical dimensions) for each type of structure are under construction. A conceptual design review was held for both the CCL and CCDTL cold models in FY 1998, and fabrication is underway. We built a detailed model of a CCDTL segment that includes the drift tube with stems as well as an independent drift-tube cooling loop. We completed computer modeling of the steadystate frequency responses and runs showing the effect of varying the coolant temperature in the drift tube. Preliminary thermal analysis of the CCL structure was completed in July 1998.

The linac is powered by an $805-\mathrm{MHz}$ rf system that will provide a peak power to the structure of over $97 \mathrm{MW}$ in 1-MW bursts. This rapid modulation has been done in the past with large energy-storage capacitor banks and complex switching of the final power output klystron tubes through direct high-voltage modulation of a tube electrode. However, Los Alamos will implement a switching system, based on the integrated-gate bipolar transistor (IGBT), to replace the conventional system presented at the $C D R$. The IGBT modulator offers improved safety, reliability, serviceability, and reduced costs. Development of this new concept is underway, and work has been initiated on many fronts: utility power system modeling, IGBT convertermodulator modeling, test fixture fabrication, circuit design, scale model prototyping, and testing and verification of electronic control circuits. After substantial circuit development, we are fabricating a reduced-scale IGBT convertermodulator. The required power supplies, filter networks, IGBT assemblies, and associated drivers have been completed and mounted into a rack assembly. We will conduct definitive tests of concept feasibility upon completion of this construction.
We are procuring prototype $805-\mathrm{MHz}$ klystrons for the if system from two vendors. The vendors have completed their design, and fabrication will commence in FY 1999. The Laboratory is setting up an area for the $805-\mathrm{MHz}$ klystron test stands that will be used for high-power structures testing as well as for lifetime test of the tubes. Work on the $805-\mathrm{MHz}$ transmitter rack is now complete and is cabled to the modulator. We have begun fabrication of the klystron water-cooling manifold. We refurbished the capacitor room, and new safety input/output hardware is being installed. Much of this effort relies on surplus Ground Test Accelerator hardware. Likewise, the Laboratory has refurbished a $402.5-\mathrm{MHz}$ test stand and delivered it to LBNL for test of its front-end systems.

An important milestone in the project will occur in January 1999 when DOE will validate the overall SNS project baseline and authorize the start of preliminary and final design, also referred to as Title I and Title II engineering. This design effort, lasting approximately 2 years, will be followed by fabrication and installation at ORNL. The linac will be installed and tested with beam in several stages with operational readiness in 2005.

-Andrew Jason (LANSCE Division) 


\section{Long-Pulse Spallation Source Proposed for LANSCE}

Over the past 4 years, LANSCE has been investigating the prospects for the construction of a 1-MW long-pulse spallation source (LPSS) based on the existing LANSCE linac. Such a spallation source would provide a cost-effective and internationally competitive new facility for a broad range of neutron research. The LPSS would be particularly well suited to cold neutron research and would complement the existing facility at the Lujan Center as well as the Spallation Neutron Source at Oak Ridge National Laboratory. Because the LPSS would use the existing Area A experimental facility, it could be built without perturbing ongoing activities at LANSCE.

To date, the exploitation of spallation neutron sources has primarily focused on the use of short-pulse spallation sources (SPSSs). A short-pulse source (of which the Lujan Center is an example) is one in which the proton pulse duration is much less than the neutron moderation time and in which the neutron moderator is "poisoned" with absorbers. The poisoning of the moderator reduces the long "tail" of the neutron timing distribution. The short neutron pulse duration from such sources has been shown to be very valuable for a wide range of applications that require moderate to high resolution. However, a large number of neutron scattering applications do not require such short pulses. For such applications, an LPSS can provide a much higher flux, substantially reduced cost, and greater simplicity of operation.

In the LPSS proposed for LANSCE, protons from the $800-\mathrm{MeV}$ linac would be sent directly to a spallation target located in Area A. The linac has a demonstrated capability of providing a proton beam with an energy of $1 \mathrm{MW}$. Because the proton beam need not be accumulated in a proton storage ring, this full power is available at the spallation target. One megawatt would provide more than one order of magnitude increase in power over the current power at the Lujan Center. In addition to having a higher spallation power, the LPSS is intrinsically a more efficient source of the neutrons, because moderator poisons, required by the SPSS to reduce the long tail of the neutron distribution, are not necessary in an LPSS. Removal of such poisons can improve the useful neutron production rate by a factor of 5. The 1-MW LPSS would have a peak neutron flux that is approximately four times that of the highest flux reactor (at the Institut Laue-Langevin). The time-averaged flux is reduced by the duty factor of approximately $1 / 16$.

The degree to which a particular instrument can benefit from the very high flux of the LPSS requires a detailed analysis of that instrument as well as the particular experiment for which it will be used. Such an analysis was carried out in a workshop convened at Berkeley in 1995. ${ }^{1}$ The results of this analysis are summarized in Table 1. As shown, there are larges classes of investigations for which an LPSS would provide a substantial improvement over the current worldstandard intruments at Institut Laue-Langevin.

Because many of the essential components of the LPSS exist (the linac, the Area A experimental hall, and much of the associated infrastructure), the LPSS could be built in a very cost-effective way in a relatively short time. A detailed study of the LPSS ${ }^{2}$ indicated that the LPSS could be constructed in approximately 3 years. A fully instrumented LPSS would enormously enhance of the U.S. capability in neutron scattering at a very modest cost.

\section{References}

1. J. Alonso, R. Pynn, T. Russell, and L. Schroeder in Proceedings of the Workshop on Neutron Instrumentation for a Long-Pulse Spallation Source, Lawrence Berkeley Laboratory document LBL-37880 (1995).

2. R. Pynn and D. Weinacht, "A Long-Pulse Spallation Source," Los Alamos National Laboratory document LA-UR-95-3904 (1995).

-Geoffrey Greene (LANSCE Division) 


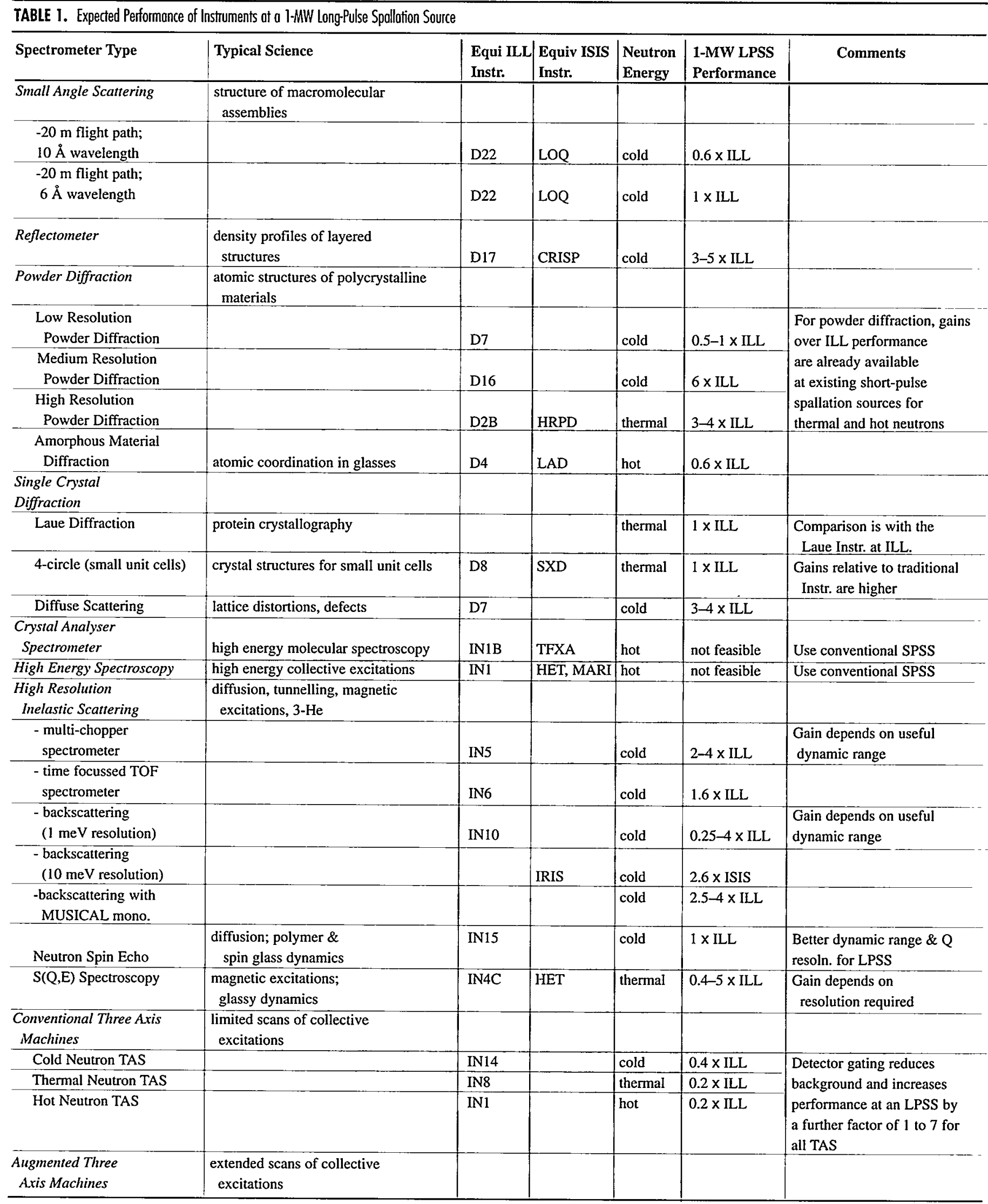




\section{New Target Irradiation Facility for Isotope Production}

A new target irradiation station for isotope production is being constructed at LANSCE. The project will modify the transition region of the existing accelerator by installing a kicker magnet to deflect beam to a new beam line. This beam line will be housed in a new facility constructed on the north side of the existing accelerator building. The beam will be delivered to a target station housed in the lower level of the new facility. A hot cell facility for handling the targets both before and after irradiation will be housed in the upper level of the new facility. When this facility becomes operational in FY 2001, isotope production will be possible whenever the accelerator is operating for other programmatic purposes.

The Isotope Production and Distribution Program at Los Alamos has been a very successful and visible program at the Laboratory for more than 20 years. It has used the unique capabilities of the Laboratory's accelerator and hot cell facilities to make more than 30 isotopes for use in medical diagnostic procedures (some of which were developed at the Laboratory) and for use in industrial applications as well. Today LANL collaborates with members of industry, research institutions, the medical community, academia, and government laboratories.

The irradiation capability that has been the foundation of the program makes use of the $800-\mathrm{MeV}$ proton beam delivered to the beam stop of the LANSCE linear accelerator. In the past, the isotope program has been very costeffective because it operated the Isotope Production Facility (IPF) in such a way that it used beam that was accelerated, but not extracted, and directed to other experimental stations. Although this arrangement made effective use of beam that might otherwise go unused, it was not optimal for two reasons:

- The beam energy is too high to produce desired isotopes selectively.

- The beam is not available on a year-round basis. Isotope production is limited to the months each year that the accelerator operates. Short-lived isotopes can only be produced and distributed at these times, and thus cannot be delivered to customers on a regular basis. While longer-lived isotopes are stockpiled and distributed year round, the lack of year-round availability of short-lived isotopes is a serious issue.

In 1995, the Isotope Production and Distribution Program proposed to optimize its program and to ensure its continued ability to produce radioisotopes by purchasing a new cyclotron that would be capable of extracting proton beams between $30 \mathrm{MeV}$ and $100 \mathrm{MeV} .{ }^{1}$ It was noted in the proposal that most of the goals could be achieved with a novel targeting technique and a $100-\mathrm{MeV}$ beam with a current of about $250 \mu \mathrm{A}$. Subsequently, the Laboratory solidified plans to convert LAMPF into a neutron science center known as LANSCE. A determination has been made that an $\mathrm{H}^{+}$beam can be extracted from the $100-\mathrm{MeV}$ transition region of the accelerator without interfering with the existing neutron science mission or new programmatic opportunities of the facility. However, the new programmatic opportunities will interfere with the current $800-\mathrm{MeV}$ IPF. Thus, the Isotope Production and Distribution Program proposes to use this $100 \mathrm{MeV}$ beam option to fulfill and enhance its isotope production commitments. ${ }^{2}$

The Laboratory and the Department of Energy, in recognition of the importance of the isotope production mission, have included the new $100-\mathrm{MeV}$ IPF in the LANSCE master plan.

The new IPF will be simple in concept. A switching magnet will be installed in the beam line between the drifttube linac and the side-coupled cavity linac where the beam energy is $100 \mathrm{MeV}$. The accelerator will simultaneously deliver $\mathrm{H}^{+}$and $\mathrm{H}^{-}$beams at different phases of the radio frequency (rf). Some of the $\mathrm{H}^{+}$beam will be extracted and sent to the IPF and the remainder of the $\mathrm{H}^{+}$beam will be accelerated to $800 \mathrm{MeV}$ for use by LANSCE experiments. A short spur for $100-\mathrm{MeV}$ beam extraction that was provided in the original LAMPF construction for $100-\mathrm{MeV}$ beam extraction will be extended with a tunnel and target room. A new beam line with steering and focusing magnets to deliver the beam to the IPF target station will be installed in the tunnel. The tunnel and target room will be below ground so that they are at the same elevation as the accelerator with earth used as radiation shielding. A facility will be built on the ground above the beam tunnel and target room. The aboveground facility will contain the power supplies and other equipment required to support the beam line and target irradiation systems. The aboveground facility will also contain a simple hot cell for extracting irradiated targets from the target chamber and transferring them to a shipping cask without exposing the staff to high levels of radiation. Access to the below-grade facilities for maintenance and repair activities will be through the aboveground facility, which will contain a stairwell and protective clothing change facilities.

The execution of the project is driven by an important constraint. The project will require 4 months for installation work in the transition region that must be scheduled around the LANSCE operating schedule, so that it does not interfere with routine beam delivery. We envision that this will occur when the accelerator will be taken out of service for upgrades. All work to be performed within the accelerator tunnel, such as installation of the $100-\mathrm{MeV}$ extraction 
system, must be accomplished when the accelerator is out of service. Capital funds were appropriated in the FY 1999 federal budget. Long-lead items, principally the extraction and focusing elements that must be installed in the accelerator tunnel, will be purchased with project funds during 1999 so that they will be available for installation on schedule.

To execute the project, the plan requires continuous design and construction without time lapses. Long-lead-time equipment will be purchased while independent design activities are being completed. This challenging plan has the competitive bid process scheduled between the title design work and the construction phase. In this way, the project can be carried out in a highly efficient, seamless stream of activity with the first isotope production run scheduled for April 2001.

The project will be executed by a team consisting of three collaborating organizations: Los Alamos National Laboratory, an architect/engineering (A/E) firm, and a constructor, under the auspices of the Department of Energy, Office of Isotope Programs. The Los Alamos team consists of the Isotope Production Program staff responsible for the irradiation and target-handling equipment; the LANSCE program staff responsible for the beam extraction and beam line equipment; and the facility engineering staff responsible for the facilities and project coordination. The A/E firm and the constructor will be responsible for facility design and construction.

\section{References}

1. R. Heaton, P. Smith, and E. Peterson, "National Biomedical Tracer Facility Project Definition Study," Los Alamos National Laboratory report LA-UR-96-0509 (May 1995).

2. R. Heaton, E. Peterson, and P. Smith, "Target Irradiation Facility for Radioisotope Production and Research," Los Alamos National Laboratory report LA-UR-96-1639 (May 1996).

-R. C. Heaton, E. J. Peterson, M. M. Fowler (CST Division), P. Wahlstrom, K. F. Johnson (LANSCE Division), R. Meyer (ESA Division), R. Bradshaw, P. A. Smith (PA Smith Concepts and Designs), S. F. Daugherty (Merrick \& Company) 


\section{Accelerator-Driven Transmutation of Waste Uses Advanced Technologies}

The Accelerator-Driven Transmutation of Waste (ATW) project is dedicated to providing the nation with a technological solution to the disposition of nuclear reactor waste. Since 1996 we have focused on a system concept that provides performance enhancement to the nuclear waste repository using advanced and substantially proven technologies. The current ATW system concept consists of an APT-class accelerator, a liquid lead-bismuth eutectic (LBE) integral spallation target and nuclear coolant reactor with solid metallic fuels, and a pyrochemical processing plant for spent reactor fuel and ATW waste treatment. We initiated development work in nuclear design, LBE technology, and pyroprocesses, and have made significant progress.

\section{ATW System Description}

An ATW facility consists of three major elements: (1) a high-power proton linear accelerator based on technology developed for the APT project; (2) a pyrochemical spent-fuel treatment/waste cleanup system; and (3) a liquid lead-bismuth cooled burner that produces and uses an intense neutron flux for transmutation in a heterogeneous (solid fuel) core. The concept is the result of many years of development at Los Alamos National Laboratory ${ }^{1}$ as well as other major international research centers. ${ }^{2}$

In the spent-fuel treatment system (Figure 1), uranium and a majority of the fission products are separated from the transuranics and the targeted long-lived fission products by pyrochemical (non-aqueous) processes. One essential requirement is the separation of enough uranium (99\%) so that no significant new plutonium or other actinides are produced during transmutation. Fission product extraction is not explicitly sought but comes out naturally from the process.

In one reference design concept, a third of the core is extracted and processed every year. In the ATW waste

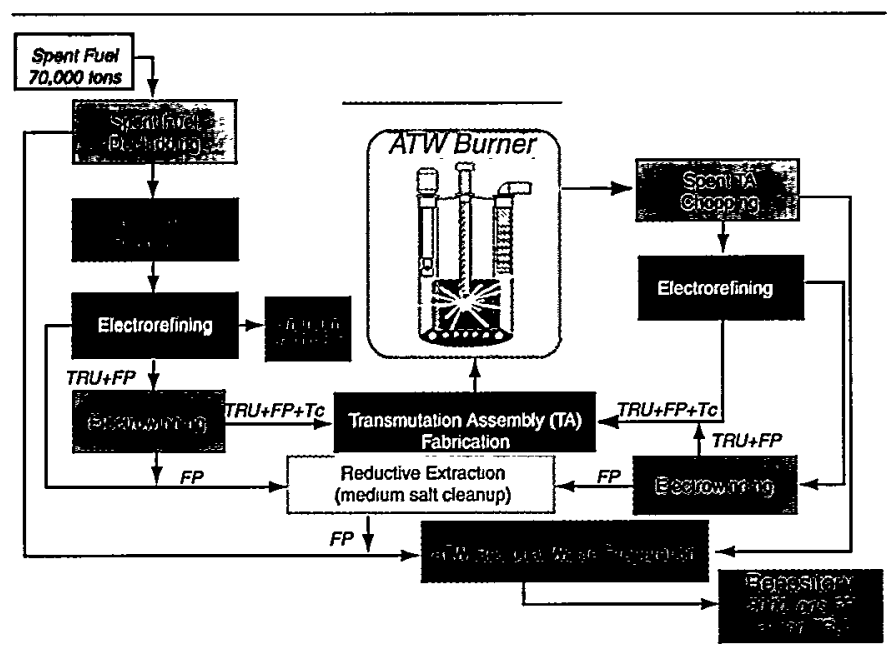

$\triangle$ FIGURE 1. Schematic ATW waste treotment flow sheet. ATW waste treatment is bosed on pyrochemicol processes developed at Los Alomos and Argonne national laboratories. cleanup process, eventually all the fission products in the irradiated waste are partitioned into three forms: active metals, noble metals, and lanthanides. This remnant waste is prepared for permanent storage as (1) oxides in engineered containers for the active metals (including strontium and cesium), (2) oxides for the lanthanides, and (3) metal ingots and oxides for the noble metals including zirconium. An average of $50 \mathrm{~kg}$ of fission products, per ton of spent fuel, is discharged as waste after transmutation (including the fission products originally present in the spent fuel), contaminated with less than $100 \mathrm{ppm}$ of transuranics (mostly in the metal oxide waste form). Most of the radioactivity in the discharges would decay within 300 years. The ability to tailor waste forms to the environment of an underground repository by segregation in the ATW waste treatment processes offers advantages over the disposition of untreated spent fuels.

The waste burner consists of a heavy-metal spallation target (liquid LBE) providing a high-intensity neutron source and the surrounding subcritical core containing the transmutation assemblies (Figure 2). Since significant neutron multiplication and heat production occurs from the fission of waste actinides in the transmutation assemblies, adequate means for heat removal must be present, analogous to the situation in critical reactors of similar power level.

ATW takes advantage of the exceptional properties of liquid LBE, both as a nuclear coolant and as a spallation neutron source, for use in the subcritical waste burner. The technology, successfully developed and used in Russia for nuclear submarine propulsion of very fast, deep diving vessels, is becoming accessible to western researchers and engineers. The subcritical liquid LBE systems presently being developed at Los Alamos operate in a fast neutron spectrum to ensure optimal destruction efficiency for the actinides and

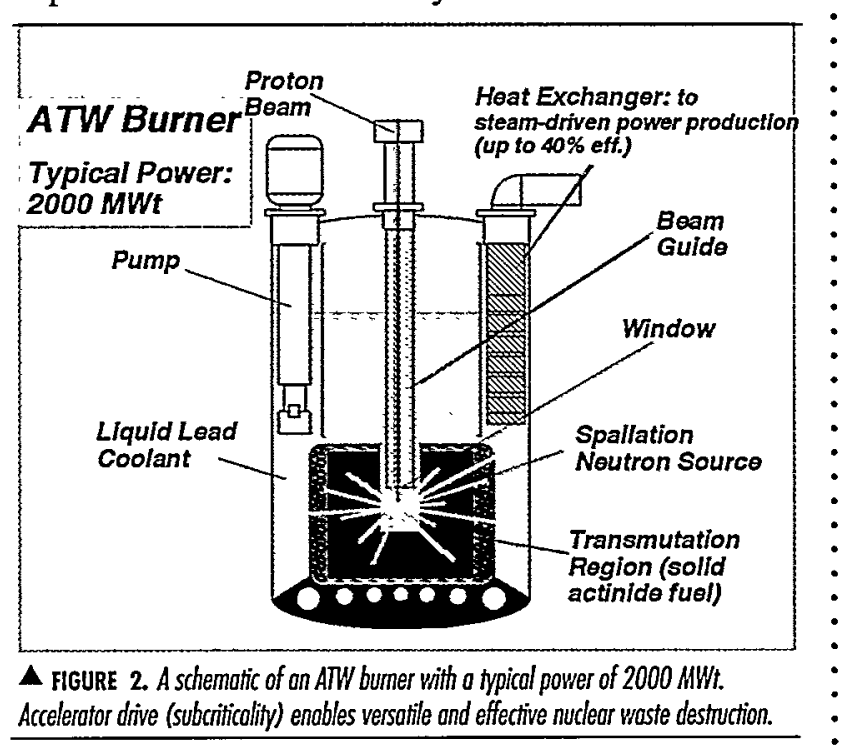

LANSCE • Activity Report 
large neutron availability for transmutation of the targeted fission products. Very low end-of-life inventories can be rapidly achieved by burn-down strategies involving gradual thermalization of the spectrum to exploit the large capture and fission cross sections of resonances.

Subcriticality does not make ATW by definition "safer" than critical reactors. Rather, subcriticality facilitates tasks that would be exceedingly difficult or inefficient in critical systems. Subcritical systems do not rely on delayed neutrons for control and power change; they are driven only by the externally generated neutron source (i.e., by the proton beam coming from the accelerator). Control rods and reactivity feedback have very low importance: these systems are neutronically (but not thermally) decoupled from their neutron source. Subcriticality therefore allows the ATW system to work with any composition of fuel (or waste) and to greatly relax the required separation in the waste treatment steps. This makes possible, in principle, the destruction of any isotopes (actinides or fission products or mixture of both) with little concern for their neutronic behavior. Fertile materials are not needed to compensate for the neutronic uncertainties or undesirable reactivity responses of the fuel, and extended burnup is achieved by increasing the power of the accelerator drive to compensate for reactivity drops.

Because of its subcritical mode of operation, ATW will be ideally suited as an "incinerator" of material that (1) is not well characterized, (2) burns very poorly or not at all in reactors, (3) has potentially unstable and hazardous reactivity responses, and (4) should not for whatever reason be isolated and placed in reactors. This includes higher actinides such as neptunium, americium, and curium, all isotopes of plutonium, and some long-lived fission products. In addition, the neutron-poor thorium-uranium fuel cycle, never successfully implemented in critical reactors, can be used rather straightforwardly in accelerator-driven subcritical systems.

\section{ATW Project Support} In January 1998, the ATW project team went to the Massachusetts Institute of Technology (MIT) and presented the program and the technical underpinnings to a nuclear engineering technical review panel. The panel concurred with our choice of technologies and system concept, and also strongly advocated our planned development program because of the tremendous spin-off benefits, such as the lead-bismuth coolant for advanced reactors, pyrochemical processes for nuclear waste treatment, and highpowered spallation neutron sources for scientific research.

The technical underpinning of these significant program development efforts was supported by a vigorous Laboratory-Directed Research and Development (LDRD) research program over the last few years. With a project team composed of members from many laboratory divisions and with the support of LANSCE Division, the ATW project has investigated several key areas, which include nuclear system design, LBE technology development, and pyrochemical processes for waste treatment.

\section{Neutronics Calculation of ATW Burner Core}

Significant progress was made in FY 1998 related to designing the ATW Waste Transmutation Region (WTR). Point designs for two WTRs were completed: one for an initial WTR demonstration and one for an operational ATW. A computer code was developed for calculating time-dependent waste compositions and physics parameters for all destruction scenarios of interest. The code divides a destruction scenario into several individual burn steps, for which the time interval, power, and material feed and reprocessing rates can be varied. Fuel shuffling schemes were devised that allow for high burnup and power flattening while maintaining linear heat rates and other parameters within demonstrated bounds. Schemes for transmuting technetium- 99 and other isotopes were devised and shown to have more than adequate performance (Figure 3). Various blanket composi-

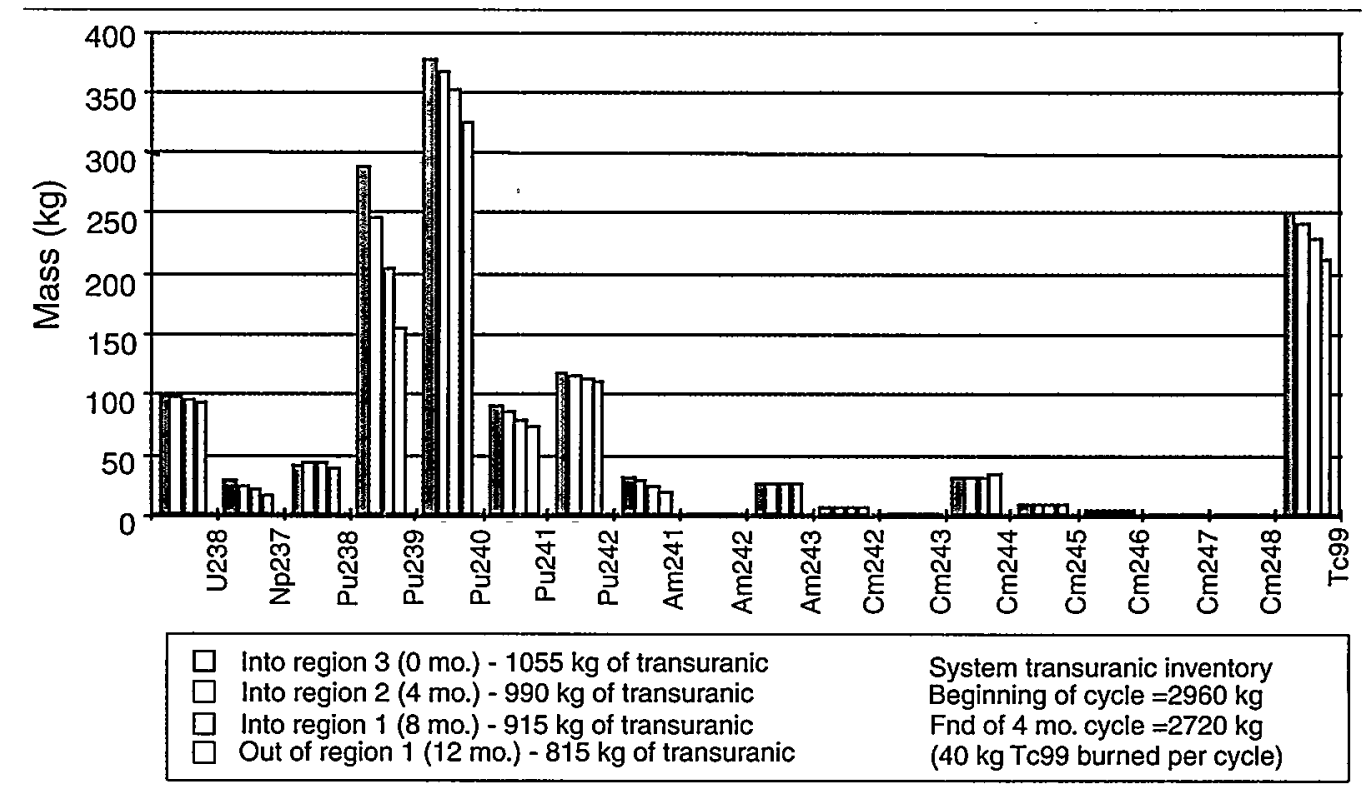

$\Delta$ FIGURE 3. Isotopic compositions and burnup in a steady-state ATW batch (2-GW, 4-month burn cyde). Batch moves from region 3 to region l; then actinides concentrated ond enriched with light woter reactor dischorge, and move into region 3 (the difference between the first and last bar is the steadystote feed rote, except for (1238). 
tions were studied to determine the effect of neutron energy spectrum on transmutation. It was found that the harder (faster) the neutron energy spectrum, the better the actinide transmutation characteristics of the system. It was demonstrated that the fast neutron spectrum of both the ATW demo and the operational ATW allows any actinide waste composition to undergo fission while generating enough excess neutrons to transmute select fission products.

Fast spectrum systems were also shown to have total waste actinide inventories comparable to or lower than those of realistically designed thermal spectrum systems and much easier waste-processing requirements. The use of LBE coolant was shown to enable both the ATW demo and operational ATW to have significant natural circulation, reducing pumping power requirements and enhancing safety. Large design simplifications were achieved by combining coolant and target fluid in a single one-liquid circuit. Fast flux, polonium production, time-dependent neutron multiplication, reactivity feedback coefficients, and other parameters of interest were also calculated and factored into the designs. Qualitative efforts to devise refueling machines and other equipment required for operation were initiated.

\section{LBE Technology Development}

The LBE technology task group successfully constructed and operated an LBE test loop during $1997 .{ }^{3}$ It is a large-scale loop that can transport LBE at over $5 \mathrm{~m} / \mathrm{s}$ speed in 2-in. inside-diameter pipes, providing a substantially realistic dynamic environment for thermohydraulic and material studies (Figure 4). The loop is the first of its kind in over 35 years outside Russia, where LBE coolant technology has been developed and deployed for submarine reactors. Our task is to transfer, verify, and extend this technology for spallation neutron source and nuclear coolant applications in ATW.

Working with our Russian partners at Obninsk (IPPE) and U.S. universities, we have acquired the basic knowledge of

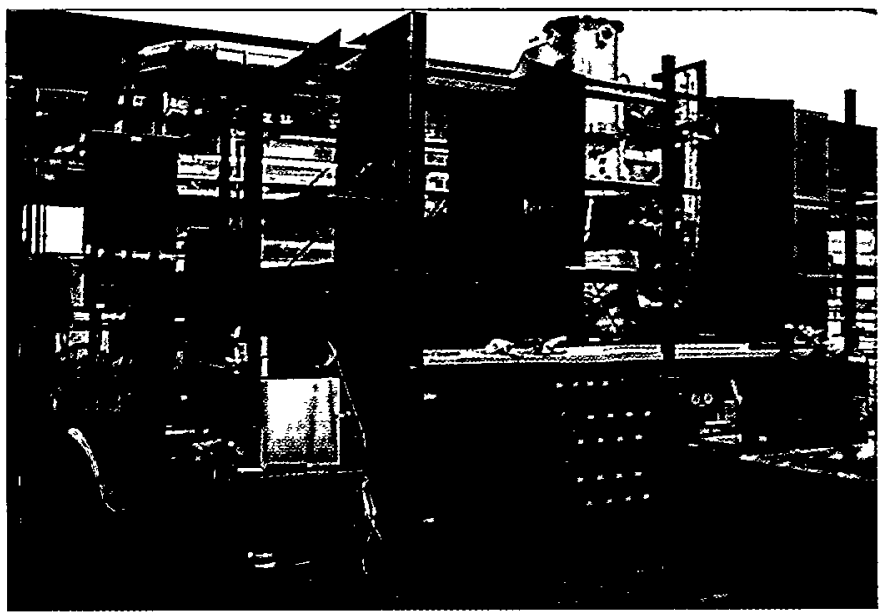

$\triangle$ FIGURE 4. The LBE test loop station before fully assembled. It wos successfully started and operated in December 1997. the corrosion control techniques employed in LBE coolant systems. This technique, through the control of the thermodynamic activities of the oxygen content, can prevent corrosion of structural materials in LBE and remove solid admixture contamination from the coolant.

We have also examined the polonium hazards associated with using LBE as a nuclear coolant. Polonium is produced in irradiated bismuth. It is a strong alpha emitter and very mobile. Working with IPPE specialists using their LBEcooled reactor experience, we investigated the polonium release mechanisms during normal conditions and accidents and found that the polonium problem can be well controlled and should not pose major radiological hazards. ${ }^{4}$

\section{1-MW LBE Test Target}

An LBE spallation target for installation at LANSCE is being constructed at IPPE (Russia) within the framework of International Science and Technology Center Project 559.
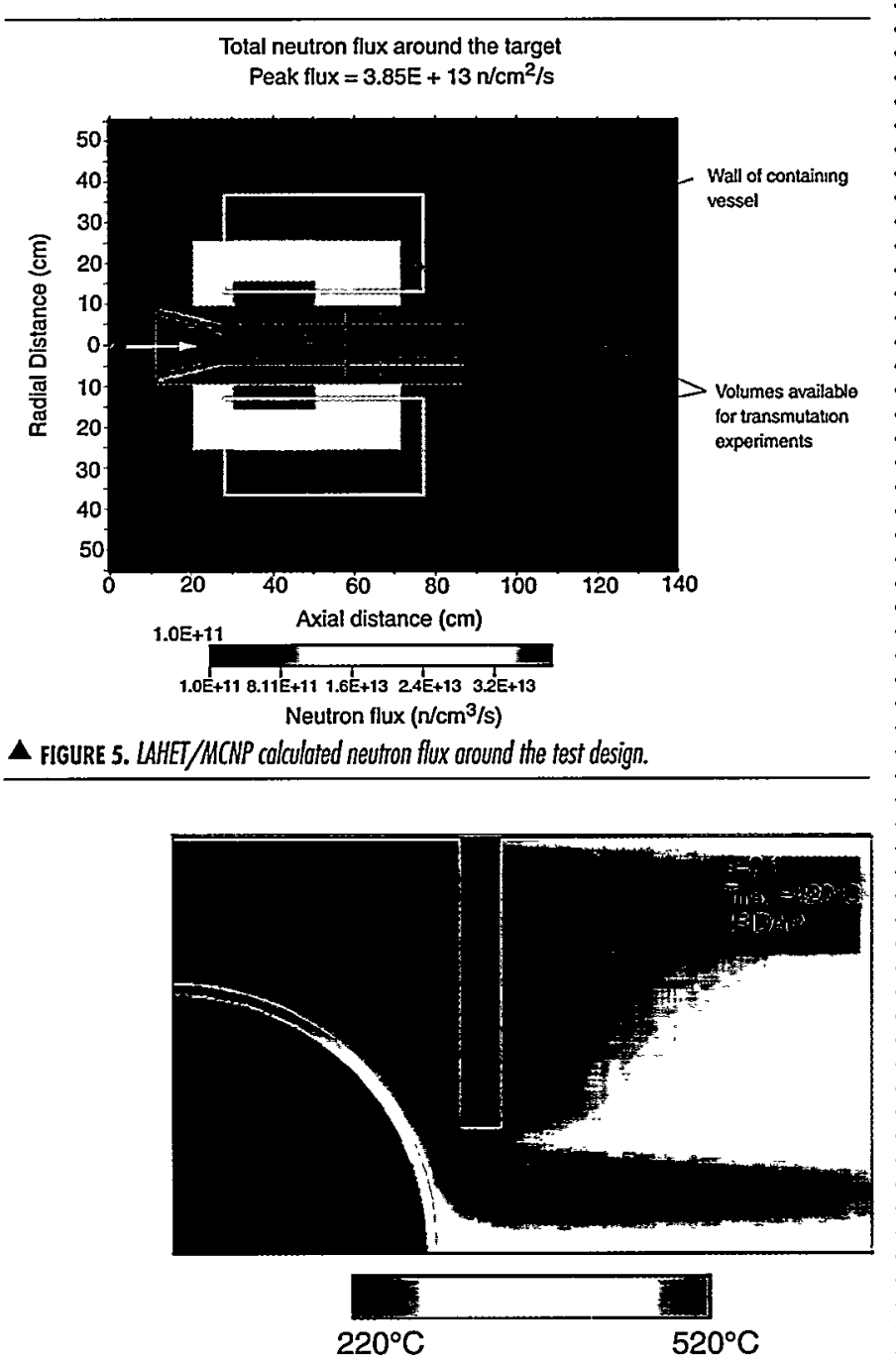

$\triangle$ FIGURE 6. Temperature field neor the target window. With more coolont flow through the center operture, the window has a lower moximum temperature. 
After finalizing the

Statement of Work and a complete set of milestones for the next 3 years, a final engineering design of the target has been produced, and detailed thermohydraulics and neutronics calculations are being performed in coordination with Russian, French, and German scientific laboratories participating in the international project (Figures 5 and 6). Arrival of the target at Los Alamos is expected in FY 2000, with first in-beam tests scheduled in the FY 2001 run cycle.

\section{Pyrochemical Processes for ATW Fuel Preparation and Cleanup Pyrochemical processing is the technology of} choice for treatment of commercial spent nuclear fuel..$^{5}$ The processes are tailored for group separations rather than the isolation of single elements, are robust, radiation resistant, and allow the process media to be recycled numerous times. A process chemistry flow sheet for the conversion of spent commercial nuclear fuel to ATW fuel was established in FY97. Work in FY98 focused on process chemistry modeling ${ }^{6}$ (Figure 7) bench-scale solvent-anode/solventcathode $\mathrm{Pu}$ electrorefining experiments, and fission product separation chemistry experiments. The process modeling effort used models currently available in the literature to develop an initial mass balance for the ATW flow sheet. Both the front-end conversion of commercial fuel to ATW fuel and the back-end recycle of ATW fuel were considered in the system mass balance. The mass balance results were the basis for the process chemistry presentation at the review by MIT. ${ }^{1}$ Experimental Pu electrorefining and fission product separation studies were initiated in FY 1998 and will be continued in FY 1999.

\section{Acknowledgments}

The authors acknowledge the entire ATW project team: K. Woloshun, V. Tcharnostskaia, M. Bjornberg, X.Y. He, D. Poston, J. King, R. Hammer, T. Langston, S. Wender, Y. C. Hu, B.G. Park, and many other individuals who contributed to the work described here: M. Carelli, W. Gudowski, Y. Orlov, E. Yefimov, A. Dedoul, N. Klimov and V. Chitaykin.

\section{References}

1. F. Venneri et al., "Accelerator-Driven Transmutation of Waste (ATW): Technical Review at MIT," Los Alamos National Laboratory technical report LA-UR-98-608 (1998).

2. C. Rubbia et al., "Conceptual Design of a Fast-Neutron-Operated High-Power Energy Amplifier," CERN/AT/95-44(ET), Geneva (1995).

3. N. Li, "Liquid Lead-Bismuth Technology Development for Accelerator-Driven Transmutation of Waste," in Proceedings of ANS Topical Meeting: Nuclear Applications of Accelerator Technology (Gatlinburg, Tennessee, 1998).

4. E. Yefimov et al., "Removal and Containment of Polonium from Liquid Lead-Bismuth Eutectic," Reg. No. 21-24/155 (1996), and "Experimental Investigation of Lead-Bismuth Coolant Quality and Its Maintenance after the Process of Polonium Alkali Extraction," Reg. No. 35-03/129 (1998), Institute of Physics and Power Engineering, Obninsk, Russia.

5. N. Li et al., "Kinetics of Pyrochemical Processes for the Los Alamos ATW Fuel Cycle," in Proceedings of Global '97: International Conference on Future Nuclear Systems-Challenge Towards Second Nuclear Era with Advanced Fuel Cycles (Yokohama, Japan, 1997).

6. M. Williamson, "Chemistry Technology Base and Fuel Cycle of the Los Alamos Accelerator-Driven Transmutation System," in Proceedings of Global '97: International Conference on Future Nuclear Systems-Challenge Towards Second Nuclear Era with Advanced Fuel Cycles (Yokohama, Japan, 1997), p. 263.

-F. Venneri (LANSCE Division), N. Li (Materials Science and Technology Division), M. Houts (Technology and Safety Assessment Division), M. Williamson (Nuclear Materials Technology Division) 



\section{Facility Upgrades Enhance LANSCE Performance}

Throughout the 1998 fiscal year, LANSCE personnel worked on a variety of upgrades to improve the safety, reliability, and performance of the facility, especially in the area of neutron scattering. As part of a $\$ 35 \mathrm{M}$, Department of Defense-funded project called the LANSCE Reliability Improvement Project, two major changes were made:

- New hardware was installed in the Proton Storage Ring (PSR) to permit direct injection of $\mathrm{H}^{-}$particles, rather than the neutral hydrogen ions that had been used in the past. This change will reduce the losses in the PSR and eventually permit higher stored currents to be achieved.

- A completely new target and moderator system was installed at the Lujan Center, together with a handling system that will allow the target to be exchanged in a few weeks rather than the 10 months that was needed when this operation was last undertaken a decade ago. The new target will also provide neutron beams for flight paths 12 through 15 at the Lujan Center. These flight paths were drilled during an earlier facility upgrade started in 1987 and have been unused since that time. For the first time, a spallation source will be able to make use of partially coupled moderators, and we can learn how these can be used to enhance instrument performance.

While the injection system for the PSR was being modified and the ring was not operational, personnel at LANSCE took the opportunity to install a replacement radio-frequency (rf) buncher that is capable of providing the voltage required to keep the proton beam out of the extraction gap. This step is part of a second, currently ongoing upgrade project-the Short-Pulse Spallation Source (SPSS) Enhancement Project-that is jointly funded by the LANSCE facility's principal sponsors, the DOE Offices of Defense Programs and Basic Energy Sciences. The rf buncher is one of several upgrades to the accelerator, described further in the section on Advanced Projects, that is supported by the Defense Programs part of the SPSS Enhancement Project.

Several upgrades to Lujan Center neutron scattering spectrometers were also made during the FY98 shutdown:

- Wide-angle detectors were installed on the PHAROS inelastic scattering spectrometer, thereby completing construction of an instrument that was started a decade ago.

- In collaboration with the National High Magnetic Field Laboratory, design was started for a 30-tesla pulsed magnet that will provide a unique capability.

- Several new neutron optical components were installed on spectrometers, including radial collimators for residual strain measurements on the neutron powder diffractometer, and a Meissner sheet polarization flipper made of a high-temperature superconducting film on the reflectometer SPEAR.

In addition, Lujan Center personnel initiated two major efforts in support of the spectrometer construction project that is funded by the Basic Energy Sciences part of the SPSS Enhancement Project:

- An extensive, user-friendly Monte Carlo simulation package was developed to facilitate the design of new neutron scattering spectrometers, both at the Lujan Center and elsewhere.

- Design was started for a new data acquisition system based on communication using the Internet.

Many of the projects described above resulted in significant construction of complex hardware-always a source of pride to the designers and builders. Photographs of much of this hardware are shown in the articles of this section.

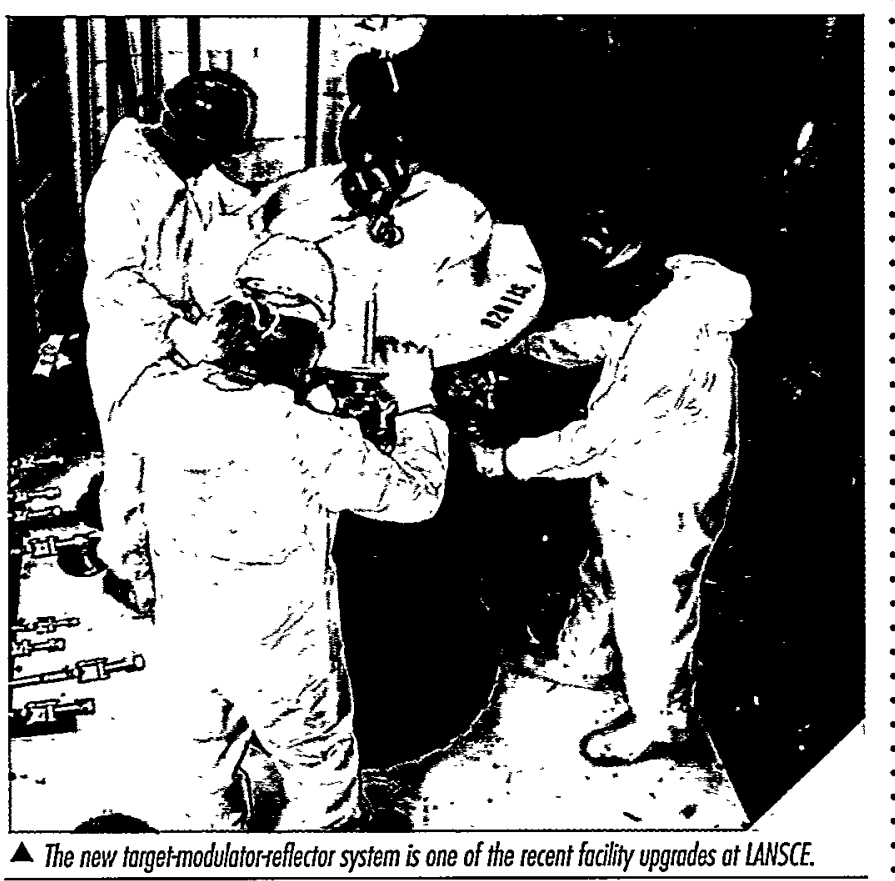




\section{Proton Storage Ring Injection Upgrade Project}

An upgrade is in progress to the Proton Storage Ring (PSR) that will allow direct injection of the $\mathrm{H}^{-}$beam into the ring, and provide a beam bump system to move the circulating beam off the stripper foil. The primary benefit of this upgrade is a reduction in beam losses in the PSR by up to a factor of 5. Beam losses at the PSR and the resulting radioactivity of ring components are the dominant factors limiting average beam current, a significant cause of equipment failure, and a major element in repair times. Reducing the beam-loss rate is key to achieving the following overall performance objectives for the PSR and the beam delivery system for the LANSCE neutron spallation source:

- routine operation at a beam current of $100 \mu \mathrm{A}$ at $20 \mathrm{~Hz}$ (a $43 \%$ increase in current)

- beam availability $>85 \%$

- less than $5 \%$ downtime from intervals $>8$ hours

There are two main contributors to beam losses in the PSR: circulating-beam losses and beam losses at injection.

Circulating-beam losses are primarily caused by nuclear and large-angle Coulomb scattering in the injection stripping foil. 1,2 These losses can be reduced by reducing the number of times the circulating beam hits the foil. In the upgrade this is accomplished by offsetting the injection horizontally and vertically and by bumping the stored beam off the foil. A vertical closed-orbit bump is introduced at the foil, and the bump is collapsed linearly to zero by the end of injection. In the old injection system, this scheme would not have been effective in reducing beam losses. A two-step injection process converted $\mathrm{H}^{-}$to $\mathrm{H}^{0}$ in a stripper magnet, then $\mathrm{H}^{0}$ to $\mathrm{H}^{+}$in the stripper foil. This process tripled the horizontal beam emittance in the stripper magnet and produced a mismatch of the injected beam. As a result, circulating-beam foil hits could not be reduced below approximately $30 \%$. To eliminate these problems, we are replacing the two-step process by direct $\left(\mathrm{H}^{-}\right.$to $\left.\mathrm{H}^{+}\right)$injection of the beam, allowing us to inject a much smaller beam that is optimally matched to the ring and to implement effective orbit bumping. Simulations predict that these measures will reduce circulating-beam foil hits by a factor of 10 .

Beam losses at injection are caused by excited $\mathrm{H}^{0}$ states produced in interactions of the injected beam in the stripper foil. These excited $\mathrm{H}^{0} \mathrm{~S}$ are stripped in the magnetic field of the PSR dipole downstream of the foil and are lost. We have demonstrated that production of excited $\mathrm{H}^{0} \mathrm{~S}$ can be suppressed by increasing the foil thickness. ${ }^{3}$ A thicker foil will, of course,

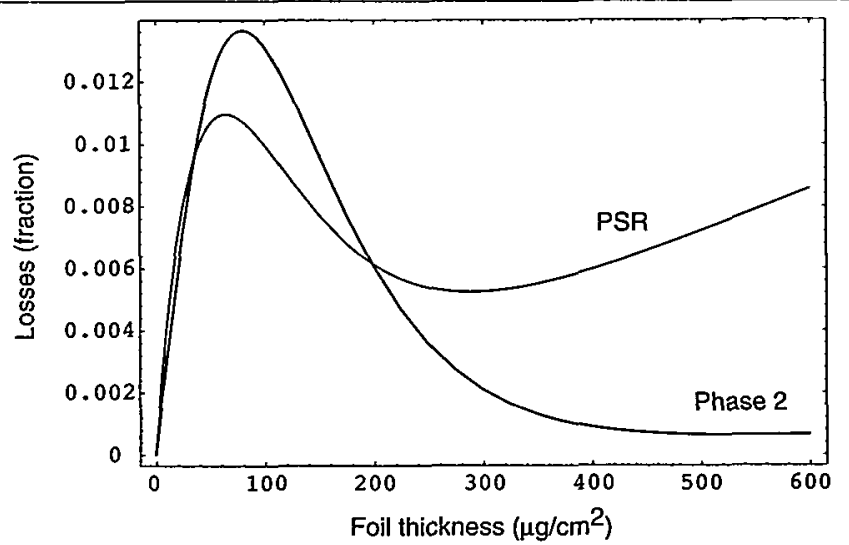

$\triangle$ FIGURE I. Totol losses in PSR versus foil thickness.

increase scattering losses in the circulating beam. Therefore, we will optimize the thickness of the stripper foil to minimize the total of injection and circulating-beam losses. Figure 1 shows the results of an optimization study of total losses versus foil thickness. With a foil thickness of 400 $\mu \mathrm{g} / \mathrm{cm}^{2}$, total losses are reduced by a factor of 5 from that for the former PSR with a $220 \mu \mathrm{g} / \mathrm{cm}^{2}$ foil.

Carbon foils produced with Dr. Isao Sugai's modified controlled ac/dc arc discharge (mCADAD) method have significantly improved the operational performance of the PSR. The mCADAD foils are produced by arc evaporating alternating layers of carbon produced with ac and then dc current. They have been tested and used for high-current $800-\mathrm{MeV}$ beam production in the PSR since 1993. The decreased shrinkage rates associated with these foils allow the use of smaller width foils, thereby decreasing storedbeam losses. Lower beam losses imply that the ring components become less radioactive, and therefore worker radiation exposures are reduced. In addition, foils produced by the mCADAD method have demonstrated lifetimes an order of magnitude higher than commercially available foils. 
TABLE 1. PSR Porometers Before ond After the Injection Upgrode

\begin{tabular}{l|l|l}
\hline Parameter & Former PSR & Upgraded PSR \\
\hline Current and repetition rate & $70 \mu \mathrm{A} @ 20 \mathrm{~Hz}$ & $100 \mu \mathrm{A} @ 20 \mathrm{~Hz}$ \\
\hline Proton beam energy & $797.2 \mathrm{MeV}$ & $798.7 \mathrm{MeV}$ \\
\hline PSR accumulation time & $650 \mu \mathrm{s}$ & $825 \mu \mathrm{s}$ \\
\hline Protons per pulse & $2.2 \times 10^{13} \mathrm{ppp}$ & $3.1 \times 10^{13} \mathrm{ppp}$ \\
\hline Injected beam offset & $\begin{array}{l}\left(\mathrm{x}_{0}, \mathrm{x}_{0}{ }^{\prime}\right)=0 \mathrm{~mm}, 0 \mathrm{mrad} \\
\left(\mathrm{y}_{0}, \mathrm{y}_{0}{ }^{\prime}\right)=8 \mathrm{~mm}, 0.9 \mathrm{mrad}\end{array}$ & $\begin{array}{l}\left(\mathrm{x}_{0}, \mathrm{x}_{0}{ }^{\prime}\right)=7.2 \mathrm{~mm},-2.0 \mathrm{mrad} \\
\left(\mathrm{y}_{0}, \mathrm{y}_{0}{ }^{\prime}\right)=22.4 \mathrm{~mm}, 3.1 \mathrm{mrad}\end{array}$ \\
\hline Closed orbit bump (linear) & None & $\begin{array}{l}\text { from }\left(\mathrm{y}_{0}, \mathrm{y}_{0}{ }^{\prime}\right)=16.0 \mathrm{~mm}, 2.2 \mathrm{mrad} \\
\text { to }\left(\mathrm{y}_{0}, \mathrm{y}_{0}\right)=0.0 \mathrm{~mm}, 0.0 \mathrm{mrad}\end{array}$ \\
\hline Foil thickness & $400 \mu \mathrm{g} / \mathrm{cm}^{2}$ \\
\hline Stored beam loss & $220 \mu \mathrm{g} / \mathrm{cm}^{2}$ & $0.046 \%$ \\
\hline Injected beam (excited $\left.\mathrm{H}^{0}\right)$ loss & $0.257 \%$ & $0.048 \%$ \\
\hline Extraction loss & $0.259 \%$ & $0.008 \%$ \\
\hline
\end{tabular}

The mCADAD technique is limited in that it is not possible to produce foils with a mass density greater than the range of 50 to $130 \mu \mathrm{g} / \mathrm{cm}^{2}$. With this limitation, a minimum of three foils will be necessary to achieve an equivalent thickness of 400 $\mu \mathrm{g} / \mathrm{cm}^{2}$ for operation at 100 $\mu \mathrm{A}$ average current. Work is currently in progress to improve on this limit.

Figure 2 is a "folded" plan

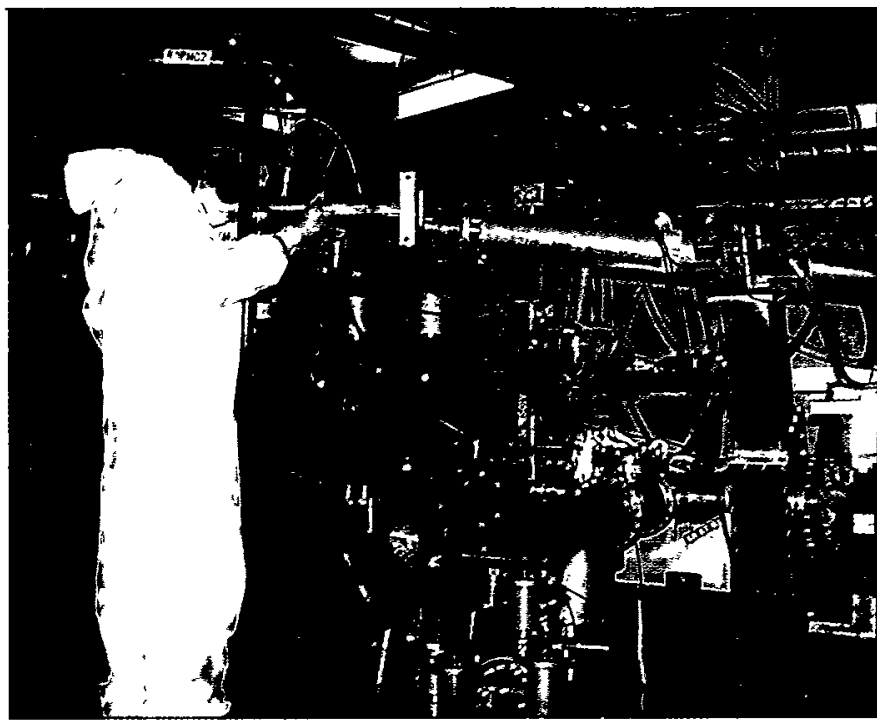

$\triangle$ FIGURE 3. A fecthicion (in ontitC clothing) adjusting a mounting brocket for a defector to monitor foil hits. The defector is located just downstream of the vocuum chamber housing the stipper foil.

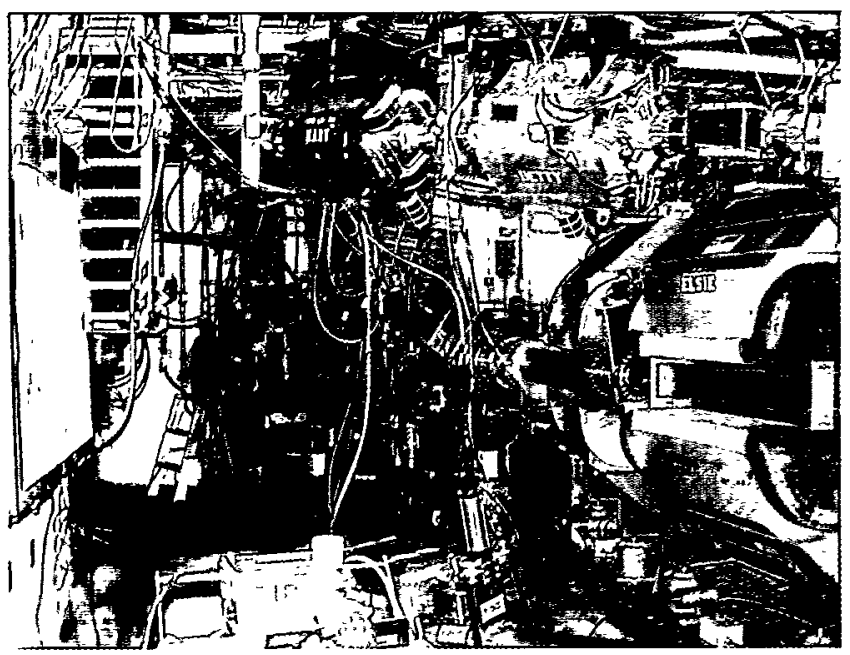

$\Delta$ FIGURE 4. View of the C-magness ond $H / H^{0}$ dump line leading to the beam dump. The magnet labeled ELSIE is the first of the two C-magnets in the ring. Those labeled MARY and BETTY in the top of the picture are part of the extroction line that transports beam from the PSR to the neution production target. view of the injection line, PSR injection section and adjacent ring sections, and the waste-beam dump line. A skew section transports the beam from the $\mathrm{H}^{-}$transfer line to the PSR level. A general drawback is that skewed beam-line elements couple the transverse planes, leading to emittance growth. In this design, skew quadrupoles upstream and downstream of the skew section, together with a quadrupole at the dispersion crossover point, uncouple the beam transfer matrix and eliminate beam emittance growth. A four-quadrupole matching section is used to tailor the beam at the stripper foil to optimize orbit bumping. The matching section is in a zero-dispersion region so transverse and longitudinal beam parameters can be tuned independently. An achromatic final bend diverts the beam around a PSR dipole and injects the beam into the merging dipole. Injection with zero dispersion reduces the size of the injected beam and thus the number of foil hits by the circulating beam.

Changes to the PSR include the merging dipole, programmed bump magnets to produce the closed-orbit bump, and using two C-magnets to replace the first dipole downstream of injection. Implementation began in August 1997 with removal of existing equipment; installation of new and upgraded equipment began 2 months later. Commissioning of the upgraded ring began in July 1998. The expected performance of the upgraded ring is summarized in Table 1. Figures 3 and 4 show a portion of the newly installed injection line near the stripper foil and downstream of the foil.

\section{References}

1. R. J. Macek, et al., Proceedings of the 1988 European Particle Accelerator Conference 1252, IEEE, Piscataway, NJ (1988).

2. R. J. Macek, et al., Proceedings of the 1993 Particle Accelerator Conference 3739, World Scientific Publishing, Singapore (1993).

3. M. S. Gulley et al., Phys. Rev. A 53, 3201 (1996).

-D. H. Fitzgerald, M. J. Borden, H. Ahn, B. Blind, R. J. Macek, F. Neri, M. A. Plum, C. R. Rose, C. A. Wilkinson, M. V. Zumbro (LANSCE Division), Isao Sugai (KEK, High-Energy Accelerator Organization, Tanashi Branch), H. A. Thiessen (Physics Division) 


\section{Radio-Frequency Buncher Refurbished for the Proton Storage Ring}

During 1997 and 1998, the radio-frequency (If) buncher in the Proton Storage Ring (PSR) was refurbished as part of the Short-Pulse Spallation Source (SPSS) Enhancement Project (see complete article on the SPSS Enhancement Project in the Advanced Projects section). The objective of this upgrade was to increase the peak rf voltage capability from $12 \mathrm{kV}$ to $18 \mathrm{kV}$ and to eliminate of instabilities in the existing system. The higher voltage is required for future $200-\mu \mathrm{A}$ (at $30 \mathrm{~Hz}$ ) operation. Major elements of the buncher refurbishment were

- upgrades to the rf amplifiers, including the development of a new intermediate power amplifier (IPA)

- relocation of the IPA from the ring equipment building to the PSR

- modification of the buncher gap, ferrite assembly, and bias circuit

- upgrades to the electrical and cooling-water utilities

We installed the refurbished buncher (Figure 1) in July 1998, and commissioned it in September 1998. The system has met or exceeded all of its design goals and is now $100 \%$ operational. In particular, testing has shown that

- the buncher can reliably provide the required $18 \mathrm{kV}$ at $2.8 \mathrm{MHz}$

- the effective output impedance is very low, resulting in minimal beam loading

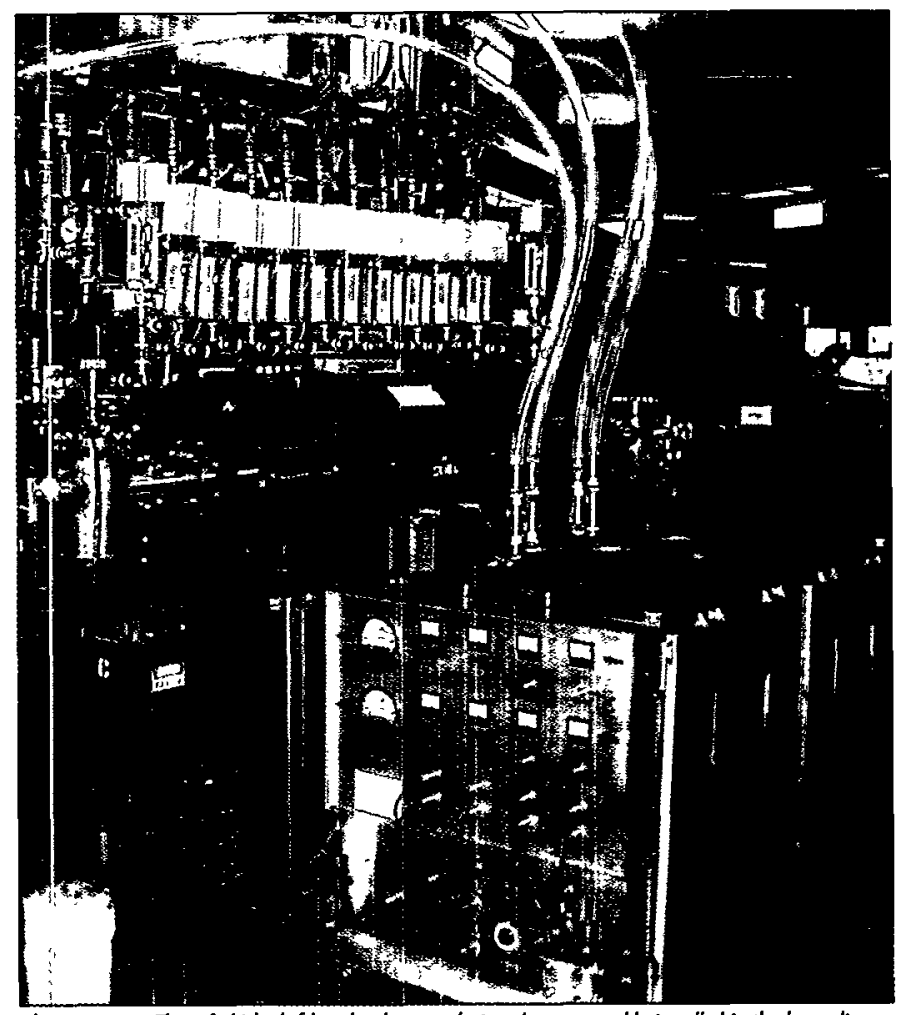

$\Delta$ FIGURE 1. The refurbished ff buncher hos o redesigned gap ossembly instolled in the beam line that sits on top of the finol power omplifier. The new intermediate power amplifier is in front.

- the If instabilities have been eliminated

-Paul Lewis (LANSCE Division) 


\section{Accelerator Upgrades Improve Reliability}

The LANSCE materials science user community has long protested the inability of the accelerator-driven spallation source to deliver the reliability, availability, and predictability that reactors produce. In 1992, the Kohn Committee expressed the dissatisfaction of the community clearly and challenged the builders of new spallation sources to meet the expressed needs of the user community. Unfortunately, the needs of the materials community for improvement occurred during a time when the sponsors of the Los Alamos Meson Physics Facility (LAMPF) were phasing out their interests in continued operation, and the materials science community was not able to fund improved operation.

The recent collaboration of the Department of Energy Offices of Defense Programs and Basis Energy Sciences to operate LANSCE provided a window of opportunity to repair some deficiencies and catch up on deferred maintenance.

\section{Accelerator and Beam Delivery Upgrades}

LANSCE responded to users' needs during the past 4 years with a number of upgrades selected to improve operational reliability and beam predictability. In addition, workers emphasized processes to increase availability and reduce operating costs.

The largest single effort has been the LANSCE Reliability Improvement Project. This project was designed to develop and install a new target-moderator-reflector system (TMRS) for the Lujan Center and to install a new direct $\mathrm{H}^{-}$injection scheme in the Proton Storage Ring (PSR) and has occupied a major fraction of the Division's resources during 1996 through 1998. As FY 1998 drew to a close, this effort paid off. On November 19, 1998, $100 \mu$ A were delivered to the Lujan Center target and moderated beams of neutrons therefore became available in the Lujan Center on a continuing basis.

The drift-tube linac accelerator has often accounted for a significant portion of unplanned downtime. Vacuum, radiofrequency (rf) power, and the mechanical structures have each contributed to the problem. A 4-year-long installation of a "soft" vacuum system for the drift-tube linac was completed in 1998. This "soft" vacuum space buffers the "hard" vacuum from atmospheric pressure within the drifttube cavity. The modification was necessary because the vacuum seals originally separating the hard vacuum space from atmosphere had deteriorated and could not be easily replaced. The vacuum integrity of the linac is now greatly improved, and recovery from venting of the vacuum space is much improved. The net result is improved availability of beams from the linac.

Several improvements to the linac if power systems have been completed. The first amplifier stage has been replaced

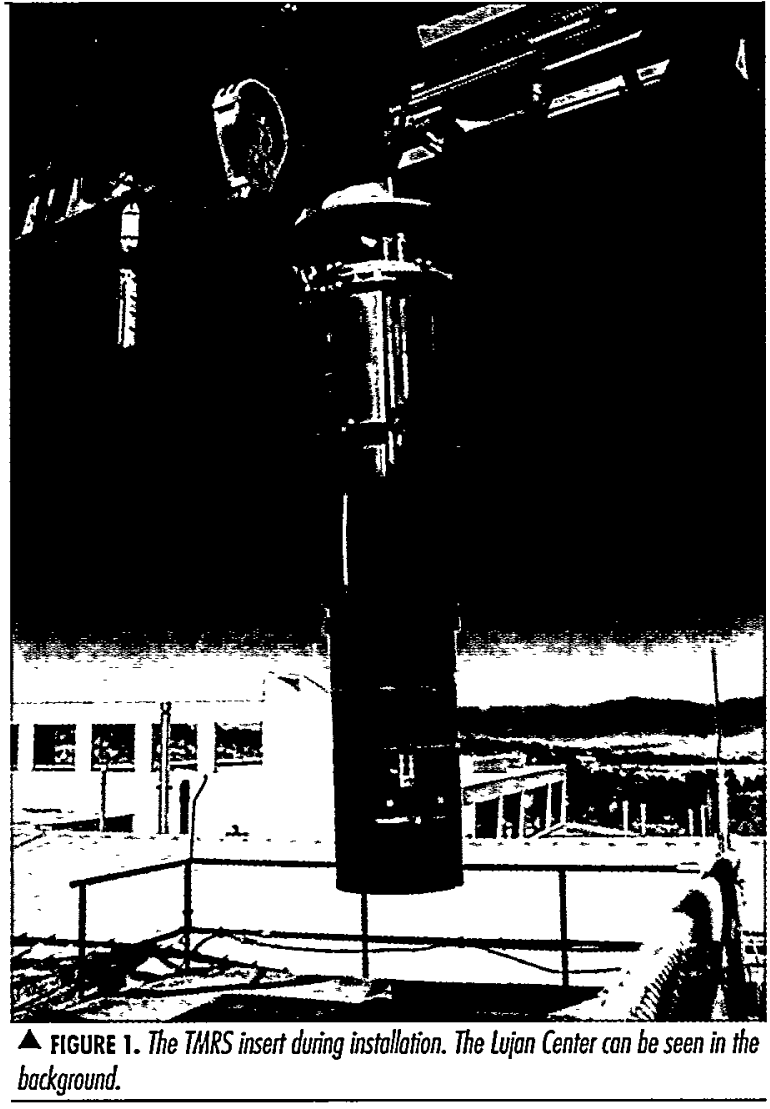

with a custom-designed, commercially constructed $5-\mathrm{kW}$, 201.25-Mhz solid-state amplifier. Operation has been highly reliable and maintenance costs have been reduced over the original vacuum tube units. The four drift-tube-linac capacitor rooms were cleaned up of PCB oil spills and modified to eliminate oil-bath cooling systems. The final and intermediate power amplifier power supplies were separated by the addition of new power supplies for the intermediate amplification stage. This modification improves operational stability by eliminating the interactions between these two stages.

An improvement program for the personnel access control systems throughout the accelerator modernized the design and technology used to ensure that personnel are excluded from hazardous areas. The implementation at LANSCE has followed Laboratory-designated modern standards that conform to the best practices in the accelerator community.

The LANSCE accelerator-control system (Figure 2) is a distributed computer-based system that has evolved from the original LAMPF system, which was the first accelerator-control system to rely entirely on a computer. Because the technology of computing and network communications is changing rapidly, a careful and continuous effort is required to avoid obsolescing. The worldwide user 
community and LANSCE staff require information from the computer system on both present and past performance of the facility as well as on maintenance and operations procedures. During the past year, the control console was extensively modernized to eliminate obsolete equipment and to incorporate modern technology. A 5-year plan integrates the controls into a single architecture, and a longterm maintenance plan ensures attention to all requirements. The Year 2000 issue was addressed to ensure that no serious consequences might interrupt operation.

Water systems have contributed to increasing linac downtime. Over the past several years, the tube-and-shell heat exchangers were replaced with stainless-steel plate and frame heat exchangers that have improved heat-exchange efficiency and maintenance characteristics. Some soldered copper piping was replaced with a Victaulic-fitting technology to reduce joint failures that have in recent years leaked water and interrupted beam delivery.

LANSCE uses over 800 magnet power supplies (up to 3,000 amperes) for operations. The power supplies for the quadrupoles in the linac were replaced with solid-state improvements to eliminate mechanical regulators. Failures of the old equipment occurred randomly with frequencies as often as once per week.

As a result of this emphasis on reliability improvements and the availability of funding to achieve them, LANSCE achieved a record performance for beam delivery during FY 1997 (see the Experimental and User Program section). The recent achievement of $100 \mu \mathrm{A}$ to the Lujan Center target simultaneous with 1-mA beam delivery to Area $\mathrm{A}$ and $2-\mu \mathrm{A}$ micro-chopped beam to WNR establishes a new level of performance for LANSCE. Higher beam current and longer operating periods are well within reach.

The results and activities of LANSCE operations, maintenance, and minor upgrades are detailed in Laboratory reports LAMPF/MLNSC/WNR Operations-FY 1994-1995 (LA-UR-96-3258), Accelerator and Beam Delivery Operations Progress Report-FY 1996 (LA-UR-97-4015), and Accelerator and Beam Delivery Operations Progress Report-FY 1997 (LA-UR-98-2264). The Accelerator and Beam Delivery Operations Progress Reports for FY 1996 and FY 1997 are on the compact disk located on the inside back cover of this report. The FY 1998 report will be published soon.

\section{Future Improvements}

LANSCE management is now faced with the task of balancing resources to maximize the productivity of the facility by continuing to improve operations while pursuing research programs that yield compelling results.

The largest single pending improvement project is the Short-Pulse Spallation Source (SPSS) Enhancement Project, which will install a higher brightness negative-ion source and an improved beam-bunching system in the PSR to allow increased average currents to $200 \mu \mathrm{A}$ at a $30-\mathrm{Hz}$ repetition rate. Some additional upgrades, such as more cooling for the target moderators, are required to support the improvements of the SPSS Enhancement Project.

The proposed long-pulse spallation source $(1,250 \mu \mathrm{A}$ at $60 \mathrm{~Hz}$ and $15 \mathrm{flight}$ paths) has the potential of making major contributions to the facility as well as fostering new directions in neutron scattering facilities.

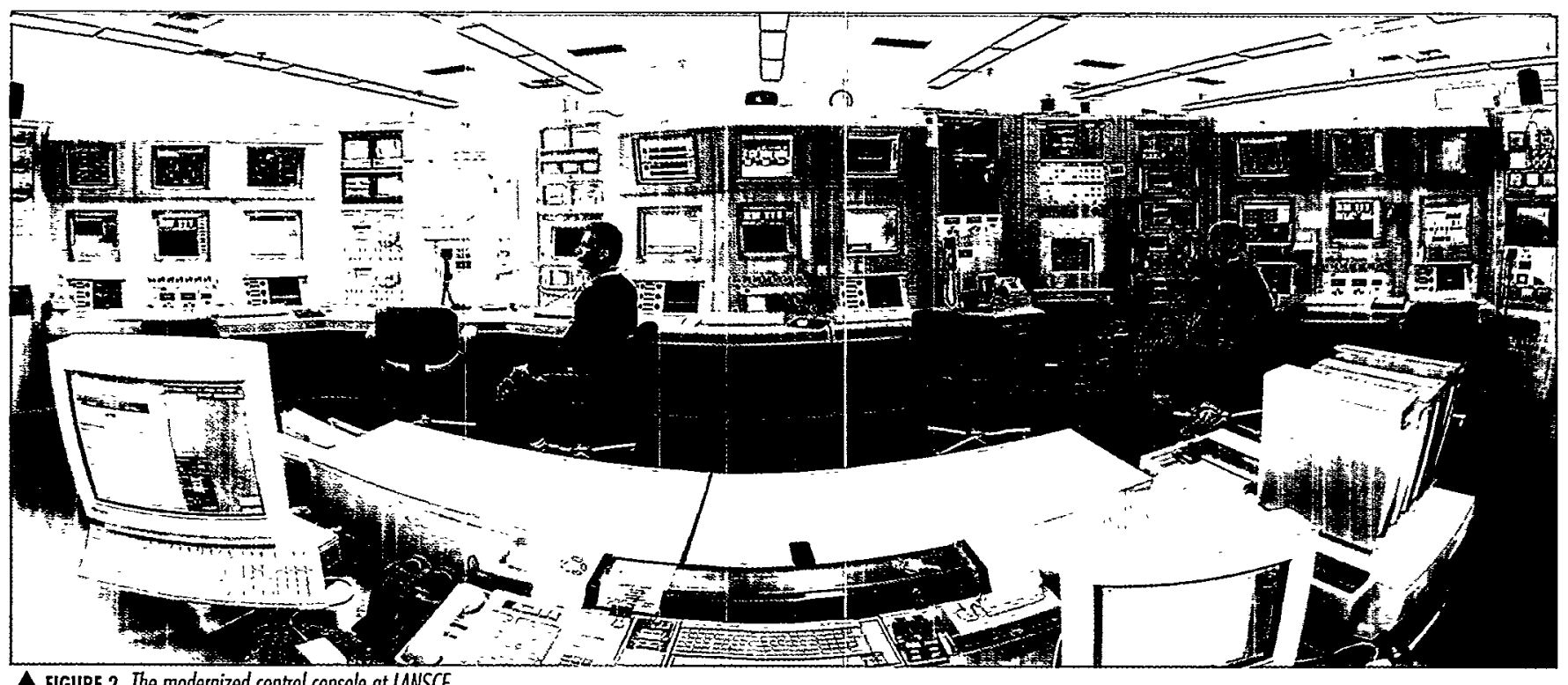

$\triangle$ FIGURE 2. The modernized control console af LANSCE. 
The long-range maintenance plans for some systems (e.g., controls, rf power, and water) will accommodate the critical need for resources to support the major upgrade projects. Some personnel and instrument access control systems will be completed. Spare magnets, especially the ring extraction septum magnet, will be procured to ensure rapid recovery from both anticipated and unanticipated failures.

The new target and beam-injection facilities call for additional improvements. A second target needs to be completed and plans developed for assembly and storage of spare devices. An irradiated materials facility will allow examination and diagnosis of radioactive components of the TMRS when failures occur. A weather-resistant enclosure for the Lujan Center target crane (Figure 3) will prolong the life of that facility and provide more expeditious repairs and maintenance when crane use is required. Additional improvements that will increase our ability to tune quickly and understand the operation of the facility include a longstroke wire scanner for the PSR, bump-tracking data acquisition, and as-built drawings and documentation.

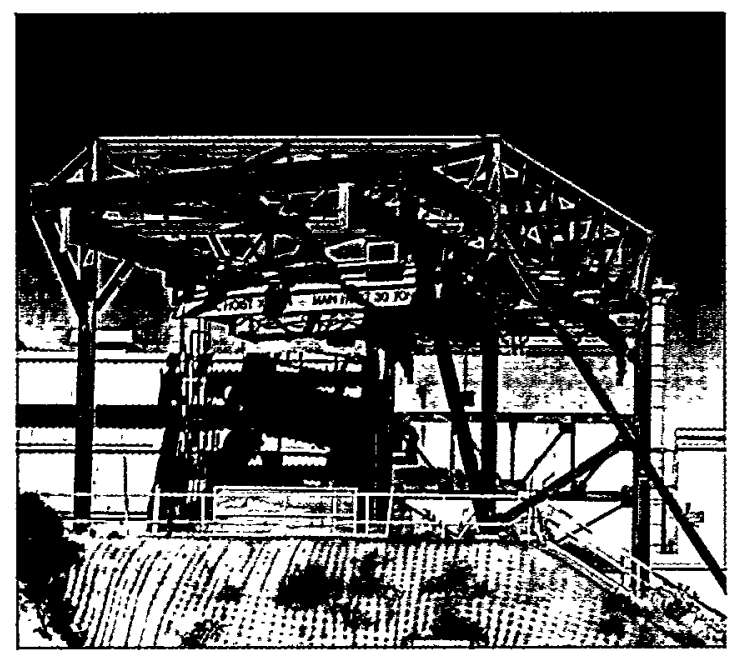

$\triangle$ FIGURE 3. Lujon Center torget crone.

The vision is clear and planning is in progress. Our goal of a flexible and highly reliable neutron science center is in sight and highly attainable.

\section{1-MHz Capacitor Room Upgrades}

Before the capacitor room upgrade, each of the four 201-MHz capacitor rooms had crowbar resistors that were immersed in oil tanks for cooling. We considered the oil tanks to be an environmental and fire hazard, so we decided to replace these units with air-cooled resistors as shown in Figure 4.

With forced-air cooling, it was necessary to reduce the heat load, so we decreased the total crowbar resistance from

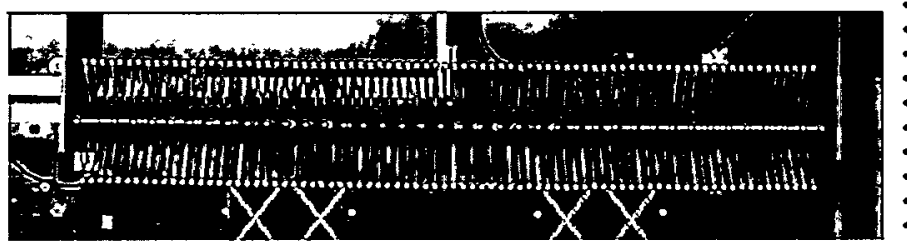

$\triangle$ FIGURE 4. This lowinductonce oir rooled resistor replaces on oilimmensed crowbor resistor.

10 to 3 ohms. This led to a need for faster crowbar triggering, which was accomplished with an improved directtrigger circuit to fire the ignitron.

A subsequent personnel safety improvement was also included in the upgrade. A combination fuse-spring apparatus was attached to each capacitor as shown in Figure 5. When a high-voltage capacitor fails, the fuse-wire opens; the spring automatically places an external short across the capacitor.

The faulty capacitor is not only removed from the highvoltage circuit, but also placed in a safe mode before personnel enter the room. Previously, we depended upon a ceramic wire-wound resistor to act as the fuse, and it was necessary for personnel to manually short each capacitor with a wire.

The initial upgrade - completed in February 1997-was on the module 2 capacitor room. Upgrading of the other three capacitor rooms was completed during December 1997.

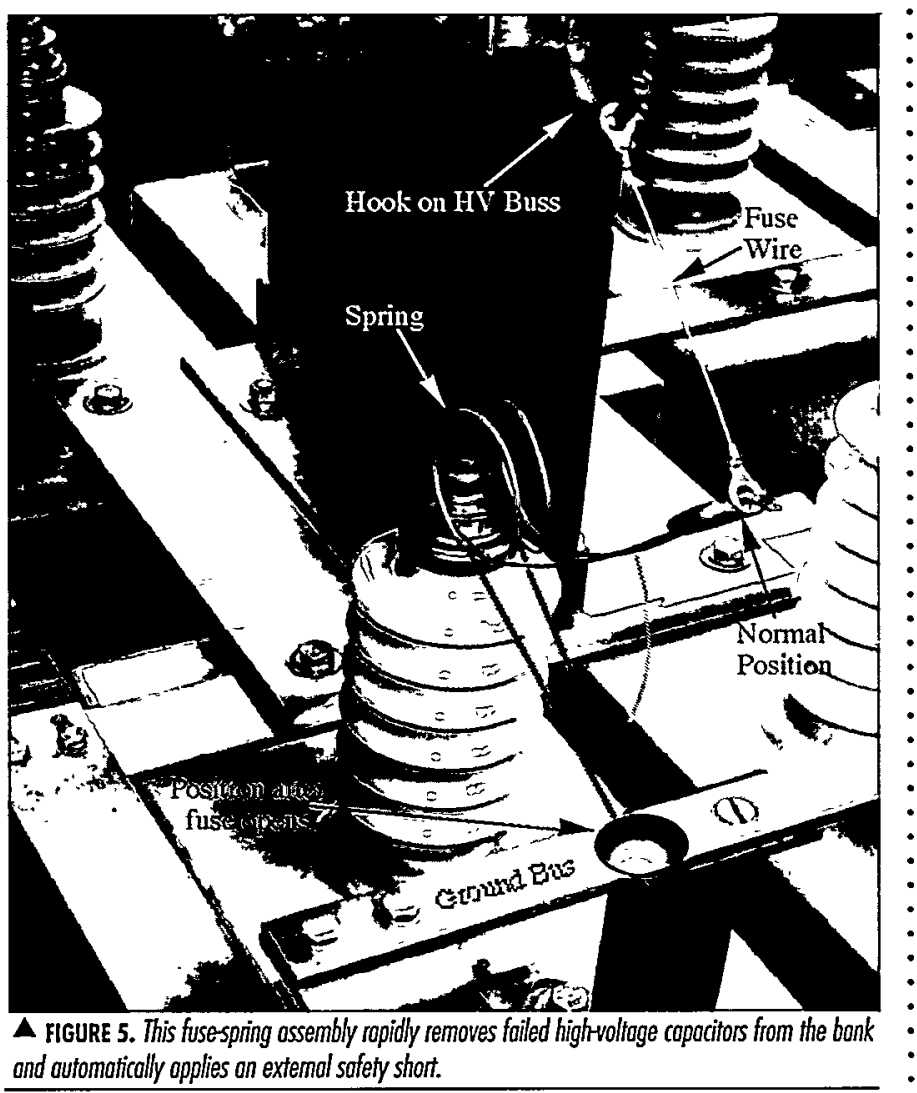

LANSCE - Activity Report 135 
This work won a 1997 Division Director's Award for the LANSCE-5 radio-frequency team.

- C. Friedrichs (LANSCE Division)

\section{New Zero-Crossing Detector for the LANSCE Accelerator Timing System}

The LANSCE linac provides pulsed beams of protons for direct use and for production of spallation neutrons. One of the instruments using the pulsed neutron beam at the Lujan Center, the PHAROS high-resolution chopper spectrometer, employs a fast monochromating Fermi chopper for neutron energy selection. For the Fermi chopper to be effective, its rotor position must remain precisely synchronized to the proton beam arrival time on the spallation target. The finite bandwidth of the combined chopper rotor and drive system implies that precise synchronization is most easily achieved when the linac reference clock frequency is fixed and stable.

However, because certain klystrons used in the accelerator exhibit gain sensitivities relative to their ac line phase operating point, optimal linac operation requires the reference clock remain well synchronized to the ac line. Previously, to satisfy this latter constraint, each machine cycle occurred a fixed delay after each zero-crossing (ZX) of the ac line voltage. Because of phase and frequency fluctuations present in the ac line, this resulted in inefficient chopper operation. In January 1997, however, the Master Timer System, which generates the accelerator timing signals, was upgraded with a new ZX detector to improve the phase and frequency stability of the linac reference clock and related timing signals without impacting accelerator performance.

A block diagram of the new $\mathrm{ZX}$ detector is shown in Figure 6. The heart of the upgrade is a narrow-band filter, which is implemented in the form of a simple phase-locked loop (PLL) circuit.

Overall the new ZX detector consists of three major components: the prefilter, the PLL, and the fault detection circuit. The prefilter section performs the functions of reducing the ac line voltage to a manageable level and curtailing the line voltage sine-wave impurities. Once the prefilter has conditioned the input, the $\mathrm{ZX}$ is detected in the PLL section using a precision comparator. The ZX pulse train is subsequently filtered with the narrow-band filter also contained in this section. The bandwidth of this filter was selected to prevent the cumulative phase error between the raw and filtered reference signals from exceeding an available window of several hundred microseconds.

Recent measurements on the linac revealed that strict synchronization to the ac line is not required, and proper beam operation is maintained as long as machine cycles occur within this window. Following the filtering action of the PLL, the pulse train enters the fault detection circuit where the time delay from pulse to pulse is checked for instantaneous frequency shifts. When a fault is detected, an oscillator, which was synchronized to the last known good ZX pulse, is switched into operation. The circuitry will automatically switch back to the detected ZX signal once the fault has cleared.

\section{- J. Rybarcyk (LANSCE Division)}

\section{Development of the Personnel Access Control System}

The personnel access control system (PACS) is an upgrade and replacement for the personnel safety systems and instrument personnel safety systems that are used at LANSCE to control access to radiological (prompt radiation) and, in some cases, high-voltage hazards. This system was designed to achieve compliance with requirements developed to standardize and improve the reliability and quality of access control systems used at accelerator facilities. These requirements are embodied in DOE Order 5480.25 and its guidance and the Los Alamos Laboratory Standard LS107-01. During the annual maintenance periods for 1995/96 and 1996/97, eighteen PACSs were

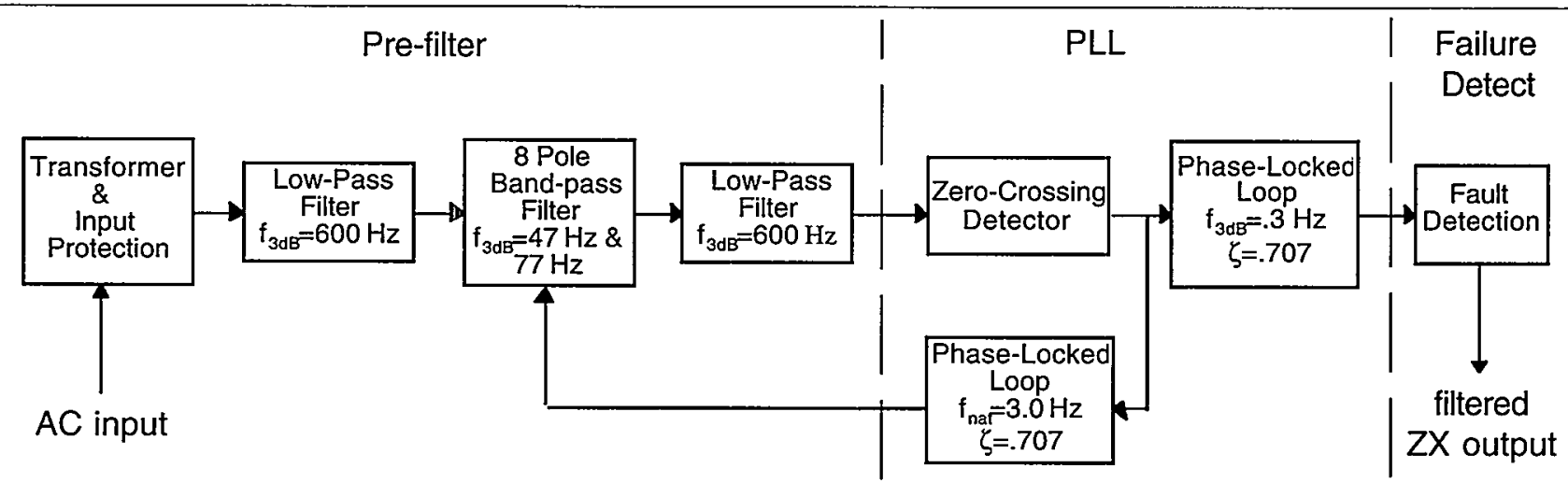

AIGURE 6. Block diogrom of new zero-crossing circuit. 
installed in the LANSCE beam-delivery system, and a plan is being developed to complete the upgrade of all accelerator facility access control systems to the PACS.

The PACS is a component of the radiation security system (RSS). The RSS is the umbrella accelerator safety system at LANSCE. As such, the PACS is subject to the same requirements as engineered safety systems. The PACS provides redundant interlock contacts indicating its protective state from redundant strings of sensors. These are processed by the RSS to provide the required protective functions. A typical PACS would include barrier sensors and personnel interface in and near the area being controlled, a main electronics enclosure, a battery backup and key release node, and an operator interface in the Central Control Room.

The PACS design was a team effort lead by the RSS team. This task was cross-organizational, very large in scope, and involved the stakeholders from the beginning of the project. Accelerator operations personnel were involved in a review of the proposed system and were included in any modifications that were felt to have an operational impact.

The PACS was designed to replace several older personnel safety systems with a single, modern, unified design. Lessons learned from the operation over the last 20 years were incorporated into a redundant-sensor, single-point failure safe, fault-tolerant, and tamper-resistant system that prevents access to the beam areas by controlling the access keys and beam stoppers. The design includes innovative concepts that are not prevalent in the accelerator community. A graded approach was applied to the design philosophy. Critical safety circuits are separated physically from circuits that control the administrative and data collection and distribution functions. This allows maintenance to be performed on the noncritical portions of the system without affecting the configuration control over those sections that provide the safety functions. Modular construction allows for rapid replacement of line-replaceable units to minimize downtime for service. The most critical assemblies are battery-backed-up, relay logic circuits; less critical devices use programmable logic controllers for timing functions and communications. Most of these replaceable units can be changed without powering down or dropping the "secured" access control state. Reviewers external to Los Alamos National Laboratory have examined the operational safety of the design.

The PACS has proven to be a reliable system in the field. The first 10 installations operated without failure during the operations period in 1996. The interlocked nature of the key release systems completely eliminated RSS trips associated with entry into PACS-controlled areas. The APT project recognized the value of the system and is installing the
PACS on the LEDA. A variation of the original PACS design, called "PACS Lite," is being developed to offer the PACS advantages to the experimental areas in the Manuel Lujan Jr. Neutron Scattering Center, WNR facility, and future LANSCE experimental areas.

\author{
- J. C. Sturrock, F. R. Gallegos, M. J. Hall (LANSCE \\ Division)
}




\section{New Spallation Target and Moderator System at the Lujan Center}

We are upgrading the short-pulse spallation target at the Manuel Lujan Jr. Neutron Scattering Center in order to provide four new flight paths and to increase the beam power capability from $56 \mathrm{~kW}$ to $160 \mathrm{~kW}$. This upgrade will improve beam availability and will reduce the target change-out time from about 1 year to about 3 weeks.

The key to a 3-week replacement time is a modular targetmoderator-reflector system (TMRS) design and a new roof penetration. Figure 1 shows a cutaway view of a target change operation. The tungsten targets, moderators, and the inner reflectors are integrated into a single TMRS module. A new bridge crane will pull the spent target module through the roof into a special transport cask.

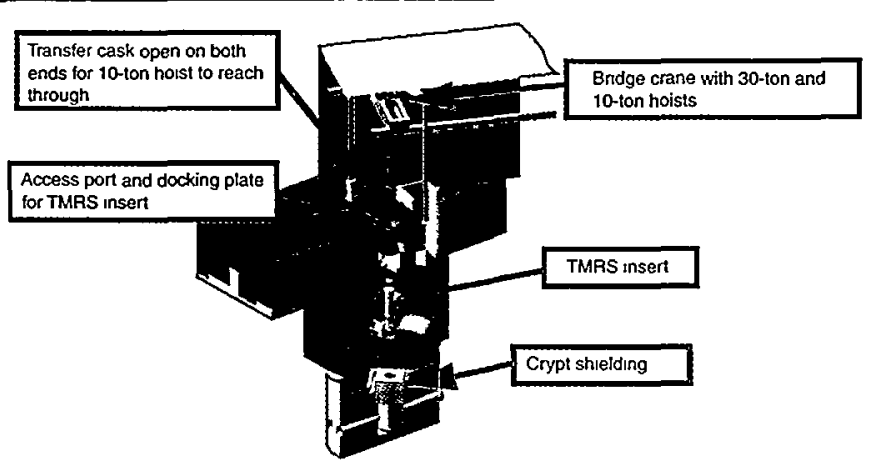

A FIGURE I. Cutawoy view of a forget change operation.

To accommodate the new, modular TMRS insert, the shielding inside the target crypt vacuum vessel had to be redesigned. All of the old target components and the crypt shielding were removed using remote handling techniques. The major vacuum leaks in the crypt were repaired during this process. The shielding was pre-assembled in a staging area before it was installed in the crypt using the new bridge crane.

Moderator performance requirements (plus the need to simultaneously service 16 neutron flight paths with highintensity neutron beams) presented a significant challenge to the Lujan spallation physics/engineering team. This team performed extensive calculations both for the neutronic performance and for support of the engineering design. Figure 2 shows the basic target/moderator geometries that are used in the design of target systems for spallation neutron sources. Los Alamos pioneered the concepts of split-target/flux-trap moderators and upstream/backscattering moderators. The new upgraded Lujan Center target system uses both of these moderator concepts in two tiers (Figure 3 ). There are four flux-trap moderators viewed in transmission (serving twelve neutron flight paths) and two upstream/backscattering moderators (serving four neutron flight paths). The designers of

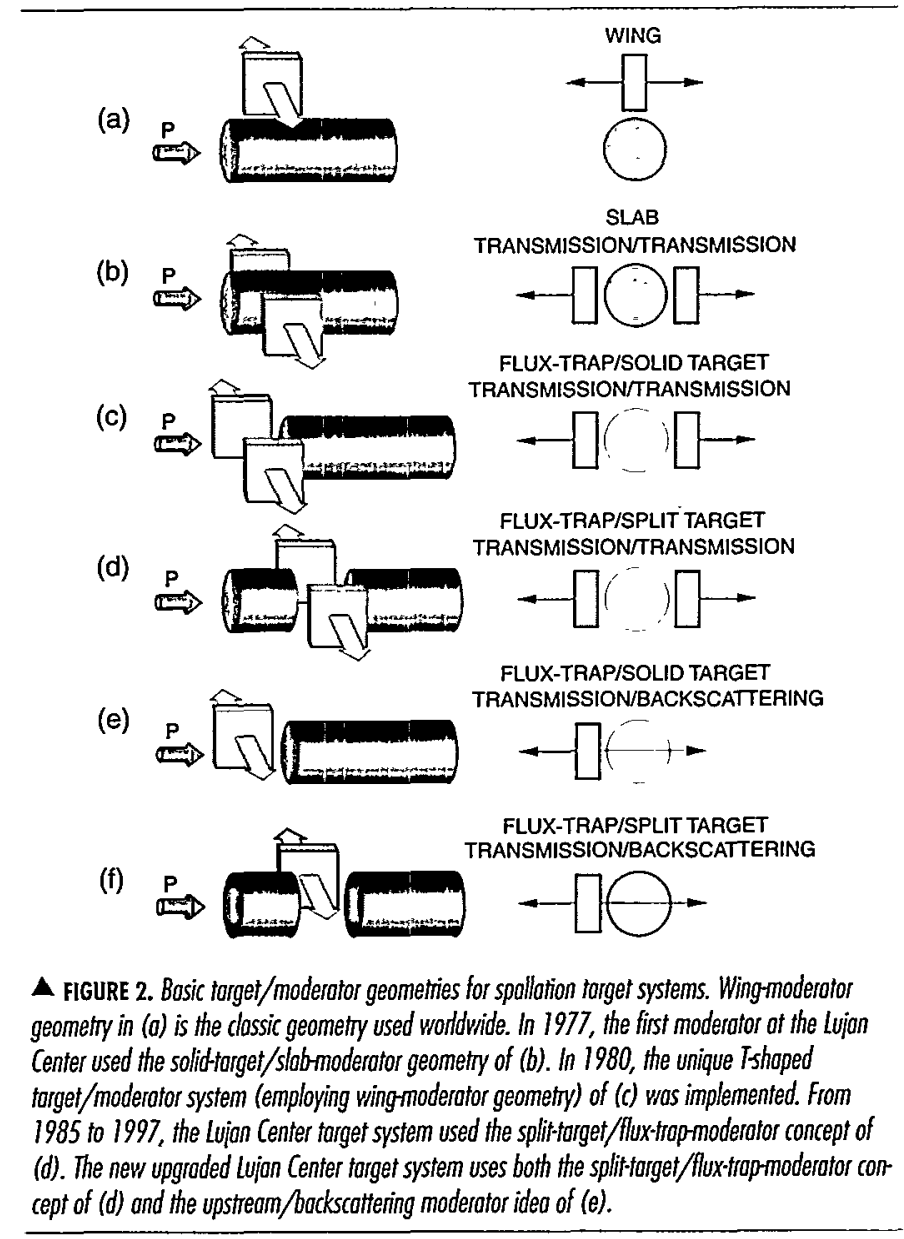

the Lujan Center scientific instruments dictated the moderator types and required neutronic performance. The design power capability of the upgraded target system is $160 \mathrm{~kW}$ (200 $\mathrm{mA}$ of $800-\mathrm{MeV}$ protons).

Moderators slow down and thermalize these fast neutrons by collisions with hydrogen atoms in the moderators, producing useful neutron probes for basic and applied research programs. Reflectors enhance neutron production by sending neutrons escaping from the target system in unwanted directions back into the moderators for other tries at becoming useful neutrons. Useful neutrons have the requisite energy, head in the right direction, and arrive at the sample location at a beneficial time. In addition to the choice of target, moderator and reflector materials, poisons, decouplers, and liners (neutron-absorbing materials) tailor the intensity, temporal, and energy characteristics of the neutron pulses emitted by a moderator.

The upgraded Lujan Center target system uses tungsten targets, light-water and liquid-hydrogen moderators, and beryllium and lead reflectors. Ambient temperature light-water moderators produce room-temperature 


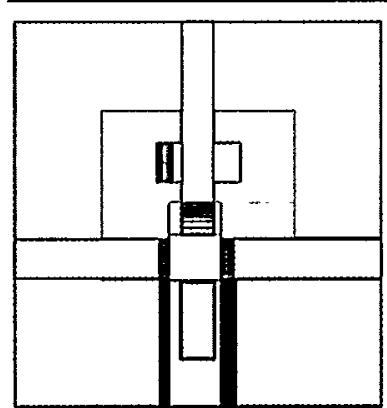

(a)

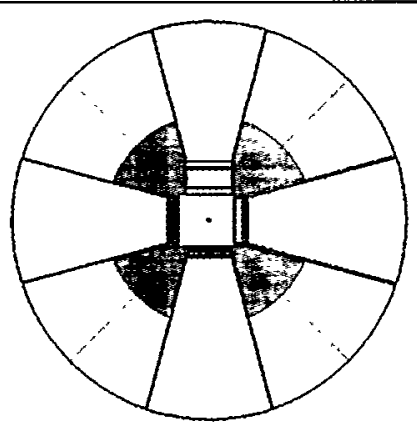

(b)

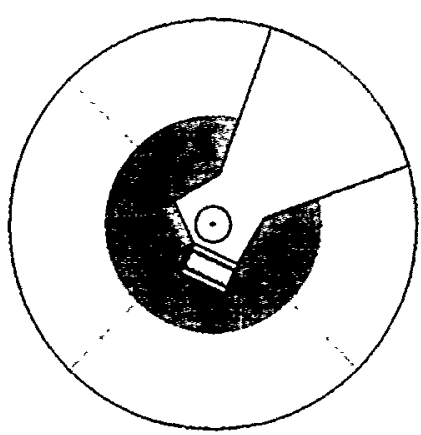

(c)

$\triangle$ FIGURE 3. Monte Casto geomerty model of the new upgraded lujon Center torget system showing (a) the elevation view, (b) the plan view through the lower moderator tier, and (c) the plan view through the upper moderator hier.

"thermal" neutrons, whereas liquid-hydrogen moderators (at $20 \mathrm{~K}$ ) produce "cold" neutrons having lower energies. In the flux-trap region of the upgraded target system, there are three ambient-temperature light-water moderators and one liquid-hydrogen moderator. In the upstream/backscattering location, there is one light-water moderator and one liquid-hydrogen moderator. The three light-water flux-trap moderators are neutronically decoupled and optimized to produce short pulses of neutrons with minimal tails in the time distributions. The liquid-hydrogen flux-trap moderator has been partially coupled to the reflector (some decoupler material removed) to gain neutron intensity at the expense of some broadening in the neutron time distributions. The upstream/backscattering moderators are partially neutronically coupled to the reflector to gain neutron intensity, and the thicknesses of the moderators are also optimized for neutron intensity. Detailed time and energy spectra for each of the moderators are provided to the scientist designing scientific instruments at the Lujan Center.

Table 1 compares the relative neutronic performance of the new upgraded moderators to the original flux-trap moderators (which have been in use since 1985). A design goal for the neutronic performance of the new target system was to prevent any reduction in the neutronic performance of the four flux-trap moderators when adding the two new upstream/backscattering moderators. As can be seen in Table 1, the new target system design achieves this goal. In addition, we performed engineering fluid dynamics calculations of rod-target thermo-hydraulics and performed an extensive set of engineering experiments for rod-target thermo-hydraulics using electrically heated rods. We carried both a bare tungsten plate and an Inconel-clad tungsten rod-target design through to final design. We have also been in close communication with people who are performing corrosion-related measurements in the LANSCE 1-mA proton beam. Our final choice was a bare tungsten plate target. This optimizes neutronic performance and simplifies the design, and the water activation is tolerable.

TABLE 1. Comparison of moderotor performance in the new upgraded target system to that of moderators in the original splitfarget/flux-trop-moderotor torget system

\begin{tabular}{l|c|c}
\hline Lujan Center Flight Paths & Relative Time Integrated Performance \\
\hline & $\begin{array}{c}\text { Original Target } \\
\text { System }\end{array}$ & $\begin{array}{c}\text { Upgraded Target } \\
\text { System }\end{array}$ \\
\hline FP 16, 1, 2 (high-resolution $\mathrm{H}_{2} \mathrm{O}$ ) & 1.0 & 1.7 \\
\hline FP 3,4, 5 (high-intensity $\mathrm{H}_{2} \mathrm{O}$ ) & 1.0 & 1.0 \\
\hline FP 6, 7, 8 (high-intensity $\mathrm{H}_{2} \mathrm{O}$ ) & 1.0 & 1.0 \\
\hline FP 9,10,11 (flux-trap $\mathrm{LH}_{2}$ ) & 1.0 & 2.5 \\
\hline FP 12,13 (upper tier $\mathrm{LH}_{2}$ ) & & $4.3^{*}$ \\
\hline FP 14,15 (upper tier $\mathrm{H}_{2} \mathrm{O}$ ) & & $4.1^{* *}$ \\
\hline
\end{tabular}

*Relative to existing FP 9, 19, 11

**Relative to existing FP $6,7,8$

Note: Integrals are for $\mathrm{E}<5 \mathrm{MeV}$ for $\mathrm{LH}_{2}, \mathrm{E}<100 \mathrm{MeV}$ for $\mathrm{H}_{2} \mathrm{O}$

The final TMRS design is shown in Figure 4. The beam enters vertically from the top and strikes the upper tungsten after passing through a profile monitor and vacuum window. The upper-tier moderators view the target in backscattered geometry. The lower-tier moderators view the flux trap between the upper and lower targets. The inner reflector is edge-cooled beryllium, and the outer reflector is lead. The edge-cooled reflector and the plate target design minimize the capture of neutrons in water and allow excellent neutronic performance.

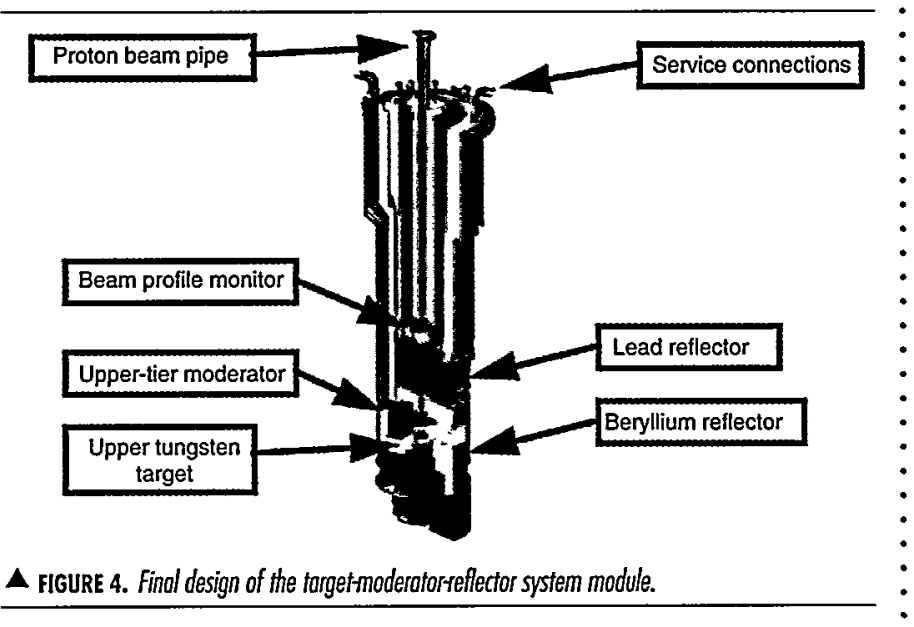

LANSCE • Activity Report 
The construction and installation process was documented with digital photographs and videotape. Figure 5 shows the TMRS insert hanging from the new bridge crane during installation. Figure 6 shows the completed TMRS insert in its support stand. Figure 7 shows the insert being installed through the seal plate in the target cell, and Figure 8 shows the target cell after the service connections were installed.

In addition to the new TMRS module and shielding, we have made improvements to the target support systems that will improve beam availability. The helium refrigerator

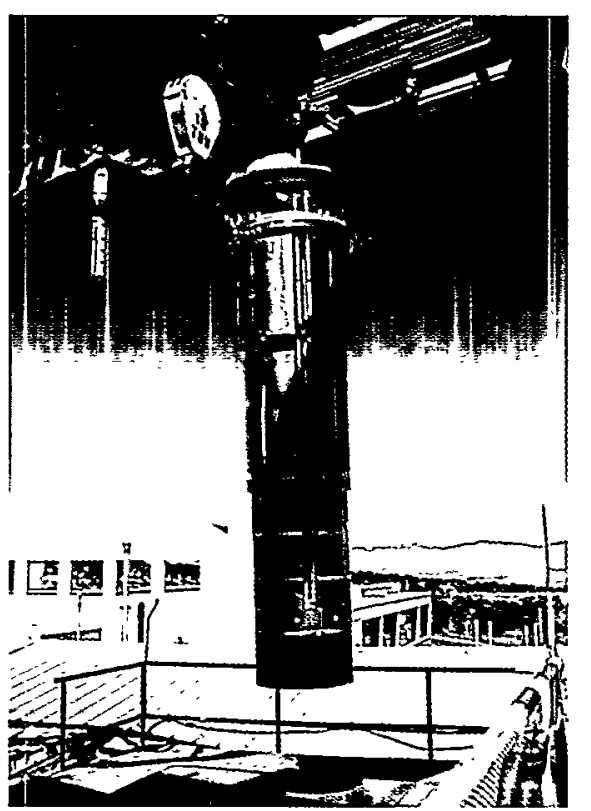

$\Delta$ FIGURE 5. The TMRS insert during installation through the roof of the torget cell.

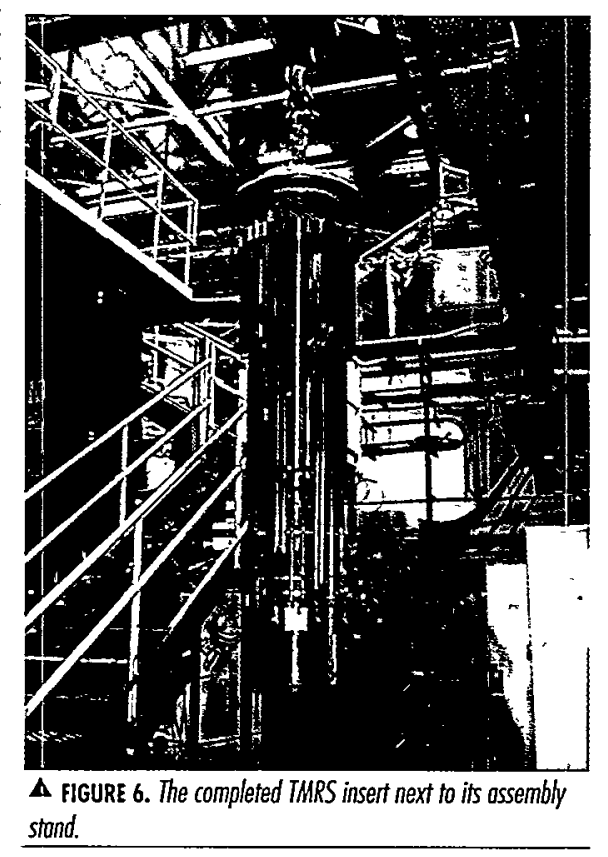

140 LANSCE • Activity Report
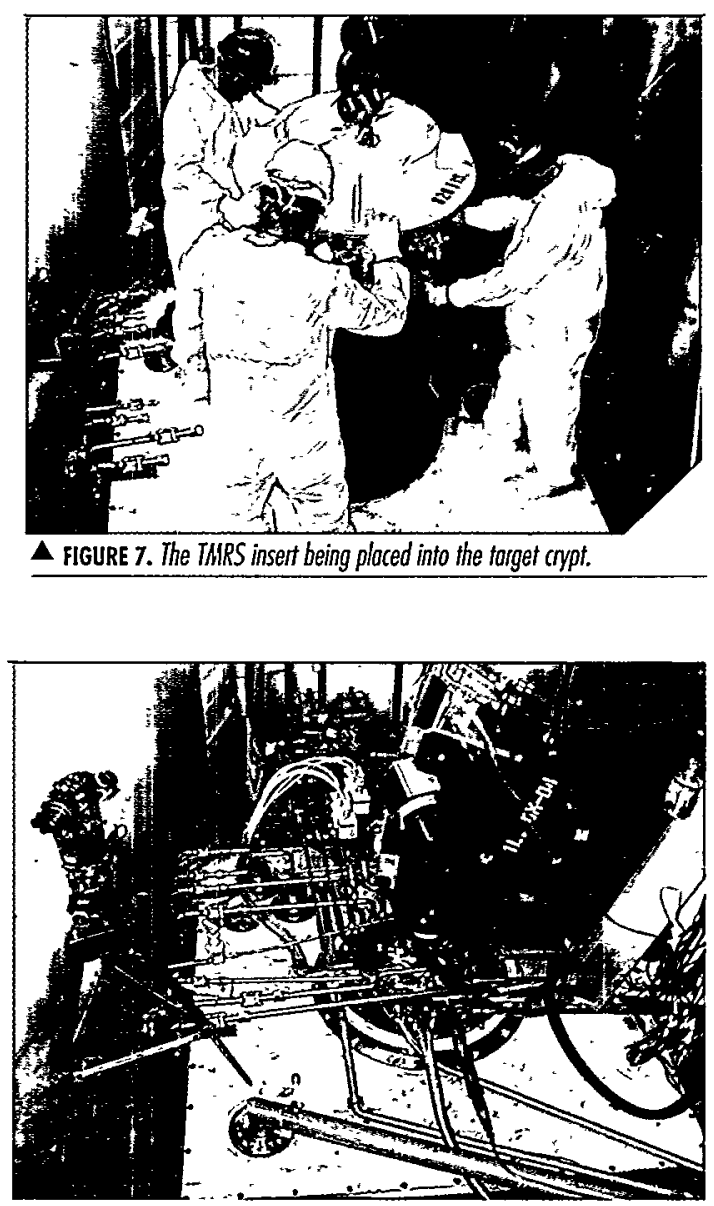

$\Delta$ FIGURE 8. Top view of the TMRS os installed in the torget cell with its service connections.

and compressors for the liquid hydrogen moderators have been moved to two new buildings on the north side of the target service area. The instrumentation panels have also been moved to this location. This move allows us to service these components without turning off the proton beam and prevents radiation damage to sensitive electronics.

The target system is now operational, and on November 19 , 1998, we raised the current delivered to the Lujan Center target to $100 \mu \mathrm{A}$.

- J. B. Donahue, T. O. Brun, P. D. Ferguson, R. J. Macek, M. A. Plum, G. J. Russell, W. M. Tuzel (LANSCE Division), G. D. Baker, J. E. Roberts (Nuclear Materials Technology Division), N. K. Bultman (Engineering Sciences and Applications Division), W. F. Sommer (Accelerator Production of Tritium Project) 


\section{Lujan Center Improves Spectrometer Capabilities}

Over the past 3 years, the Manuel Lujan Jr. Neutron Scattering Center has obtained funding to upgrade the capabilities of its existing spectrometers. The Department of Energy's (DOE's) Scientific Facilities Initiative in 1996 funded three major new capabilities at the Lujan Center. These include a wide-angle capability on PHAROS in conjunction with five U.S. universities; a 30-tesla pulsed magnet for neutron diffraction in conjunction with the National High Magnetic Field Laboratory (NHMFL); and new supermirror polarizers for our reflectometer SPEAR in conjunction with University of California at San Diego. A brief report on each of these developments is given in this section.

For several instruments, we made significant improvements in detector capabilities, shielding improvements, and sample environments. In particular, the high-intensity powder diffractometer (HIPD) increased its detector coverage by approximately a factor of two, allowing for the first time near-real-time experiments to be conducted on this diffractometer. This also greatly improved our capability to conduct high-pressure experiments, which require small samples. We upgraded the detector for the small-angle, low-Q diffractometer (LQD) to operate at the higher count rates possible with the beam current increase to 70 microamperes.

Additionally, a chopper to eliminate the initial high-energy neutrons from the pulsed source decreased the background in this spectrometer and extended the size range over which data could be collected. On the filter difference spectrometer (FDS), we installed 60 new detectors to eliminate the random noise problems that have previously existed.

With the increasing beam current, we determined the shielding on some of the older spectrometers was no longer sufficient for the projected $100-\mu \mathrm{A}$ current. An extensive effort in modeling studies by the spallation physics team at the Lujan Center incorporated effects from neighboring flight paths. This effort resulted in a new design for shielding on FDS, the neutron powder diffraction (NPD), PHAROS, and

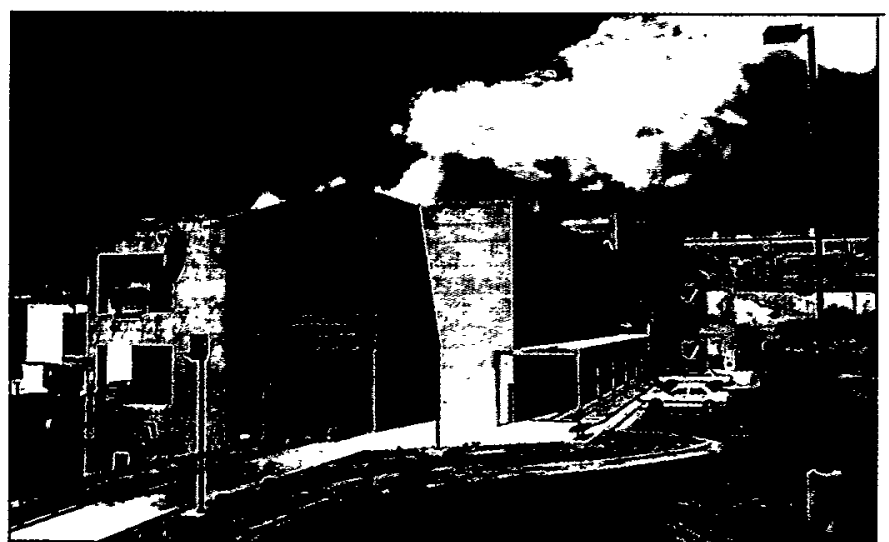

$\Delta$ figure 1. Manuel Lujon Jr. Neuton Scottering Center. the single-crystal diffractometer (SCD). The reduction in radiation levels will reduce not only personnel dose levels, but also backgrounds in the spectrometer detectors.

As upgrades to the sample environment capability at the Lujan Center, we purchased a three-stage displex to reach temperatures below $10 \mathrm{~K}$ without cryogens, a room-temperature access displex, and radial collimators and focusing optics for spatially-resolved strain measurements. We are purchasing a 12-tesla superconducting magnet, a cryo-displex and a cryo-furnace to use particularly with catalysis experiments, and an open-cycle cryogenic system for temperatures down to $2 \mathrm{~K}$.

\section{Installation of the Wide-Angle Detector Bank on PHAROS}

During the latest long shutdown, the existing low-angle secondary spectrometer on PHAROS was dismantled and the vacuum vessel for Phase II was installed. This increases the available solid angle by an order of magnitude and has the potential to increase data rates by the same ratio, in addition to opening up completely new fields of study for the community.

LANSCE's high-resolution chopper spectrometer PHAROS $^{1}$ has now run successfully for 3 years with $\sim 1 \mathrm{~m}^{2}$ of detectors in the forward direction. However, it was planned from the beginning ${ }^{2}$ that the spectrometer should have a wide-angle secondary flight path ranging from forward scattering to $140^{\circ}$, with a total detector area of $\sim 10 \mathrm{~m}^{2}$. In fact, the $34-$ ton $20-\mathrm{m}^{3}$ vacuum vessel for this flight path was delivered in 1991, but progress toward installation of the wide-angle capability was halted because of uncertainties in future funding for the neutron program at LANSCE. Nevertheless, in 1996, a University-Los Alamos National Laboratory collaboration ${ }^{3}$ succeeded in obtaining funds from the Scientific Facilities Initiative for the installation of the wide-angle bank along with $33 \%$ of its detector coverage.

The difference in the spectrometer before and after this installation is shown schematically in Figure 2, and Figure 3 shows a photograph of the outside portion of the wide-angle vacuum vessel before fitting of its vacuum windows, detectors, and external shielding.

During the same time, improvements were made to the $\mathrm{T}_{0}$ chopper bearings. Also, a larger Roots-blower pumping system is being installed, and a dry-air venting system is being implemented to shorten turnaround time when changing samples. In addition, the crane in Experiment Room (ER) 2 has been modified, and it can now reach the $\mathrm{T}_{0}$ chopper shielding. 
(a)

\section{Bulk shieid}

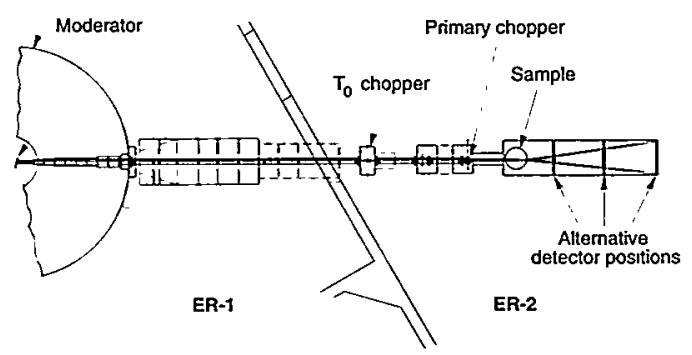

Bulk shield

Moderator

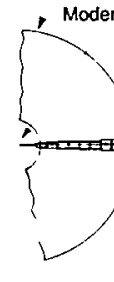

Phase II (1998 onwards)
$: \quad$ :$$
\text { : }
$$$$
\vdots
$$

A FIGURE 2. Diagrom showing PHAROS before and ofter installotion of the videangle bank.

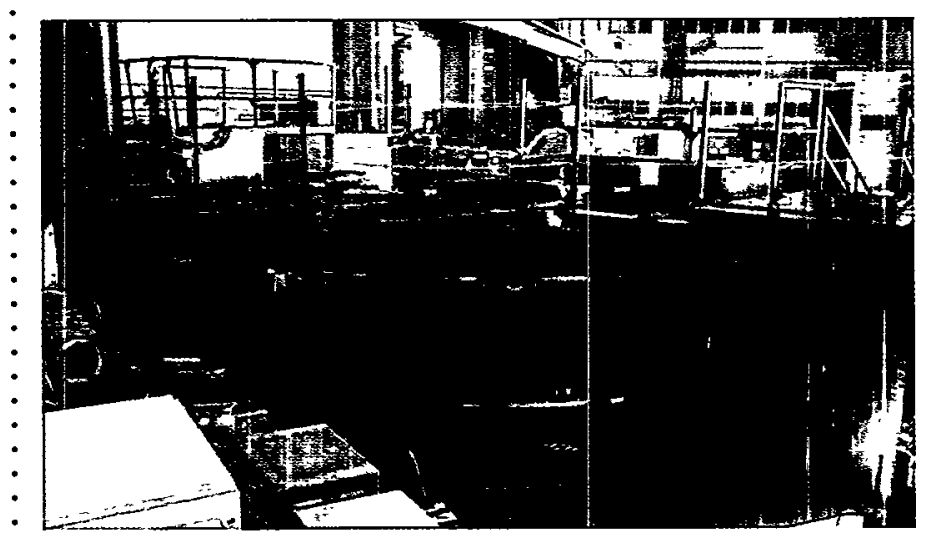

$\triangle$ FIGURE 3. The wide-ongle vocuum vessel of PHAROS after plocement, alignment, and installation of $B_{4} C$ intemol shielding. The vertical elements ore the boron-fibre aluminum-alloy ribs that support the 3 large $\left(-3 m^{2}\right.$ each) aluminum-llloy vocuum windows. Neither the windows nor the exfernal shielding had been installed ot the time that this photograph wos token (March 1998).

\section{References}

1. R. A. Robinson, M. Nutter, R. L. Ricketts, E. Larson, J. P. Sandoval, P. Lysaght, and B. J. Olivier, "A New Chopper Spectrometer for Neutron Brillouin Scattering and Low-Angle Neutron Inelastic Scattering: PHAROS (Phase I)," in Proceedings of ICANS-XII, Abingdon, United Kingdom, May 24-28, 1993, Rutherford Appleton Laboratory report 94-025, Vol. I, pp. 44-51 (available on the Web at http://www.lansce.lanl.gov/mlnsc/scientists/robinson.html).

2. J. M. Carpenter, K. Crawford, R. Kleb, C. -K. Loong, G. Ostrowski, D. L. Price, M. Nutter, R. Pynn, R. A. Robinson, R. N. Silver, J. M. Rowe, P. Sokol, and A. D. Taylor, "Report on a Workshop to Discuss a Chopper Spectrometer for LANSCE," Los Alamos National Laboratory document LA-UR-87-2582 (1987).
3. Proposal to the U.S. Department of Energy by M. C. Aronson (University of Michigan), S. J. L. Billinge (Michigan State University), C. Broholm (Johns Hopkins University), T. Egami (University of Pennsylvania), M. B. Maple (University of California, San Diego), and R. A. Robinson and J. Eckert (Los Alamos Neutron Science Center, Los Alamos National Laboratory), January 1996.

\section{-R. A. Robinson, K. J. Lovell, E. Larson, M. Velarde (LANSCE Division)}

\section{0-T Pulsed Magnet for Neutron Diffraction}

Scientists and engineers from LANSCE and the NHMFL are collaborating on the construction of a novel high-field pulsed magnet for neutron diffraction. This project is funded at the $\$ 1.1 \mathrm{M}$ level and should be ready for use in a neutron beam by the end of 1999 . The high-field pulsed magnet is being designed to reach a maximum field of $30 \mathrm{~T}$, has a split in its midplane for the incident and scattered neutrons, and will be water-cooled.

At present, materials research with neutrons is limited to magnetic fields of around $16 \mathrm{~T}$. To reach the higher magnetic fields ${ }^{1}$ that presently are available for complementary resistance, magnetization, and optical measurements, one needs to use resistive magnets made from high-strength copper alloys. Furthermore, power and cooling costs can be kept down by pulsing the magnet, using a capacitor bank as driver. Los Alamos National Laboratory is a partner in the NHMFL, and is host to its pulsed magnet facility. Combining pulsed magnetic fields with pulsed neutrons at LANSCE is a sensible approach. In fact, collaboration between the NHMFL and LANSCE has been funded by the DOE, under the Scientific Facilities Initiative, to build such a magnet for neutron-scattering studies. ${ }^{2,3}$

The project consists of three major components: the magnet, the capacitor power supply and charging system, and interfaces to the LANSCE building facilities/spectrometer/dataacquisition system. Table 1 lists key technical parameters.

Several factors lead to this specification. First, we chose a vertical-field split-pair geometry. This is the most flexible geometry for a wide range of scattering angles, which are horizontal or near-horizontal in most neutron instruments. This geometry also ensures perpendicularity between $\mathbf{Q}$ and the field direction, for all scattering angles. Second, we quickly discovered that the magnet must be cooled continuously with water-this is very different from most pulsedfield magnets, which are effectively single-shot devices in which the energy is dissipated in the thermal mass of the magnet, which is in turn immersed in liquid nitrogen. Third, given the high repetition rate and the length of typical neutron experiments, the whole system will experience 
TABLE 1. Current Parameters for the 30-T Pulsed Magnet for Neution Diffraction

\begin{tabular}{l|l}
\hline Parameter & \\
\hline Maximum magnetic field & $30 \mathrm{~T}$ \\
\hline Field pulse length & $2-3 \mathrm{~ms}$, half-sinusoidal \\
\hline Magnet repetition rate & $2 \mathrm{~Hz}$ \\
\hline Gap & $5 \mathrm{~mm}$ \\
\hline Vertical divergence & $\pm 4^{\circ}$ \\
\hline Horizontal divergence & $4 \times 73^{\circ}$ \\
\hline Bore & $20 \mathrm{~mm}$ \\
\hline Magnet inductance & $107 \mu \mathrm{H}$ \\
\hline Capacitance & $5 \mathrm{mF}$ \\
\hline Peak voltage & $14.1 \mathrm{kV}$ \\
\hline Peak current & $90 \mathrm{kA}$ \\
\hline Stored energy in capacitor bank & $500 \mathrm{~kJ}$ \\
\hline Average heat dissipation & $262 \mathrm{~kW}$ \\
\hline
\end{tabular}

$10^{7}$ cycles in 2 months at the full repetition rate. This is far beyond what most pulsed-field facilities ever experience and it imposes constraints on the type of capacitors and the nature of the main electrical switch, and makes fatigue performance of all the materials critical. We measured several candidate high-strength conductors ${ }^{4}$ (Figure 3 ), in addition to reinforcement and insulation materials. Materials strength is the limiting factor for the fields in pulsed magnets, while cooling provides the constraint on fields from dc-resistive magnets; in this magnet, we are pushing on both limits. The present plan is to fabricate the magnet in Tallahassee, using CuAg conductors and MP35N steel reinforcement, and to use the four-coil design shown in Figure 4 . The purchase request for magnet construction was submitted in March 1998.

While the magnet design is technically the most challenging part of the project, it accounts only for $\sim 15 \%$ of the cost. The major cost components are the capacitor-bank

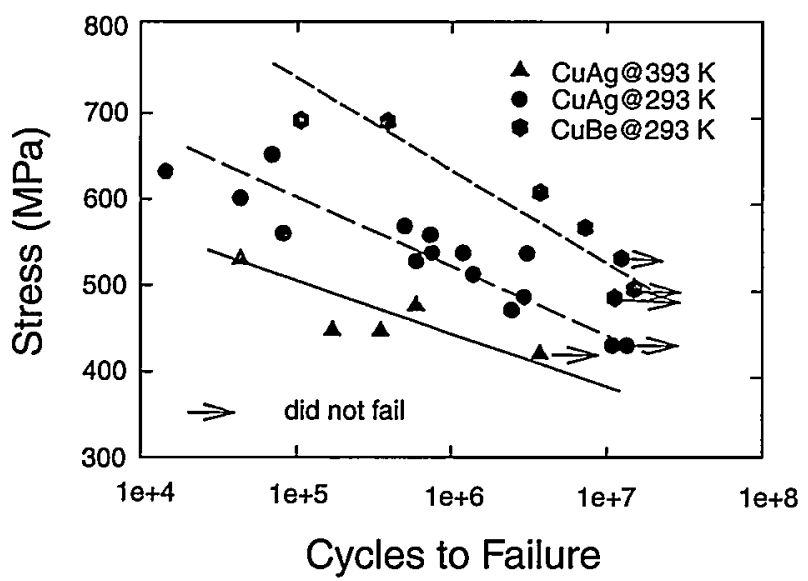

$\Delta$ FIGURE 3. Typical fotigue testing data token by our colleagues at NHMFL-Tallahossee for several possible highstrength conductor materials. Our design is such that the von-Mises stresses are below $400 \mathrm{MPa}$ in the conductor, of full field.

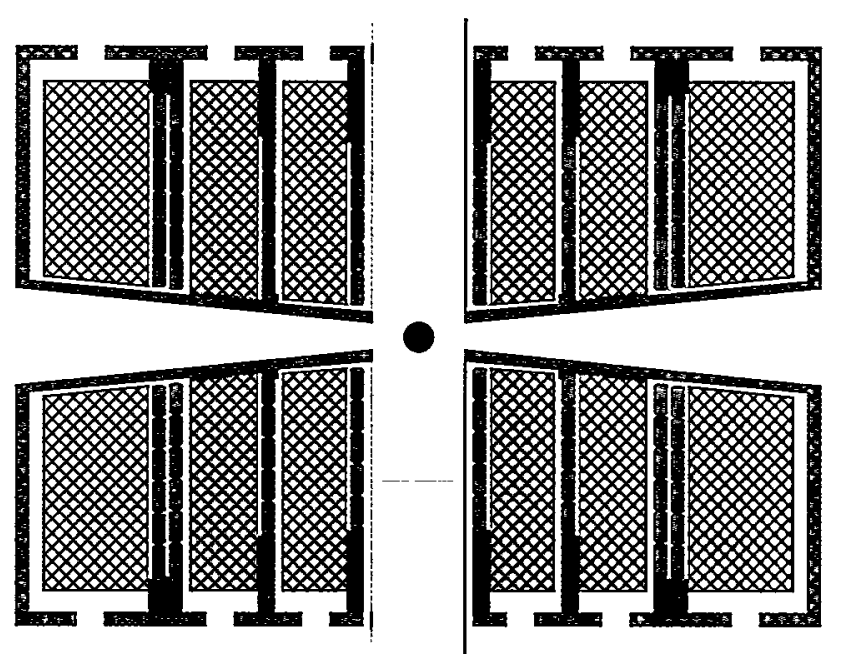

A FIGURE 4. Schematic diagram of the present 30-T, 20-mmbore mognet design, showing the fourcoil system, each loyer with its own reinforcement shell, to fake the radial load. Each coil is watercooled on its inner conductor surfoce, and the electric current flows through oll eight coils (four in each half of the mognet) in series. The neutrons illuminote the somple by entering wittin the horizontol plane; neutron detectors can observe the neutrons scottered within the gop.

power supply and recharging system. We anticipate the magnet initially will be on a dedicated cold-neutron beam line, and will be used primarily for the study of magnetic phase diagrams and the structures that occur above $16 \mathrm{~T}$. However, we plan to build the power supply, which likely will be the size of a medium-sized container, in such a way that it can be moved onto other diffractometers and spectrometers at LANSCE. We expect to have the magnet ready to install in a neutron beam line early in 2000 .

\section{References}

1. See for instance, G. Boebinger, A. Passner, and J. Bevk, Scientific American 272, 58 (1995).

2. R. A. Robinson, Y. M. Eyssa, H. J. Schneider-Muntau, and H. J. Boenig, "A 30-T Pulsed Magnet Suitable for Neutron Scattering Experiments," in Proceedings of Conference on Physical Phenomena at High Magnetic Fields II, Tallahassee FL, May 6-9 1995, Z. Fisk, L. Gor'kov, D. Meltzer, and R. Schrieffer, Eds. (World Scientific, Singapore, 1996), pp. 735-738.

3. R. A. Robinson, Y. M. Eyssa, H. J. Schneider-Muntau, and H. J. Boenig, "Proposal for a 30-T Pulsed Magnet Suitable for Neutron Scattering Experiments," in Proceedings of ICANS-XIII, Villigen, Switzerland, October 11-14, 1995, in PSI-Proceedings 95-02, I, pp. 300-306.

4. Y. M. Eyssa et al., "2-Hz, 30-T Split Pulse Water-Cooled Magnet for Neutron Scattering Experiments-Materials Characterization and Design Options," National High-Magnetic Field Laboratory report (August 1997).

- R. A. Robinson (LANSCE Division), H. J. Boenig, C. Mielke (National High-Magnetic Field Laboratory, Los Alamos), Y. M. Eyssa, H. J. Schneider-Muntau, T. Painter (National High-Magnetic Field Laboratory, Tallahassee), H. Nakotte (New Mexico State University and LANSCE Division) 


\section{YBCO Cryo-Flippers for Polarized Neutrons}

The SPEAR polarization neutron reflectometry user program requires a method for reversing the polarization of a neutron beam that is independent of neutron wavelength. This is accomplished using a high-temperature superconducting thin film of YBCO as a cryo-flipper. Advantages of the new approach include passive and maintenance-free operation; compatibility with polarized neutron beams of large cross section, while compact in size $\left(\sim 0.02 \mathrm{~m}^{3}\right)$; and best of all, low cost (the cryo-flipper is very inexpensive).

Magnetic and nuclear scattering can be determined independently by measuring the intensities of the scattered neutron beams whose polarizations are parallel and antiparallel to the sample magnetization. On the reflectometer SPEAR at the Lujan Center, magnetic and nuclear scattering is accomplished using a broad band of neutron wavelengths polarized with supermirrors ${ }^{1}$ in reflection geometry. ${ }^{2}$ The experiments require a method for reversing the polarization of the neutron beam.

One method, tried previously on SPEAR, used a Mezei $\pi$-flipper ${ }^{3}$ to reverse the neutron spin relative to the direction of the fields that magnetized the polarizers and sample. This approach has two disadvantages: a source of small-angle scattering is introduced, and the process used to determine the currents in the coils of the flipper is time consuming. Recently, the Meissner effect produced by a thin superconducting YBCO film called a Meissner screen has been used to create a discontinuity in the magnetic field through which the neutrons travel. By reversing the current through the electromagnet that magnetizes the polarizer, spin-flip of the neutron beam in the reference frame of the sample can be made to occur in a manner that is wavelength independent.

The Meissner screen consisted of a 5-cm-diameter YBCO film (c-axis parallel to the surface normal) grown on a I-mm-thick single-crystal substrate. The critical current densities, $\mathrm{J}_{\mathrm{c}}$, of the film parallel to the ab-plane (in the plane of the film) exceeded $2 \cdot 10^{10} \mathrm{~A} / \mathrm{m}^{2}$ at $77 \mathrm{~K}\left(\mathrm{~T}_{\mathrm{c}} \sim 87 \mathrm{~K}\right)$. A YBCO-coated wafer was mounted separately in a $\mathrm{Cu}$ holder, which was attached to a Displex cryostat in an ultra highvacuum (UHV) tee (Figure 5). The film was cooled to $20 \mathrm{~K}$. The neutron beam entered and exited the UHV tee through Suprasil windows. Magnetic fields generated by solenoids placed between $\mu$-metal sheets defined the quantization of the neutron spin within the tee. The solenoids in the guide on the polarizer side of the film were connected to a dc bipolar supply and produced a field of about $\mathrm{H}_{\mathrm{b}}= \pm 25 \mathrm{Oe}$. The solenoids in the guide on the sample side of the film were connected to a dc unipolar supply and produced a field of $\mathrm{H}_{\mathrm{s}}=+25 \mathrm{Oe}$ in the direction parallel to the field that would be applied to a sample. The device consisting of

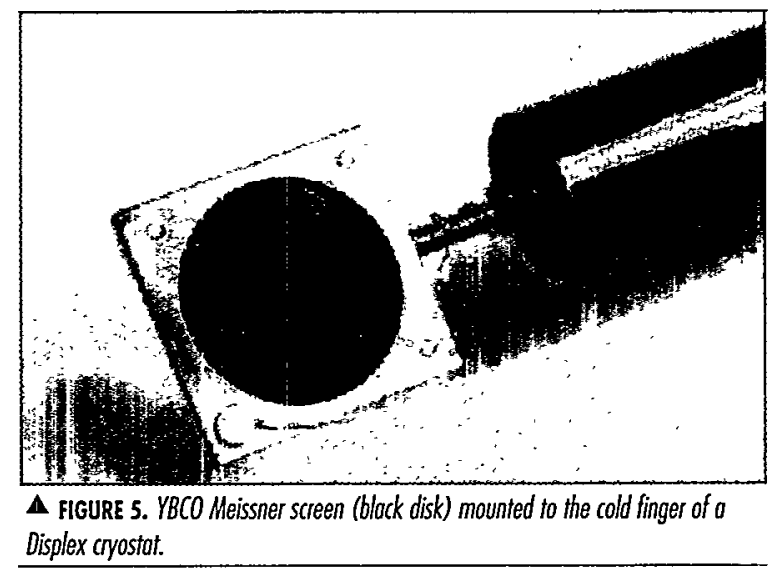

UHV tee, guides, cryostat, and Meissner screen is called a cryo-flipper. Tasset earlier developed a cryo-flipper using superconducting niobium $(\mathrm{Nb})$ for applications with monochromated neutron beams. ${ }^{4}$

The performance of the YBCO cryo-flipper was determined from a neutron scattering experiment in which the intensity of the neutron beam was measured after reflection from two polarizing supermirrors. Two measurements were made: one when the magnetic fields on the polarizers were aligned-a condition requiring the guide fields before and after the Meissner screen to be parallel, and a second when the fields were opposite. (The ratio of the two measured intensities is called the flipping ratio.) Figure 6 is an example of one such measurement.

Thin YBCO c-axis-grown films performed well as Meissner screens when they were cooled in near-zero fields or uniform fields, and the fields to which they were exposed were applied along the ab-plane of the film. The

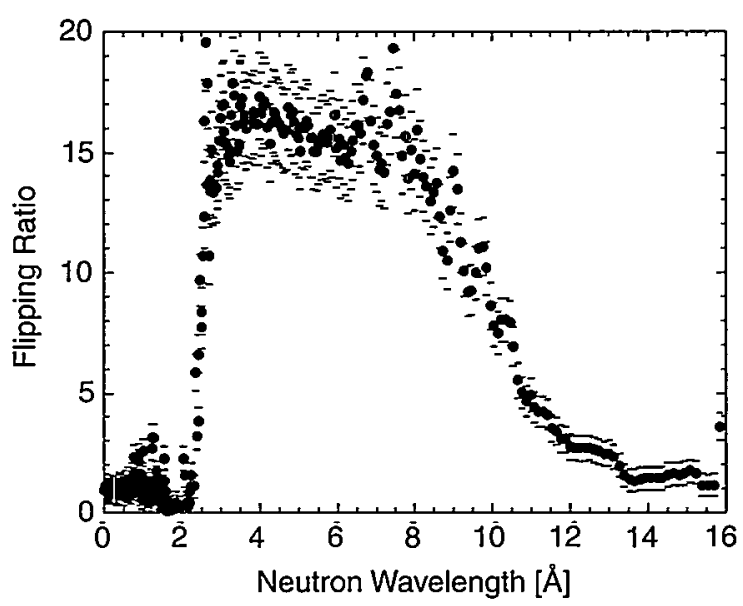

$\Delta$ FIGURE 6. Flipping ratio measured for the polarized neutron reflectometer. The polanizing supermirrors were adjusted to reflect neutrons with wavelengths greater thon $2.8 \dot{A}$. The decay in the flipping ratio at $10 \AA$ was caused by reflection of the spindown stote from the glass substrate of the polorizing supermirror. In the future, the polorizing supermirors will be replaced vith ones grown on Si substrates rother than glass, thus extending the polorized neutron wovelength band to $16 \dot{A}$. 
advantages of the YBCO cryo-flipper over the more conventional $\mathrm{Nb}$ cryo-flipper include near-zero small-angle scattering (YBCO and its substrate are single crystals), less susceptibility to the magnetic field environment during cooling $\left(J_{c}\right.$ is much larger for YBCO than pure $\mathrm{Nb}$ ), and affordability and ease-of-use (a closed-cycle cryostat is less expensive than a liquid-helium cryostat to purchase and maintain). Since $\mathrm{J}_{c}$ is so large for $\mathrm{YBCO}$ (compared with pure $\mathrm{Nb}$ ), the YBCO cryo-flipper can be used in reasonably close proximity (within $1.5 \mathrm{~m}$ ) to superconducting magnets, whose fringe fields may quench a $\mathrm{Nb}$ cryoflipper, or introduce perturbations to the magnetic field gradient of more conventional electromagnetic flippers (e.g., Mezei spin-turn coils, Drabkin flippers, or radiofrequency-flippers), producing a loss in flipping efficiency.

\section{References}

1. D. Clemens et al., "Polarizing $\mathrm{Ti}_{1-\mathrm{u}} \mathrm{X}_{\mathrm{u}} / \mathrm{Fe}_{\mathrm{x}} \mathrm{Co}_{\mathrm{y}} \mathrm{V}_{\mathrm{z}}$ Supermirrors," International Conference on Neutron Scattering, Sendai, Japan, October 11-14, 1994, Physica B 213 \& 214, 942 (1995).

2. M. R. Fitzsimmons et al., ICANS-12, Rutherford Appleton Laboratory report 94-025, 355 (1994).

3. O. Schärpf, "The Polarized Neutron Techniques of Neutron Spin Echo," in Proceedings of a Laue-Langevin Institut Workshop on Neutron Spin Echo, Grenoble, October 15-16, 1979, published as Lecture Notes in Plyysics (Springer Verlag, Berlin, 1980), Vol. 128, p. 27.

4. F. Tasset, "Zero Field Neutron Polarimetry," Physica B 156 \& 157,627 (1989).

\section{-M. R. Fitzsimmons (LANSCE Division)}

\section{Achievement of Simultaneous High Pressure and High Temperature}

Structural and physical properties of materials under highpressure $(\mathrm{P})$ and high-temperature $(\mathrm{T})$ conditions are of great interest in condensed-matter physics, materials science, and earth science. High-pressure/temperature techniques are being used to create new and exotic materials with industrial applications. The possibility of making better ceramics, superhard substances, and materials with superconductive properties has already stimulated collaboration among different scientific fields. Diffraction experiments at simultaneous high P-T conditions provide the means of studying the structural response of different materials to pressures and temperatures as described by thermoelastic equation of state (EOS), crystal structural parameters, and atomic thermal vibrations (Debye-Waller factors). A full description of the thermal expansion and bulk modulus over P-T-X space (where X stands for composition) is the primary thermodynamic data for metals, alloys, ceramics, and minerals. The thermoelastic EOS data and Debye-Waller factors are essential for computer modeling the response of materials to static and dynamic changes in P-T conditions.
Compared with synchrotron $\mathrm{x}$-ray diffraction, the high $\mathrm{P}-\mathrm{T}$ neutron diffraction experiments require much larger sample volumes $\left(100 \mathrm{~mm}^{3}\right.$ versus $\left.1 \mathrm{~mm}^{3}\right)$, longer data collection times (hours versus minutes), and very restricted scattering geometry (only for $2 \theta=90^{\circ}$ diffraction optics). All of these impose tremendous experimental difficulties on the design of a cell assembly for neutron diffraction working under simultaneous high $\mathrm{P}$ and $\mathrm{T}$ conditions. The development of the high P-T cell assembly needs to optimize the dimension, material, and shape of the gasket and furnace for pressure and heating efficiency, for thermal and electrical conduction and insulation, and for neutron scattering and absorption properties.

We have successfully developed a cell assembly (Figure 7) for neutron diffraction experiments capable of performing simultaneous high $\mathrm{P}$ and $\mathrm{T}$ conditions up to $10 \mathrm{GPa}$ and $1500 \mathrm{~K}$. We have collected high-quality neutron diffraction data of metals, oxides, and halides at the HIPD beam line of LANSCE using this cell assembly under stable, uniform temperature and sustained hydrostatic pressure. Our cell assembly (Figure 8) uses a ceramic gasket that has the mechanical strength as well as plastic deformation necessary to build up sustained hydrostatic pressure on a sample at high temperatures. The ceramic gasket is of great thermal/electric insulating properties, and it is also somewhat transparent to neutrons. We designed a disk-capped cylindrical furnace made of amorphous carbon that heats the sample to high temperatures in a stable and uniform manner at high pressures. The accurate measurement of pressure, temperature, and diffraction peak positions depends on the success of thermocouples and the calibration of the diffraction optics. We have successfully worked out an analytical method and experimental procedure to obtain correct P-V-T data from the high P-T neutron diffraction spectrum of multiple phases.

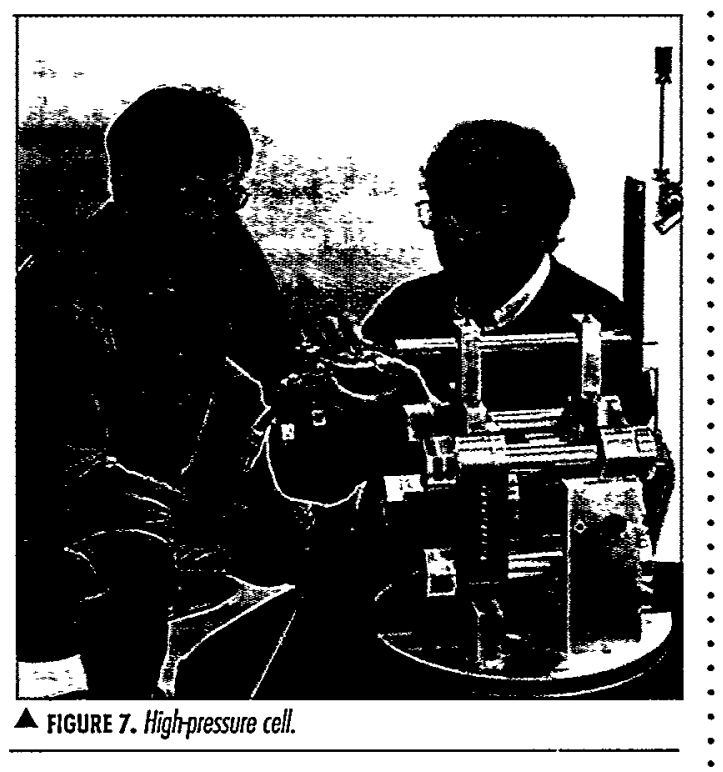

LANSCE • Activity Report 145 


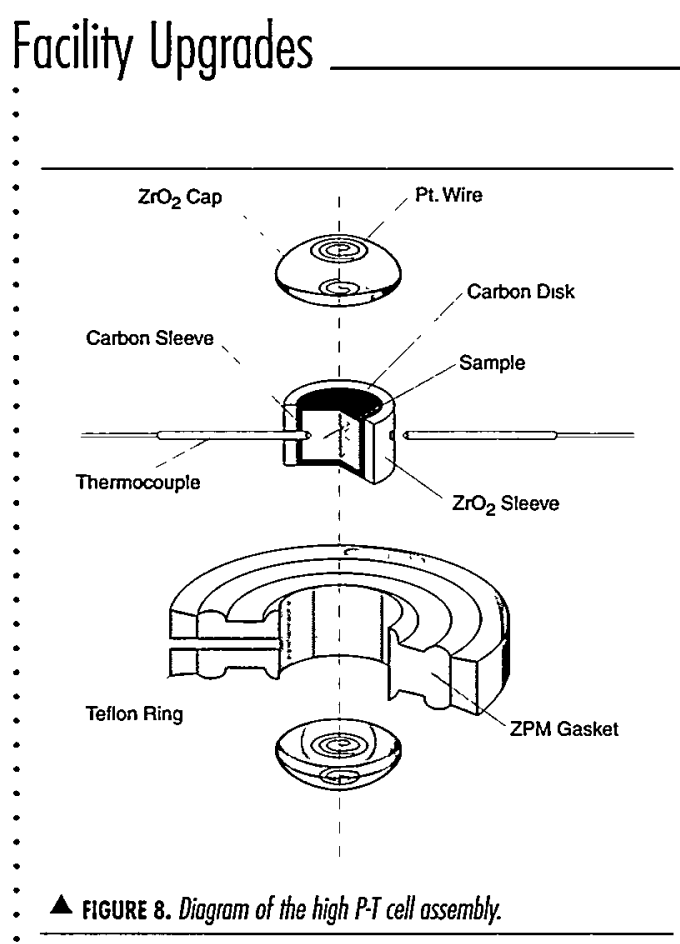

The in-situ high P-T neutron diffraction experiments provide a unique opportunity to study texture, hydrogen bonding, magnetic moments, and structural and thermal parameters of light elements (e.g., hydrogen, lithium, carbon) and heavy elements (e.g., tantalum, uranium, plutonium), which are virtually impossible to determine by $\mathrm{x}$-ray diffraction techniques. We expect to extend the high P-T neutron diffraction capability at LANSCE up to $30 \mathrm{GPa}$ and $2000 \mathrm{~K}$ with our ongoing design of a new diffractometer called HIPPO (for high-pressure preferred orientation) and a new toroidal-anvil press referred to as TAP-98.

\section{-Y. Zhao, R. B. Von Dreele (LANSCE Division)}

\section{Macrostrain Measurement Using Radial Collimators}

We have implemented a series of "short" radial collimators in the $90^{\circ}$ scattering geometries on NPD at the Lujan Center. The capability to perform macrostrain measurements has been improved by the ability to rapidly select a sampling volume appropriate to the specimen. The compact design of the collimators was dictated by the need to fit them in a cylindrical vacuum chamber as well as provide space in which to manipulate a specimen in three dimensions. Collimators of different vane lengths were fabricated to give four different resolutions for which $2 / 3$ of the diffracted intensity comes from distances of $0.75,1.25$, 2.5 , and $4 \mathrm{~mm}$ along the incident beam. We performed qualifying scans and a demonstration on a cracked ring, containing a steep stress gradient.

At LANSCE we perform spatially resolved measurements of macro-residual strains by masking a sampling volume (from which diffraction to a detector is possible), moving a specimen through it, and examining different internal positions. Definition of the incident and diffracted neutron beams is a prerequisite but, for the diffracted beam, is performed differently at a pulsed than at a steady-state source because of the different detection philosophy of the scattered radiation. In either case, incident collimation is simple using an aperture in a cadmium or boron mask to define an approximately parallel incident beam. However, at a pulsed source, neutrons scattered in all directions are eligible for detection in contrast with a steady-state source for which the neutron intensity is scattered at discrete angular positions. Using the time-of-flight (TOF) method, the wavelength of a neutron detected in any direction can be determined. Thus, individual detectors that collectively subtend a continuous range of angles simultaneously record spectra that are combined to give a single spectrum. Summation over a range of angles is usually necessary for effective count times.

The distinction between having usable diffracted intensity over an angular range instead of concentrated at one angle affects the manner in which collimation is used to define the sampling volume dimension parallel to the incident beam. For monochromatic diffraction at an approximately fixed angle, a single slit aperture is sufficient and may be several centimeters from the object (although it is preferable to keep it closer). For comparable count times at a pulsed source, radiation over a spread of angle must be collected simultaneously. Thus a single aperture is ineffective unless it is very close to the incident beam because parallax rapidly expands the sampling volume.

At LANSCE, early attempts to use a single diffracted beam slit close to the incident beam did give good results for measurements in flat specimens. The averaging in strain direction of $5.5^{\circ}$ that results from summing over an $11^{\circ}$ detector proved negligible compared to changes in the macrostrains between principal directions. Nevertheless, the approach was limited to in-plane profiles in plate specimens because the aperture was usually placed in contact with the surface (to minimize the parallax effect). This aperture impeded the manipulation options in one dimension precluding throughthickness scans or objects with irregular geometries.

At ISIS in the United Kingdom, these problems were overcome using radial collimators pioneered on the ENGIN instrument. Using two permanently mounted radial collimators, spatial resolution along the incident beam of $3 \mathrm{~mm}$ was possible with a distance of $15 \mathrm{~cm}$ between the sampling volume and the front of the collimator. This space buffer allowed objects of irregular geometry to be easily translated and rotated into various positions.

Specifications for the LANSCE radial collimators were chosen to give spatial resolution along the incident beam (XMAX) of $4,2.5,1.25$, and $0.75 \mathrm{~mm}$. The inner radius of 
the NPD chamber is $370 \mathrm{~mm}$ and each $90^{\circ}$ detector subtends $84.5^{\circ}$ to $95.5^{\circ}$. For simplicity of manufacture, a constant angular vane separation of $0.31^{\circ}$ was used, and vane lengths were altered to give the nominal spatial resolutions. The principal difference between the NPD collimators and those at ISIS is that the vanes at ISIS extend all the way between collimator and detector-offering ultrafine angular resolution at the expense of mobility. Installation of ISISstyle collimators on the NPD would be impractical because the detectors are $1.5 \mathrm{~m}$ from the sample position and are separated by the wall of a vacuum chamber. Fortunately, in almost all problems, ultrafine angular resolution is not needed. We could place the collimators inside or outside the chamber. A collimator outside the sample chamber would have been substantially long and would have been difficult to install between the detectors and the chamber wall, precluding rapid manual interchange. Fortunately, short vanes can provide the required spatial resolution without impeding the free volume around the specimen.

A standard choice for vane materials is mylar coated with gadolinium oxide paint. The techniques used to precisely stretch large numbers of mylar vanes on a divergent pattern without wrinkling or kinking (which would render the collimators useless) are difficult to achieve and require considerable experience. Three manufacturers were approached: Eurocollimators (formerly L\&H designs) in the United Kingdom, JJ X Ray in Denmark, and Ohashi Industries in Japan. The manufacturing approach of each company differed in their choice of materials, and we selected Ohashi industries on the basis of cost, speed of delivery, and lightness of each collimator. Their construction used a frame and spacers made of epoxy-glass fiber, and vanes comprising mylar sheets $50 \mu \mathrm{m}$ in thickness with $25 \mu \mathrm{m}$ of $\mathrm{GdO}_{2}$ paint on both sides. Each collimator was manufactured with a carrying handle, and the weight of the longest was less than $5 \mathrm{~kg}$, making the collimators easy to maneuver. The four collimators are shown in Figure 9.

One concern in interchanging the collimators for different experiments is the need for accurate alignment and repositioning. The center of the sampling volume must be reproducible to at least $0.1 \mathrm{~mm}$. Because the back face of each collimator is $350 \mathrm{~mm}$ from the sampling volume, even small errors in angle or position can displace the actual from the nominal center of the sampling volume. To minimize this possibility, each collimator is mounted on a precision inverted V-base that mates with permanently mounted supports on the equipment. These supports provide $\mathrm{XYZ}$ motion for the specimen. Each support can be precisely moved parallel, perpendicular, or rotated using optical slides. The supports are shown in Figure 10.

Before use, we assessed the performance of each collimator by moving a $3.15-\mathrm{mm}$-diameter steel pin parallel to the
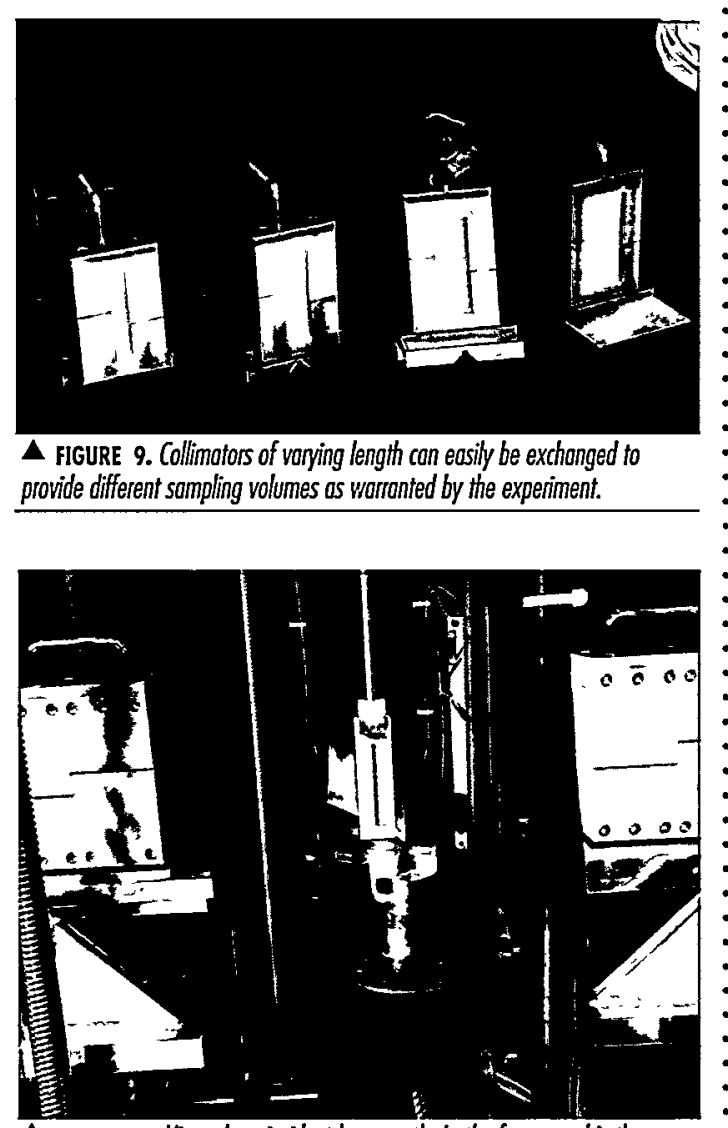

$\triangle$ FIGURE 10. View along incident beom poth. In the foreground is the incident boron nitride mosk. The rodial collimotors ore mounted on either side of the specimen (a fitanium fan blade in the background).

incident beam through each defined volume. We evaluated the diffracted intensity (measured as an integral over a range that included several reflections) profile by numerically convoluting a triangular resolution function with a function describing the circular (in the horizontal plane) pin. Convoluted intensity profiles are plotted from "first pick up" to "last pick up" in Figure 11. The lines are dotted for the experimental measurements and solid for the predicted profiles. For all four collimators, the measured and calculated profiles are in close agreement.

To evaluate the performance on a real specimen, we examined a cracked ring. The ring was $5 \mathrm{~mm}$ thick and contained a fatigue crack partially propagated through an autofrettage residual stress distribution. The resulting redistribution produces a steep strain gradient around a radial position of $16 \mathrm{~mm}$. In that region, over approximately $4 \mathrm{~mm}$, the hoop strain increases from a compressive value of $-400 \mu$ strain to a tensile value of close to $2000 \mu$ strain. This increase demands small sampling volumes to satisfactorily resolve the change between extremes, but also provides a test specimen in which the increasing spatial resolution provided by the collimators can be monitored. In practice we only made measurements using three collimators and did not use the finest spatial resolution (XMAX=0.75). In Figure 12, the 


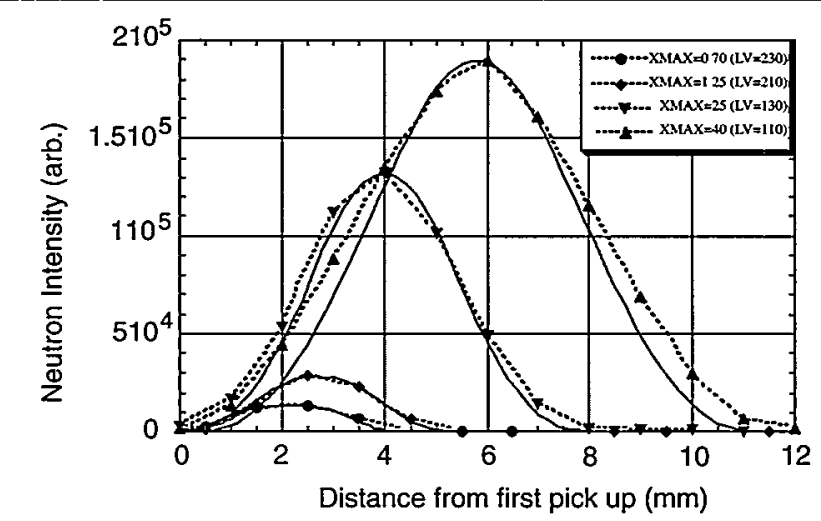

A FIGURE II. Meosured (dotted) and colculated (solid) profiles for the convoluted intensity of the resolution function with o $3.15 \mathrm{~mm}$ pin moved along the incident beam for each collimator. Colculated profiles were evaluated numerically.

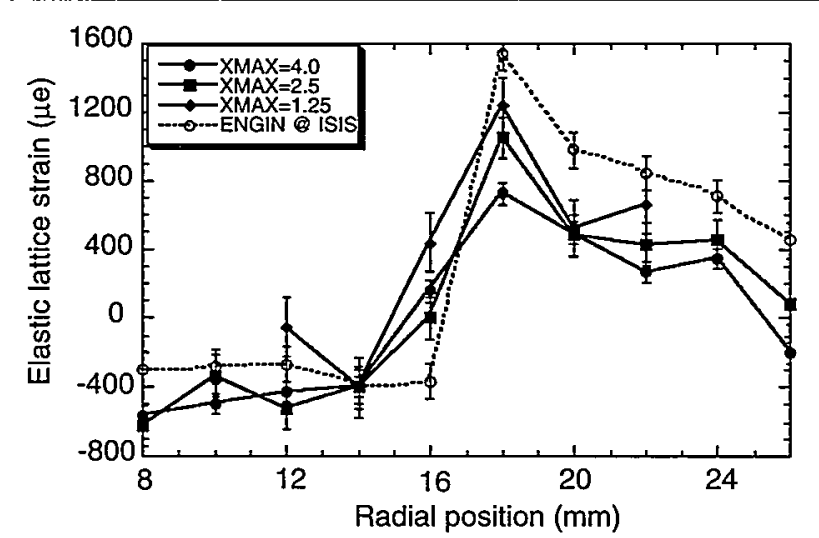

A FIGURE 12. Hoop strain versus rodial position in a crocked ferritic steel ring become progressively sharper near the crack tip with increasing spatial resolution. The NPD dato ore from o single peok fit [I10] whereos the ISIS doto are calculated from a Rietveld refinement of the whole pattern.

maximum to minimum strain variation increases as XMAX is reduced. Also shown in the graph is a comparable scan obtained using the ENGIN instrument.

\section{- M. A. M. Bourke (Materials Science and Technology Division and LANSCE Division), J. A. Roberts, D. Davis (LANSCE Division)}

\section{Monte Carlo Tool for Simulations of Neutron Scattering Instruments}

The design of neutron scattering instruments and the exploration of novel techniques in neutron scattering are activities that have recently picked up pace. The trend is expected to continue with the recent endorsement by the U.S. Congress of the Spallation Neutron Source (SNS) project and with support for a number of pulsed or continuous sources enhancement projects at Los Alamos, Oak Ridge, and Brookhaven national laboratories. Meanwhile, the European Spallation Source project is well underway:
Germany is building a new research reactor in Munich, and the Paul Scherrer Institute in Switzerland is about to go into production. Japan is also planning a 5-MW spallation source. Given the cost of these sources, carefully choosing and optimizing the performance of their associated suite of instruments is highly desirable. This task is less costly and more efficient than optimizing the neutron production target itself. At pulsed sources, however, the neutron pulse emitted by a moderator has a complex dependence on time, neutron energy, and direction of emission, all of which directly affect the performance of a given instrument. This greatly complicates the task of the instrument designer, who now needs design tools beyond the traditional raytracing techniques of conventional optics. Even at continuous sources, there are many aspects of instrument design and optimization that cannot be addressed through raytracing techniques.

Analog Monte Carlo techniques provide a powerful complement to analytical design tools such as ray-tracing methods. Monte Carlo consists of sampling at random, but in a statistically representative manner, the phase space of the moderator to select the characteristics of a neutron. This neutron is then transported down the instrument axis and undergoes along the way all the interactions an actual neutron would feel in the real instrument until it is either lost or detected. The interactions in such a history can be deterministic or statistical models of the relevant physical processes. The final answer is obtained by combining the results of many histories. While this approach has been used in the past to simulate isolated optical components or, less frequently, entire instruments, the associated codes were very much ad hoc. They lacked generality, took an excessive amount of time to program, and required advanced knowledge of Monte Carlo techniques.

The main purpose of our Neutron Instrument Simulation Package (NISP) was to overcome these shortcomings by providing a general, easy-to-use simulation tool to the general user with little or no experience with Monte Carlo techniques. This tool would allow the user to produce or modify a computer model of an instrument and simulate its performance on widely available computer platforms. The effort to make the code general started in 1994 and produced a package that consists of three components:

- MCLIB: a library of Fortran subroutines that deals with elementary tasks such as geometry representation and neutron transport and that contains all the models for optical elements. Associated with this library is a set of source files representing more than 25 different types of moderators and a set of scattering kernels for sample simulation.

- MC_RUN: a Monte Carlo engine that runs the Monte Carlo simulation itself and produces a series of output files with detector output and general information about the outcome of the simulation. 
- MC_Web: a Web-based application that allows the user to set up the instrument geometry interactively without having to learn any of the data structures of MCLIB.

NISP has been used extensively during the past few years to design instrumentation for a number of LANSCE-based projects including the long-pulse spallation source project and the LANSCE Enhancement project. NISP has many uses beyond instrumentation design. It can be used to optimize optical components or entire instruments, analyze the performance of existing instruments, test novel ideas and concepts for components or instruments, verify design specifications, analyze experimental results, plan experiments on existing instruments, and teach neutron scattering techniques. NISP currently has about 50 registered users in the neutron scattering community and is available at the LANSCE Web site at strider.lansce.lanl.gov/mlnsc/computing.html.

The following example illustrates the power of NISP: The addition of a large mirror to the LQD at LANSCE would increase the intensity of the beam at the sample location and would greatly increase the range of feasible small-angle scattering experiments. Ideally, an ellipsoidal mirror should be used to retain a sufficiently well-focused beam and thus resolution. Large ellipsoidal mirrors are difficult and expensive to make, and one may ask whether a simpler mirror made of more easily fabricated sections of cylinders or toroidal surfaces would emulate the effect of the ellipsoidal mirror reasonably well. Figure 13 shows that is not the case. Figure 13(a) is the beam spot produced by the ellipsoidal mirror. Figure 13(c) is the beam spot produced by a single toroidal surface. It is rather clear that the latter produces a beam spot that is not tightly focused. Other arrangements of sections of surfaces were tested, and the conclusion reached was that the ellipsoidal mirror was definitely superior. While the ellipsoidal mirror was a more expensive solution, NISP showed that the benefit justifies the cost. Figure 13(b) illustrates another use of NISP: namely, the quantitative evaluation of the effect of imperfections on the performance of the mirror. The calculation of the effect of surface roughness, surface distortions, or uncertainties in the value of the ellipsoidal axes would be difficult by means of other techniques. Using NISP, we quickly concluded that distortions well above the tolerance that can be achieved during fabrication would not result in excessive growth of the beam spot.

NISP is designed to allow users to add new optical elements and new features to MCLIB, thus making NISP a highly flexible tool for the designer. Collaborative efforts under LANSCE leadership with Oak Ridge and Brookhaven national laboratories, the National Institute of Standards and Technology, and the Rutherford-Appleton Laboratory in the United Kingdom, have helped us-and continue to help us-expand MCLIB. NISP is now being

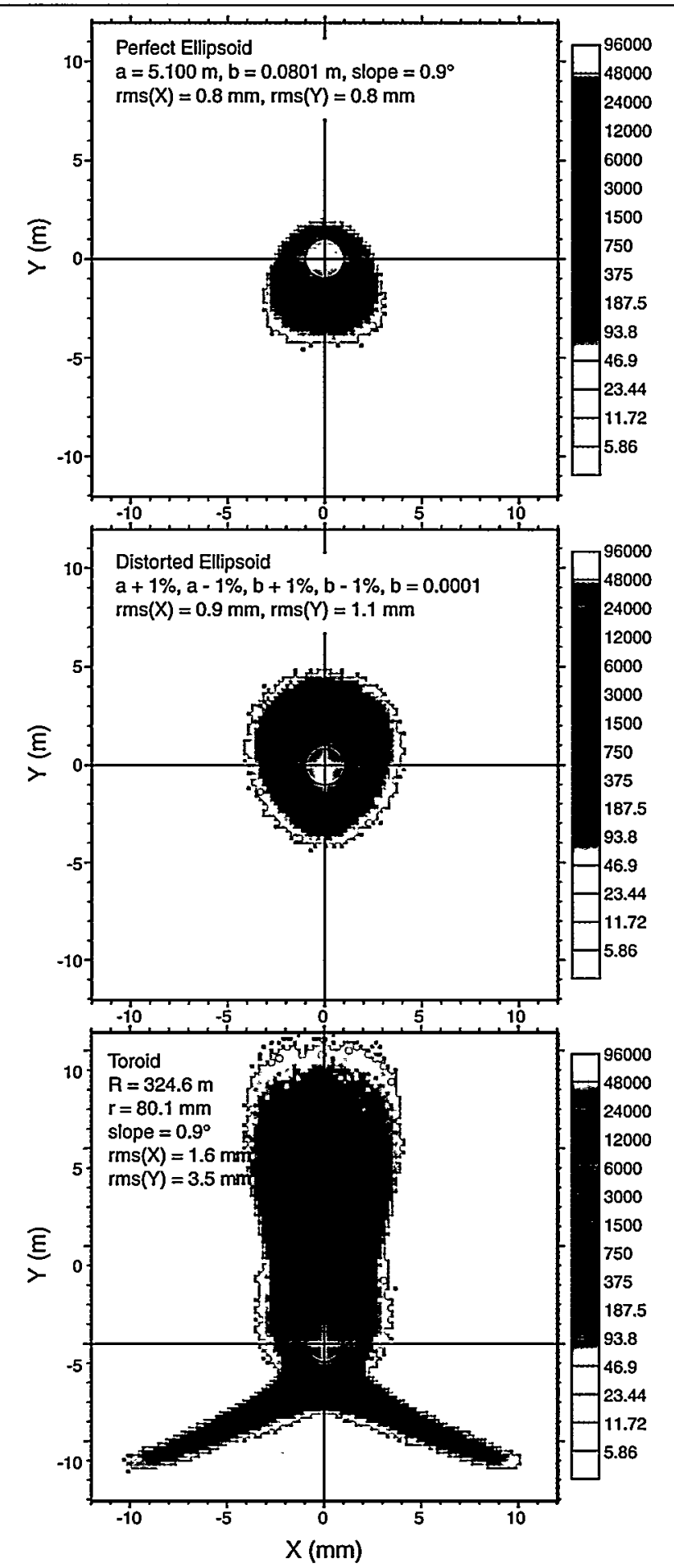

$\Delta$ FIGURE 13. (a) Beam spot projected by a $4 \mathrm{~m}$-ong ellipsoidal mirror (major ond minor axes are $a$ and $b$, respectively) on the defector of a smallongle scattering instrument. The beam spot is the imoge of a circular operture $2 \mathrm{~mm}$ in diometer. The yellowrolored disk (black circle perimeter) is what a simple andyfical calculation would predict. (b) The mirnor was divided in four segments, and the major or minor axis in each segment wos varied by $1 \%$. The beam spot remoins well focused. (c) Beam spot produced by a toroidal mirror. 
considered as the basis for an international standard for the construction of a Monte Carlo library of subroutines for neutron scattering instrument simulation. This effort, under the guidance of the SNS project and Argonne National Laboratory, would provide the neutron scattering community with a much-needed tool and a unified approach to instrument design. At the same time, it would avoid duplication of efforts and guarantee a rapid and universal benchmarking and validation of the final code. This approach is similar to that followed extraordinarily successfully by the SHADOW code-the analog of NISP for $x$-ray instrumentation at synchrotron sources-now in use by thousands of users and the recognized authoritative tool in the field. This is our ultimate goal for NISP.

\section{-L. L. Daemen, P. A. Seeger, R. P. Hjelm (LANSCE \\ Division), T. G. Thelliez (Computing, Information, and Communications Division)}

\section{Web-Based Data Acquisition}

In response to the need for new instruments at the Lujan Center, we have designed a new data acquisition system based upon neutron scattering requirements, market trends, and developments within the data acquisition community. We placed high value on designs that facilitated experimental scalability, user flexibility, and hardware/software availability. These values lead us to Web-based software systems and modular hardware systems.

We chose our new data acquisition hardware to be commercially successful VXI and VME systems, so that we may build our systems with any of the off-the-shelf processor modules incorporating virtually any microprocessor chip (PowerPC, Intel, Alpha, MIPS) and with other numerous VXI/VME modules useful for instrument control. In collaboration with scientists from Argonne National Laboratory's Intense Pulsed Neutron Source, we participated in the design of the single custom VXI module required to time stamp and buffer events from our scattering experiments.

Just as the hardware leverages commercial products, we want to exploit successful commercial and public domain software products. The three data acquisition components shown in Figure 14, the user interface, the data acquisition Web server, and the real-time system, will employ a generic Web browser, WindowsNT, and VxWorks as these software operating systems, respectively. WindowsNT is the industrial-strength version of the Windows system from Microsoft. VxWorks, the real-time kernel used throughout the high-energy and nuclear physics data acquisition communities, will work in conjunction with the Experimental Physics and Industrial Control System (EPICS) software to control environmental conditions for the sample and the configuration of the neutron beamline.

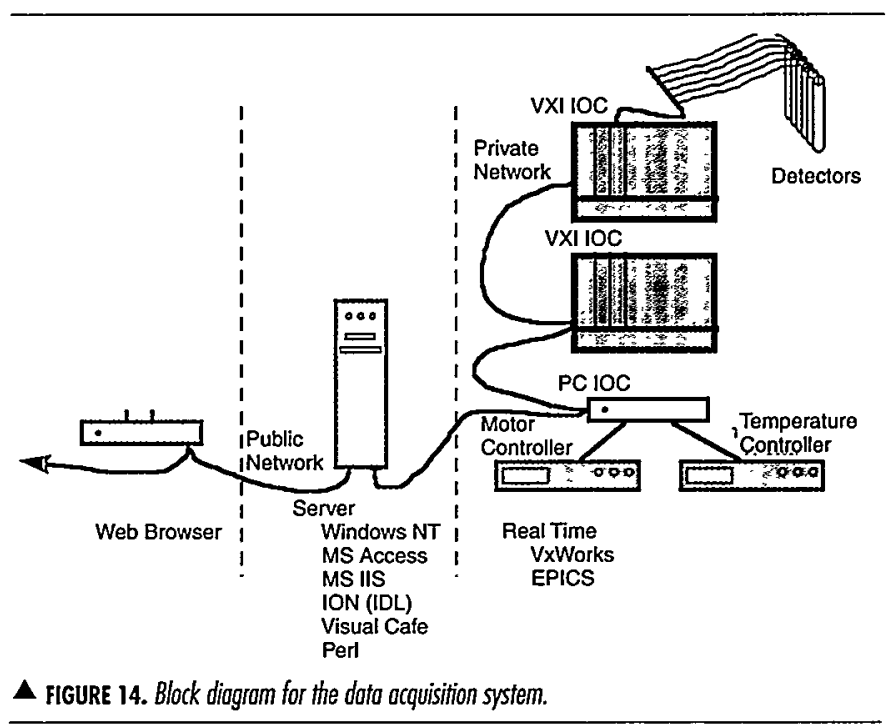

We will develop extensions to EPICS to support event counting and histogram construction.

As indicated in Figure 14, the data acquisitions configuration consists of the user's Web browser, the data acquisition Web server, and the real-time systems. Thanks to the flexibility offered through the Internet, the experimenter may be located in a data room, office, another laboratory, home, or hotel. The real-time systems are tied to the Web server through a separate private network. These real-time systems are either VXI crates or PC IOCs (input/output computers). Each VXI crate will include up to 11 custom VXI TOF modules to capture the data from a pulse of neutrons and one microprocessor to handle the real-time event processing, histogram construction, and storage of the histograms. Optional PC IOCs may share the private network and provide controls to temperature controllers, motors, shutters, etc. In addition to serving the graphical user interface for Web users for data inspection and run control, the server also prepares the databases, histogramming algorithms, and software support for the real-time systems.

In December 1997 we demonstrated a prototype system at the NOBUGS ' 97 conference held at the Argonne National Laboratory. This prototype incorporated features of run control, data monitoring, and control of EPICS channels. While the prototype system did not include any VXI crates or TOF modules, it demonstrated the feasibility of our approach. In October 1998, we plan to field a partial implementation for use of the PHAROS wide-angle bank that includes VXI crates and TOF modules. We expect full production systems by the end of 1999 .

-R. O. Nelson, R. G. Aguilar, G. M. Cooper, T. J. Kluegal, J. J. Ross, J. P. Sandoval, J. R. Seal, B. C. Williams, A. Gjovig-retired (LANSCE Division), P. J. Trujillo (Computing, Information, and Communications Division) 
The LANSCE User Program

Sofety Training

Student Training and Education

Experiment Reports

The LANSCE User Office
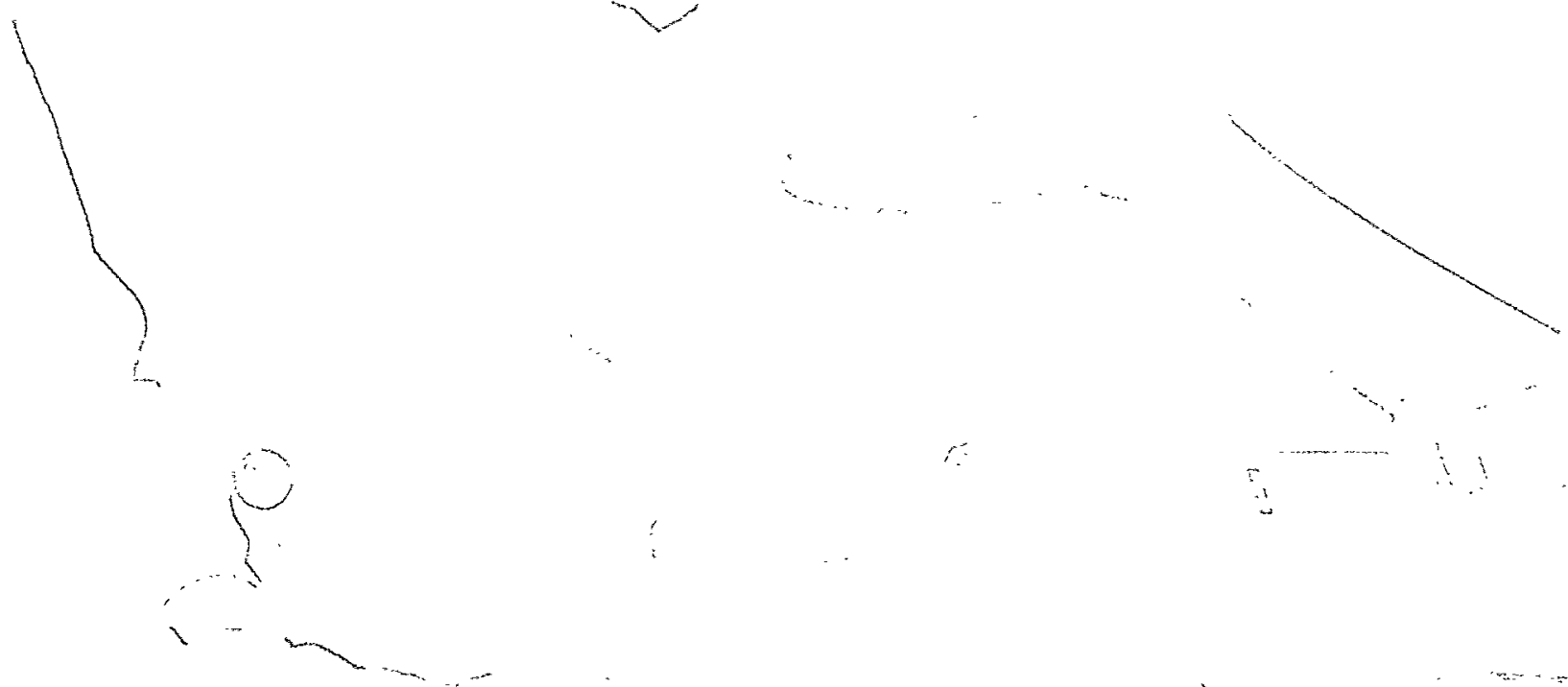



\section{LANSCE: A User Facility for Defense and Civilian Research}

LANSCE is a National User Facility whose goal is to provide state-of-the-art neutron instrumentation; safe, reliable operation; and expert collaborators for the defense and civilian research communities.

LANSCE has two spallation neutron sources: the Manuel Lujan Jr. Neutron Scattering Center (Lujan Center) and the Weapons Neutron Research (WNR) facility. Together they provide neutrons over an unprecendented range of energies -extending from sub-milli-electron-volts to hundreds of mega-electron-volts-that are used for both neutron scattering and nuclear physics research. In addition, LANSCE provides protons for radiography of dynamically evolving systems as well as for materials irradiation and isotope production. Users of the facility span a broad spectrum of institutions in the U.S. and abroad (Figure 1) and scientific disciplines (Figure 2).

Experiments at the Lujan Center and WNR are selected on the basis of proposals that are peer reviewed by the appropriate subcommittee of our Program Advisory Committee (PAC). The areas of expertise of these subcommittees and the current membership are shown in Table 1.

By charter, members of the PAC are selected by LANSCE management for 3-year terms on the basis of recommendations by members of the LANSCE User Group (LUG) through the LUG Executive Committee.

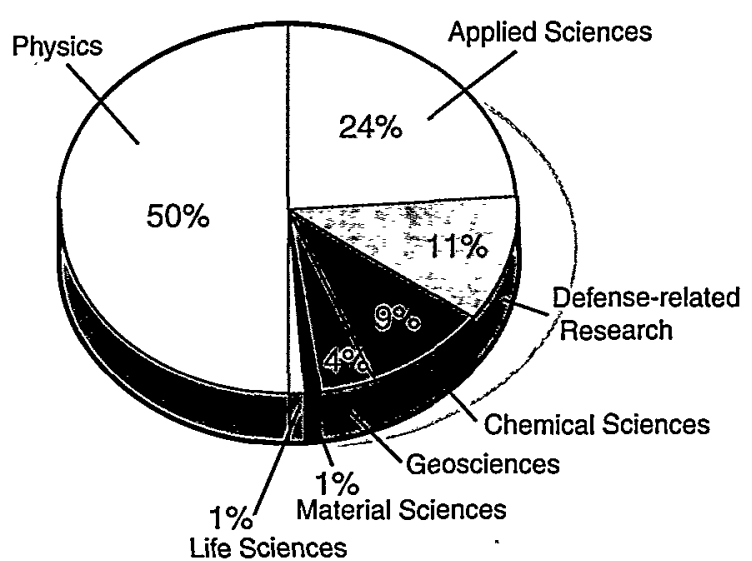

$\triangle$ FIGURE 2. Primary disciplines as reported by LANSCE users during 1995 to 1998. The physics cotegory indudes condensedmotter physics.

\section{Accelerator and Beam Delivery}

The accelerator and beam delivery facilities at LANSCE are operated for proton ion beam delivery to and/or neutron production at the Lujan Center, the WNR facility, the high-intensity experimental Area $\mathrm{A}$, and experimental Areas $B$ and C. Performance generally improved starting in 1995 as shown in Figures 3 and 4, and Table 2.

Performance peaked in FY 1997 when beam was delivered to the Lujan Center spallation target for 3604 hours

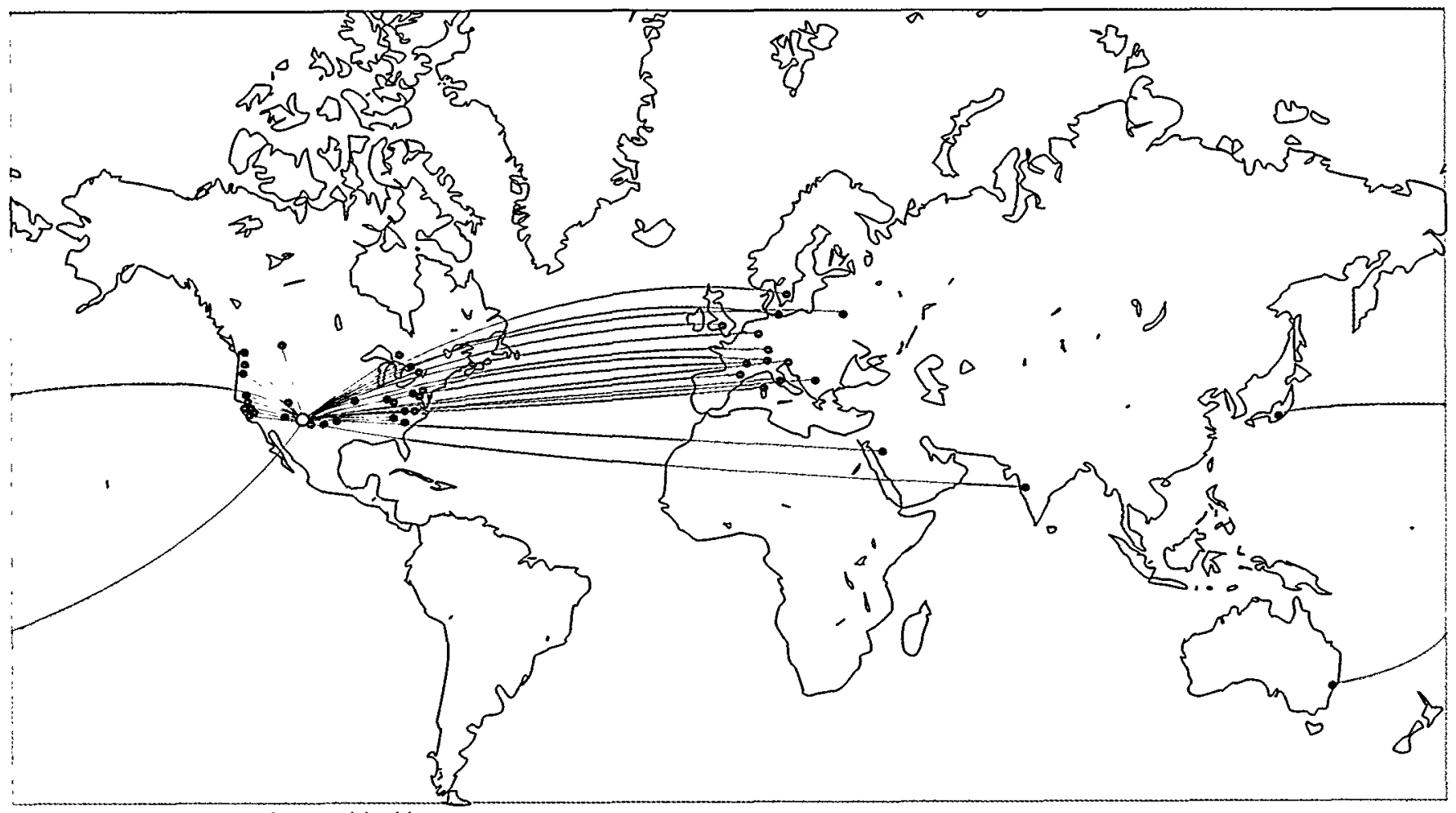

A FIGURE 1. LANSCE users come from oround the globe. 
TABLE 1. 1998 LANSCE Progrom Advisory Subcommittees.

\begin{tabular}{l|l|l}
\hline \multicolumn{1}{c|}{ Materials_Elastic } & \multicolumn{1}{c}{ Materials_-Inelastic } & \multicolumn{1}{c}{ Materials-Large Scale } \\
\hline $\begin{array}{l}\text { Dimitri Argyriou } \\
\text { Los Alamos National Laboratory }\end{array}$ & $\begin{array}{l}\text { Meigan Aronson } \\
\text { University of Michigan }\end{array}$ & $\begin{array}{l}\text { Shenda Baker, Chair } \\
\text { Harvey Mudd College }\end{array}$ \\
\hline $\begin{array}{l}\text { Despo Louca } \\
\text { Los Alamos National Laboratory }\end{array}$ & $\begin{array}{l}\text { Brent Fultz } \\
\text { California Institute of Technology }\end{array}$ & $\begin{array}{l}\text { Gian Felcher } \\
\text { Argonne National Laboratory }\end{array}$ \\
\hline James Richardson, Chair & Kenneth Herwig & Mike Fitzsimmons \\
Argonne National Laboratory & Argonne National Laboratory & Los Alamos National Laboratory \\
\hline Joseph Smyth & Dan Neumann, Chair & William Hamilton \\
University of Colorado & National Inst. of Standards \& Technology & Oak Ridge National Laboratory \\
\hline $\begin{array}{l}\text { Andy Winholtz } \\
\text { University of Missouri }\end{array}$ & Rob Robinson & Mike Kent \\
\hline Thomas Koetzle & Los Alamos National Laboratory & Sandia National Laboratories \\
Brookhaven National Laboratory & Marie-Louise Saboungi \\
Argonne National Laboratory & Thomas Rieker \\
\hline
\end{tabular}

Materials-Defense-Related

\begin{tabular}{|c|c|c|}
\hline $\begin{array}{l}\text { Bard Bennett, Chair } \\
\text { Los Alamos National Laboratory }\end{array}$ & $\begin{array}{l}\text { Alan Patterson } \\
\text { Los Alamos National Laboratory }\end{array}$ & $\begin{array}{l}\text { Steve Valone } \\
\text { Los Alamos National Laboratory }\end{array}$ \\
\hline $\begin{array}{l}\text { Lloyd Chase } \\
\text { Lawrence Livermore National Laboratory }\end{array}$ & $\begin{array}{l}\text { Gordon Pike } \\
\text { Sandia National Laboratories }\end{array}$ & \\
\hline $\begin{array}{l}\text { Bob Von Dreele (materials advisory/nonvoting) } \\
\text { Los Alamos National Laboratory }\end{array}$ & \multicolumn{2}{|c|}{$\begin{array}{l}\text { Gene Farnum (defense programs advisory/nonvoting) } \\
\text { Los Alamos National Laboratory }\end{array}$} \\
\hline $\begin{array}{l}\text { Basic Nuclear } \\
\text { and Particle Physics }\end{array}$ & Nuclear Technology & $\begin{array}{l}\text { Nuclear Science-_ } \\
\text { Defense-Related }\end{array}$ \\
\hline $\begin{array}{l}\text { Jolie Cizewski } \\
\text { Rutgers University }\end{array}$ & $\begin{array}{l}\text { Arnold Sierk } \\
\text { Los Alamos National Laboratory }\end{array}$ & $\begin{array}{l}\text { Paul Bradley } \\
\text { Los Alamos National Laboratory }\end{array}$ \\
\hline $\begin{array}{l}\text { Dan Strottman } \\
\text { Los Alamos National Laboratory }\end{array}$ & $\begin{array}{l}\text { Benjamin Gibson } \\
\text { Los Alamos National Laboratory }\end{array}$ & $\begin{array}{l}\text { Stephanie Frankle } \\
\text { Los Alamos National Laboratory }\end{array}$ \\
\hline $\begin{array}{l}\text { Blayne Heckel } \\
\text { University of Washington }\end{array}$ & $\begin{array}{l}\text { Michael Kreisler } \\
\text { Lawrence Livermore National Laboratory }\end{array}$ & $\begin{array}{l}\text { Michael Kreisler, Chair } \\
\text { Lawrence Livermore National Laboratory }\end{array}$ \\
\hline $\begin{array}{l}\text { Benjamin Gibson } \\
\text { Los Alamos National Laboratory }\end{array}$ & $\begin{array}{l}\text { S. Raman } \\
\text { Oak Ridge National Laboratory }\end{array}$ & $\begin{array}{l}\text { Charlie Miller } \\
\text { Los Alamos National Laboratory }\end{array}$ \\
\hline $\begin{array}{l}\text { June Matthews, Chair } \\
\text { Massachusetts Institute of Technology }\end{array}$ & $\begin{array}{l}\text { Stan Roeske/Fred Sexton } \\
\text { Sandia National Laboratories }\end{array}$ & $\begin{array}{l}\text { Mohammed Mustafa } \\
\text { Lawrence Livermore National Laboratory }\end{array}$ \\
\hline $\begin{array}{l}\text { Lee Riedinger } \\
\text { University of Tennessee }\end{array}$ & $\begin{array}{l}\text { Lynn Veeser, Chair } \\
\text { Los Alamos National Laboratory }\end{array}$ & $\begin{array}{l}\text { Lynn Veeser } \\
\text { Los Alamos National Laboratory }\end{array}$ \\
\hline
\end{tabular}

(7 months). During FY 1997, an additional month of beam was delivered to all areas except the Lujan Center.

During FY 1998, operation of the facility was interrupted to install a new injection system in the Proton Storage Ring (PSR) and to construct and install a new target/moderator system for the Lujan Center. In June 1998, beam was delivered to support proton radiography and continued as other LANSCE facilities prepared to accept beam. The year ended with these new facilities being commissioned and beam delivery in progress to WNR and Area A.

\section{Commissioning of the New Facilities}

A major activity in 1998 was the startup and commissioning of the upgraded PSR injection line, beam buncher, and the new, re-engineered Target/Moderator/Reflector System at the Lujan Center. The PSR received beam August 1, 1998, and beam was directed to the new target October 24, 1998.

\section{Neutron Production}

During FY 1995 through FY 1997, the reliability of $\mathrm{H}^{-}$ beam delivery to the neutron spallation target steadily increased as a result of our maintenance and improvement efforts. The average beam current was limited to $70 \mu \mathrm{A}$ because of the assessed capability of the target system.

\section{$\mathrm{H}^{+}$Beam Delivery}

The reliability of beam delivery to users in the high-intensity experimental area declined from FY 1996 through FY 1997. Accelerator performance remained higher than in the recent past, but the reduced support for this experimental area resulted in an increasing number of equipment 


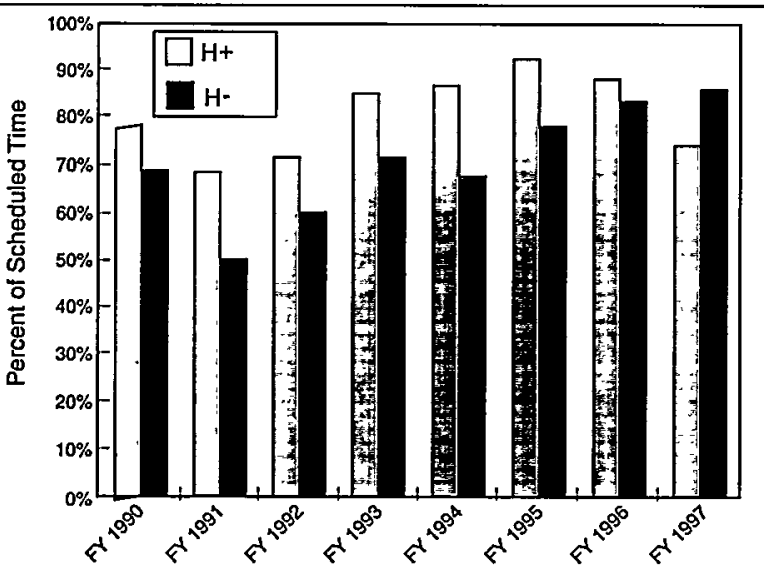

$\triangle$ FIGURE 3. Beom availability by yeor for Area $A H^{+}$, WNR $H$, and Lujon $H$ beams. (Availability is defined os the percentage of scheduled beom hours that are actually delivered. It does not indude unscheduled beam hours delivered to compensate for downtime.)

failures. Frequent arcs in the high-voltage injector that marred the performance of beam delivery in FY 1997 were cured and absent during FY 1998.

\section{Areas $B$ and $C$}

The beam lines served by Line $X$ were restored in 1995 for several projects requiring $800-\mathrm{MeV}$ proton beams at low to average intensity. These areas had been removed from service in 1993 to reduce operating costs. In 1997, 25 beam pulses were delivered for proton radiography to Area B in synchronism with the detonation of experimental samples. Close teamwork between the experimental team and the accelerator operators allowed a perfect record of beam synchronism with the detonation process. During 1998, 20 days of beam delivery to users at Areas $\mathrm{B}$ and $\mathrm{C}$ were provided.

\section{Weapons Neutron Research Facility}

Beam hours, current, and reliability for Target 4 at WNR improved between FY 1995 and FY 1998. Additionally, work on Neutron Resonance Spectroscopy (NRS) in the

\begin{tabular}{|c|c|}
\hline \multicolumn{2}{|c|}{ Useful World Wide Web Addresses } \\
\hline Helpful Information & hthp://... \\
\hline LANSCE Home Page & lansce.lanl.gor \\
\hline Beom Availobility \& Schedules & lansce.lonlggov/operations/index_op.htm \\
\hline Instrument Avoilability & bayberry.lanl.gov//ml/Welcome.hml \\
\hline Facility Upgrades & lanscelonnlgov/focilities/upgrades.htm \\
\hline Lujon Instrument Specifications & lansce.lanl.gov/lujan/instruments.hmm \\
\hline WNR Flight Path Information & wnrlonl.gov/facility/index.hm \\
\hline Getting To \& Around LANSCE & lansce.lonl.gov/users/visitors.hm \\
\hline Proposal Submission & lansce.lanl.gov/users/proposals/index_prop.hm \\
\hline Proposal Writing Tips & lanscelonl_gov/users/proposals/index_prop.htmtthints \\
\hline User Home Page & lansce.lonl.gov/users/index_users.hm \\
\hline User Group & lansce.lonl.gov/users/ug/ug_index.htm \\
\hline User Training & unw.atdivlanl.gov/ooffm/factro/Userstrain.hm \\
\hline
\end{tabular}

Blue Room (Target 2) at WNR required synchronizing beam delivery from the PSR to the detonation of small explosive charges.

\section{Operations Progress Reports}

Detailed beam delivery statistics for FY 1996 and FY 1997 , including goals and measures, accelerator control system maintenance, and beam diagnostics are available in the Operations Progress Reports included on the compact disk (CD) located on the inside back cover of this Activity Report.

\section{Instrument Availability at the Lujan Center}

In 1997, the Lujan Center initiated an automated system for tracking instrument and hydrogen moderator availability during scheduled beam time. The goal is $95 \%$ reliability for the instruments and the moderators with respect to the schedule. We measure the data-collection time (run time); the setup time (changing environmental conditions, sample, instrument configuration, and so forth); and the

TABLE 2. 1995-1997 Operations Performance Summory.

\begin{tabular}{l|c|c|c|c|c|c|c|c|c}
\hline & \multicolumn{3}{|c|}{ FY 1995 } & \multicolumn{3}{c|}{ FY 1996 } & \multicolumn{3}{c}{ FY 1997 } \\
\cline { 2 - 11 } & $\begin{array}{c}\text { Lujan } \\
\mathbf{H}^{-}\end{array}$ & $\begin{array}{c}\text { WNR } \\
\mathbf{H}^{-}\end{array}$ & $\mathbf{H}^{+}$ & $\begin{array}{c}\text { Lujan } \\
\mathbf{H}^{-}\end{array}$ & $\begin{array}{c}\text { WNR } \\
\mathbf{H}^{-}\end{array}$ & $\mathbf{H}^{+}$ & $\begin{array}{c}\text { Lujan } \\
\mathbf{H}^{-}\end{array}$ & $\begin{array}{c}\text { WNR } \\
\mathbf{H}^{-}\end{array}$ & $\mathbf{H}^{+}$ \\
\hline Scheduled Hours & 1095 & 2241.6 & 2773.3 & 2808 & 2324 & 2074.4 & 3604.3 & 3073.9 & 4192.3 \\
\hline Scheduled Current $(\mu \mathrm{A})$ & - & & 70 & - & 1000 & 70 & - & 1000 & \\
\hline Delivered Hours & 849 & 1855.6 & 2558.2 & 2341.8 & 1922.8 & 1831 & 3240.4 & 2695.7 & 3092.4 \\
\hline Ave. Delivered Current $(\mu \mathrm{A})$ & 62.9 & 2.2 & 981.8 & 69.3 & 2 & 957.2 & 70.3 & 2.36 & 9214 \\
\hline mA.hr Scheduled & & - & 196.6 & - & 2074.4 & 252.3 & - & 4192.3 & \\
\hline mA.hr Delivered & 53 & 4 & 2512 & 163.8 & 2.4 & 1739.8 & 217.1 & 5.9 & 2828.9 \\
\hline Availability (hours) & $78 \%$ & $83 \%$ & $92 \%$ & $83.4 \%$ & $83.2 \%$ & $88.3 \%$ & $90 \%$ & $87.7 \%$ & $73.8 \%$ \\
\hline
\end{tabular}

Note: $\mathrm{H}^{+}$was available on schedule in 1998, but experiments were delayed by 3 months. 
Lujan Center Target

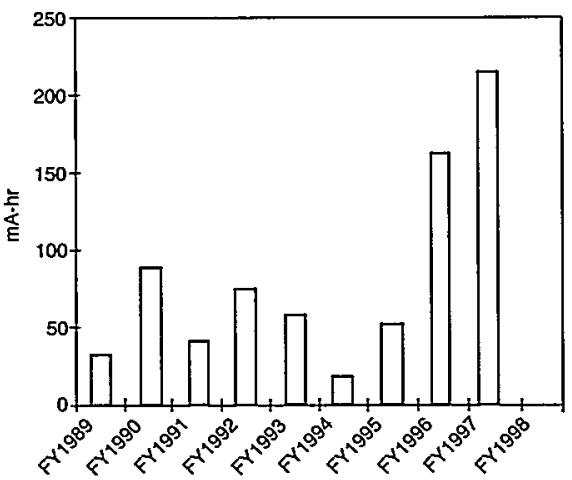

WNR

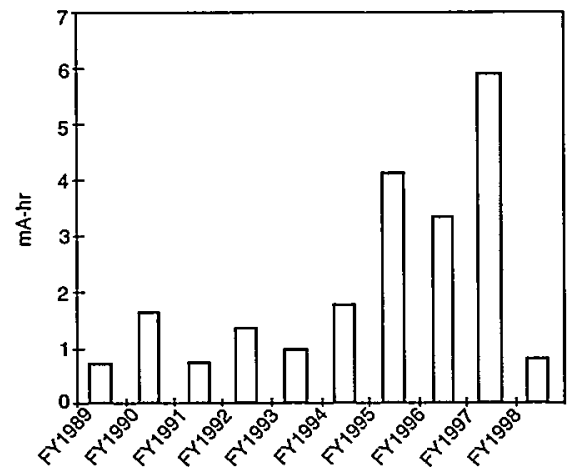

Area A

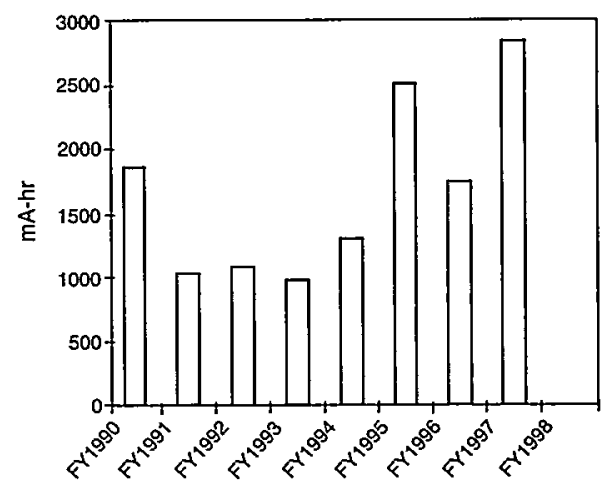

$\triangle$ FIGURE 4. H beam charge delivered to the Lujan Center target, $H$ beam chorge delivered to WNR, and $H^{+}$beam charge delivered to Area $A$..

unscheduled downtime for each instrument. For the hydrogen moderator, we track whether the moderator was operating properly or not operating.

Figure 5 represents 1997 run cycle data at the Lujan Center. These data, as well as additional useful information, are accessible on the LANSCE World Wide Web site at bayberry.lanl.gov/ml/Welcome.html.

\section{Neutron Scattering Spectrometers}

On the left part of the bar graph in Figure 5, green indicates the time period when the instrument was collecting

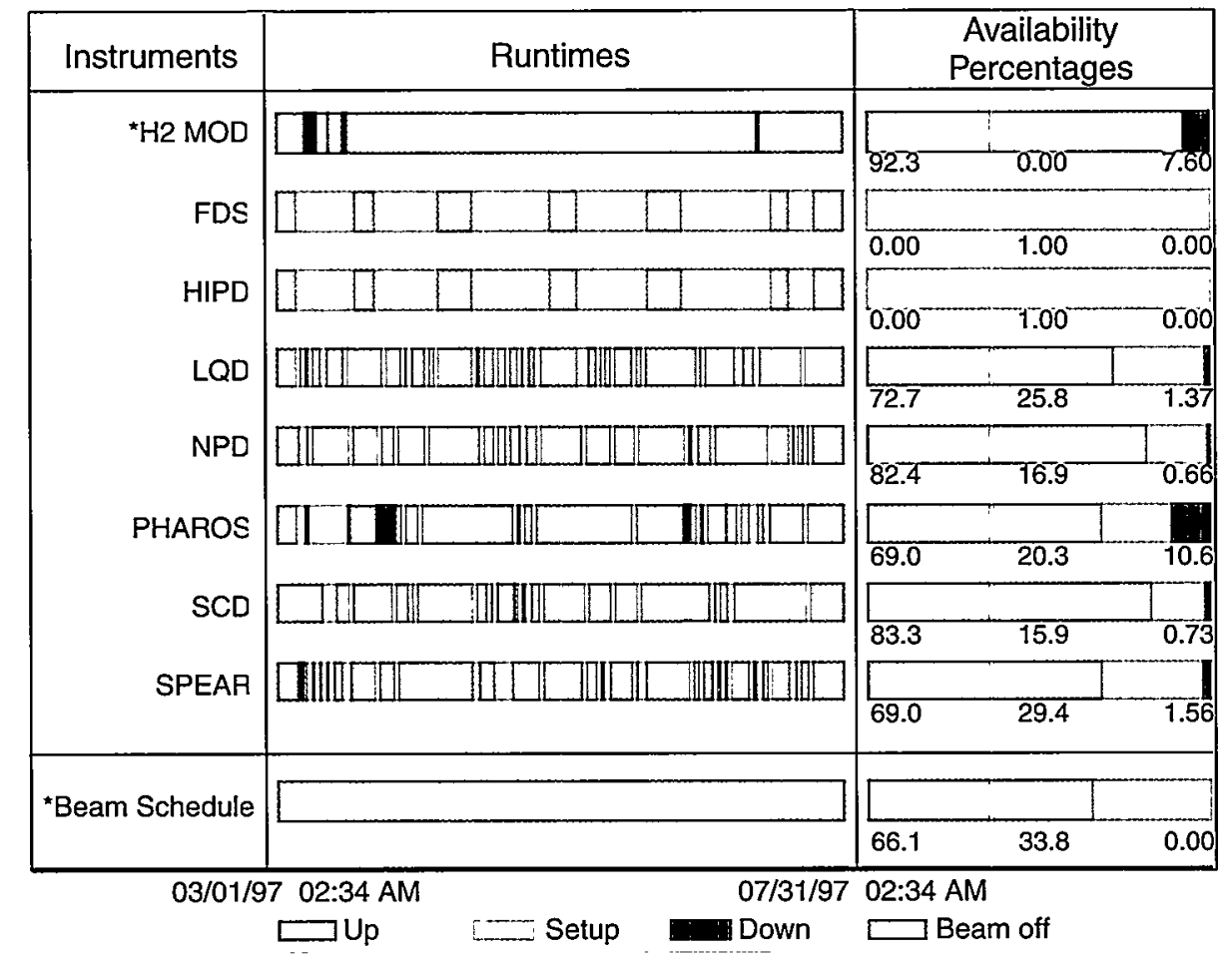

A FIGURE 5. Instrument and hydrogen moderator availability at the Lujan Center for the 1997 run cycle from March 1, 1997, through July 31, 1997. Informotion for interprefing the bar grophs is provided in the text. data, yellow when the instrument was in setup mode, and red when there was unscheduled downtime. Blue indicates the instrument was not scheduled to operate at that time. On the right, a summary of the time each instrument spent collecting data (green), being set up (yellow), or in unscheduled downtime (red) is given as a percentage of the total scheduled instrument time. The three percentage values below the bars on the right correspond, respectively. For the 1997 run cycle, all but one of the instruments were available to users (collecting data or in setup mode) greater than $95 \%$ of the time.

Liquid Hydrogen Moderator

The liquid hydrogen moderator availability (H2-MOD) is also tracked as shown in Figure 5. The moderator only allows binary states-it is filled and at temperature (green) or it is not (red). The moderator was a little shaky at the beginning of the 1997 run cycle, but its availability improved significantly after the first month to an overall value of $92.4 \%$.

Figure 6 shows the percentage of total instrument time affected by various unscheduled downtimes incidents. All incidents greater than 24 hours were investigated. A report was written describing the incident and the remedy in order to document and incorporate lessons learned.

\section{Beam Schedule Definition}

The bottom row in Figure 5 indicates the beam schedule, which is usually completely overlapped by the 
Percent Instrument Time Affected

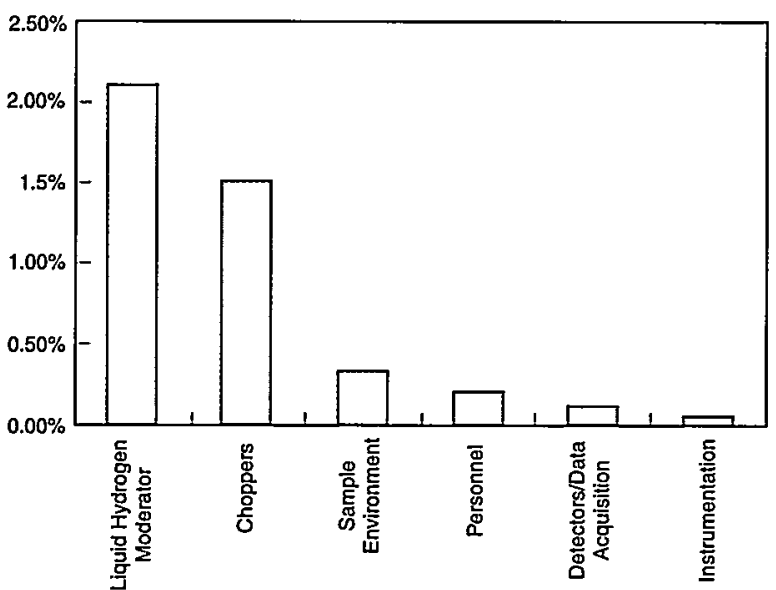

$\triangle$ FIGURE 6. The percentoge of total instrument time offected by unscheduled downtime as a result of various factors (i.e., moderator, choppers, somple environment, etc.). The percentoge is the product of the number of instruments affected by the total number of beam days the instrument was unavailable, divided by fotal instrument time. The totol instrument time is the number of instruments muliplied by the number of scheduled beom doys.

instrument schedule. The green indicates that the beam was scheduled to be delivered to the Lujan Center. The blue indicates times when the beam is not scheduled. Activities during "blue" periods include $\mathrm{H}^{-}$source recycling, proton radiography experiments, and NRS experiments.

\section{The LANSCE User Program}

The major portion of the funding for the LANSCE facility is provided by the Department of Energy (DOE) Office of Defense Programs (DP), which uses LANSCE in support of its stockpile stewardship mission to ensure that U.S. nuclear weapons remain safe, secure, and reliable without nuclear testing. Work at LANSCE in support of the DP mission includes

- neutron scattering measurements that interrogate materials aging, equations of state, and so forth

- nuclear cross section measurements required, for example, for more extensive interpretation of radiochemical data from past nuclear tests

- proton radiography of dynamically evolving systems such as implosions

- NRS measurements of temperature and particle velocity in dynamically evolving systems

- materials irradiation experiments required to certify materials for a new Accelerator Production of Tritium (APT) plant at the Savannah River site

Most of the DP users of LANSCE are from one of the three defense laboratories: Lawrence Livermore, Los Alamos, and Sandia as shown in Figure 7.

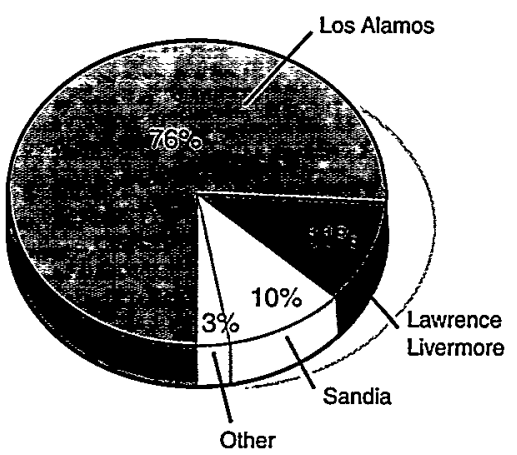

$\triangle$ FIGURE 7. Defense Program users during 1997 run cycle.

The obvious synergy between the DP uses of LANSCE and the areas of basic research that have traditionally used reactor or spallation neutron sources, particularly in the area of condensed-matter science, has led to a strong alliance between DP and the DOE Office of Basic Energy Sciences (BES) to support and use LANSCE (Figure 8 and Table 3). BES provides funding for a National User

\section{The DOE Offices of Defense Programs and Energy Research have long-term synergistic needs for accelerator and neutron science.}

Program at the Lujan Center that attracts scientists in various disciplines who use neutron scattering in support of their research (Figure 9). The synergy between DP and BES allows scientists from different institutions (Figure 10) to perform defense, basic, and industrial research (Figure 11) at LANSCE.

\section{Published versus Proprietary Research}

Experiments at the Lujan Center or WNR that involve either results that are publishable in the open literature or support the DOE's stockpile stewardship mission receive beam time from LANSCE at no cost to the user. Beam time for proprietary work can be purchased by a special user agreement under DOE's full-cost recovery rules.

Major Civilian Companies Performing Work at LANSCE from 1995-1998

Aerospatiale Aeronautique
Boeing Company
Digital Equipment Corporation
Fuïtsu Laboratories Limited
General Electric
Hewlitt Packord

Hitachi limited Honeywell BCAS Intel Corporation Lockheed Martin PPG Industries Incorporated Texos Instruments Incorporated 


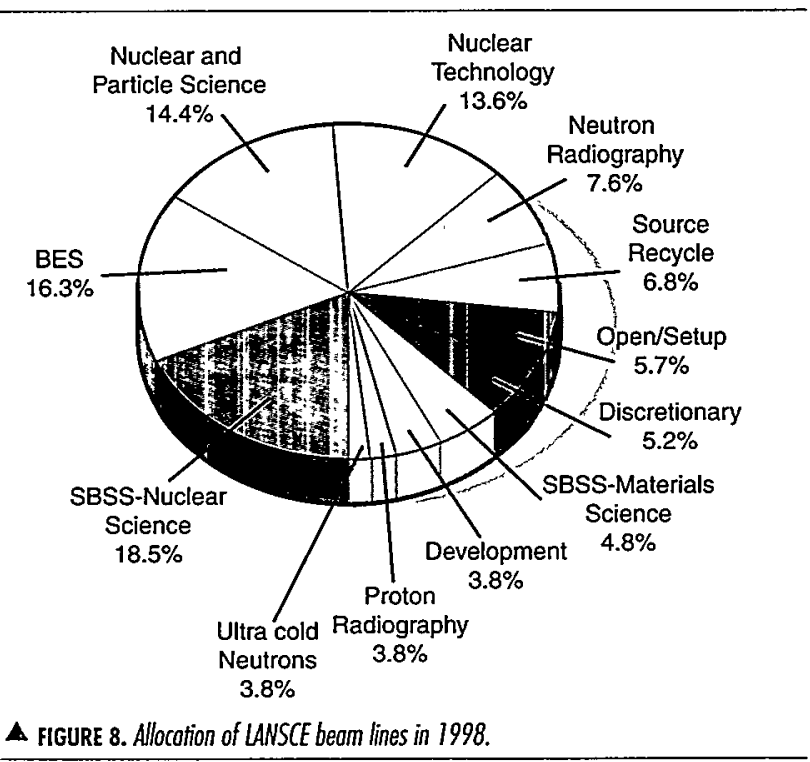

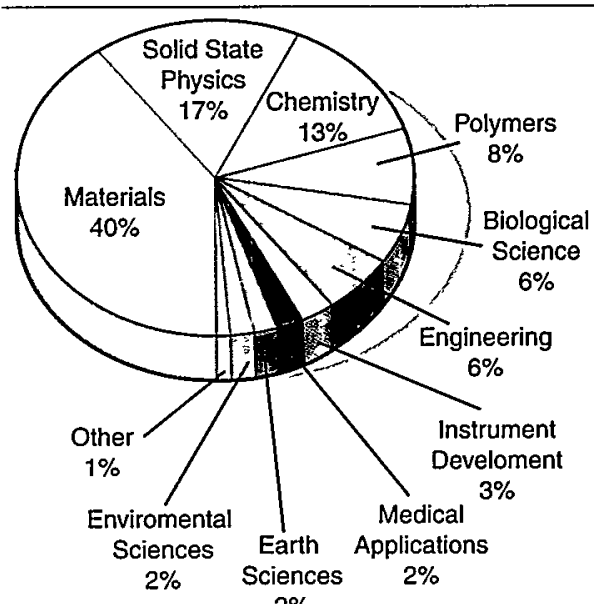

$\Delta$ fIGURE 9. Basic reseorch disciplines corried out of the Lujon Center, as reported by users on 1998 proposals for beam time.

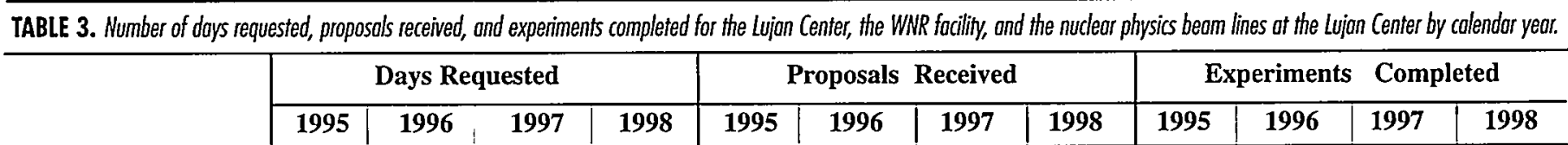

\section{Lujan Center}

(\#:\# = 1997 instrument oversubscription rate)

\begin{tabular}{|c|c|c|c|c|c|c|c|c|c|c|c|c|c|}
\hline $\begin{array}{l}\text { FDS } \\
(1: 1)\end{array}$ & $\begin{array}{l}\text {-Basic } \\
\text {-Defense }\end{array}$ & 57 & $\begin{array}{l}80.5 \\
14\end{array}$ & $\begin{array}{l}66 \\
20\end{array}$ & $\begin{array}{r}86 \\
5\end{array}$ & 7 & $\begin{array}{r}12 \\
2 \\
\end{array}$ & $\begin{array}{l}9 \\
3 \\
\end{array}$ & $\begin{array}{r}12 \\
1 \\
\end{array}$ & 7 & $\begin{array}{l}9 \\
1 \\
\end{array}$ & $\begin{array}{l}6 \\
2 \\
\end{array}$ & $*$ \\
\hline $\begin{array}{l}\text { HIPD } \\
(3: 1)\end{array}$ & $\begin{array}{l}\text {-Basic } \\
\text {-Defense }\end{array}$ & 110 & $\begin{array}{l}155.5 \\
121\end{array}$ & $\begin{array}{r}139 \\
62\end{array}$ & $\begin{array}{r}129 \\
92\end{array}$ & 29 & $\begin{array}{l}38 \\
11\end{array}$ & $\begin{array}{l}46 \\
10\end{array}$ & $\begin{array}{l}34 \\
14\end{array}$ & 21 & $\begin{array}{r}17 \\
7\end{array}$ & $\begin{array}{r}32 \\
7\end{array}$ & $*$ \\
\hline $\begin{array}{l}\text { LQD } \\
(2: 1)\end{array}$ & $\begin{array}{l}\text {-Basic } \\
\text {-Defense }\end{array}$ & 27 & $\begin{array}{l}45.5 \\
23\end{array}$ & $\begin{array}{l}49 \\
25\end{array}$ & $\begin{array}{l}77 \\
20\end{array}$ & 8 & $\begin{array}{r}10 \\
6\end{array}$ & $\begin{array}{r}11 \\
6\end{array}$ & $\begin{array}{r}19 \\
3\end{array}$ & 0 & $\begin{array}{l}8 \\
5\end{array}$ & $\begin{array}{r}16 \\
5\end{array}$ & * \\
\hline $\begin{array}{l}\text { NPD } \\
(2: 1)\end{array}$ & $\begin{array}{l}\text {-Basic } \\
\text {-Defense }\end{array}$ & 82 & $\begin{array}{c}152.5 \\
32\end{array}$ & $\begin{array}{r}149 \\
29\end{array}$ & $\begin{array}{r}133 \\
57\end{array}$ & 20 & $\begin{array}{r}18 \\
5\end{array}$ & $\begin{array}{r}24 \\
4\end{array}$ & $\begin{array}{r}22 \\
6\end{array}$ & 19 & $\begin{array}{r}11 \\
3\end{array}$ & $\begin{array}{r}19 \\
3 \\
\end{array}$ & $*$ \\
\hline $\begin{array}{l}\text { PHAROS } \\
(2: 1)\end{array}$ & $\begin{array}{l}\text {-Basic } \\
\text {-Defense }\end{array}$ & 96 & $\begin{array}{r}77 \\
7 \\
\end{array}$ & $\begin{array}{r}139 \\
0 \\
\end{array}$ & $\begin{array}{r}199 \\
20 \\
\end{array}$ & 9 & $\begin{array}{l}7 \\
1 \\
\end{array}$ & $\begin{array}{r}10 \\
0 \\
\end{array}$ & $\begin{array}{r}19 \\
2 \\
\end{array}$ & 5 & $\begin{array}{l}6 \\
1 \\
\end{array}$ & $\begin{array}{l}8 \\
0 \\
\end{array}$ & $*$ \\
\hline $\begin{array}{l}\text { SCD } \\
(2: 1) \\
\end{array}$ & $\begin{array}{l}\text {-Basic } \\
\text {-Defense }\end{array}$ & 95 & $\begin{array}{l}68 \\
24 \\
\end{array}$ & $\begin{array}{r}101 \\
0\end{array}$ & $\begin{array}{r}72 \\
0 \\
\end{array}$ & 7 & $\begin{array}{l}5 \\
1 \\
\end{array}$ & $\begin{array}{r}12 \\
0 \\
\end{array}$ & $\begin{array}{l}8 \\
0 \\
\end{array}$ & 5 & $\begin{array}{l}1 \\
1 \\
\end{array}$ & $\begin{array}{l}6 \\
0\end{array}$ & $*$ \\
\hline $\begin{array}{l}\text { SPEAR } \\
(2: 1)\end{array}$ & $\begin{array}{l}\text {-Basic } \\
\text {-Defense }\end{array}$ & 69 & $\begin{array}{r}163 \\
25\end{array}$ & $\begin{array}{l}96.5 \\
25.5\end{array}$ & $\begin{array}{r}215 \\
10\end{array}$ & 18 & $\begin{array}{r}28 \\
7\end{array}$ & $\begin{array}{r}16 \\
5\end{array}$ & $\begin{array}{r}30 \\
2\end{array}$ & 10 & $\begin{array}{r}16 \\
3\end{array}$ & $\begin{array}{r}19 \\
5\end{array}$ & $*$ \\
\hline
\end{tabular}

\section{WNR and Nuclear Physics Beam Lines at Lujan Center}

\begin{tabular}{l|c|c|c|c|c|c|c|c|c|c|c|c}
\hline $\begin{array}{l}\text { Defense Nuclear } \\
\text { Physics }\end{array}$ & 198 & 327 & 482 & 349 & 6 & 7 & 12 & 11 & 5 & 6 & 11 & 8 \\
\hline Nuclear Technology & 122 & 312 & 442 & 244 & 19 & 16 & 18 & 24 & 17 & 14 & 12 & 13 \\
\hline Basic Physics & 331 & 284 & 200 & 700 & 4 & 6 & 20 & 18 & 3 & 8 & 14 & 6 \\
\hline
\end{tabular}

*At time of printing, the 1998 Lujan Center User Program had not commenced.

Note: Breakout for defense and basic research proposals not available for 1995 . 


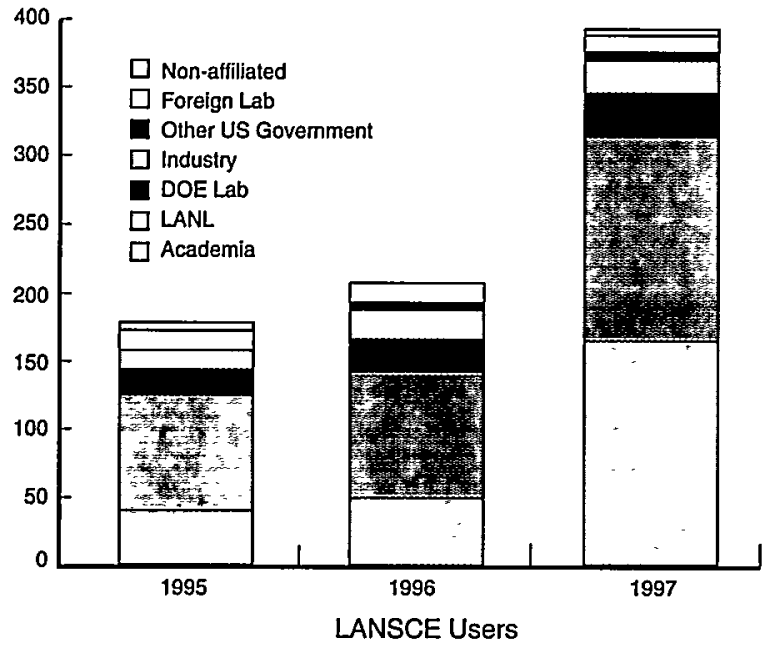

A FIGURE 10. Number of LANSCE users by affiliation. Eoch user is counted only once, regordless of the number of experiments and/or visits. All LANSCE experimental areas are represented.

\section{Safety Training}

No activity is permitted at LANSCE that could in any way jeopardize the safety of users, visitors, or employees. The facility is complex, posing a number of potential hazards that are mitigated both by engineered controls and appropriate training. Although users are exposed to only a small part of the overall facility during their visit, it is important that they understand how to use equipment safely and what to do under both normal and abnormal circumstances.

A variety of training methods are used to accommodate different learning styles and provide preparatory materials to make the training process as efficient as possible.

Methods include:

- User Training Study Booklet

- On-line web-based training

- Interactive Computer-Based Training

- Quick Reference Job Aids

- Challenge Exams

Presently, five computer terminals are dedicated to computer-based and web-based training applications in the LANSCE Training Lab. Training sessions are offered three times daily. Written study materials are available to users before their scheduled visits in order to minimize their training time when they arrive. On average, the entire training and check-in process takes no more than 2 to 3 hours once the user arrives at LANSCE.

To provide valuable training information to users who have English comprehension difficulties, a new program was developed to assist Russian visitors with basic environment, safety, and health terminology. The Russian Training Introduction is the first in a series of translated training

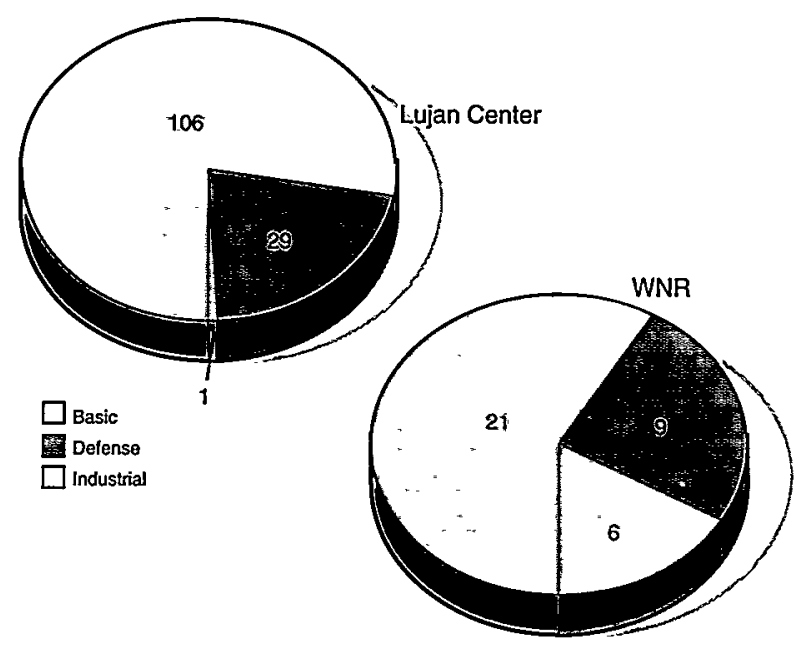

$\triangle$ FIGURE II. Numbers of defense, bosic, and industrial research experiments run during 1997 on neutron scottering spectrometers at the Lujon Center (left), and nuclear physics flight paths ot the Lujon Center and WNR (right).

materials to be developed, and includes graphics and onscreen text.

\section{Student Training and Education}

LANSCE recognizes that it has a key role to play in science education, not only of the graduate students who use the facility as part of their higher education, but also of younger students, especially those from Northern New Mexico. Students, as well as postdoctoral fellows, learn from and contribute to the research at LANSCE (Figure 12). A listing of theses completed that used LANSCE facilities is available following the Publications section.

The Lujan Center takes great pride in serving as a training ground for undergraduate and graduate students in the use of neutron scattering techniques. Many students spend summer months as Los Alamos National Laboratory employees working at the Lujan Center, sometimes at the recommendation of their thesis advisor. Students who avail themselves of this opportunity are paid a salary during their visit. More information on student employment programs available at the Laboratory can be obtained from the Laboratory's Human Resources Division Web site at www.hr.lanl.gov/Students/.

\section{Experiment Reports}

Experiment reports are required for all experiments performed at the Lujan Center or WNR. (We "encourage" those who forget this responsibility by reminding them that their approved experiments cannot be scheduled until the report from their last experiment is received!) Experiment Reports from 1995 to 1997, which describe what was done 


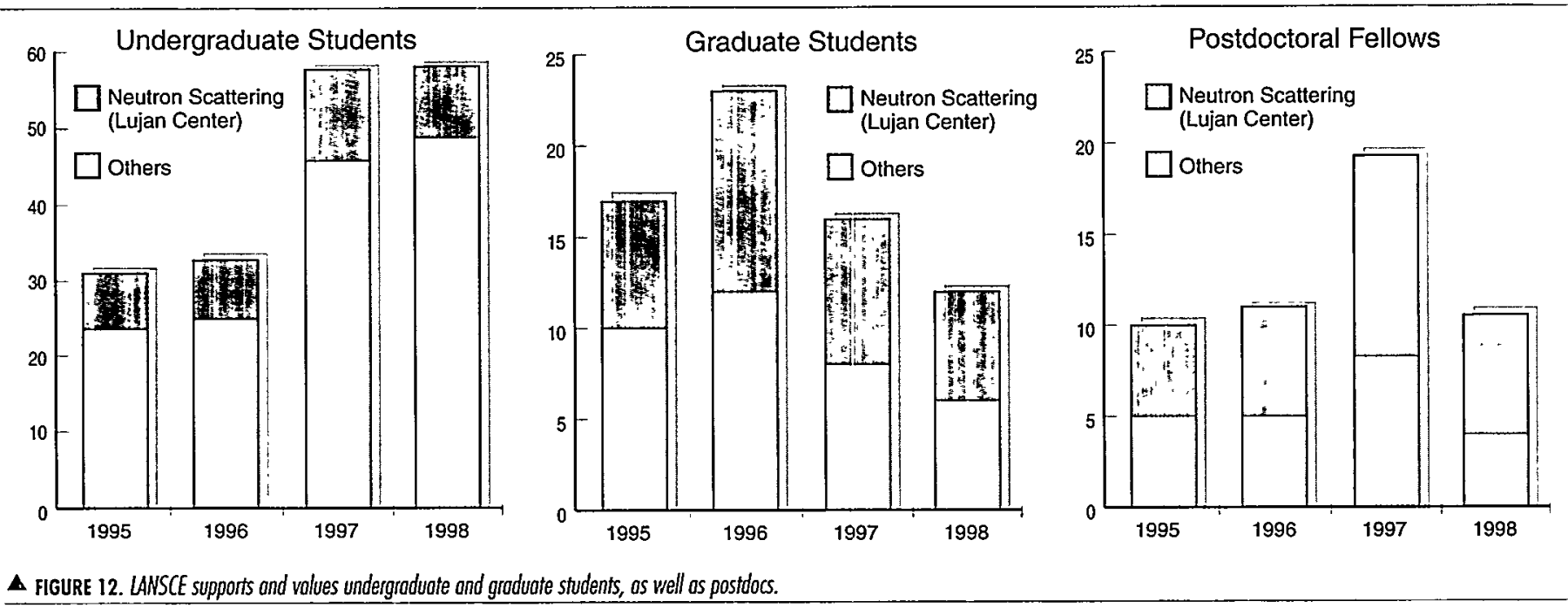

with allocated beam time, are included on the $C D$ in the inside back cover of this Activity Report. Current reports can be obtained by contacting the LANSCE User Office.

\section{The LANSCE User Office}

The LANSCE User Office (Figure 13) develops, organizes, and administers the user program, including:

- assisting users and visitors with planning and coordinating their visit

- administering the proposal, review, and scheduling processes

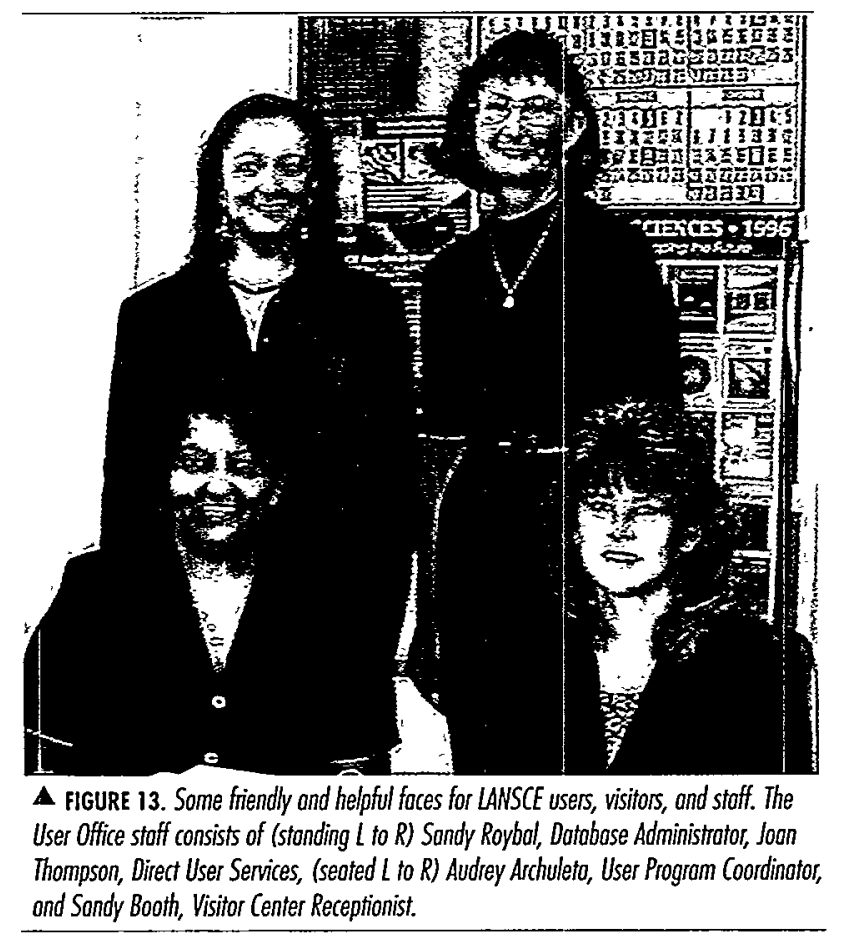

- improving processes for user access, orientation, training, and services

- communicating beam-schedule information to users

- supporting conferences and workshops

- supporting the User Group and other committees integral to the user program

- organizing and supporting the Annual User Meeting

- collecting and reporting statistical information about the facility and users

- assessing and reporting user satisfaction

- arranging travel support and funding support for students

- coordinating user/visitor communications

- reception for all visitors and users to the facility

- arranging facility tours

\section{The User Program Evolves}

In 1997, a formal User Satisfaction Survey was developed to better evaluate the needs and concerns of users and to establish benchmarks for continuous improvement. The survey, piloted in 1997 with Lujan Center and industrial users, covers aspects of administrative and technical support, beam reliability, instrument and equipment, data collection and analysis, and user amenities. Survey results (Figure 14) have been used to prioritize funding for ancillary equipment, improve facility access procedures, and to set priorities for user program improvement projects.

As a result of the 1997 User Survey, LANSCE has:

- improved safety training by designing a user-specific facility training module

- produced a study guide that users can read before their arrival, reducing the amount of time spent in training

- centralized the check-in process to reduce time users must spend attending to administrative issues

- improved the quality and quantity of information available about the facility on the Web 
(2) Check-in Procedures

(3) Training

(4) Instrument/FP Readiness

(5) Beam Reliability

(6) Lost time/equipment

(7) Sample \& Support Labs

(8) Sample Environment Equip.

(9) Instrument/FP User-friendliness

(10) Time Allocated

(11) Facilities for Data Analysis

(12) Sending Data

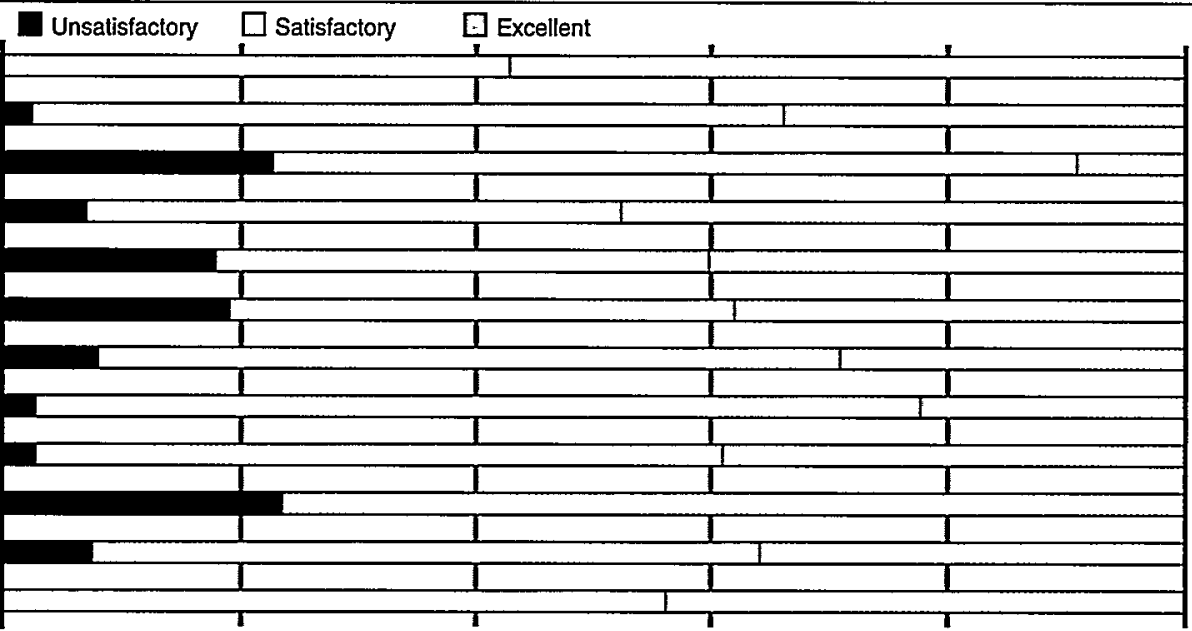

$\triangle$ FIGURE 14. User Salisfaction Survey results for 1997 users.

\section{The Voice for Users}

In 1996, the LANSCE User Group (LUG) was established to provide users and potential users of LANSCE with an opportunity to influence the way in which the facility is managed and improved. To ensure that we only hear from active users, biennial renewal of membership is required to maintain active status in the LUG. As of September 1998, membership included 487 individuals from across the diverse spectrum of facility users (Figure 15). The formally adopted charter of the LUG is available on the Web at lansce.lanl.gov/users/lug/charter.rtf.

The User Group is represented by an 11-member Executive Committee (ExecCom). ExecCom membership (Figure 16) is structured so there is appropriate representation of the principal activities at LANSCE: neutron scattering, defense-related research, and nuclear physics and technology. Additionally, there is at least one graduate student or postdoctoral fellow on the ExecCom. The ExecCom holds monthly conference calls and meets at LANSCE on a quarterly basis.

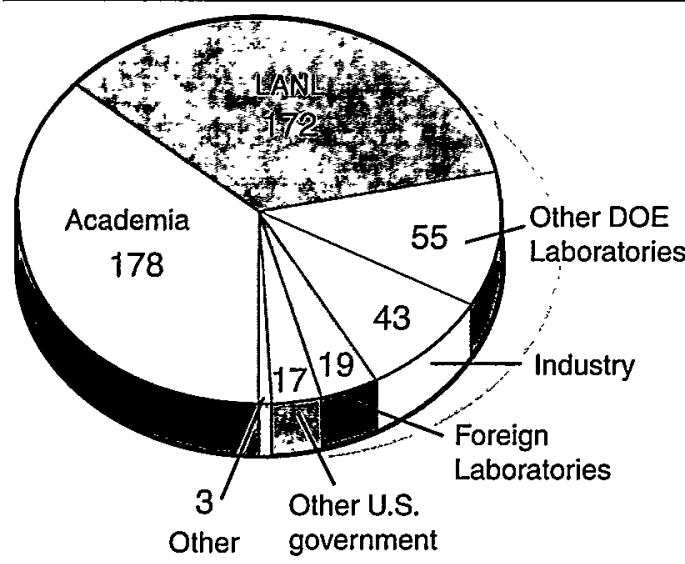

A FIGURE 15. LANSCE User Group membership by offiliation os of September 1998.

\section{Annual User Meetings}

Annual User Meetings are organized by the LUG ExecCom. The annual meeting, usually held in August, brings together current and potential users of LANSCE to share recent scientific discoveries and to discuss instrumentation upgrades and other changes that could enhance scientific output.

Scientific areas represented at the meeting include materials science, condensed-matter physics, defense-related research,

LANSCE User Group Executive Committees

\section{6-1997}

Alan Hurd, Chair

Collin Broholm, Vice Chair

Ronald Fleming

Philip Howe

Tonya Kuhl

Stephen Nogler

William Nellis

John Szymanski

Merri Wood

Kennard Wright

\section{8}

Collin Broholm, Chair

Frank Dietrich, Vice Choir

David Dunand

Ronold Fleming

Philip Howe

Tonya Kuhl

June Mathews

Stephen Nagler

Eugene Normand

John Sorracino

Alan Hurd, Past Chair, Ex-Officio
Sandia National Laboratories

Johns Hopkins University

University of Michigon

Los Alamos National Laboratory

University of Colifornia of Santo Barbara

Ook Ridge National Loborotory

Lawrence Livermore National Laboratory

Indiana University

Los Alamos National Laboratory

GE Aircroft Engines

Johns Hopkins University

Lawrence Livermore National Laboratory

Northwestern University

University of Michigon

Los Alomos National Laboratory

University of Callfornia at Santa Barbara

Massachusetts Instifute of Technology

Oak Ridge National Laboratory

The Boeing Company

Los Alamos National Laboratory

Sandia National Laborafories 


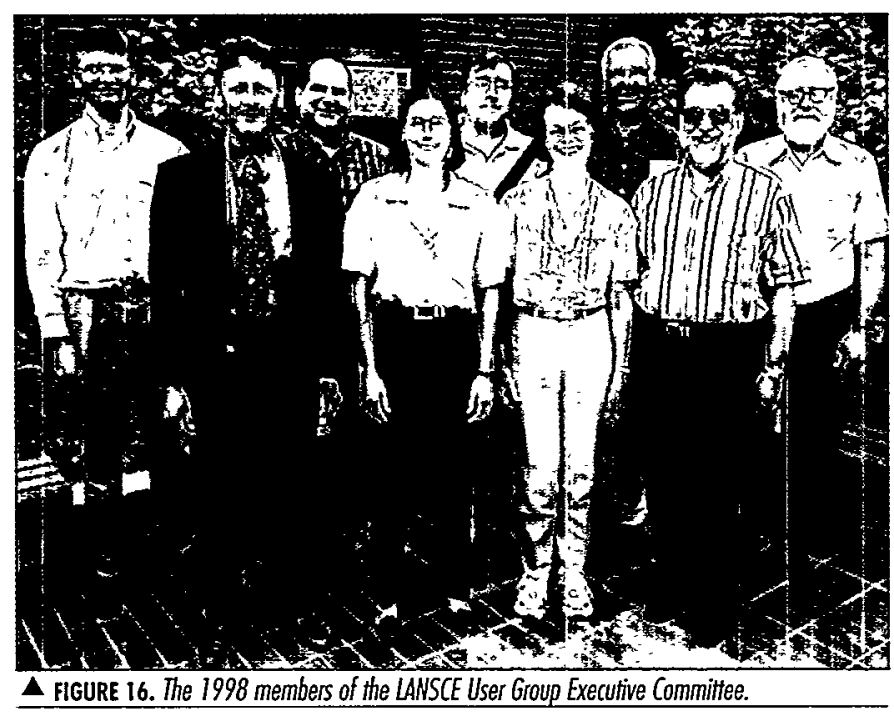

engineering, nuclear technology, and nuclear and particle physics. Summaries of the 1996 to 1998 Annual User Meetings, including plenary talks and abstracts, are available on the Web at lansce.lanl.gov/users/lug/news.htm.

Student participation is encouraged through special social activities, travel support, reduced registration fees, and student poster prizes (Figure 17). Additionally, the annual Louis Rosen prize, established by the LAMPF Users Group, Inc., in 1983 to recognize the outstanding contributions made by Louis Rosen in the development and leadership of what is now the Los Alamos Neutron Science Center, is awarded at the Annual Meeting (Figure 18). The LUG awards the Rosen Prize for the outstanding thesis based on experimental or theoretical research performed at LANSCE.

At the 1997 and 1998 Annual User Meetings, short courses were offered to introduce new users to various aspects of neutron scattering research. These workshops have proven popular with the student meeting attendees as they also provide the opportunity to interact with local scientists and guest lecturers from the neutron scattering community.

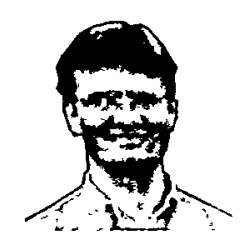

Comments from the Chair

Collin L Broholm, Professor of Physics, Johns Hopkins University

1998 LANSCE User Group Execufive Committee Chair
Following a period of much uncertointy, neutron science in the United States now seems headed for a renaissance. One of the important signs of this hos been the commitment of BES and DP to fund the development of LANSCE into a world-class user facility for neutron science. We are fortunate that John Browne, Roger Pynn, and LANSCE management in general went out of their way to involve the general user community in this process right from the beginning. They established the LANSCE User Group, started a user support office that Audrey Archuleta so cleverly hos headed, supported our annual meetings, and chose to involve users in the design and construction of five new spectrometers ot the lujan Center through Spectrometer Development Teams. If LANSCE operations are successful, os we certainly expect, in generating a reliable pulsed neutron beam with brightness similar to the ISIS source, then everything should be in place for spectacular scientific productivity from LANSCE as we enter the next millennium.

Of course, the other important data point that has us bullish on the future is the fact that a second generation Spallation Neutron Source (SNS) is now a line item in the notion's budget. LANSCE is an important player in this $\$ 1.3 \mathrm{~B}$ project; it will be delivering the $7-G e V$ linac. But I think LANSCE will be playing an equally important role in developing instrumentation technology as well as the user community that is required to turn that investment into scientific results. How about after 2005 when SNS turns on, will LANSCE still be a competitive facility? Definitely! The experience in Europe hos been thot the high-intensity sources (ISIS and ILL) can only be used efficiently when there is a network of smaller neutron sources that can educote users and where innovative and exploratory experiments can be initioted. Generally, it is only when you know how an experiment needs to be done and that it will work that you move to the expensive and highly competitive maximum intensity sources. As a university-based neutron scatterer, I am particularly anxious that we maintain medium-size facilities even os SNS comes online. There is a human time scole for moving up the learning curve as well, and it is often best matched to the pace of experiments at medium flux sources.

One of the things that I have enjoyed in working on the Executive Committee over the post 2 years has been to learn obout the many interesting activities outside of my professional area of neutron scattering in materials science. It is a challenge but also a strength that LANSCE encompasses such a wide range of science ond technology. I was very pleased to hear from Stephen Younger and Robin Staffin af the 1998 annual LUG meeting that stockpile stewardship activities at LANSCE have been very successful. It wos olso clear from the fechnical talks at the meeting that there is a vibront nuclear physics and technology program being conducted ot the WNR facility. LANSCE is an expensive facility to run and it would be hard to justify its cost for one segment of users only. Therefore, it is vital for each of us that all the programs at LANSCE remain highly productive in their respective areas.

These are my last months as the last oppointed choirman of the LUG Executive Committee. I think we have established a solid foundation for an institution that will help users realize the potentiol of LANSCE. I thank you for your support and I look forward to seeing the new 1999 Chair, Frank Dietrich, and Vice Chair, Christopher Durning, take over in this exciting period for LANSCE and for neutron scattering in general. 


\section{Student Poster Prize}

for student science posters at LANSCE User Group Meetings

\section{7}

First Prize, 10-troy-ounce silver ingot

Neil Dilley, University of Colifornia ot San Diego

Magnetic Structure and Lattice Distortion in UPd $\mathrm{P}_{2} \mathrm{~Pb}$

Runners-up, silver coins

Biorn Clousen, Technical University of Denmark

Validation of a Self Consistent Modeling Scheme Using Neutron Diffraction

Paul Hubbard, University of New Mexico

A Neutron Reflectivity Study on the Aging of Tontalum Nitide

in Harsh Environments

1998

First Prize, 10-troy-ounce silver ingot

Michoel Manley, California Institute of Technology

Low-Temperature Inelastic Neuton Scattering Study of Phoses of Cerium

Runner-up, silver coin

Andrew Christianson, Colorado State University

Low-Energy Excitations, Symmetry Breaking, and Specific Heat in YbBiPF

During the 1997 meeting, a 5-hour General Structure Analysis Systems (GSAS) workshop was offered that was designed to increase awareness of the GSAS application and to encourage collaboration between users in the fields of crystal structure refinement, neutron scattering, materials research and geoscience. The eight separate modules were presented by Linda Mansker, University of New Mexico; Davor Balzar, National Institute of Standards and Technology; George Kwei (now in the Director's Office) and Angus Lawson, Materials Science and Technology

\section{Louis Rosen Prize}

for the outstanding thesis based on experimental or theoretical research performed at LANSCE

\section{5}

Linh Phuong Nguyen, University of Maryland

$$
p i-p \rightarrow \text { piO } n \text { Cross Sections in the Region of the } \Delta \text { (1232) Resonance }
$$

1996

Rathnoyaka Gunasingho, Louisiona State University

Search for Neutrino Oscillations in the Appearance Channel

numubar $>$ nuebar and Measurements of Neutrino-Carbon Cross Sections

1997

Sharon Stephenson, North Carolina State University

Pority Violation in 232Th-A Study of the 'Sign Effect'

1998

Carole Gaulard, Arizona State University

Anolyzing Powers for the $\pi-p \rightarrow p$ in Reaction Across the

$\Delta$ (1232) Resonance

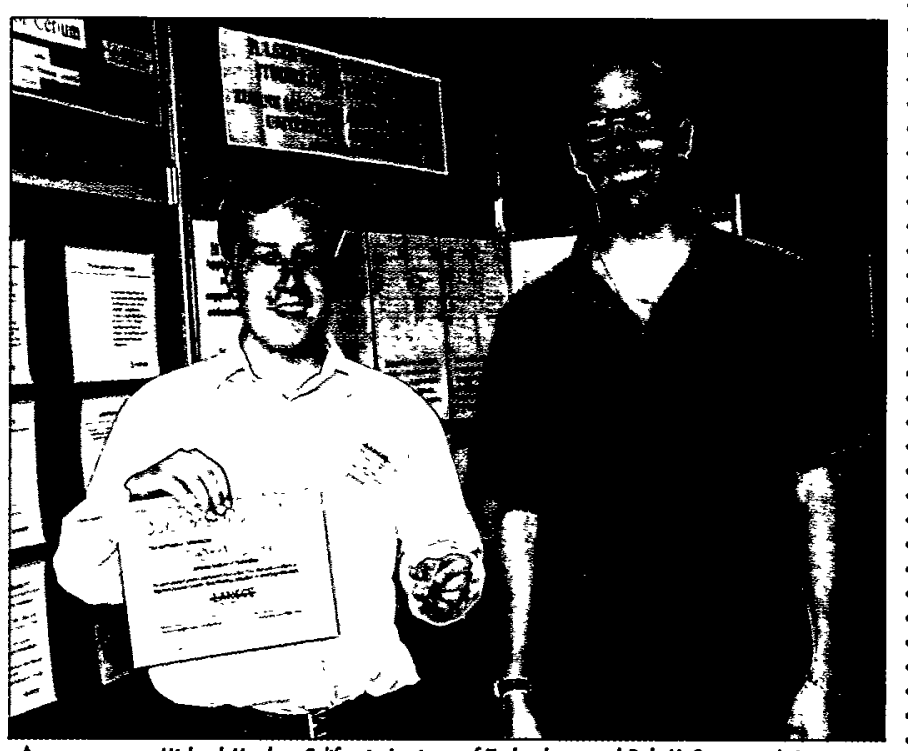

$\triangle$ FIGURE 17. Michoel Monley, Coliformia Institute of Technology, and Rob McQueeney, Lujon Center, proudly disploying the l0-troy-oz silver ingot given for the fisst place student poster prize.

Division at Los Alamos; and Kristin Bennett, Dimitri Argyriou, Mark Daymond (now at ISIS), and Yusheng Zhao, the Lujan Center.

Also offered was a 6-hour course on Long Length Scale Structures taught in six units by Christopher Durning, Columbia University; and Rex Hjelm and Gregory Smith, Lujan Center. The units were organized to provide the novice with an introduction into neutron scattering probes of long length scale structure.

Two courses were offered in conjunction with the 1998 Annual User Meeting: Use of Neutrons for Probing Long

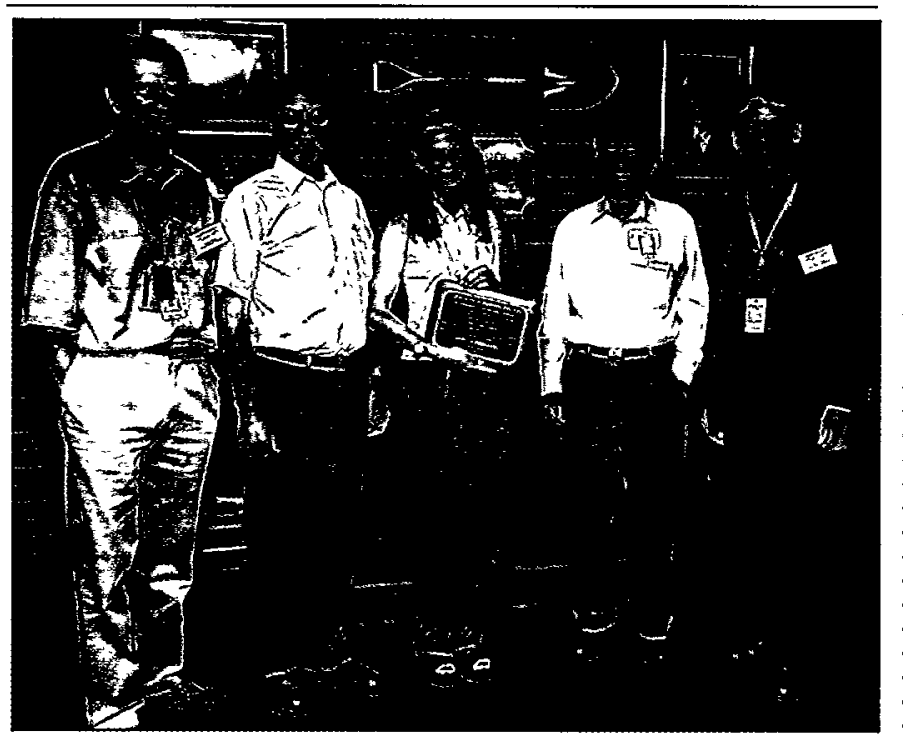

$\triangle$ FIGURE 18. Roger Pynn, LANSCE Division Director, Joseph Comfort (Gaulard's PhD advisor), Anizono Stote University; Corole Gaulard, 1998 Rosen Prize recipient; Lovis Rosen; and Alan Hurd (Rosen Prize Committee Choir), Sondio Nationol Laborotories; at the entronce of the Louis Rosen Auditorium offer the Rosen oword presentation. 


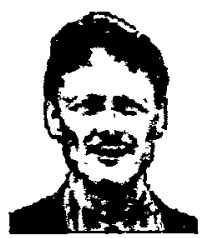

A History of the LANSCE User Group

Alan J. Hurd, Sandia National Laboratories

1996-1997 LANSCE User Group Executive Committee Chair
I first heard of something special happening at LANSCE when I got a call one ofternoon in December 1994 from Roger Pynn and Steve Sterbenz. LANSCE planned a new life around its new science-based defense mission. While the ideo sounded interesting, I struggled with how I could help.

The Los Alamos February 1995 conference on stockpile stewardship was a great success. An extraordinary strategic vision emerged, and it was remorkable how well nevtron science fit the mission. Even more energizing wos the proposed close coupling between defense and basic research, entailing openess and collaboration on unclassified issues. Intrigued, I still wondered how I could help.

Over the next few months, I was able to help organize visits by LANSCE staff to Sandia National Laboratories, helping to educate staff on the advantages of nevtrons and culminaling in a Sandia meeting on October 3, 1995. Our choice of date was terrible: not only were we pre-empted by an emergency meeting of the Sandia executive management, Judge lto chose that day to announce the 0 .J. Simpson trial verdict. Aftention was a bit diffuse.

The basic research and neutron communities began to pull together for LANSCE as a rejuvenated user facility. Indeed, the press referred to LANSCE as a phoenix rising from the ashes-quite a change from 1994 talk of closing. By May 1996 the LANSCE Advisory Board (LAB) begon to meet biannually, and in August the first LANSCE User Group (LUG) Meeting was held in Los Alamos. The talk by Patricia Dehmer of the DOE Office of Basic Energy Sciences (BES) was memorable during that first meeting: the lack of solidarity in the neutron community flew in the face of heary annual BES investments, and Dehmer made it clear that she expected a turnoround.

Having been involved with LANSCE over some years, I was pleased to accept John Browne's invitation to serve on the first Executive Commiltee (hey, free dinner!)

Length Scale Structure (LLSS) and Introduction to Monte Carlo Techniques for Neutron Scattering Instrument Design. The objective of the LLSS course was to provide a basic introduction to neutron scattering techniques used to probe length scales from 10 to $1000 \AA$ to students and scientists interested in applying neutron scattering methods to structural problems. Participants were asked to select specific scientific areas of interest before the workshop so the instructors could customize the examples presented. The course was taught by Christopher Durning, Columbia University; Brent Heuser, University of Illinois; and Rex Hjelm, Gregory Smith, and Jarek Majewski, the Lujan Center.

The Monte Carlo workshop consisted of morning lectures presenting the basics of the Monte Carlo techniques as embodied in the Los Alamos Neutron Instrument
As I expected, it was a pleasure to serve over the next 2 years with highly capable people, both on the LANSCE staff and on the ExecCom. In the first meeting, we each tied to avoid being appointed chair, but I blinked and found myself leading the group.

An important order of business during the first few months of life in LUG wos the establishment of bylows. Here we borrowed heavily from the previous user group, LUGI, from the LAMPF days, relying on Jim Bradbury (former LAMPF Director) who worked closely with us on details during many conference calls. As a result, we now own a very flexible structure that guarantees foir representation accoss the rainbow of researchers of LANSCE, from students to defense to nuclear physics. The reins were passed from LUGl to LUG of a dinner meeting on October 25 , 1996-the evening of a terifici New Mexico snowstorm - 0 which time LUG! donated \$2000 to LUG to continue the Rosen Prize for the outstanding thesis on the mesa.

As chair of the LUG Executive Committee, I held on ex-officio position on the LANSCE Advisory Board (LAB). This board advises the highest levels of Laboratory management on LANSCE activities and it has always paid keen ottention to User Group concerns. User metrics, for example, were deemed highly important to LANSCE success, and the addition of Audrey Archuleta to the LANSCE staff in 1996 os User Coordinator guaranteed that such concerns would be given full attention.

Of course, the greatest attention of the LUG Executive Committee, the LAB, and LANSCE management since 1996 has been toword the multiple concerns of the accelerator upgrade and spectrometer development projects. It is surpising how complex and interlocking these pieces of LANSCE are and how the pieces' fit will be explored over the next decade.

Simulation Package code and the code's use. (More information, as well as the code, is available on the Web at bayberry.lanl.gov/lansce/Welcome.html.) In the afternoon, hands-on portion of the workshop, participants used the code and its Web-based interface to produce a computer model of a neutron scattering instrument and to simulate the operation of this instrument. Lecturers included Thom Mason, Oak Ridge National Laboratory; Phil Seeger, Sumner Associates; Thierry Thelliez of Los Alamos' Computing Division; and Luke Daemen and Rex Hjelm, Lujan Center. 

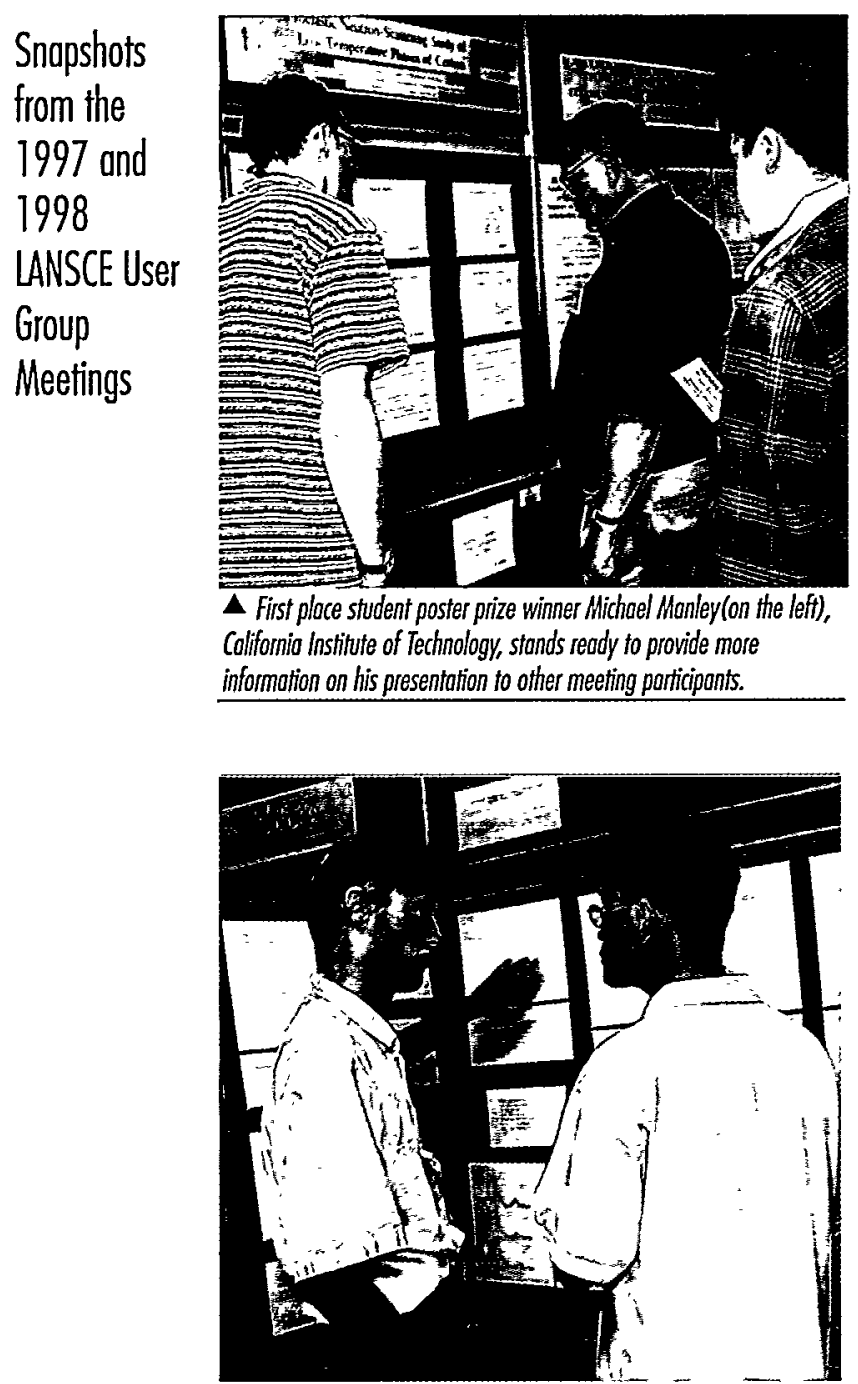

$\Delta$ Andrew Christionson, Colorodo Stote University, second place student poster prize winner, discussing his poster with fellow meeting attendee Yicheng Zhong, City College of New York.

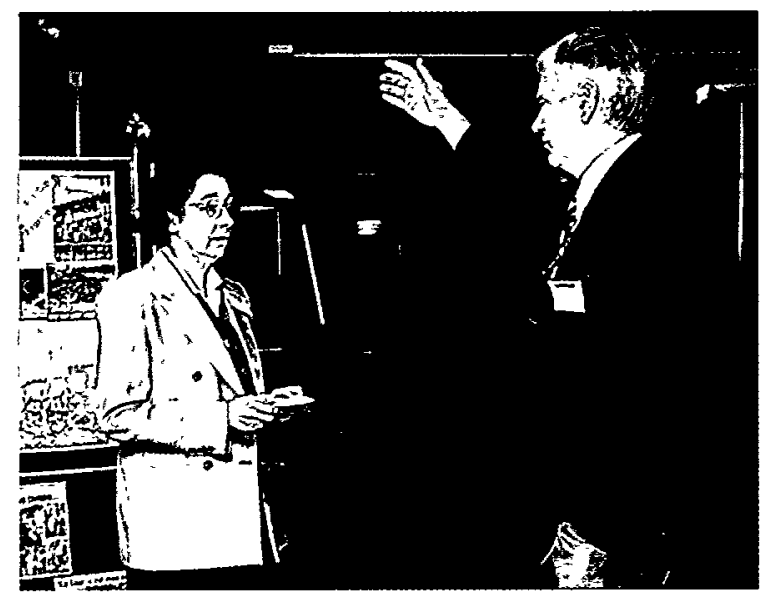

$\triangle A$ lively discussion between Associote Director of DOE's Office of Bosic Energy Sciences, Potricia Dehmer, ond LANSCE Director, Roger Pynn, during the evening's student poster presentation ond reception.

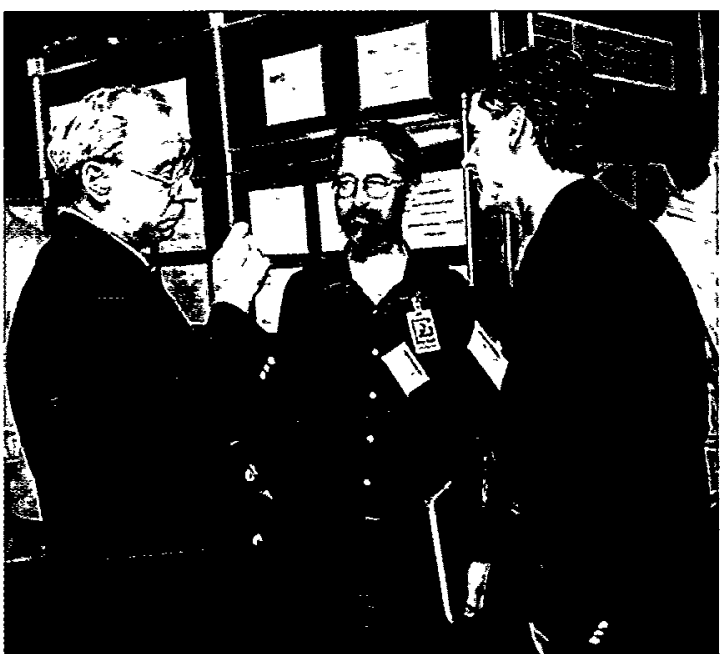

A Iran Thomos, DOE Office of Basic Energy Sciences, Brent Fultz, Colifornio Institute of Technology, and Alon Hurd, Sandia National Laboratories and post LUG Chair, discuss the doy's presentotions.

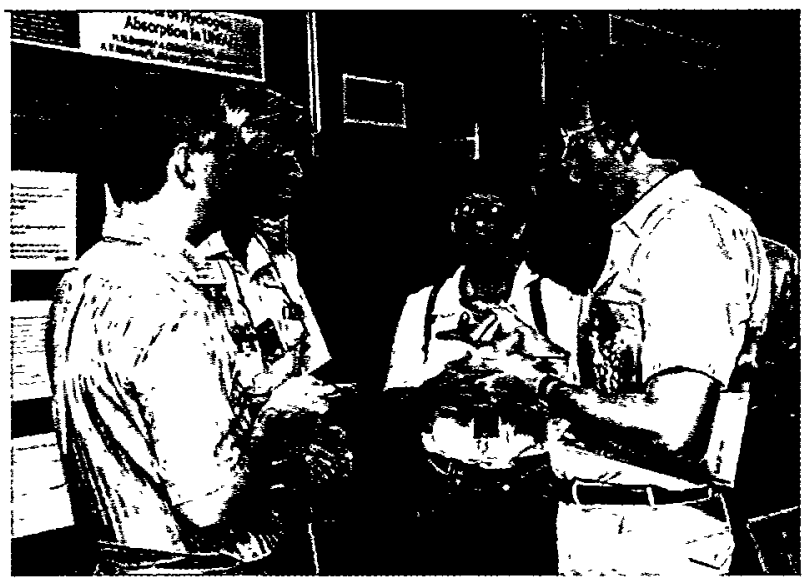

A The poster session reception provided on excellent opportunity for impromptu discussions. From L to R, Ray Osborn, Argonne National Laborotory; Ron Nelson and Kristin Benneit, Lujon Center; and Brion Annis, Oak Ridge Notionol Laboratory.

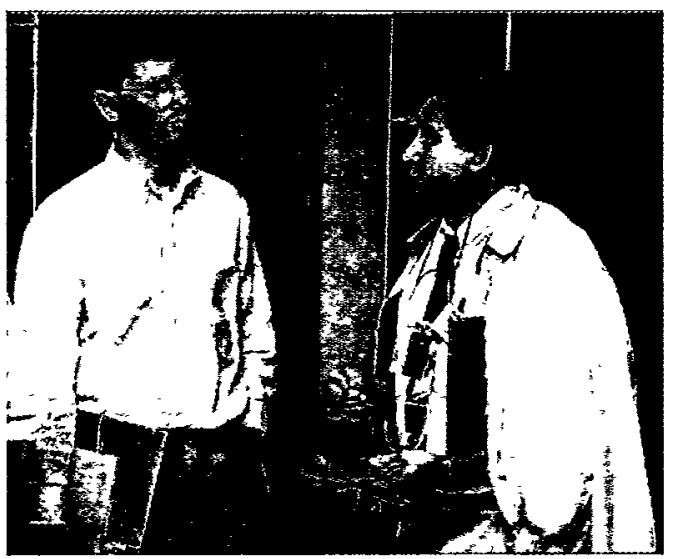

A LUG Executive Committee Choir, Collin Broholm, Johns Hopkins University, and the Loborotory's John Wheatley Scholar, Ferenc Mezei, enjoy a privote discussion during the poster reception. 


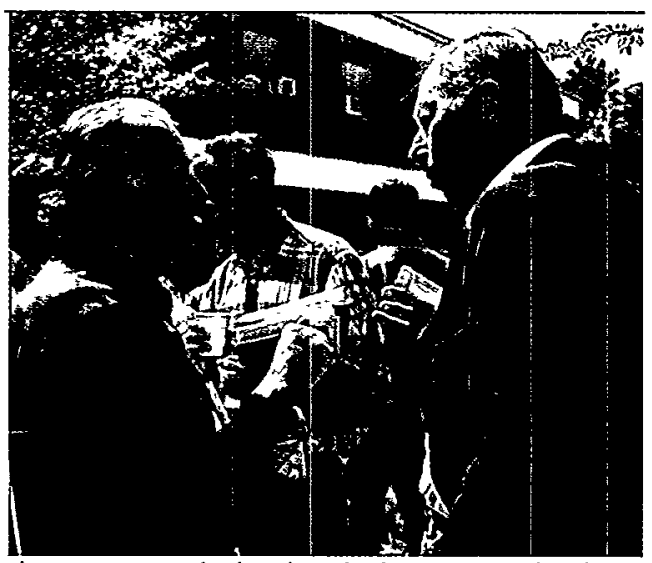

A During o morning break, Andre Michoudon, LANSCE's Nuclear Physics Group, and Arthur Kermon, Mossochusetts Institute of Technology, have on onimated discussion about the day's program.

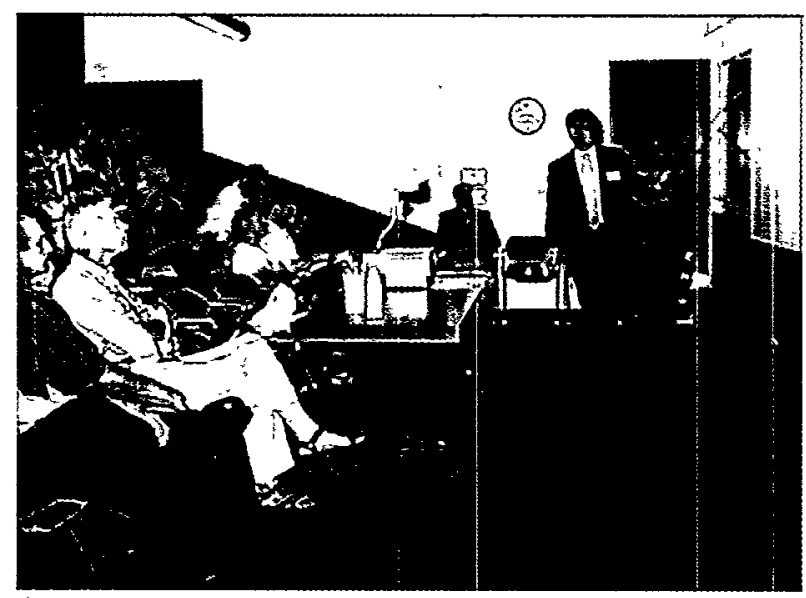

A Massachusetts Institute of Technology postdoctural fellow, Dorrell Irvine, presents a talk on "Design of Tailored Polymeric Materials for Cell Biology and Tissue Engineering" during the soft condensedmatter session. Parallel sessions for condensed matter and nuclear physics, which allowed LANSCE's diverse user community to toke advantage of a foll progrom, worked well for the 1998 meeting.

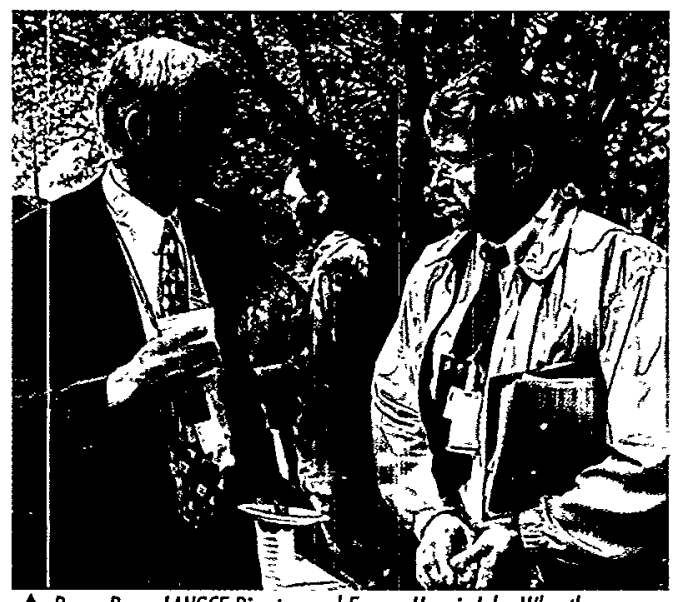

$\Delta$ Roger Pynn, LANSCE Director, and Ferenc Mezei, John Wheatley Scholar, toke odvanfage of an outdoor break.

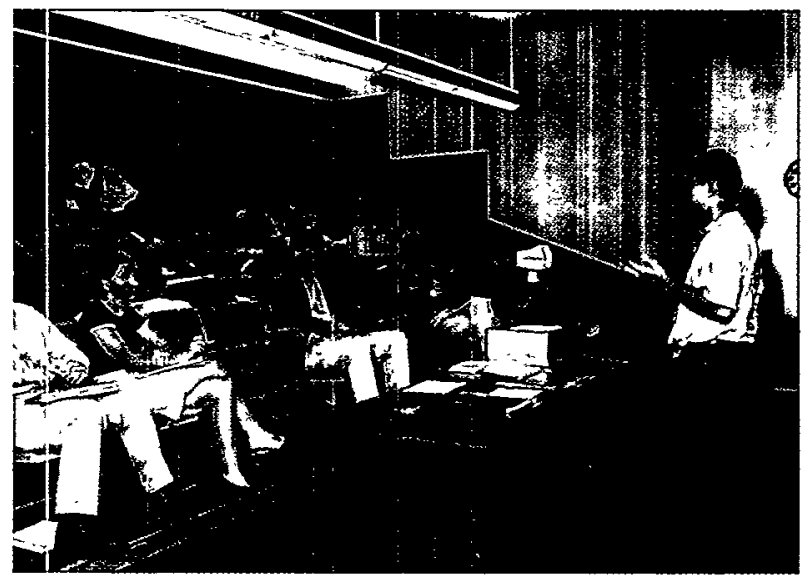

A Dimitri Argyriou, Lujan Center, presents "A Practical Guide to Rietveld Refinement of Latice Anomalies and Magnetic Structure in the Loyered CMR Manganites" to GSAS workshop attendees.

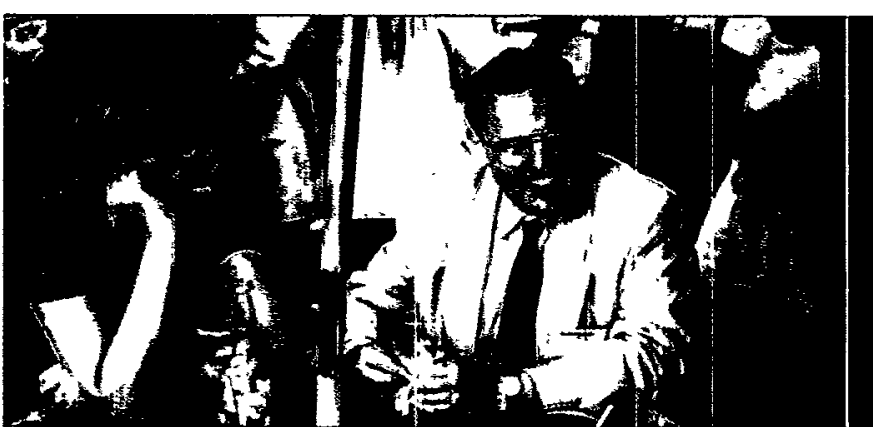

$\Delta$ A recent addition to LANSCE is our Chief of Stoff, Mouri Kotz (formerly of DOE's Defense Programs), here sharing a lighter moment with banquet guests.

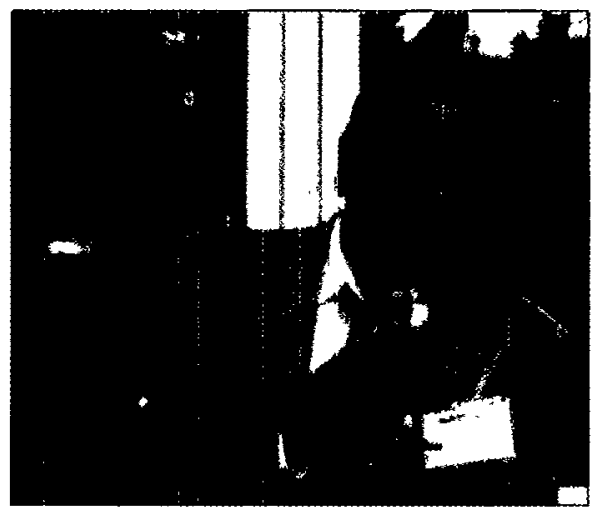

1998 Keynote speaker and "fother of spallation neution sources," Jack Corpenter, Argonne Nationol Loborotory, oppears quite reloxed at the bonquet reception shortly before delivering o top-notch presentation on the "History of Spallation Neutron Source Development." 


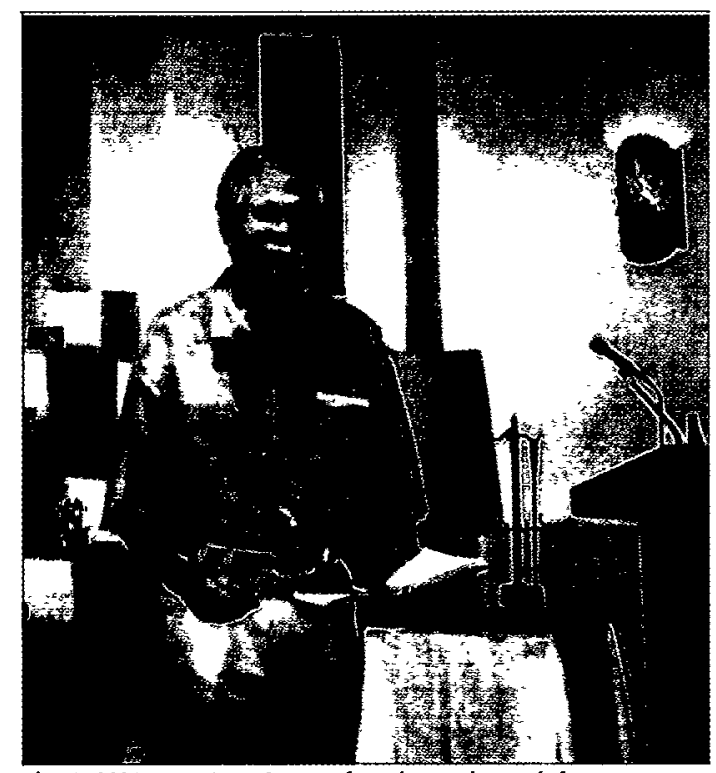

$\Delta$ LANSCE Director, Roger Pynn, performs his standup act before presenting awards to the 1998 User Group Executive Committee members.

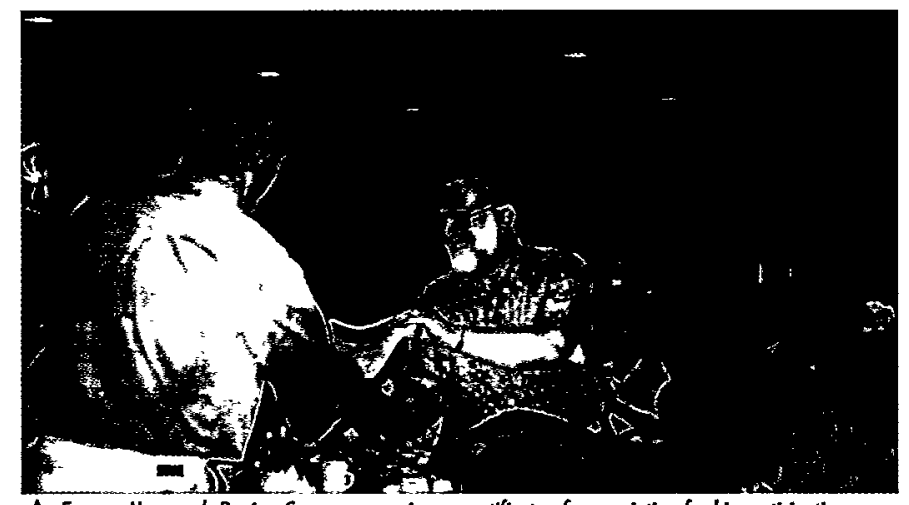

A Eugene Normond, Boeing Compony, receives a certificate of appreciation for his porticipation on the 1998 User Group Executive Committee.

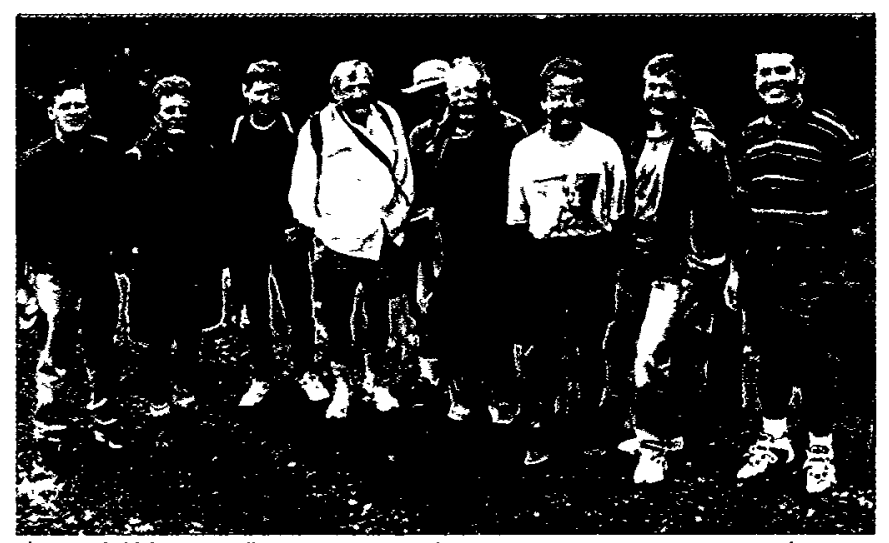

$\Delta$ A guided hike to McCulley Worm Springs in the picturesque Jemez Mountoins aftracted some adventurous meeting attendees: L Loughlin, NMSU; Guide J. Studeboker, Los Alomos Mountaineers Club; V. Gudkov, U. of South Carolina; J. Carpenter, Argonne; B. Moranville, UCSD; Y. Alexandrov, IINR; A. Pichlmoier, ILl; R. Osborm, Argonne; ond J. Hanan, Caltech. Photo by Joan Thompson, LANSCE User Office, who olso joined the hike. 



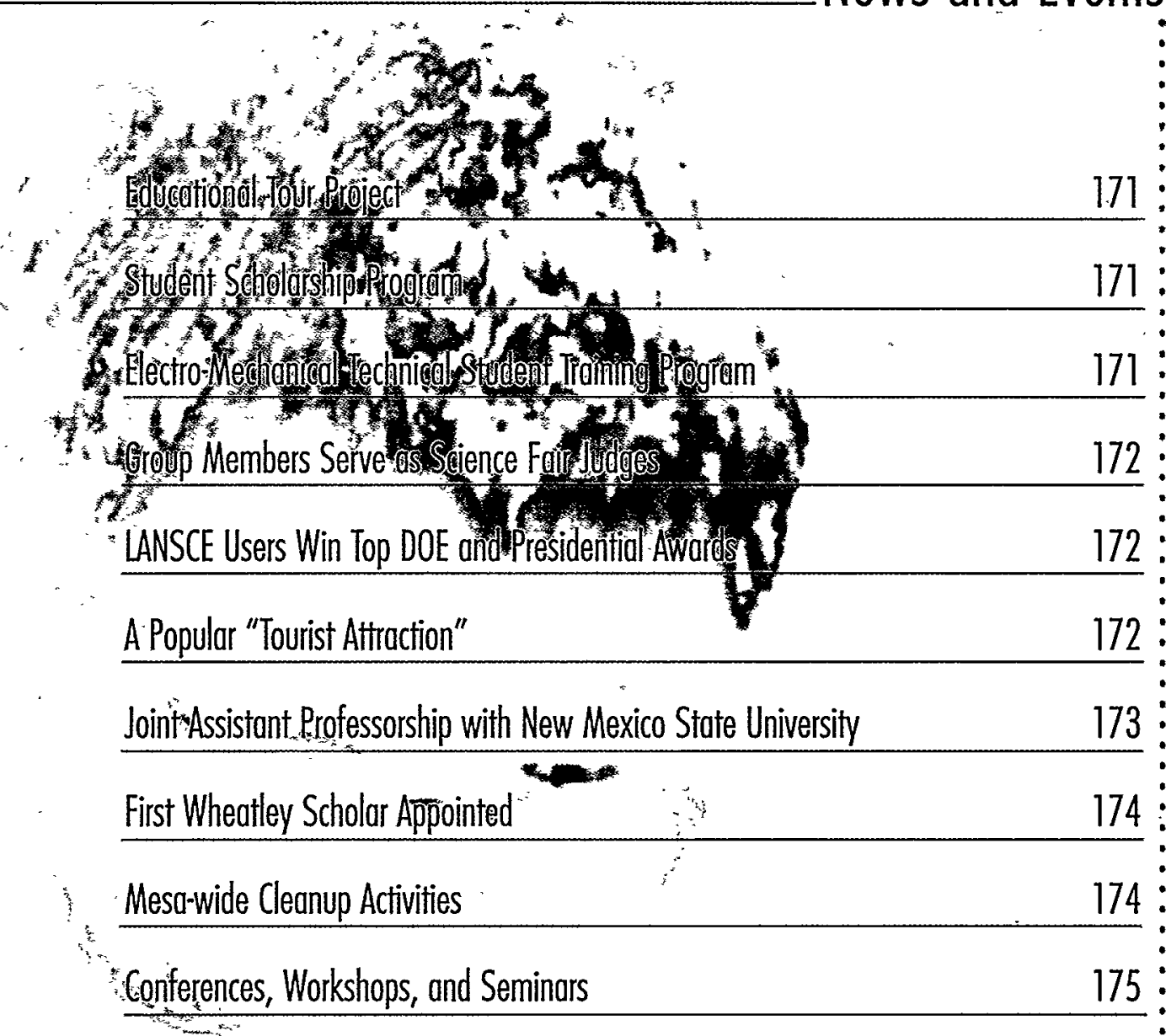

\begin{tabular}{ll}
\hline First Wheatley Scholar Appointed & 173 \\
\hline
\end{tabular}

Mesa-wide Cleanup Activities
Conferences, Workshops, and Seminars

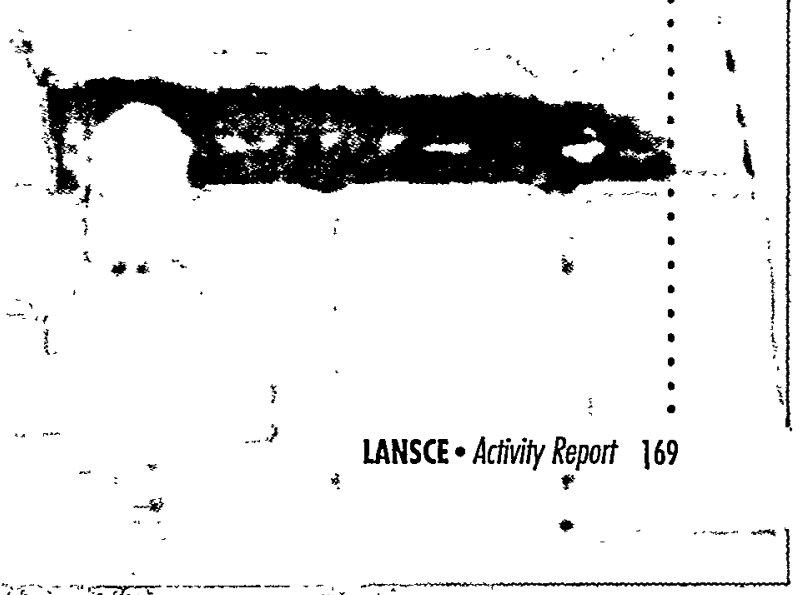




\section{Educational Tour Project}

The LANSCE Educational Tour project was created in 1998 to bring classroom teachers together with LANSCE staff to create a guide for student tours of the facility along with pre- and post-tour classroom activities. This educational outreach program highlights the many research uses and practical applications of the linear accelerator and of neutron science.

This year's project focused on grades 4 through 8 . The teachers came from local school districts: Phyllis Stahm, Pojoaque Schools; Marilyn Peabody, McCurdy School;

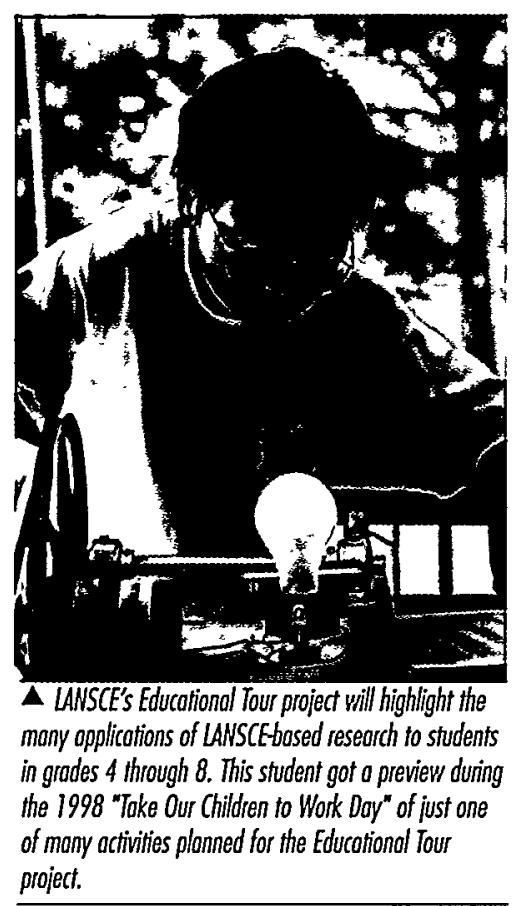

Tom Sisneros, Belen Schools; and Ted Greer, Jemez Valley Schools. During the summer, these four teachers worked at LANSCE developing the tour curriculum, classroom activities, a booklet, a teacher recruitment brochure, and a Web site with mail links to LANSCE scientists to allow students and teachers to ask questions. Also, tour stations, which will be based on information in the tour booklet, are being designed using cartoon characters that help explain the science related to each tour stop. Each station will provide visual aids to help the tour guide and participants interpret the facilities and functions linked to that area of the tour.

The teachers recommended LANSCE conduct between 20 and 30 of these specialized tours per year. Future plans for this program include

- having high school teachers come to LANSCE to continue development of the program and to adapt a similar program for grades 9 through 12

- creating a tour station with a permanent collection of working models and hands-on activities to illustrate science concepts

- creating a short video introducing students to the site and giving a brief virtual tour with connections to science concepts used in different parts of the facility

\section{Student Scholarship Program}

In 1998, LANSCE created the LANSCE Student Education Scholarship Program and awarded $\$ 8,000$ in scholarships to 19 undergraduate students who worked in the Division. Approximately 80 students worked in the Division this summer.

Of the 19 undergraduate students who received scholarships, 15 attend New Mexico schools. Scholarship amounts ranged from $\$ 250$ to $\$ 500$ per student. The scholarship funds came from annual patent royalties derived from LANSCE-developed technologies. All student scholarship funds went to the students' respective universities and were used to credit their educational accounts.

The students' areas of study encompass a wide range of disciplines, including electrical, computer, civil, mechani$\mathrm{cal}$, and optical engineering; political science; accounting; environmental science; communication disorders; and electronics. Award criteria included overall performance and contributions to their respective groups, academic promise, and level of community involvement.

1998 LANSCE Student Education Scholarship Program

Recipients and their Educational Institutions
Crystal Archuleta

Ken Cardell

Evereft Espinosa

Julianne Konkel

Erin Moes

Georgette Maestas

Carlos Manzanares

Gene Martinez

Tim Martinez

Carly MoNeese

Jessica Montoya

Eric Olivas

Stephanie Radzinski

Dan Riley

Eric Salgado

Holly Sanchez

Richard Stein

Francisco Varela

Marsho Wenzel

Northern New Mexico Community College
University of Arizona
University of New Mexico
Technical Vocational Institute
New Mexico State University
Northern New Mexico Community College
New Mexico State University
New Mexico Highlonds University
University of New Mexico
New Mexico State University
University of New Mexico
University of New Mexico
Texos Tech University
University of New Mexico
University of California at San Diego
University of New Mexico
University of New Mexico of Los Alamos
Texas Christion University
University of New Mexico at Los Alamos

Northern New Mexico Community College

Technical Vocational Institute

New Mexico State University

Northern New Mexico Community College

ew Mexico State University

University of New Mexico

New Mexico State University

niversity of New Mexico

University of New Mexico

University of California at San Diego

University of New Mexico

Texas Christion University

University of New Mexico at Los Alamos

\section{Electro-Mechanical Technical Student Training Program}

The Electro-Mechanical Technical Student Training Program, developed by the University of New Mexico at Los Alamos (UNM-LA) and LANSCE, is designed to help participants develop new skills in order to make them more 
marketable in the business community. The program was created by LANSCE's Diversity Committee, consists of courses developed and taught by LANSCE technical staff, and is administered by Marilyn Thomas in the LANSCE Dvision Office. Students spend a half-day in class at UNMLA attending courses especially designed for their jobs at the Laboratory. The second half of the day is spent working at the Laboratory as an entry-level technician partnered with a mentor. At the conclusion of the two-year program, students receive a certificate in Electro-Mechanical Technology from UNM-LA; in December 1998, six of the original ten students will complete the school's certificate requirements.

The first group of 10 students was hired on January 13, 1997, and since inception, the program has grown to 25 students. In August 1998, LANSCE hired nine new students in addition to the ten already employed at LANSCE through this program. In 1998, the local ABC affiliate television station featured the program and interviewed students about the educational and practical portions. After the program aired, LANSCE received calls from throughout New Mexico for information on how to implement a similar program at other companies.

\section{Group Members Serve as Science Fair Judges}

In April 1998, several LANSCE engineers and a LANSCE property specialist served as judges for a science fair at the Larragoite Elementary School in Santa Fe. The science fair included entries from approximately 300 students, who presented projects in such areas as electricity, computers, solar energy, biology, and waste management. Judges were Patrick Kelly, Paul Leslie, and Robert Valdiviez of the LANSCE-1 Accelerator Physics and Engineering Group and Chris Vigil of the LANSCE-4 Division Services Group.

\section{LANSCE Users Win Top DOE and Presidential Awards}

Two long-term LANSCE users were honored by the Department of Energy's (DOE's) Office of Defense Programs when they received the DOE Defense Programs Early Career Scientist and Engineer Award. Shenda Baker, Harvey Mudd College, was the 1996 recipient, and Tonya Kuhl, University of California at Santa Barbara, received her award in 1998. Both Baker and Kuhl were then nominated by the DOE for the Presidential Early Career Award for Scientists and Engineers. Baker was selected for the Presidential award, and she received recognition at a White House ceremony. (The 1998 recipients of the Presidential award had not been announced when this report went to print.) Both Baker and Kuhl were nominated for the DOE award by Greg Smith of the Lujan Center.

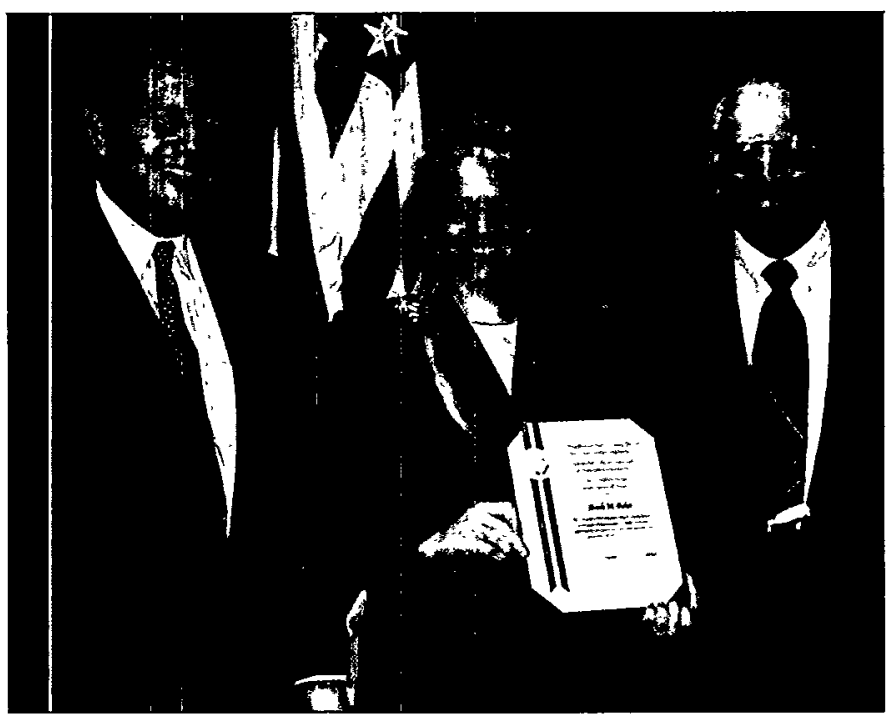

- Shenda Boker (center) receiving the Presidenfial award from Charles Curtis (leff), Deputy Seccetary of Energy, and Jack Gibbons (right), former Presidential Science Advisor.

Baker is not only an active user of the LANSCE facility but is also a member of the LANSCE Division Review Committee and the LANSCE Materials Program Advisory Committee. She was recognized by this award for her work at LANSCE in developing techniques for the examination of advanced materials (polymers) under shear.

Kuhl has served on the LANSCE User Group Executive Committee for the past 2 years and has just been elected to another term. She is also the User Group's representative on the Proposal Evaluation Committee, which reviews and recommends new instruments for the Spectrometer Development Project. Kuhl was cited by DOE for her studies of "smart" materials and surface coatings.

\section{A Popular "Tourist Attraction"}

LANSCE's tour program plays a key role in increasing awareness of the facility and in educational programs. A recent tour in September 1998 of the Utah, Colorado, Arizona, and New Mexico Navajo Nation Coalition Advisory Board, which helps targeted schools strengthen their math, science, and technology programs, was treated to a detailed overview during their tour by Deputy Division Director Stan Schriber. Many LANSCE tours are custom tailored to meet the individuals' interests.

One of the more interesting tours conducted here was a visit by the local Public Broadcasting System (PBS) channel star, Bill Nye "the Science Guy," who conducts a weekly television program aimed at increasing awareness in the sciences for children ages 10 to 16 . The program is syndicated out to other PBS channels and the Discovery Channel. Nye visited LANSCE in 1997 to shoot footage for use in an episode on 


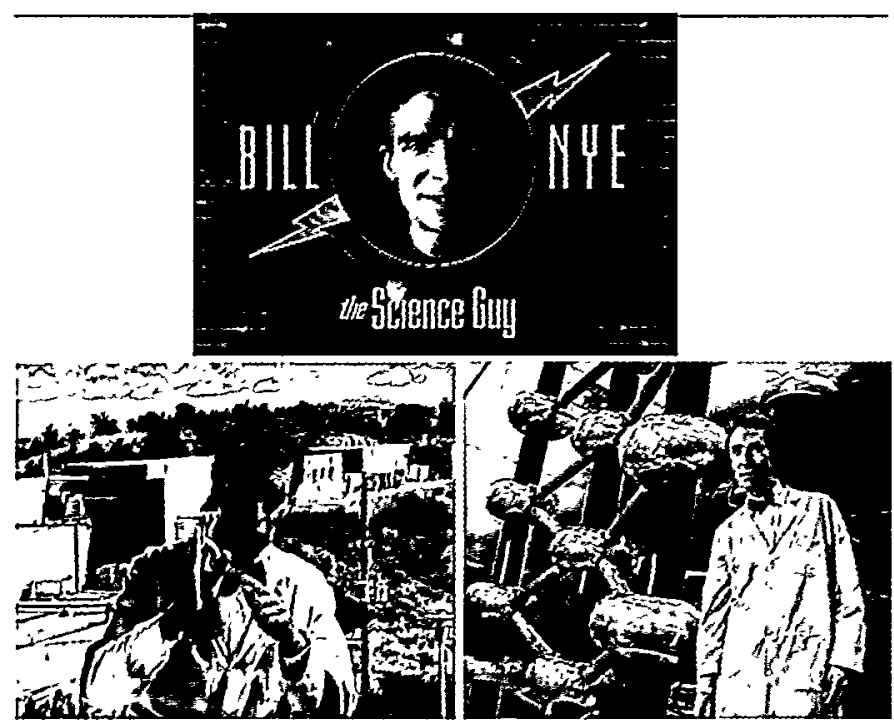

$\Delta$ Bill Nye "the Science Guy" visited LANSCE for on episode of his science show for children.

"smashing atoms." The LANSCE accelerator was used as a backdrop for "the Science Guy" to demonstrate how protons and neutrons are separated from atoms. One of the shots included Nye running down one-third the length of the halfmile-long accelerator tunnel, but with the camera speeding up his run to about 5 seconds, to show how far and how fast the protons must travel. During the filming, this particular shot required three takes, making for one very winded television personality!

In May 1998, several science fiction writers from across the nation visited LANSCE to gather information on the science behind the fiction they create. The writers were in Santa Fe for a weekend conference.

A number of tours involved Laboratory sponsors reviewing projects; for example, the Accelerator Production of

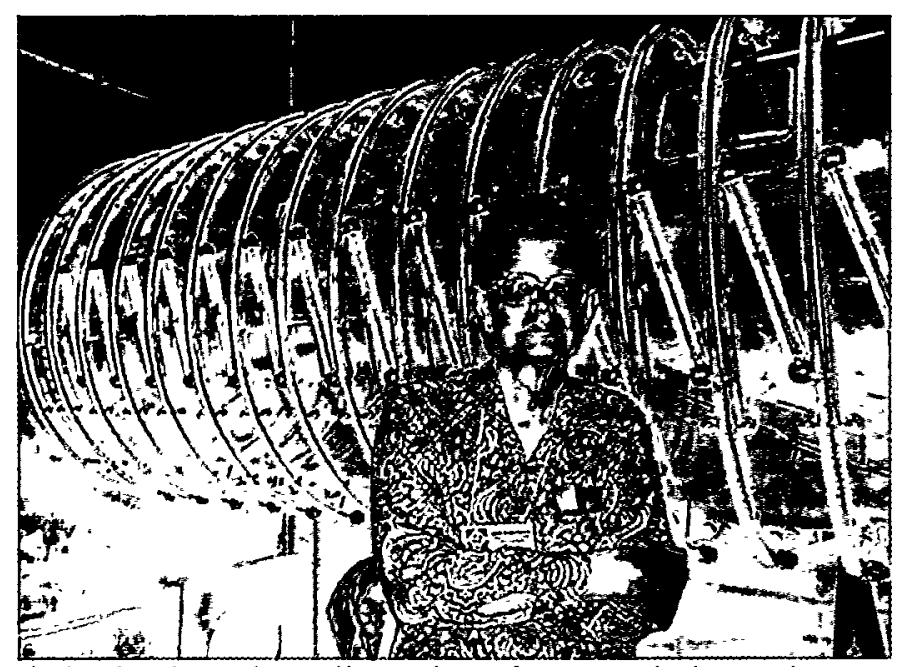

$\Delta$ This enhanced picture shows worldrenowned science fiction vinter Poul Anderson standing next to the Cockroff-Walton injector of LANSCE.
Tritium project. Tours have included representatives of the Economic Development Partnership from South Carolina, the New Mexico Environmental Department, the American Society of Medical Association Counsel, and schools from Texas and New Mexico. The Laboratory's Public Affairs Office often showcases LANSCE for photo opportunities, from the Cockcroft-Walton injector to the suite of instruments at the Lujan Center.

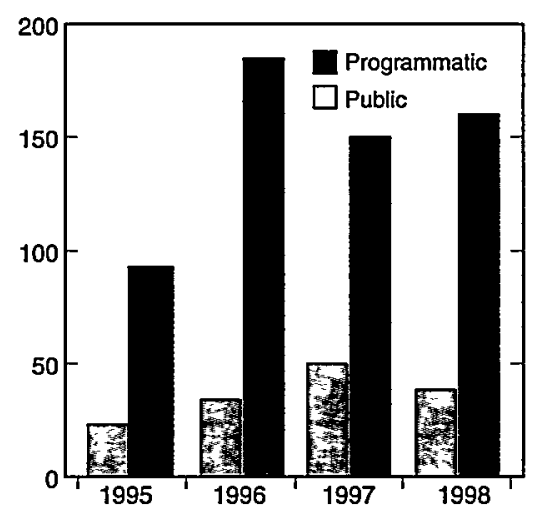

$\Delta$ Requests for fours come from groups induding the media, governmen officials, sponsors, academia, industry, and the public sector. These dato reflect the number of public ond programmotic fours conducted from 1995 to 1998. Progrommatic tours usudlly involve smaller groups of 2 to 6 people while public fours tend to ronge from 5 to 30 people per four.

\section{Joint Assistant Professorship with New Mexico State University}

As part of a broader effort to strengthen the relationships between the Laboratory and nearby universities, a joint position has been established between the Physics Department at New Mexico State University (NMSU) in Las Cruces and LANSCE. A Memorandum of Understanding to this effect was signed by the president of NMSU and former Laboratory Director Sig Hecker in September 1996. A wide search conducted by a committee of representatives of both institutions commenced in December 1996. From a broad field of excellent

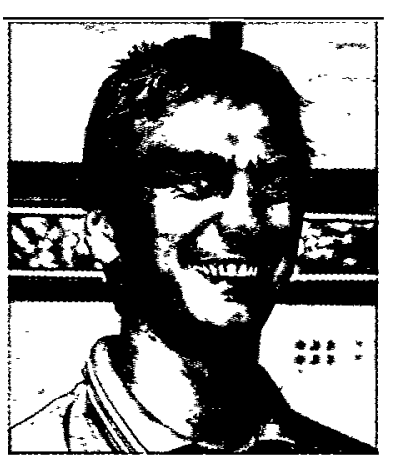

$\Delta$ Heinz Nakotte, the New Mexico State University-LANSCE Joint Assistant Professor. candidates, Dr. Heinrich "Heinz" Nakotte was appointed to the position and began work at NMSU in August 1997.

Nakotte started his career at the University of Amsterdam. In addition to his work at the High Magnetic Field Laboratory in Amsterdam, he performed neutron scattering experiments at the Institut Laue-Langevin, LANSCE, and the National Institute of Standards and Technology. 
Nakotte worked at the Lujan Center starting as a postdoctoral fellow in 1994 until taking the NMSU-LANSCE position. More information on Nakotte is available on the Lujan Center Web pages and information on the NMSU Physics Department can be found at thor.nmsu.edu/index.html.

Similar joint professorships with the University of California and the University of New Mexico are also being discussed.

\section{First Wheatley Scholar Appointed}

A new scientific position named for the late John Wheatley has been established at Los Alamos National Laboratory. The Wheatley Scholar position is designated for visiting scientists at the Los Alamos Neutron Science Center, the Laboratory's Materials Science and Technology Division, or the Laboratory's Theoretical Division.

The recipients are distinguished researchers who bring expertise to Los Alamos in the areas of neutron scattering, thermal physics, or condensed-matter science and whose career achievements are recognized as outstanding by the scientific community. The position will generally be for 1 year but can be extended for another year with approval of the Laboratory Director.

The first Wheatley Scholar is Ferenc Mezei of the Hahn Meitner Institute in Berlin. Mezei is renowned for his innovative contributions to neutron scattering research. He has joined LANSCE at a time of significant growth in the use of spallation neutron sources for neutron scattering research, a field to which he has already made substantial contributions. Mezei is a member of Academia Europaea

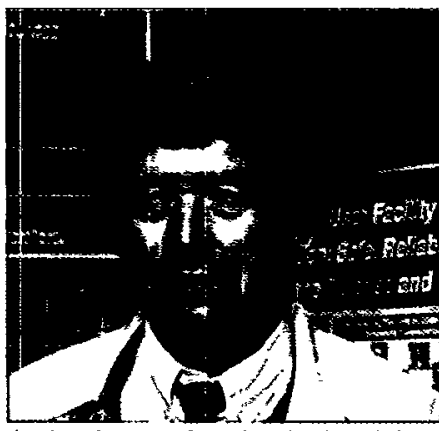

A The Loboratory's first John Wheatley Scholor, Ferenc Mezei, joined the LANSCE stoff in 1997. and the Hungarian

Academy of Sciences and a recipient of the Hewlett Packard Europhysics Prize for the invention of Neutron Spin Echo spectroscopy.

John Wheatley was a leading low-temperature experimental physicist who made major contributions to the understanding of the physics of liquid helium and low-temperature technology. He was at Los Alamos from 1981 to 1985, working on conventional and natural heat engines, superfluid helium-3, and other low-temperature phenomena. He held the first joint fellowship between the University of California at Los Angeles (UCLA) and Los Alamos and was working at UCLA at the time of his death in 1986.

\section{Mesa-wide Cleanup Activities}

LANSCE is a facility with a long, multi-use history. Until September 1998, it was plagued with a legacy of unused and abandoned equipment, supplies, and even cars left by visiting researchers. In January 1998, the LANSCE Facility Management Office took a hard look at what could be done to improve its general "housekeeping" and to formulate a plan to avoid this problem in the future. It was discovered that most of the unused items could be removed as reuseable salvage, but the groups on the mesa did not have the time nor manpower necessary to get the items removed. Authorization was obtained to use the proceeds generated from the sale of recycled materials to pay for the cleanup.

A major, coordinated effort was carried out by Facility Management's Bernadette Pesenti-Valdes, along with members of the local contractor company Johnson Controls Northern New Mexico. Cleanup at LANSCE began on February 18, 1998, including a building-by-building sweep and removal from parking lots and storage yards. At
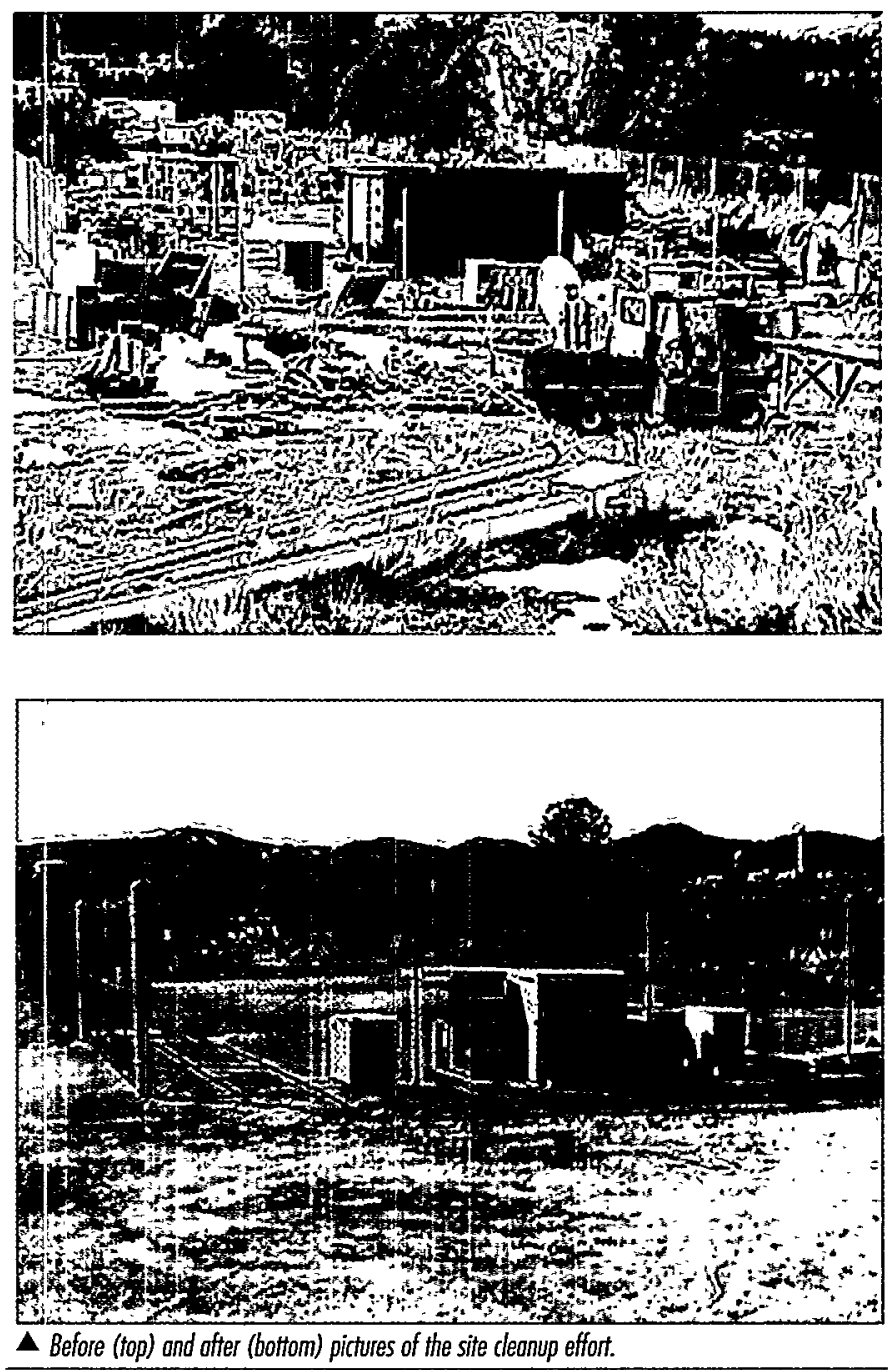
the end of September 1998, approximately 1,600 tons of scrap, at least 30 truckloads of equipment that was later reused or resold, and minimal loads of trash were removed. To ensure that the problem of improper storage and accumulation of excess does not reoccur, LANSCE created 10 salvage collection points that are serviced every week. LANSCE Facility Management has also taken a proactive approach by constructing a warehouse for the storage of property for future projects. The result is a more attractive and organized place in which to work.

\section{Conferences, Workshops, and Seminars}

LANSCE sponsors or supports a variety of conferences, workshops, and seminars to educate and inform the scientific community about accelerator science and technology and the uses of neutrons and neutron scattering techniques. An average of 20 to 25 major conferences and workshops per year are hosted by the staff at LANSCE. A few key meetings are summarized.

LANSCE also provides opportunities at the formal and informal level to train newcomers and students in the applications of neutrons in their research. The Lujan Center conducts a weekly seminar series, with speakers from around the world presenting various topics of interest to the neutron scattering community. The speakers are hosted by LANSCE scientists.

\section{Defense, Basic, and Industrial Research at the Los} Alamos Neutron Science Center

A workshop on "Defense, Basic, and Industrial Research at the Los Alamos Neutron Science Center" was held February 12-15, 1995, and has helped to identify and clarify many key stockpile stewardship needs that can be addressed at LANSCE. This workshop gathered scientists from DOE national laboratories, other federal institutions, universities, and industry to discuss the use of neutrons in science-based stockpile stewardship.

The workshop began with presentations by government officials (M. Greenwood, Associate Director of the Office of Science and Technology Policy; V. Reis, Assistant Secretary, DOE Office of Defense Programs; and I. Thomas, DOE's Office of Basic Energy Sciences), senior representatives from the three weapons laboratories (R. Fortner, Associate Director of Physics and Space Technology at Livermore; D. Hayes, Director of Defense Programs Capabilities at Sandia; and S. Hecker, Los Alamos Director), and scientific opinion leaders (A. Cheetham, Director of the Materials Research Laboratory at UC Santa Barbara; G. Aeppli, AT\&T Bell Labs; S. Freedman, UC Berkely Department of Physics; and F. Dietrich, Physics and Space Technology Division of Livermore). Then the approximately 200 workshop participants met in breakout sessions on materials science

\section{Conferences and Workshops}

1995

February 6-10 Materials for Spallation Neutron Sources Workshop

February 12-15 Defense, Basic, and Industrial Research of the Los Alomos Neution Science Center

April 18-21 Neuton Instrumentotion for a Long Pulse Spallation Source Workshop

May 8-10

September 11-13

December 4-6 The High-Intensity SRF Proton Linoc Workshop

December 13-15

E-MF Accelerotor Teom Meeting Workshop

Workshop on Critical Beam Intensity Issues in Cyclotrons

Hydrogen and Quantum Mechonical Phenomeno in the

Coordination Sphere of Transition Metals Workshop

1996

June 5

July 31-August 1 Ook Ridge Spallation Source Workshop

August

August 19-20

September

October

December 4-5

1997

February 7

February 27

March 3-4

Morch 4-7

Joly 14-August 1

July 28-30

July 31-August 2

August 6-8

August 8

August 8

August 25-29

September 9-11

0tober 6-10

November 3-7

November $6-7$

November 16-20

December 2

December 11

December 12-14

December 16-17

Technicol Advisory Committee on Accelerator Production of Tititium Superconducting Linoc Design

Texture Andysis Workshop

First Annual LANSCE User Group Meeting

Workshop on Numericol Methods for Simulating Neutron Scattering Instruments

DOE Residual Stress Workshop

Workshop on Criticol Beam Intensity Issues in Cyclotons

1996 LANSCE Run Cyde Roundup

Diffuse Scattering in Plutonium, Mesoscopic Working Group Seminar National Spallation Source Design Meeting

Election Effects in High Current Proton Rings Workshop

First Neutron Scattering Summer School

Research Workshop on Residual Stresses

Netional Spollation Neutron Source Collaboration Workshop

Second Annual LANSCE User Group Meeting

Generol Structure Anolysis System Workshop

Long Length Scole Structures Workshop

EPICS Troining Workshops

EPICS Collaboration

RF Superconductivity Workshop

Internationol Conference on Accelerator and Large Experimental Control Systems

Beam Diagnostics Workshop

1997 American Nudear Society Winter Meeting

Spectrometer Development Teom Workshop

Small Angle Neutron Scattering Spectrometer Development Team Workshop Probing Frontiers in Matter with Neutron Scattering Workshop

Spollation Neutron Sources and Export Control lssues

1998

Januory 22

Jonuary 23-24

March 4-5

March 9-13

April 20

April 29

April 29-Moy 1

May 10-14

June 20

August 10-11

August 12

August 12

October 26-27
Spallahion Neutron Source Project Manogement Workshop

Spallation Neutton Source Colloboration Workshop

Beam Halo Workshop

EPICS Troining Workshops

WNR Workshop for High Energy Neutron Irradiation of Electronics

1997 LANSCE Run Cycle Roundup

EPICS Collaboration

DOE Council on Chemical Sciences Workshop

Electric Dipole Moment Colloboration

Third Annual LANSCE User Group Meeting

Use of Neutrons for Probing Long Length Scale Structure Workshop

Introduction to Monte Carlo Techniques for Neutron Scattening Instrument Design Workshop

E866 Colloboration 
Lujan Center Seminar Series

1997

April 4

D. Clemens

Instrumentation for Reffectometry and Neutron Polarizotion at SINQ/PSI

May 7

R. PYmn, LANSCE

Low Energy Spinfluctuations in Chromium Metal

May 16

1. Grigorov, Johns Hopkins University

Magnetic and Structural Properties of Expanded Manganese

June 13

D. Argyriou, LANSCE

Two-Dimensiond Ferromognefic Clusters Above ond Below $I_{c}$ in the Layered Manganite $\mathrm{LA}_{2 \cdot 2 \mathrm{~S}} \mathrm{SR}_{\mathrm{I}+2} / \mathrm{Hn}_{2} \mathrm{O}_{7}$

July 18

I. Eyssa, National High Field Magnetic Loboratory

Conceptual Design for a 30-T Neuton-Scattering SplitPair Mognet

September 12

T. Kelley, LANSCE

Recent Experiments on PHAROS

October 10

P. Ferguson, LANSCE

An Overview of the Lujon Center Spollation Physics Team Activities

October 14

W. Li, Comegie Institution of Woshington

Synthesis of Diamonds and Hydrous Magnesium Silicote Minerols Under High Pressure

October 17

P. Seeger, Sumner Associates

MCUB: A Monte Carto Library for Neuton Scattering Instrument Design ond Analysis

October 24

M. Luett, LANSCE

Growth Behovior of Self-Assembled Polymer Multilayers

October 25

H. Bordallo, LANSCE

Structure and Dymomics in BaZnF 4 Crystals

October 31

T. Brun, LANSCE

Thermal Diffuse Scattering in Pb

November 7

J. Loshley, Los Alamos National Laboratory

Preparation of Gollium Stabilized Delta-Plutonium Single Crystals

November 14

G. Cocognoni, Institut Laue-Langevin

A Two-Dimensional Microstip Gaseous Detector for Thermal Neutrons

November 21

F. Mezei, LANSCE

How to Beat the Ill on its Own Game?

1998

January 16

C. Marques, Rhone Poulenc/CNRS

Polymer-membrane Interactions: Embedding of Polysoops in Lyotropic Phases

January 23

R. Heffner, Los Alomos National Laboratory

Effects of Polaronic Degrees of Freedom on the Spin Dynomics in the Magneforesistive

Monganites $(L a, A) M n \mathrm{O}_{3}$ and $(\mathrm{La}, \mathrm{A})_{3} / \mathrm{Mn}_{2} \mathrm{O}_{7}, \mathrm{~A}=\mathrm{Ca}$ and $\mathrm{Sr}$
1998 , continued

Jonuary 30

A. Sharma, Los Alamos National Loboratory

Ultrosonic Spectroscopy of Solid-liquid Suspensions

February 6

K. Bennett, LANSCE

Applications in Texture Analysis of the Lujon Center

February 13

R. Robinson, LANSCE

Neutron Scattering from Heovy Fermions

February 27

M. Costantino

Livermore High Pressure Gas Installation

S. Stishov, Institute for High Pressure Physics

Versatile High Pressure Gas Apparotus

March 6

S. Schorr, Hohr-Meitner-Institut

Critical Dynamics in Heisenberg Ferromagnets Above and Below the Curie Point

March 11

D. Argyriou,H. Bordallo, R. McQueeney,and R. Robinson, LANSCE

Preview of the American Physical Society Meeting Talks

March 13

R. Hielm, LANSCE

Partide Form and Stability in Mixed Colloids: A Problem in Complex Fluids from Medicine to Physics

March 20

C. Durning, Columbia University

Surface Archifectures from Polymers Irreversibly Adsorbed from Melf

March 31

J. Lipkowski, University of Guelph

Electrochemical and Spectroscopic Studies of Ionic Adsorption at the (Au(11I) Electrode Suface

April 3

H. Warriner, LANSCE

$X$ ray Scattering Studies of on Undulation-Driven Long-Ranged

April 10

M. Torikachvili, San Diego State University

Kondo Insulators

April 17

J. Sarrao, Los Alomos National Laboratory

Physics of $\mathrm{VblnCU}_{4}$ and Related Compounds

April 24

C. Mielke, Notionol High Magnetic Field Loboratory

Highly Correlated Moterials of Very High Magnetic Fields

May 8

D. Louca, Los Alamos National Loboratory

Locol Lottice Effects in Mognetic Oxides

Moy 15

N. Harrison, National High Magnetic Field Laboratory

Quantum Intefference in the Spin-Polorised Heavy Fermion Compound Ceb_6: Evidence for

Topological Deformation of the Fermi-Surface in Strong Magnetic Fields

May 22

H. Bordallo, LANSCE

Effect of Hydrogen Absorption in TbNiAl and UNiAl 
Lujan Center Seminar Series, continued

1998 continued

May 29

B. Von Dreele, LANSCE

HIPPO: High Intensity Powder Diffroction for Texture and High Pressure

M. Bourke, LANSCE

SMARTS: A Next Generation Instrument for Studying Stress in Engineering Materials

F. Trouw, Argonne Nationol Laboratory

Neutron Scaltering and Molecular Dynomics Simulation of Molecular Sieve Compounds

June 1

D. Wechsler, Hohn-Meiner-Institut

Powder Dirfaction on a Long-Pulse Spallation Source

June 3

H. Roder, Los Alomos Nationol Loboratory

Theoretical Studies of Electron-Lattice Interoctions in CMR Materials

June 5

G. Bendele, State University of New York

Alkoli Fullerides: Effect of the Chorge State on Interfullerence Bonding Geometry

June 12

P. Rangoswomy, LANSCE

How Lujan Center's Xrays and Neutrons Impact MST Residual Stress/Strain Problems

June 19

R. Movshovich and A. Balatsky, Los Alamos National Loboratory

Instobility of the High-Temperature Superconducting Order Porometers Due to Magnefic Impurities

June 8

M. Bourke and K. Benneft, LANSCE

SMARTS \& HIPPO: Án Informotion Overview

July 31

J. Smith, Los Alomos Nationol Laboratory

Heary Fermion Materials and Plutonium

August 7

J. Cooley, Los Alomos

Gap Physics in Kondo Insulators

August 14

A. Christionson, Colorodo State University

Low-Energy Excitations, Symmetry Breaking and Specific Heot in YbBiPt and Crystal Field

Spectrum of PrAg 2 In

August 21

M. Shima, Univ. of Manyland

Structural and Magnefic Properties of Electrodeposite Co/Cu Multilayers

August 28

G. Kwei, LANSCE

The Dynamics of Methane Trapped in Fullerene Interstices

September 4

C. Booth, Los Alomos National Loboratory

Reloting Electronic, Mognefic and Structurol Properties of Partially-Locolized F-Election

Systems Using X-Ray-Absorption Spectroscopy

September 8

H. Riegler, Max-Planck-Institut

How Well is the Wetting Behovior of Simple nalkones Understood?

September 11

L. Bulaevskii, Los Alomos National Loboratory

Josephson Plasmo Resononce os a Tool to Study Vortex State in Layered Superconductors

September 18

A. Lawson, LANSCE

Exheme Neutron Diffraction
1998 continued

September 25

H. Green II, University of Colifornia at Riverside

Deep Earthquakes: A Shearing Instability Triggered by on Exothermic Phose

Transformation Under Conditions Where the Product Phase is NonocrystallineSeptember

30

P. Doi, Oak Ridge National Loboratory

Spin and Lattice Dynamics of the Collossal Magnetoresistance Perouskite Manganites

October 2

M. Rodriguez, Sandia National Loborotories

Characterization Via In-Situ Analysis Techniques

October 9

M. Daymond, ISIS, Rutherford Appleton Loboratory

Engineering Science of ISIS

October 16

P. Longan, Los Alamos National Laboratory

Neutron and X-ray Fibre Diffraction Studies of ONA

October 23

G. Smith, LANSCE

The Stucture of Organized Lipid Bilayers

0ctober 30

R. Von Dreele, LANSCE

Protein Crystal Structures from Powder Diffraction Dato

Other LANSCE Seminars

1998

May 26

D. Bar

Embedded Systems and Signol Processing: A Work in Progress

September 23

1. Lozarev, Russion Federal Nuclear Center

A Super Light Source as a Means for Solving the Problems of Present Microwave Technologies

P. Petrov, Russion Federal Nudear Center

A Charged Porticle Microwove Accelerofor

September 24

R. Dovidson, Princeton University

EP Instability

October 29

1. Garrett, Oak Ridge National Laboratory

Plans for Constructing a Radioactive Ion Beam Focility at the Spallotion Neutron Source

Site in Ook Ridge

October 30

J. Nolen, Argonne Nationol Laboratory

The Argonne Advanced ISOL Focility Concept

November 2-4

D. Louca, Los Alamos, and F. Mezei, LANSCE

Troditional ond New Techniques for Studying Short Ronge Atomic Order in Crystalline Solids

November 2

P. Colestock, Fermi National Accelerator Loborotory

Nonlinear Coherent Phenomena in Storage Rings

November 3

R. McCurdy, Camegie Mellon University

Spin-Pority Anolysis of p-bor $p-w \pi+\pi \pi^{\circ}$ 
and engineering; polymers, complex fluids, and biomaterials; fundamental neutron physics; applied nuclear physics; condensed-matter physics and chemistry; and nuclear weapons research.

The conclusion reached at the workshop was that neutrons will play an essential role in science-based stockpile stewardship and that there is overlap and synergy between defense and other uses of neutrons in basic, applied, and industrial research from which defense and civilian research can benefit. Since the workshop, ideas and potentialities have been consolidated into a program of experimental and analytical efforts.

\section{Electron Effects Workshop}

An Electron Effects in High-Current Proton Rings workshop was held March 4-7, 1997, in Santa Fe, New Mexico. The planned scheme for Spallation Neutron Source (SNS) is similar to that used at LANSCE's Proton Storage Ring (PSR), which experiences peak intensity limitations from a fast loss of particles during accumulation believed to be an electron-proton (e-p) instability. The workshop was sponsored by the SNS project to aid the SNS and the LANSCE PSR upgrade project in understanding and remediating this instability. The approximately 50 attendees came from the laboratories participating in the SNS project (Brookhaven, Berkeley, Argonne, Oak Ridge, and Los Alamos) as well as strong representation from institutions including Fermi National Laboratory, KEK, CERN, Rutherford Appleton Laboratory, TRIUMF, JAERI, the University of Maryland, and SAIC.

The first day was devoted to plenary talks that included a review of e-p instability theory, an overview of PSR observations, observations at other laboratories, reviews of electron-production mechanisms, theoretical studies on the PSR instability, and design issues of the SNS ring. For the second day, the workshop was organized into three working groups: theory and computation, past experiences and proposed experiments, and SNS design strategy. The groups' charters were to summarize previous work in their respective areas and to originate a course for future progress. The results of the working groups and the workshop were summarized in a joint session the morning of the third day.

\section{Methods for Neutron Scattering Instrument Design}

The future of neutron and $x$-ray scattering instrument development and international cooperation was the focus of the workshop "Methods for Neutron Scattering Instrument Design" held September 23-25, 1996, at the E.O. Lawrence Berkeley National Laboratory. The meeting was organized by staff from the Institut Laue-Langevin, National Institute of Standards and Technology, Argonne National Laboratory, Oak Ridge National Laboratory, Rutherford-Appleton Laboratory, and LANSCE.
This international gathering, representing 15 national facilities, universities, and corporations, looked at a number of issues concerning neutron scattering instruments and the tools used in instrument design. Objectives included

- determining the needs of the neutron scattering community in instrument design computer code and information sharing to aid future instrument development,

- providing for a means of training scientists in neutron scattering and instrument techniques,

- facilitating the involvement of other scientists in determining the characteristics of new instruments that meet future scientific objectives, and

- fostering international cooperation in meeting these needs.

The meeting comprised six sessions covering a review of $\mathrm{x}$-ray scattering instrument design tools, the present status of neutron scattering instrument design tools and models of neutron optical elements, and discussions of the present and future needs of the neutron scattering community. 
Neutron Scattering - Materials Science

Neutron Scattering-Instrumentation

Accelerator Science

Spallation Science and Techínology

Nuclear Physics

Other

Theses
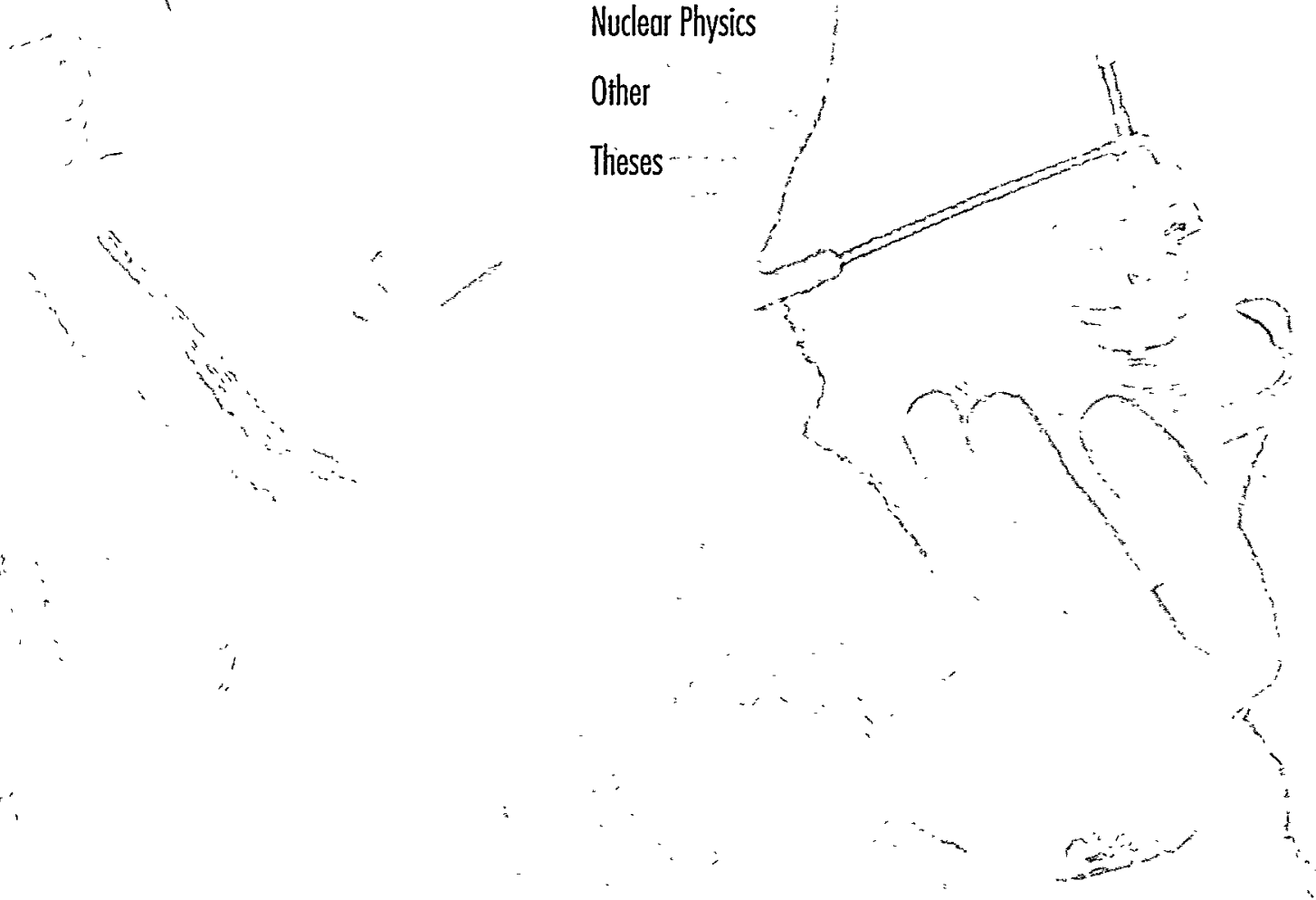
. 


\section{ACRONYMS}

A

ACL

ADC

A/E

AFEL

AGS

ANL

APS

APT

ASCI

ATW

B

BES

BNL

C

CA

CCD

CCDTL

CCL

CD

CD

CDEV

CDR

CIT

CMR

CRITS

CRL

CT

CVS

$\mathrm{CW}$

D

D

dc

DDS

DMPC

DoD

DOE

DP

DSP

d-spacing

DTL

E

ED\&D

EIS
Advanced Computing Laboratory

analog-to-digital converters

architect/engineering

advanced free-electron laser

alternating gradient synchrotron

Argonne National Laboratory

Advanced Photon Source

Accelerator Production of Tritium

Advanced Scientific Computing

Initiative

Accelerator-Driven Transmutation

of Waste

Basic Energy Sciences

Brookhaven National Laboratory

channel access

charge-coupled device

coupled-cavity drift tube linac

conventional coupled-cavity linac

compact disk

competency development

object-oriented device support layer

conceptual design review

California Institute of Technology

colossal-magneto resistance

Chalk River Injection Test Stand

Chalk River Laboratories

compact tension

Concurrent Version System

continuous wave

dimensional, as in 3-D

direct current

damped and detuned structure

dimyristoylphospatidylcholine

Department of Defense

Department of Energy

Office of Defense Programs

digital signal processing

lattice spacing

drift-tube linac

engineering design and development

electrochemical impedance

spectroscopy e-p

EPICS

ER

ESA

ESS

EW

ExecCom .

F

FCG

FDS

FE

FEL

FEM

FeS-1

FP

FPA

FTIR

FY

G

GA

GEANIE

GFLOPS

GNP

GSAS

H

HELIOS

HEPA

HERA

HERF

HERMES

HIPD

HIPPO

HPRF

HVDC

HVPS

I

IAP

IF

IGBT

IHE electron-proton

Experimental Physics and

Industrial Control System

Experiment Room

Engineering Sciences and

Applications

European Spallation Source

equation of state

Executive Committee

flux compression generator

filter difference spectrometer

finite element

free-electron laser

finite element model

iron silicalite-1

flight path

final power amplifier

Fourier transform infrared

fiscal year

\section{General Atomics}

Germanium Array for NeutronInduced Excitations

billions of floating point operations per second gross national product

Generalized Structure Analysis System

high-intensity chopper spectrometer high-frequency particulate air filter High-Energy Resolution Array high-energy rate forging

high resolution backscattering spectrometer

high-intensity powder diffractometer

High Pressure and Preferred

Orientation (Diffractometer)

.high-power radio-frequency

high-voltage direct current

high-voltage power supply

Institute of Applied Science

intermediate frequency

integrated-gate biopolar transistor

insensitive high explosives 


\begin{tabular}{|c|c|c|c|}
\hline ILL & Institut Laue-Langevin & $\mathbf{M}$ & \\
\hline INS & inelastic neutron scattering & MEU & multiple-event upset \\
\hline IOC & input/output controller & MIT & Massachusetts Institute of Technology \\
\hline IP & Individual Projects & MLNSC (now & Manuel Lujan Jr. Neutron \\
\hline IP & industry packs & Lujan Center) & Scattering Center \\
\hline IPA & intermediate power amplifier & MRS & Materials Research Society \\
\hline IPF & Isotope Production Facility & MST & Material Science and Technology \\
\hline IPNS & Intense Pulsed Neutron Source & & Division \\
\hline IPPE & $\begin{array}{l}\text { Institute of Physics and Power } \\
\text { Engineering }\end{array}$ & & \\
\hline I/Q (I\&Q) & in-phase and quadrative & $\mathbf{N}$ & \\
\hline ISIS & Rutherford-Appleton Laboratory & $\begin{array}{l}\mathrm{Nb} \\
\mathrm{NC}\end{array}$ & $\begin{array}{l}\text { niobium } \\
\text { normal-conducting }\end{array}$ \\
\hline & & NCMS & $\begin{array}{l}\text { National Center for Manufacturing } \\
\text { Sciences }\end{array}$ \\
\hline $\mathbf{J}$ & & NERSC & $\begin{array}{l}\text { National Energy Research Scientific } \\
\text { Computing Center }\end{array}$ \\
\hline $\mathbf{K}$ & & $\mathrm{nH}$ & nanoHenries \\
\hline KEKB & Japanese B-Factory & NHMFL & $\begin{array}{l}\text { National High Magnetic Field } \\
\text { Laboratory }\end{array}$ \\
\hline $\mathbf{L}$ & & NISP & Neutron Instrumentation Simulation \\
\hline$L(30 \infty L)$ & left of the incident proton beam & & Package \\
\hline LAB & LANSCE Advisory Board & NIST & National Institute of Standards and \\
\hline LAMPF & Los Alamos Meson Physics Facility & & Technology \\
\hline LANL & Los Alamos National Laboratory & NLC & next linear colider \\
\hline LANSCE & Los Alamos Neutron Science Center & NMSU & New Mexico State University \\
\hline LASREF & Los Alamos Spallation Radiation & NPD & neutron powder diffractometer \\
\hline & Effects Facility & NRS & Neutron Resonance Spectroscopy \\
\hline LB & Langmuir-Blodgett & & \\
\hline LBE & lead-bismuth eutectic & $\mathbf{O}$ & \\
\hline LBNL & Lawrence Berkeley National & OBES & Office of Basic Energy Science \\
\hline LCLS & $\begin{array}{l}\text { Laboratory } \\
\text { linac coherent light source }\end{array}$ & OBER & Office of Biological and \\
\hline LDRD & $\begin{array}{l}\text { laboratory directed research and } \\
\text { development }\end{array}$ & $\begin{array}{l}\text { Oe } \\
\text { OFE }\end{array}$ & $\begin{array}{l}\text { Environmental Research } \\
\text { oersted } \\
\text { copper }\end{array}$ \\
\hline LEBT & low-energy beam transport & ORNE & $\begin{array}{l}\text { copper } \\
\text { Oak Ridge National Laboratory }\end{array}$ \\
\hline LEDA & low-energy demonstration accelerator & UKIVL & \\
\hline LHC & large hadron collider & & \\
\hline linac & linear accelerator & $\mathbf{P}$ & \\
\hline LLNL & $\begin{array}{l}\text { Lawrence Livermore National } \\
\text { Laboratory }\end{array}$ & $\begin{array}{l}\mathrm{P} \\
\mathrm{PAC}\end{array}$ & $\begin{array}{l}\text { high pressure } \\
\text { Program Advisory Committee }\end{array}$ \\
\hline LLRF & low-level radio-frequency & PACS & personnel access control system \\
\hline LLSS & Long Length Scale Structure & PARMELA & Phase and Radial Motion in \\
\hline LPSS & long-pulse spallation source & & Electron Linear Accelerators \\
\hline LQD & low-Q diffractometer & PBS & Public Broadcasting System \\
\hline LRIP & $\begin{array}{l}\text { LANSCE Reliability Improvement } \\
\text { Project }\end{array}$ & $\begin{array}{l}\text { PD } \\
\text { PEI }\end{array}$ & $\begin{array}{l}\text { Program Development } \\
\text { polyethyleneimine }\end{array}$ \\
\hline LS & Life Sciences (Division) & PET & positron-emitter-tomography \\
\hline LSND & liquid scintillator neutrino detector & PHAROS & high-resoluition chopper spectrometer \\
\hline LUG & LANSCE User Group & PID & proportional integral differential \\
\hline Lujan Center & Manuel Lujan Jr. Neutron & PLL & phase-locked loop \\
\hline & Scattering Center & PSI & Paul Scherrer Institute \\
\hline & & PSR & proton storage ring \\
\hline
\end{tabular}




\section{Q}

R

RAFEL

rf

RFQ

RHEED

RHIC

RSS

S

SASE

SBSS

SC

SCD

SCL

SCR

SEU

SLAC

SLS

SMARTS

SNS

SPA

SPEAR

SPSS

SPSS-EP

STD

\section{$\mathbf{T}$}

$T$

T/B

TCP/IP

TFLOPS

3-D

$\mathrm{TiO}_{2}$

TJNAF

TMD

TMRS

TNS

TOF

TS-1

TSZ
$\mathbf{U}$

radio-frequency

radio-frequency quadrupole

Reflection High-Energy Electron

Diffraction

relativistic heavy ion collider

radiation security system

self-amplified spontaneous-emission

Science-Based Stockpile Stewardship superconducting

single-crystal diffractometer

side-coupled linac

silicon-controlled rectifier

single-event upset

Stanford Linear Accelerator Center

Swiss Light Source

Spectrometer for Materials Research at Temperature and Stress

Spallation Neutron Source

Sub-Picosecond Accelerator

Surface Profile Analysis Reflectometer

short-pulse spallation source

Short Pulse Spallation Source

Enhancement Project

Spectrometer Development Teams
UCLA

UCR

UCSD

UHV

UNM-LA

University of Califomia at Los Angeles University of California at Riverside University of California at San Diego ultra-high-vacuum

University of New Mexico at Los Alamos

V

VME

VXI

W

WNR

WTR

Weapons Neutron Research (facility) Waste Transmutation Region

$\mathbf{X}$

$\mathrm{X}$

composition

$\mathbf{Y}$

YBCO

$\mathrm{YBa}_{2} \mathrm{Cu}_{3} \mathrm{O}_{7}$

Z

$\mathrm{ZX} \quad$ zero-crossing high temperature

target/blanket

transmission-control-protocol/

internet protocol

trillions of floating point operations

per second

three-dimensional

anatove

Thomas Jefferson National

Accelerator Facility

theoretical maximum density

target-moderator-reflector-system

tungsten neutron source

time-of-flight

titanium silicalite-1

titanosilicate zeolites 



\section{Publications}

The following publications relate to all work carried out at LANSCE. Work performed elsewhere by LANSCE staff is included where relevant. This list does not include publications submitted or in press.

Publications are an important "product" of LANSCE and a valuable metric to its sponsors and review boards. All facility users are requested to send complete references of all publications resulting from work done at LANSCE to the LANSCE User Office as soon as the publications appear.

Authors should remember that LANSCE is funded by the United States Department of Energy and an appropriate acknowledgement is required. A recommended acknowledgement citation is:

This work has benefited from the use of the Los Alamos Neutron Science Center at Los Alamos National Laboratory. This facility is funded by the U.S. Department of Energy under Contract W-7405-ENG-36.

\section{Neutron Scattering-Materials Science}

ALBINATI, A., CHALOUPKA, S., ECKERT, J., VENANZI, L. M., and WOLFER, M., "Synthetic, Structural, and Spectroscopic Studies of the Hydrido-Bridged Cationic Complexes $\mathrm{L}_{2} \mathrm{YPt}(\mathrm{m}-$ $\left.\mathrm{H}) \mathrm{PtYL}_{2}\right]+\left(\mathrm{L}=\mathrm{PMe}_{3}, \mathrm{Y}=\mathrm{C}_{6} \mathrm{H}_{5}\right.$ and $\mathrm{C}_{6} \mathrm{~F}_{5} ; \mathrm{L}=\mathrm{PEt}_{3}, \mathrm{Y}=\mathrm{C}_{6} \mathrm{H}_{5}$ and $\left.\mathrm{C}_{6} \mathrm{~F}_{5}\right)$ and $\left[\left(\mathrm{PEt}_{3}\right)_{2} \mathrm{HPt}(\mathrm{m}-\mathrm{H}) \mathrm{Pt}\left(\mathrm{C}_{6} \mathrm{H}_{5}\right)\left(\mathrm{PEt}_{3}\right)_{2}\right]+$," Inorganica Chimica Acta 259, 305 (1997)

ALBINATI, A., ECKERT, J., PREGOSIN, P. S., RUEGGER, H., SALZMANN, R., and STOESSEL, C., "NMR Studies of Chiral P,S Chelate Pt, Rh, and Ir Complexes and the X-Ray Structure of the Pd(II) Allyl Derivative," Organometallics 16, 579 (1997)

ALBINATI, A., KLOOSTER, W. T., KOETZLE, T. F., BORTIN, J. B., RICCI, J. S., ECKERT, J., FONG, T. P., LOUGH, A. J., MORRIS, R. H., and GOLOBEK, A. P., "Single-Crystal X-Ray and Neutron Diffraction Structure Determination and Inelastic Neutron Scattering Study of the Dihydrogen Complex Trans $\left[\mathrm{RuH}\left(\mathrm{H}_{2}\right)(\mathrm{dppe})_{2}\right]\left[\mathrm{BPh}_{4}\right]$," Inorganica Chimica Acta 259, 351 (1997)

ANDERSON, C., GOLDMAN, P., RANGASWAMY, P., PETRUS, G., FERGUSON, B. L., LATHROP, J., NIKKEL, D., and LOWE, T., "Development of a Carburizing and Quenching Simulation Tool: Numerical Simulation of Rings and Gears," In Proceedings of the 2nd International Conference on Quenching and Control of Distortion, Totten, G. E., Howes, S. J., Sjostrom, Funatani, K., Eds. (ASM Heat Treating Society, 1996) 377-383

ANDERSON, W. W., ZHAO, Y., and AHRENS, T. J., "Equation of State of Clay, Shale, and Slate," Defense Nuclear Agency Report, DNA001-92-C-0128, 1-45 (1995)

ARANDA, M. A. G., BRUQUE, S., ATTFIELD, J. P., PALACIO, F., and VON DREELE R. B., "Changes in Magnetic Couplings After Chimi Douce Reactions: Magnetic Structures of $\mathrm{LiMnXO}_{4}(\mathrm{OD})$ (X=P, As)," J. Solid State Chem. 132, 202-212 (1997)

ARGYRIOU, D. N, and ELCOMBE, M. M., "A NeutronScattering Investigation Of Cubic Stabilized Zirconia (Csz) .2. Lattice-Dynamics of Y-CSZ and Ca-CSZ," Journal of Physics and Chemistry of Solids 57, 343-351 (1996)
ARGYRIOU, D. N., and HOWARD, C. J., "Reinvestigation of Yttria-Tetragonal Zirconia Polycrystal (Y-Tzp) By Neutron Powder Diffraction: A Cautionary Tale," Journal of Applied Crystallography 28, 206-208 (1995)

ARGYRIOU, D. N., ELCOMBE, M. M., and LARSON, A C., "A Neutron-Scattering Investigation of Cubic Stabilized Zirconia (CSZ) .1. the Average Structure Of Y-CSZ," Joumal of Physics and Chemistry of Solids 57, 183-193 (1996)

ARGYRIOU, D. N., GARCIA, J. A., MITCHELL, J. F., JORGENSEN, J. D., and HINKS, D. G., "Phase Development of Bi-2212 Superconductor: A Time-Resolved Neutron Powder Diffraction Investigation," Journal of Materials Research 11, 277-280 (1996)

ARGYRIOU, D. N., HINKS, D. G., MITCHELL, J. F., POTTER, C. D., SCHULTS, A. J., YOUNG, D. M., JORGENSEN, J. D., and BADER, S. D., "The Room-Temperature Crystal-Structure of the Perovskite $\mathrm{Pr}_{0.5} \mathrm{Sr}_{0.125} \mathrm{MnO}_{3+\text { Delta, Journal of Solid State }}$ Chemistry 124, 381-384 (1996)

ARGYRIOU, D. N., JORGENSEN, J. D., HITTERMAN, R. L., HIROI, Z., KOBAYASHI, N., and TAKANO, M., "Structure and Superconductivity without Apical Oxygens in $(\mathrm{Ca}, \mathrm{Na})_{2} \mathrm{CuO}_{2} \mathrm{Cl}_{2}$," Physical Review B 51, 8434-8437 (1995)

ARGYRIOU, D. N., KELLEY, T., MITCHELL, J. F., ROBINSON, R. A., OSBORN, R., ROSENKRANZ, S. and JORGENSEN, J. D., "Two-Dimensional Ferromagnetic Correlations Above $T_{c}$ in the Naturally Layered CMR Manganite $\mathrm{La}_{2-2 x} \mathrm{Sr}_{1-2 x} \mathrm{Mn}_{2} \mathrm{O}_{7}(\mathrm{x}=0.3-0.4)$," J. Appl. Physics 83 11, 6374-6378 (1998)

ARGYRIOU, D. N., MITCHELL, J. F., GOODENOUGH, J. B., CHMAISSEM, O., SHORT, S., and JORGENSEN, J. D., "Sign Reversal of the Mn-O Bond Compressibility In $\mathrm{La}_{1.2} \mathrm{Sr}_{1.8} \mathrm{Mn}_{2} \mathrm{O}_{7}$ Below $\mathrm{T}_{\mathrm{c}}$ : Exchange Striction In the Ferromagnetic State," Physical Review Letters 78, 1568-1571 (1997)

ARGYRIOU, D. N., MITCHELL, J. F., POTTER, C. D., BADER, S. D., KLEB, R., and JORGENSEN, J. D., "Unconventional Magnetostriction In Layered $\mathrm{La}_{1.2} \mathrm{Sr}_{1.8} \mathrm{Mn}_{2} \mathrm{O}_{7}$. Evidence For Spin-Lattice Coupling Above $\mathrm{T}_{c}$, Physical Review B 55, 11965-11968 (1997) 
ARGYRIOU, D. N., MITCHELL, J. F., POTTER, C. D., HINCKS, D. G., JORGENSEN, J. D., and BADER, S. D., "Lattice Effects and Magnetic Order In the Canted Ferromagnetic

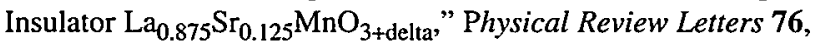
3826-3829 (1996)

ARONSON, M. C., OSBORN, R., ROBINSON, R. A., LYNN, J. W., CHAU, R., SEAMAN, C. L., and MAPLE, M. B., "NonFermi Liquid Scaling in $\mathrm{UPd}_{\mathrm{x}} \mathrm{Cu}_{5-\mathrm{x}}(\mathrm{x}=1,1.5)$," Physica $B 206$ \& 207, 108-111 (1995).

ARONSON, M. C., OSBORN, R., ROBINSON, R. A., LYNN, J. W., CHAU, R., SEAMAN, C. L., and MAPLE, M. B., "NonFermi Liquid Scaling of the Magnetic Response in $\mathrm{UCu}_{5-\mathrm{x}} \mathrm{Pd}_{\mathrm{x}}$ $(\mathrm{x}=1,1.5)$," Physical Review Letters 75, 725 (1995)

BAKER, S. M., CALLAHAN, A., SMITH, G. S., TOPRAKCIOGLU, C., and VRADIS, A. A., "Shear Effects on the Geometry of Polystyrene-Polyethylene Oxide Copolymers at the Solid-Solvent Interface," Physica B 241-243, 1041-1047 (1997)

BAKER, S. M., SMITH, G. S., BROWN, J. J. S., HUBBARD, K., and NASTASI, M., "Observation of Non-Standard Fickian Diffusion at the Interface of Isotopically Pure Amorphous ${ }^{11} \mathrm{~B}$ on ${ }^{10} \mathrm{~B}$ by Neutron Reflectometry," Phys. Rev. B 55, 7255-63 (1997)

BAKER, S. M., WU, K., SMITH, G. S., HUBBARD, K. M., NASTASI, M., DOWNING, R. G., and LAMAZE, G. P., "NonStandard Fickian Self-Diffusion of Isotopically Pure Boron Observed by Neutron Reflectometry and Depth Profiling," In Proceedings of Neutron Scattering in Materials Science II Symposium, Neumann, D. A., Russell, T. P., and Wuensch, B. J., Eds. (Mater. Res. Soc., 1995) 209-15

BALZAR, D. and LEDBETTER, H., "Elastic Strain and Stress by Rietveld Refinement, Materials Structure in Chemistry, Biology, Physics, and Technology," Bulletin of the Czech and Slovak Crystallographic Association 5A, 28 (1998)

BALZAR, D., VON DREELE, R., BENNETT, K. A., and LEDBETTER, H., "Elastic-Strain Tensor by Rietveld Refinement of Diffraction Measurements," Journal of Applied Physics 84, 4822-4833 (1998)

BARANOV, N., MARKIN, P. E., NAKOTTE, H., and LACERDA, A., "Magnetic and Transport Properties of $\mathrm{Tb}_{3}$ Co Studied on Single Crystals," J. Magn. Mater. 177-181, 1133-1134 (1998)

BARDEAU, J-F., BULOU, A., KLOOSTER, W., KOETZLE, T. F., JOHNSON, S., SCOTT, B., SWANSON, B. I., and ECKERT, J., "Neutron Diffraction Study of $\left[\mathrm{Pt}(\mathrm{en})_{2}\right]\left[\mathrm{Pt}(\mathrm{en})_{2} \mathrm{I}_{2}\right]\left(\mathrm{ClO}_{4}\right)_{4}$ at $20 \mathrm{~K}$ : Structure and Evidence of a New Phase Transition," Acta Crystallographica B 52, 854 (1996)

BARTHES, M., BORDALLO, H. N., ECKERT, J., MAURUS, D., DE NUNZIO, G., and LEON, J., "Dynamics of Crystalline $\mathrm{N}$-Methlacetamide: Temperature Dependence of Infrared and Inelastic Neutron Scattering Spectra," Journal of Physical Chemistry 102, 32, 6177-6183 (1998)
BENDER, B. R., JONES, L. H., SWANSON, B. I., ECKERT, J. , KUBAS, G. J., KAPPS, K. B., and HOTT, C. D., "Why Does $\mathrm{D}_{2}$ Bind Better Than $\mathrm{H}_{2}$ ? A Theoretical and Experimental Study of the Equilibrium Isotope Effect on $\mathrm{H}_{2}$ Binding in a $\mathrm{M}\left(\mathrm{eta}_{2}-\mathrm{H}_{2}\right)$ Complex, Normal Coordinate Analysis of W(CO) $)_{3}\left(\mathrm{PCy}_{3}\right)_{2}\left(\mathrm{eta}_{2}-\mathrm{H}_{2}\right)$," Journal of Am. Chem. Soc. 119, 9179 (1997)

BENMORE, C. J., OLIVIER, B. J., SUCK, J. B., ROBINSON, R. A., and EGELSTAFF, P. A., "A Neutron Brillouin Scattering Study of the Amorphous Metal $\mathrm{Mg}_{70} \mathrm{Zn}_{30}$," Journal of Physics: Condensed Matter 7, 4775-4785 (1995)

BENMORE, C. J., SWEENEY, S., ROBINSON, R. A., EGELSTAFF, P. A., and SUCK, J. B., "Constant-Q Measurements of Collective Modes in $\mathrm{Mg}_{70} \mathrm{Zn}_{30}$ Glass," Physica B 241-243, 912-914 (1996)

BENMORE, C., FORMISANO, F., MAGLI, R., BAROCCHI, F., EGELSTAFF, P. A., ROBINSON, R. A., and VERKERK, P., “An Experimental Investigation of the Interaction Law of $\mathrm{Kr}$ Atoms through Small-Angle Neutron Scattering," Physica B 234-236, 313-315 (1997)

BENNETT, K,. WENK, H. R., BURHAN, W. B., STERN, L. A., and KIRBY, S. H., "Preferred Crystallographic Orientation in the Ice I-II Transformation and the Flow of Ice II," Phil. Mag. A76, $413-435$ (1997)

BENNETT, K., VON DREELE, R. B., BAI, Q., and GREEN, H. R., "Preferred Orientation in a-b Olivines: Implications for Seismic Anisotropy in the Upper Mantle," EOS Transactions of the American Geophysical Union 77 (1996)

BERRET, J-F., ROUX, D. C., PORTE, G., and LINDNER, P., "Tumbling Behavior of Neumatic Worm-Like Micelles Under Shear Flow," Europhys. Lett. 32, 2, 137 (1995)

BILLINGE, S. J. L., and KWEI, G., "Probing the Short Range Order and Dynamics of Phase Transitions Using Neutron Powder Diffraction," J. Phys. Chem. 57, 1457 (1996)

BILLINGE, S. J. L., and KWEI, G., "Determination of the Local Atomic Structure of $\mathrm{La}_{2-\mathrm{x}}(\mathrm{Sr}, \mathrm{Ba})_{x} \mathrm{CuO}_{4}$ Materials from Neutron Powder Diffraction Data," Neumann, D. A., Russell, T. P., and Wuensch, B. J., Eds., Mater. Res. Soc. Proc. 376, 523 (1995)

BILLINGE, S. J. L., DIFRANCESCO, R. G., KWEI, G., NEUMEIER, J. J., and THOMPSON, J. D., "Direct Observation of Lattice Polaron Formation in the Local Structure of $\mathrm{La}_{1-\mathrm{x}} \mathrm{Ca}_{\mathrm{x}} \mathrm{MnO}_{3}$," Phys. Rev. Lett. 77, 715 (1996)

BILLINGE, S. J. L., "The Structure of Real Materials Using $\mathrm{X}$-Ray and Neutron Scattering," Current Opinion in Solid State \& Materials Science 1, 477-84 (1996)

BINBREK, O. S., TORRIE, B., VON DREELE, R. and POWELL, B. M., "The Two Solid Phases of Chlorodifluoromethane," Molec. Phys. 90, 1, $49-54$ (1997) 
BORDALLO, H. N., ARGYRIOU, D. N., MITCHELL, J. F., and STROUSE, G. F., "Effects of Dynamic Jahn-Teller Distortions by Raman Spectroscopy in the Layered CMR LA 2-2X $\mathrm{Sr}_{1+2 \mathrm{X}} \mathrm{Mn}_{2} \mathrm{O}_{7}$," Bulletin of the American Physical Society 43, 1, 163 (1998)

BORDALLO, H. N., BARTHES, M., and ECKERT, J., "Dynamics of Peptide Chains and Nucleic Acid Constituents," Physica B 241-243 (1998)

BORDALLO, H. N., BARTHES, M., and ECKERT, M., "Vibrational Dynamics of Crystalline L-Alanine," Physica B 241-243, 1138-1140 (1997)

BORDALLO, H. N., ECKERT, J., and BARTHES, M., "Dynamic Disorder in a Crystallized Peptide Chain," Bulletin of the American Physical Society 42, 1, 154 (1997)

BORDALLO, H. N., NAKOTTE, H., KOLOMIETS, A., HAVELA, L., ECKERT, J., ANDREEV, A. V., and DRULIS, H., "Hydrogen Induced Changes in the Structural and Magnetic Properties of TbNiAl," Joumal of Applied Physics 83, 11, 6986 (1998)

BOURKE, M. A. M., GOLDSTONE, J. A., and ROBINSON, R. A., "Strain Measurement by Diffraction at LANSCE," Physica B 213 \& 214, 806-808 (1995)

BOURKE, M. A. M., MALDONADO, J. G., MASTERS, D., MEGGERS, K., and PRIESMEYER, H. G., "Real Time Measurement of Bragg Edge Diffraction of the Reverse (alpha to gamma) Transformation in a Deformed 304 Stainless Steel," Journal of Materials Science \& Engineering A221, 1-10 (1996)

BOURKE, M. A. M., "Phase Transformations in Engineering Materials," In Proceedings of the SPIE (SPIE, 1997) 2867, 131-135

BOURKE, M. A. M., ROBERTS, J. A., DAVIS, D., "Macrostrain Measurement Using Radial Collimators at LANSCE," In Proceedings of the SPIE (SPIE, 1997) 2867, 136-140

BOURKE, M. A. M., VAIDYANATHAN, R., and DUNAND, D. C., "Neutron Diffraction Measurements of Stress-Induced

Transformations in Superelastic NiTi," Appl. Phys. Lett. 69, 21 (1996)

BOZIN, E. S., BILLINGE, S. J. L., and KWEI, G., "Reexamination of the Second Order Structural Phase Transition in $\mathrm{La}_{2-\mathrm{x}} \mathrm{A}_{\mathrm{x}} \mathrm{CuO}_{4}$ ( $\left.\mathrm{A}=\mathrm{Ba}, \mathrm{Sr}\right), "$ Physica B 241-24, 795-797 (1997)

BOZIN, E. S., BILLINGE, S. J. L. and KWEI, G., "Understanding the Role of the Local Structure in the Second Order Structural Phase Transition of $\mathrm{La}_{2-\mathrm{x}} \mathrm{A}_{\mathrm{x}} \mathrm{CuO}_{4}(\mathrm{~A}=\mathrm{Ba}, \mathrm{Sr})$," Solid State Phenomena 61-62, 271-274 (1998)

CAMPBELL, J. P., HWANG, J., YOUNG, V. G., VON DREELE, R., CRAMER, C. J., and GLADFELTER, W. L., "Crystal Engineering Using the Unconventional Hydrogen Bond; Synthesis, Structure, and Theoretical Investigation of Cyclotrigallazane," J. Am. Chem. Soc. 120, 521-531 (1998)

CARTER, D. H., and BOURKE, M. A. M., "Neutron Diffraction Study of the Co-Deformation Behavior of a Beryllium-Aluminum Composite," In Proceedings of the Eleventh International Conference on Composite Materials III, 736-745 (1997)
CHAU, R., MAPLE, M., and ROBINSON, R. A., "Crystallographic Evidence for Chemical Ordering in $\mathrm{UCu}_{4} \mathrm{Pd}$," Physical Review B 58, 139-143 (1998)

CHAU, R., MAPLE, M., and ROBINSON, R. A., "Evidence of Atomic Ordering in $\mathrm{UCu}_{4} \mathrm{Pd}$," Bulletin of the American Physical Society 43, 182 (1998)

CHMAISSEM, O., ARGYRIOU, D. N., HINKS, D. G., JORGENSEN, J. D., STOREY, B. G., ZHANG, H., MARKS, L. D., WANG, Y. Y., DRAVID, V. P., and DABROWSKI, B., "Chromium Clustering and Ordering In $\mathrm{Hg}_{1-\mathrm{x}} \mathrm{Cr}_{\mathrm{x}} \mathrm{Sr}_{2} \mathrm{CuO}_{4+\text { delta," }}$ Physical Review B 52, 21, 15636-43 (1995)

CHO, J. H. SAFAR, H., MALEY, M. P., WILLIS, J. O., COULTER, J., STEEL, D. G., and GRAY, K. E., "Effects of Unidirectional and Isotropic Columnar Defects in $\mathrm{Bi}_{2} \mathrm{Sr}_{2} \mathrm{CaCu}_{2} \mathrm{O}_{8}$ Single Crystals," Physica C 302, 113 (1998)

CLAUSEN, B., and LORENZEN, T., "Experimental Evaluation of a Polycrystal Deformation Modeling Scheme Using Neutron Diffraction Measurements," Metallurgical and Materials Transactions 28A, 2537-2541 (1997)

CLINE, J. P., VON DREELE, R. B., VAUDIN, M. D., and MINOR, D. B., "Certificate of Analysis: Standard Reference Material 656-Silicon Nitride Powders for Quantitative Analysis by Powder Diffraction," National Institute of Standards and Technology (1995)

COHEN, I., WEISSBUCH, R. POPOVITZ-BIRO, MAJEWSKI, J., MAUDER, H. P., LAVI, R., LEISEROWITZ, L., and LAHAV, M., "Spontaneous Assembly in Organic Thin-Films Spread on Aqueous Subphase: A Scanning Force Microscope (SFM) Study," Israel Journal of Chemistry 36, 1, 97-110 (1996)

COOKE, D. W., BENNETT, B. L., LAWSON, A. C., HUBER, J. G., COSTENS, J., BOEKEMA, C., FLINT, J. A., and LICHTI, R. L., "Spontaneous Magnetic Ordering in Cerium Rhodium Boride Observed by Zero-Field Muon-Spin Relaxation," Philosophical Magazine B 74, 3, 259-67 (1996)

DAYMOND, M. R., BOURKE M. A. M., VON DREELE, R. B., CLAUSEN B., and LORENTZEN, T., "Use of Rietveld Refinement for Elastic Macrostrain Determination and for Evaluation of Plastic Strain History from Diffraction Spectra," Journal of Applied Physics 82, 4, 1554-1562 (1997)

DE BOER, F. R., KINDO, K., NAKOTTE, H., PROKES, K., and SECHOVSKY, V., " $\mathrm{U}_{2} \mathrm{~T}_{2} \mathrm{X}$ ( $\left.\mathrm{T}=\mathrm{Co}, \mathrm{Ni}, \mathrm{Rh}, \mathrm{Pd}, \mathrm{Ir}, \mathrm{Pt}, \mathrm{X}=\mathrm{In}, \mathrm{Sn}\right)$ Compounds in High Magnetic Fields," Physica B A67 (1997)

DE CHATEL, P. F., PROKES, K., NAKOTTE, H., PURWANTO, A., SECHOVSKY, V., HAVELA, L., BRUCK, E., ROBINSON, R. A., and DE BOER, F. R., "Anisotropic Exchange Interactions in UNiGe," J. Magn. Magn. Mater. 177, 785-786 (1998)

DICK, J. J., "Anomalous Shock Initiation of Detonation in Pentaerythritol Tetranitrate Crystal," Journal of Applied Physics 81, 2, 601-612 (1997) 
DMOWSKI, W., MCQUEENEY, R. J., EGAMI, T., FENG, Y. P., SINHA, S., HINATSU, T., and UCHIDA, S., "Temperature Dependent X-Ray Diffuse Scattering from Single Crystals of $\mathrm{La}_{2-\mathrm{x}} \mathrm{Sr}_{\mathrm{x}} \mathrm{CuO}_{4}$," Physical Review B 52, 6829 (1995)

DUNAND, D. C. , MARI, D., BOURKE, M. A. M., and ROBERTS, J. A., "NiTi and NiTi-TiC Composites: IV. Neutron Diffraction Study of Twinning and Shape Memory Recovery," Metallurgical and Materials Transactions 27A, 2820-2836 (1997)

DUNAND, D., FUKAMI-USHIRO, K. L., MARI, D., ROBERTS, J. A., and BOURKE, M. A. M., "Mechanical Properties of NiTi-TiC Shape-Memory Composites," Mat. Res. Soc. Symp. Proc. 459, 131-142 (1997)

DUNAND, D., MARI, D., BOURKE, M. A. M., and GOLDSTONE, J. A., "Neutron Diffraction Study of NiTi During Compressive Deformation and After Shape Memory Recovery," Journal de Physique IV C8-653 (1995)

EASTMAN, J. A. and FITZSIMMONS, M. R., "On the TwoState Microstructure of Nanocrystalline Chromium," Journal of Applied Physics 77, 2, 522 (1995)

EASTMAN, J. A., FITZSIMMONS, M. R., THOMPSON, L. J., BENO, M. A., and KNAPP, G. S., "High Angle Diffraction Studies of Defects and Phase Stability in Nanocrystalline Materials," Nanostr. Materials 6, 543 (1995)

ECKERT, J., ALBINATI, A., BUCHER, U. E., and VENANZI, L. M., "The Nature of the Rh- $\mathrm{H}_{2}$ Bond in $\mathrm{LRhH}_{2}\left(\mathrm{~h}_{2}-\mathrm{H}_{2}\right), \mathrm{L}=$ $\mathrm{HB}\left(3,5-\left(\mathrm{CH}_{3}\right)_{2}-\mathrm{py}\right)_{3}$, The First $\mathrm{H}_{2}$-Dihydrogen Complex Stabilized by a Nitrogen Donor Ligand: An Inelastic Neutron Scattering Study," Inorganic Chemistry 35, 1292 (1996)

ECKERT, J., "Dynamics of Hydrogen Ligands in Metal Complexes," In Proceedings of the Transactions of the American Crystallographic Association 31, 45, Montreal, Canada (1995)

ECKERT, J., JENSEN, C. M., KOETZLE, T. F., LE-HUSEBO, T., NICOL, J. M., and WU, P., "Inelastic Neutron Scattering Studies and Neutron Diffraction Structure Determination of $\mathrm{IrIH}_{2}\left(\mathrm{~h}_{2}-\mathrm{H}_{2}\right)\left(\mathrm{PPri}_{3}\right)_{2}$ : Implications on the Mechanism for Interconversion of Dihydrogen and Hydride Ligands," Journal American Chemical Society 117, 7271 (1995)

ECKERT, J., NICOL, J. M., HOWARD, J., and TROUW, F. R., "Adsorption of Hydrogen in $\mathrm{Ca}^{2}+$ Exchanged Zeolite As Probed by Inelastic Neutron Scattering Spectroscopy," Journal of Physical Chemistry 100, 10646 (1996)

ECKERT, J., VARMA, R., DIEBOLT, L., and REID, M., "Effects of Cycling Conditions of Active Material from Discharged Ni Positive Plates Studied by Inelastic Neutron Scattering Spectroscopy," J. Electrochem. Soc. 144, 1895 (1997)

EGAMI, T., DMOWSKI, W., MCQUEENEY, R. J., ARAI, M., SEIJI, N., and YAMAUCHI, H., "Dynamic Local Lattice Distortion in $\mathrm{YBa}_{2} \mathrm{Cu}_{4} \mathrm{O}_{8}$," J. Supercond. 8, 587 (1995)
EGAMI, T., LOUCA, D., and MCQUEENEY, R. J., "Nonuniform Metallic State in Manganites and Cuprates," J. Supercond. 10, 323 (1997)

EGAMI, T., MCQUEENEY, R. J., and ISHIHARA, S., "Lattice Effects in HTSC Cuprates Observed by Neutron Scattering," Czech. J. of Phys. 46, 1249 (1996)

EGAMI, T., MCQUEENEY, R. J., DMOWSKI, W., SEIJI, N., YAMAUCHI, H., ARAI, M., ISHIHARA, S., and TACHIKI, M., "Unconventional Electron-Phonon Interaction and Anharmonicity in Superconducting Cuprates," Physica B 219 \& 220, 145 (1996)

FITZSIMMONS, M. R., EASTMAN, J. A, ROBINSON, R. A., and LYNN, J. W., "The Néel Temperature of Nanocrystalline Chromium," Journal of Applied Physics 78, 1364 (1995)

FITZSIMMONS, M. R., EASTMAN, J. A., ROBINSON, R. A., and LYNN, J. W., "On the Possibility of a Two-State Magnetic Structure for Nanocrystalline Chromium," Nanostructured Materials 7, 179-184 (1996)

FITZSIMMONS, M. R., LUTT, M., PYNN, R., KINDER, H., and PRUSSEIT, W., "YBCO Films as Meissner Screens in the Control of Polarized Neutron Beams, Observations, and Calculations," Nuclear Instr. and Methods A411, 401 (1998)

FITZSIMMONS, M. R., ROLL, A., BURKET, E., SICKAFUS, K. E., NASTASI, M., SMITH, G. S., and M., PYNN, R., "The Magnetization of a Grain Boundary in Nickel," Nanostructured Materials 6, 539-542 (1995)

FITZSIMMONS, M. R., PYNN, R., LUTT, M., KINDER, H., and PRUSSEIT, W., "Use of YBCO Films for Applications with Polarized Neutrons," Physica B 241-243, 121-123(1997)

FUKUSHIMA, T., NAKOTTE, H., MATSUYAMA, S., KUMADA, T., KINDO, K., PROKES, K., DE BOER, F. R., HAVELA, L., SECHOVSKY, V., WINAND, J. M., REBIZANT, J., and SPIRLET, J. C., "High-Field Magnetization Studies of U2T2Sn ( $=\mathrm{Co}, \mathrm{Ir}, \mathrm{Pt})$ Compounds," In Proceedings of the Conference on Physical Phenomena in High Magnetic Fields II (World Scientific 1996) 215-220

GOLDSTONE, J. A., BOURKE, M. A. M., and SHI, N., "Internal Strain Measurement Using Pulsed Neutron Diffraction at LANSCE," Neumann, D. A., Russell, T. P., and Wuensch, B. J., Eds., Mater. Res. Soc 376, 391-396 (1995)

GRIGOROV, I. L., WALKER, J. C., HAWLEY, M., BROWN, G. W., FITZSIMMONS, M. R., and LUTT, M., "Structural and Magnetic Properties of "Expanded" MN," Journal of Applied Physics 83, 11, 7010 (1998)

HAGMUSA, I. H., KLAASSE, J. C. P., BRUCK, E., PROKES, K., DE BOER, F. R., and NAKOTTE, H., "Specific Heat of UNiGe in High Magnetic Fields," Journal of Applied Physics 81, 4157-4159 (1997) 
HARLOW, R. L., KWEI, G. H., SURYANARAYANAN, R., and SUBRAMANIAN, M. A., "Structure of $Y \mathrm{Sr}_{2} \mathrm{Cu}_{3-\mathrm{x}} \mathrm{M}_{\mathrm{x}} \mathrm{O}_{7+\mathrm{d}} \mathrm{M}=$ $\mathrm{Mo}, \mathrm{W}$ and $\mathrm{Re}$ ) from Single Crystal $\mathrm{X}$-Ray and Powder Neutron Diffraction: Substituent Site Preference and Oxygen Defect Structure," Physica C 257, 125 (1996)

HAVELA, L., SECHOVSKY, V., NAKOTTE, H., LACERDA, A., PROKES, K., and BRUCK, E., "Low-Temperature Behavior of Electrical-Resistivity in UTX Compounds," Czechoslovak Journal of Physics 46, S4, 2043-2044 (1996)

HAVELA, L., SECHOVSKY, V., PROKES, K., NAKOTTE, H., FUJII, H., and LACERDA, A., "Giant Magnetoresistance Effects in 5f-Materials," Physica B, 223-224, 245 (1996)

HAVELA, L., SECHOVSKY, V., SVOBODA, P., NAKOTTE, H., PROKES, K., DE BOER, F. R., SERET, A., WINAND, J. M., REBIZANT, J., SPIRLET, J. C., PURWANTO, A., and ROBINSON, R. A., "Magnetism in $\mathrm{U}_{2} \mathrm{~T}_{2} \mathrm{X}$ Compounds," Journal of Magnetism and Magnetic Materials 140-144, 1367-1368 (1995)

HJELM, R. P., "Summary on Neutron Source Concepts," In Proceedings of the Thirteenth Meeting of the International Collaboration on Advanced Neutron Sources 17-18 (1995)

HJELM, R. P., DOUGLAS, E. P., and BENICEWICZ, B. C., "The Solution Structure of Liquid-Crystal Polymers with Small Liquid-Crystal Thermoset Maleimides and Nadimides," International Journal of Thermophysics 16, 309-317 (1995)

HJELM, R. P., MANG, J. T., HOFMANN, A. F., SCHTEINGART, C., ALKAN-ONYUKSEL, H., and AYD, S., "Formation of Drug-Bearing Vesicles in Mixed Colloids of Bile Salts and Phosphatidylcholine," DOE Office of Scientific and Technical Information Report DE98001202 (1997)

HJELM, R. P., SCHTEINGART, C., HOFMANN, A. F., and SIVIA, D. S., "Form and Structure of Self-Assembling Particles of Monoolein-Bile Salt Mixtures," Journal of Physical Chemistry 99, 16395-16406 (1995)

HJELM, R. P., THIYAGARAJAN, P., and CHAIKO, D. J., "A Neutron Scattering Study of Poly(ethylene glycol) in Electrolyte Solutions," Macromolecules 28, 7730-7736 (1995)

HJELM, R. P., THIYAGARAJAN, P., SCHTEINGART, C., HOFMANN, A. F., and ALKAN-ONYUKSEL, M. H., "Structure of Mixed Micelles Present in Bile and Intestinal Content Based on Studies of Model Systems," Falk Symposium No. 80: Bile Acids in Gastroenterology: Basic and Clinical Advances (Kluwer, 1995) 41-58

HJELM, R. P., THIYAGARAJAN, P., SCHTEINGART, C., HOFMANN, A. F., ALKAN-ONYUKSEL, M. H., "Probing Self Assembly in Biological Mixed Colloids by SANS, Deuteration and Molecular Manipulation," Neutrons in Biology (Plenum, 1996) $175-190$

HJELM, R. P., WAMPLER, W. A., and GERSPACHER, M., "The Structure of Carbon Black-Elastomer Composites by SmallAngle Neutron Scattering and the Method of Contrast Variation," In Proceedings of the International Conference on Neutrons in Research and Industry (SPIE, 1997) 2867, 144-147
HJELM, R. P., WAMPLER, W. A., and GERSPACHER, M., "Variation of Solvent Scattering-Length Density in Small-Angle Neutron Scattering as a Means of Determining Structure of Composite Materials," In Proceedings of the Materials Research Society Symposium Proceedings on Neutron Scattering in Materials Science II 376, 303-307 (1995)

JAVORSKY, P., NAKOTTE, H., ROBINSON, R. A., and KELLEY, T. M., "Crystal Field in ErNiAl Studied by Inelastic Neutron Scattering," J. Magn. Mater. 186, 373-376 (1998)

JAYARAMAN, N., and RANGASWAMY, P., "Oxide Scale Stresses in Polycrystalline $\mathrm{Cu} / \mathrm{Cu}_{2} \mathrm{O}$ System," Advances in X-Ray Analysis 39, 421-432 (1997)

JOHNSON, C. S., DEES, D. W., MANSUETTO, M. F., THACKERAY, M. M., VISSERS, D. R., ARGYRIOU, D., LOONG, C. K., and CHRISTENSEN, L., "Structural and Electrochemical Studies of Alpha-Manganese Dioxide (Alpha$\mathrm{MnO}_{2}$ ), Journal of Power Sources 68, 570-577 (1997)

JOHNSON, S. W., BARTHES, M., MCMULLAN, R. K., ECKERT, J., and MULLER, M., "Comment on Dynamical Test of Davydov-Type Solitons in Acetanilide Using a Picosecond Free-Electron Laser: Neutron Diffraction Study of Acetanilide at 15 and 295K," Physical Review Letters 74, 2844 (1995)

JOHNSON, S. W., ECKERT, J., BARTHES, M., MCMULLAN, R. K., and MULLER, M., "The Crystal Structure of Acetanilide at $15 \mathrm{~K}$ and $295 \mathrm{~K}$ by Neutron Diffraction: Lack of Evidence for Proton Transfer Along the N-H $\cdots$ O Hydrogen Bond," Journal of Physical Chemistry 99, 16253 (1995)

JORGENSEN, J. D., HINKS, D. G., CHMAISSEM, O., ARGYRIOU, D. N., MITCHELL, J. F., and DABROWSKI, B., "Structural Features that Optimize High Temperature Superconductivity," In Proceedings of the First Polish-US Conference on High Temperature Superconductivity (SpringerVerlag, 1995) 1-15

KELLEY, T. M., ARGYRIOU, D. N., ROBINSON, R. A., NAKOTTE, H., MITCHELL, J. F., OSBORN, R., and JORGENSEN, J. D., "Short-Range Spin Correlations in the CMR Material La ${ }_{1.4} \mathrm{Sr}_{1.6} \mathrm{Mn}_{2} \mathrm{O}_{7}$," Physica B 241-243, $439-441$ (1997)

KENT, M. S., FACTOR, B. J., SATIJA, S., GALLAGHER, P., and SMITH, G. S., "Structure of Bimodal Polymer Brushes in a Good Solvent by Neutron Reflectivity," Macromolecules 29, 8, 2843 (1996)

KENT, M. S., LEE, L. T., FACTOR, B. J., RONDELEZ, F., and SMITH, G. S., "Tethered Chains in Good Solvent Conditions: An Experimental Study Involving Langmuir Diblock Copolymer Monolayers," J. Chem. Phys. 103, 2320 (1995)

KENT, M. S., MAJEWSKI, J. P., SMITH, G. S., LEE, L., and SATIJA, S. K., "Tethered Chains in Theta Solvent Conditions: An Experimental Study Involving Langmuir Diblock Copolymer Monolayers," J. Chem. Phys. 108, 13, 5635-5645 (1998) 
KENT, M. S., SAUNDERS, R. S., NELSON, G. C., SMALL, J. H., WONG, A. P. Y., SMITH, G. S., MAJEWSKI, J., "SelfAssembling Monolayers of Functionalized Diblock Copolymers," Macromolecules 30, 3942-5 (1997)

KENT, M. S., SMITH, G. S., BAKER, S., NITRAY, A., BROWNING, J., MOORE, G., and HUA, D-W., "The Effect of a Silane Coupling Agent on Water Adsorption at a Metal/Polymer Interface Studied by Neutron Reflectometry and Angle Resolved X-Ray Photoelectron Spectroscopy," Journal of Materials Science 31, 927 (1996)

KINDO, K., FUKUSHIMA, T., KUMADA, T., DE BOER, F. R., NAKOTTE, H., PROKES, K., HAVELA, L., SECHOVSKY, V., SERET, A., WINAND, J. M., and SPIRLET, J. C., "ElectronicProperties of $\mathrm{U}_{2} \mathrm{Ni}_{2} \mathrm{SN}$," Journal of Magnetism and Magnetic Materials 140, 2, 1369-1370 (1995)

KING, W. A., KUBAS, G. J., and ECKERT, J., "Application of a Gallium Based Low Interacting Anion to the Study of Cationic Manganese Dihydrogen Complexes," in Abstracts of Papers of the American Chemical Society 214, 31 (1997)

KWEI, G. H. and MOROSIN, B., "Structures of the Boron-Rich Boron Carbides from Neutron Diffration: Implications for the Nature of Intericosahedral Chains," Journal of Physical Chemistry 100, 8031-8039 (1996)

KWEI, G. H., ARGYRIOU, D. N., LAWSON, A. C., NEUMEIER, J. J., THOMPSON, J. D., BILLINGE, S. J. L., SUBRAMANIAN, M. A., and RAMIREZ, A. P., "Lattice Effects in Perovskite and Pyrochlore CMR Materials," In Proceedings of Materials Research Symposium Proceedings 475, 533 (1997)

KWEI, G. H., JORGENSEN, J. D., SCHIRBER, J. E., and MOROSIN, B., "Inert Gas Intercalation into Fullerene Interstices," Fullerene Science \& Technology 5, 243-256 (1997)

KWEI, G. H., LAWSON, A. C., LARSON, A. C., MOROSIN, B., LARSON, E. M., and CANFIELD, P. C., "Structure of $\mathrm{Ce}_{2} \mathrm{Pt}_{6} \mathrm{Ga}_{15}$ : Interplanar Disorder from the $\mathrm{Ce}_{2} \mathrm{Ga}_{3}$ Layers," Acta Cryst. B 52, 4, 580-585 (1996)

KWEI, G., BILLINGE, S. J. L., CHEONG, S. -W., and SAXTON, J. G., "Pair Distribution Functions of Ferroelectric Perovskites: Direct Observation of Structural Ground States," Ferroelectrics 164, 57 (1995)

KWEI, G., LOUCA, D. A., BILLINGE, S. J. L., and ROSENFELD, H. D., "Recent 'Local' Structural Studies: Metallic Alloys, Superconductors and Proteins," In Local Structure from Diffraction, Billinge, S. J. L., and Thorpe, M. F., Eds. (Plenum, 1998) 323

LAGER, G. A., and VON DREELE, R. B., "Neutron Powder Diffraction Study of Hydrogarnet to $9.0 \mathrm{GPa}$," American Mineralogist 81, 1097-1104 (1996)

LANGLOIS, D. A., SMITH, M. E., BENICEWICZ, B. C., DOUGLAS, E. P., and HJELM, R. P., "Properties of Liquid Crystal Thermosets in High Temperature Polymers" (CRC Press, 1996) 79-96
LAWSON, A. C., GOLDSTONE, J. A., CORT, B., MARTINEZ, R. J., and ZOCCO, T. G., "Structure of z-phase PlutoniumUranium," Acta Crystallographica B 52, 32-37 (1996)

LEINENWEBER, K., PARTIN, D. E., SCHUELKE, U., O'KEEFFE, M. A., VON DREELE, R. B., "The Structure of High Pressure $\mathrm{Ca}(\mathrm{OD})_{2}$ II from Powder Neutron Diffraction: Relationship to the $\mathrm{ZrO}_{2}$ and EuI $\mathrm{EI}_{2}$ Structures," Journal of Solid State Chemistry 132, 267-273 (1997)

LI, D., LUTT, M., FITZSIMMONS, M. R., SYNOWICKI, R., HAWLEY, M., and BROWN, G. W., "Preparation, Characterization, and Properties of Mixed Organic and Polymeric Self-Assembled Multilayers," J. Am. Chem. Soc. 120, $8797-8804$ (1998)

LI, D., LUTT, M., SHI, X. B., and FITZSIMMONS, M. R., "Multilayer Self-Assemblies as Electronic and Optical Materials," In Proceedings of the Materials Research Society Symposium 488, 401-406 (1998)

LIU, Y., LI, N., and ECKE, R. E., "Dynamics of Surface Patterns in Salt-Crystal Dissolution," Physical Review E 53, R5572-R5575 (1996)

LUTT, M., FITZSIMMONS, M. R., and LI, D., "X-Ray Reflectivity Study of Self-Assembled Multilayers of Macrocycles and Macromolecules," Journal of Physical Chemistry B 102, 400 (1998)

MAJEWSKI, J. P., KUHL, T. L., KJAER, K., GERSTENBERG, M., ALS-NIELSEN, J., ISRAELACHVILI, J. N., and SMITH, G. S., "An X-Ray Synchrotron Study of Packing and Protrusions: Polymer-Lipid Monolayers at the Air-Water Interface," J. Am. Chem. Soc. 120, 1469-1473 (1998)

MAJEWSKI, J., KUHL, T. L., GERSTENBERG, M. C., ISRAELACHVILI, J. N., and SMITH, G. S., "The Structure of Phospholipid Monolayers Containing Polyethylene Glycol Lipids at the Air-Water Interface," Journal of Physical Chemistry $B$ 101, 3122-3129 (1997)

MAJEWSKI, J., POPOVITZ-BIRO, R., EDGAR, R., ARBEL-HADDAD, M., KJAER, K., BOUWMAN, W., ALS-NIELSEN, J., LAHAV, M., and LEISEROWITZ, L., "An Insight into the Ice Nucleation Process via Design of Crystalline Ice Nucleators of Variable Size," Journal of Physical Chemistry B 101, 8874-8877 (1997)

MALEY, M. P., WILLIS, J. O., BULAEVSKII, L. N., CHO, J. H., SAFAR, H. F., WU, X. D., FOLTYN, S. R., and ARENDT, P. N., "Effects of Splayed Columnar Defects Produced by Heavy Fission Fragments on Vortex Dynamics in BSCCO Conductors," In Physical Phenomena at High Magnetic Fields II, Fisk, Z., Gor'kov, L., Meltzer, D., and Schrieffer, R., Eds. (World Scientific, 1996) 467

MANG, J. T. and HJELM, R. P., "SANS Investigation of the Pressure and Temperature-Dependent Structure of the Bile Salt Lecithin System," Molecular Crystals and Liquid Crystals 299, 439-443 (1997)

MANG, J. T., HJELM, R. P., SKIDMORE, C. B., and HOWE, P. M., "Parameterization of Structures in He Composites Using Surrogate Materials: A Small Angle Neutron Scattering Investigation," US Department of Defense Report DE96012857 (1996) 
MARASINGHE, G. K., JAMES, W. J., EZEKWENNA, P. C., LUO, R., YELON, W. B., ZHAO, Y., VON DREELE, R., ELLOUZE, M., and I'HERITIER, P., "Pressure Induced Reversal of the Volume Expansion Caused by Interstitial Nitrogen in $\mathrm{Nd}_{2} \mathrm{Fe}_{17} \mathrm{~N}_{3}$," Journal of Applied Physics 83, 6911 (1998)

MCQUEENEY, R. J., "A Local Dynamic Correlation Function from Inelastic Neutron Scattering," Physica B 241-243, 412-414 (1998)

MCQUEENEY, R. J., "Dynamic Radial Distribution Function from Inelastic Neutron Scattering," Physical Review B 57, 17, 10650-10658 (1998)

MCQUEENEY, R. J., EGAMI, T., SHIRANE, G., and ENDOH, Y., "Wide and Asymmetric Oxygen Bond-Stretching Phonons in $\mathrm{La}_{1.85} \mathrm{Sr}_{0.15} \mathrm{CuO}_{4}$, " Physical Review B 54, R9689 (1996)

MEGGERS, K., PRIESMEYER, H. G., STALDER, M., VOGEL, S., and TRELA, W., "Single-Shot Neutron Transmission Diffraction," Physica B 234-236, 1160-1162 (1997)

MEI, J., COOPER, B. R., LAWSON, A. C., ROBERTS, J. A., and COTTON, J. D., "Atom-Specific Thermal Vibration Amplitudes in Stoichiometric NiAl: Agreement of Neutron Diffraction and Ab-Initio-Based Molecular Dynamics," Philosophical Magazine B 74, 169-175 (1996)

MENTINK, S., NIEUWENHUYS, G. J., NAKOTTE, H., MENOVSKY, A. A., DROST, A., FRIKKEE, E., and MYDOSH, J. A., "Magnetization and Resistivity of UN14B in High Magnetic-Fields," Physical Review B 51, 17, 11567-11573 (1995)

MITCHELL, J. F., ARGYRIOU, D. N., JORGENSEN, J. D., HINKS, D. G., POTTER, C. D., and BADER, S. D., "Charge Delocalization and Structural Response In Layered $\mathrm{La}_{1,2} \mathrm{Sr}_{1.8} \mathrm{Mn}_{2} \mathrm{O}_{7}$ : Enhanced Distortion, In the Metallic Regime," Physical Review B 55, 63-66 (1997)

MITCHELL, J. F., ARGYRIOU, D. N., POTTER, C. D., HINKS, D. G., JORGENSEN, J. D., and BADER, S. D., "Structural PhaseDiagram of $\mathrm{La}_{1-x} \mathrm{Sr}_{x} \mathrm{MnO}_{3+\delta}$ : Relationship to Magnetic and Transport-Properties," Physical Review B 54, 6172-6183 (1996)

MOGILEVSKY, R., MITCHELL, J. F., HINKS, D. G., ARGYRIOU, D. N., and JORGENSEN, J. D., "Ternary and Quaternary Thallium-Containing High- $\mathrm{T}_{\mathrm{c}}$ Superconductors Synthesized at Low-Temperature and Elevated Pressure," Physica C 250, 15-24 (1995)

MORGAN, J. G., VON DREELE, R. B., WOCHNER, P., and SHAPIRO, S. M., "Inelastic Neutron Scattering from Single Crystal Zn Under High Pressure," Physical Review B 54, $812-18$ (1996)

MOROSIN, B., ASSINK, R. A., DUNN, R. G., MASSIS, T. M., SCHRIBER, J. E., and KWEI, G. H., "Methane Intercalated $\mathrm{C}_{60}$," Physical Review B 56, 13611-13614 (1997)

MOROSIN, B., JORGENSEN, J. D., SHORT, S., KWEI, G. H., and SCHRIBER, J. E., "Ne Intercalated $\mathrm{C}_{60}$ : Pressure Dependence of Ne Site Occupancy," Physical Review B 53, 16751678 (1995)
MOROSIN, B., KWEI, G. H., LAWSON, A. C., ASELAGE, T. L., and EMIN, D., "Neutron Powder Diffraction Refinement of Boron Carbides: Nature of Interciosahedral Chains," J. of Alloys and Compounds 226, 121-125 (1995)

NAKOTTE, H., BUSCHOW, K. H. J., BRUCK, E., KLAASSE, J. C. P., PROKES, K., DE BOER, F. R., ANDREEV, A. V., and LACERDA, A., "Non-Fermi-Liquid Scaling in U(Cu,Al $)_{5}$ Compounds," Physica B 230, 616-619 (1997)

NAKOTTE, H., BUSCHOW, K. H. J., KLAASE, J. C. P., PROKES, K., DE BOER, F. R., ANDREEV, A. V., SUGIYAMA, K., KURODA, T., and DATE, M., "Possible Heavy-Fermion Behavior of $\mathrm{New} \mathrm{U}(\mathrm{Cu}, \mathrm{Al})_{5}$ Compounds," Journal of Magnetism and Magnetic Materials 140-144, 1261 (1995)

NAKOTTE, H., HAVELA, L., SECHOVSKY, V., LACERDA, A., PURWANTO, A., PROKES, K., BRUCK, E., DE BOER, F. R., and TORIKACHVILI, M. S., "Magnetoresistance Behavior of UNiGe," In Proceedings of the Conference on Physical Phenomena at High Magnetic Fields-II (World Scientific, 1996) 209-214

NAKOTTE, H., KAWANAKA, H., BRUCK, E., PROKES, K., KIM-HGAN, N. H., TAKABATAKE, T., FUJII, H., and SAKURAI, J., "Thermal Properties of UPdSn and UCuSn," Physica B 237-238, 226-228 (1997)

NAKOTTE, H., PROKES, K., BRUCK, E., BUSCHOW, K. H. J., DE BOER, F. R., ANDREEV, A. V., LACERDA, A., TORIKACHVILI, M. S., ROBINSON, R. A., BOURKE, M. A. M., and SCHULTZ, A. J., "Non-Fermi-Liquid Scaling in Heavy-Fermion $\mathrm{UCU}_{3.5} \mathrm{AL}_{1.5}$ and $\mathrm{UCU}_{3} \mathrm{AL}_{2}$," Physical Review $B$ $54,12176-12183$ (1996)

NAKOTTE, H., PROKES, K., DE BOER, F. R., HAVELA, L., SECHOVSKY, V., SVOBODA, P., WINAND, J. M., REBIZANT, J., SPIRLET, J. C., and HU, X., "Electronic Properties of U2Pt2Sn," Journal of Applied Physics 79, 6408-6410 (1996)

NAKOTTE, H., PURWANTO, A., ROBINSON, R. A., PROKES, K., DE BOER, F. R., HAVELA, L., SECHOVSKY, V., and SWAINSON, I. P., "Electronic Properties of UCuSn," Journal of Applied Physics 79, 6408-6410 (1996)

NAKOTTE, H., PURWANTO, A., ROBINSON, R. A., PROKES, K., KLAASSE, J. C. P., DE CHATEL, P. F., DE BOER, F. R., HAVELA, L., SECHOVSKY, V., PEREIRA, L. C. J. , SERET, A., REBIZANT, J., SPIRLET, J. C., and TROUW, F., "Hybridization Effects in $\mathrm{U}_{2} \mathrm{~T}_{2} \mathrm{X}$ Compounds: MagneticStructures of $\mathrm{U}_{2} \mathrm{Rh}_{2} \mathrm{Sn}$ and $\mathrm{U}_{2} \mathrm{Ni}_{2} \mathrm{In}$," Physical Review B 53, 3263-3271 (1996)

NAKOTTE, H., PURWANTO, A., ROBINSON, R. A., TUN, Z., PROKES, K., LARSON, A. C., HAVELA, L., SECHOVSKY, V., MALETTA, H., BRUCK, E., and DE BOER, F. R.,

"Commensurate and Incommensurate Magnetic-Structures of UNiGe," Physical Review B 54, 7201-7207 (1996)

NAKOTTE, N., PURWANTO, A., ROBINSON, R. A., VON DREELE, R. B., HAVELA, L., PROKES, K, SECHOVSKY, V., PEREIRA, L. C. J., SPIRLET, J. C., and REBIZANT, J., "On the Relationship Between Crystal-Structure and Magnetism in $\mathrm{U}_{2} \mathrm{Pd}_{2} \mathrm{Sn}$ and $\mathrm{U}_{2} \mathrm{Ni}_{2}$ In," Joumal of Applied Physics 81, 4927 (1997) 
NAKOTTE, H., ROBINSON, R. A., BULL, M., MCEWEN, K. A., ECCELSTONE, R. A., and BRUCK E., "Crystal-Field Excitations in UPdSn," Bull. Am. Phys. Soc. 41, 470 (1996)

NAKOTTE, H., ROBINSON, R. A., BULL, M., MCEWEN, K. A., SWAN, T., KELLEY, T. M., ECCELSTON, R. A., and BRUCK, E., "Localized Excitations in UPdSN," Physica B 241243, 675-677 (1997)

NAKOTTE, H., ROBINSON, R. A., PURWANTO, A., TUN, Z., PROKES, K., BRUCK, E., and DE BOER, F. R.,

"Antiferromagnetism and Domain Effects in UPdSn," Phys Rev. B 58, 14, 9269-9275 (1998)

NGUYEN, B., CLARKE, C. J., EISENBERG, A., RAFAILOVICH, M. H., SOKOLOV, J., and SMITH, G. S., "Investigation of Polymer Brushes and Adsorbed Monolayers Under Shear," Journal of Applied Crystallography 30, 680 (1997)

OLAH, G. A., and HJELM, R. P., "Analysis and Simulations of a Small-Angle Neutron Scattering Instrument on a $1 \mathrm{MW}$ Long Pulsed Spallation Source," Journal of Neutron Research 6, 79-93 (1997)

OLAH, G. A., HJELM, R. P., and SEEGER, P.A., "Small-Angle Scattering Instruments on a $1 \mathrm{MW}$ Long Pulse Spallation Source," In Proceedings of the 13th Meeting of the International Collaboration on Advanced Neutron Sources 5232, 370-380 (1995)

PAFFETT, M., SZANYI, J., JACUBINAS, R. M., OTT, K. C., VON DREELE, R., HUGHES, C. D., and EARL, W. L., "Characterizing the Framework Demetallation of Environmentally Relevant Zeolites Using IR, NMR, and Neutron Diffraction Techniques," Studies in Surface Science and Catalysis III (1998)

PROKES, K., DE BOER, F. R., NAKOTTE, H., HAVELA, L., SECHOVSKY, V., SVOBODA, P., WINAND, J. M., REBIZANT, J., SPIRLET, J. C., and HU, X., "Electronic Properties of $\mathrm{U}_{2} \mathrm{Pt}_{2} \mathrm{Sn}$," Journal of Applied Physics 79, 6361-6363 (1996)

PROKES, K., NAKOTTE, H., HAVELA, L., SECHOVSKY, V., PEREIRA, L. C. J., RIJKEBOER, C., SERET, A., SPIRLET, J. C., SVOBODA, P., DE BOER, F. R., "Magnetic Anisotropy in $\mathrm{U}_{2} \mathrm{Pd}_{2} \mathrm{In}$, , Physica B 223-224, 225-227 (1995)

PROKES, K, SECHOVSKY, V., BRUCK, E., DE BOER, F. R, BUSCHOW, K. H. J., SONNTAG, R., ROBINSON, R. A., and MALETTA, H., "Magnetism in UPdSi," Physica B 229, 101-112 (1997)

PROKES, K., SECHOVSKY, V., ROBINSON, R. A., SONNTAG, R., SVOBODA, P., and DE BOER, F. R., "Commensurate and Incommensurate Magnetic Order of UPdSi," Physica B 241-243, 687-689 (1997)

PURWANTO, A., ROBINSON, R. A., NAKOTTE, H., LARSON, A. C., TUN, Z., SECHOVSKY, V., HAVELA, L., PROKES, K., BRUCK, E., and DE BOER, F. R., "Incommensurate Magnetic Structure of UNiGe," Bull. Am. Phys. Soc. 41, 471 (1996)
PURWANTO, A., ROBINSON, R. A., NAKOTTE, H., SWAINSON, I. P., and TORIKACHVILI, M. S., "Crystallographic and Magnetic Structure of $\mathrm{UCu}_{1.5} \mathrm{Sn}_{2}$," Journal of Applied Physics 79, 6411-6413 (1996)

PURWANTO, A., SECHOVSKY, V., HAVELA, L., ROBINSON, R. A., NAKOTTE, H., LARSON, A. C., PROKES, K., BRUCK, E., and DE BOER, F. R., "Low-Temperature Magnetic Structure of UNiGe," Physical Review B 53, 2, 758-765 (1996)

PYNN, R., "What Can We Learn from Off-Specular Neutron Reflection?," In Proceedings of the Materials Research Society Symposium 376, 165-173 (1995)

RADAELLI, P. G., IANNONE, G., MAREZIO, M., HWANG, H. Y., CHEONG, S. W., JORGENSEN, J. D., and ARGYRIOU, D. N., "Structural Effects on the Magnetic and Transport Properties of Perovskite $\mathrm{La}_{1-\mathrm{X}} \mathrm{Ca}_{\mathrm{x}} \mathrm{MnO}_{3}(\mathrm{X}=0.25 ; 0.30)$," Physical Review B 56, 8265-8276 (1997)

RANGARAJAN, G., HABIB, S., and RYNE, R., "Lyapunov Exponents without Rescaling and Reorthogonalization," Physical Review Letters 80, 3747-3750 (1998)

RANGASWAMY, P., and JAYARAMAN, N., "Issues Related to Prediction of Residual Stresses in Ti-Alloy Matrix Composites," In Proceedings of the ASTM Symposium on Life Prediction Methodology for Titanium Matrix Composites, (ASTM-STP, 1996) $1253,66-82$

RANGASWAMY, P., and JAYARAMAN, N., "Residual Stresses in SCS-6/Beta-21S Composites," Journal of Composites Technology \& Research 17, 43-49 (1995)

RANGASWAMY, P., BOURKE, M. A. M., LAWSON, A. C., O'ROURKE, J., and GOLDSTONE, J. A., "Residual Stress and Microstructural Characterization Using Rietveld Refinement of a Carburized Layer in a 5120 Steel," In Proceedings of Advances in X-Ray Analysis 39, 319-329 (1997)

RANGASWAMY, P., BOURKE, M. A. M., WRIGHT, P. K., JAYARAMAN, N., KARTZMARK, E., and ROBERTS, J. A., "The Influence of Thermomechanical Processing on Residual Stresses in Titanium Matrix Composites," Materials Science and Engineering A224, 200-209 (1997)

RICHARDSON, T. B., DE GALA, S., CRABTREE, R. H., and SIEGBAHN, P. E. M., "Dynamics of Novel M-H·H-O Hydrogen Bonds," J. Am. Chem. Soc. 117, 12875-12876 (1995)

ROBERTS, J. A., "Close-Up: Manuel Lujan Jr. Neutron Scattering Center," MRS Bulletin 22, $42-46$ (1997)

ROBINSON, R. A., "Neutron Scattering from Heavy Fermions," Book Chapter, World Scientific (1997)

ROBINSON, R. A., "SANS and the Sax," Neutron News 7, 3, 39 (1996)

ROBINSON, R. A., and BOURKE, M. A. M., "Measurement of Strains on Cubic Polycrystals Using Pulsed Neutron Diffraction," Bull. Am. Phys. Soc. 41, 13 (1996) 
ROBINSON, R. A., KOHGI, M., OSAKABE, T., TROUW, F., LYNN, J. W., CANFIELD, P. C., THOMPSON, J. D., FISK, Z., and BEYERMANN, W. P., "Low-Energy Excitations and the Electronic Specific Heat of YbBiPt," Physical Review Letters 75, 1194-1197 (1995)

ROBINSON, R. A., LAWSON, A. C., LARSON, A. C., VON DREELE, R. B., and GOLDSTONE, J. A., "Rietveld Refinement of Magnetic Structures from Pulsed-Neutron-Source Powder Diffraction Data," Physica B 213-214, 985-989 (1995)

ROBINSON, R. A., MCQUEENEY, R. J., and KELLEY, T. M., "Observation of Transverse Phonon Modes in the First Brillouin Zone in Aluminum," Physica B 241-243, 161-163 (1997)

ROBINSON, R. A., NAKOTTE, H., DILLEY, N. R., MAPLE, M. B., LYNN, J. W., and SKANTHAKUMAR, S. K., "Structural and Magnetic Transitions in $\mathrm{UPd}_{2} \mathrm{~Pb}$," Bulletin of the American Physical Society 42, 75 (1997)

ROSENKRANZ, S., OSBORN, R., MITCHELL, J. F., VASILIU-DOLOC, L., LYNN, J. W., SINHA, S. K., and ARGYRIOU, D. N., "Magnetic Correlations in the Bilayer Manganite $\mathrm{La}_{1.2} \mathrm{Sr}_{1.8} \mathrm{Mn}_{2} \mathrm{O}_{7}$," J. Appl. Phys. 83, 7345 (1998)

ROSSETTI, G. A., RODRIGUEZ, M. A., NAVROTSKY, A., CROSS, L. E., and NEWNHAM, R. E., "Structure of the Defect Perovskite $\left(\mathrm{Pb}_{0.85} \mathrm{La}_{0.10}\right) \mathrm{TiO}_{3}$ between 10 and $1023^{\circ} \mathrm{K}$," J. Appl. Phys. 77, 4, 1683-1689 (1995)

SAWHNEY, U., DURNING, C. J., O'SHAUGHNESSY, B., SMITH, G., and MAJEWSKI, J., "Dynamics of Adsorbed Polymer Layers: Melt Exchange Kinetics," In MRS Symp. Proc., 464, 219 (1997)

SAWHNEY, U., DURNING, C. J., O'SHAUGHNESSY, B., SMITH, G., and MAJEWSKI, J., "Irreversible Adsorption of Polymer Melts, Morphological Control in Multiphase Polymer Mixtures," In Proceedings of the Fall MRS Meeting, 461 (1996)

SCHLOMKA, J., PRESS, W., FITZSIMMONS, M. R., LUTT, M., and GRIGOROV, I. L., "Structural and Magnetic Properties of Ion Beam Sputtered NiMnSb Films," Physica B 248, 140-145 (1998)

SCHLOMKA, J-P., FITZSIMMONS, M. R., PYNN, R., STETTNER, J., SEECK, O. H., TOLAN, M., and PRESS, W., "Characterization of Si/Ge Interfaces by Diffuse X-Ray Scattering in the Region of Total External Reflection," Physica B 221, 44-52 (1996)

SCHWARTZ, K. B., and VON DREELE, R. B., "Structure Refinement of High-Density Polyethylene Using X-Ray Powder Diffraction Data and the Rietveld Method," Adv. In X-Ray Analysis 39, 515-521 (1997)

SECHOVSKY, V., HAVELA, L., SVOBODA, P., ANDREEV, A. V., BURLET, P., PROKES, K., NAKOTTE, H., DE BOER, F. R., BRUCK, E., ROBINSON, R. A., and MALETTA, H., "Magnetic Phase Diagram of UNiGa," Journal of Magnetism and Magnetic Materials 1379, 140-144 (1995)
SECHOVSKY, V., HAVELA, L., NAKOTTE, H., and BRUCK, E., "GMR in Intermetallics," IEEE Transactions on Magnetics 32, 4687-4691 (1996)

SECHOVSKY, V., HAVELA, L., NAKOTTE, H., PROKES, K., BRUCK, E., DE BOER, F. R., BUSCHOW, K. H. J., and ANDREEV, A. V., "Magnetism and Hybridization in UTIn Compunds ( $\mathrm{T}=\mathrm{Rh}, \mathrm{Pt}, \mathrm{Pd})$," Journal of Magnetism and Magnetic Materials 140, 2, 1391-1392 (1995)

SHI, N., ARSENAULT, R. J., BOURKE, M. A. M., and GOLDSTONE, J. A., "Relaxation of Thermal Mismatch in Discontinuously Reinforced Composites," Micromechanics of Advanced Materials, 471-474 (1995)

SHI, N., BOURKE, M. A. M., ROBERTS, J. A., and ALLISON, J. E., "Influence of Thermal Residual Stresses on the Elastic Phase-Strain Development in Metal Matrix Composites," Modeling of Composites: Processing and Properties, 47-56 (1996)

SHI, N., BOURKE, M. A. M., ROBERTS, J. A., and ALLISON, J. E., "Phase Stress Partition During Uniaxial Tensile Loading of a TiC-Particulate-Reinforced Al Composite," Metallurgical and Materials Transactions A28, 2741-2753 (1997)

SHI, N., BOURKE, M. A. M., and GOLDSTONE, J. A., "A Study of Internal Damage of Metal Matrix Composites by Neutron Diffraction," In Proceedings of the Neutron Scattering in Materials Science II Symposium (MRS 1995) 441-446.

SHI, N., SONG, S., GRAY, G. T. II, ROBERTS, J. A., ARSENAULT, R. J., COLE, D., GOSS, T., SIZEK, H., LIAW, P., PARMESWARAN, S., and KOSTORZ, G., "Particle Shape Effects on the Fracture of Discontinuously Reinforced 6061-Al Matrix Composites," In Proceedings of the Johannes Wertman Symposium (TMS, 1996) 243-254

SHI, X. B., LI, D., LUTT, M., FITZSIMMONS, M. R., and VAN PATTEN, G. P., "Self-Assembled Multilayers and Photoluminescence Properties of a New Water-Soluble Poly (p-phenylene)," Mater. Res. Soc. Symp. Proc. 488, 133-138 (1998)

SHIMAKAWA, Y., KUBO, Y., MANAKO, T., SUSHKO, Y. V., ARGYRIOU, D. N., and JORGENSEN, J. D., "Structural and Magnetotransport Properties of the Colossal Magnetoresistance Material $\mathrm{T}_{12} \mathrm{Mn}_{2} \mathrm{O}_{7}$," Physical Review B 55, 6399-6404 (1997)

SICKAFUS, K. E., LARSON, A. C., YU, N., NASTASI, M., HOLLENBERG, G. W., GARNER, F. A., and BRANDT, R. C., "Neutron Scattering Measurements of the Atomic Structure of Magnesio-Aluminate Spinal Compounds," Journal of Nuclear Materials 219, 128 (1995)

SICKAFUS, K. E., LARSON, A. C., YU, N., NASTASI, N. Y. M., HOLLENBERG, G. W., GARNER, F. A., and BRADT, R. C., "Cation Disorder in High Dose, Neutron-Irradiated Spinel," Journal of Nuclear Material 219, 138-134 (1995)

SMITH, G. S., and MAJKRZAK, C. F., "Neutron Reflectometry," International Crystalographic Tables (1995) 
SONG, S. G., SHI, N., GRAY, G. T., and GOLDSTONE, J. A., "Reinforcement Shape Effect on the Fracture Behavior and Ductility of the Particle-Reinforced 6061-Al Matrix Composites," Acta Metallurgica et Materialia 27A, 3739-3746 (1996)

STEINER, M., KAKURAI, K., DORNER, B., PYNN, R., and AKIMITSU, J., "The Change of Symmetry of the Soft-Mode Spin Wave at the Spin-Flop Transition as Observed in MnTiO3 Using Inelastic Polarized Neutron Scattering," J. Phys. Condensed Matter 8, 5905 (1996)

STROUSE, G. F., CUMBERLAND, S., BORDALLO, H. N., and ARGYRIOU, D. N., "Local Lattice Dynamics in CMR Manganites: Probing Local Jahn-Teller Effects," Bulletin of the American Physical Society 43, 1, 163 (1998)

SUBRAMANIAN, M. A., RAMIREZ, A. P., and KWEI, G., "Colossal Magnetoresistance Without Double-Exchange: Pyrochlores," Solid State Ionics 108, 185 (1998)

SVOBODA, P., JAVORSKY, P., DIVIS, M., SECHOVSKY, V., PROKES, K., DE BOER, F. R., ANDREEV, A. V., BARTASHEVICH, M. I., TORIKACHVILI, M. S., NAKOTTE, H., LACERDA, A., and ONUKI, Y., "High Field Magnetization in $\mathrm{NdCu}_{2}$ Single Crystal," Physica B 211, 172 (1995)

SWOPE, R. J., SMYTH, J. R., and LARSON, A. C., "H in Rutile-Type Compounds: I. Single-Crystal Neutron and X-Ray Diffraction Study of $\mathrm{H}$ in Rutile," American Mineralogist 80, 448-453 (1995)

TAM, C. N., BOUR, P., TROUW, F. R., and ECKERT, J., "Inelastic Neutron Scattering Study of Hydrogen-Bonded Solid Formamide at 15K," Journal of Physical Chemistry 101, 33, 5877-5883 (1997)

THIYAGARAJAN, P., CHAIKO, D. J., and HJELM, R. P., “A Neutron Scattering Study of Poly(ethylene glycol) in Electrolyte Solutions Under Static and Shearing Conditions,"

Macromolecules 28, 7730-7736 (1995)

TODD, R. I., BORSA, C., DERBY, B., and BOURKE, M. A. M., "Analysis of Neutron Diffraction Peak Broadening Caused by Internal Stresses in Composite Materials," Nuclear Instruments \& Methods, Physics Research, Section A 354, 1, 139-44 (1995)

TODD, R. I., BOURKE, M. A. M., BORSA, C. E., and BROOK, R. J., "Measurement and Role of Residual Stresses in Alumina/ SiC Nanocomposites," Fourth Euro-Ceramics 4, 217-224 (1995)

TODD, R. I., BOURKE, M. A. M., BORSA, C. E., and BROOK, R. J., "Neutron Diffraction Measurements of Residual Stresses in Alumina/ SiC Nanocomposites," Acta Materialia 45, 1791-1800 (1997)

TOPOR, L., NAVROTSKY, A., ZHAO, Y., and WEIDNER, D. J., "Thermochemistry of Fluoride Perovskites: Heat Capacity, Enthalpy of Formation, and Phase Transition of $\mathrm{NaMgF}_{3}$," Journal of Solid State Chemistry 132, 131-138 (1997)
USTUNDAG, E., ZHANG, Z., STOCKER, M. L., RANGASWAMY, P., BOURKE, M. A. M., SUBRAMANIAN, S., SICKAFUS, K. E., ROBERTS, J. A., and SASS, S. L., "Influence of Residual Stress on the Evolution of Microstructure During the Partial Reduction of $\mathrm{NiAl}_{2} \mathrm{O}_{4}$," Materials Science \& Engineering A238, 50-65 (1997)

VAIDYA, R. U., RANGASWAMY, P., BOURKE, M. A. M., and BUTT, D. P., "Measurement of Bulk Residual Stresses in Molybdenum Disilicide/Stainless Steel Joints Using Neutron Scattering," Acta. Mater. 46, 6, 2047-2061 (1998)

VON DREELE, R. B., "High Pressure Neutron Powder Diffraction at LANSCE," High Pressure Research 14, 13-19 (1995)

VON DREELE, R. B., "Quantitative Texture Analysis by Rietveld Refinement," Journal of Applied Crystallography 30, 517-525 (1997)

VON DREELE, R. B., "Rietveld Analysis of High Pressure Powder Diffraction Data," High Pressure Research 14, 321-326, (1996)

VON DREELE, R. B., and CLINE, J. P., "The Impact of Background Function on High Accuracy Quantitative Rietveld Analysis (QRA): Application to NIST SRMs 676 and 656," Advances in X-Ray Analysis 38, 59-68 (1995)

VORDERWISCH, P., HAUTECLER, S., and ECKERT, J., "Neutron Spectroscopy of Localized Modes in $\mathrm{CeD}_{2-\mathrm{x}} \mathrm{H}_{\mathrm{y}}$," Journal of Alloys and Compounds 252-254 (1997)

ZHANG, J., VON DREELE, R. B., and EYRING, L., "Structures in the Oxygen-Deficient Fluorite-Related $\mathrm{R}_{\mathrm{n}} \mathrm{O}_{2 \mathrm{n}-2}$ Homologous Series: $\mathrm{Pr}_{9} \mathrm{O}_{16}$, Journal of Solid-State Chemistry 118, 133-140 (1995)

ZHANG, J., VON DREELE, R. G., and EYRING, L., "Structures in the Oxygen-Deficient Fluorite-Related $\mathrm{R}_{\mathrm{n}} \mathrm{O}_{2 \mathrm{n}-2}$ Homologous Series: $\mathrm{Pr}_{10} \mathrm{O}_{18}$," Journal of Solid-State Chemistry 118, 141-147 (1995)

ZHANG, J., VON DREELE, R. G., and EYRING, L., "Structures in the Oxygen-Deficient Fluorite-Related $\mathrm{R}_{\mathrm{n}} \mathrm{O}_{2 \mathrm{n}-2}$ Homologous Series: $\mathrm{Pr}_{12} \mathrm{O}_{22}$," Journal of Solid-State Chemistry 122, 53-58 (1996)

ZHAO, Y., SCHIFERL, D., and ZAUG, M., "RSCU-SO: A Rapid Searching and Centering Utility Routine for Single-Crystal X-ray Diffraction Studies at Simultaneous High-Pressures and Temperatures," Journal of Applied Crystallography 29, 71-80 (1996)

ZHAO, Y., SCHIFERL, D.I., and SHANKLAND, T. J., "A High P-T Single-Crystal X-Ray Diffraction Study of Thermoelasticity of $\mathrm{MgSiO}_{3}$ Orthoenstatite," Physics and Chemistry of Minerals 22, 393-398 (1995)

ZHAO, Y., VON DREELE, R. B., SHANKLAND, T. J., and WEIDNER, D. J., "Thermoelastic Equation of State of Jadeite $\mathrm{NaAlSi}_{2} \mathrm{O}_{6}$ : An Energy-Dispersive Rietveld Refinement Study of Low Symmetry and Multiple Phases Diffraction," Geophysical Research Letters 24, 5-8 (1997)

ZHAO, Y., VON DREELE, R. B., WEIDNER, D. J., and SCHIFERL, D., "P-V-T Data of Hexagonal Boron Nitride hBN and Determination of Pressure and Temperature Using Thermoelastic Equations of State of Multiple Phases," High Pressure Research 15, 369-386 (1997) 
ZHAO, Y., VON DREELE, R., ZHANG, J., and WEIDNER, D. J., "Thermal Equation of State of Monoclinic Pyroxene: CaMgSi ${ }_{2} \mathrm{O}_{6}$ Diopside," Review of High Pressure Science \& Technology 7, 25-27 (1998)

ZHU, Y., VALDEZ, J. A., SHI, N., LOVATO, M. L., STOUT, M. G., ZHOU, S., BLUMENTHAL, W. R., and LOWE, T., "Influence of Reinforcement Morphology on the Mechanical Properties of ShortFiber Composites," Processing of Metals and Advanced Materials: Modeling, Design, and Properties, 251-259 (1998)

\section{Neutron Scattering-Instrumentation}

DAEMEN, L. L., ECKERT, J., PYNN, R., and SEEGER, P. A., "Monte Carlo Study of the Performance of a Time-of-Flight Multichopper Spectrometer," In Proceedings of the Thirteenth Meeting of the International Collaboration on Advanced Neutron Sources 1, 388-399 (1995)

FITZSIMMONS, M. R., and PYNN, R., "The Performance of Reflectometers at Continuous Wave and Pulsed-Neutron Sources," In Proceedings of the 13th Meeting of the International Collaboration on Advanced Neutron Sources 1, 381-387 (1995)

FITZSIMMONS, M. R., "Performance of a Reflectometer at Continuous Wave and Pulsed Neutron Sources," In Proceedings of the Workshop on Neutron Instrumentation for a Long-Pulse Spallation Source III, 53-60 (1995)

FITZSIMMONS, M. R., "Reflectometry at Continuous Wave and Pulsed-Neutron Sources," Nuclear Instruments and Methods A383, 549-565 (1996)

HJELM, R. P. (Ed.), "Proceedings of the Workshop on Methods for Neutron Scattering Instrumentation Design," E.O. Lawrence Berkeley National Laboratory Report No. 9609353, Berkeley, CA (1997)

HJELM, R. P., "Introduction and Summary, Proceedings of a Workshop on Methods for Neutron Scattering Instrumentation Design," E.O. Lawrence Berkeley National Laboratory Report No. 9609353, Berkeley, CA, 8-11 (1997)

HJELM, R. P., "Neutron Scientists Discuss Instruments, Design, Needs, and the Future," Neutron News 8, 2, 6-7 (1997)

PATTON, B. W., RUSSELL, G. J., GILMORE, J. S., FERGUSON, P. D., COURT, J. D., and PITCHER, E. J., "Benchmarking of the Fertile to Fissile Conversion (FERFICON) Water Bath Experiments: Integral n/p Measurements," In Proceedings of the Topical Meeting on Nuclear Applications of Accelerator Technology (ANS, 1997) 229-234

PITCHER, E., RUSSELL, G., SEEGER, P. A., and FERGUSON, P. D., "Performance of Long-Pulse Source Reference TargetModerator-Reflector Configurations," In Proceedings of the 13th Meeting of the International Collaboration on Advanced Neutron Sources 323-329 (1995)

PYNN, R., and DAEMEN, L., "The Performance of Neutron Spectrometers at a Long-Pulse Spallation Source," In Proceedings of the Thirteenth Meeting of the International Collaboration on Advanced Neutron Sources 1, 330-338 (1995)
ROBINSON, R. A., EYSSA, Y. M., SCHNEIDER-MUNTAU, H. J., and BOENIG, H. J., "Proposal for a 30-T Pulsed Magnet Suitable for Neutron Scattering Experiments," In Proceedings of the 13th Meeting of the International Collaboration on Advanced Neutron Sources 1, 300-306 (1995)

ROBINSON, R. A., EYSSA, Y. M., SCHNEIDER-MUNTAU, H. J., BOENIG, H. J., FISK, Z., GORKOV, L., MELTZER, D., and SCHRIEFFER, R., "A 30-T Pulsed Magnet Suitable for Neutron Scattering Experiments," In Proceedings of the Physical Phenomena at High Magnetic Fields (World Scientific, 1996) 2, 735-738

ROBINSON, R. A., "On the Relationship Between Intensity and Resolution of Pulsed-Source Back-Scattering Spectrometers," Physica B 213, 1002-1004 (1995)

ROBINSON, R. A., Report of Excitations Group at NSNS Workshop on Instrumentation Needs and Performance Metrics, Oak Ridge, October 31-November 1 (1996)

ROBINSON, R. A., "Analytical Techniques for Instrument Design - Matrix Methods," In Proceedings of the Workshop on Methods for Neutron Scattering Instrument Design, Lawrence Berkeley Report (LBNL-40816, CONF-9609353, 1996) 45-54

SEEGER, P. A., "The MCLIB Library: Monte Carlo Simulation of Neutron Scattering Instruments," In Proceedings of the Thirteenth International Collaboration on Advanced Neutron Sources 1, 194-212 (1995)

THELLIEZ, T., DAEMEN, L. L., SEEGER, P. A., and HJELM, R. P., "A User-Friendly, Graphical Interface for the Monte Carlo Neutron Optics Code MCLIB," In Proceedings of the Thirteenth Meeting of the International Collaboration on Advanced Neutron Sources 1, 307-311 (1995)

\section{Accelerator Science}

BAKER, S. I., BULL, J. S., and GOSS, D. L., "Leaching of Accelerator-Produced Radionuclides," Health Physics 73, 6, 912-918 (1997)

BARLOW, D. B., BORDEN, M. J., and KRAUS, R. H., "Radiation Hardness Measurements of New Permanent Magnet Materials for High-Intensity LINAC Applications," DOE Office of Scientific and Technical Information (1997)

BILLEN, J. H., NATH, S., STOVALL, J. E., TAKEDA, H., WOOD, R. L., and YOUNG, L. M., "A Versatile, High-Power Proton Linac for Accelerator-Driven Transmutation Technologies," In Proceedings of the 1995 Particle Accelerator Conference (IEEE, 1995) 1137-1139

BOLME, G. O., KEFFELER, D. R., BROWN, V. W., CLARK, D. C., HODGKINS, D., LARA, P. D., MILDER, M. L., REES, D. E., SCHAFSTALL, P. J., SCHNEIDER, D. J., SHERMAN, J. D., STEVENS, R. R., ZAUGG, T. J., SHEIKH, J. Y., DAVIDSON, A. D., and SMITH, B. H., "High Power RF Operations Studies with the CRITS RFQ," In Proceedings of the 1995 Particle Accelerator Conference (IEEE, 1995) 2, 1161-1163 
BOWMAN, C. D., "Basis and Objectives of the Los Alamos Accelerator-Driven Transmutation Technology Project, Transmutation Technologies and Applications," In Proceedings of the AIP Conference (AIP, 1995) 346, 22-43

BROWN, S. K., MEAD, W. C., BOWLING, P. S., and JONES, R. D., "G2.14 Engineering: Adaptive Control of a Negative Ion Source," Handbook of Neural Computation (1995)

BUTT, D. P., KANNER, G. S., and LILLARD, R. S., "Corrosion of Target and Structural Materials in Water Irradiated by an 800 MeV Proton Beam," In Proceedings of the Accelerator-Driven Transmutation Technologies and Applications 2, $947-952$ (1997)

CAPPIELLO, M. W., ROSE, S., EDWARDS, J., GREENE, G., YOUNGBLOOD, R., and BAKER, D., "Design and Safety Features of the APT Target/Blanket System," Transactions of the American Nuclear Society (ANS, 1996) 74, 250-251

CAPPIELLO, M. W., SMITH, B., and RUSSELL, G. J., "Design of the APT Target/Blanket and Support Systems," In Proceedings of the 1996 ANS/ENS International Meeting, Trans. Am. Nucl. Soc. (ANS, 1996) 75, 256-257

CARLSTEN, B. E., "Axial Free-Electron Laser Interaction Between an Annular Electron Beam and an Axisymmetric TM Mode," IEEE Journal of Quantum Electronics 31, 1753-1763 (1995)

CARLSTEN, B. E., "Calculation of the Noninertial Space-Charge Force and the Coherent Synchrotron Radiation Force for Short Electron Bunches in Circular Motion Using the Retarded Green's Function Technique," Physical Review E 54, 1, 838-845 (1996)

CARLSTEN, B. E., "Centrifugal Space-Charge Force of an Electron Beam in a Focusing Element," Physical Review E 55, 5 , R4893-R4896 (1997)

CARLSTEN, B. E., "Discrete Monotron Oscillator," IEEE

Transactions on Plasma Science 24, 4, 1249-1258 (1996)

CARLSTEN, B. E., "Enhanced Phase Stability of a Raman FreeElectron Laser Amplifier in the Exponential Growth Regime," Physics of Plasmas 2, 3880-3892 (1995)

CARLSTEN, B. E., "Nonlinear Subpicosecond Electron-Bunch Compressor," Nuclear Instruments and Methods in Physics Research A 380, 3, 505-516 (1996)

CARLSTEN, B. E., "Space-Charge-Induced Emittance Compensation in High-Brightness Photoinjectors," Particle Accelerators 49, 27-65 (1995)

CARLSTEN, B. E., "Stable Off-Axis Electron Orbits in a Longitudinal-Wiggler Free-Electron Laser," Journal of Appl. Physics 78, 2811-2816 (1995)

CARLSTEN, B. E., "Rippled Beam Free-Electron Laser Amplifier Using the Axial Free-Electron Laser Interaction," Journal of Applied Physics 81, 10, 6570-6578 (1997)
CARLSTEN, B. E., FAZIO, M. V., HAYNES, W. B., MAY, L., and POTTER, J. M., "Phase-Stable, Microwave FEL Amplifier," In Proceedings of the 1995 Particle Accelerator Conference (IEEE, 1995) 3, 1494-1496

CARLSTEN, B. E., FELDMAN, D. W., KINROSS-WRIGHT, J. M., MILDER, M. L., RUSSELL, S. J., PLATO, J. G., SHERWOOD, B. A., WEBER, M. E., COOPER, R. G., and STURGES, R. E., "Subpicosecond Compression Experiments at Los Alamos National Laboratory," In Proceeding of 1995 Micro Bunches Workshop (AIP, 1996) 367, 21-35

CARLSTEN, B. E., and FERGUSON, P., "Numerical Determination of the Matching Conditions and Drive Characteristics for a Klystron Input Cavity with Beam," IEEE Transactions on Electron Devices $44,5,894-900$ (1997)

CARLSTEN, B. E., and GOLDSTEIN, J. C., "Emittance Growth of a Short Electron Bunch in Circular Motion," Nuclear Instruments \& Methods in Physics Research A 393, 1-3, 490-493 (1997)

CARLSTEN, B. E., HAYNES, W. B., FORTGANG, C. M., FAZIO, M. V., MAY, L., and POTTER, J. M., "Microwave Axial FreeElectron Laser with Enhanced Phase Stability," Nuclear Instruments \& Methods in Physics Research A 375, 1-3, 171-174 (1996)

CARLSTEN, B. E., HAYNES, W. B., and SHEFFIELD, R. L., "Particle Acceleration by the Inverse Axial-Free-Electron Laser Interaction," Particle Accelerators 51, 2, 121-33 (1995)

CARLSTEN, B. E., MILDER, M. L., KINROSS-WRIGHT, J. M., RUSSELL, S., PLATO, J. G., SHERWOOD, B., SHAPIRO, A., LOVATO, R., WARREN, D., FELDMAN, D., COOPER, R., STURGESS, R., and WILIIAMS, M., "Subpicosecond, UltraBright Electron Injector," In Proceedings of the 1995 Particle Accelerator Conference (IEEE, 1995) 2, 985-987

CARLSTEN, B. E., and NYUGEN, D. C., "Electron Bunch Compression Due to RF Forces in an RF Photoinjector for Small RF Launch Phases," Particle Accelerators 56, 3, 127-145 (1996)

CARLSTEN, B. E., and RUSSELL, S. J., "Subpicosecond Compression of 0.1-1 nC Electron Bunches Using a Magnetic Chicane at $8 \mathrm{MeV}$," Physical Review E 53, R2072-2075 (1996)

CHAN, K. C. D., BROWMAN, A., HUTSON, R. L., MACEK, R. J., TALLERICO, P. J., and WILKINSON, C. A., "Development of a RAMI Program for LANSCE Upgrade," In Proceedings of the 1995 Particle Accelerator Conference (IEEE, 1995) 2, 822-824

CHAN, K. C. D., GOLDSTEIN, J. C., NGUYEN, D. C., and TAKEDA, H., "A Chirped-Pulse Regenerative-Amplifier FEL for the Gamma-Gamma Collider," In Proceedings of the 1995 Particle Accelerator Conference and International Conference on High Energy Accelerators (IEEE, 1995) 1, 228-230

CHAN, K. C. D., HILL C., and VRETENAR, M., "Conceptual Design of a Superconducting High Intensity Proton Linac," In Proceedings of the XVII International Linac Conference, (CERN, 1996) 2, 580-584 
COURT, J. D., PITCHER, E. J., FERGUSON, P. D., RUSSELL, G. J., and PATTON, B. W., "Preliminary Earth Berm Shielding Calculations for the Accelerator Production of Tritium 1700-MeV Accelerator," In Proceedings of the Topical Meeting on Nuclear Applications of Accelerator Technology, ISBN: 0-89448-692-2, 481-486 (1997)

COURT, J. D., PITCHER, E. J., RUSSELL, G. J., FERGUSON, P. D., and PATTON, B. W., "LAHET Code System Benchmarking in the Accelerator Production of Tritium November 1995 Small Scale Tritium Production Experiment," In Proceedings of the Topical Meeting on Nuclear Applications of Accelerator Technology, ISBN: 0-89448-692-2, 219-228 (1997)

CUMMINGS, K., RISBUD, S., METZGER, D., and BULTMAN, N., "Experimental and Analytical Study of Heating in RF Accelerator Windows," Journal of Microwave Power and Electromagnetic Energy 32, 2, 101-108 (1997)

FAZIO, M. V., CARLSTEN, B. E., HAYNES, W. B., and POTTER, J., "Design for a High Power, Phase-Stable, Microwave FEL Amplifier," In Proceedings of the 22nd IEEE International Conference on Plasma Science (IEEE, 1995) 246

FAZIO, M. V., COONS, J., DYER, T., GRAHAM, A., STEELE, R. D., and STRINGFIELD, R. M., "Advanced Technology for Nitrogen Oxide (NOX) Abatement in Flue Gas Using Microwave Irradiation of Coal Char," DOE Office of Scientific and Technical Information Report DE96014724 (1996)

FERGUSON, P., RUSSELL, G. J., and PITCHER, E., "Reference Moderator Calculated Performance for the LANSCE Upgrade Project," In Proceedings of the Thirteenth Meeting of the International Collaboration on Advanced Neutron Sources 2, 510-517 (1995)

FORTGANG, C. M., "A Pure Permanent Magnet-Two Plane Focusing-Tapered Wiggler for a High Average Power FEL," Nuclear Instruments and Methods A 393, 1-3, 385-388 (1997)

FRIEDRICHS, C. C., and LYLES, J. T. M., "LANSCE 201.25 Mhz Drift Tube Linac RF Power Status," In Proceedings of the 18th International Linac Conference (CERN, 1996) 2, 698-700

FULTON, R. D., GOLDSTEIN, J. C., JONES, M. E., KINROSS-WRIGHT, J., KONG, S. H., and NGUYEN, D. C., "Enhanced Energy Loss of Short Pulses of Electrons in Plasma," Journal of Applied Physics 82, 6, 2822-2825 (1997)

GALLEGOS, F. R., 'LANSCE Beam Current Limiter," In Proceedings of the 7th Workshop on Beam Instrumentation 390, 446-453 (1996)

GARNETT, R. W., and WANGLER, T. P., "A Design Approach for Superconducting High-Current Ion Linacs," In Proceedings of the 18th International Linac Conference (CERN, 1996) 2, 707-709

GARNETT, R. W., BILLEN, J. H., CHANG, K. C. D., GENTZLINGER, R., GRAY, E. R., NATH, S., RUSNAK, B., SCHRAGE, D. L., STOVAL, J. E., TAKADA, H., WOOD, R. L., WANGLER, T. P., and YOUNG, L. M., "Linear Accelerator for Tritium Production," In Proceedings of the Space Charged Dominated Beams and Applications of High Brightness Beams (AIP, 1996) 377, 60-73
GARNETT, R. W., GRAY, E. R., RYBARCYK, L. J., and WANGLER, T. P., "Simulation Studies of the LAMPF Proton Linac," In Proceedings of the 1995 Particle Accelerator Conference (IEEE, 1995) 5, 3185-3187

GARNETT, R. W., GRAY, E. R., RYBARCYK, L. J., and WANGLER, T. P., "Simulations Studies of the LAMPF Proton Linac," Bulletin American Phys. Society 40, 1188 (1995)

GILPATRICK, J., KHAN, S., and KRAMER, D., "A State Variable Approach to the Bessy II Local Beam-Position-Feedback System," In Proceedings of EPAC96: the Fifth European Particle Accelerator Conference (Institute of Physics Publishing, 1997) 3, 1902-1904

GOLDSTEIN, J. C., NGUYEN, D. C., and SHEFFIELD, R. L., "Theoretical Study of the Design and Performance of a High-Gain, High-Extraction-Efficiency FEL Oscillator," Nuclear Instruments and Methods in Physics Research 393, 137-141 (1997)

HAINES, J., EARL, D. D., and TSAI, C.-C., "Summary of Mercury Target Thermal Shock Tests Conducted at Accelerator Facilities in FY 1997," Spallation Neutron Source Target Systems Report SNS/TSR-0041 (1997)

HAYNES, W. B., CARLSTEN, B. E., FAZIO, M. V., and STRINGFIELD, R. M., "Conditioning and Simulations for the One-Microsecond, One Kilojoule Per-Pulse L-Band Relativistic Klystron," In Proceedings of the International Conference on Plasma Science (IEEE, 1995) 242

HOFMANN, I., JOHNSON, K. F., SPILLER, P., EICKHOFF, H., KALISCH, G., LAUX, W., and STECK, M., "Analysis of the Thermal Equilibrium State of Bunched Beams with a Streak Camera," Phys. Rev. Lett. 75, 3842 (1995)

HOGAN, M. J., PELLEGRINI, C., ROSENZWEIG, J., ANDERSON, S., FRIGOLA, P., TREMAINE, A., FORTGANG, C., NGUYEN, D. C., SHEFFIELD, R. L., KINROSS-WRIGHT, J., VARFOLOMEEV, A. A., TOLMACHEV, S., and CARR, R., "Measurement of Gain Larger than $10^{5}$ at $12 \mu \mathrm{m}$ in a SelfAmplified Spontaneous Emission Free-Electron Laser," Phys. Rev. Lett. 81, 22, 4867 (1998)

JASON, A., BLIND, B., CHANNEL, P., and GARNETT, R., "A High Power Accelerator Driver System for Spallation Neutron Sources," DOE Office of Scientific and Technical Information report DE96012869 (1996)

JOHNSON, K. F., CONNOLLY, R. C., GARCIA, R. C., RUSTHOI, D. P., SANDER, O. R., SANDOVAL, D. P., SHINAS, M. A., SMITH, M., and YUAN, V. W., "Application of a Transverse Phase-Space Measurement Technique for HighBrightness, $\mathrm{H}^{-}$Beams to the GTA $\mathrm{H}^{-}$Beam," In Proceedings of the 1995 Particle Accelerator Conference (IEEE, 1995) 4, 2607-2609

JOHNSON, K. F., SANDER, K. F., BOLME, G. O., BOWLING, S., CONNOLLY, R. C., GILPATRICK, J. D., LYSENKO, W. P., POWER, J., WADLINGER, A. E., and YUAN, V., "Phase-Scan Analysis Results for the First Drift Tube Linac Module in the Ground Test Accelerator: Data Reproducibility and Comparison to Simulations," In Proceedings of the 1995 Particle Accelerator Conference (IEEE, 1995) 2, 1161-1163 
KEATING, P. B., GULLEY, M. S., BRYANT, H. C., MAC KERROW, E. P., MILLER, W. A., RISLOVE, D. C., COHEN, S., DONAHUE, J. B., FITZGERALD, D. H., FRANKLE, S. C., FUNK, D. J., HUTSON, R. L., MACEK, R. J., PLUM, M. A., STANCIU, N. G., VAN DYCK, O. B., WILKINSON, C. A., and PLANNER, C. W., "Electric-Field-Induced Electron Detachment of $800-\mathrm{MeV} \mathrm{H}$-Ions," Physical Review A 52, 6, 4547-4555 (1995)

KURENNOY, S., "Beam Coupling Impedances of Obstacles Protruding into Beam Pipe," Physical Review E 55, 3, 3529-3532 (1997)

LAWRENCE, G. P., "Transmutation and Energy Production with High Power Accelerators," In Procedings of the 1995 Particle Accelerator Conference, 35 (1995)

LAWRENCE, G., BARLOW, D., BILLEN, J., BLIND, B., CHAN, K. C. D., GARNETT, R., GENTZLINGER, R., GRAY, E., GURD, D., KRAWCYZK, F., LYNCH, M., NATH, S., REGAN, A., REES, D., ROHLEV, A., RUSNAK, B., RYNE, R., SCHNEIDER, J.D., SCHRAGE, D., SHAFER, R., SHERMAN, J., STOVALL, J., TAKEDA, H., TALLERICO, P., WANGLER, T., WOOD, R., YOUNG, L., HILL, C., and VRETENAR, M., "Conventional and Superconducting RF Linac Designs for the APT Project," In Procedings of 18th International LINAC Conference (CERN, 1996) 2, 710-12

LINDEMUTH, I. R., REINOVSKY, R. E., CHRIEN, R. E., CHRISTIAN, J. M., EKDAHL, C. A., GOFORTH, J. H., HAIGHT, R. C., IDZOREK, G., KING, N. S. P., KIRKPATRICK, R. C., LARSON, R. E., MORGAN, G. L., OLINGER, B. W., OONA, H., SHEEHEY, P. T., SHLACHTER, J. S., SMITH, R. C., VEESER, L. R., WARTHEN, B. J., YOUNGER, S. M., CHERNYSHEV, V. K., MOHKOV, V. N., DEMIN, A. N., DOLIN, Y. N., GARANIN, S. F., IVANOV, V. A., KORCHAGIN, V. P., MIKKHAILOV, O. D., MOROZOV, I. V., PAK, S. V., PAVLOVSKI, E. S., SELEZNEV, N. Y., SKOVELEV, A. N., VOLKOV, G. I., and YAKUBOV, V. A., "Target Plasma Formation for Magnetic Compression/Magnetized Target Fusion," Physical Review Letters 75, 1953 (1995)

LYLES, J., REGAN, A., and BOLME, G., "LANSCE Linac RF Performance for a Long Pulse Spallation Source," In Proceedings of I8th International Linac Conference (CERN, 1996) 2, 695-697

LYNCH, M. T., TALLERICO, P. J., and LAWRENCE, G. P., "Important Requirements for RF Generators for AcceleratorDriven Transmutation Technologies (ADTT)," In Proceedings of the International Conference on Accelerator-Driven

Transmutation Technologies and Applications (AIP, 1995) 346. 446-452

LYNCH, M., REES, D., TALLERICO, P., and REGAN, A., "The RF System for the Accelerator Production of Tritium (APT) Low Energy Demonstration Accelerator (LEDA) at Los Alamos," In Proceedings of the I8th International Linac Conference (CERN, 1996) 2, 692-694

MERRILL, F., and RYBARCYK, L., "Transverse Match of High Peak-Current Beam into the LANSCE DTL Using PARMILA," In Proceedings of the 18th International Linac Conference (CERN, 1996) 1, 231-233
NATH, S., BILLEN, J. H., STOVAL, J. E., TAKEDA, H., and YOUNG, L. M., "Front-End Physics Design of APT Linac," XVIII International Linac Conference (CERN, 1996) 2, 680

NATH, S., BILLEN, J., STOVAL, J., TAKEDA, H., and YOUNG, L., "Physics Design of APT Linac with Normal Conducting RF Cavities," In Proceedings of the XVIII International Linac Conference (CERN, 1996) 2, 689-691

NELSON, E. M., "High Accuracy 3D Electromagnetic Finite Element Analysis," In Proceedings of the 1996 Computational Accelerator Physics Conference (AIP, 1997) 391, 77-82

NGUYEN, D. C., "Coherent Smith-Purcell Radiation as a Diagnostic for Subpicosecond Electron Bunch Length," Nuclear Instruments \& Methods A 393, 1-3, 514-518 (1997)

NGUYEN, D. C., "High-Power FEL Based on Seeded SinglePass Amplifier Using Prebunched Electron Beams," in CLEO '96: Summaries of Papers Presented at the Conference on Lasers and Electro-Optics (Optical Society of America, 1996) 9, 467-468

NGUYEN, D. C., and CARLSTEN, B. E., "Amplified Coherent Emission from Electron Beams Prebunched in a Masked Chicane," Nuclear Instruments Methods A 375, 597-601 (1996)

NGUYEN, D. C., EBRAHIM, N., FORTGANG, C., GOLDSTEIN, J. C., HAYES, K. L., KINROSS-WRIGHT, J. M., and SHEFFIELD, R. L., "Initial Results of the Infrared Regenerative Amplifier Free-Electron Laser Experiment," In Proceedings of the SPIE International Symposium on Coherent Electron-Beam X-Ray Sources (SPIE, 1997) 3154, 39-50

NGUYEN, D., FORTGANG, C., GOLDSTEIN, J., KINROSSWRIGHT, J., and SHEFFIELD, R., "Synchronously Injected Amplifiers, A Novel Approach To High-Average-Power FEL," Nuclear Instruments \& Methods A, 393 1-3, 252-256 (1997)

NGUYEN, D. C., SHEFFIELD, R. L., FORTGANG, C. M., GOLDSTEIN, J. C., KINROSS-WRIGHT, J. M. and EBRAHIM, N. A., "Self-Amplified Spontaneous Emission Driven by a HighBrightness Electron Beam," Physics Review Letter 81, 4, 810 (1998)

PITCHER, E. J., RUSSELL, G. J., KIDMAN, R. B., FERGUSON, P. D., and TODOSOW, M., "Neutronic Design of the APT Target/Blanket," In Proceedings of the Topical Meeting on Nuclear Applications of Accelerator Technology (ANS, 1997) 136-145

REES, D., KEFFELER, D., ROYBAL, W., and TALLERICO, P. J., "Characterization of a Klystrode as a RF Source for HighAverage-Power Accelerators," In Proceedings of the 1995 Particle Accelerator Conference (IEEE, 1995) 3, 1521-1523

REES, D. E., TALLERICO, P. J., and LYNCH, M. T., "The RF System for Accelerator Production of Tritium," IEEE Transactions on Plasma Science 24, 1033-1040 (1996)

REGAN, A. H., REES, D., ROYBAL, W. T., PALOMARES, M. POLSTON, C., TISCHLER, D., and ORTEGA, R., "In Situ RF/Microwave Remediation of Soil Experiment Overview," In Proceedings of the 6th Spectrum International Conference on Nuclear and Hazardous Waste (ANS, 1996) 1, 331-336 
REGAN, A. H., ROHLEV, A., and ZIOMEK, C., "APT LLRF Control System Functionality and Architecture," In Proceedings of the 18th International Linac Conference (CERN, 1996) 1, 225-227

ROYBAL, W. T., REES, D. E., TALLERICO, P. J., and GAHL, J. M., "Characterization of a High-Power Klystrode Amplifier as a RF Source for Particle Accelerators," Journal of Microwave Power and Electromagnetric Energy 30, 158-164 (1995)

RUSNAK, B., and SHAPIRO, A. H., "Test Results for a HeatTreated 4-Cell $805 \mathrm{MHz}$ Superconducting Cavity," In Proceedings of the 1995 Particle Accelerator Conference (IEEE, 1995) 3, 1636-1638

RUSSELL, G. J., TODOSOW, M., FERGUSON, P. D., KIDMAN, R. B., and PITCHER, E. J., "Accelerator-Driven Targets: Understanding and Analyzing the Spallation Process," the American Nuclear Society and the European Nuclear Society 1996 International Conference (ANS, 1996) 75, 254-256

RUSTHOI, D. P. and CRANDALL, K., "TRACE 3-D Documentation," Third Edition, Los Alamos National Laboratory Report, May (1997)

RYNE, R., "Beam Dynamics Simulations Using a Parallel Version of PARMILA," In Proceedings of the 18th International LINAC Conference (CERN, 1996) 1, 234-236

RYNE, R, COOPER, F. M., HABIB, S., and DAWSON, J., "Chaos in Time-Dependent Variational Approximations to Quantum Dynamics," Physical Review E 57, 1489-1498 (1998)

RYNE, R. D., and HABIB, S., "Beam Dynamics Calculations and Particle Tracking Using Massively Parallel Processors," In Proceedings of the Second International Workshop on Single Particle Effects in Large Hadron Collides (Gordon \& Breach, 1996) $54,365-374$

RYNE, R., and HABIB, S., "Parallel Beam Dynamics Calculations on High Performance Computers," In Proceedings of the 1996 Computational Accelerator Physics Conference (AIP, 1997) 391, 377-388

SCHNEIDER, J. D., and STEVENS, R. R., "Design and Construction of a New DC Proton Injector," In Proceedings of Accelerator-Driven Transmutation Technologies and Applications (AIP, 1995) 324, 1133-1135

SCHNEIDER, J. D., HILL, C., and VRETENAR, M., “APT Accelerator Technology," In Proceedings of the 18th International LINAC Conference (CERN, 1996) 1, 22-26

SCHRIBER, S. O., "Overview of Advanced Proton Driver for Nuetron Transmutation Systems," Nuclear Physics B 51A, 109-114 (1996)

SHAPIRO, A., CARLSTEN, B. E., MILDER, M. L., KINROSS-WRIGHT, J., RUSSELL, S. J., PLATO, J. G., SHERWOOD, B. A., STUDEBAKER, J., TIMMER, C., WILLIAMS, M., LOVATO, R. D., WARREN, D. S., FELDMAN, D. W., COOPER, R., and STURGES, R., "Subpicosecond, UltraBright Electron Injector," In Proceedings of the Particle Accelerator Conference (IEEE, 1995) 2, 985-987
SHEFFIELD, R., "High-Brightness Electron Sources," In Proceedings of the 1995 Particle Accelerator Conference (IEEE, 1995) 2, 882-886

SHEFFIELD, R., "Report of the Working Group on Production and Dynamics of High Brightness Beams," In Proceedings of the ICFA Workshop on Nonlinear and Collective Phenomena in Beam Physics (AIP, 1997) 11-18

SHEFFIELD, R. L., NGUYEN, D. C., GOLDSTEIN, J. C., EBRAIM, N. A., FORTGANG, C. M., and KINROSS-WRIGHT, J. M., "A Compact $1 \mathrm{~kW}$ Infrared Regenerative Amplifier FEL," In Proceedings of the SPIE (SPIE-Int. Soc. Opt. Eng., 1997) 2988, 28-37

SHERMAN, J. D., HODGKINS, D. J., LARA, P. D., SCHNEIDER, J. D., and STEVENS, R. Jr., "Lifetime Test on a High Performance Direct Current Microwave Proton Source," In Proceedings of the Particle Accelerator Conference (IEEE, 1995) 2, 867-870

SHERMAN, J. D., ARVIN, A. H., HANSBOROUGH, L. D., HODGKINS, D. J., MEYER, E. A., SCHNEIDER, J., SMITH, H., STETTLER, M. W., STEVENS, R. R., THUOT, M., ZAUGG, T. J., and FERDINAND, R., "Status Report on a dc 130-mA, 75-keV Proton Injector," Review of Scientific Instruments 69, 1003-1008 (1998)

SHERMAN, J. D., ARVIN, A. H. HANSBOROUGH, L. D., HODGKINS, D. J., MEYER, E. A, SCHNEIDER, J., STEVENS, R. R., THUOT, M., and ZAUGG, T. J., "Development of a 130-mA, $75 \mathrm{kV}$ High Voltage Column for High-Intensity DC Proton Injectors," Review of Scientific Instruments 69, 1017-1019 (1998)

SHERMAN, J., BOLME, J., GEISIK, C., GILPATRICK, D., HANSBOROUGH, L., HODGKINS, D., LARA, P., MEYER, E., NEEDES, M., POWER, J., SANDOVAL, D., ROSE, C. R., SCHAFSTALL, P., SCHNEIDER, J. D., STETTLER, M., STEVENS, R. Jr., THUOT, M., WRIGHT, R., ZAUGG, T., SPENCE, D., TAYLOR, T., and MCMICHAEL, G. E., "Microwave Proton Source Development for a High-Current Linac Injector," Review of Scientific Instruments 67, 1296-1301 (1996)

SHERMAN, J. D., BOLME, G. O., HANSBOROUGH, L. D., HODGKINS, D. J., LIGHT, M. E., MEYER, E. A., SCHNEIDER, J. D., SMITH, H.V., JR, STETTLER, M. W., STEVENS, R. R. Jr, THUOT, M. E., ZAUGG, T. J., FERDINAND, R., HILL, C., VRETENAR, M., "Development of a 110-MA, 75-keV Proton Injector for High-Current, CW Linacs," In Proceedings of the XVII International Linac Conference (CERN, 1996) 2, 701-703

SHERMAN, J. D., HODGKINS, D. J., LARA, P. D., SCHNEIDER, J. D., and STEVENS, R. Jr., "Lifetime Test on a High Performance Direct Current Microwave Proton Source," In Proceedings of the 1995 Particle Accelerator Conference (IEEE, 1995) $2,867-870$

SMITH, H. V., ALLISON, P., SCHNEIDER, J. D., STELZER, J. E., and STEVENS, R. R., "Evaluation of a Simple Method for Chopping Penning Surface-Plasma Source $\mathrm{H}^{-}$Beams," Review of Scientific Instruments 66, 1024-1027 (1995) 
STEVENS, R. R., GEISIK, C., YORK, R. L., and SWENSON, D. R., "Development of a Toroidal-Filter $\mathrm{H}^{-}$Ion Source," Review of Scientific Instruments 67, 1042-1044 (1996)

STEVENS, R. R., SWENSON, D. R., YORK, R. L., GEISIK, C., INGALLS, W. B., STELZER, J. E., and FITZGERALD, D. H., "Development of a Volume $\mathrm{H}^{-}$Ion Source for LAMPF," In Proceedings of the 1995 Particle Accelerator Conference (IEEE, 1995) 2, 1016-1018

STINTZ, A., BRYANT, H. C., RISLOVE, D. C., MILLER, W. A., STRAUSS, C. E. M., FUNK, D., INGALLS, W., ZHAO, X., and KYRALA, G., "Multiphoton Electron Detachment from $\mathrm{H}^{-}$," In Proceedings of the XIX International Conference on the Physics of Electronic and Atomic Collisions, 642 (1995)

STINTZ, A., ZHAO, X. M., STRAUSS, C. E. M., INGALLS, W. B., KYRALA, G. A., FUNK, D. J., and BRYANT, H. C., "Resonant Two-Photon Detachment Through the Lowest Singlet D State in $\mathrm{H}^{-}$," Physical Review Letters 75, 16, 2924-2927 (1995)

STOVALL, J., GRAY, E., NATH, S., TAKEDA, H., WOOD, R., YOUNG, L., and CRANDALL, K., HILL, C., and VRETENNNAR, M., "Alignment and Steering Scenarios for the APT Linac," In Proceedings of the 18th International Linac Conference 2, 686-688 (1996)

SUGAI, I., OYAIZU, M., KAWAKAMI, H., OHMORI, C., HATTORI, T., KAWASAKI, K., BORDEN, M. J., MACEK, R. J., "Development of Thick, Long-Lived Carbon Stripper Foils for PSR of LANL," Nuclear Instruments \& Methods in Physics Research A 362, 1, 70-76 (1995)

TAKEDA, H., and STOVALL, J. E., "Modified Parmila Code for New Accelerating Structures," In Proceedings of the Particle Accelerator Conference (IEEE, 1995) 4, 2364-2365

TAKEDA, H., BILLEN, J. H., NATH, S., STOVALL, J. E., WOOD, R. L., and YOUNG, L. M., "A Compact High-Power Proton Linac for Radioisotope Production," In Proceedings of the Particle Accelerator Conference (IEEE, 1995) 2, 1140-1142

TAKEDA, H., YOUNG, L., NATH, S., BILLEN, J., and STOVALL, J., HILL, C., and VRETENAR, M., "Linac Design Algorithm with Symmetric Segments," In Proceedings of the 17th International Linac Conference 12, 683-685 (1996)

TALLERICO, P. J., "Gyrotrons, Magnicons, and Ubitrons," Handbook of Microwave Technology (1995)

TALLERICO, P. J., "Klystron,” Encyclopedia of Electrical \& Electronic Engineering (1997)

TALLERICO, P., REES, D., YOUNG, A., LAYCOCK, D., and SIMONS, R., "Techniques for Maintaining Design Efficiency When Operating Klystron Amplifiers at Levels Below the Maximum Output Power," IEEE Trans. on Electron Devices 42, 780-784 (1995)

THODE, L., CHAN, K. C. D., and SCHMIDTT, M. J., "Free Electron Laser Physical Process Code (FELPC)," In Proceedings of the SPIE-International Society for Optical Engineering (SPIE, 1995) 2376, 104-105
THOMAS, J. R., Jr., "Particle Size Effect in MicrowaveEnhanced Catalysis," Catalysis Letters 49, 137 (1997)

THOMAS, J. R., NELSON, E. M., KARES, R. J., and STRINGFIELD, R. M., "Temperature Distribution in a Flowing Fluid Heated in a Microwave Resonant Cavity," Mat. Res. Soc. Symp. Proc. 430, 565 (1996)

TREMAINE, A., ROSENZWEIG, J. B., ANDERSON, S., FRIGOLA, P., HOGAN, M., MUROKH, A., PELLEGRINI, C., NGUYEN, D. C., and SHEFFIELD, R. L., "Oberservation of Self-Amplified Spontaneous-Emission-Induced Electron-Beam Microbunching Using Coherent Transition Radiation," Physical Review Letters 81, 26, 5816-5819 (1998)

VAN DYCK, O., "Trends in Prompt Radiation Risk Management at DOE Accelerator Facilities," In Proceedings of the Fourteenth International Conference on the Application of Accelerators in Research and Industry (AIP, 1996) 346, 1416-1419

WADLINGER, A., "Beam-Bunching with a Linear-Ramp Including Space-Charge Force Effects Cylinder Model," In Proceedings of the 18th International Linac Conference (CERN, 1996) 1, 151-153

WADLINGER, A., and GARNETT, R., "The Brown-Servanckx Matching Transformer for Simultaneous RFQ to DTL $\mathrm{H}^{+}$and $\mathrm{H}^{-}$ Matching," In Proceedings of the 18th International Linac Conference (CERN, 1996) 1, 146-147

WADLINGER, A., LYSENKO, W., RUSNAK, B., and SAADATMAND, K., "Beam Dynamics of a Double-Gap Acceleration Cell for Ion Implantation with Multiple Atomic Species," In Proceedings of the14th International Conference on the Application of Accelerators in Research \& Industry (AIP, 1996) 392, 941-944

WANG, T.-S. F., "A Theoretical Study of the Electron-Proton Instability in a Long Proton Pulse," In Proceedings of the Particle Accelerator Conference (IEEE, 1995) 5, 3143-3144

WANG, T.-S. F., CHANNELL, P. J., COOPER, R. K., FITZGERALD, D. H., HARDEK, T. W., HUTSON, R. L., JASON, A. J., MACEK, R. J., PLUM, M. A., WILKINSON, C. A., and COLTON, E. P., "Recent Progress on Beam Stability Studies in the PSR,' In Proceedings of the Particle Accelerator Conference (IEEE, 1995) 5, 3146-3148

WANG, T.-S. F., JASON, A. J., and STEVENS, R. R., "Quadrupole Slow-Wave Deflector for Chopping ChargedParticle Beams," In Proceedings of the 18th International Linac Conference (CERN, 1996) 1, 219-221

WANGLER, T. P., "Emittance Concept and Growth Mechanisms," In Proceedings of the Space Charged Dominated Beams and Applications of High Brightness Beams (AIP, 1996) 377, 3-22

WANGLER, T. P., "Strong Focusing and the Radiofrequency Quadrupole Accelerator," American Journal of Physics 64, 177-182 (1996) 
WANGLER, T. P., GARNETT, R. W., GRAY, E. R., RYNE, R., and WANG, T. S., "Dynamics of Beam Halo in Mismatched Beams," In Proceedings of the 18th International Linear Accelerator Conference (CERN, 1996) 1, 372-374

WECHSLER, M. S., LIN, C., SOMMER, W. F., DAEMEN, L. L., FERGUSON, P. D., "Radiation Effects. in Materials for Accelerator-Driven Neutron Technologies," Journal of Nuclear Materials 244, 3, 177-84 (1997)

WILLIAMS, H. E., and PAUL, J. D., "Filament Heater Current Modulation for Increased Filament Lifetime," In Proceedings of the 1996 International Power Modulator Symposium (IEEE, 1996) 239-242

WOOD, R., CLARK, W., MARTINEZ, F., and SIGLER, F., "Thermal/Structural Design and Fabrication Development of High Power CCDTL and CCL Structures," In Proceedings of the XVIII International Linac Conference (CERN, 1996) 2, 752-754

YUAN, V. W., CONNOLLY, R. C., JOHNSON, K. F., GILPATRICK, J. D., LYSENKO, W. P., RUSTHOI, D. P., SANDER, O. R., SMITH, M., and WEISS, R., "Unexpected Matching Insensitivity in DTL of GTA Accelerator," In Proceedings of the Particle Accelerator Conference (IEEE, 1995) 2, 1167-1169

\section{Spallation Science and Technology}

BOWMAN, C. D., “Accelerator-Driven Transmutation Technologies for Resolution of Long-Term Nuclear Waste Concerns," In Proceedings of the Inst. Phys. \& Power Eng. 2, 660-674 (1997)

BURMAN, R. L., and DAEMEN, L. L., "Water Target at the ISIS Spallation Source: Neutrino and Neutron Fluxes," Nucl. Instr. Meth. A370, 335 (1996)

BUTT, D. P., KANNER, G. S., DAEMEN, L. L., and LILLARD, R., "In Situ Studies of the Aqueous Corrosion of Target and Structural Materials in Water Irradiated by an $800 \mathrm{MeV}$ Proton Beam," In Materials for Spallation Neutron Sources, TMS Publication 93-98 (1998)

DAEMEN, L. L., FERGUSON, P. D., SOMMER, W. E., and WECHSLER, M. S., "Radiation Damage Effects at Spallation Neutron Sources," In Proceedings of the International Conference on Accelerator-Driven Transmutation Technologies and Applications (AIP, 1995) 346, 488-494

DAEMEN, L. L., KANNER, G. S., LILLARD, R., BUTT, D. P., BRUN, T. O., and SOMMER, W. F., "Radiation-Enhanced Corrosion of Water-Cooled Targets for Spallation Neutron Sources," In Materials for Spallation Neutron Sources, TMS Publication 93-98 (1998)

DOOLEN, G., VENNERI, F., WILLIAMSON, M. A., and LI, N., "The Los Alamos Accelerator-Driven Transmutation of Nuclear Waste Concept (ATW)," In Proceedings of the International Workshop: Nuclear Methods for Transmutation of Nuclear Waste 26 (1996)
FERGUSON, P. D., SOMMER, W. F., DUDZIAK, D. J., WECHSLER, M. S., BARNETT, M. H., and CORZINE, R. K., "Radiation Damage Calculations for the LANSCE Degrader," In Proceedings of the 1998 Annual Meeting of the American Nuclear Society (ANS, 1998) 78, 291-293

HOUTS, M. G., and POSTON, D. I., "Accelerator Transmutation of Waste Blanket Considerations," In Proceedings of the 1997 Annual Meeting of the American Nuclear Society 76, 100-101 (1997)

KELLEY, J. P., CLAUSEN, J. J., KUENDIG, A., SENN, A. E., and DIXON, K. D., "Deep Discharge Recovery with the AML\&P SMES Helium Refrigeration System," Advances in Cryogenic Engineering 43 (1998)

KELLEY, J. P., KRAL, S. F., KARASIK, V., GRUT, K. E., DIXON, K. D., RIBIERO, P. F., and KUNZ, R., "Alaska SMES: Form and Function for the World's Largest Magnet," Advances in Cryogenic Engineering 43 (1998)

LI, N., CAMASSA, R. A., ECKE, R. E., and VENNERI, F., "Solutal Separation Under Centrifugation," Separation Science and Technology 33, 551-567 (1998)

LI, N., CAMASSA, R., ECKE, R., and VENNERI, F., "Decontamination of Nuclear Fuels with Centrifugal Separation," In Proceedings of the International Conference on Evaluation of Emerging Nuclear Fuel Cycle Systems 1, 1059 (1995)

LILLARD, R., KANNER, G. S., and BUTT, D. P., "The Nature of Oxides on Tungsten in Acidic and Alkaline Solutions," $J$. Electrochem. Soc. 145, 8, 2718-2725 (1998)

PYNN, R., and WEINACHT, D., "A Proposal for a Long-Pulse Spallation Source at Los Alamos National Laboratory," In Proceedings of the Thirteenth Meeting of the International Collaboration on Advanced Neutron Sources 1 (1995)

RUSSELL, G. J., FERGUSON, P. D., PITCHER, E. J., and COURT, J. D., "Split-Target Neutronics and the MLNSC Spallation Target System," In Proceedings of the 14th International Conference on the Application of Accelerators in Research and Industry (AIP, 1997) 392, 361-364

RUSSELL, G. J., PITCHER, E. J., and DAEMEN, L. L., "Introduction to Spallation Physics and Spallation-Target Design," Accelerator-Driven Transmutation Technologies \& Applications (AIP 1995) 346, 93-104

RUSSELL, G. J., PITCHER, E., and FERGUSON, P., "Coupled Moderator Neutronics," In Proceedings of the 13th Meeting of the International Collaboration on Advanced Neutron Sources (Paul Scherrer Institut 1995) 2, 495-509

RUSSELL, G. J., PITCHER, E., FERGUSON, P., and PATTON, B. W., "Long-Pulse Spallation Source Neutronic Performance," J. Neutron Research 6, 33-62 (1997)

STUBBINS, J. F., WECHSLER, M. S., BORDEN, M., and SOMMER, W. F., "Behavior of Structural and Target Materials Irradiated in Spallation Neutron Environments," In Proceedings of the International Conference on Accelerator-Driven Transmutation Technologies and Applications (AIP, 1995) 346, 879-888 
VENNERI, F. and BOWMAN, C. D., "Physics Design of Accelerator-Driven Transmutation Systems," In Proceedings of the International Conference on Accelerator-Driven Transmutation Technologies and Applications (AIP, 1995) 346, 117-137

WANGLER, T., BILLIN, J., JASON, A., SHAFER, R., STAPLES, J., TAKEDA, H., and TALLERICO, P., "High-Power Linac for a US Spallation-Neutron Source," In Proceedings of the 18th International Linac Conference (CERN, 1996) 2, 749-751

WECHSLER, M. S., LIN, C., and SOMMER, W. F., "Basic Aspects of Spallation Radiation Damage to Materials," In Proceedings of the International Conference on Accelerator-Driven Transmutation Technologies and Applications (AIP, 1995) 346, 466-475

WECHSLER, M. S., LIN, C., FERGUSON, P. D., MANSUR, L. K., and SOMMER, W. F., "Calculations of Radiation Damage in Target, Container, and Window Materials for Spallation Neutron Sources," In Proceedings of the Second International Conference on Accelerator-Driven Transmutation Technologies and Applications 2, 968-974 (1997)

\section{Nuclear Physics}

ABFALTERER, W. P., BATEMAN, F. B., DIETRICH, F. S., ELSTER, C., FINLAY, R., GLOCKLE, W., GOLAK, J., and HAIGHT, R. C., "Inadequacies in the Nonrelativistic $3 \mathrm{~N}$ Hamiltonian Describing the $\mathrm{n}+\mathrm{d}$ Total Cross Section," Physical Review Letters 81, 57 (1998)

ALBERT, M., ATHANASSOPOULOS, C., AUERBACH, L. B., BAUER, D., BOLTON, R., BOYD, B., BURMAN, R. L., COHEN, I., CALDWELL, D. DIETERLE O., B. D., DONAHUE, J. B., EISNER, A. M., FAZELY, A., FEDERSPIEL, F. J., GARVEY, G. T., GUNASINGHA, R. M., HIGHLAND, V., HILL, J., IMLAY, R., JOHNSTON, K., LOUIS. W. C., LU, A., MANN, A. K., MARGULIES, J., MCILHANY, K., METCALF, W., REEDER, R. A., SANDBERG, V., SCHILLACI, M., SMITH, D., STANCU, I., STROSSMAN, W., SULLIVAN, M. K., VAN DALLEN, G. J., VERNON, W., WANG, Y.-X., WHITE, D. H., WHITEHOUSE, D., WORKS, D., XIAO, Y., and YELLIN, S., "Measurement of the Reaction ${ }^{12} \mathrm{C}$ (formula)X Near Threshold," Physical Review C 51, R1065 (1995)

ALLEN, G. E., and CHEN, M.-L., "A Study of Large Zenith-Angle Air Shower with the CYGNUS Experiment," In Proceedings of the International Cosmic-Ray Conference 1, 321 (1995)

ALLEN, G. E., and CHEN, M.-L., "Gamma-Ray Bursts: Detection and Distance Estimates with Milagro," In Proceedings of the International Cosmic-Ray Conference 3, 561 (1995)

ALLEN, G. E., and CHEN, M.-L., "Search for Gamma-Ray Bursts with Water Cherenkov Detector Single-Particle Rate," In Proceedings of the Intemational Cosmic-Ray Conference 2, 140 (1995)

ALLEN, G. E., and CHEN, M.-L., "Search for Ultra High Energy Radiation from Gamma-Ray Bursts," In Proceedings of the International Cosmic-Ray Conference 2, 124 (1995)

ALLEN, G. E., and CHEN, M.-L., "Search for Ultra High Energy Radiation from Supernova Remnants," In Proceedings of the International Cosmic-Ray Conference 2, 443 (1995)
ALLEN, G. E., and CHEN, M.-L., "Source Search Using the CYGNUS Water Cherenkov Array," In Proceedings of the International Cosmic-Ray Conference 2, 401 (1995)

ALLEN, G. E., and CHEN, M.-L., "The Milagro Data Acquisition System," In Proceedings of the International CosmicRay Conference 1, 914 (1995)

ALLEN, G. E., and CHEN, M.-L., "The Milagro Detector," In Proceedings of the International Cosmic-Ray Conference 1, 942 (1995)

AMBROSE, D. A., BACHMAN, M. G., COFFEY, W. P., GLASS, G., McNAUGHTON, K. H., RILEY, P. J., ADAMS, D. L., GAUSSIRAN, T. L., HUNGERFORD, E. V., LAN, K. A., JOHNSTON, K., McNAUGHTON, M. W., PENTTILA, S. I., and SUPEK, I., "A Large Acceptance Cylindrical Drift Chamber Detector," Nucl. Instrum. and Methods A364, 265 (1995) ARCHER, D. E., BECKER, J. A., BERNSTEIN, L. A., YOUNES, W., DRAKE, D. M., JOHNS, G. D., and NELSON, R. O., "Capabilities of GEANIE at LANSCE/WNR: Spectroscopy of 238U," Bull. Am. Phys. Soc. 42, 1072 (1997)

ARCHER, D. E., HENRY, E. A., and YOUNES, W., "Measuring Lu(n,xny) with GEANIE," Bull. Am. Phys. Soc. 42, 1687 (1997)

ATHANASSOPOULOS, C., AUERBACH, L. B. , BURMAN, R. L., COHEN, I., CALDWELL, D. I., DIETERLE, B. D., DONAHUE, J. B., EISNER, A. M., FAZELY, A., FEDERSPIEL, F. J., GARVEY, G. T., GRAY, M., GUNASINGHA, R. M., IMLAY, R., JOHNSTON, K., KIM, H. J., LOUIS, W. C., MAJKIC, R., MARGULIES, J., MCILHANY, K., METCALF, W., MILLS, G. B., REEDER, R. A., SANDBERG, V., SMITH, D., STANCU, I., STROSSMAN, W., TAYLOE, R., VANDALEN, G. J., VERNON, W., WADIA, N., WALTZ, J., WANG, Y.-X., WHITE, D. H., WORKS, D., XIAO, Y., and YELLIN, S., "Evidence for (nu)Over-Bar(mu)-(nu)Over-Bar(E) Oscillations from the LSND Experiment at the Los Alamos Meson Physics Facility," Physical Review Letters 77, 15, 3082-3085 (1996)

ATHANASSOPOULOS, C., AUERBACH, L. B., BAUER, D., CALDWELL, D. O., GRAY, M., LU, A., YELLIN, S., BOLTON, R. D., BURMAN, R. L., DONAHUE, J. F., FEDERSPIEL, F. J., GARVEY, G. T., LOUIS, W. C., SANDBERG, V. D., SCHILLACI, M. E., WHITE, D. H., WHITEHOUSE, D. A., BOYD, B., JOHNSTON, K., COHEN, I., DIETERLE, B. D., REEDER, R. A., EISNER, A. M., SULLIVAN, M. K., WANG, Y.-X., FASELY, A., GUNASINGHA, R. M., IMLAY, R. L., METCALF, W., HIGHLAND, V. L., MARGULIES, J., WORKS, D., MCILHANY, O. K., STANCY, I., STROSSMAN, W., VAN DALEN, G. J., SMITH, D., and VERNON, W., "Candidate Events in a Search for $\mathrm{nu}_{\mathrm{mu}}$ to $\mathrm{nu}_{\mathrm{e}}$ Oscillations," Physical Review Letters 75, 2650-2653 (1995)

ATHANASSOPOULOS, C., AUERBACH, L. B., BURMAN, R. L., CALDWELL, D. O., CHURCH, E., COHEN, I., DIETERLE, B. D., DONAHUE, J. B., EISNER, A. M., FAZELY, A., FEDERSPIEL, F. J., GARVEY, G. T., GRAY, M., GUNASINGHA, R. M., IMLAY, R., JOHNSTON, K., KIM, H. J., LOUIS, W. C., MAJKIC, R., MCILHANY, K, METCALF, W., MILLS, G. B., REEDER, R. A., SANDBERG, V., SMITH, D., STANCU, I., STROSSMAN, W., TAYLOE, R., and VANDALEN, G. J., "Measurements of the Reactions ${ }^{12} \mathrm{C}(\mathrm{ge}, \mathrm{e}-)^{12} \mathrm{~N}$ g.s. and ${ }^{12} \mathrm{C}$ (ge,e-) ${ }^{12} \mathrm{~N}^{*}, "$ Physical Review C 55, 4, 2078-2091 (1997) 
ATHANASSOPOULOS, C., AUERBACH, L. B., BURMAN, R. L., CALDWELL, D. O., CHURCH, E., COHEN, I., DIETERLE, B. D., DONAHUE, J. B., EISNER, A. M., FAZELY, A., FEDERSPIEL, F. J., GARVEY, G. T., GRAY, M., GUNASINGHA, R. M., IMLAY, R., JOHNSTON, K., KIM, H. J., LOUIS, W. C., MAJKIC, R., MCILHANY, K., METCALF, W., MILLS, G. B., REEDER, R. A., SANDBERG, V., SMITH, D., STANCU, I., STROSSMAN, W., TAYLOE, R., and VANDALEN, G. J., "Measurements of the Reactions ${ }^{12} \mathrm{C}\left(\mathrm{nu}_{\mathrm{mu}}\right.$, mu) ${ }^{12} \mathrm{~N}_{\mathrm{g} . \mathrm{s}}$ and ${ }^{12} \mathrm{C}\left(\mathrm{nu}_{\mathrm{mu}}, \mathrm{mu}\right) \mathrm{X}$," Physical Review $C$ 56, 5 , 2806-2819 (1997)

ATHANASSOPOULOS, C., AUERBACH, L. B., MAJKIC, R., WORKS, D. L., XIAO, Y., BURMAN, R. L., DONAHUE, J. B., FEDERSPIEL, F. J., GARVEY, G., LOUIS, W. C., MILLS, G. B., SANDBERG, V. D., TAYLOE, R. L., WHITE, D., CALDWELL, D. O., YELLIN, S. J., CHURCH, E. D., MCILHANY, K., STANCU, I., STROSSMAN, W. H., VAN DALEN, G. J., COHEN, I. M., REEDER, R., FAZELY, A. R., GUNASINGHA, R., IMLAY, R. L., KIM, H. J., METCALF, W. J., WADIA, N. D., JOHNSTON, K., SMITH, D., and VERNON, W., "Evidence for $\mathrm{nu}_{\mathrm{mu}}$ to $\mathrm{nu}_{\mathrm{e}}$ Oscillations from the LSND Detector at LAMPF," Physical Review Letters 81, 1774 (1998)

ATHANASSOPOULOS, C., AUERBACH, L. B., BAUER, D., BOLTON, R. D., BURMAN, R. L., COHEN, I., CALDWELL, D. O., DIETERLE, B. D., DONAHUE, J. B., EISNER, A. M., FAZELY, A., FEDERSPIEL, F. J., GARVEY, G. T., GRAY, M., GUNASINGHA, R. M., HIGHLAND, V., IMLAY, R., JOHNSTON, K., KIM, H. J., LOUIS, W. C., LU, A., MARGULIES, J., MILLS, G. B., MCILHANY, K., METCALF, W., REEDER, R. A., SANDBERG, V., SCHILLACI, M., SMITH, D., STANCU, I., STROSSMAN, W., TAYLOE, R., VANDALEN, G. J., VERNON, W., WANG, Y.-X., WHITE, D. H., WHITEHOUSE, D., WORKS, D., XIAO, Y., and YELLIN S., "The Liquid Scintillator Neutrino Detector and LAMPF Neutrino Source," Nuclear Instruments \& Methods in Physics Research A388, 1-2, 149-172 (1997)

ATHANASSOPOULOS, C., AUERBACH, L. B., BURMAN, R. L., COHEN, I., CALDWELL, D. O., DIETERLE, B. D., DONAHUE, J. B., EISNER, A. M., FAZELY, A., FEDERSPIEL, F. J., GARVEY, G. T., GRAY, M., GUNASINGHA, R. M., IMLAY, R., JOHNSTON, K., KIM, H. J., LOUIS, W. C., MAJKIC, R., MARGULIES, J., MCILHANY, K., METCALF, W., MILLS, G. B., REEDER, R. A., SANDBERG, V., SMITH, D., STANCU, I., STROSSMAN, W., TAYLOE, R., VANDALEN, G. J., VERNON, W., WADIA, N., WALTZ, J., WANG, Y.-X., WHITE, D. H., WORKS, D., XIAO, Y., and YELLIN, S., "Evidence for Neutrino Oscillations from Muon Decay at Rest," Physical Review C 54, 5, 2685-2708 (1996)

BARAKAT, M., CHEN, Y., CONRAD, S., COOPER, M. D., DZEMIDZIC, M., FLICK, J., GAGLIARDI, C. A., HUNGERFORD, E. V., JOHNSTON, K., KIM, G., LAN, K. J., LIU, F., LU, Y., MAYES, B. W., MISCHKE, R. E., PHELPS, R. A., PINSKY, L. S., SEMON, D., TRIBBLE, R. E., TANG, L. G., TU, X., VANAUSDELN, L., VONWITSCH, W., WANG., H., YAO, X., YI, W., "Construction of the MEGA Photon Detector," Nucl. Inst. and Meth. A268, 118 (1995)
BATEMAN, F. B., GRIMES, S. M., BOUKHAROUBA, N., MISHRA, V., BRIENT, C. E., PEDRONI, R. S., MASSEY, T. N., and HAIGHT, R. C., "Determination of the ${ }^{29}$ Si Level Density from 3 to $22 \mathrm{MeV}$," Physical Review C 55, 133 (1997)

BATEMAN, F. B., HAIGHT, R. C., CHADWICK, M. B., YOUNG, P. G., VOGT, M., FELDMAN, G., and SKOPIK, D. M., " $208 \mathrm{~Pb}$ Photoneutron Cross Sections Obtained Using Tagged Photons," Bull. Am. Phys. Soc. 41, 5, 1235 (1996)

BEARD, C. A., and BELYAKOV-BODIN, V. I., "Comparison of Energy Deposition Calculations by the LAHET Code System with Experimental Results," Nuclear Science and Engineering 119, 2, 87-96 (1995)

BECKER, J. A., and NELSON, R. O., "New Opportunities in Nuclear Science with GEANIE at LANSCE/WNR," Nuclear Physics News International 7, 2, 11 (1997)

BERLEY, D. E., and CHEN, M.-L., "First Light in the Milagrito Detector," In Proceedings of the 25th International Cosmic-Ray Conference 5, 201 (1997)

BERLEY, D. E., and CHEN, M.-L., "Results from the Milagrisimo Air Shower Detector," In Proceedings of the 25th International Cosmic-Ray Conference 3, 285 (1997)

BERNSTEIN, L. A., ARCHER, D. E., BECKER, J. A., DRAKE, D., GARRETT, P. E., JOHNS, G. D., NELSON, R. O., WILBURN, W. S., YATES, S. W., and YOUNES, W., "Probing the ${ }^{196} \mathrm{Pt}(\mathrm{n}, \mathrm{xng})$ Reactions Using GEANIE at LANSCE/WNR," In Proceedings of the International Conference on Nuclear Data for Science and Technology 1, 450-454 (1997)

BERNSTEIN, L. A., ARCHER, D. E., BECKER, J. A., YOUNES, W., DRAKE, D. M., JOHNS, G. D., NELSON, R. O., WILBURN, W. S., and YATES, S. W., "Probing Neutron-Induced Nuclear Reactions up to En=200 MeV Using GEANIE at LANSCE/WNR," Bull. Am. Phys. Soc. 42, 1686 (1997)

BERNSTEIN, L. A., ARCHER, D. E., BECKER, J. A., YOUNES, W., DRAKE, M., JOHNS, G. D., and NELSON, R. O., "The Search for Rigid Triaxiality in Pt with GEANIE," Bull. Am. Phys. Soc. 42, 1073 (1997)

BERNSTEIN, L. A., BECKER, J. A., ARCHER, D. E., HAUSCHILD, K., YOUNES, W., DRAKE, D. M., JOHNS, G. D., NELSON, R. O., and WILBURN, W., "Probing Reaction Dynamics Using the ${ }^{196} \mathrm{Pt}(\mathrm{n}, \mathrm{xn})$ Reactions for $\mathrm{x}<15$," Physical Review C 57, 6, R2799-R2803 (1998)

BOWMAN, C. D., "Current Status and Recommended Future Studies of Underground Supercriticality of Fissile Material," Inst. Phys. \& Power Eng. 2, 743-753 (1997)

BOWMAN, J. D., "Experiments on Parity Violation in the Compound Nucleus," In Proceedings of the International Workshop on Parity and Time Reversal Violation in Compound Nuclear States and Related Topics (World Scientific, 1996) 65-82 
BOWMAN, J. D., FRANKLE, C. M., GREEN, A. A., KNUDSON, J. N., PENTTILA, S. I., SEESTROM, S. J., YEN, Y.-F., YUAN, V. W., CRAWFORD, B. E., ROBERSON, N. R., GOULD, C. R., HAASE, D. G., LOWIE, L. Y., MITCHELL, G. E., STEPHENSON, S. L., DELHEIJ, P. P. J., SHARAPOV, E. I., POSTMA, H. MASUDA, Y., SHIMIZU, H. M., INUMA, M., MASAIKE, A., MATSUDA, Y., and FUKUDA, K., "Parity Violation in the Compound Nucleus," In Proceedings of the Fifth Conference on Intersections of Particle \& Nuclear Physics (AIP, 1995) 338, 78-90

BOWMAN, J. D., LOWIE, L. Y., and SHARAPOV, E. I., "Likelihood Analysis of Parity Violating Asymmetries Measured for Compound Nuclear Resonances," Phys. Part. Nucl. 27, 398 (1996)

BOWMAN, J. D., LOWIE, L. Y., MITCHELL, G. E., SHARAPOV, E. I., and YEN, Y.-F., "Analysis of Parity Violation in Neutron Resonances," Physical Review C 53, 285 (1996)

BOWMAN, J. D., LOWIE, L. Y., MITCHELL, G. E., SHARAPOV, E. I., and YEN, Y.-F., "Extraction of Parity Violating Matrix Element from Data on Neutron Resonances," In Proceedings of the Third International Seminar on Neutron-Nucleus Interactions (JINR, 1995) 57

BOWMAN, J. D., PENTTILA, S. I., and TIPPENS, W. B., "A Spin Reversal System for Polarized Epithermal Neutrons," Nucl. Instrum. Methods A369, 195 (1996)

BRYANT, H. C., "H- Spectroscopy," XIV Atomic Physics Program Contractor's Workshop of the Department of Energy, Office of Basic Energy Sciences, Division of Chemical Sciences, OBES Book of Abstracts, 53-56 (1995)

BRYANT, H. C., and HALKA, M., "H' Spectroscopy," Chapter 4: Coulomb Interactions in Nuclear and Atomic Few-Body Collisions (Plenum Press, 1996), ISBN 0-306-45149-2, 221-280

BRYANT, H. C., and MACKERROW, E. P., "Modeling Multiphoton Measurements on $\mathrm{H}^{-}$," Atomic and Molecular Physics C Morgan, J., and Alvarez, J. Eds. (World Scientific, 1995), ISBN 981-02-2370-6

BRYANT, H. C., and MACKERROW, E. P., "Relativistic Neutral Hydrogen Beams," AIP Press Instrumentation Series: Accelerator-Based Atomic Physics Techniques and Applications (AIP, 1997) 8, 181-212

BUDHANI, R. C., WILLIS, J. O., SUENAGA, M., MALEY, M. P., COULTER, J. Y., SAFAR, H., ULLMANN, J. L., and HALDAR, P., "Studies of Flux Pinning by Proton-induced Fission Tracks in Multifilamentary Tapes of (Bi,Pb ${ }_{2} \mathrm{Sr}_{2} \mathrm{Ca}_{2} \mathrm{Cu}_{3} \mathrm{O}_{10} / \mathrm{Ag}$ Superconductors," J. Appl. Phys. 82, 6, 3014-18 (1997)

CARLSON, V., GARNETT, R., HILL, D., JOHNSON, K. F., LOPIANO, D., OHASHI, Y., SHIMA, T., SPINKA, H., STANEK, R., UNDERWOOD, D., YOKOSAWA, A., BEDDO, M., BURLESON, G., FAUCETT, J. A., KYLE, G., RAWOOL-SULLIVAN, M., SHIMIZU, H., GLASS, G., NATH, S., NORTHCLIFFE, G. E., JARMER, J. J., JEPPESEN, R. H., and TRIPARD, G. E., "Neutron-Proton Elastic Scattering Spin-Spin Correlation Parameter Measurements Between 500 and $800 \mathrm{MeV}$. III. Mixtures of CSS, CLL, and CNN," Physical Review D 53, 3506 (1996)
CHADWICK, M. B., DELUCA, P. M., and HAIGHT, R. C., "Nuclear Data Needs for Neutron Therapy and Radiation Protection," Radiation Protection Dosimetry 70, 1 (1997)

CHADWICK, M. B., HAIGHT, R. C., BATEMAN, F. B., YOUNG, P. G., STERBENZ, S. M., GRIMES, S. M., WASSON, O. A., and VONACH, H., "Nuclear Level Densities Determined by Theoretical Analysis of (n,alpha) Measurements," Bull. Am. Phys. Soc., 41, 1235 (1996)

CHADWICK, M. B., REFFO, G., VENTURA, A., and GRANDI, C., "Calculation of Nuclear Data for Fast Neutron and Proton Radiotherapy: A New ICRU Report," In Proceedings of the International Conference on Nuclear Data for Science and Technology (Italian Phys. Soc., 1997) 2, 1683-1687

CHEN, C. M., ERNST, D. J., JIANG, M. F., JOHNSON, M. B., "Pion-Nucleus Scattering and Baryon Resonances in the Nuclear Medium," Physical Review C 52, R485-R489 (1995)

CHEN, Y., COOPER, M. D., COOPER, P. S., DZEMIDZIC, M., GAGLIARDI, C. A., HOGAN, G. E., HUNGERFORD, E. V., KIM, G. J., KNOTT, J. E., LAN, K. J., LIU, F., MAYES, B. W., MISCHKE, R. E., PHELPS, R. A., PINSKY, L. S., STANTZ, K. M., SZYMANSKI, J. J., TANG, L. G., TRIBBLE, R. E., TU, $X$. L., VANAUSDELN, L. A., VONWITSCH, W., and WRIGHT, C. S., "Architecture of the MEGA Detector Trigger," Nucl. Inst. and Meth. A372, 195 (1996)

CHURCH, E. D., "Measurements of the Reactions ${ }^{12} \mathrm{C}$ (numu,mu-) ${ }^{12} \mathrm{~N}_{\mathrm{gs}}$ and ${ }^{12} \mathrm{C}\left(\mathrm{nu}_{\mathrm{mu}}, \mathrm{mu}^{-}\right) \mathrm{X}$," In Proceedings of the 6 th Conference on Intersections Between Particle and Nuclear Physics, 570-572 (1997)

CRAWFORD, B. E., BOWMAN, J. D., FRANKLE, C. M., HASEYAMA, T., MASAIKE, A., MATSUDA, Y., PENTTILA, S. I., ROBERSON, N. R., SEESTROM, S. J., SHARAPOV, E. I., and STEPHENSON, S. L., "Development of Apparatus for Capture-g Studies of Parity Violation at Los Alamos," In Proceedings of the Fourth International Seminar on NeutronNucleus Interactions (JINR, 1996) 268

CRAWFORD, B. E., BOWMAN, J., DELHEIJ, P., FRANKLE, C. M., IINUMA, M., KNUDSON, J. N., LOWIE, L. Y., MASAIKE, A., MATSUDA, Y., MITCHELL, G., PENTTILA, S. I., POSTMA, H., ROBERSON, N., SEESTROM, S. J., SHARAPOV, E. I., STEPHENSON, S. L., YEN, Y, and YUAN, V. W., "Parity Violation in Neutron Resonances in ${ }^{238} \mathrm{U}$," Phys. Rev. C 58, 2, 1225-1235 (1998)

CRAWFORD, B. E., BOWMAN, J., DELHEIJ, P., HASEYAMA, T., KNUDSON, J. N., LOWIE, L. Y., MASAIKE, A., MATSUDA, Y., MITCHELL, G. E., PENTTILA, S. I., POSTMA, H., ROBERSON, N., SEESTROM, S. J., SHARAPOV, E. I., STEPHENSON, S. L., and YUAN, V. W., "Neutron Resonance Spectroscopy of ${ }^{106 P d}$ and ${ }^{108} \mathrm{Pd}$ from $20-2000 \mathrm{eV}$," Phys. Rev. C $\mathbf{5 8 , 2}, \mathbf{7 2 9 - 7 3 8}$ (1998)

CSOTO, A., GIBSON, B. F., and PAYNE, G. L., "Parity Conserving g Asymmetry in n-p Radiative Capture," Physical Review C 56, 2, 631-634 (1997) 
DELHEIJ, P. P. J., BOWMAN, J. D., FRANKLE, C. M., HAASE, D. G., LANGSTON, T., MORTENSEN, R., PENTTILA, S., POSTMA, H., SEESTROM, S. J., and YEN, Y.-F., "The TRIPLE Spin Filter," Nucl. Instrum. Methods A356, 120 (1995)

DEVINE, R. T., WALKER, S., STAPLES, P., MUNDIS, R., MILLER, J., and DURAN, M., "Etched Track Dosemeter Response to High Energy Neutrons," Radiation Protection Dosimetry 66, 1-4, 349-352 (1996)

DEVINE, R. T., WALKER, S., STAPLES, P., MUNDIS, R., MILLER, J., and DURAN, M., "Track Etch Dosimeter Response to Neutrons up to $300 \mathrm{MeV}$," In Proceedings of the 1996 International Congress on Radiation Protection 4, 398 (1996)

DIETRICH, F. S., ABFALTERER, W., HAIGHT, R. C., MORGAN, G. L., BATEMAN, F. B., and FINLAY, R. W., "Recent Measurements of Neutron Total Cross Sections on a Wide Range of Targets from 5 to $600 \mathrm{MeV}$," In Proceedings of the International Conference on Nuclear Data for Science and Technology, 402-406 (1997)

DIETRICH, F. S., and BECKER, J. A., "The GEANIE Project at Los Alamos LANSCE/WNR Facility," Summary Report of the 2nd Research Coordination Meeting on Measurement, Calculation, and Evaluation of Photon Production Data (LAEA, 1996)

EBRAHIM, A. A., and PETERSON, R. J., "Parametrization of PionNucleon Phase Shifts and Effects Upon Pion-Nucleus Scattering Calculations," Physical Review C 54, 5, 2499-2508 (1996)

EDWARDS, C. M., GAULARD, C. V., COMFORT, J. R., AMANN, J. F., BEDDO, M. E., BOUDRIE, R. L., BURLESON, G. R., COLE, P. L., CRAIG, K. K., ESPY, M. A., ISENHOWER, L. D., KASPRZYK, T. E., KNIGHT, K. R., MORRIS, C. L., PENTTILA, S., RIGSBY, D., SADLER, M. E., SIX, E., SPINKA, H. M., SUPEK, I, WAGNER, G. J., and ZHAO, Q., "Analyzing Powers for the $\pi$ " $\rightarrow \pi$ n Reaction Across the $\Delta$ (1232) Resonance" (World Scientific, 1997) 289

ESPY, M. A., BLANCHARD, S. P., BRASH, J. E., BRINKMOLLER, B., BURLESON, G. R., CUMMINGS, W. J., DAVIS, B. J.,

DEHNHARD, D., DELHEIJ, P. P. J., EDWARDS, C. M., HAUSSER, O., HENDERSON, R., JENNINGS, B. K., JONES, M. K., LAIl, B. A., LANGENBRUNNER, J. L., LARSON, B., LORENZEN, W., MAEDA, K., MORRIS, C. L., NELSON, B., O'DONNELL, J. M., PALARCZYK, M. A., PARK, B. K., PENTTLLA, S. I., SWENSON, D. R., THIESSEN, D., TUPA, D., and ZHAO, Q., "Elastic p ${ }^{+}$ Scattering on Polarized 3He," In Proceedings of the 8th International Symposium on Polarization Phenomena in Nuclear Physics, Stephenson, E. J., and Bigdor, J., Eds. (AIP, 1995) 339, 349

ESPY, M. A., DEHNHARD, D, EDWARDS, C. M., PALARCZYK, M. A., LANGENBRUNNER, J. L., DAVIS, B., BURLESON, G. R., BLANCHARD, S. P., GIBBS, W. R., LAIL, B., NELSON, B., PARK, B. K., ZHAO, Q., CUMMINGS, W. J., DELHEIJ, P. P. J., JENNINGS, B. K., HENDERSON, R., HAUSSER, O., THIESSEN, D., BRASH, J. E., JONES, M. K., LARSON, B., BRINKMOLLER, B., MAEDA, K., MORRIS, C. L., O'DONNELL, J. M., PENTTILA, S. I., SWENSON, D. R., TUPA, D., BENNHOLD, C., and KAMALOV, S. S., "Asymmetries for Elastic Scattering of $\mathrm{p}^{+}$from Polarized ${ }^{3} \mathrm{He}$ and the $\Delta$-neutron Spin-Spin Interaction," Phys. Rev. Lett. 76, 3667 (1996)
ESPY, M. A., O'DONNELL, J. M., DAVIS, B., DEHNHARD, D., LANGENBRUNNER, J. L., LARSON, B., PALARCZYK, M., RIEDEL, C. M., WICK, K., BLANCHARD, S., BURLESON, G. R., GIBBS, W. R., LAIL, B., NELSON, B., PARK, B. K., ZHAO, Q., CUMMMNGS, W. J., DELHEIJ, P. P. J., JENNINGS, B. K., HAUSSER, O., HENDERSON, R., THIESSEN, D., BRASH, E., JONES, M. K., BRINKMOLLER, B., MAEDA, K., MERRULL, F., MORRIS, C. L., PENTTILAE, S., SWENSON, D., TUPA, D., BENNHOLD, C., and KAMALOV, S. S., "Asymmetries for Elastic Scattering of $\mathrm{pi}^{\mathrm{i} / \text { - }}$ from Polarized ${ }^{3} \mathrm{He}$ at Delta Resonance Energies," Physical Review C 56, $2607-2620$ (1997)

FABRIS, D., NEBBIA, G., VIESTI, G., LUNARDON, M., CINAUSERO, M., FIORETTO, E., NAPOLI, D. R., PRETE, G., HAGEL, K., NATOWITZ, J. B., WADA, R., GONTHIER, P., MAJKA, Z., ALFARO, R., ZHAO, Y., MDEIWAYEH, N., and HO, T., "Energy Deposition in ${ }^{209} \mathrm{Bi}(\mathrm{a}, \mathrm{a} 1)$ Reactions at $60 \mathrm{~A}$ MeV," Bull. Am. Phys. Soc. 42, 7, 1687 (1997)

FEDERSPIEL, F., "Results of the LSND Search for $\mathrm{nu}_{\mathrm{mu}}$ to $\mathrm{nu}_{\mathrm{e}}$ Oscillation," In Proceedings of the XXXI Rencontres de Moriond: Electroweak Interactions and Unified Theories, 271-276 (1996)

GISLER, G., and CHEN, M.-L., "Solar Physics with the MILAGRO Telescope," In Proceedings of the International Cosmic-Ray Conference 4, 12308 (1995)

GREENE, M. A., AKDOGAN, T., DA GRACA, J., KWOK, L. L., MATTHEWS, J. L., SAFKAN, Y. N., SCHMITT, W. M., ZELEVINSKY, V. V., GRAM, P. A., TADDEUCCI, T. N., WENDER, S., and PATE, S. F., "Measurement of the Differential Cross Section for Pion Production in the Neutron-Proton System," Bull. Am. Phys. Soc. 43, 6, 1580 (1998)

GRIMES, S. M., BRIENT, C. E., GOECKNER, F. C., BATEMAN, F. B., CHADWICK, M. B., HAIGHT, R. C., LEE, T. M., STERBENZ, S. M., YOUNG, P. G., WASSON, O. A., and VONACH, H., "The ${ }^{59} \mathrm{Co}(\mathrm{n}$,alpha) Reaction from 5 to $50 \mathrm{MeV}$," Nuclear Science Engineering 124, 271 (1996)

GULLEY, M. S., KEATING, P. B., BRYANT, H. C., MACKERROW, E. P., MILLER, W. A., RISLOVE, D. C., COHEN, S., DONAHUE, J. B., FITZGERALD, D. H., FRANKLE, S. C., FUNK, D. J., HUTSON, R. L., MACEK, R. J., PLUM, M. A., STANCIU, N. G., VAN DYCK, O. B., WILKINSON, C. A., and PLANNER, C. W., "Measurement of $\mathrm{H}^{-}, \mathrm{H}^{0}$, and $\mathrm{H}^{+}$Yields Produced by Foil Stripping of $800-\mathrm{MeV}$ $\mathrm{H}^{-}$Ions," Physical Review A 53, 5, $3201-3210$ (1996)

GULLEY, M. S., ZHAO, X. M., STRAUSS, C. E. M., FUNK, D. J., KYRALA, G. A., INGALLS, W. B., BRYANT, H. C., RISLOVE, D. C., MILLER, W. A., and STINTZ, A., "NonResonant Two-Photon Detachment of $\mathrm{H}^{-}$Ions with $1.17 \mathrm{eV}$ Photons;" Bull. Am. Phys. Soc. 42, 985 (1997)

GULLEY, M., ZHAO, X. M., BRYANT, H. C., STRAUSS, C. E. M., FUNK, D. J., STINTZ, A., RISLOVE, D. C., KYRALA, G. A., INGALLS, W. B., MILLER, W. A., and BILLINGE, S. J. L., "Excess Photon Detachment of $\mathrm{H}^{-}$Ions with 1.17 EV Photons," Bull. Am. Phys. Soc. 44, 1086 (1996) 
HAGBERG, E., TOWNER, I. S., HARDY, J. C., KOSLOWSKI, V. T., SAVARD, G., and STERBENZ, S., "Beta Decays of Vanadium-44 and Cobalt-52," Nuclear Physics A 613, 183-198 (1997)

HAIGHT, R. C., "Charged Particles Produced in Neutron Reactions on Nuclei from Beryllium to Gold," In Proceedings of the International Conference on Nuclear Data for Science \& Technology 1, 500-504 (1997)

HAIGHT, R. C., BATEMAN, F. B., GRIMES, S. M., BRIENT, C. E., MASSEY, T. N., WASSON. O. A., CARLSON, A. D., and ZHOU, H., "Measurement of the Angular Distribution of Neutron-Proton Scattering at $10 \mathrm{MeV}$," Fusion Engineering and Design 37, 49-56 (1997)

HAIGHT, R. C., BATEMAN, F. B., STERBENZ, S. M., CHADWICK, M. B., YOUNG, P. G., GRIMES, S. M., WASSON, O. A., MAIER-KOMOR, P., VONAHC, H., REFFO, G., VENTURA, A., and GRANDI, C., "The $58,60 \mathrm{Ni}$ (n,xalpha) Reactions from Threshold to $50 \mathrm{MeV}$," In Proceedings of the International Conference on Nuclear Data for Science and Technology (Italian Phys. Soc., 1997) 1, 603-606

HAIGHT, R. C., BATEMAN, F. B., STERBENZ, S. M., CHADWICK, M. B., YOUNG, P. G., GRIMES, S. M., WASSON, O. A., VONACH, H., and MAIER-KOMOR, P., "The $58,60 \mathrm{Ni}$ (n,alpha) Reaction from Threshold to $50 \mathrm{MeV}$," Bull. Am. Phys. Soc. 41, 5, 1235 (1996)

HAIGHT, R. C., BATEMAN, F. B., STERBENZ, S. M., GRIMES, S. M., WASSON, O. A., MAIER-KOMOR, P., and VONACH, H., "An Update on (n,charged particle) Research at WNR," Fusion Engineering and Design 37, 73-77 (1997)

HAIGHT, R. C., KNEFF, D. W., OLIVER, B. M., GREENWOOD, L. R., and VONACH, H., "Helium Production by $10 \mathrm{MeV}$ Neutrons in Elemental Iron, Nickel, and Copper, and in ${ }^{56 \mathrm{Fe}}$ and ${ }^{58,60,61} \mathrm{Ni}$," Nucl. Sci. Eng. 124, 219 (1996)

HOGAN, G. E., AHMED, M., AMANN, J. F., BARLOW, D., BLACK, K., BOLTON, R. D., BROOKS, M., CARIUS, S., CHEN, Y., CHERNYSHEV, A., COOPER, M. D., COOPER, P. S., CROCKER, J., DZEMIDZIC, M., EMPL, A., FISK, R. J., FLICK, J., FOREMAN, W., GAGLIARDI, C. A., HAIM, D., HALLIN, A., HARRISON, R., HART, G., HOFFMAN, C. M., HUGHES, E. B., HUNGERFORD, E. V., III, JOHNSTON, K., JUI, C., KIM, G. J., KNOTT, J. E., KOETKE, D. D., KROUPA, M. A., KOZLOWSKI, T., LAN, K., LAPTEV, V., LEE, D., LEE, F. S., LIU, F., MANWEILER, R., MARSHALL, R., MAYES, B. W., II, MISCHKE, R. E., NAIVAR, F. J., NEFKENS, B. M. K., NOVAK, J.. OOTHOUDT, M. A., OTIS, J. N., PHELPS, R., PILONEN, L. E., PILLAI, C., PINSKY, L., PRICE, J., RITTER, M. W., SCHILLING, S., STANISLAUS, T. D. S., STANTZ, K. M., SZYMANSKI, J. J., STEPHENS, W., STURROCK, J., TANG, L., TIPPENS, B., TRIBBLE, R. E., TU, X.-L., VAN AUSDELN, L. A., VONWITSCH, W., WHITEHOUSE, D., WILKINSON, C., WRIGHT, S. C., ZHANG, Y., ZHOU, X-G., ZIOCK, K. O. H., AJDUK, Z., and WROBLEWSKI, A. K., "A Study of the Decay mu to e gamma by the MEGA Experiment," In Proceedings of the 28th International Conference on High-Energy Physics (World Scientific, 1997) 2, 1450-1452
HORIKAWA, Y. and TANAKA, Y., "Charge Conservation for Nonrelativistic RPA Theory," Phys. Lett. B 409, 1 (1997)

HSU, H.-H., and DEVINE, R. T., "Neutron Charged Particle Reactions in CR-39 Using MCNP and LAHET," In Proceedings of the 30th Midyear Topical Meeting: Health Physics of Radiation-Generating Machines, 181 (1997)

HUGHES, R. J., BUTTLER, W. T., KWIAT, P. G., LUTHER, G. G., MORGAN, G. L., NORDHOLT, J. E., PETERSON, C. G., and SIMMONS, C. M., "Secure Communications Using Quantum Cryptography," In Proceedings of the SPIE-The International Society for Optical Engineering (SPIE, 1997) 3076, 2-11

HUGHES, R. J., GULLEY, M. S., HOLZSCHEITER, M. H., KWIAT, P. G., LAMOREAUX, S. K., PETERSON, C. G., SANDBERG, V. D., SCHAUER, M. M., THORNBURN, C., TUPA, D., GOMEZ, J. J., and SIMMONS, C. M., "The Los Alamos Trapped Ion Quantum Computer Experiment," Fortschritte der Physik 46, 4-5, 329-261 (1998)

IMLAY, R. L., "The LSND Experiment," In Proceedings of the International Conference on Nuclear Physics, 679-689 (1996)

JOHNS, G. D., NELSON, R. O., WILBURN, W. S., ARCHER, D. E., BECKER, J. A., BERNSTEIN, L. A., STOYER, M. A., and YOUNES, W., "Excitation Functions for ${ }^{238} \mathrm{U}(\mathrm{n}, \mathrm{xng})$ Reactions from eN=1-30 MeV," Bull. Am. Phys. Soc. 42, 1687 (1997)

JOHNSON, M. B., AUERBACH, N., and BOWMAN, J. D., "Model-Space Approach to Parity Violation in Heavy Nuclei," In Proceedings of the International Workshop on Parity and Time Reversal Violation in Compound Nuclear States and Related Topics (World Scientific, 1996) 253-262

JOHNSON, M. B., and BOWMAN, J. D., "Microscopic, ModelSpace Approach to Parity Nonconservation in Compound Nuclei," Physical Review C 51, 999 (1995)

KAHRIMANIS, G., BURLESON, G., CHEN, C. M., CLARK, B. C., DHUGA, K., ERNST, D. J., FAUCETT, J. A., FORTUNE, H. T., HAMA, S., HUSSEIN, A., JIANG, M. F., JOHNSON, K. W. R., KERR, L., MATHEWS, S., McGILL, J., MOORE, C. F., MORDECHAI, S., MORRIS, C. L., O'DONNELL, J., SNELL, M., RAWOOL-SULLIVAN, M., RAY, L., WHITLEY, C. and WILLIAMS, A., "Pion-Nucleus Elastic Scattering on ${ }^{12} \mathrm{C}$, ${ }^{40} \mathrm{Ca},{ }^{90} \mathrm{Zr}$, and ${ }^{208} \mathrm{~Pb}$ at 400 and $500 \mathrm{MeV}$," Physical Review $C$ 55, 2533-2540 (1997)

KAPUSTINSKY, J. S., BOISSEVAIN, J. G., ZIOCK, H. J., MUCK, R. C., SMITH, G. D., and WONG-SWANSON, B. G., "Multichip Module Technology Development," DOE Office of Scientific and Technical Information Report DE98000074 (1997)

KEATING, P. B., GULLEY, M. S., BRYANT, H. C., MACKERROW, E. P., MILLER, W. A., RISLOVE, D. C., COHEN, S., DONAHUE, J. B., FITZGERALD, D. H., FRANKLE, S. C., FUNK, D. J., HUTSON, R. L., MACEK, R. J., PLUM, M. A., STANCIU, N. G., VAN DYCK, O. B., and WILKINSON, C. A., "Electric-Field-Induced Detachment of 800 MeV H Ions," Physical Review A 52, 4547 (1995) 
KEATING, P. B., GULLEY, M. S., BRYANT, H. C., MACKERROW, E. P., MILLER, W. A., RISLOVE, D. C., COHEN, S., DONAHUE, J. B., FITZGERALD, D. H., FUNK, D. J., FRANKLE, S. C., HUTSON, R. L., MACEK, R. J., PLUM, M. A., STANCIU, N. G., VAN DYCK, O. B., and WILKINSON, C. A., "Field Ionization of $800 \mathrm{MeV} \mathrm{H}^{-}$ Ions," In Proceedings of the XIX International Conference on the Physics of Electronic and Atomic Collisions, 674 (1995)

KEATING, P. B., GULLEY, M. S., BRYANT, H. C., MACKERROW, E. P., MILLER, W. A., RISLOVE, D. C., COHEN, S., DONAHUE, J. B., FITZGERALD, D. H., FUNK, D. J., FRANKLE, S. C., HUTSON, R. L., MACEK, R. J., PLUM, M. A., STANCIU, N. G., VAN DYCK, O. B., and WILKINSON, C. A., "Field-Assisted Start State $\mathrm{H}^{0}$ Production by $800 \mathrm{MeV} \mathrm{H}^{-}$Ions Passing Through an $\mathrm{A} 12 \mathrm{O} 3$ Foil," In Proceedings of the XIX Intemational Conference on the Physics of Electronic and Atomic Collisions, 675 (1995)

KEATING, P. B., GULLEY, M. S., BRYANT, H. C., MACKERROW, E. P., MILLER, W. A., RISLOVE, D. C., COHEN, S., DONAHUE, J. B., FITZGERALD, D. H., FUNK, D. J., FRANKLE, S. C., HUTSON, R. L., MACEK, R. J., PLUM, M. A., STANCIU, N. G., VAN DYCK, O. B., and WILKINSON, C. A., "Magnetic-Field Enhancement of $\mathrm{m}-0$ States Produced in the Interaction of $800-\mathrm{MeV}$ $\mathrm{H}^{-}$Ions with an $\mathrm{Al}_{2} \mathrm{O}_{3}$ Foil," Bull. Am. Phys. Soc. 44, 1144 (1996)

KEITH, C. D., CHOWDHURI, Z., RICH, D. R., SNOW, W. M., BOWMAN, J. D., PENTTILA, S. I., SMITH, D. A., LEUSCHNER, M. B., POMEROY, V. R., SHARAPOV, E. I., and JONES, G. L., "Measurement of the Neutron Total Cross Section of ${ }^{3} \mathrm{He}$ in the Energy Range 0.1-500 eV," Bulletin of the American Physical Society 42, 1686 (1997)

KETLEROV, V. V:; GOVERDOVSKI, A. A., KHRYACHKOV, V. A., MITROFANOV, V. F., OSTAPENKO, Y. B., HAIGHT, R. C., KOEHLER, P. E., GRIMES, S. M., and SMITH, R. S., "Detailed Study of Double Differential Cross-Sections for the ${ }^{17} \mathrm{O}\left(\mathrm{n}\right.$,alpha) ${ }^{14} \mathrm{C}$ Reaction," Nuclear Physics A 621, 243-246 (1997)

KHRIPLOVICH, I. B., CHAICHIAN, M., HUITU, K., and ORAVA, R., "CP-Violation Without Strangeness or Electric Dipole Moments of a Neutron, Nuclei, Atoms, Molecules" (World Scientific, 1995) 214-220

KIM, H. J., "Evidence for Neutrino Oscillations from the LSND Experiment," In Proceedings of the International Workshop on Dark Matter in Astro-and Particle Physics, 665-669 (1996)

KIM, H. J., "Inclusive and Exclusive nue and numuC Cross Section Measurements from LSND," In Proceedings of the 14th International Conference on Particles and Nuclei, 583-584 (1996)

KNUDSON, J. N., BOWMAN, J. D., CRAWFORD, B. E., DELHEI, P. J., FRANKLE, C., GOULD, C. R., HAASE, D. G., ILNUMA, M., LOWIE, L., MASAIKE, A., MASUDA, Y., MATSUDA, Y., MITCHELL, G. E., PENTTILA, S. I., POSTMA, H., ROBERSON, N. R., SEESTROM, S. J., SHARAPOV, E. I., SHIMIZU, H., STEPHENSON, S. L., YEN, Y.-F., YUAN, V. W., and YOO, S. H., "A High-Rate Detection System to Study Parity Violation with Polarized Epith Neutrons at LANSCE," Journal of Neutron Res. 4, 209 (1996)
KRUSIN-ELBAUM, L., LOPEZ, D., THOMPSON, J. R., WHEELER, R., ULLMANN, J., CHU, C. W., and LIN, Q. M., "Superconductivity Enhanced by Hg Fission," Nature 389, 243 (1997)

KRUSIN-ELBAUM, L., LOPEZ, D., THOMPSON, J. R., WHEELER, R., ULLMANN, J., TSUEI, C. C., CHU, C. W., and LIN, Q. M., "A Path to High-Field Performance of Cuprate Superconductors Above 100K With Fission-Induced Defects," Physica C 282-287, 375-378 (1997)

LEE, W., "A Preliminary Measurement of the u-bar/d-bar Asymmetry in the Proton Sea," In Proceedings of the Intersections Between Particle and Nuclear Physics (AIP, 1997) 412, 643

LEE, W., "Measuring the u-bar/d-bar Asymmetry in the Proton Sea: Fermilab E866," In Proceedings of the '97 QCD and High Energy Hadronic Interactions (Editions Frontieres, 1997) 387

LEE, W. M., "d-bar/u-bar Asymmetry and the Origin of the Nucleon Sea," Phys. Rev. D 58 (1998)

LEE, W. M., "Measurement of the Light Antiquark Flavor Asymmetry in the Nucleon Sea," Phys. Rev. Lett. 80, 3715 (1998)

LOUIS, W. C., "LSND Neutrino Oscillations Results," In Proceedings of the First International Conference on Particle Physics Beyond the Standard Model, 923-930 (1997)

LOUIS, W. C., "LSND Neutrino Oscillations Results," In Proceedings of the Orbis Scientiae: Neutrino Mass, Dark Matter, Gravitational Waves, 103-109 (1996)

LOUIS, W. C., "LSND Neutrino Oscillations Results," In Proceedings of the Rencontres de Blois: Neutrinos, Dark Matter and the Universe, 67-74 (1996)

LOUIS, W. C., "Neutrino Oscillation Studies at LAMPF," Nuclear Physics B 38, 229-234 (1995)

LOWIE, L. Y., BOWMAN, J. D., CRAWFORD, B. E., DELHEIJ, P. P. J., HASEYAMA, T., KNUDSON, J. N., MASAIKE, A., MASUDA, Y., MATSUDA, Y., MITCHELL, G. E., PENTTILA, S., POSTMA, H., ROBERSON, N. R., SEESTROM, S. J., SHARAPOV, E. I., STEPHENSON, S. L., YEN, Y.-F., and YUAN, V. W., "Neutron Resonance Spectroscopy of ${ }^{107} \mathrm{Ag}$ and ${ }^{109} \mathrm{Ag}$," Physical Review C 56, 90 (1997)

MACFARLANE, R. E., CHADWICK, M. B., REFFO, G., VENTURA, A., and GRANDI, C., "Working with 150-MeV Evaluations: NJOY and KERMA," In Proceedings of the International Conference on Nuclear Data for Science and Technology (Italian Phys. Soc., 1997) 2, 1443-1445

MCKEE, W. R., MC ADAMS, H. P., SMITH, E. B., MC PHERSON, J. W., JANZEN, J. W., ONDRUSEK, J. C., HYSLOP, A. E., RUSSELL, D. E., COY, R. A., BERGMAN, D. W., NGUYEN, N. Q., ATON, T. J., BLOCK, L. W., and HUYNH, V. C., "Cosmic Ray Neutron Induced Upsets as a Major Contributor to the Soft Error Rate of Current and Future Generation DRAMs," In Proceedings of the 1996 IEEE International Reliability Physics Symposium (IEEE, 1996) 1-6 
MEADOWS, J. W., SMITH, D. L., GREENWOOD, L. R., HAIGHT, R. C., IKEDA, Y., and KONNO, C., "Measurement of Fast-Neutron Activation Cross Sections for Copper, Europium, Hafnium, Iron, Nickel, Silver, Terbium, and Titanium at 10.0 and $14.7 \mathrm{MeV}$ and for the Be(d,n) Spectrum," Annals of Nuclear Energy 23, 877-899 (1996)

MITCHELL, G. E., BOWMAN, J. D., CRAWFORD, B. E., DELHEIJ, P. P. J., HASEYAMA, T., KNUDSON, J. N., LOWIE, L. Y., MASAIKE, A., MASUDA, Y., MATSUDA, Y., PENTTILA, S., POSTMA, H., ROBERSON, N. R., SEESTROM, S. J., SHARAPOV, E. I., STEPHENSON, S. L., YEN, Y.-F, and YUAN, V. W., "Parity Violation with Epithermal Neutrons," In Proceedings of the Ninth International Conference on Capture Gamma-Ray Spectroscopy 616 (1997)

MORRIS, C. L., ARMIJO, V., ATENCIO, L. G., BRIDGE, A., GAVRON, A., HART, G. W., MORLEY, K B., MOTTERSHEAD, C. T., YATES, G. J., and ZUMBRO, J. D., "An Integrating Image Detector for High Energy Neutrons," In Proceedings of the 5th International Conference on Neutrons in Research and Industry (SPIE, 1997) 2867, 351-357

NAVRATIL, G. A., CATES, C., MAUEL, M. E., MAURER, D., NADLE, D. L., TAYLOR, E., XIAO, Q., WURDEN, G. A., and REASS, W. A., "Active Control of $2 / 1$ Magnetic Islands in the HBT-EP Tokamak," In Proceedings of the 39th Annual Meeting of the Division of Plasma Physics of the American Physical Society (AIP, 1998) 5, 1855-1863

NELSON, R. O., DRAKE, D. M., JOHNS, G. D., WILBURN, W. S., ARCHER, D. E., BECKER, J. A., BERNSTEIN, L. A:, STOYER, M. A., YOUNES, W., YATES, S. W., and GARRETT, P. E., "Nuclear Physics with GEANIE at LANSCE/WNR," Bull. Am. Phys. Soc. 42, 1686 (1997)

NELSON, R. O., JOHNS, G. D., WILBURN, W. S., DRAKE, D. M., RUNDBERG, R. S., ARCHER, D. E., BECKER, J. A., BERNSTEIN, L. A., YOUNES, W., HAUSCHILD, K., MITCHELL, G. E., ROBERSON, N. R., YATES, S. W., and GARRETT, P. E., "Performance and Status of GEANIE," Bull. Am. Phys. Soc. 42, 1072 (1997)

NGUYEN, D., SHEFFIELD, R., FORTGANG, C. M., GOLDSTEIN, J. C., KINROSS-WRIGHT, J. M., and EBRAHIM, A. A., "Self-Amplified Spontaneous Emission Driven by a HighBrightness Electron Beam," Phys. Rev. Lett. 84, 4, 810 (1998)

OSSANDON, J. G. and THOMPSON, J. R., "Stability of Supercurrents in $\mathrm{BiSrCaCuO}(\mathrm{Bi}-2212)$ High- $\mathrm{T}_{\mathrm{c}}$ Superconductor with Artificially Created Defects," Superlattices and Microstructures 23, 543 (1998)

PARK, H. T., MATTHEWS, J. L., PATE, S. F., AMANN, J. F., MORRIS, C. L., WHITTON, R. M., KINNEY, E. R., MERTZ, C., and REDMON, J., "Pion-Induced Single Charge Exchange in Deuterium," Physical Review C 51, R1613 (1995)

PATE, S. F., FONG, W., HARVEY, M. T., MATTHEWS, J. L., PARK, H. T., VIDOS, L. L., ZELEVINSKY, V. V., SAUNDERS, A., HOLCOMB, M. D., KINNEY, E. R., KRISS, B. J., GRAM, P. A. M., and ROBERTS, D. A., "Two-Nucleon Processes in Pion-Induced DoubleCharge-Exchange in ${ }^{4} \mathrm{He}$ : A Coincidence Measurement of the ${ }^{4} \mathrm{He}\left(\mathrm{pi}^{+}, \mathrm{pi}^{-}\right.$ p) 3p Reaction," In Proceedings of the XIV International IUPAP Conference on Few Body Problems in Physics (AIP, 1995) 334, 542
PAVLIK, A., HITZENBERGER-SCHAUER, H., VONACH, H., CHADWICK, M. B., HAIGHT, R. C., NELSON, R. O., and YOUNG, P. G., "27Al(n,xgamma) Reactions for Neutron Energies from 3 to $400 \mathrm{MeV}$," Physical Review C 57, 2416 (1998)

PENTTILA, S., BOWMAN, J. D., DELHEIJ, P. P. J., FRANKLE, C. M., HAASE, D. G., POSTMA, H., SEESTROM, S. J., and YEN, Y.-F., "A Neutron Beam Polarizer for Study of Parity Violation in Neutron-Nucleus Interactions," In Proceedings of the Ilth International Symposium on High Energy Spin Nuclear Physics 343, 532-539 (1995)

PETERSON, R. J., EBRAHIM, A. A., BHANG, H. C., "Observable Sensitivities to Scaling of K+-Nucleon Interactions Within Nuclei," Nuclear Physics A 625, 1-2, 261-271 (1997)

PILING, S., and CHEN, M.-L., "An Experiment to Detect Correlations Between Cherenkov and Muon Lateral Distributions," In Proceedings of the International Cosmic-Ray Conference 3, 408 (1995)

POSTMA, H., BOWMAN, J. D., CORVI, F., CRAWFORD, B. E., DELHEIJ, P. P. J., GUNSING, F., HASEYAMA, T., KNUDSON, J. N., LOWIE, L. Y., MASAIKE, A., MASUDA, Y., MATSUDA, Y., MITCHELL G. E., PENTTLLA, S., ROBERSON, N. R., SEESTROM, S. J., SHARAPOV, E. I., SHIMIZU, H. M., STEPHENSON, S. L., YEN, Y.-F., YUAN, V. W., and ZANINI, L., "Some Aspects of the Analysis of Parity Violation Effects Observed at Neutron p-wave Resonances," In Proceedings of the Fourth International Seminar on Neutron-Nucleus Interactions 101 (1996)

POSTMA, H., BOWMAN, J. D., CRAWFORD, B. E., CORVI, F., DELHEIJ, P. P. J., GUNSING, F., HASEYAMA, T., KNUDSON, J. N., LOWIE, L. Y., MASSAKE, A., MASUDA, Y., MATSUDA, Y., MITCHELL G. E., PENTTILA, S., ROBERSON, N. R., SEESTROM, S. J., SHARAPOV, E. I., STEPHENSON, S. L., YEN, Y.-F., and YUAN, V. W., "Parity Violation Effects Observed at Neutron p-wave Resonances, Their Analysis and Interpretation," In Proceedings of the North-West Europe Nuclear Physics Conference B5, 55 (1996)

PRIESMEYER, H. G., STALDER, M., MEGGERS, K., and TRELA, W., "Progress in Single-Shot Neutron Transmission Diffraction," In Proceedings of the International Conference: Neutrons in Research and Industry (SPIE, 1996) 2867, 164-167

PROUT, D. L., DELUCIA, S., COOPER, D., LUTHER, B., SUGARBAKER, E., TADDEUCCI, T. N., RYBARCYK, L. J., RAPAPORT, J., PARK, B. K., GOODMAN, C. D., EDWARDS, G., GLASHAUSSER, C., SAMS, T., UDAGAWA, T., and OSTERFELD, F., "Spin Decomposition of the Delta Resonance Cross Section Using the ${ }^{12} \mathrm{C}(\mathrm{p}, \mathrm{n})$ Reaction at Ep=795 MeV," Phys. Rev. Lett. 76, 4488 (1996)

PROUT, D. L., ZAFIRATOS, C., TADDEUCCI, T. N., ULLMANN, J., BYRD, R. C., CAREY, T. A., LISOWSKI, P., MCCLELLAND, J. B., RYBARCYK, L. J., SAILOR, W., AMIAN, W., BRAUNSTEIN, M., LIND, D., MERCER, D. J., COOPER, D., DELUCIA, S., LUTHER, B., MARCHLENSKI, D. G., SUGARBAKER, E., RAPAPORT, J., PARK, B. K., GULMEZ, E., WHITTEN, C. A., GOODMAN, C. D., HUANG, W., CISKOWSKI, D., and ALFORD, W. P., "Cross Sections and Analyzing Powers for Quasielastic Scattering at 795 and 495 MeV Using the (p,n) Reaction," Phys. Rev. C 52, 228 (1995) 
RICH, D. R., KEITH, C. D., SNOW, W. M., BOWMAN, J. D., PENTTILA, S., SMITH, D. A., LEUSCHNER, M. B., POMEROY, V. R., HASEYAMA, T., MASAIKE, A., and JONES, G. L., "Accurate Absolute Measurements of the Neutron Polarization Obtained Via Transmission Through Polarized ${ }^{3} \mathrm{He}$ Spin Filters at LANSCE," Bulletin of the American Physical Society 42, 1647 (1997)

RISLOVE, D. C., BRYANT, H. C., GULLEY, M. S., STRAUSS, C. E. M., FUNK, D. J., MILLER, W. A., ZHAO, X. M., STINTZ, A., KYRALA, G. A., and INGALLS, W. B., "Isotope-Dependent Effects in an Autodetaching Resonance in Hydrogen Minus," In Proceedings of the Twentieth International Conference on the Physics of Electronic and Atomic Collisions, 23-29 (1997)

RISLOVE, D. C., BRYANT, H. C., MILLER, W. A., STINTZ, A., GULLEY, M. S., ZHAO, X. M., STRAUSS, C. E. M., FUNK, D. J., KYRALA, G. A., and INGALLS, W. B., "Resonant TwoPhoton Detachment in Negative Hydrogen Atoms," Bulletin of the American Physical Society 42, 1136 (1997)

RISLOVE, D. C., STRAUSS, C. E. M., FUNK, D. J., GULLEY, M. S., ZHAO, X. M., BRYANT, H. C., STINTZ, A., KYRALA, G. A., INGALLS, W. R., MILLER, W. A., "Detachment of $\mathrm{H}^{-}$ Using a Broadly-Tunable VUV Source," Bulletin of the American Physical Society 44, 1095 (1996)

RUSEK, A., BASSALLECK, B., BERDOZ, A., BURGER, T., BURGER, M., CHRIEN, R. E., DIEBOLD, G. E., EN'YO, H., FISCHER, H., FRANKLIN, G. B., FRANZ, J., IIJIMA, T., IMAI, K., LOWE, J., MAGAHIZ, R., MASAIKE, A., MEYER, C. A., MCCRADY, R., MERRILL, F., MIHARA, S., HELSON, J. M., OKADA, K., PILE, P. H., QUINN, B., ROSSLE, E., SAITO, N., SAWAFTA, R., SCHMITT, H., SCHUMACHER, R. A., STERNS, R. L., STOTZER, R., SUKATON, R., SUTTER, R., TAKEUTCHI, F., WOLFE, D. M., YAMAMOTO, K., YAMASHITA, S., YOKKAICHI, S., ZEPS, V., and ZYBERT, R., "Strangelet Search and Light Nucleus Production Relativistic Si+Pt and $\mathrm{Au}+\mathrm{Pt}$ Collisions," Physical Review C 54, 1, R15-19 (1996)

SAFAR, H., CHO, J. H., FLESHLER, S., MALEY, M. P., WILLIS, J. O., COULTER, J. Y., ULLMANN, J. L., LISOWSKI, P. W., RILEY, G. N., RUPICH, M. W., THOMPSON, J. R., and KRUSIN-ELBAUM, L., "Enhancement of Transport Critical Current Densities at $75 \mathrm{~K}$ in Bi-2223/Ag Tapes by Means of Fission Tracks from Irradiation by $0.8 \mathrm{GeV}$ Protons," Applied Physics Letters 67, 130 (1995)

SAFKAN, Y. N., AKDOGAN, T., DA GRACA, J., GREENE, M. A., KWOK, L. L., MATTHEWS, J. L., SCHMITT, W. M., ZELEVINSKY, V. V., GRAM, P. A., TADDEUCCI, T. N., WENDER, S., and PATE, S. F., "Measurement of the Differential Cross Section for Neutron-Proton Bremsstrahlung," Bull. Am. Phys. Soc. 43, 6, 1580 (1998)

SANDBERG, V., "Neutrino Cross Sections on Carbon," In Proceedings of the XVII International Conference on Neutrino Physics and Astrophysics, 164-168 (1996)
SEESTROM, S. J., ALDE, D., BOWMAN, J. D., CRAWFORD, B. E., DELHEIJ, P. P. J., FRANKLE, C. M., FUKUDA, K., GOULD, C. R., GREEN, A. A., HAASE, D. G., IINUMA, M., KNUDSON, J. N., LOWIE, L. Y., MASAIKE, A., MASUDA, Y., MITCHELL, G. E., PENTTILA, S., POPOV, Y. P., POSTMA, H., ROBERSON, N. R., SHARAPOV, E. I., SHIMIZU, H. M., STEPHENSON, S. L., YEN, Y-F., and YUAN, V. W., "New Results from Los Alamos in the Study of Parity Violation in Neutron Resonances," In Proceedings of the Second International Seminar on Neutron-Nucleus Interactions 50 (1995)

SEESTROM, S. J., BOWMAN, J. D., CRAWFORD, B. E., DELHEIJ, P. J., HASEYAMA, T., KNUDSON, J. N., LOWIE, L., MASAIKE, A., MATSUDA, Y., MITCHELL, G. E., PENTTILA, S., POSTMA, H., ROBERSON, N. R., SHARAPOV, E. I., STEPHENSON, S., YEN, Y. F., and YUAN, V., "Study of Parity Nonconservation with Epithermal Neutrons," In Proceedings of the International Conference on Neutrons in Research and Industry (SPIE, 1997) 2867, 388-397

SHARAPOV, E. I., BOWMAN, J. D., CRAWFORD, B. E., DELHEIJ, P. P. J., FRANKLE, C. M., FUKUDA, K., GOULD, C. R., GREEN, A. A., HAASE, D. G., IINUMA, M., KNUDSON, J. N., LOWIE, L. Y., MASAIKE, A., MASUDA, Y., MATSUDA, Y., MITCHELL, G. E., PENTTILA, S. I., POPOV, Y. P., POSTMA, H., ROBERSON, N. R., SEESTROM, S. J., SHIMIZU, H. M., STEPHENSON, S. L., YEN, Y.-F., and YUAN, V. W., "Parity Violation in the Compound Nucleus," In Proceedings of the Third International Seminar on NeutronNucleus Interactions (JINR, 1997) 27

SHARAPOV, E. I., BOWMAN, J. D., CRAWFORD, B. E., DELHEIJ, P. P. J., FRANKLE, C. M., FUKUDA, K., GOULD, C. R., GREEN, A. A., HAASE, D. G., IINUMA, M., KNUDSON, J. N., LOWIE, L. Y., MASAIKE, A., MASUDA, Y., MATSUDA, Y., MITCHELL, G. E., PENTTILA, S., POPOV, Y. P., POSTMA, H., ROBERSON, N. R., SEESTROM, S. J., SHIMIZU, H. M., STEPHENSON, S. L., YEN, Y.-F., OGANESSIAN, Y., KALPAKCHIEVA, R., VON OERTZEN, W., and YUAN, V. W., "Parity Violation in Neutron Resonances: The TRIPLE Collaboration Recent Results," In Proceedings of the 15th Divisional Conference on Low-Energy Nuclear Dynamics (World Scientific, 1995) 138-146

SHEN, B. C., "The Milagro Gamma Ray Observatory," In Proceedings of the International Workshop on New Worlds in Astroparticle Physics, Mourao, A. M., Pimenta, M., Potting, R., and Sonderegger, P., Eds. (1996) 255-262

SILK, J. D., BURLEIN, M., FORTUNE, H. T., INSKO, E., KARGARLIS, M., KUTT, P. H., O'DONNELL, J. M., ZUMBRO, J. D., FAUCETT, J. A., GARNETT, R., RAWOOL-SULLIVAN, M., MORRIS, C. L., and SAINI, S., "Nonanalog ${ }^{40,44} \mathrm{Ca}\left(\mathrm{p}^{+}, \mathrm{p}^{-}\right)^{40,44} \mathrm{Ti}$ (g.s.) Reactions at Low Energy," Physical Review C 51, 665 (1995)

SKOY, V. R., SHARAPOV, E. I., GUNDORIN, N. A., POPOV, Y. P., PROKOFICHEV, Y. V., ROBERSON, N. R., and MITCHELL, G. E., "Isotopic Identification of the ParityViolating Neutron p-wave Resonance at Energy $E_{0}=3.2 \mathrm{eV}$ in Xe," Physical Review C 53, 6, R2573-R2575 (1996) 
SMITH, D. A. and SMOTRITSKY, L. M., "Parity Violating Asymmetries in ${ }^{117}$ Sn," Bull. Am. Phys. Soc. 42, 1071 (1997)

SMITH, D., "Candidate Events in a Search for Neutrino Oscillations," In Proceedings of the International Workshop for Double Beta Decay, 240-255 (1995)

STANCU, I., "Evidence for Neutrino Oscillations from the LSND Experiment," In Proceedings of the First International Workshop on the Identification of Dark Matter, 487-492 (1996)

STANCU, I., "Evidence for Neutrino Oscillations," In Proceedings of the Rencontres de Moriond: Electroweak Interactions and Unified Theories, 497-504 (1995)

STANCU, I., "Searching for Neutrino Oscillations with LSND," In Proceedings of the International Symposium on Weak and Electromagnetic Interactions in Nuclei, 156-163 (1995)

STEPHENSON, S. L., BOWMAN, J. D., POSTMA, H., SEESTROM, S. J., and SHARAPOV, E. I., "Multiple Scattering Effect in (n,y) Resonance in 108Pd," In Proceedings of the Fourth Intemational Seminar on Neutron-Nucleus Interactions (JINR, 1996) 171

STEPHENSON, S. L., BOWMAN, J., CRAWFORD, B. E., DELHEIJ, P., FRANKLE, C. M., INUMA, M., KNUDSON, J. N., LOWIE, L. Y., MASAIKE, A., MATSUDA, Y., MITCHELL, G. E., PENTTILA, S. I., POSTMA, H., ROBERSON, N., SEESTROM, S. J., SHARAPOV, E. I., YEN, Y., and YUAN, V. W., "Parity Nonconservation in Neutron Resonances in 232Th," Phys. Rev. C 58, 2, 1236-1246 (1998)

STOTZER, R. W., BURGER, T., BARNES, P. D., BASSALLECK, B., BERDOZ, A. R., BURGER, M., CHRIEN, R. E., DAVIS, C. A., DIEBOLD, G. E., ENYO, H., FISCHER, H., FRANKLIN, G. B., FRANZ, J., GAN, L., GILL, D. R., IIJIMA, T., IMAI, K, KORAN, P., LANDRY, M., LEE, L., LOWE, J., MAGAHIZ R, MASAIKE, A., MCCRADY, R., MERRILL, F., MEYER, C. A., NELSON, J. M., OKADA, K., PAGE, S. A., PILE, P. H., QUINN, B. P., RAMSAY, W. D., ROSSLE, E., RUSEK, A., SAWAFTA, R., SCHMITT, H., SCHUMACHER, R. A., STEARNS, R. L., SUKATON, I. R., SUTTER, R., SZYMANSKI, J. J., TAKEUTCHI, F., VANOERS, W. T. H, WOLFE, D. M., YAMAMOTO, K., YOSOI, M., ZEPS, V. J., and ZYBERT, R., "Search for the $\mathrm{H}^{-}$Dibaryon in ${ }^{3} \mathrm{He}\left(\mathrm{K}, \mathrm{K}^{+}\right) \mathrm{Hn}$," Physical Review Letters 78, 19, 3646-49 (1997)

SZYMANSKI, J. J., SNOW, W. M., BOWMAN, J. D., CAIN, B., CRAWFORD, B. E., DELHEIJ, P. P. J., HARTMAN, R. D., HASEYAMA, T., KEITH, C. D., KNUDSON, J. N., KOMIVES, A., LEUSCHNER, M., LOWIE, L. Y., MASAIKE, A., MATSUDA, Y., MITCHELL, G. E., PENTTILA, S., POSTMA, H., RICH, D., ROBERSON, N. R., SEESTROM, S. J., SHARAPOV, E. I., STEPHENSON, S. L., YEN, Y.-F., and YUAN, V. W., "Parity Violation in Neutron Transmission Through Xenon," In Proceedings of the Fourth International Seminar on Neutron-Nucleus Interactions (JINR, 1996) 113
SZYMANSKI, J. J., SNOW, W. M., BOWMAN, J. D., CAIN, B., CRAWFORD, B. E., DELHEIJ, P. P. J., HARTMAN, R. D., KEITH, C. D., KNUDSON, J. N., KOMIVES, A., LEUSCHNER, M., LOWIE, L. Y., MASAIKE, A., MATSUDA, Y., MITCHELL, G. E., PENTTILA, S., POSTMA, H., RICH, D., ROBERSON, N. R., SEESTROM, S. J., SHARAPOV, E. I., STEPHENSON, S. L., YEN, Y.-F., and YUAN, V. W., "Observation of a Large Parity NonConserving Effect in Xe," Physical Review C 53, R2576-2580 (1996)

TADDEUCCI, T. N., ULLMANN, J., RYBARCYK, L. J., BUTLER, G. W., and WARD, T. E., "Total Cross Sections for Production of ${ }^{7} \mathrm{Be},{ }^{22} \mathrm{Na},{ }^{24} \mathrm{Na}$ in $\mathrm{p}+{ }^{7} \mathrm{Li}$ and $\mathrm{p}+{ }^{27} \mathrm{Al}$ Reactions at 495 and 795 MeV," Physical Review C 55, 155 I-1554 (1997)

TAYLOE, R., "Evidence for Neutrino Oscillations from Muon Decay at Rest," In Proceedings of the 14th International Conference on Particles and Nuclei, 581-582 (1996)

THOMPSON, J. R., "Mercury Cuprate High-T ${ }_{c}$ Superconductors: What Do Their Magnetic Properties Reveal?" Studies of High Temperature Superconductors 26 (1998)

THOMPSON, J. R., CHRISTEN, D. K., PARANTHAMAN, M., KRUSIN-ELBAUM, L., MARWICK, A. D., CIVALE, L., WHEELER, R., OSSANDON, J. G., LISOWSKI, P., and ULLMANN, J., "The Impact of Tailored Defects on Length Scales and Current Conduction in High- $\mathrm{T}_{\mathrm{c}}$ Superconductors," In Proceedings of the Ist Polish-US Conference on Recent Developments in High Temperature Superconductivity (SpringerVerlag, 1996) 321-335

THOMPSON, J. R., KRUSIN-ELBAUM, L., KIM, Y. C., CHRISTEN, D. K., MARWICK, A. D., WHEELER, R., LI, C., PATEL, S., SHAW, D. T., LISOWSKI, P., and ULLMANN, J., "Persistent Current Density and Flux Creep in Bi-Sr-Ca-Cu-O/Ag Tapes with Splayed Columnar Defects from $0.8 \mathrm{GeV}$ Proton Irradiation," IEEE Transactions on Applied Superconductivity 5 , 1876-1879 (1995)

THOMPSON, J. R., KRUSIN-ELBAUM, L., CHRISTEN, D. K., SONG, K. J., PARANTHAMAN, M., ULLMANN, J. L., WU, J. Z., REN, Z. F., WANG, J. H., TKACZYK, J. E., and DELUCA, J. A., "Current Density Enhancements of the Highest-Tc Superconductors with GeV Protons," Applied Physics Letters 71, 536 (1997)

TOMSOVIC, S., "Foundations of Statistical Spectroscopy," In Proceedings of the Parity and Time Reversal Vilation in Compound Nuclear States and Related Topics, Auerbach, N., and Bowman, J. D., Eds. (World Scientific, 1996) 29

TOMSOVIC, S., "Implementing Statistical Spectroscopy," In Proceedings of the Parity and Time Reversal Vilation in Compound Nuclear States and Related Topics, Auerbach, N., and Bowman, J. D., Eds. (World Scientific, 1996) 263 
TORNOW, W., CARMAN, T., CHEN, Q., GIBBS, W., GIBSON, B. F., GONZALES TROTTER, D. E., HOWELL, C. R., HUSSEIN, A. H., MERTENS, G., MOORE, C., MORRIS, C., OBST, A. W., PASYUK, E. A., ROPER, C. D., SALINAS, F., SCHMIDT, D., SETZE, H. R., SLAUS, I., STERBENZ, S. M., TANG, H., WALTER, R. L., WHITELEY, C. R., WITALA, H., and ZHOU, Z., "Scattering Length Measurements from Radiative Pion Capture and Neutron-Deuteron Breakup," Nuclear Physics A 631, 421c-425c (1998)

TOSAKA, Y., KANATA, H., ITAKURA, T., SUZUKI, K., and SATOH, S., "Simplified Simulator for Neutron-Induced Soft Errors Based on Modified BGR Model," In Proceedings of the 1998 International Conference on Simulation of Semiconductor Processes and Devices, Technical Digest 117 (1998)

TOSAKA, Y., SATOH, S., and ITAKURA, T., "Neutron Induced Soft Error Simulator and Accurate Predictions," In Proceedings of the International Conference on Simulation of Semiconductor Processes and Devices, Technical Digest 253 (1997)

TOSAKA, Y., SATOH, S., ITAKURA, T., EHARA, H., UEDA, T., WOFFINDEN, G. A., and WENDER, S., "Measurements and Analysis of Neutron-Induced Soft Errors in Sub-Half Micron CMOS Circuits," IEEE Trans. Electron. Dev. 45, 7, 1453-1458 (1998)

TOSAKA, Y., SATOH, S., ITAKURA, T., SUZUKI, K., SUGII, T., EHARA, H., and WOFFINDEN, G. A., "Cosmic Ray Neutron Induced Soft Errors in Sub-Half Micron CMOS Circuits," IEEE Electron Dev. Lett. 18, 99 (1997)

TOSAKA, Y., SATOH, S., SUZUKI, K., SUGII, T., EHARA, H., WOFFINDEN, G. A., and WENDER, S. A., "Impact of Cosmic Ray Neutron Induced Soft Errors in Advanced Submicron CMOS Circuits," Technical Digest 148 (1996)

TOSAKA, Y., SATOH, S., SUZUKI, K., SUGII, T., NAKAYAMA, N., EHARA, H., WOFFINDEN, G. A., and WENDER, S. A., "Measurements and Analysis of NeutronReaction-Induced Charges in Silicon Surface Region," IEEE Trans. Nucl. Sci. A1025, 173 (1997)

VONACH, H., PAVLIK, A., WALLNER, A., DROSG, M., HAIGHT, R. C., DRAKE, D. M., and CHIBA, S., "Spallation Reaction in $27 \mathrm{Al}$ and ${ }^{56} \mathrm{Fe}$ Induced by $800 \mathrm{MeV}$ Protons," In Proceedings of the International Conference on Nuclear Data for Science and Technology, 1431-1433 (1997)

VONACH, H., PAVLIK, A., WALLNER, A., DROSG, M., HAIGHT, R. C., DRAKE, D. M., and CHIBA, S., "Spallation Reactions in ${ }^{27} \mathrm{Al}$ and ${ }^{56} \mathrm{Fe}$ Induced by $800 \mathrm{MeV}$ Protons," Physical Review 55, 2458-2467 (1997)

WANG, Y., YANG, X., RAPAPORT, J., GOODMAN, C. D., DELUCIA, S. L., LUTHER, B. A., MARCHLENSKI, D. G., PARK, B. K., RYBARCYK, L., SMITH, A., SUGARBAKER, E., TADDEUCCI, T., and WANG, L., "Study of the $(1 / 2)_{\text {g.s. }}$ - to $(1 / 2)_{1}{ }^{+}$Transition in the ${ }^{13} \mathrm{C}(\mathrm{p}, \mathrm{n})^{13} \mathrm{~N}$ Reaction at Intermediate Energies," Physical Review C 51, 1345 (1995)
WENDER, S. A., VENNERI, F., BOWMAN, C. D., ARTHUR, E. D., HEIGHWAY, E., BEARD, C. A., BRACHT, R. R., BUKSA, J. J., CHAVEZ, W., DEVOLDER, B. G., PARK, J. J., PARKER, R. B., PILLAI, C., PITCHER, E., POTTER, R. C., REID, R. S., RUSSELL, G. J., TRUJILLO, D. A., WEINACHT, D. J., WILSON, W. B., and WOLOSHUN, K. A, "A Small Scale Accelerator Driven Subcritical Assembly Development and Demonstration Experiment at LAMPF," In Proceedings of the International Conference on Accelerator-Driven Transmutation Technologies and Applications (AIP, 1996) 346, 663-672

WHITE, D. H., "LSND Neutrino Oscillations Results," In Proceedings of the 28th International Conference on High Energy Physics, 1274-1277 (1996)

WHITE, D. H., "LSND Neutrino Oscillations Results," In Proceedings of the XXXII Rencontres de Moriond: Electroweak Interactions and Unified Theories, 275-282 (1997)

WILBURN, W. S., BOWMAN, J. D., PENTTILA, S. I., YUAN, V., LEUSCHNER, M. B., POMEROY, V. R., RICH, D. R., SNOW, W. M., and SHARAPOV, E. I., "Low-Noise Photocathode Preamplifiers for Measuring the Gamma Asymmetry in Neutron Capture on Protons," Bull. Am. Phys. Soc. 42, 1667 (1997)

WILBURN, W. S., DRAKE, D. M., JOHNS, G. D., NELSON, R. O., ARCHER, D. E., BECKER, J. A., BERNSTEIN, L. A., YOUNES, W., MITCHELL, G. E., ROBERSON, N. R., and SHRINER, J. R., Jr., "Level Structure Studies with the GEANIE Detector," Bull. Am. Phys. Soc. 42, 1073 (1997)

WILLIS, J. O., SAFAR, H., CHO, J. H., COULTER, J. Y., MALEY, M. P., SMITH, P. A., PHILLIPS, D. S., ULLMANN, J. L., RILEY, G. N., RUPICH, M. W., and FLESHLER, S., "High Energy Proton Irradiation Induced Pinning Centers in Bi-2212 and Bi-2223 Superconductors," In Advances in Superconductivity VIII, Hayakawa, H., and Enomoto, Y., Eds. (Springer-Verlag, 1996) 509

YANG, X., WANG, L., RAPAPORT, J., GOODMAN, C. D., FOSTER, C. C., WANG, Y., SUGARBAKER, E., MARCHLENSKI, D., DE LUCIA, S., LUTHER, B., RYBARCYK, L., TADDEUCCI, T. N., and PARK, B. K., "Dipole Excitations in P-Shell Nuclei Via (p,n) Reactions," Phys. Rev. C 52, 2534 (1995)

YEN, Y.-F., BOWMAN, J. D., CRAWFORD, B. E., DELHEIJ, P. P. J., FRANKLE, C. M., FUKUDA, K., GOULD, C. R., GREEN, A. A., HAASE, D. G., IINUMA, M., KNUDSON, J. N., LOWIE, L. Y., MASAIKE, A., MASUDA, Y., MATSUDA, Y., MITCHELL, G. E., PENTTILA, S., POSTMA, H., ROBERSON, N. R., SEESTROM, S. J., SHARAPOV, E. I., SHIMIZU, H. M., STEPHENSON, S. L., and YUAN, V. W., "Study of Parity and Time-Reversal Violation in Neutron-Nucleus Interactions," In Proceedings of the 8th International Conference on Polarization Phenomena in Nuclear Physics, Stephenson, E. J., and Vigdor, S. E., Eds. (AIP, 1995) 339, 120-125 
YEN, Y.-F., BOWMAN, J. D., LOWIE, L. Y., MASUDA, Y., PENTTILA, S. I., SEESTROM, S. J., and YOO, S. H., "Studies of Gamma Background in a Pulsed Epithermal Neutron Beam and the Neutron-Boron Capture in a 10B-Loaded Hydrogenous Scintillator," In Proceedings of the First International Workshop on Accelerator-Based Neutron Sources for Boron Neutron Capture Therapy (INEL, 1995) 351

YEN, Y.-F., BOWMAN, J. D., LOWIE, L. Y., MITCHELL, G. E., MASUDA, Y., and PENTTILA, S., "Determination of the Gamma-Ray Background in a Pulsed Epithermal Neutron Beam," Nuclear Instruments \& Methods in Physical Research A 397, 365370 (1997)

YODH, G. B. and CHEN, M-L., "Mass Resolution of GroundBased Air Shower Experiments in the 10 to 10000 TeV Range," In Proceedings of the International Cosmic-Ray Conference 1, 903 (1995)

YOUNES, W., ARCHER, D. E., BECKER, J. A., BERNSTEIN, L. A., YATES, S. W., JOHNS, G. D., and NELSON, R. O., "Search for Multiphonon Structures in ${ }^{170} \mathrm{Er}$, , Bull. Am. Phys. Soc. 42, 1687 (1997)

YUAN, V. W., BOLME, G. O., ERICKSON, J. L., JOHNSON, K. F., MOTTERSHEAD, T., SANDER, O. R., and SMITH, M. T., "Simulation and Steering in the Intertank Matching Section of the Ground Test Accelerator," In Proceedings of the Beam Instrumentation Workshop (AIP, 1995) 333, 536-42

ZHAO, X. M., GULLEY, M. S., BRYANT, H. C., STRAUSS, C. E. M., FUNK, D. J., STINTZ, A., RISLOVE, D. C., KYRALA, G. A., INGALLS, W. B., and MILLER, W. A., "Nonresonant Excess Photon Detachment of Negative Hydrogen Ions," Physical Review Letters 78, 1656 (1997)

\section{Other}

BULL, J. S., ROMERO, V. D., BAKER, S. I., STAPLETON, G. B., GOSS, D. L., and COULSON, L. V., "Groundwater Activation at the Superconducting Supercollider: A New Design Model," Health Physics 73, 5, 800-807 (1997)

PAUL, J. D., EARLEY, L. M., ROHLEV, L. E., GOFORTH, J. H., and HALL, C. R., "Optically Powered Firing System for the Procyon High Explosive Pulse Power System," In Proceedings of the Optical Network Engineering and Integrity Conference 2611, 138-145 (1996)

SHAFER, R. E., “Cricket Research,” Science 268, 13-14 (1995)

THUOT, M. E., CLAUSEN, M., DALESIO, L. R., KATOH, T., KRAIMER, M. E., MUELLER, R., SHOAEE, H., WATSON, W. A., HILL, C., VRETENAR, M., "The Success and the Future of Epics," In Proceedings of the XVII International Linac Conference (CERN, 1996) 2, 611-615

\section{Theses}

AYD, S. W., "Phospholipid/Bile Salt Mixed Micelles and Their Use in Predictive Models of Solubility Improvement," University of Illinois, USA (1997)

BLESS, R., "Construction and Test of a Triggerable Uniaxial Stress Rig for Neutron Transmission Diffraction Applications," University of Kiel, Germany (1996)

BROWN, L. A., "Redox Rearrangement and Molecular Intercalation Reactions of Titanium and Zirconium Disulfide," Arizona State University, USA (May 1997)

CHOO, H., "Synthesis, Processing, and Characterization of NiAlAIN-Al ${ }_{2} \mathrm{O}_{3}$ Composites," Illinois Institute of Technology, USA (May 1998)

CLAUSEN, B., "Characterization of Polycrystal Deformation by Numerical Modeling and Neutron Diffraction Measurements," Riso National Laboratory, Denmark (1997)

ESPY, M. A., "Asymmetries for Pion Elastic Scattering from Polarized ${ }^{3} \mathrm{He}$ in the Energy Region of the $[3,3]$ Resonanc," University of Minnesota, USA (July 1996)

GAULARD, C. V., "Analyzing Powers for the $\pi^{-} p \rightarrow \pi^{\circ} \mathrm{n}$ Reaction Across the $\Delta(1232)$ Resonance," Arizona State University, USA (November 1997)

GERSTENBERG, M. C., "A Neutron Scattering Study of Triblock Copolymer Micelles," University of Copenhagen, Denmark (November 1997)

GULLEY, M. S., "Non-resonant Two photon Detachment of $\mathrm{H}^{-}$ Ions with $1.165 \mathrm{eV}$ Photons," University of New Mexico, USA (August 1997)

JARMER, J. J., "Effects of Engineering Controls on Radioactive Air Emissions from the Los Alamos Neutron Science Center," Masters Thesis. Texas A\&M University, USA (1996)

KEILMAN, M., "Pion Single Charge Exchange on Deuterium at Low Energies," University of Colorado, USA (1998)

KRISS, B. J., "Pion-Proton Integral Cross Sections," University of Colorado, USA (1997)

LIU, W., "High Precision Measurement of Muonium Ground State Hyperfine Interval and Muon Magnetic Moment," Yale University, USA (April 1997)

LUND, C., "A Study of Deformation in Metal-Matrix Composites at Room- and Elevated Temperature," Masters Thesis, Massachusetts Institute of Technology, USA (February 1997)

MALDONADO, J. G., "Corrosion Behavior and Mechanical Properties of Liquid-Nitrogen-Deformed 304 Stainless Steel," University of Texas at El Paso, USA (December 1997) 
MAYO, D. R., "Neutron-Proton Bremsstrahlung Inclusive Photon Measurements from 100 to $280 \mathrm{MeV}$," University of California at Davis, USA (June 1997)

MEGGERS, K., "Real-Time Neutron Transmission Investigation of the Kinematics of the Austenite-Bainite Phase Transformation in Cast Iron," University of Kiel, Germany (1995)

PURWANTO, A., "Magnetic Neutron Diffraction on Uranium Intermetallics," New Mexico State University, USA (December 1995)

RUSSELL, S. J., "Measuring the Transverse RMS Emittance and RMS Pulse Length of a Short Pulse, Photoinjector Produced Electron Beam with the Second Moment of its Image Charge," Michigan State University, USA (1998)

STALDER, M., "Neutron Transmission Investigations on Steel and Temperature Measurements Based on Resonance Doping," University of Kiel, Germany (1996)

STEPHENSON, S. L., "Parity Violation in 232Th: A Study of the 'Sign Effect'," North Carolina State University, USA (December 1996)

VAIDYANATHAN, R., "Mechanical Properties of Shape Memory and Superelastic $\mathrm{NiTi}$ and $\mathrm{NiTi}-\mathrm{Ti}_{\mathrm{c}}$ Composite Studied by Neutron Diffraction," Massachusetts Institute of Technology, USA (1998)

VOGEL, S., "Single-Shot Neutron Transmission Diffractometry Using a Transient Recorder," University of Kiel, Germany (1996) 